ENGINEERING DATA TRANSMITTAL I

\begin{tabular}{|l|l|}
\hline $\begin{array}{l}\text { 2. To: (Receiving organization) } \\
\text { Distribution }\end{array}$ & $\begin{array}{l}\text { 3. From: Coriginating Organization) } \\
\text { Restoration and Upgrade } \\
\text { Programs }\end{array}$ \\
\hline $\begin{array}{l}\text { 5. Proj.fProg./Dept./Div.: } \\
\text { W-242 Thermal Treatment }\end{array}$ & $\begin{array}{l}\text { 6. Design Authority/ Design Agent/Cog. } \\
\text { Engr.: B. G. Place }\end{array}$ \\
\hline $\begin{array}{l}\text { 8. originator Remarks: } \\
\text { The existing thermaliy treatable radioactive mixed waste } \\
\text { inventory is ident ified and characterized to support the } \\
\text { solid waste thermal treatment activity. }\end{array}$ \\
\hline
\end{tabular}

11, Receiver Remarks: 11A. Design Baseline Document? [] Yes [X] No none
4. Related EDT NO.:
\[ -10488-\lim _{9 / 24 / 96 d} \]

7. Purchase Order No.:

MW6-SBV-357079

9. Equip./Component No.:

$\mathrm{n} / \mathrm{a}$

10. System/3ldg./Facility:

$\mathrm{n} / \mathrm{a}$

12. Major Assm. Dwg. No.:

$n / a$

13. Permit/Permit Application No.: $n / a$

14. Required Response Date:

$\mathrm{n} / \mathrm{a}$

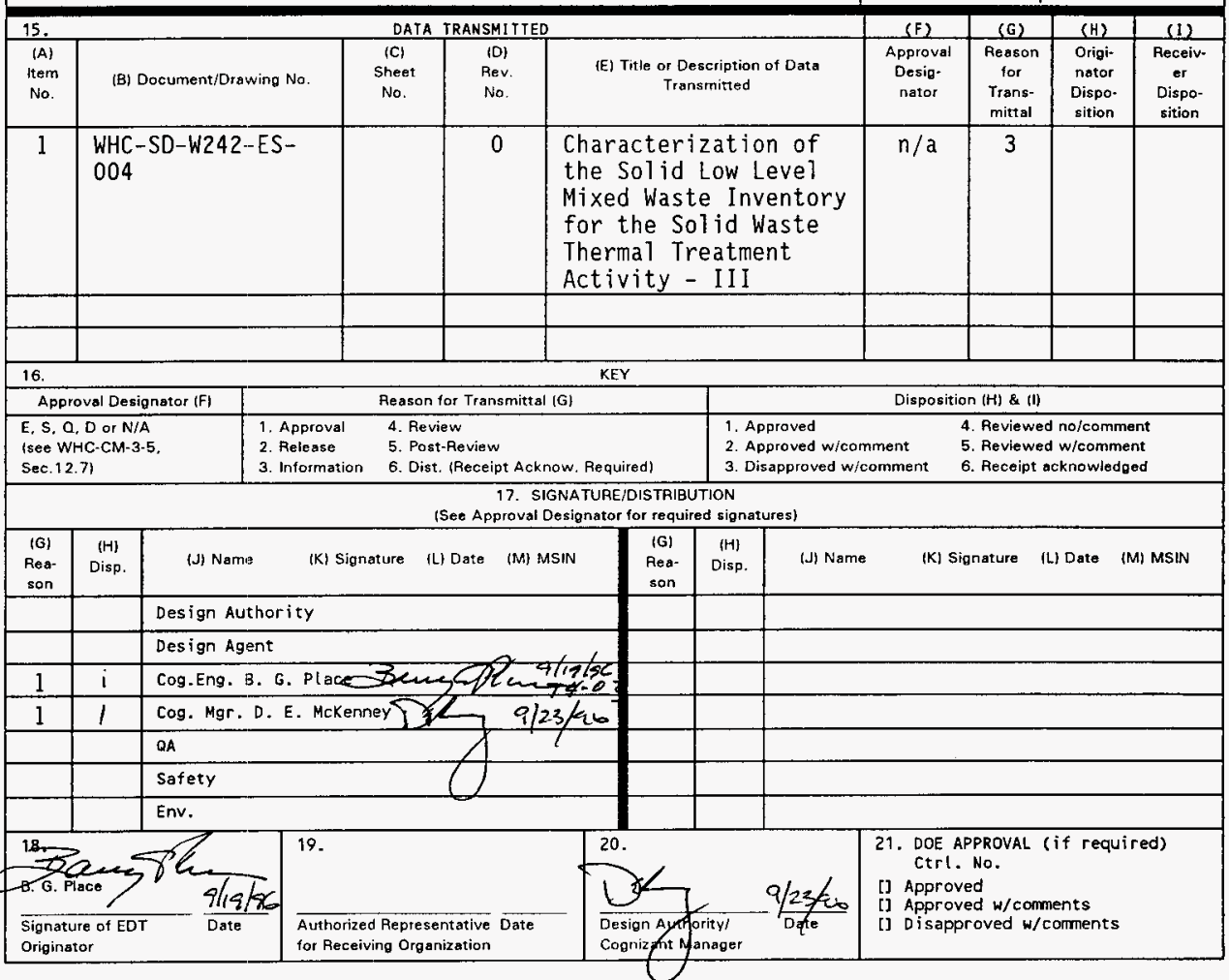


WHC-SD-W242-ES-004, Rev. 0

poge lof 2 (Coversheet)

\section{Characterization of the Solid Low Level Mixed Waste Inventory for the Solid Waste Thermal Treatment Activity - III}

Barry G. Place

Westinghouse Hanford Co. Richland, WA 99352

U.S. Department of Energy Contract DE-AC06-87RL10930

chase Lola34!

EDT/ECN: 610488

Org Code: 87330

B\&R Code: EW3130020

UC: 2070

Charge Code: A1074

Total Pages: $200167 \mathrm{kms} 9 / 24 / 96$

Key words: Thermal treatment, solid waste, incineration, radioactive mixed waste, low-1evel mixed waste, LLMW. MLLW. W-242, waste characterization, commercial thermal treatment, thermal treatment contract, statistical analysis, hazardous materials content, radionuclides content, radioactive isotopes, physical properties content, generators, container types, RCRA Timits, TSCA Timits, PCBs, dangerous waste codes, performance assessment, mobile isotopes, dose equivalent curies content, tritium content, fissile isotope content, Hanford Site Solid Waste Acceptance Criteria, DOT limits.

Abstract: The existing thermally treatable, radioactive mixed waste inventory is characterized to support implementation of the commercial, thermal treatment contract. The existing thermally treatable waste

TRADEMARK DISCLAIMER. Reference herein to any specific commercial product, process, or service by trade name, trademark, manufacturer, or otherwise, does not necessarily constitute or imply its endorsement, recommendation, or favoring by the United States Government or any agency thereof or its contractors or subcontractors.

Printed in the United States of America. To obtain copies of this document, contact: WHC/BCS Document Control Services, P.0. Box 1970, Mailstop H6-08, Richland WA 99352, Phone (509) 372-2420, Fax (509) 376-4989.
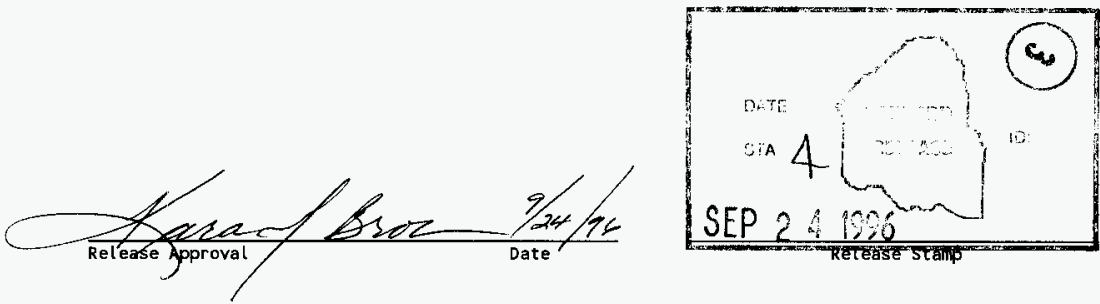

Approved for Public Release 


$$
\begin{aligned}
& \text { WHC-SO-WHAD-ES-OO4, RCV. } \\
& \text { Page 2of2 (Cover shest) }
\end{aligned}
$$

inventory has been identified using a decision matrix developed by Josephson et al. (1996). Similar to earlier waste characterization reports (Place 1993 and 1994), hazardous materials, radionuclides, physical properties, and waste container data are statistically analyzed. In addition, the waste inventory data is analyzed to correlate waste constituent data that are important to the implementation of the commercial thermal treatment contract for obtaining permits and for process design. The specific waste parameters, which were analyzed, include the following: "dose equivalent" curie content, polychlorinated bipheny? (PCB) content, identification of containers with PA-related mobile radionuclides $\left({ }^{14} \mathrm{C}\right.$, ${ }^{129} \mathrm{I},{ }^{79} \mathrm{Se},{ }^{99} \mathrm{TC}$, and $\mathrm{U}$ isotopes), tritium content, debris and non-debris content, container free liquid content, fissile isotope content, identification of dangerous waste codes, asbestos containers, high mercury containers, beryllium dust containers, lead containers, overall waste quantities, analysis of container types, and an estimate of the waste compositional split based on the thermal treatment contractor's proposed process. A qualitative description of the thermally treatable mixed waste inventory is also provided.

IRADEMARK DISCLAIMER. Reference herein to any specific comercial product, process, or service by trade name, trademark, manufacturer, or otherwise, does not necessarily constitute or imply its endorsement, recommendation, or favoring by the United States Government or any agency thereof or its contractors or subcontractors.

Printed in the United States of America. To obtain copies of this document, contact: WHC/BCS Document Control Services, P.O. Box 1970, Mailstop H6-08, Richland WA 99352, Phone (509) 372-2420; Fax (509) 376-4989.
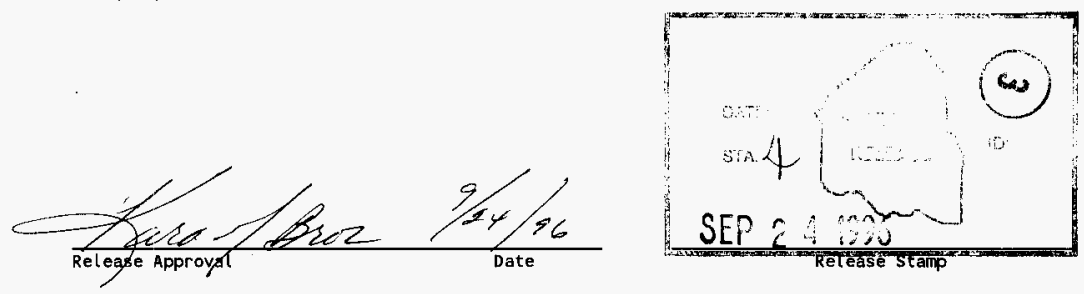

\section{Approved for Public Release}


WHC-SD-W-242-ES-004, Rev. 0

\section{EXECUTIVE SUMMARY}

The existing thermally treatable, radioactive mixed waste inventory is characterized to support implementation of the commercial, thermal treatment contract. The existing thermally treatable waste inventory has been identified using a decision matrix developed by Josephson et al. (1996). Similar to earlier waste characterization reports (Place 1993 and 1994), hazardous materials, radionuclides, physical properties, and waste container data are statistically analyzed. In addition, the waste inventory data is analyzed to correlate waste constituent data that are important to the implementation of the commercial thermal treatment contract for obtaining permits and for process design. The specific waste parameters, which were analyzed, include the following: "dose equivalent" curie content, polychlorinated biphenyl (PCB) content, identification of containers with PA-related mobile radionuclides $\left({ }^{14} \mathrm{C},{ }^{129} \mathrm{I},{ }^{79} \mathrm{Se},{ }^{99} \mathrm{Tc}\right.$, and $\mathrm{U}$ isotopes $)$, tritium content, debris and non-debris content, container free liquid content, fissile isotope content, identification of dangerous waste codes, asbestos containers, high mercury containers, beryllium dust containers, lead containers, overall waste quantities, analysis of container types, and an estimate of the waste compositional split based on the thermal treatment contractor's proposed process. A qualitative description of the thermally treatable mixed waste inventory is also provided. 
WHC-SD-W-242-ES-004, Rev. 0

\section{CONTENTS}

1.0 INTRODUCTION $\ldots \ldots \ldots \ldots \ldots \ldots \ldots \ldots \ldots \ldots \ldots \ldots \ldots$

2.0 OBJECTIVE ...................... 1

2.1 IDENTIFICATION OF THE THERMALLY TREATABLE

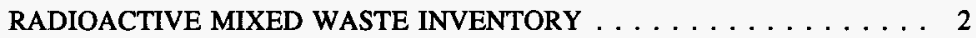

3.0 ANALYSIS APPROACH $\ldots \ldots \ldots \ldots \ldots \ldots \ldots \ldots \ldots \ldots \ldots \ldots$

3.1 GLOBAL QUERYING AND SORTING OF

THE HAZARDOUS MATERIALS . . . . . . . . . . . . . 4

3.2 GLOBAL QUERYING AND SORTING OF THE RADIONUCLIDES . . . . 4

3.3 GLOBAL QUERYING AND SORTING OF THE PHYSICAL

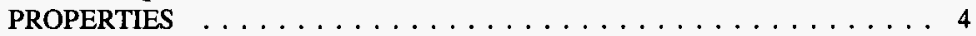

3.4 ANALYSIS OF "DOSE EQUIVALENT" CURIE CONTENT . . . . . . . 5

3.5 ANALYSIS OF POLYCHLORINATED BIPHENYL CONTAINER

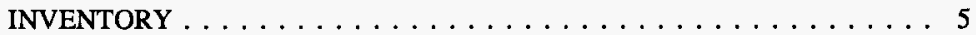

3.6 ANALYSIS OF COMPOSITIONAL DATA RELATED TO PERFORMANCE ASSESSMENT REQUIREMENTS $\ldots \ldots \ldots \ldots \ldots$

3.7 ANALYSIS OF TRITIATED WASTE CONTAINERS $\ldots \ldots \ldots \ldots \ldots 7$

3.8 ANALYSIS OF DEBRIS WASTE IN THE THERMALLY TREATABLE INVENTORY $\ldots \ldots \ldots \ldots \ldots \ldots$

3.9 ANALYSIS OF FREE LIQUID CONTENT $\ldots \ldots \ldots \ldots \ldots \ldots$

3.10 ANALYSIS OF FISSILE WASTE COMPOSITION $\ldots \ldots \ldots \ldots \ldots$

3.11 IDENTIFICATION OF LOW ACTIVITY CONTAINERS . . . . . . . . . . 9

3.12 IDENTIFICATION OF UNIQUE DANGEROUS WASTE CODES . . . . 10

3.13 IDENTIFICATION OF ASBESTOS, HIGH MERCURY, AND BERYLLIUM DUST CONTAINERS $\ldots \ldots \ldots \ldots \ldots \ldots$

3.14 IDENTIFICATION OF LEAD CONTAINERS . . . . . . . . . . . . 10

3.15 GLOBAL QUERYING AND SORTING OF WASTE QUANTITIES . . . . 10

3.16 ANALYSIS OF CONTAINER TYPES $\ldots \ldots \ldots \ldots \ldots \ldots \ldots \ldots$

4.0 SIMULATED APPLICATION OF THE COMMERCIAL THERMAL

TREATMENT PROCESS TO THE EXISTING THERMALLY TREATABLE WASTE INVENTORY $\ldots \ldots \ldots \ldots \ldots \ldots \ldots \ldots \ldots \ldots \ldots \ldots \ldots \ldots$

5.0 FORMULATION OF A SEMI-QUANTITATIVE DESCRIPTION OF THE THERMALLY TREATABLE MIXED WASTE INVENTORY $\ldots \ldots \ldots \ldots 12$

6.0 DISCUSSION OF WASTE FORECAST DATA $\ldots \ldots \ldots \ldots \ldots$

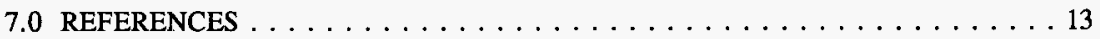

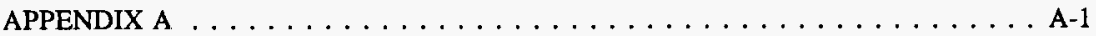


WHC-SD-W-242-ES-004, Rev. 0

\section{LIST OF TERMS}

$\begin{array}{ll}\text { DOT } & \text { U.S. Department of Transportation } \\ \text { FY } & \text { Fiscal Year } \\ \text { LDR } & \text { Land Disposal Restricted } \\ \text { PA } & \text { Performance Assessment } \\ \text { PCB } & \text { polychlorinated biphenyl } \\ \text { PIN } & \text { Package Identification Number } \\ \text { SWITS } & \text { Solid Waste Information Tracking System }\end{array}$


WHC-SD-W-242-ES-004, Rev. 0

This page intentionally left blank. 


\subsection{INTRODUCTION}

Early in Fiscal Year (FY) 1996, a commercial contract was awarded for the thermal treatment of Hanford Site radioactive mixed waste (WHC 1995). The contract award was the final step in a procurement process that spanned approximately four years. At the beginning of the procurement process, a comprehensive data file of the then-existing lowlevel mixed waste inventory stored in Central Waste Complex was compiled and collated by querying the Solid Waste Information Tracking System (SWITS) database. This data file was the basis for all issues related to waste characterization during the procurement process. Although "representative" was used frequently to describe the data during negotiations with contractors, it was emphasized that future waste could differ significantly in composition. Thus, the procurement scope is based on a waste inventory database compiled in FY 1992 (WHC 1994).

Waste characterization reports analyzing the SWITS database were issued in FY 1993 and FY 1994 (Place 1993 and 1994). These reports analyze the thermally treatable, radioactive, mixed waste inventory using the SWITS database and waste projection data. The reports were used to support internal Westinghouse Hanford Company activities. They could not be referenced formally as part of the thermal treatment procurement process. Cursory comparison of the results of the reports with the FY 1992 procurement basis data indicate compositional consistency. However, no detailed comparison has been performed.

\subsection{OBJECTIVE}

A primary objective of this report is to provide characterization information that supports the implementation of the thermal treatment contract (WHC 1995). To accomplish this objective, data analyses were performed that are relevant to thermal treatment contractor needs. On a number of occasions during the procurement process, waste characterization data was required and referenced. Usually it was required that the data be queried into a format to answer specific questions about the waste. For example, it was required that all container types be identified and that containers with specific contaminants, such as PCBs and asbestos, also be identified. The FY 1992 procurement basis data was queried for answers to these questions. These questions have been compiled, and it is a report objective to answer these questions using a more recent database. 


\subsection{IDENTIFICATION OF THE THERMALLY TREATABLE RADIOACTIVE MIXED WASTE INVENTORY}

Previous characterizations of existing and projected radioactive mixed waste inventories used the same criterion to identify the thermally treatable mixed waste. Generally, waste containers containing organic constituents were included in the thermally treatable inventory. A recently conducted value engineering seminar produced a decision matrix to identify the thermally treatable, radioactive mixed waste (Josephson et al. 1996). This decision matrix has been used in this report to identify the thermally treatable, radioactive mixed waste inventory. The SWITS data analyzed in this report was downloaded in January 1995 to a format amenable to initial screening using Paradox ${ }^{1}$. It is the most recent database available for the type of analysis in this report. More recent abbreviated downloads of the SWITS data were compared to the subject data for consistency. Such comparisons exhibited satisfactory consistency in constituent distribution for the three compositional variables considered.

The decision matrix developed by Josephson et al. (1996) was used to identify the thermally treatable, radioactive mixed waste inventory. To implement the decision matrix, the parameters identified in the decision matrix were correlated with the SWITS data. Similar to previous characterization reports, the SWITS data was collated into the following data groups: hazardous materials, radionuclides, physical properties, and general container information. The criteria for determining thermal treatability included (1) the presence of a sufficient quantity (greater than or equal to $1.0 \mathrm{~kg}$ ) of organic or combustible constituents including organic constituents identified in the hazardous material database and combustible hazardous constituents identified in the physical properties database, (2) the presence of PCBs in any quantity and identified in the hazardous material or the physical properties databases, and (3) the presence of a sufficient quantity (greater than 50 volume percent) of combustible debris. Using these criteria as a basis, the SWITS data base was queried, and 2,815 containers were identified as being considered thermally treatable. The waste categories and the number of containers are identified below.

$\begin{array}{lc}\text { Waste Category } & \begin{array}{c}\text { Number of Containers } \\ \text { Identified }\end{array} \\ \text { Hazardous Materials } & 2,788 \\ \text { Radionuclides } & 2,815 \\ \text { Physical Properties } & 2,815 \\ \text { Container Data }^{2} & 2,762\end{array}$

'Paradox is a trademark of Borland International, Inc., Scotts Valley, California.

${ }^{2}$ Container data consist of container volume and weight and generator identification. 
Only containers with complete and consistent data sets for a given waste category were analyzed. The differences between the number of containers included in a given waste category can be attributed to inconsistencies or unreported data. The analyses in this report emphasize compositional variations that are statistically significant. The deletion of a small percent of inconsistent data will have a negligible effect on the results.

Tables 1, 2, and 3 list the constituents for the three waste categories: hazardous materials, radionuclides, and physical properties, respectively. These tables include classification data for each waste category. Table 1 identifies 719 hazardous materials, Table 2 identifies 99 radionuclides, and Table 3 identifies 118 physical properties.

\subsection{ANALYSIS APPROACH}

Similar to the analyses conducted in Place (1993 and 1994), the data were analyzed using Paradox" and $\mathrm{Excel}^{3}$ database management systems. For the waste parameters, hazardous materials, radionuclides, and physical properties, global analyses were performed which correlate accumulative and statistical quantities to identify quantitatively significant contaminants. Using querying and sorting algorithms provided in the above programs, the SWITS data were correlated in the following ways:

1. The data were correlated in ascending order based on the container count for a given constituent.

2. The data were correlated in ascending order based on the total accumulated weight of a given constituent.

3. The data were correlated in ascending order based on the maximum weight of a given constituent reported in an individual container.

The above correlation schemes were selected because they were thought to anticipate the permit and process design needs of the thermal treatment contractor.

${ }^{3}$ Excel is a trademark of the Microsoft Corporation, Redmond, Washington. 


\subsection{GLOBAL QUERYING AND SORTING OF THE HAZARDOUS MATERIALS}

Table 4 shows the results of a statistical analysis of uniquely-named ${ }^{4}$ hazardous materials in ascending order based on container count. It includes the following information: the complete reported name of each hazardous material, container count, accumulated weight, average weight, standard deviation, minimum reported weight, and maximum reported weight. Tables 5 and 6 show the same statistical data identified in Table 4 sorted in ascending order based on the total accumulated quantity and on the maximum quantity, respectively. The analyses in Tables 4,5 , and 6 identify the statistically significant hazardous materials by sorting the data in ascending order based on a given parameter.

\subsection{GLOBAL QUERYING AND SORTING OF THE RADIONUCLIDES}

Table 7 shows the results of a statistical analysis of uniquely named alpha bearing radionuclides in ascending order based on container count. The parameters in Table 7 include the following: container count, accumulated weight, average weight, standard deviation, minimum reported weight, and maximum reported weight. Tables 8 and 9 show the same statistical data, identified in Table 7, sorted in ascending order based on total accumulated weight and on maximum weight, respectively.

Table 10 shows the results of a statistical analysis of uniquely-named fission products in ascending order based on container count. Fission products are reported in units of curies. The parameters in Table 10 include the following: container count, accumulated activity, average activity, standard deviation, minimum reported activity, and maximum reported activity. Tables 11 and 12 show the same statistical data, identified in Table 10, sorted in ascending order based on the total accumulated activity and on the maximum activity, respectively.

\subsection{GLOBAL QUERYING AND SORTING OF THE PHYSICAL PROPERTIES}

Table 13 shows the results of a statistical analysis of uniquely-named physical properties in ascending order based on container count, and it shows the complete reported name of each physical property. The parameters in Table 13 include the following: container count, accumulated weight, average weight, standard deviation, minimum reported weight, and maximum reported weight. Tables 14 and 15 show the same statistical data, identified in Table 13, sorted in ascending order based on the total accumulated quantity and on the maximum quantity, respectively.

\footnotetext{
${ }^{4}$ A computer sorting algorithm was used to identify uniquely named hazardous materials, radionuclides, and physical properties. This algorithm considers all character differences that are unique, including trivial differences such as spaces and hyphens. Therefore, manual inspection of the hazardous material list will always be required to avoid redundancy in component naming.
} 


\subsection{ANALYSIS OF "DOSE EQUIVALENT" CURIE CONTENT}

To assess the probable radiation dose for a given waste container storage configuration, Solid Waste Disposal has defined a "dose equivalent" curie for each isotope. WHC (1993) provides conversion tables for the calculation of isotope-specific dose equivalent curie content. The analytical results discussed below assess the dose equivalent curie content for the subject inventory.

Table 16 shows the results of a statistical analysis of all identified radionuclides in ascending order based on container count. Activities are reported in units of "dose equivalent" curies. The parameters in Table 16 include the following: container count, accumulated activity, average activity, standard deviation, minimum activity, and maximum activity.

Tables 17 and 18 show the same statistical data identified in Table 16 sorted in ascending order based on the total accumulated activity and on the maximum activity, respectively.

In addition to the above statistical analyses, the total "dose equivalent" curie content of each container was determined. The isotope-specific contribution for containers with a total "dose equivalent" curie content greater than or equal to 1.0 are shown in Table $19^{5}$.

\subsection{ANALYSIS OF POLYCHLORINATED BIPHENYL CONTAINER INVENTORY}

Table 20 calculates data on all PCB containers identified in the subject inventory. The Package Identification Numbers (PINs) listed in Table 20 have a PCB constituent reported in the hazardous material database or in the physical property database. A total of 743 PCB containers were identified. Table 20 parameters includes the following: PIN, the PCB component description, the component weight, the PCB component count in the individual container inventory, the total number of hazardous material components reported, the total component weight, the total PCB component weight, and the parts per million (ppm, weight basis) of PCB on a calculated component weight basis. Table 20 was sorted in ascending order based on ppm of PCB on a reported waste weight basis. The Toxic Substances Control Act of 1976 regulations regarding PCBs require that waste with greater than $50 \mathrm{ppm}$ be thermally treated. Of the 743 containers identified, 490 containers will require thermal treatment according to the $50 \mathrm{ppm}$ criteria. Sampling will be required on $175 \mathrm{PCB}$ containers because PCBs have been identified, but quantities were not reported.

"The tabulation of the total "dose Equivalent" curies for all the containers in the subject inventory will be provided by the author on request. 


\subsection{ANALYSIS OF COMPOSITIONAL DATA RELATED TO PERFORMANCE ASSESSMENT REQUIREMENTS}

Specific requirements about the performance of the final waste form for immobilizing radionuclides were included in the thermal treatment contract. The performance requirements concern the following mobile radionuclides: ${ }^{14} \mathrm{C},{ }^{129} \mathrm{I},{ }^{79} \mathrm{Se},{ }^{99} \mathrm{Tc}$, and all isotopes of uranium. The performance requirements are related to the concentration of these radionuclides in the final waste form which, for the purposes of the subject analysis, is assumed to consist of all inorganic (noncombustible) physical properties and one weight percent of the organic physical properties. The criteria for performance stated in the thermal treatment contract, which are concentration-specific, are given below.

1. The ash/residue final waste form shall be a stabilized solid, or the treatment process shall produce a stabilized, solid waste form such as a glass or a slag. The stabilized, solid waste form shall exhibit a compressive strength greater than 50 psi.

2. The stabilized, solid waste form shall be packaged in containers having a void volume percent of less than 10 percent.

3. If the ash/residue contains any of the five radionuclides, ${ }^{99} \mathrm{Tc},{ }^{79} \mathrm{Se},{ }^{129} \mathrm{I},{ }^{14} \mathrm{C}$, or uranium and has a total activity concentration for these radionuclides greater than or equal to $0.0001 \mathrm{Ci} / \mathrm{m}^{3}$ and less than or equal to $0.001 \mathrm{Ci} / \mathrm{m}^{3}$, the stabilized waste form shall exhibit a diffusion coefficient less than or equal to $10^{-10} \mathrm{~cm}^{2} / \mathrm{sec}$ when subjected to leach testing in accordance with American Nuclear Society Procedure 16.1 (ANSI 1986).

4. If the ash/residue contains any of the five radionuclides, ${ }^{99} \mathrm{Tc},{ }^{79} \mathrm{Se},{ }^{129} \mathrm{I},{ }^{14} \mathrm{C}$, or uranium and has a total activity concentration for these radionuclides greater than or equal to $0.001 \mathrm{Ci} / \mathrm{m}^{3}$ and less than or equal to $0.01 \mathrm{Ci} / \mathrm{m}^{3}$, the stabilized waste form shall exhibit a diffusion coefficient less than or equal to $10^{-12} \mathrm{~cm}^{2} / \mathrm{sec}$ when subjected to leach testing in accordance with American Nuclear Society Procedure 16.1 (ANSI 1986).

5. If the ash/residue contains uranium in activity concentrations greater that $0.01 \mathrm{Ci} / \mathrm{m}^{3}$, the ash/residue must be encapsulated in a Portland cement-based grout matrix. Any treatment process that produces a glass or a slag final waste form will require administrative controls of the feed composition on an individual container basis to avoid this requirement. Analysis of the waste inventory suggests that only a few containers will require such administrative controls.

Table 21 tabulates the PIN-specific concentration of Performance Assessment- (PA-) related radionuclides in curies per cubic meter on an ash volume basis. The volume of ash for each 
PIN was calculated according to the above physical property criteria. The container count for each concentration range is as follows.

$\begin{array}{cl}\text { PIN Count } & \begin{array}{l}\text { Concentration Range, } \\ \text { Curies per Cubic Meter }\end{array} \\ 85 & \text { greater than } 0.01 \\ 95 & \text { less than } 0.01 \text { and greater than } 0.001 \\ 207 & \text { less than } 0.001 \text { and greater than } 0.0001 \\ 1,049 & \text { less than } 0.0001\end{array}$

A total of 995 containers contain PA-related radionuclides. The final residue from thermal treatment of 387 containers may require leach testing under the performance assessment requirements. Table 22 summarizes the inventories of the PA-related radionuclides.

\subsection{ANALYSIS OF TRITIATED WASTE CONTAINERS}

Tritiated waste is waste containing greater than 0.02 curies of tritium per cubic meter of waste. The disposal acceptance criteria for tritiated waste is in WHC (1993). A total of 571 PINs were reported to contain tritium. Of these, 48 contained tritium in concentrations close to or greater than 0.02 curies per cubic meter and should be considered tritium waste according to WHC (1993). Table 23 shows an analysis of the tritium waste PINs. WHC (1993) also requires case-by-case reporting of containers having greater than 100 curies per cubic meter. The maximum concentration of tritium is approximately 3 curies per cubic meter and therefore case-specific reporting should not be required for the subject inventory.

\subsection{ANALYSIS OF DEBRIS WASTE IN THE THERMALLY TREATABLE INVENTORY}

The identification of hazardous debris waste must be considered in addition to the selection criteria for identifying the subject waste inventory discussed above. Hazardous debris is to be treated by stabilization (Josephson et al. 1996). The Resource Conservation Recovery Act of 1976 (RCRA 1976) defines hazardous debris as waste contaminated with land disposal restricted (LDR) constituents and containing at least 50 volume percent debris constituents. Table 3 indicates the physical properties that would probably be considered debris constituents. The SWITS database reports the constituent volume as a percent of the total waste volume. Cursory examination of the reported component-specific volumetric data indicates that much of it may be in error. The probable volume is estimated by calculation using a surrogate density. Comparing the reported volume to the calculated volume reveals many discrepancies in the determined volume. As a measure of how significant these discrepancies are, Table 24 compares the reported component volume percent to the 
calculated component volume percent. The data in Table 24 is sorted in ascending order based on the difference between these two values of determined volume. Table 24 is an abridged listing including only containers for which the difference between the volume determinations is greater than 25 volume percent. Of 1,650 PINs containing debris constituents, 1,362 are considered non-debris according to the Resource Conservation and Recovery Act of 1976 "less than 50 volume percent" criteria ${ }^{6}$. The volumetric data discrepancy averages 13 percent for containers that would be considered debris and should be sufficiently significant to require additional characterization.

\subsection{ANALYSIS OF FREE LIQUD CONTENT}

Analysis of the existing thermally treatable mixed waste inventory for liquid content suggests that a significant number of containers should be considered for possible free liquid content. According to WHC (1993), the free liquid must be minimized in containers of mixed waste. The minimum free liquid requirement is not to exceed one percent. Secondary containment for liquid containing mixed waste in drums shall be a 90 -mil high density polyethylene rigid liner if the liquid exceeds one percent of the waste (WHC 1993). For low-level waste disposal, free liquid shall be less than one percent of the volume of the waste when the waste is in a disposal package, or one-half percent of the volume of waste processed to a stable form (WHC 1993). Table 3 identifies physical properties that would be considered liquid. The physical property data was analyzed for liquid composition with the results that approximately 2,300 containers contain at least one volume percent liquid components and approximately 1,700 containers contain at least 10 volume percent liquid. Table 25 summarizes a statistical analysis of the liquid physical property components. The table is arranged in ascending order based on average volume percent. The parameters in Table 25 include the container count, the average volume percent, the standard deviation, the minimum volume percent, and the maximum volume percent.

\subsection{ANALYSIS OF FISSILE WASTE COMPOSITION}

Solid Waste Disposal acceptance criteria and U.S. Department of Transportation (DOT) regulations include waste composition specifications for fissile isotopes. Currently, WHC (1993) discusses a single waste acceptance criterion related to nuclear criticality. Criticality safety limits for waste packages that contain more than $15 \mathrm{gm}$ of ${ }^{235} \mathrm{U}$ will be determined by Westinghouse Hanford Company Criticality Engineering Analysis on a case-by-case basis. These limits will be indicated in the storage/disposal approval record. Waste packages containing $15 \mathrm{gm}$ or less of ${ }^{235} \mathrm{U}$ will not require a separate criticality safety analysis. Table 26 lists the PINs containing ${ }^{235} \mathrm{U}$. Only two containers have ${ }^{235} \mathrm{U}$ in excess of the 15 gm limit.

\footnotetext{
${ }^{6} \mathrm{~A}$ listing of all debris-containing containers in the subject inventory will be provided by the author on request.
} 
The DOT regulations are more inclusive with regard to fissile isotopes. The "Definitions" (49 CFR 173.403) identifies ${ }^{238} \mathrm{Pu},{ }^{239} \mathrm{Pu},{ }^{241} \mathrm{Pu},{ }^{233} \mathrm{U}$ and ${ }^{235} \mathrm{U}$ as fissile, either individually or in mixtures. The DOT's fissile definition excludes natural uranium and depleted uranium. The DOT has packaging regulations for which the total fissile material quantity limit is $15 \mathrm{gm}$ (49 CFR 173.403). Table 27 lists the PINs containing fissile isotopes ${ }^{233} \mathrm{U}$, ${ }^{235} \mathrm{U}$, and ${ }^{239} \mathrm{Pu}$ in excess of $15 \mathrm{gms}$. Eighteen PINs contain fissile material in excess of $15 \mathrm{gm}$, and these PINs, as well as the final waste form containers derived from them, should be considered for fissile material packaging. Isotopes ${ }^{238} \mathrm{Pu}$ and ${ }^{241} \mathrm{Pu}$ are not included in this calculation because they are in negligible quantities.

The DOT regulations also consider as critical the atomic ratio of hydrogen-to-fissile isotopes ( $\mathrm{H} / \mathrm{X}$ ratio) in liquid solutions. This ratio has been estimated for PINs containing aqueous solutions of fissile isotopes; the results are shown in Table 28. Containers with a ratio less than 5,200 must be considered for fissile material packaging (49 CFR 173.403). As Table 28 indicates, no PIN contains solution with an $H / X$ ratio less than 5,200 , and the ratios generally are many orders of magnitude greater than 5,200.

The fissile $\mathrm{H} / \mathrm{X}$ ratio for PINs containing organic solvents may have to be performed on a case-by-case basis as the calculation below demonstrates. Table 29 lists the hazardous material and physical property components in the PINs with the $\mathbf{5 0}$ highest quantities of fissile isotopes. Table 29 indicates that PCBs are a commonly present organic liquid. If the maximum quantity of DOT-regulated fissile material is 554 grams (see Table 27), which is equivalent to about 2.31 gram atoms of a nominal fissile isotope, it would require at least $2.31 \times 5,200=12,000$ gram atoms of hydrogen to satisfy the DOT specification. A typical PCB is about 33 atomic percent hydrogen, so 12,000 gram atoms of hydrogen corresponds to approximately 36,000 gram moles of PCB, which is equivalent to about $450 \mathrm{~kg}$ of PCB. As Table 29 indicates, the maximum quantity of PCB is $0.25 \mathrm{~kg}$, many orders of magnitude less than the required limit. The results of this calculation suggest that organic solvents contaminated with DOT-regulated fissile material will not be excepted from fissile packaging.

\subsection{IDENTIFICATION OF LOW ACTIVITY CONTAINERS}

Based on the definition of radioactive waste outlined in DOE (1988), no minimum activity exists below which waste is considered nonradioactive. Nevertheless, it should be advantageous to have an inventory of low-activity containers identified. It may be desirable to handle only low-activity containers during prototypical waste processing operations. Table 30 lists the first 200 low-activity containers in the thermally treatable mixed waste inventory. These 200 containers correspond to approximately two truckloads of packaged waste. 


\subsection{IDENTIFICATION OF UNIQUE DANGEROUS WASTE CODES}

Table 31 lists the dangerous waste codes identified for the thermally treatable inventory.

\subsection{IDENTIFICATION OF ASBESTOS, HIGH MERCURY, AND BERYLLIUM DUST CONTAINERS}

The commercial thermal treatment contract has excluded the following waste streams from the thermally treatable waste inventory:

Asbestos Containers

High Mercury Containers (greater than 260 ppm mercury)

Containers with beryllium dust

Both high mercury and beryllium dust waste have technology-specific LDR standards. Asbestos is not a RCRA-regulated waste and does not have LDR standards. Asbestos is an OSHA-regulated waste, and the reason for destructive treatment is basically to reduce worker exposure.

Table 32 lists reported asbestos containers, Table 33 lists high mercury containers, and Table 34 lists containers reported to contain beryllium dust waste although no quantity has been reported for any container.

\subsection{IDENTIFICATION OF LEAD CONTAINERS}

Table 35 lists all containers in the subject inventory that contain lead. Under the Resource Conservation and Recovery Act of 1976, lead is a characteristic waste with concentrationbased and technology-specific LDR standards. The concentration-based standard is a $5.0 \mathrm{ppm}$ technology characteristic leaching procedure in the final waste form. This is determined by leaching soluble contaminants from the final waste form using at least a 20:1 leach water-to-solid dilution ratio. The 20:1 dilution ratio allows the average concentration in the media to be approximately $100 \mathrm{ppm}$. Of 584 lead containers, 323 exhibit a lead concentration greater than $100 \mathrm{ppm}$. The treatment of these containers should be managed so that the lead concentration in the leachate remains less than $5 \mathrm{ppm}$.

\subsection{GLOBAL QUERYING AND SORTING OF WASTE QUANTITIES}

Tables 36 to 40 summarize the following waste container quantities: container volume, container gross weight, container tare weight, reported waste weight, and calculated waste weight. The reported waste weights in Table 39 exhibit reasonable agreement with the calculated waste weights in Table 40 . The total waste volume is $1,516 \mathrm{~m}^{3}$. The total reported waste weight is $440,920 \mathrm{~kg}$, and the total container gross weight is $638,090 \mathrm{~kg}$. 


\subsection{ANALYSIS OF CONTAINER TYPES}

Tables 41 to 44 show a statistical analysis of the thermally treatable inventory container types. Table 41 shows an analysis of container volumes. Tables 42 to 44 show analyses of container gross, tare, and waste weight.

\subsection{SIMULATED APPLICATION OF THE COMMERCIAL THERMAL TREATMENT PROCESS TO THE EXISTING THERMALLY TREATABLE WASTE INVENTORY}

The commercial thermal treatment contract was awarded at the beginning of FY 1996 (WHC 1995). The contractor proposed a treatment process that included waste sorting so that only waste requiring thermal treatment would be incinerated. The balance of the waste will be processed by stabilization treatment. The proposed process separates the waste into four streams: combustible non-debris; combustible debris; non-combustible, non-metallic debris; and metallic debris. The quantity indicated below for each stream has been estimated by querying the database and correlating the query with the characterization data shown in Table 3.

$\begin{array}{lcc}\text { Waste Stream } & \text { Quantity, kg } & \text { Percent } \\ \text { Combustible non-debris } & 231,720 & 56.68 \\ \text { Combustible debris } & 44,680 & 10.93 \\ \text { Non-combustible, non-metallic debris } & 28,475 & 6.97 \\ \text { Metallic debris } & 103,926 & 25.42\end{array}$

Both the hazardous material database and the physical property data base were queried. Because the querying algorithm is stream-specific, some "overlap" exists in the calculated stream quantities, but the relative quantities are probably accurate. 


\subsection{FORMULATION OF A SEMI-QUANTTTATIVE DESCRIPTION OF THE THERMALLY TREATABLE MIXED WASTE INVENTORY}

The SWITS database analyzed for this report allows component-specific, detailed characterization of the waste inventory. An emphasis was made to obtain as detailed a characterization as possible because of permit and facility design requirements. Recently, however, a number of communications were made regarding the scope of the contract for which a qualitative or semi-quantitative description of the waste inventory is more appropriate. In an effort to address this need, a waste inventory description discussed below was formulated.

Table 45 lists generator-specific waste volumes sorted in ascending order based on accumulated volume. For this analysis, significant generators are defined as those contributing to the first 90 percent of the existing accumulated waste volumes. Therefore, the "break off" in Table 45 occurs at Generator 241B.

The generators are organized into groups in the SWITS database. Table 46 shows the waste volume by group and by generator, and Table 47 shows the waste weight by group and by generator. The discrepancies between Table 46 and 47 regarding listed groups and generators are associated with differences in reported data. The primary sort on the data in Tables 46 and 47 is alphabetically by group. The secondary sort is by ascending order based on generator quantity within a given group. Table 48 shows group facility descriptions.

The data in Tables $45,46,47$, and 48 include all groups and generators that contribute to the thermally treatable inventory. To provide a global, semi-quantitative, summary description of the waste, an algorithm was established by which only groups and generators contributing significantly to the total waste inventory were included. Table 49 lists the significant groups and generators.

Table 50 shows waste quantity data for significant groups, generators, and physical properties sorted by a "one percent rule," that is, each listed parameter contributed greater than or equal to one percent of the total quantity.

In addition to container-specific physical property data, the SWITS database also includes container-specific waste descriptions. Not all containers have waste descriptions, but an analysis of the waste descriptions allows a representative qualitative assessment of the waste produced by a given generator. Table 51 , which gives a count of the waste descriptions provided by groups and by generators, indicates which groups and generators included container-specific waste descriptions in their SWITS data. For a given group or generator, the waste descriptions tend to be repetitive, and the frequency with which individual descriptive words (primarily nouns) are used provides a qualitative measure of the total waste inventory. Table 52 counts the frequency of individual words. It also indicates that much of the waste is overpacked and includes a significant number of boxes; drums are mentioned less frequently. 


\subsection{DISCUSSION OF WASTE FORECAST DATA}

The Solid Waste Disposal's Systems Engineering section issues a number of annual forecast characterization reports (Valero 1996a, 1996b, and 1996c). The correlation parameters in these reports include waste volumes, container types, and characteristics. Although the forecasted waste constitutes approximately 80 percent of the anticipated thermally treatable inventory, this report will only reference the Valero 1996a, 1996b, and 1996c reports.

\subsection{REFERENCES}

49 CFR 173.403, "Definitions," Federal Code of Regulations, as amended.

ANSI, 1986, Measurement of the Leachability of Solidified Low-Level Radioactive Waste by a Short Term Test Procedure, ANS 16.1, American Nuclear Society, La Grange Park, Illinois.

DOE, 1988, Radioactive Waste Management, DOE Order 5820.2A, U.S. Department of Energy, Washington D.C.

Josephson, W. S., R. E. Flora, and B. L. Lenzer, 1996, Mixed Waste Treatment Sampling and Analysis Plan, Westinghouse Hanford Company, Richland, Washington.

Place, B. G., 1993, W-242 Thermal Treatment Facility Technical Basis Document, WHC-SD-W242-ES-002, Westinghouse Hanford Company, Richland, Washington.

Place, B. G., 1994, Low-Level Mixed Waste Thermal Treatment Technical Basis Report, WHC-SD-W242-ES-003, Westinghouse Hanford Company, Richland, Washington.

RCRA, Resource Conservation and Recovery Act of 1976, 42 U.S.C. 6901 et seq.

TSCA, Toxic Substances Control Act of 1976, 15 U.S.C., 2601 et seq.

Valero, O. J., K. J. Templeton, J. R. Brichoux, M. J. Do, J. T. Hauth, M. Skumanich, 1996a, FY 1996 Solid Waste Integrated Life-Cycle Forecast Characteristics Summary, WHC-EP-0906 Westinghouse Hanford Company, Richland, Washington.

Valero, O. J., K. J. Templeton, J. R. Brichoux, M. J. Do, J. T. Hauth, M. Skumanich, 1996b, FY 1996 Solid Waste Integrated Life-Cycle Forecast Container Summary, WHC-EP-0903 Westinghouse Hanford Company, Richland, Washington. 
Valero, O. J., K. J. Templeton, C. D. Westra, M. J. Do, M. Skumanich, 1996c, FY 1996 Solid Waste Integrated Life-Cycle Forecast Volume Summary, WHC-EP-0900, Westinghouse Hanford Company, Richland, Washington.

WHC, 1993, Hanford Site Solid Waste Acceptance Criteria, WHC-EP-0063-4, Westinghouse Hanford Company, Richland, Washington.

WHC, 1994, Low-Level Mixed Waste Thermal Treatment Service, Request For Proposal No. W-357079-JW, Westinghouse Hanford Company, Richland, Washington.

WHC, 1995, Low-Level Mixed Waste Thermal Treatment Service, Purchase Order No. MW6-SBV-357079, Westinghouse Hanford Company, Richland, Washington. 
WHC-SD-W-242-ES-004, Rev. 0

APPENDIX A

A-1 
WHC-SD-W-242-ES-004, Rev. 0

This page intentionally left blank. 
TABLE 1 HAZARDOUS MATERIALS

(10 SHEET)

\begin{tabular}{|c|c|c|c|}
\hline COMPONENT NAME & ORGANIC/INORGANIC & METALANON-METAL & STRONG ACID OR BASE \\
\hline 11-BUTYLOCTYLIBENZENE & 0 & $N$ & $\mathbf{N}$ \\
\hline (1-ETHYLDECYLIBENZENE & 0 & $N$ & $\mathbf{N}$ \\
\hline (1-METHYLUNDECYLIBENZENE & 0 & $\mathbf{N}$ & $\mathbf{N}$ \\
\hline (1-PENTYLHEPTYL)BENZENE & 0 & $\mathbf{N}$ & $\mathbf{N}$ \\
\hline 11.PROPYLNONYLIBENZENE & 0 & $\mathbf{N}$ & $\mathbf{N}$ \\
\hline O-XYLENE & 0 & $\mathbf{N}$ & $\mathbf{N}$ \\
\hline $1,1,1$ TRICHLORETHANE & 0 & $\mathbf{N}$ & $\mathbf{N}$ \\
\hline $1,1,1$ TRICHLOROEHTANE & 0 & $N$ & $\mathbf{N}$ \\
\hline $1,1,1$ TRICHLOROETHANE & 0 & $\mathbf{N}$ & $\mathbf{N}$ \\
\hline $1,1,1$-DICHLOROETHANE & 0 & $\mathbf{N}$ & $\mathbf{N}$ \\
\hline 1,1,1-TRICHLORETHANE & 0 & $\mathbf{N}$ & $\mathbf{N}$ \\
\hline 1,1,1-TRICHLOROETHANE & 0 & $\mathbf{N}$ & $\mathbf{M}$ \\
\hline $1,1,2,2$-TETRABROMOETHANE & 0 & $\mathbf{N}$ & $\mathbf{N}$ \\
\hline 1,1,2-TAICHLORO 1,2,2-TRIFLUOROETHANE & 0 & $\mathbf{N}$ & $\mathbf{N}$ \\
\hline 1,1,2-TRICHLOAO-1,2,2-TRIFLUOROETHANE & 0 & $\mathbf{N}$ & $\mathbf{N}$ \\
\hline 1,1,2-TRICHLOROETHANE & 0 & $\mathbf{N}$ & $\mathbf{N}$ \\
\hline 1,1,2.TRICHLOAOTRIFLUOROETHANE & 0 & $\mathbf{N}$ & $\mathbf{N}$ \\
\hline 1,1,2-TRICHLOROTRIFLUROETHANE & 0 & $\mathrm{~N}$ & $\mathbf{N}$ \\
\hline 1,1,DICHLOROETHYLENE & O & $\mathbf{N}$ & $\mathbf{N}$ \\
\hline 1.1-OICHLOROETHANE & 0 & $\mathbf{N}$ & $\mathbf{N}$ \\
\hline 1,1-DICHLOROETHENE & 0 & $\mathbf{N}$ & $\mathbf{N}$ \\
\hline 1,1-DICHLOROETHYLENE & 0 & $N$ & $\mathbf{N}$ \\
\hline 1,2 BUTLYENE OXIOE & 0 & $\mathbf{N}$ & $\mathbf{N}$ \\
\hline 1,2,3-TRIMETHYLBENZENE & 0 & $\mathbf{N}$ & $\mathbf{N}$ \\
\hline 1,2,4-TRIMETHYY BENZENE & 0 & $\mathbf{N}$ & $\mathbf{N}$ \\
\hline 1.2,4-TRIMETHYLBENZENE & 0 & $\mathbf{N}$ & $\mathbf{N}$ \\
\hline 1,2-B ENZEN EDICARBOXYLIC ACID & 오 & $\mathbf{N}$ & $\mathbf{N}$ \\
\hline 1,2-BENZISOTHIAZOLIN-3-ONE, 1,1 -OIOXIDE, & 0 & $\mathbf{N}$ & $\mathbf{N}$ \\
\hline 1,2-DICHLOAETHYLENE & 0 & $\mathbf{N}$ & N \\
\hline 1,2-DIETHYLBENZENE & o & $\mathbf{N}$ & $\mathbf{N}$ \\
\hline 1,2-EPOXY BUTANE & 0 & $\mathbf{N}$ & N \\
\hline 1,2-ETHANEDIAMINE, N-12-AMINOETHYLL-N'-2-AMINOETHYLIAMINE & o & $\mathbf{N}$ & $\mathbf{N}$ \\
\hline 1,3 BUTADIENE AND ACRYLONITRILE & 0 & $\mathbf{N}$ & $\mathbf{N}$ \\
\hline 1,3,6-TRUMETHYLBENZENE & 0 & $\mathbf{N}$ & $\mathbf{N}$ \\
\hline 1,3-DIETHYLBENZENE & 0 & $\mathrm{~N}$ & $\mathbf{N}$ \\
\hline 1,3-PFIOPANEDIAMINE & 0 & $\mathbf{N}$ & $\mathbf{N}$ \\
\hline $1,4,7,10,13,16$-HEXANOXACYCLOOCTADECANE & 0 & N & $\mathbf{N}$ \\
\hline 14-DIOXANE & 0 & $\mathbf{N}$ & N \\
\hline 1,4BIS2-6PHENYLOXAZOLYLBENZENE & 0 & $\mathbf{N}$ & $\mathbf{N}$ \\
\hline 1-BUTANOL & 0 & $\mathbf{N}$ & $\mathbf{N}$ \\
\hline 1-CHLORO-2,3-EPOXY PROPANE & 0 & $\bar{N}$ & $\mathbf{N}$ \\
\hline 1-ETHYL-2-METHYLBENZENE & 0 & $N$ & $\mathbf{N}$ \\
\hline 1-ETHYL-4-METHYLBENZENE & 0 & $\mathbf{N}$ & $\bar{N}$ \\
\hline 1-METHOXY 2-PAOPANOL & 0 & $\mathbf{N}$ & $\mathbf{N}$ \\
\hline 1-METHYL-2-PYAROLIDINONE & 0 & $N$ & $\ddot{N}$ \\
\hline 1-NITAOPROPENE & 0 & $\mathrm{~N}$ & $\mathrm{~N}$ \\
\hline 2BUTOXYETHANOL & 0 & $\mathrm{~N}$ & $\mathbf{N}$ \\
\hline 2 HEPTANONE & 0 & $\mathrm{~N}$ & $\mathbf{N}$ \\
\hline 2,3-DIHYDRO-1H-INDENE & 0 & $\mathbf{N}$ & $\mathbf{N}$ \\
\hline 2,4,6-TRI(DIMETHYLAMINOMETHYL) PHENOL & 0 & $\mathbf{N}$ & $\mathbf{N}$ \\
\hline 2,4-D\|SOCYANATO-1-METHYL BENZENE & 0 & $\mathbf{N}$ & $\mathrm{N}$ \\
\hline 2,6-DI-TERT-BUTYLHYDROOUINONE & 0 & N & $\mathrm{N}$ \\
\hline 2,6-DIPHEAYLOXAZOLE & 0 & $\mathrm{~N}$ & $\mathbf{N}$ \\
\hline 2-(2-BUTOXYETHOXY]ETHANOL & 0 & N & $\mathrm{N}$ \\
\hline 2-(2-BUTOXYETHOXY)-ETHANOL & 0 & $\mathrm{~N}$ & $\mathrm{~N}$ \\
\hline 2. IMETHOXYETHOXYI ETHANOL. & 0 & $\mathbf{N}$ & $\mathrm{N}$ \\
\hline 2-BIPHENYLOL SODIUM SALT & 0 & $\mathrm{~N}$ & $\mathrm{~N}$ \\
\hline 2-BROMO-2-BAOMOMETHYL-GLUTRARONITRILE & 0 & $\mathbf{N}$ & $\bar{N}$ \\
\hline 2-BUTANONE & 0 & $\mathrm{~N}$ & N \\
\hline 2-BUTANONE OXIME & 0 & $\mathbf{N}$ & $\mathrm{N}$ \\
\hline 2-BUTANONE PEROXIDE & $a$ & $N$ & $N$ \\
\hline 2-BUTOXETHANOL & 0. & $\mathrm{~N}$ & $N$ \\
\hline 2-BUTOXY ETHANOL & 0 & $\mathrm{~N}$ & $\mathbf{N}$ \\
\hline 2-BUTOXYETHANOL & 0 & $\mathrm{~N}$ & $\mathbf{N}$ \\
\hline 2-BUTYOXYETHANOL & 0 & $N$ & $\mathbf{N}$ \\
\hline 2-ETHOXY BUTANOL & 0 & $\mathbf{N}$ & $\mathrm{N}$ \\
\hline 2-ETHOXYETHANOL & 0 & $N$ & $\mathrm{~N}$ \\
\hline 2.ETHYL-1-HEXANOL & 0 & $\mathbf{N}$ & $\mathrm{N}$ \\
\hline 2-HEPTANONE & 0 & $\mathrm{~N}$ & $\mathrm{~N}$ \\
\hline 2-METHYLNAPHTHALENE & 0 & $\mathrm{~N}$ & $N$ \\
\hline 2-PROPANONE & 0 & $\mathrm{~N}$ & $\mathrm{~N}$ \\
\hline 2-PROPOXYETHANOL & 0 & $\mathbf{N}$ & $\mathbf{N}$ \\
\hline 3-1ODO-2-PROPYNYL BUTYL CAREAMATE & 0 & $\mathbf{N}$ & $N$ \\
\hline 4,4-METHYLENEDIANILINE & o. & N & $N$ \\
\hline 4-METHYL-2-PENTANONE & 0 & $\mathbf{N}$ & $N$ \\
\hline
\end{tabular}


WHC-SD-W-242-ES-004, Rev. 0

TABLE 1 HAZARDOUS MATERIALS

(10 SHEET)

\begin{tabular}{|c|c|c|c|}
\hline COMPONENT NAME & ORGANICINORGANIC & METAL/NON-METAL & STRONB ACID OR BASE \\
\hline 4-TERTBUTYLPYROCATECHOL & 0 & $\mathbf{N}$ & $\mathbf{N}$ \\
\hline ACETALDEHYDE-1-2-C14 & 0 & $\mathbf{N}$ & $\mathbf{N}$ \\
\hline ACETAMIDE & 0 & $\mathbf{N}$ & $N$ \\
\hline ACETIC ACID & 0 & $\mathbf{N}$ & $\mathbf{N}$ \\
\hline ACETIC ACID ETHYL ESTER & 0 & $\mathbf{N}$ & $\mathbf{N}$ \\
\hline ACETIC ACID SODIUM SALT & 1 & $\mathbf{N}$ & $\mathbf{N}$ \\
\hline ACETIC ACID VINYL ESTER & 0 & N & $\mathbf{N}$ \\
\hline ACETIC ACID VINYL ESTER POLYMER WICHLOROETHYLENE & 0 & $\mathrm{~N}$ & $\mathbf{N}$ \\
\hline ACETIC ACID VINYL ESTER POLYMER WITH CHLORETHYLENE & 0 & $\mathbf{N}$ & $\mathbf{N}$ \\
\hline ACETIC ACID VINYL POLYMER WITH CHLOROETHYLENE & 0 & $\mathbf{N}$ & $\mathbf{N}$ \\
\hline ACETONE & 0 & $\mathbf{N}$ & $\mathbf{N}$ \\
\hline ACETONITRILE & 0 & $\ddot{N}$ & $\mathbf{N}$ \\
\hline ACETYL CHLORIDE & o & $\mathbf{N}$ & $\mathbf{N}$ \\
\hline ACETYLENE TETRABROMIDE & 0 & $\mathbf{N}$ & $\mathbf{N}$ \\
\hline$A C \mid D$ & 1 & $\mathbf{N}$ & $\mathbf{N}$ \\
\hline ACID SODIUM SALT & 1 & $\mathbf{N}$ & $Y$ \\
\hline ACRYLAMIOE & 0 & $\mathbf{N}$ & $\mathbf{N}$ \\
\hline ACRYLIC ACID & 0 & $\mathbf{N}$ & $\mathbf{N}$ \\
\hline ALIPHATIC PETROLEUM DISTILLATE & 0 & $\mathbf{N}$ & $\mathbf{N}$ \\
\hline ALIPHATIC PETROLEUM OISTILLATES & 0 & $\mathbf{N}$ & $\mathbf{N}$ \\
\hline ALIQUAT 336 & 0 & $\mathrm{~N}$ & $\mathbf{N}$ \\
\hline ALKALENE CARBONATE & $!$ & $\mathbf{N}$ & $N$ \\
\hline ALKYL BENZENES & 0 & $\mathbf{N}$ & N \\
\hline ALKYLBENZENE & 0 & $\mathbf{N}$ & $\mathbf{N}$ \\
\hline ALKYLNAPHTHALENE & 0 & $N$ & $\mathbf{N}$ \\
\hline ALUMINUM CHLORIDE & 1 & $\mathrm{~N}$ & $\mathbf{N}$ \\
\hline ALUMINUM NITRATE & 1 & $\mathrm{~N}$ & $\mathbf{N}$ \\
\hline ALUMINUM NITRATE MONOHYORATE & I & $\mathrm{N}$ & $\mathbf{N}$ \\
\hline ALUMINUM SIEICATE & 1 & $\mathrm{~N}$ & $\mathbf{N}$ \\
\hline AMERCOAT 234 & 0 & $\mathrm{~N}$ & $\mathbf{N}$ \\
\hline AMINOETHYLPIPERAZINE & 0 & $\mathrm{~N}$ & $\mathbf{N}$ \\
\hline AMINOGUANIDINE HEMISULFAT $E$ & 0 & $\mathrm{~N}$ & $\mathbf{N}$ \\
\hline AMMONIA & 1 & $\mathrm{~N}$ & $N$ \\
\hline AMMONIUM BICARBONATE & 1 & $\mathrm{~N}$ & N \\
\hline AMMONIUM CARBONATE & 1 & $\mathrm{~N}$ & $\mathbf{N}$ \\
\hline AMMONIUM CHLORIDE & 1 & $\mathbf{N}$ & $N$ \\
\hline AMMONIUM CITRATE & 1 & $\mathrm{~N}$ & $\mathbf{N}$ \\
\hline AMMONIUM CITRATE DIBASIC & 1 & $\mathbf{N}$ & $\mathbf{N}$ \\
\hline AMMONIUM HYDROXIDE & 1 & $\mathrm{~N}$ & N \\
\hline AMMONIUM MOLYBDATE & 1 & $\mathbf{N}$ & $\mathrm{N}$ \\
\hline AMMONIUM NITRATE & 1 & $\mathrm{~N}$ & $\mathrm{~N}$ \\
\hline AMMONIUM PERSULFATE & 1 & $\mathrm{~N}$ & $\mathbf{N}$ \\
\hline AMMONIUM SULFATE & $i$ & $\mathrm{~N}$ & $\mathbf{N}$ \\
\hline AMONIUM CHLORIDE & 1 & $\mathrm{~N}$ & $\mathbf{N}$ \\
\hline AMORPHOUS SILICA & 1 & $\mathrm{~N}$ & $\mathbf{N}$ \\
\hline ANTIMONY & 1 & $Y$ & $\mathbf{N}$ \\
\hline ANTIMONY TRICHLORIDE & 1 & $\dot{Y}$ & $\mathbf{N}$ \\
\hline AASENAZO I & 1 & $Y$ & $\mathrm{~N}$ \\
\hline ARSENIC & 1 & $Y$ & $\mathbf{N}$ \\
\hline ARSENIC PENTOXIDE & $i$ & $Y$ & $N$ \\
\hline ARSENIC TRIOXIDE & 1 & $r$ & $N$ \\
\hline ASBESTOS & 1 & $Y$ & $\mathrm{~N}$ \\
\hline ASCOABIC ACID & 0 & $\mathbf{N}$ & $N$ \\
\hline ASPMALT & 0 & $\mathbf{N}$ & $\mathbf{N}$ \\
\hline AZOBENZENE & 0 & $\mathbf{N}$ & $\mathbf{N}$ \\
\hline BAMBP & 0 & $\mathbf{N}$ & $\mathbf{N}$ \\
\hline BARIUM & $i$ & $Y$ & $N$ \\
\hline BARIUM CARBONATE & 1 & $Y$ & $\mathbf{N}$ \\
\hline BARIUM CHLORIOE & $!$ & $Y$ & $N$ \\
\hline BARIUM CHROMATE & i & $Y$ & $\mathbf{N}$ \\
\hline BARIUM HYOROXIDE & 1 & $\mathbf{Y}$ & $\mathbf{N}$ \\
\hline BARIUM NITAATE & 1 & $Y$ & $\mathbf{N}$ \\
\hline BARIUM OXIDE & 1. & $\underline{Y}$ & $\mathbf{N}$ \\
\hline BASE METAL ILEADI & $i$ & $y$ & N \\
\hline BASIC ZINC CHROMATE & 1 & $Y$ & $\mathbf{N}$ \\
\hline BATOHOPHENATHROLLNE & 0 & $\mathbf{N}$ & $\mathbf{N}$ \\
\hline BATTERIES CONTAINING LEAD, LEAD OXIOES, AND SULFURIC ACID & 1 & $Y$ & $\mathbf{N}$ \\
\hline BATTERIES CONTAINING POTASSIUM HYDROXIDE, NICKEL & i & Y & $\mathbf{N}$ \\
\hline BENZENE & 0 & $\mathbf{N}$ & $\mathbf{N}$ \\
\hline BENZENEARSEN|C ACID & 0 & $\mathrm{~N}$ & $\mathbf{N}$ \\
\hline BENZENESULFONIC ACID DODECYL SODIUM SALT & 0 & $\mathbf{N}$ & $\mathbf{M}$ \\
\hline BENZIN-(NAPHTHA) & O & $\mathbf{N}$ & $\mathbf{N}$ \\
\hline BENZOIC ACIO & 0 & $N$ & $\mathbf{N}$ \\
\hline BENZYL BUTYL ESTER & 0 & $\mathrm{~N}$ & $\mathbf{N}$ \\
\hline BEAYLLIUM DUST & 1 & $\gamma$ & $\mathrm{N}$ \\
\hline
\end{tabular}

A- 4 
WHC-SD-W-242-ES-004, Rev. 0

TABLE 1 HAZARDOUS MATERIALS

(10 SHEET)

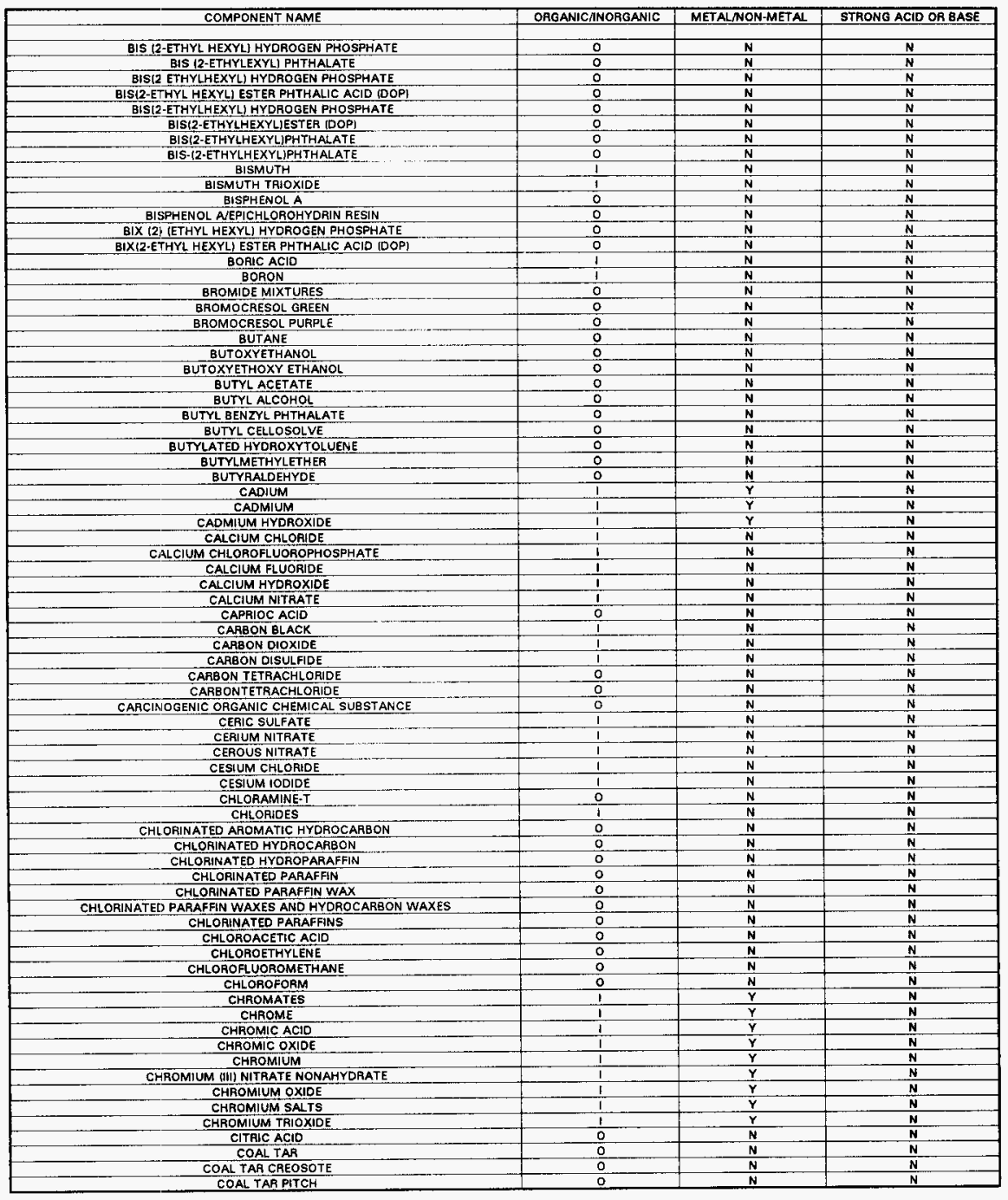


WHC-SD-W-242-ES-004, Rev. 0

TABLE 1 HAZARDOUS MATERIALS

(10 SHEET)

\begin{tabular}{|c|c|c|c|}
\hline COMPONENT NAME & ORGANICANORGANIC & METALNON-METAL & STRONO ACID OR BASE \\
\hline COBALT HYDROXIDE & 1 & $\mathbf{N}$ & $\mathbf{N}$ \\
\hline COBALTOUS NITRATE & 1 & $\mathbf{N}$ & $\mathbf{N}$ \\
\hline COBALTOUS SULFATE & 1 & $N$ & $\mathbf{N}$ \\
\hline COLLODION & 0 & $N$ & $\mathbf{N}$ \\
\hline COPPER & 1 & N & $\mathbf{N}$ \\
\hline COPPER CYANIDE & 1 & N & $\mathbf{N}$ \\
\hline CORROSIVE REACTOR ANALYSIS SOLUTION & 1 & N & $\mathbf{N}$ \\
\hline CREOSOTE & 0 & $\mathbf{N}$ & $\mathbf{N}$ \\
\hline CRESOL & 0 & $\mathbf{N}$ & $\mathbf{M}$ \\
\hline CRESOLS & 0 & $\mathbf{N}$ & $\mathbf{N}$ \\
\hline CRESYUIC ACID & 0 & $\mathbf{N}$ & $\mathbf{N}$ \\
\hline CRYSTALLINE-QUARTZ SILICA & 0 & $\mathbf{N}$ & $\mathbf{N}$ \\
\hline CUMENE HYDROXIDE & 0 & $\mathbf{N}$ & $\mathbf{N}$ \\
\hline CUPFERAON & 1 & $\mathbf{N}$ & $\mathbf{N}$ \\
\hline CUPAIC NITRATE & 1 & $\mathbf{N}$ & $\mathbf{M}$ \\
\hline CUPRIC SULFATE & 1 & $\mathbf{N}$ & $\mathbf{N}$ \\
\hline CYANIDE COMPOUNDS & 1 & $Y$ & $\mathbf{N}$ \\
\hline CYCLOHEXANE & $\underline{0}$ & $\mathbf{N}$ & $\mathbf{N}$ \\
\hline CYCLOHEXANONE & 0 & $\mathbf{N}$ & $\mathbf{N}$ \\
\hline O.TAR & $\underline{0}$ & $\mathbf{N}$ & $\mathbf{N}$ \\
\hline DEBRIS & 0 & $\mathrm{~N}$ & $N$ \\
\hline DEBAIS CONTAINING METHYL ETHYL KETONE & 0 & $\mathbf{N}$ & N \\
\hline DECANE & 0 & $\mathbf{N}$ & $\mathbf{N}$ \\
\hline DI(2-ETHYLHEXYLI PHTHALATE & 0 & $\mathbf{N}$ & $\mathbf{N}$ \\
\hline DI\{2-ETHYLHEXYLIPHTHALATE & 0 & $\mathbf{N}$ & $\mathbf{N}$ \\
\hline DI(2-ETHYLHEXYLIPHTHATATE & $\underline{Q}$ & $\mathbf{N}$ & N \\
\hline DI-2-ETHYLMEXYL PHOSPHOHIC ACID & 0 & $\mathbf{N}$ & $\mathbf{N}$ \\
\hline DI.N+BUTYL PHTHALATE & 0 & $\mathbf{N}$ & $\mathbf{N}$ \\
\hline DI-SEC-OCTYL PHTHALATE & 0 & $\mathbf{N}$ & N \\
\hline OIACETONE ALCOHOL & $\underline{0}$ & $\mathbf{N}$ & N \\
\hline DIBUTYL PHTHALATE & 0 & $\mathbf{N}$ & $\mathbf{N}$ \\
\hline DICHLORODIFLUOROMETHANE & 0 & $\bar{N}$ & N \\
\hline DICHLORODIMETHYL SILANE & 0 & $\mathbf{N}$ & $\mathbf{N}$ \\
\hline DICHLOROMETHANE & 0 & $\mathbf{N}$ & $\mathbf{N}$ \\
\hline DICHLOROOCTAMETHYLTETRA SFLOXANE & $\mathbf{Q}$ & $\mathrm{N}$ & $\mathbf{N}$ \\
\hline DICHLOROTRIFLUROETHANE & 0 & $\mathrm{~N}$ & $\mathbf{N}$ \\
\hline DICYCLOHEXANO-18-CROWN-6 & 0 & $\mathbf{N}$ & $\mathbf{N}$ \\
\hline DICYCLOPENTADIENE & 0 & $\mathbf{N}$ & $\mathbf{N}$ \\
\hline DIESEL & 0 & $\mathbf{N}$ & $\mathbf{N}$ \\
\hline DIESEL FUEL & 0 & $N$ & $\mathbf{N}$ \\
\hline DIETHYL ETHEA & 0 & $\mathbf{N}$ & $\mathrm{N}$ \\
\hline DIETHYL OXALATE & a & $N$ & $\mathbf{N}$ \\
\hline DIETHYLENE GLYCOL & 0 & $\mathbf{N}$ & $\mathbf{N}$ \\
\hline DIETHYLENE GLYCOL MONOETHYLETHER & 0 & $\mathbf{N}$ & $\mathbf{N}$ \\
\hline DIETHYYLENE TRIAMINE & 0 & $\mathrm{~N}$ & $\mathbf{N}$ \\
\hline DIETHYLENETAIAMINE & $\underline{0}$ & $\mathrm{~N}$ & $\mathbf{N}$ \\
\hline DIGLYCOLIC ACIO & 0 & $\mathbf{N}$ & $\overrightarrow{\mathbf{N}}$ \\
\hline DIMETHOXYMETHANE & 0 & $N$ & $\mathbf{N}$ \\
\hline DIMETHTYL OXALATE & 0 & $\mathbf{N}$ & $\mathbf{N}$ \\
\hline DIMETHYL BENZENE & 0 & $\mathrm{~N}$ & $N$ \\
\hline OIMETHYL FORMAMIDE & 0 & $N$ & N \\
\hline DIMETHYL PHLHATE & 0 & $\mathrm{~N}$ & $\mathrm{~N}$ \\
\hline DIMETHYL SULFATE & 0 & N & $\mathrm{N}$ \\
\hline DIMETHYL SULFOXIDE & 0 & $\mathrm{~N}$ & $\mathbf{N}$ \\
\hline DIMETHYYLGLOXIME & 0 & $\mathbf{N}$ & $N$ \\
\hline DIMETHYLGLYOXIME & 0 & $\mathbf{N}$ & $\mathbf{N}$ \\
\hline DIMETHYLSUFOXIDE & O & $\mathrm{N}$ & $\mathbf{N}$ \\
\hline DIMETHYLSULFOXIDE & 0 & $\mathbf{N}$ & $N$ \\
\hline DIOCTYL PHTHALATE & 0 & $\mathbf{N}$ & $\mathbf{N}$ \\
\hline DIOCTYL PHTHALATE (DOP) & 0 & $\mathrm{~N}$ & $\mathbf{N}$ \\
\hline DIOXOLANE & 0 & $N$ & $\mathbf{N}$ \\
\hline OIPHENYLAMINE & 0 & N & $\mathbf{N}$ \\
\hline DIPRDPYLENE GLYCOL MONOMETHYL ETHER. & 0 & $N$ & $\mathbf{N}$ \\
\hline DITHIOXAMIOE & o & $\mathrm{N}$ & $N$ \\
\hline DODECYLBENZENE SULFONIC ACID & 0 & $\mathbf{N}$ & $N$ \\
\hline DOP & 0 & $\mathbf{N}$ & $\mathbf{N}$ \\
\hline DUSOCYANATE & 0 & $\mathbf{N}$ & $\mathbf{N}$ \\
\hline ECOLITE & 0 & $\mathbf{N}$ & $\mathbf{N}$ \\
\hline EDETIC ACID & 0 & $\mathbf{N}$ & $\mathbf{N}$ \\
\hline EPOXXY RESIN & 0 & $\mathbf{N}$ & $\mathbf{N}$ \\
\hline ETHANOL & 0 & $\mathbf{N}$ & $\mathbf{N}$ \\
\hline ETHANOL (ETHYL ALCOHOL) & 0 & $\mathbf{N}$ & $N$ \\
\hline ETHANOL DIBENZYL MERCURY & 0 & $Y$ & $N$ \\
\hline ETHANOLAMINE & 0 & $\mathbf{N}$ & $\mathbf{N}$ \\
\hline EIHANOLAMINE DIMETHYLSULFOXIDE & 0 & $\mathrm{~N}$ & $\mathbf{N}$ \\
\hline
\end{tabular}


TABLE 1 HAZARDOUS MATERIALS

(10 SHEET)

\begin{tabular}{|c|c|c|c|}
\hline COMPONENT NAME & ORGANICINORGANIC & METAL/NON-METAL & STRONG ACID OR BASE \\
\hline ETHIDIUM BROMIDE & 0 & $\mathbf{N}$ & $\mathbf{N}$ \\
\hline ETHOXYETHANOL & 0 & $\mathbf{N}$ & $\mathbf{N}$ \\
\hline ETHYL ACETATE & 0 & $\mathrm{~N}$ & $\bar{N}$ \\
\hline ETHYL ACAYLATE & O & $\mathbf{N}$ & $\mathbf{N}$ \\
\hline ETHYL ALCOHOL & 0 & $\mathbf{N}$ & $\mathbf{N}$ \\
\hline ETHYL BENZENE & $\underline{0}$ & $\mathbf{N}$ & $\mathbf{N}$ \\
\hline ETHYL CYANIDE & 0 & $\mathbf{N}$ & $\mathbf{N}$ \\
\hline ETHYLETHER & 0 & $\mathbf{N}$ & $\mathbf{N}$ \\
\hline ETHYL MERCURIC CHLOAIDE & 0 & $\mathbf{Y}$ & $\mathbf{N}$ \\
\hline ETHYLBENZENE & 0 & $\mathbf{N}$ & $N$ \\
\hline ETHYLENE GLYCOL & o & $\mathbf{N}$ & $\mathbf{N}$ \\
\hline ETHYLENE GLYCOL MONOBUTYL ETHER & 0 & $\mathbf{N}$ & $\mathbf{N}$ \\
\hline ETHYLENE GLYCOL MONOETHYL ETHER ACETATE & 은 & $\mathbf{N}$ & $\mathrm{N}$ \\
\hline ETHYLENE GLYCOLAMONOBUTYL ETHER & 0 & $N$ & N \\
\hline ETHYLENEDIAMINETETRAACETIC ACID DISODIUM SALT (EDTA) & 0 & $\mathbf{N}$ & $\mathbf{N}$ \\
\hline ETHYLENEDIAMINETRA ACIDIC ACID & $\underline{\mathbf{O}}$ & $\ddot{N}$ & $\mathbf{N}$ \\
\hline ETHYLENEGLYCOL-MONOBUTYL ETHER & $\circ$ & $\hat{N}$ & $\underline{N}$ \\
\hline ETHYLHEXYLPHOSPHORIC ACID & o & $N$ & $\mathbf{N}$ \\
\hline FERRIC OXIOE & 1 & $\mathbf{N}$ & $\mathbf{N}$ \\
\hline FERROCENE & 0 & $\mathbf{N}$ & $\mathbf{N}$ \\
\hline FERROUS SULFATE & 1 & $N$ & $\mathbf{N}$ \\
\hline FLUOORS & 0 & $N$ & $\mathbf{N}$ \\
\hline FORMALDEHYDE & 0 & $N$ & $\mathbf{N}$ \\
\hline FORMALIN & 0 & $\mathbf{N}$ & $\mathbf{N}$ \\
\hline FORMAMIDE & 0 & $\mathrm{~N}$ & $\mathbf{N}$ \\
\hline FORMIC ACID & o. & $\mathrm{N}$ & $\mathbf{N}$ \\
\hline FREON & 0 & $N$ & $N$ \\
\hline FREON 113 & 0 & $\mathrm{~N}$ & $N$ \\
\hline FURFURYL-ALCOHOL & 0 & $\mathbf{N}$ & $\mathrm{N}$ \\
\hline GADOLINIUM NITRATE & 0 & $\mathbf{N}$ & $N$ \\
\hline GALLIC ACID & 1 & $N$ & $\mathbf{N}$ \\
\hline GLACIAL ACETIC ACID & 0 & $\mathrm{~N}$ & $\mathbf{N}$ \\
\hline GLYCOLS & o. & $\mathbf{N}$ & $\mathbf{N}$ \\
\hline GLYCOLS, POLYETHYLENE MONO (NONYLPHENYL) & 0 & $\mathrm{~N}$ & $\mathbf{N}$ \\
\hline GRAPHITE & 1. & $\mathrm{~N}$ & $\mathbf{N}$ \\
\hline HALOGENATED HYDROCARBON & 0 & $\underline{N}$ & $\mathbf{N}$ \\
\hline HALOGENATEO HYOROCARBONS & 0 & $N$ & $\mathbf{N}$ \\
\hline HALOGENATED ORGANIC COMPOUNDS & 0 & $\mathbf{N}$ & $\mathbf{N}$ \\
\hline HEXACHLOROBENZENE & 0 & $\mathrm{~N}$ & $N$ \\
\hline HEXACHLOROBENZNE & 0 & $\mathrm{~N}$ & $\mathrm{~N}$ \\
\hline HEXANE & 0 & $\mathrm{~N}$ & $\mathbf{N}$ \\
\hline HEXANOL & o & $\mathbf{N}$ & $\mathbf{N}$ \\
\hline HEXCHLOROBENZENE & 0 & $\mathbf{N}$ & $\mathbf{N}$ \\
\hline HEXONE & 0 & $\mathbf{N}$ & $\mathbf{N}$ \\
\hline HEXYLENE GLYCOL & 0 & $\mathbf{N}$ & $\mathbf{N}$ \\
\hline HYDRAULIC FLUID & 0 & $\mathbf{N}$ & $\mathbf{N}$ \\
\hline MYDRAULIC OHL & 0 & $\mathrm{~N}$ & $\mathbf{N}$ \\
\hline HYORAZINE & 0 & $\mathbf{N}$ & $\mathbf{N}$ \\
\hline HYDAOBROMIC ACID & $\mathrm{O}$ & $\mathbf{N}$ & $\mathbf{N}$ \\
\hline HYDROCARBON TAR & 0 & $\mathbf{N}$ & $\mathbf{N}$ \\
\hline HYDROCARBONS & 0 & $N$ & $\mathbf{N}$ \\
\hline HYOROCHLORIC ACID & 1 & $\mathbf{N}$ & $Y$ \\
\hline HYDROFLUOAIC ACID & I & $\mathbf{N}$ & $Y$ \\
\hline HYDROGEN IOOATE & $!$ & $N$ & $Y$ \\
\hline HYDROGEN PEROXIDE & i & $N$ & $\mathbf{N}$ \\
\hline HYDROQUINONE & 0 & $\mathbf{N}$ & $\mathbf{N}$ \\
\hline HYDROTREATED HEAVY NAPHTA & 0 & $\mathrm{~N}$ & $\mathbf{N}$ \\
\hline HYDROTREATED HEAVY NAPHTHA & 0 & $\mathbf{N}$ & $\mathbf{N}$ \\
\hline HYOROXYQUINOUINE & 0 & $\mathrm{~N}$ & $\mathbf{N}$ \\
\hline LII TRICHLOROETHANE & 0 & $\mathbf{N}$ & $\mathbf{N}$ \\
\hline INDENE & 0 & $\underline{N}$ & $\mathbf{N}$ \\
\hline INSTAGEL XF & 0 & N & $\mathbf{N}$ \\
\hline IODIC ACID & 1 & $\mathbf{N}$ & $\underline{Y}$ \\
\hline IRON & 1 & N & $\bar{N}$ \\
\hline IRON NITRATE & $\mathrm{I}$ & $\mathbf{N}$ & $N$ \\
\hline JRON OXIDE & 1 & $\mathbf{N}$ & $\mathbf{N}$ \\
\hline IRON III\} CHLORIDE & 1 & $\mathbf{N}$ & $\mathbf{N}$ \\
\hline ISOAMYL ALCOHOL & 0 & $\mathbf{N}$ & $\mathbf{N}$ \\
\hline ISOBUTANOL & 0 & $\mathbf{N}$ & $\mathbf{N}$ \\
\hline ISOBUTYL ALCOHOL & 0 & $\mathbf{N}$ & $\mathbf{N}$ \\
\hline ISOBUTYL ISOBUTYAATE & 0 & $N$ & $\mathbf{N}$ \\
\hline ISOFARAFFINIC PETAOLEUM HYOROCARBCN & 0 & $\mathbf{N}$ & $\mathbf{N}$ \\
\hline ISOPROPANOL & 0. & $N$ & $\mathrm{~N}$ \\
\hline ISOPROPLY ALCOHOL & 0 & $N$ & $\mathbf{N}$ \\
\hline ISOPROPYL ACETONE & 0 & $\mathbf{N}$ & $\mathbf{N}$ \\
\hline
\end{tabular}


TABLE 1 HAZARDOUS MATERIALS

(10 SHEET)

\begin{tabular}{|c|c|c|c|}
\hline COMPONENT NAME & ORGANICANORGANIC & METAL/NON-METAL & STRONB ACID OR BASE \\
\hline ISOPROPYL ALCOHOL & 0 & $\mathbf{N}$ & $\mathbf{N}$ \\
\hline ISOPROPYLACETONE & 0 & $\mathbf{N}$ & $\mathbf{N}$ \\
\hline KEROSENE & 0 & $\mathbf{N}$ & $\mathbf{N}$ \\
\hline KEROSINE & 0 & $\mathbf{N}$ & $\mathbf{N}$ \\
\hline KETONE & 0 & $\mathbf{N}$ & $\mathbf{N}$ \\
\hline KLEEN-O-BOWL & 0 & $\mathbf{N}$ & $\mathbf{N}$ \\
\hline LEAD & 1 & $Y$ & $\mathbf{N}$ \\
\hline LEAD ACID & 1 & $Y$ & $N$ \\
\hline LEAD BRICK & i & $Y$ & $\mathbf{N}$ \\
\hline LEAD CHROMATE & 1 & $\bar{Y}$ & $\mathbf{N}$ \\
\hline LEAD CHROMATE BASED PAINT & 1 & $Y$ & $\mathbf{N}$ \\
\hline LEAD CHROMATE MOLYBDATE & 1 & $\mathbf{Y}$ & $\mathbf{N}$ \\
\hline LEAD CHROMATE PAINT & 1 & $\mathbf{Y}$ & $\mathbf{N}$ \\
\hline LEAD CHROMATE,CHLORIN.PARAFFIN & $i$ & $\mathbf{Y}$ & $\mathbf{N}$ \\
\hline LEAD DIOXIDE & $i$ & $Y$ & $\mathbf{N}$ \\
\hline LEAD NAPHTHENATE & 1 & $\mathbf{Y}$ & $\mathbf{N}$ \\
\hline LEAD OXIDE & 1 & $Y$ & $\mathbf{N}$ \\
\hline LEAD SALTS & 1 & $\mathbf{Y}$ & $\mathbf{N}$ \\
\hline LEAD SHIELDING & 1 & $Y$ & $\mathbf{N}$ \\
\hline LEAD SOLUTION & 1 & $\mathbf{Y}$ & $\mathbf{N}$ \\
\hline LEAD TELLURIDE & 1 & $Y$ & $\mathbf{N}$ \\
\hline LEAD TETROXIOE & 1 & $\mathbf{Y}$ & $\mathbf{N}$ \\
\hline LEAKING BALLASTS CONTAINING $>500$ PPM PCBS & 0 & $\mathbf{N}$ & $\mathbf{N}$ \\
\hline LEAKING LIGHT BALLASTS CONTAINING PCB & 0 & $\mathbf{N}$ & N \\
\hline LEAKING LIGHT FIXTURES CONTAMINATED WITH POLYCHLORINATEO BIPHENYLS & 0 & $\mathbf{N}$ & $\mathbf{N}$ \\
\hline LECTRA CLEANER & 0 & $\mathbf{N}$ & $\mathbf{N}$ \\
\hline LIGHT BALLASTS CONTAINING PCE & 0 & $\mathbf{N}$ & $\mathbf{N}$ \\
\hline LIGROINE & 0 & $\mathbf{N}$ & $\mathbf{N}$ \\
\hline LIGAOINE INAPHTHAI & 0 & $\mathbf{N}$ & $\mathbf{N}$ \\
\hline LIQUID WASTE & 0 & $\mathbf{N}$ & $\mathbf{N}$ \\
\hline LIOUIFIED PETROLEUM GAS & 0 & $\mathbf{N}$ & $N$ \\
\hline LITHIUM CARBONATE & 1 & $N$ & $N$ \\
\hline LITHIUM CHLORIDE & $\mathrm{i}$ & $\mathbf{N}$ & $\mathbf{N}$ \\
\hline LITHIUM CHROMATE & 1 & $Y$ & $\mathbf{N}$ \\
\hline LITHIUM HYDROSTEARATE & 1 & $\mathbf{N}$ & $\mathbf{N}$ \\
\hline LITHIUM HYDROXIOE & 1 & $\mathbf{N}$ & $Y$ \\
\hline M-XYLENE & 0 & $\mathbf{N}$ & $\mathbf{N}$ \\
\hline MAGNESSIUM CHLORIDE & 1 & $\mathbf{N}$ & $\mathbf{N}$ \\
\hline MAGNESIUM HYOROXIDE & $\mathrm{i}$ & $\mathbf{N}$ & $Y$ \\
\hline MAGNESIUM METAL & 1 & $\mathbf{N}$ & $\mathbf{N}$ \\
\hline MAGNESIUM PERCHLORATE & 1 & $\mathbf{N}$ & $\mathbf{N}$ \\
\hline MANGANESE & 1 & $\mathbf{N}$ & $\mathbf{N}$ \\
\hline MANGANESE DIOXIDE & 1 & $N$ & $\mathrm{~N}$ \\
\hline MED ALIPHATIC SOLVENT NAPHTA & 0 & $\mathbf{N}$ & $\mathbf{N}$ \\
\hline MEOIUM ALIPHATIC SOLV NAPHTHA & 0 & N & $\mathbf{N}$ \\
\hline MEK & 0 & $N$ & $\mathbf{N}$ \\
\hline MERCURIC HYDROXIDE & 1 & $Y$ & $\mathbf{N}$ \\
\hline MEFCUAY & 1 & $Y$ & $\mathbf{N}$ \\
\hline METAL & 1 & $\mathbf{N}$ & $\mathbf{N}$ \\
\hline METALLIC MERCURY & 1 & $Y$ & $\mathbf{N}$ \\
\hline METHANOL & $\mathrm{O}$ & $\mathbf{N}$ & $\mathbf{N}$ \\
\hline METHLY ETHYL KETONE & 0 & $\mathrm{~N}$ & $\mathbf{N}$ \\
\hline METHLY ISOBUTYL KETONE & 0 & $N$ & $\mathbf{N}$ \\
\hline METHYL ALCOHOL & 0 & $N$ & N \\
\hline METHYL ALCOHOL SOLUTION & 0 & $N$ & $\mathbf{N}$ \\
\hline METHYL BUTYL KETONE & 0 & $\mathbf{N}$ & $\mathbf{N}$ \\
\hline METHYL ETHYL KETONE & 0 & $\mathbf{N}$ & $\mathbf{N}$ \\
\hline METHYL ETHYL KETONE PEROXIDE & 0 & $N$ & $\mathrm{~N}$ \\
\hline METHYL ISOBUTYL KETONE & 0 & $\mathrm{~N}$ & $\mathbf{N}$ \\
\hline METHYL METHACRYLATE & 0 & $\mathrm{~N}$ & $\mathbf{N}$ \\
\hline METHYL ORANGE & 0 & $\mathrm{~N}$ & $\mathbf{N}$ \\
\hline METHYL PENTYL KETONE & 0 & $\mathbf{N}$ & $N$ \\
\hline METHYL-1-PHENYL-2-PYAAZOLIN & 0 & $\mathbf{N}$ & $\mathbf{N}$ \\
\hline METHYLCYCLOHEXANONE & 0 & $\mathbf{N}$ & $N$ \\
\hline METHYLENE CHLORDIE & 0 & $\mathbf{N}$ & $N$ \\
\hline METHYLENE CHLORIDE & 0 & $\mathbf{N}$ & $N$ \\
\hline METHYLNAPHTHALENE & 0 & $N$ & $\mathbf{N}$ \\
\hline METHYLTAIACETOXY SILANE & 0 & $\mathbf{N}$ & $\mathbf{N}$ \\
\hline METHYLTRICAPRYLALAMMONIUM CHLORIDE & 0 & $\mathrm{~N}$ & $N$ \\
\hline METHYLTRICAPRYLYLAMMONIUM CHLORIOE & 0 & $\mathbf{N}$ & $\mathbf{N}$ \\
\hline METHYLTRIOCTYLAMMONIUM CHLORIDE & Q & $\mathrm{N}$ & $\mathbf{N}$ \\
\hline MIBK & 0 & $\mathbf{N}$ & $\mathbf{N}$ \\
\hline MINERAL OIL & 0 & $\mathrm{~N}$ & $\mathbf{N}$ \\
\hline MINERAL OIL PETROLEUM DISTILLATE HYDROTREATED & 0 & $\mathbf{N}$ & $\mathbf{N}$ \\
\hline MINERAL OIL PETROLEUM DISTILLATES & 0 & $\mathrm{~N}$ & $N$ \\
\hline
\end{tabular}


TABLE 1 HAZARDOUS MATERIALS

(10 SHEET )

\begin{tabular}{|c|c|c|c|}
\hline COMPONENT NAME & ORGANIC/INORGANIC & METALNON-METAL & STRONG ACID OA BASE \\
\hline COMPONENT NAME & ORGANICANORGANIC & METALINON-METAL & STRONG ACID OR BASE \\
\hline & & & \\
\hline MINERAL OIL, PETROLEUM DISTILALTE IMILDF & o & $\mathbf{N}$ & $\mathbf{N}$ \\
\hline MINERAL OILPETROLEUM DISTILLATES & 0 & $\mathbf{N}$ & $\mathbf{M}$ \\
\hline MINERAL SPIRITS & 0 & 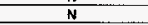 & $\mathbf{N}$ \\
\hline MISCELLANEOUS MATERIALS & 0 & $\mathbf{N}$ & N \\
\hline MOL YEDATE ORANGE & 0 & $\mathbf{N}$ & $\mathbf{N}$ \\
\hline MOLYBDIC ACID HEXAMMONIUM SALT & 1 & $\mathbf{N}$ & $\mathbf{M}$ \\
\hline MONOINONYLPHENYLI POLYETHYLENE GLYCOLS & 0 & $\mathbf{N}$ & $\mathbf{N}$ \\
\hline MONOBUTYL ETHER & O & $\mathbf{N}$ & $\mathbf{N}$ \\
\hline MONOETHANOLAMINE & 0 & $\mathbf{N}$ & N \\
\hline MONOETHANOLAMINE DIMETHYL SULFOXIDE & 9 & $\mathbf{N}$ & $N$ \\
\hline MORFHOLINE & 0 & $\mathbf{N}$ & $\mathbf{N}$ \\
\hline MULTISOL & o & $\mathbf{N}$ & $\mathbf{N}$ \\
\hline MURIATIC ACID & 1 & $\mathbf{N}$ & $Y$ \\
\hline N,N DIMETHYL-FORMANIDE & $\underline{a}$ & $\mathbf{N}$ & $\mathbf{N}$ \\
\hline$N, N, N$, N'-TETRAMETHYL-1,3-BUTANEDIAMINE & 0 & N & $N$ \\
\hline N,N-OIMETHYL FORMANIDE & o & $\mathrm{N}$ & $\mathbf{N}$ \\
\hline N,N-DIMETHYL-FORMANIDE & 0 & N & $\mathbf{N}$ \\
\hline N,N-DIMETHYLANILINE & 0 & $\mathbf{N}$ & $\mathbf{N}$ \\
\hline N-BUTANOL & 0 & $\mathrm{~N}$ & $\mathbf{N}$ \\
\hline N-BUTYL ACRYLATE & 0 & $\mathrm{~N}$ & $\mathbf{N}$ \\
\hline N-BUTVL ALCOHOL & 0 & $\mathrm{~N}$ & $\mathbf{N}$ \\
\hline N-DODECANE METHANOL & 0 & $\mathbf{N}$ & $\mathbf{N}$ \\
\hline N-HEPTANE & 0 & $\mathrm{~N}$ & $\mathbf{N}$ \\
\hline N-METHYL-2-PYRROLIDONE & 0 & $\mathbf{N}$ & $\mathbf{N}$ \\
\hline NAPHTA & 0 & $N$ & $\mathbf{N}$ \\
\hline NAPHTHA & 0 & $\mathbf{N}$ & $\mathbf{N}$ \\
\hline NAPHTHALENE & 0 & $\mathbf{N}$ & $\mathbf{N}$ \\
\hline NAPHTHENIC ACID LEAD SALT & 0 & $\mathbf{N}$ & $\mathbf{N}$ \\
\hline NAPHTHENIC PETROLEUM OLL & 0 & $\mathrm{~N}$ & $\mathbf{N}$ \\
\hline NAPTHA & 0 & $N$ & $\mathbf{N}$ \\
\hline NAPTHALENE & 0 & $N$ & $\mathbf{N}$ \\
\hline NAPTHENIC DISTILLATE & 0 & $\mathbf{N}$ & $\mathbf{N}$ \\
\hline NICKEL HYDROXIDE & 1 & $Y$ & $\mathbf{N}$ \\
\hline NICKEL MONOXIDE & $i$ & $\bar{Y}$ & $\mathbf{N}$ \\
\hline NICKEL-CADMIUM BATTERIES & 1. & $Y$ & $\mathbf{N}$ \\
\hline NITRATES & 1 & $\mathbf{N}$ & $\mathbf{N}$ \\
\hline NITRIC ACID & 1 & $\mathbf{N}$ & $Y$ \\
\hline NITRILOTRIACETIC ACID & o & $\mathbf{M}$ & $\mathbf{Y}$ \\
\hline NITROBENZENE & o & $\mathbf{M}$ & $\mathbf{N}$ \\
\hline NITROCELLULOSE & 0. & $\mathbf{N}$ & $\mathbf{N}$ \\
\hline NON-IONIC EMULSIFIERS & 0 & $\mathbf{N}$ & $\mathbf{N}$ \\
\hline NONREGULATED WATER MISCIBLE LIOUID WISOLID SLUDGE RESIDUE & 0 & $\mathbf{N}$ & $\mathbf{N}$ \\
\hline NONYLPHENOL & o & $\mathbf{N}$ & N \\
\hline NONYLPHENOXYPOLY [ETHYLENEOXY) ETHANOL & 0 & $\mathbf{N}$ & $\mathbf{N}$ \\
\hline NONYLPHENOXYPOLY ETHANOL & 0 & $\mathbf{N}$ & $\mathbf{N}$ \\
\hline NONYLPHENOXYPOLYIETHYLENEOXYIETHANOL & 0 & $\mathbf{N}$ & $\mathbf{N}$ \\
\hline NOAMAL PARAFFIN HYDAOCARBON & 0 & $\mathbf{N}$ & $\mathbf{N}$ \\
\hline O-CRESOL & 0 & $\mathbf{N}$ & $\mathbf{N}$ \\
\hline O.XYLENE & 0 & N & $N$ \\
\hline OCTYLIPHENYLI-N.N-DUSOBUTYL-CARBAMOYLMETHYLPHOSPHINE OXIDES & o & $\mathbf{N}$ & $\mathbf{N}$ \\
\hline OCTYLFHENOXYPOLYETHOXETHANOL & 0 & $\bar{N}$ & $\mathbf{N}$ \\
\hline OCTYLPHENOXYPOLYETHOXYETHANOL. & 0 & $\mathbf{N}$ & $\mathbf{N}$ \\
\hline Olt & 0 & $\mathbf{N}$ & $\mathbf{N}$ \\
\hline OXALIC ACID & i. & $\mathbf{N}$ & $\mathbf{N}$ \\
\hline OXINE & 0 & $\mathbf{N}$ & $\bar{N}$ \\
\hline P-(SEC-BUTYL)-PHENOL & 0 & $\mathbf{N}$ & $\mathbf{N}$ \\
\hline P-DIOXANE & 0 & $\mathbf{N}$ & $N$ \\
\hline P-FORMALOEHYDE & 0 & $\mathbf{N}$ & $\mathbf{N}$ \\
\hline P-NITROPHENOL & 0 & $\mathrm{~N}$ & $\mathbf{N}$ \\
\hline P.XYLENE & 0 & $\mathbf{N}$ & $N$ \\
\hline PAINT CONTAINING LEAD CHROMATE & 0 & $\mathbf{N}$ & $\mathbf{N}$ \\
\hline PAINT CONTAINING NONYLPHENOL & 0 & $\mathbf{N}$ & $\mathbf{N}$ \\
\hline PCB & $\mathrm{O}$ & $N$ & $\mathbf{N}$ \\
\hline PCB BALLASTS & 0 & $\mathbf{N}$ & $\mathbf{N}$ \\
\hline PCB OIL CONTAINING PCB'S & o & $\mathbf{N}$ & $\mathbf{N}$ \\
\hline PENTACHLOROPHENOL & 0 & $\mathbf{N}$ & $\overline{\mathbf{N}}$ \\
\hline PENTAERYTHAITOL & 0 & $\mathbf{N}$ & $N$ \\
\hline PENTAETHYLENE-HEXAMINE & 0 & $\mathbf{N}$ & $\mathrm{N}$ \\
\hline PERCHLORIC ACID & 1 & $N$ & $\bar{Y}$ \\
\hline PERCHLOROETHYLENE & 0 & $\mathbf{N}$ & $\mathbf{N}$ \\
\hline PETROLEUM DISTILIATE & $\ddot{0}$ & N & $\mathbf{N}$ \\
\hline PETROLEUM DISTILLATES & 0 & $N$ & $\mathbf{N}$ \\
\hline PETROLEUM OIL & 0 & $\mathbf{N}$ & N \\
\hline
\end{tabular}


TABLE I HAZARDOUS MATERIALS

(10 SHEET)

\begin{tabular}{|c|c|c|c|}
\hline COMPONENT NAME & ORGANICINORGANIC & METAL/NON-METAL & STRONO ACID OR BASE \\
\hline PHENOL & 0 & $\mathbf{N}$ & $\mathbf{N}$ \\
\hline PHENYL GLYCIDYL ETHER & 0 & N & $\mathbf{N}$ \\
\hline PHENYLXYLYLETHANE & 0 & $\mathbf{N}$ & $\mathbf{N}$ \\
\hline PHOSPHORIC ACID & $\mathrm{I}$ & $\mathbf{N}$ & $\mathbf{Y}$ \\
\hline PHTHALIC ACID & 0 & $\mathbf{N}$ & N \\
\hline PHTHALIC ACID BIS|2-ETHYLHEXYLIESTER & 0 & $\mathbf{N}$ & $\mathbf{N}$ \\
\hline PHTHALIC ACID, BISI2-ETHYLHEXYLIESTER (DOP) & 0 & $\mathbf{N}$ & $\mathbf{N}$ \\
\hline PAPERAZINE & 0 & $\mathbf{N}$ & $\mathbf{N}$ \\
\hline PLASTIC & 0 & $\mathbf{N}$ & $\mathbf{N}$ \\
\hline PLASTIC ACID SUIT/GLOVES/ROPE/RAGS & 0 & $\mathbf{N}$ & $\mathbf{N}$ \\
\hline POLY ARO HYOROCARBONS & 0 & $\mathbf{N}$ & $\mathbf{N}$ \\
\hline POLYCHLORINATED BINPHENYLS & 0 & $\mathbf{N}$ & $\mathbf{N}$ \\
\hline POLYCHLORINATED BIPHENYLS & 0 & $\mathbf{N}$ & $\mathbf{N}$ \\
\hline POLYCHLORINATED BIPHENYLS \{PCB\} & 0 & $\mathbf{M}$ & $\mathbf{N}$ \\
\hline POLYCYCLIC AROMATIC HYOROCARBONS & 0 & $\mathbf{N}$ & $\mathbf{N}$ \\
\hline POLYETHYLENE & 0 & $\mathbf{N}$ & $\mathbf{N}$ \\
\hline POLYETHYLENE MONO(NONYLPHENYL) GLYCOLS & 0 & $\mathbf{M}$ & $\mathbf{N}$ \\
\hline POLYOXPROPYLENE-POLYOXETHYLENE & 0 & $\mathbf{N}$ & $\mathbf{N}$ \\
\hline POLYOXYALKYLENE GLYCOL & 0 & $\mathbf{N}$ & $\mathbf{N}$ \\
\hline POLYPROPYLENE GLYCOL & 0 & $\mathbf{N}$ & $\mathbf{N}$ \\
\hline POLYPROPYLENE GLYCOLS & 0 & $\mathbf{N}$ & $\mathbf{N}$ \\
\hline POLYVINYL ALCOHOL & 0 & $\mathbf{N}$ & $\mathbf{N}$ \\
\hline POLYVINYL CHLORIDE POLYMER & 0 & $\mathbf{N}$ & $\mathbf{N}$ \\
\hline POTASSIUM ACETATE & 1 & $\mathbf{N}$ & $\mathbf{N}$ \\
\hline POTASSIUM BROMATE & 1 & $\mathbf{N}$ & $\mathbf{N}$ \\
\hline POTASSIUM CARBONATE & 1 & $\mathbf{N}$ & $\mathbf{N}$ \\
\hline POTASSIUM CHLORATE & 1 & N & $\mathbf{N}$ \\
\hline POTASSIUM CHLORIDE & 1 & $\mathrm{~N}$ & $\mathbf{N}$ \\
\hline POTASSIUM CHROMATE & $i$ & $\bar{Y}$ & $\mathbf{N}$ \\
\hline POTASSIUM CYANIDE & 1 & $Y$ & $\mathbf{N}$ \\
\hline POTASSIUM DICHROMATE & 1 & $Y$ & $\mathbf{N}$ \\
\hline POTASSIUM FLUORIOE & 1 & $\mathbf{N}$ & $\mathbf{N}$ \\
\hline POTASSIUM HYDROXIDE & 1 & $\mathbf{N}$ & $\mathbf{Y}$ \\
\hline POTASSTUM IODIDE & 1 & $\mathbf{N}$ & $\mathbf{N}$ \\
\hline POTASSIUM METAGISULFATE & 1 & $\mathbf{N}$ & $\mathbf{N}$ \\
\hline POTASSIUM NITRATE & + & $N$ & $\mathbf{N}$ \\
\hline POTASSIUM NITRITE & 1 & $\mathbf{N}$ & $\mathbf{N}$ \\
\hline POTASSIUM PERIODATE & 1 & $\mathbf{N}$ & $\mathbf{N}$ \\
\hline POTASSIUM PERMANGANATE & 1 & $\mathbf{N}$ & $\mathbf{N}$ \\
\hline POTASSIUM PERMANGANTE & 1 & $\mathbf{N}$ & $\mathbf{N}$ \\
\hline POTASSIUM PHOSPHATE & 1 & $\mathbf{N}$ & $\mathbf{N}$ \\
\hline POTASSIUM ZINC CHROMATE & 1 & $Y$ & $N$ \\
\hline PROPANE, 1-NITRO & 0 & $\mathbf{N}$ & $\mathbf{N}$ \\
\hline PAOPARGYL ALCOHOL & 0 & $\mathbf{N}$ & $\mathbf{N}$ \\
\hline PROPIONIC ACID & 0 & $\mathbf{N}$ & $\mathbf{N}$ \\
\hline PROPYDIAMINE & 0 & $\mathbf{N}$ & $\mathbf{N}$ \\
\hline PAOPYLDIAMINE & 0 & $\mathbf{N}$ & $\mathbf{N}$ \\
\hline PROPYLENE GLYCOL & 0 & $\mathbf{N}$ & $\mathbf{N}$ \\
\hline PROPYLENE GLYCOL MONOMETHYL ETHER & 0 & $\mathbf{N}$ & $\mathbf{N}$ \\
\hline PROPYLENEGLYCOL & 0 & N & $\mathbf{N}$ \\
\hline PSEUDOCUMENE & 0 & $\mathbf{M}$ & $\mathbf{N}$ \\
\hline PSEUDOCUMENE-XYLENE & 0 & $\mathbf{N}$ & $\mathbf{N}$ \\
\hline PSUEDOCUMENE & 0 & $\mathbf{N}$ & $\mathbf{N}$ \\
\hline PYDAAUL/FYAGUEL (HYDRAULIC FLUID) & 0 & $\mathbf{N}$ & $\mathbf{N}$ \\
\hline PYRIDINE & 0 & N & $N$ \\
\hline REMAINDER NON HAZARDOUS INGREDIENTS & 0 & N & $\mathbf{N}$ \\
\hline RESIN & 0 & $\mathbf{N}$ & N \\
\hline RUTHENIUM CHLORIDE & O & $\mathbf{N}$ & $\mathbf{N}$ \\
\hline SALICYLIC ACID & 0 & $\mathbf{N}$ & $\mathbf{N}$ \\
\hline SCINTILLATION LIOUID & 0 & N & N \\
\hline SEC BUTYL ALCOHOL & 0 & $\mathrm{~N}$ & N. \\
\hline SEC-BUTYL ALCOHOL & 0 & $\mathbf{N}$ & $\mathbf{N}$ \\
\hline SELENIUM & 1 & $Y$ & $\mathrm{~N}$ \\
\hline SILICA & 1 & $\mathrm{~N}$ & $\mathbf{N}$ \\
\hline SILICA CRYSTALLINE QUAFTZ & 1 & $\mathbf{N}$ & $\mathbf{N}$ \\
\hline SILICON & 1 & $\mathbf{N}$ & $\mathbf{N}$ \\
\hline SILICON DIOXIDE & 1 & $\mathbf{N}$ & $\mathbf{N}$ \\
\hline SILVER & 1 & $\ddot{Y}$ & $\mathbf{N}$ \\
\hline SILVER CHLORIDE & 1 & $Y$ & $\mathbf{N}$ \\
\hline SILVER NITRATE & $t$ & $Y$ & N. \\
\hline SILVER ZEOLITE. & 1 & $Y$ & $\mathbf{N}$ \\
\hline SODA ASH & 1 & $\mathrm{~N}$ & $\mathbf{N}$ \\
\hline SODIUM & 1 & $N$ & $\mathbf{N}$ \\
\hline SODIUM ACETATE & 1 & N & $\mathbf{N}$ \\
\hline SODIUM ALUMINATE & 1 & $N$ & $\mathbf{N}$ \\
\hline
\end{tabular}


WHC-SD-W-242-ES-004, Rev. 0

TABLE 1 HAZARDOUS MATERIALS

(10 SHEET)

\begin{tabular}{|c|c|c|c|}
\hline COMPONENT NAME & ORGANICINORGANIC & METALNON-METAL & STRONC ACID OA BASE \\
\hline SODIUM BICARBONATE & 1 & $\mathbf{N}$ & $\mathbf{N}$ \\
\hline SODIUM CARBONATE & 1 & $N$ & $\mathbf{N}$ \\
\hline SODIUM CHLOFIOE & 1 & $\mathbf{N}$ & $N$ \\
\hline SODIUM CHAOMATE & 1 & $Y$ & $\mathbf{N}$ \\
\hline SODIUM CYANIDE & 1 & $Y$ & $\mathbf{N}$ \\
\hline SODIUM DICHROMATE & 1 & $Y$ & $\mathbf{N}$ \\
\hline SODIUM DIOCTYLSULFOCCINATE & $i$ & $N$ & $\mathbf{N}$ \\
\hline SODIUM DODECYLBENZENESULFONATE & 1 & N & $\mathbf{N}$ \\
\hline SODIUM FLUORIDE & 1 & $\mathbf{N}$ & $\mathbf{N}$ \\
\hline SODIUM HYOROXIDE & 1 & $N$ & $Y$ \\
\hline SODIUM IODIDE & 1 & $\mathbf{N}$ & $\mathbf{N}$ \\
\hline SODIUM LAURYL SULFATE & 1 & $\mathbf{N}$ & $\mathbf{N}$ \\
\hline SODIUM METABISULFITE & 1 & $\mathbf{N}$ & $\mathbf{N}$ \\
\hline SODIUM METABOAATE OCTAHYORATE & 1 & $\mathbf{N}$ & $\mathrm{N}$ \\
\hline SODIUM METASILICATE & 1 & $\mathbf{N}$ & $\mathbf{N}$ \\
\hline SODIUM NITRATE & 1 & $\mathbf{N}$ & $\mathbf{N}$ \\
\hline SODIUM NITRITE & 1 & $\mathbf{N}$ & $\mathbf{N}$ \\
\hline SODIUM NITAOFEARICYANIDE & 1 & $\mathbf{N}$ & $\mathbf{N}$ \\
\hline SODIUM PHOSPHATE & 1 & $\mathbf{N}$ & $\mathbf{N}$ \\
\hline SODIUM PHOSPHATE TRIBASIC & $i$ & $\mathbf{N}$ & $\mathbf{N}$ \\
\hline SODIUM PYROPHOSPHATE & 1 & $\mathbf{N}$ & $\mathbf{N}$ \\
\hline SODIUM SILICATE & 1 & $\mathbf{N}$ & $\mathbf{N}$ \\
\hline SODIUM SULFATE & 1 & $\mathbf{N}$ & $\mathbf{N}$ \\
\hline SODIUM SULFIDE B-HYDRATE & 1 & $\mathbf{Y}$ & $\mathbf{N}$ \\
\hline SODIUM SULFITE & 1 & $N$ & $\mathbf{N}$ \\
\hline SODIUM TRIPOLY PHOSPHATE & 1 & $\mathbf{N}$ & $\mathbf{N}$ \\
\hline SOIL & 1 & $\mathbf{N}$ & $\mathbf{N}$ \\
\hline SOLVENT PETROLEUM DISTULLATE & 0 & $\mathbf{N}$ & $\mathbf{N}$ \\
\hline $\begin{array}{l}\text { SOLVENTS } \\
\end{array}$ & 0 & $\mathbf{N}$ & $\mathbf{N}$ \\
\hline STANNOUS SULFATE & 1 & $\mathbf{N}$ & $\mathbf{N}$ \\
\hline STARGLAZE & 0 & N & $\mathbf{N}$ \\
\hline STODDARD SOLVENT & 0 & N & $\mathbf{N}$ \\
\hline STRIPCOAT & 0 & $\mathbf{N}$ & $N$ \\
\hline STRONTIUM NITRATE & 1 & N & $\mathbf{N}$ \\
\hline STYRENE & 0 & $\mathbf{N}$ & $\mathrm{N}$ \\
\hline STYRENE MONOMER & 0 & $\mathbf{N}$ & $\mathbf{N}$ \\
\hline SULFAMIC ACID & 0 & N & $\mathbf{N}$ \\
\hline SULFATES & i. & N & $\mathrm{N}$ \\
\hline SULFURIC ACIO & 1 & $\mathbf{N}$ & $Y$ \\
\hline TALC & i & $N$ & $\mathbf{N}$ \\
\hline TAR & 0 & $\mathbf{N}$ & $\mathbf{N}$ \\
\hline TARTARIC ACID & 0 & N & $N$ \\
\hline TELLURIUM METAL & 1 & $\mathbf{N}$ & $\mathbf{N}$ \\
\hline TERT BUTYL ALCOHOL & 0 & $\mathbf{N}$ & $\mathbf{N}$ \\
\hline TERT-BUTYL METHYLETHER & 0 & $\mathbf{N}$ & N \\
\hline TETRAACETIC ACID & 0 & $\mathrm{~N}$ & $\mathbf{N}$ \\
\hline TETRACHLORETHYLENE & 0 & $\mathbf{N}$ & $\mathbf{N}$ \\
\hline TETRACHLOROETHENE & 0 & $\mathbf{N}$ & $\mathbf{N}$ \\
\hline TETRACHLOROETHYLENE & 0 & $\mathbf{N}$ & N \\
\hline TETRAETHYL AMMONIUM BROMIOE & 0 & $\mathbf{N}$ & $\mathbf{N}$ \\
\hline TETRAETHYLENEPENTAMINE & 0 & $\mathbf{N}$ & $\mathbf{N}$ \\
\hline TETAAHYOAOFURAN & 0 & N & $\mathbf{N}$ \\
\hline TETRAMETHYL 1,3-BUTANEDIAMINE & 0 & N & $\mathbf{N}$ \\
\hline TETRAMETHYL AMMONIUM HYDROXIDE & 0 & $\mathrm{~N}$ & $\mathbf{N}$ \\
\hline TETRAMETHYLAMMONIUM BROMIDE & 0 & $\mathbf{N}$ & $N$ \\
\hline TETRAPHYENYLARSONIUM HYDROCHLORITE & 0 & $\mathbf{N}$ & $\mathrm{N}$ \\
\hline TETPAFFOPYL AMMONIUM HYOROXIDE & 0 & $\mathrm{~N}$ & N \\
\hline THENOLYTRIFLUOROACETONE & 0 & $\mathbf{N}$ & $\mathbf{N}$ \\
\hline THENOYLTRIFL UORACETONE & 0 & $\mathbf{N}$ & N \\
\hline THENOYLTRIFLUOROACETATE & 0 & $\mathrm{~N}$ & N \\
\hline THENOYLTRIFLUOROACETONE & 0 & $\mathbf{N}$ & $\mathbf{N}$ \\
\hline THINNER & 0 & N & $N$ \\
\hline THIOACETAMIDE & 0 & $N$ & $\mathbf{N}$ \\
\hline THIONYL CHLORIDE & 0 & $\mathbf{N}$ & $\mathbf{N}$ \\
\hline THORIUM NITRATE TETRAYYDRATE & 1 & $\mathbf{N}$ & $\mathbf{N}$ \\
\hline TITANIUM DIOXIDE & 1 & $\mathbf{N}$ & $\mathbf{N}$ \\
\hline TOLUENE & $a$ & $\mathbf{N}$ & $N$ \\
\hline TOTAL ORGANIC HALOGENS. & 0 & $\mathbf{N}$ & $\mathbf{N}$ \\
\hline TOX/EOX & o & N & $\mathbf{N}$ \\
\hline TRI BUTYL PHOSPHATE & 0 & $\mathbf{N}$ & $\mathbf{N}$ \\
\hline TRIBUTYL PHOSPHATE & 0 & $\mathrm{~N}$ & $\mathbf{N}$ \\
\hline TRIBUTVL PHOSPHATES & 0 & $\mathrm{~N}$ & $\mathbf{N}$ \\
\hline TRIBUTYLPHOSPHATE & o & $\underline{N}$ & $\mathbf{N}$ \\
\hline TRICHLOROETHANE & 0 & $N$ & $\mathbf{N}$ \\
\hline TRICHLOROETHENE & 0 & $N$ & $\mathbf{N}$ \\
\hline
\end{tabular}


WHC-SD-W-242-ES-004, Rev. 0

TABLE 1 HAZARDOUS MATERIALS

(10 SHEET )

\begin{tabular}{|c|c|c|c|}
\hline COMPONENT NAME & ORGANIC $N$ NORGANIC & METALINON-METAL & STRONG ACID OR BASE \\
\hline TRICHLOROETHYLENE & 0 & $\mathbf{N}$ & $\mathbf{N}$ \\
\hline TRICHLOROFLUOROMETHANE & 0 & $\mathbf{N}$ & $\mathbf{N}$ \\
\hline TRICHLOROTRIFLUOROETHANE & 0 & $\mathbf{N}$ & $\mathbf{N}$ \\
\hline TRIETHYLAMINE & 0 & $\mathbf{N}$ & $\mathbf{N}$ \\
\hline TRIETHYLENERETRAMINE & 0 & $\mathbf{N}$ & $\mathbf{N}$ \\
\hline TRIETHYLENETETRAMINE & 0 & $\mathbf{N}$ & $\mathbf{N}$ \\
\hline TRFFLUOROACETIC ACIO & 0 & $\mathbf{N}$ & $\mathbf{N}$ \\
\hline TREISOOCTYLAMINE & o & N & $\mathbf{N}$ \\
\hline TRIMETHYL BENZENE & Q & $\mathbf{N}$ & $\mathbf{N}$ \\
\hline TRIMETHYLBENZENE & 0 & $\mathbf{N}$ & $N$ \\
\hline TRIOCTYLAMINE & 0 & $\mathbf{N}$ & N \\
\hline TRIOCTYLPHOSPHINE OXIDE & 0 & $\mathbf{N}$ & $\mathbf{N}$ \\
\hline TRIPHENYL PHOSPHATE & o & $\mathbf{N}$ & $N$ \\
\hline TRISODIUM PHOSPHATE & 1 & $\mathbf{N}$ & $Y$ \\
\hline TURCO & 0 & $\mathbf{N}$ & N \\
\hline UNDECANE & 0 & $N$ & $N$ \\
\hline URANYL ACETATE & 1 & N & $\mathbf{N}$ \\
\hline URANYL NITRATE HEXAHYDRATE & 1 & N & N \\
\hline VANADIUM PENTOXIDE & 1 & $\mathbf{Y}$ & $\mathbf{N}$ \\
\hline VANDYL SULFATE & 1 & $Y$ & $\mathbf{N}$ \\
\hline VINYL ACETATE & 0 & $\mathbf{N}$ & $\mathbf{N}$ \\
\hline VINYL CHLORIDE & O & $\mathbf{N}$ & $\overline{\mathbf{N}}$ \\
\hline VINYL CHLORIDE RESIN & 0 & $\mathbf{N}$ & $\mathrm{N}$ \\
\hline VINYL ESTER & 0 & $\mathrm{~N}$ & $\mathbf{N}$ \\
\hline VINYLESTER RESIN & 0 & $\mathbf{N}$ & $\mathbf{N}$ \\
\hline VINYLIDENE-4, 1-DIPHOSPHONIC ACID & 0 & $\mathbf{N}$ & $\mathbf{N}$ \\
\hline WATER & 1 & $\mathbf{N}$ & N \\
\hline WHITE POWDER CONTAINING SILOXANES & 0 & $\mathbf{N}$ & $\mathbf{N}$ \\
\hline XYLENE & o & $\mathbf{N}$ & $\mathbf{N}$ \\
\hline XYLENE SULFONIC ACIO SODIUM SALT & 0 & $\mathbf{N}$ & $\mathbf{N}$ \\
\hline XYLENE-P & 0 & $\mathbf{N}$ & N \\
\hline XYLENE.TOLUENE & ? & N & $\mathbf{N}$ \\
\hline YTTRIUM NITAATE & 1 & $\mathbf{N}$ & N \\
\hline ZINC & $i$ & $\mathbf{Y}$ & $\mathbf{N}$ \\
\hline ZINC CHLORIDE & I. & $\ddot{\gamma}$ & $\mathbf{N}$ \\
\hline ZINC CHAOMATE & I & $Y$ & $\mathbf{N}$ \\
\hline ZINC NAPHTHENATE & 1 & $Y$ & $\mathbf{N}$ \\
\hline ZINC SALT & 1 & $\bar{Y}$ & $\mathbf{N}$ \\
\hline ZIRCONIUM SULPHATE & 1 & $\mathbf{N}$ & $\mathbf{N}$ \\
\hline ZIRCONYL NITRATE & 1 & $\mathbf{N}$ & $\mathbf{N}$ \\
\hline
\end{tabular}


WHC-SD-W-242-ES-004, Rev. 0

TABLE 2

\section{RADIONUCLIDES}

(3 SHEET)

ISOTOPE

TOTAL BETA/GAMMA
H-3
BE-7
C-14
NA-22
P-32
S-35
CL-36
K-40
CA-45
SC-47
V-49
CA-51
MN-54
FE-55
CO-56
CO-57
CO-58
FE-59
N1-59
CO-60
N1-60
N1-63
NI-63 METAL
ZN-65
SE-75
SE-79
KR-85
SR-90-Y90
MO-93
NB-93M
ZR-93
NB-94
NB-95
NB-95M
ZR-95 - NB-95M
TC-99

TC-99

\begin{abstract}
UNIT T/NT PAISOTOPE FISSILE(N/Y/XI
\end{abstract}

$\begin{array}{lll}\text { Cl } & \text { NT } & \text { N } \\ \text { Cl } & \text { NT } & \text { N } \\ \text { Cl } & \text { NT } & \text { N } \\ \text { Cl } & \text { NT } & \text { N } \\ \text { Cl } & \text { NT } & \text { N } \\ \text { Cl } & \text { NT } & \text { N } \\ \text { Cl } & \text { NT } & \text { N } \\ \text { Cl } & \text { NT } & \text { N } \\ \text { Cl } & \text { NT } & \text { N } \\ \text { Cl } & \text { NT } & \text { N } \\ \text { Cl } & \text { NT } & \text { N } \\ \text { Cl } & \text { NT } & \text { N } \\ \text { Cl } & \text { NT } & \text { N } \\ \text { Cl } & \text { NT } & \text { N } \\ \text { Cl } & \text { NT } & \text { N } \\ \text { Cl } & \text { NT } & \text { N } \\ \text { Cl } & \text { NT } & \text { N } \\ \text { Cl } & \text { NT } & \text { N } \\ \text { Cl } & \text { NT } & \text { N } \\ \text { Cl } & \text { NT } & \text { N } \\ \text { Cl } & \text { NT } & \text { N } \\ \text { Cl } & \text { NT } & \text { N } \\ \text { Cl } & \text { NT } & \text { N } \\ \text { Cl } & \text { NT } & \text { N } \\ \text { Cl } & \text { NT } & \text { N } \\ \text { Cl } & \text { NT } & \text { N } \\ \text { Cl } & \text { NT } & \text { Y } \\ \text { Cl } & \text { NT } & \text { N } \\ \text { Cl } & \text { NT } & \text { N } \\ \text { Cl } & \text { NT } & \text { N } \\ \text { Cl } & \text { NT } & \text { N } \\ \text { Cl } & \text { NT } & \text { N } \\ \text { Cl } & \text { NT } & \text { N } \\ \text { Cl } & \text { NT } & \text { N } \\ \text { Cl } & \text { NT } & \text { N } \\ \text { Cl } & \text { NT } & \text { N } \\ \text { Cl } & \text { NT } & \text { Y } \\ & & \\ & & \text { NT }\end{array}$
HALF LIFE

$1.0000 E+00$
$1.2260 E+01$ $1.4600 \mathrm{E} \cdot 01$

$5.7300 \mathrm{E}+03$

$2.6020 \mathrm{E}+00$

$2.41000-01$

3.1000E + 05

1. $2800 \mathrm{E}+09$

4.5200E-01

9.3970E-03

9.0400E-01

7.6160E-02

8.3000E.03

2. $6000 \mathrm{E}+O O$

$2.1100 \mathrm{E}-01$

7.3970E-O1

1.9530 E-O1

1.2360E-01

$8.0000 E+04$
$5.2600 E+00$

9. $2000 \mathrm{E}+01$

9. $2000 E+01$

6.6740E-01

3. 2990E-O1

$6.5000 \mathrm{E}+04$

$1.0760 E+01$

$2.8100 E+01$

$1.0000 E+02$

1. $5000 \mathrm{E}+06$

$2.0000 \mathrm{E}+04$

9.6300E-02

$1.7800 \mathrm{E}-01$

1.7800E-01

2.1200E + 05

ASS NU
1
1
3
7
14
22
32
35
36
40
45
47
49
51
54
55
56
57
58
59
59
60
60
63
63
65
75
79
65
90
93
93
93
94
95
95
95
99

DOT A2 LIMIT, CURIES

1. $0000 \mathrm{E}+00$

$1.0000 E+\infty 0$

$1.0000 E+\infty$

$1.0000 E+\infty 0$

$1.0000 E+\infty 0$

$1.0000 E+\infty 0$

$1.0000 E+\infty 0$

$1.0000 E+00$

$1.0000 E+\infty$

$1.0000 E+\infty 0$

$1.0000 E+\infty 0$

$1.0000 E+00$

$1.0000 E+\infty 0$

$1.0000 E+\infty 0$

$1.0000 E+\infty$
$1.0000 E+\infty$

$1.0000 E+\infty$
$1.0000 E+\infty$

$1.0000 E+00$

$1.0000 E+\infty 0$

$1.0000 E+\infty$

$1.0000 E+\infty$

$1.0000 E+\infty 0$

$1.0000 E+\infty 0$

$1.0000 E+\infty$

$1.0000 E+\infty$
$1.0000 E+\infty$

$1.0000 E+\infty$

$1.0000 E+\infty 0$

$1.0000 E+00$

$1.0000 E+\infty 0$

1. $0000 E+\infty$

$1.0000 E+\infty 0$

$1.0000 E+\infty$

$1,0000 E+\infty$

$1.0000 E+\infty 0$

$1.0000 E+00$

$1.0000 E+\infty 0$

$1.0000 E+\infty$

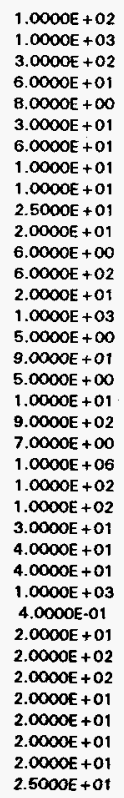

DE CURIES/CI

DE CURIES/UNIT

\begin{tabular}{cc}
$1.0000 E+00$ & $1.0000 E+00$ \\
$1.1000-03$ & $1.1000 E-03$ \\
$1.0000 E-06$ & $1.0000 E-06$ \\
$6.5000 E-06$ & $6.5000 E-06$ \\
$5.4000 E-04$ & $5.4000 E-04$ \\
$4.9000 E-05$ & $4.9000 E-05$ \\
$7.8000 E-06$ & $7.8000 E-06$ \\
$6.0000 E-04$ & $6.0000 E-04$ \\
$1.9000 E-04$ & $1.9000 E-04$ \\
$2.1000 E-05$ & $2.1000 E-05$ \\
$5.9000 E-06$ & $5.9000 E-06$ \\
$1.1000 E-06$ & $1.1000 E-06$ \\
$1.1000 E-06$ & $1.1000 E-06$ \\
$2.2000 E-05$ & $2.2000 E-05$ \\
$8.5000 E-06$ & $8.5000 E-06$ \\
$1.3000 E-04$ & $1.3000 E-04$ \\
$2.7000 E-05$ & $2.7000 E-05$ \\
$3.6000 E-05$ & $3.6000 E-05$ \\
$4.9000 E-05$ & $4.9000 E-05$ \\
$4.0000 E-06$ & $4.0000 E-06$ \\
$6.3000 E-04$ & $6.3000 E-04$ \\
$0.0000 E+00$ & $0.0000 E+00$ \\
$9.4000 E-06$ & $9.4000 E-06$ \\
$9.4000 E-06$ & $9.4000 E-06$ \\
$6.7000 E-05$ & $6.7000 E-05$ \\
$2.7000 E-05$ & $2.7000 E-05$ \\
$9.0000 E-04$ & $9.0000 E-04$ \\
$2.1000 E-05$ & $2.1000 E-05$ \\
$4.3000 E-03$ & $4.3000 E-03$ \\
$9.0000 E-05$ & $9.0000 E-05$ \\
$7.8000 E-06$ & $7.8000 E-06$ \\
$2.5000 E-03$ & $2.5000 E-03$ \\
$1.2000 E-03$ & $1.2000 E-03$ \\
$1.9000 E-05$ & $1.9000 E-05$ \\
$7.8000 E-06$ & $7.8000 E-06$ \\
$1.2000 E-04$ & $1.2000 E-04$ \\
$1.3000 E-04$ & $1.3000 E-04$ \\
\hline
\end{tabular}


WHC-SD-W-242-ES-004, Rev. 0

TABLE 2

\section{RADIONUCLIDES}

(3 SHEET)

\begin{abstract}
ISOTOPE
\end{abstract}
103 - RH-103M

RU-106 - RH-106

PD-107

CD-109

AG-11OM - AG-110

SB-1 24

SB-124
+125
SB-125
TE-125M

1-129

1.131

XE-131M

BA-1 33

CS-134

CS-137 -. BA-137M

CE-141

CE-144 - PP-144

PM-147

SM-151

EU-152

EU-154

GD-154

EU-155

HF-181

TA-182

TA-183

W-185

AU-195

PT-195M
TL-206

PO-210

Bl-212

PB-212

(日-214

PB-214
UNIT T/NT PA ISOTOPE FISSILE(N/Y/X)

\begin{tabular}{|c|c|c|}
\hline $\mathrm{Cl}$ & NT & $\mathbf{N}$ \\
\hline $\mathrm{Cl}$ & NT & $\mathrm{N}$ \\
\hline Cl & NT & $\mathrm{N}$ \\
\hline $\mathrm{Cl}$ & NT & $\mathrm{N}$ \\
\hline $\mathrm{Cl}$ & NT & $\mathrm{N}$ \\
\hline $\mathrm{Cl}$ & NT & $N$ \\
\hline $\mathrm{Cl}$ & NT & $\mathrm{N}$ \\
\hline $\mathrm{Cl}$ & NT & $N$ \\
\hline $\mathrm{Cl}$ & NT & $\mathrm{N}$ \\
\hline $\mathrm{Cl}$ & NT & $\mathrm{N}$ \\
\hline Cl & NT & $Y$ \\
\hline $\mathrm{Cl}$ & NT & $\mathbf{N}$ \\
\hline $\mathrm{Cl}$ & NT & $N$ \\
\hline $\mathrm{Cl}$ & NT & $N$ \\
\hline $\mathrm{Cl}$ & NT & $\mathrm{N}$ \\
\hline $\mathrm{Cl}$ & NT & $\mathrm{N}$ \\
\hline $\mathrm{Cl}$ & NT & $\mathbf{N}$ \\
\hline $\mathrm{Cl}$ & NT & $\mathrm{N}$ \\
\hline $\mathrm{Cl}$ & NT & $\mathbf{N}$ \\
\hline $\mathrm{Cl}$ & NT & $\mathrm{N}$ \\
\hline $\mathrm{Cl}$ & NT & $\mathrm{N}$ \\
\hline $\mathrm{Cl}$ & NT & $\mathrm{N}$ \\
\hline $\mathrm{Cl}$ & NT & $N$ \\
\hline $\mathrm{Cl}$ & NT & $\mathrm{N}$ \\
\hline $\mathrm{Cl}$ & NT & $N$ \\
\hline $\mathrm{Cl}$ & NT & $\mathbf{N}$ \\
\hline $\mathrm{Cl}$ & NT & $\mathrm{N}$ \\
\hline $\mathrm{Cl}$ & NT & $\mathrm{N}$ \\
\hline $\mathrm{Cl}$ & NT & $\mathrm{N}$ \\
\hline $\mathrm{Cl}$ & NT & $\mathbf{N}$ \\
\hline $\mathrm{Cl}$ & NT & $\mathbf{N}$ \\
\hline $\mathrm{Cl}$ & NT & $N$ \\
\hline $\mathrm{Cl}$ & NT & $N$ \\
\hline CI & NT & $N$ \\
\hline $\mathrm{Cl}$ & NT & $\mathrm{N}$ \\
\hline $\mathrm{Cl}$ & NT & $\mathrm{N}$ \\
\hline $\mathrm{Cl}$ & NT & $\mathrm{N}$ \\
\hline
\end{tabular}

MASS NUMBER CURIES/UNIT $1.0050 E+00$ $7.0000 E+06$

$1.2300 E+\infty$

1.6500E-01

\section{2100E-02}

$3.23005-02$

$1.0500 E+01$

$3.0000 E+06$

(n)

$1.1600 E \cdot 0$

5.0100E-01

103
106
107
109
110
110
124
125
125
125
129
131
131
133
134
135
137
141
144
147
151
152
154
154
155
181
182
183
185
195
195
208
210
212
212
214
214

103
106
107

07

110

24

125
125

129

31

131
133
134

135
137
141

141

47

$(51$

154

55

81

182

185

195

208

14

214
$1.0000 E+\infty$

$1.0000 E+\infty$

$1.0000 E+\infty 0$

$1.0000 E+\infty$

$1.0000 \mathrm{E}+\infty$

$.0000 E+\infty$

$1.0000 E+\infty$

$1.0000 E+\infty$

$1.0000 E+\infty$

$1.0000 E+\infty$

$1.0000 E+00$

$1.0000 \mathrm{E}+\infty$

$1.0000 E+\infty$

$1000 E+\infty$

$1.0000 E+\infty$

$1.0000 E+00$

.OOOOE $+\infty 0$

1.0000E +OO

$1.0000 E+\infty$

$1,0000 E+\infty$

$1.000 \mathrm{E}+\infty$

$1.0000 E+\infty$

$1.0000 E+\infty$

$1.0000 E+\infty 0$

$1.0000 E+\infty 0$

$1.0000 E+\infty$

$1.0000 E+\infty$

$10000+\infty$

.

$1.0000 E+\infty$

$1.0000 E+\infty$

$1.0000 E+\infty$

$1.0000 E+\infty$

$1.0000 E+\infty$

$\infty 00 \mathrm{~F}+\infty$

$1.0000 E+\infty$
DOT A2 LIMIT, CURIES

DE CURIES/CI

DE CURIES/UNIT

$2.5000 E+01$
$7.0000 E+00$
$7.0000 E+00$
$7.0000 E+01$
$7.0000 E+\infty 0$
$7.0000 E+00$
$5.0000 E+00$
$7.0000 E+01$
$2.5000 E+01$
$1.0000 E+02$
$2.0000 E+00$
$1.0000 E+01$
$1.0000 E+01$
$1.0000 E+01$
$1.0000 E+01$
$2.5000 E+01$
$1.0000 E+01$
$2.5000 E+01$
$7.0000 E+\infty 0$
$2.5000 E+01$
$9.0000 E+01$
$1.0000 E+01$
$5.0000 E+00$
$1.0000 E+06$
$6.0000 E+01$
$2.5000 E+01$
$2.0000 E+01$
$2.0000 E+01$
$2.5000 E+01$
$2.0000 E+02$
$2.0000 E+01$
$2.0000 E+01$
$2.0000 E-01$
$6.0000 E+00$
$5.0000 E+00$
$6.0000 E+00$
$5.0000 E+00$

2.9000E-05

$1.5000 \mathrm{E}-03$

4.0000E-05

4.5000E-04

$1.2000 \mathrm{E}-03$

1. $200 \mathrm{OE}-03$

$8.3000 E-05$

8.500OE-01

4.0000E-05

2.3000E-05

1. $0000 E+01$

$1.2000 E+\infty 0$

$6.1000 \mathrm{E}-05$

2.5000E-05

1.4000E-O4

1.4000 E- 05

9.6000E-05

$2.7000 \mathrm{E}-05$

1.2000 E- 03

1. $2000 \mathrm{E}-04$

$1.6000 E-04$

6.5000E-04

8.5000E-04

$0.0000 E+\infty 0$

$1.7000 E-04$

9.6000E-05

1.4000E-O4

1.7000E-05

5000

2.5000E-06

4.1000E-05

3.9000E-06

1.0000E-O3

3.1000E-02

7.100OE-05

5.6000E-O4

2. 2000E-05

2.5000E-05 
WHC-SD-W-242-ES-004, Rev. 0

TABLE 2

\section{RADIONUCLIDES}

(3 SHEET)

\begin{tabular}{|c|c|c|c|c|c|c|c|c|c|c|}
\hline ISOTOPE & UNIT & T/NT & PA ISOTOPE & FISSILE(N/Y/X) & HALF LIFE & MASS NUMBER & CURIES/UNIT & DOT A2 LIMIT, CURIES & DE CURIES/CI & DE CURIES/UN/T \\
\hline RA-224 & GM & NT & $\mathbf{N}$ & $\mathbf{N}$ & $9.9726 \mathrm{E}-03$ & 224 & $1.6000 E+05$ & $5.0000 \mathrm{E}-01$ & $9.4000 \mathrm{E} \cdot 03$ & $1.5040 E+03$ \\
\hline RA-226 & GM & $T$ & $\mathbf{N}$ & $\mathbf{N}$ & $1.6000 E+03$ & 226 & $9.9100 \mathrm{E}-01$ & $5.0000 E-02$ & $2.5000 \mathrm{E}-02$ & $2.4775 \mathrm{E}-02$ \\
\hline$A C-228$ & Cl & NT & $N$ & $\mathbf{N}$ & & 228 & $1.0000 \mathrm{E}+00$ & $4.0000 E+00$ & $1.7000 \mathrm{E}-03$ & $1.7000 E-03$ \\
\hline FA-228 & $\mathrm{Cl}$ & NT & $\mathbf{N}$ & $\mathbf{N}$ & $5.7500 E+\infty$ & 228 & $2.7300 E+02$ & $5.0000 E-02$ & 1. $3000 \mathrm{E}-02$ & $3.5490 E+00$ \\
\hline $\mathrm{TH}-228$ & GM & NT & $\mathbf{N}$ & $\ddot{x}$ & $1.9130 E+00$ & 228 & $8.2100 E+02$ & $8.0000 E-03$ & $1.6000 E+\infty$ & $1.3136 E+03$ \\
\hline PA-231 & $\mathrm{Cl}$ & NT & $N$ & $N$ & & 231 & $1.0000 E+00$ & $2.0000 E-03$ & $1.0000 E+01$ & $1.0000 E+01$ \\
\hline TH-232 & GM & NT & $\mathbf{N}$ & $x$ & $1.4100 E+10$ & 232 & 1. $1000 \mathrm{E}-07$ & $1.0000 E+06$ & $1.3000 E+01$ & $1.4300 \mathrm{E}-06$ \\
\hline $\mathrm{U}-232$ & GM & NT & Y & $x$ & $7.3600 E+01$ & 232 & $2.0985 E+01$ & $3.0000 E-02$ & $2.1000 E+00$ & $4.4068 E \div 01$ \\
\hline $\mathrm{U}-233$ & GM & $T$ & $Y$ & $\gamma$ & $1.6200 E+05$ & 233 & $9.6400 E-03$ & $1.0000 E-01$ & 4.3000E-01 & 4.1452E-03 \\
\hline TH-234 & $G M$ & NT & $\mathrm{N}$ & $\mathrm{x}$ & $6.6030 \mathrm{E}-02$ & 234 & $2.3200 E+04$ & $1.0000 E+01$ & $1.1000 \mathrm{E}-04$ & $2.5520 E+00$ \\
\hline$U-234$ & GM & NT & $Y$ & $x$ & $2.4700 E+05$ & 234 & 6. $2500 \mathrm{E}-03$ & $1.0000 \mathrm{E}-01$ & $4.3000 E-01$ & $2.6875 E-03$ \\
\hline U.235 & GM & NT & $Y$ & $Y$ & $7.1000 E+08$ & 235 & $2.1600 \mathrm{E}-06$ & 2.0000E-01 & 3.8000E-01 & 8. 2080E-07 \\
\hline $\mathrm{U}-236$ & GM & NT & $Y$ & $x$ & $2.3900 E+07$ & 236 & $6.3527 E-05$ & $2.0000 E-01$ & 4.3000E-01 & $2.7317 E-05$ \\
\hline NP-237 & GM & $T$ & $N$ & N & $2.1400 E+06$ & 237 & $7.0500 E-04$ & 5.0000E-O3 & $4.5000 E+\infty 0$ & $3.1725 \mathrm{E}-03$ \\
\hline PU-238 & GM & $T$ & $\mathrm{~N}$ & $\mathbf{N}$ & $8.6000 E+01$ & 238 & $1.7100 E+01$ & $3.0000 E-03$ & 9.1000E-01 & $1.5561 E+01$ \\
\hline $\mathrm{U}-238$ & GM & NT & $Y$ & $\mathbf{Y}$ & $4.5100 E+09$ & 238 & $3.3600 €-07$ & $1.0000 E+06$ & 3.8000E-01 & $1.2768 \mathrm{EE}-07$ \\
\hline URANIUM-DEPLETED & GM & NT & $Y$ & $Y$ & $4.5100 E+09$ & 238 & $3.6407 \mathrm{E}-07$ & $1.0000 E+06$ & 3.800OE-01 & $1.3835 E-07$ \\
\hline URANIUM-ENRICHED & $G M$ & NT & $Y$ & $\dot{Y}$ & $4.5100 E+09$ & 238 & $3.6407 \mathrm{E}-07$ & $1.0000 E+06$ & $3.8000 \mathrm{E} \cdot 01$ & $1.3835 \mathrm{E}-07$ \\
\hline $\mathrm{PE}-\mathrm{Cl}$ & $\mathrm{Cl}$ & $T$ & $\mathbf{N}$ & $\mathbf{Y}$ & $2.4400 E+04$ & 239 & $1.0000 E+00$ & $2,0000 E-03$ & 6.5500E-02 & 8.6500E-02 \\
\hline PU & $G M$ & $\mathbf{T}$ & $\mathbf{N}$ & $Y$ & $2.4400 E+04$ & 239 & 6. 2000E-02 & 2.0000E-03 & 8.5500E-02 & 5.3010E-03 \\
\hline PU-239 & GM & $T$ & $\mathbf{N}$ & $\mathrm{Y}$ & $2.4400 E+04$ & 239 & 6. 2000E-02 & 2.0000E-03 & $1.0000 E+00$ & 6. 2000E-02 \\
\hline PU239 FSL GR EQUIV & GM & $\mathbf{T}$ & N & $Y$ & $2.4400 E+04$ & 239 & $6.2000 \mathrm{E}-02$ & $2.0000 E-03$ & $8.5500 \mathrm{E}-02$ & 5.3010E-03 \\
\hline TOTAL-ALPHA & $\mathrm{Cl}$ & NT & $\mathbf{N}$ & N & $2.4400 E+04$ & 239 & $1.0000 E+\infty 0$ & $2.0000 E-03$ & $8.5500 E-02$ & 6.5500E-02 \\
\hline PU-240 & $G M$ & $T$ & $\mathbf{N}$ & $\mathbf{N}$ & $6.5800 E+03$ & 240 & $2.2700 \mathrm{E}-01$ & $2.0000 E-03$ & $1.0000 E+\infty 0$ & 2.2700E-01 \\
\hline$A M-241$ & $\mathrm{GM}$ & $\mathbf{T}$ & $\mathbf{N}$ & $\mathbf{N}$ & $4.5800 E+02$ & 241 & $3.4200 E+\infty 0$ & $8.0000 E-03$ & $2.5000 E+\infty 0$ & $8.5500 E+\infty 0$ \\
\hline PU-241 & GM & NT & $\mathbf{N}$ & $\mathbf{N}$ & $1.3200 E+01$ & 241 & $2.5000 E-03$ & $1.0000 E-01$ & $6.5920 E+O 2$ & $1.6480 E+\infty$ \\
\hline $\mathrm{CM}-242$ & GM & NT & $\mathbf{N}$ & $\mathbf{N}$ & $4.4660 E-01$ & 242 & $3.3100 E+03$ & 2.0000E-01 & $5.6000 E-02$ & $1.6536 \mathrm{E}+02$ \\
\hline PU-242 & GM & $\mathbf{T}$ & $\mathbf{N}$ & $\mathbf{N}$ & $3.7900 E+05$ & 242 & $3.9300 \mathrm{E}-03$ & $3.0000 E-03$ & $9.1000 E-01$ & $3.5763 E-03$ \\
\hline$A M-243$ & GM & $\mathbf{T}$ & N & $\mathbf{N}$ & $7.3700 E+03$ & 243 & $2.0000 E-01$ & 8.0000E-03 & $2.5000 E+\infty 0$ & 5.0000E-01 \\
\hline$C M-243$ & GM & $\mathbf{T}$ & N & $\mathbf{N}$ & 3. $2000 E+01$ & 243 & $5.1700 E+01$ & $9.0000 E-03$ & $1.7000 E+\infty$ & $8.7890 E+01$ \\
\hline CM-244 & GM & NT & $\mathbf{N}$ & $\mathbf{N}$ & 1. $7600 E+01$ & 244 & $8.1000 E+01$ & $1.0000 E-02$ & $1.3000 E+\infty$ & $1.0530 E+02$ \\
\hline CM-245 & GM & $T$ & $\mathbf{N}$ & $\mathbf{N}$ & $9.3000 E+03$ & 245 & $1.5726 \mathrm{E}-01$ & 6.0000E-03 & $2.5000 E+\infty 0$ & $3.9315 E-01$ \\
\hline
\end{tabular}


WHC-SD-W-242-ES-004, Rev. 0

TABLE 3

PHYSICAL PROPERTIES

(2 SHEETS)

\begin{tabular}{|c|c|c|c|c|c|c|c|}
\hline PHYYSICAL COMPONENT NAME & SURROGATE & NOMINAL DENSITY, LB/CU FT & HAZARDOUS & DEBAIS & ORGANIC/INORGANIC & ASH VOLUMAE FACTOR & LOUID/SOLIDNAPOR \\
\hline TO MIL LNER & PVC & 80.00 & $\mathbf{N}$ & $\mathrm{Y}$ & 0 & 0.01 & 5 \\
\hline 90 MIL LNER & PVC & 80.00 & $\frac{N}{N}$ & $\bar{Y}$ & 0 & 0.01 & $\frac{3}{2}$ \\
\hline ABSORBENT/KITY LTR/VERMICULTE & DIATOM. EARTH & 30.00 & $\mathrm{~N}$ & $\mathbf{N}$ & 1 & 1.00 & $\mathbf{s}$ \\
\hline ACID & WATER & 62.40 & $\mathbf{Y}$ & $\bar{N}$ & 1 & 1.00 & $\mathrm{~L}$ \\
\hline AlR & AIR & 0.05 & $\mathrm{~N}$ & N & 1 & 1.00 & $\mathrm{v}$ \\
\hline ALUMINUM & ALUMINUM & 165.00 & $\mathrm{~N}$ & $\mathbf{Y}$ & 1 & 1.00 & s \\
\hline ANEMAL WASTE & CALCIUM & 160.00 & $Y$ & $\overline{\mathrm{N}}$ & 0 & 0.01 & 5 \\
\hline ANTI-CORAOSIVE RADPAD & STEEL & 500.00 & $\mathrm{~N}$ & $\mathbf{Y}$ & 1 & 1.00 & s \\
\hline ANTIFREEZE & ANT\&FREEZE & 68.00 & $r$ & $\mathrm{~N}$ & 0 & 0.01 & L \\
\hline ASBESTOS & ASBESTOS & 36.00 & $\mathrm{Y}$ & $\mathrm{N}$ & 1 & 1.00 & s \\
\hline ASHES & SUUCA & 100.00 & $\dot{\gamma}$ & $\mathrm{N}$ & 1 & 1.00 & $\mathbf{s}$ \\
\hline ASPHALT/BLACKTOP & ASPHALT & 130.00 & $\mathbf{Y}$ & $\mathbf{N}$ & 0 & 0.01 & $\overline{\mathrm{s}}$ \\
\hline BATTERIES & LEAD & 710.00 & $Y$ & $\mathrm{~N}$ & $I$ & 1.00 & $\mathbf{5}$ \\
\hline BRASS METAL & BRASS & 530.00 & $Y$ & $\dot{Y}$ & 1 & 1.00 & s \\
\hline CEMENT & CEMENT & 150.00 & $\mathbf{N}$ & $Y$ & I & 1.00 & $\mathbf{s}$ \\
\hline CERAMACS & CEMENT & 150.00 & $\mathbf{N}$ & $Y$ & 1 & 1.00 & $\mathbf{s}$ \\
\hline CHARCOAL & CARBON & 97.00 & $\mathbf{N}$ & $\mathbf{N}$ & 0 & 0.01 & $\mathrm{~s}$ \\
\hline CHEMICALS & SOLVENT & 56.00 & Y & N & 0 & 0.01 & s \\
\hline CHEMSEARCH SS.80 & WATER & 62.40 & $\mathrm{~N}$ & $\mathrm{~N}$ & 0 & 0.01 & L \\
\hline CLAY & CLAY & 95.00 & N & $\mathbf{N}$ & 1 & 1.00 & $\mathbf{s}$ \\
\hline CLOTH/RAGSINYION & COTTON & 95.00 & $\mathrm{~N}$ & $Y$ & 0 & 0.01 & $\mathbf{s}$ \\
\hline CONCRETE & CONCRETE & 150.00 & $N$ & $\bar{Y}$ & 1 & 1.00 & $\mathbf{s}$ \\
\hline CONTAMINATES & SOLVENT & 97.00 & $\mathbf{N}$ & $\mathbf{N}$ & $i$ & 1.00 & L. \\
\hline CONVEB PADS & COTTON & 5.00 & $\mathbf{N}$ & $\mathbf{N}$ & 0 & 0.01 & s \\
\hline COPPER METAL & COPPER & 556.00 & N & $\mathbf{Y}$ & 1 & 1.00 & $s$ \\
\hline CORK & COAK & 10.00 & $\mathbf{N}$ & $Y$ & 0 & 0.01 & s \\
\hline COTTON/KOTEX & COTTON & 95.00 & $\mathbf{N}$ & $Y$ & 0 & 0.01 & $\mathbf{s}$ \\
\hline CREOSOIE & ASPHALT & 130.00 & $Y$ & $\mathbf{N}$ & 0 & 0.01 & L \\
\hline DIRT/SOILOIATOMACEOUS EARTH & DLATOM. EARTH & 30.00 & $\mathrm{~N}$ & $\mathrm{~N}$ & 1 & 1.00 & 5 \\
\hline DOT 34-5 POLY CONTAINER & PLASTIC & 60.00 & $\mathrm{~N}$ & $Y$ & 0 & 0.01 & s \\
\hline EPOXY/PAINT/OILBASE & SOLVENT & 56.00 & $\mathrm{Y}$ & $\mathrm{N}$ & 0 & 0.01 & $\mathbf{L}$ \\
\hline EOUIPMENT & STEEL & 500.00 & $\mathbf{N}$ & $Y$ & 1 & 1.00 & $\mathbf{s}$ \\
\hline FECES & WATER & 62.40 & $Y$ & $\mathbf{N}$ & 0 & 0.01 & $\mathbf{s}$ \\
\hline FIBERGLASS & GLASS & 140.00 & $\mathbf{N}$ & $Y$ & $i$ & 1.00 & $\mathbf{s}$ \\
\hline FiLTERS & GLASS & 140.00 & $\mathbf{N}$ & $Y$ & 1 & 1.00 & $\mathbf{s}$ \\
\hline FLOOR TILE & ASPHALT & 130.00 & $\mathbf{N}$ & $\mathbf{Y}$ & 0 & 0.01 & s \\
\hline FOAMSTTYROFOAM & STYROFOAM & 10.00 & N & $\mathbf{Y}$ & 0 & 0.01 & $\mathrm{~s}$ \\
\hline GLASS & GLASS & 140.00 & $\mathbf{N}$ & $Y$ & 0 & 0.01 & $\mathbf{s}$ \\
\hline GREASE & SOLVENT & 56.00 & $\mathbf{Y}$ & $\mathbf{N}$ & 0 & 0.01 & $\mathrm{~L}$ \\
\hline HAZARDOUS CONSTITUENTS & SOLVENT & 56.00 & $\mathrm{Y}$ & $\mathbf{N}$ & 0 & 0.01 & L \\
\hline PHYSICAL COMPONENT NAME & SURROGATE & NOMANAL DENSITY, LB/CU FT & MAZARDOUS & DEBRIS & ORGAMICINORGANIC & ASH VOLUME FACTOA & LOUID/SOUDNAAPCR \\
\hline
\end{tabular}




\begin{tabular}{|c|c|c|c|c|c|c|c|}
\hline 7 & 100 & o & $\mathbf{N}$ & $\hat{A}$ & $\infty .99$ & INBKTOS & 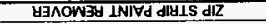 \\
\hline s & 100 & 0 & $\lambda$ & $\mathrm{N}$ & $0^{\circ} 0 \mathrm{~g}$ & $000 \mathrm{M}$ & 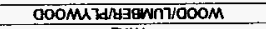 \\
\hline $\mathbf{s}$ & $\infty+1$ & 1 & N & $\mathbf{N}$ & $\infty 0^{\prime} 009$ & 79315 & $3 \mathrm{Hm}$ \\
\hline $\mathbf{s}$ & 100 & 0 & $\mathrm{~N}$ & $\mathrm{~N}$ & $\infty .05$ & $000 \mathrm{M}$ & NOIIVLIDJNSGI3M \\
\hline 7 & $\infty \div$ & 1 & $\bar{N}$ & $\mathbf{N}$ & $00 \cdot 29$ & $8 \exists \perp \nabla M$ & YIIVM \\
\hline $\mathbf{s}$ & 100 & 0 & $\mathbf{N}$ & $\lambda$ & 00051 & ב1Z3ONOJ & 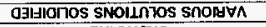 \\
\hline s & $10^{\circ} 0$ & 0 & $\lambda$ & $\mathbf{N}$ & $.00 \mathrm{9} 6$ & sषayn aganow & JNУHIBYI \\
\hline $\mathbf{s}$ & $10^{\circ} 0$ & 0 & A & $\hat{\Lambda}$ & 00.99 & 3NJIAdO\&dATOOd & SINGTUdOYdATOd TVSUGAIN \\
\hline 7 & 100 & 0 & $\mathbf{N}$ & $\bar{i}$ & 0009 & aJd & 3.USNVZII \\
\hline $\mathbf{s}$ & 100 & o & $i$ & $\mathbf{N}$ & $\infty$ sel & 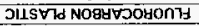 & NO7-931 \\
\hline $\mathbf{s}$ & 100 & 0 & $\mathbf{N}$ & $\lambda$ & 00081 & $17 \mathrm{VHASV}$ & HV1 \\
\hline $\mathbf{s}$ & $00 \mathrm{~L}$ & 1 & $\lambda$ & $\mathbf{N}$ & 0000 & $73 \exists \perp S$ & TEIIS SSITNIVIS \\
\hline 7 & 100 & 0 & $\mathrm{~N}$ & A & $\infty 99$ & $\perp N \exists \wedge 70 S$ & SHINNIHL/SLNGATOS \\
\hline $\mathbf{s}$ & 100 & 0 & $\mathbf{N}$ & $\mathbf{N}$ & $0 b^{\prime} 29$ & $\mathrm{y} \exists \perp \nabla M$ & $d 70 s$ \\
\hline 7 & 100 & 0 & N & $\mathrm{N}$ & Ot'Z9 & $\forall \exists \perp \forall M$ & S390ก7s \\
\hline $\mathbf{s}$ & $00 \%$ & $\mathrm{I}$ & $\mathrm{N}$ & $\mathrm{N}$ & 00.96 & anvs & ONVS \\
\hline $\mathbf{s}$ & 100 & 0 & A. & $\mathrm{N}$ & $00 \Omega L$ & y3gent & yagent \\
\hline $\mathbf{s}$ & $00^{\circ}$ & I & $\mathrm{N}$ & $\mathbf{N}$ & $000+1$ & JNOIS ONVS & $73 \wedge$ HO/XIOOU \\
\hline s & $10^{\circ} 0$ & 0 & $\mathrm{~N}$ & A & 00211 & JLSVId OTONBHA & SNISIY \\
\hline $\mathbf{s}$ & 100 & 0 & A & $\mathbf{N}$ & 0008 & JISVId & 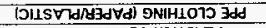 \\
\hline $\mathbf{s}$ & 100 & 0 & $\bar{N}$ & $\mathbf{N}$ & 00.96 & sषayn ago7ow & 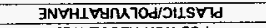 \\
\hline 7 & $10 \%$ & 0 & $\mathbf{N}$ & $\hat{A}$ & 0009 & $82 d$ & OJIVINDOA-NON $82 \mathrm{~d}$ \\
\hline 7 & $10 \%$ & 0 & $\mathrm{~N}$ & $\lambda$ & 00.09 & 8כd & gDd \\
\hline $\mathrm{s}$ & 100 & 0 & $\lambda$ & $\mathbf{N}$ & 0002 & yad $d \mathrm{~d}$ & OUVOBCYWDRJAVA \\
\hline 7 & 100 & 0 & N & $\lambda$ & $00.9 L$ & 7ซOIGIOL-INIVd & 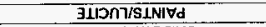 \\
\hline $\mathbf{s}$ & 001 & $i$ & N & $\mathrm{N}$ & 00.96 & aNVS & SUIOMOd/SJOIXO \\
\hline 7 & 100 & o & $\ddot{N}$ & ㅅ․ & 00.95 & LNGATOS & SDINVפYO \\
\hline 7 & $10 \%$ & 0 & N & $\hat{A}$ & 0009 & 710 & \$710 \\
\hline 7 & 100 & 0 & $\bar{N}$ & ᄉ & 00.09 & 710 & HEIVM aN \\
\hline $\mathbf{s}$ & $\overline{\mathrm{DO}} \overline{\mathrm{L}} \mathrm{L}$ & I & N & N & 00009 & 7315 & 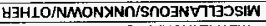 \\
\hline $\mathrm{s}$ & $00 \div$ & I & $\ddot{\Lambda}$ & $\mathrm{N}$ & 00.009 & $7 \mathrm{gIIS}$ & 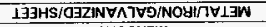 \\
\hline 7 & $00 \div$ & 1 & $\mathrm{~N}$ & A & 00.098 & 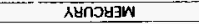 & גUกวษ \\
\hline 7 & $00 \%$ & $i$ & $\mathrm{~N}$ & $\hat{A}$ & 0099 & $\perp \mathrm{N} \boxminus \wedge 70 \mathrm{~S}$ & JINFSUO OInOก \\
\hline 7 & 100 & 0 & $\mathrm{~N}$ & $\bar{N}$ & $0+29$ & घIIVM & ainon \\
\hline$s$ & 100 & 0 & $\bar{N}$ & $\Lambda$ & 00801 & JNOLSIWIT & 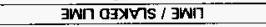 \\
\hline $\mathrm{s}$ & 001 & $T$ & $\mathbf{N}$ & $N$ & 00.005 & $73 \mathrm{BIS}$ & sg7ng $\perp$ HOT \\
\hline 7 & $10 \%$ & 0 & $\mathrm{~N}$ & 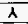 & 0009 & 710 god & ISVTVVO IHOT \\
\hline $\mathrm{s}$ & 100 & 0 & $\lambda$ & $\bar{N}$ & $0+Z 9$ & 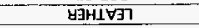 & 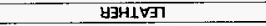 \\
\hline $\mathbf{s}$ & 00.1 & 1 & A & A & 00016 & $0 \nabla \Omega 1$ & SNITIJIHS OVIT \\
\hline s & $00 \div$ & $i$ & A & A & 00014 & ave & at97 \\
\hline $\mathbf{s}$ & 00.1 & 1 & $\Lambda$ & $\mathrm{N}$ & 00001 & ssviา & SOLSJES $\forall-N O N$ NOIIVIIISNI \\
\hline$i$ & 100 & 0 & $N$ & A & 0029 & aInT: כחกण & 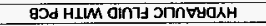 \\
\hline 7 & 100 & 0 & $\mathrm{~N}$ & A & 00.99 & IN $\exists \wedge 70$ S & 7ONVXIH \\
\hline
\end{tabular}

(SIGaHS 2)

SAIIIAJOOd TVDISXHd

$\varepsilon$ GTgVI

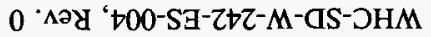


TABLE 4

STATISTICAL ANALYSIS OF HAZARDOUS MATERIALS

IN ASCENDING ORDER BASED ON CONTAINER COUNT

(* INDICATES DATA EXHIBITED NEGATIVE VARIANCE)

(10 SHEETS)

COMPONENT INAME

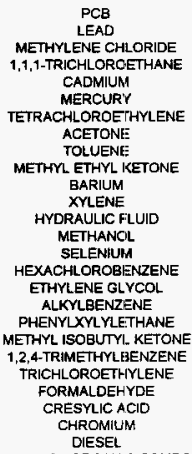

HALOGENATED ORGANIC COMPOUNDS

TETRAHYDROFURAN

1,1,1 TRICHLORCETHANE

$$
\text { ARSENIC; }
$$

SODIUM HYDROXIDE

CARBON DISULFIDE

LEAD CHROMATE

1,1,DICHLOROETHYLENE

1,4-DIOXAINE

1-BUTANOL

2-BLTANONE PEROXIDE

ACETALDEHYOE -1-2-C14

CHLOROACETIC ACID

ETHYLENE GLYCOL MONOETHYL ETHER ACETATE

HALOGENATED HYCIROCARBON

2,5-DIPHENYLOXXZOLE

2-ETHOXYETHANOL

CARBON TETRACHLORIOE

PERCHLOROETHYLENE

SODIUM CHLORIDE

ETHYL BENZZENE

CHLOROFCIRM

SILVER

OSPHORIC: ACID

4-METHYL-2-PENITANONE

HYDROCHLOR C ACID

TRIPHENYL PHCISPHATE

\begin{tabular}{|c|c|c|}
\hline COUNT & ACCUM WT, KG & AVERAGE WT. KG \\
\hline 672 & 1.3004E+03 & $2.2078 \mathrm{E}+00$ \\
\hline 423 & $9.6972 E+03$ & $2.3089 E+01$ \\
\hline 346 & $8.8554 \mathrm{E}+02$ & $2.8484 \mathrm{E}+00$ \\
\hline 327 & $4.3043 \mathrm{E}+02$ & $1.3163 E+00$ \\
\hline 279 & 4.3095E+00 & $1.5558 \mathrm{E}-02$ \\
\hline 282 & $7.9173 \mathrm{E}+02$ & $3.0219 E+00$ \\
\hline 252 & $1.2318 \mathrm{E}+03$ & $5.3006 \mathrm{E}+\infty$ \\
\hline 232 & $9.7276 \mathrm{E}+01$ & 4.1829E-01 \\
\hline 205 & $1.0789 E+03$ & $5.2886 E+\infty 0$ \\
\hline 188 & $5.8801 E+01$ & 2.9728E-01 \\
\hline 181 & $1.3756 \mathrm{E}+01$ & 7.3169E-02 \\
\hline 169 & $9.1173 E+02$ & $5.5256 E+00$ \\
\hline 160 & $37801 \mathrm{E}+03$ & 2.3501E+01 \\
\hline 154 & $4.4951 \mathrm{E}+02$ & 2.9573E+00 \\
\hline 127 & $2.4504 E+00$ & $1.9603 E-02$ \\
\hline 124 & $1.8170 E-01$ & 1. $4653 E=03$ \\
\hline 120 & $4.2835 E+02$ & $3.5779 E+\infty$ \\
\hline 117 & $2.9407 \varepsilon+03$ & $2.5134 E+01$ \\
\hline 117 & $9.4292 E+02$ & $8.0591 E+00$ \\
\hline 114 & $7.5529 E+01$ & 8.8253E-01 \\
\hline 113 & $1.7500 E+03$ & $1.5705 E+01$ \\
\hline 111 & 1.1169E+01 & 1.3789E-01 \\
\hline 99 & $1.7922 \mathrm{E}+02$ & 1.8103E+ +00 \\
\hline 97 & $7.8020 \mathrm{E}+00$ & $8.0433 \mathrm{E}-02$ \\
\hline 94 & $92783 E+01$ & $1.0085 E+00$ \\
\hline 85 & $1.3744 E+03$ & 1. $6170 E+01$ \\
\hline 78 & 7.0023E+00 & $9.3364 \mathrm{E}-02$ \\
\hline 67 & 6. $1717 \mathrm{E}+00$ & 9.2115E-02 \\
\hline 62 & $6.8513 E+00$ & 1.1050E-0 \\
\hline 61 & 8.971CE-01 & $1,4707 \mathrm{E}-02$ \\
\hline 61 & $7.1893 E+02$ & 1. 1786E+01 \\
\hline 58 & $5.0000 \mathrm{E}-04$ & 8.6207E-06 \\
\hline 58 & 3. 2057E+02 & $55271 E+00$ \\
\hline 57 & $1.0000 \mathrm{E}-04$ & 1.7544E-06 \\
\hline 56 & $0.0000 E+00$ & $0.0000 E+C 0$ \\
\hline 56 & $8.4000 E-02$ & $1.5000 \mathrm{E}-03$ \\
\hline 56 & 8. $4000 \mathrm{E}-02$ & $1.5000 \mathrm{E}-03$ \\
\hline 56 & $0.0000 E+00$ & $0.0000 E+\infty 0$ \\
\hline 56 & $0.0000 E+00$ & $0.0000 E+00$ \\
\hline 56 & $8.4000 \mathrm{E}-02$ & $1.5000 \mathrm{E}-03$ \\
\hline 55 & $3.0020 \mathrm{E}+01$ & 5.4582E-01 \\
\hline 44 & 1. $8810 \mathrm{E}+01$ & 4.2750E-01 \\
\hline 44 & 2.1729E +01 & 4.9383E-01 \\
\hline 44 & $4.8930 E+01$ & $1.1379 E+\infty 0$ \\
\hline 44 & $2.6204 E+01$ & 5.9554E-01 \\
\hline 42 & $5.3830 E+03$ & $1.2817 \mathrm{E}+02$ \\
\hline 40 & 2.3791E+01 & $5.0477 \mathrm{E}-01$ \\
\hline 39 & 1.3575E+ +0 & $3.4808 E-02$ \\
\hline 39 & 6.3271E+01 & $1.6223 E+00$ \\
\hline 38 & $8.2361 E+00$ & 2.1674E-01 \\
\hline 38 & 2.3646E+02 & $6.3908 E+\infty 0$ \\
\hline 37 & $4.44335+\infty 0$ & 1.2009E-01 \\
\hline 37 & $2.1803 E+02$ & $5.8386 E+\infty 0$ \\
\hline 37 & $4.2252 \mathrm{E}+02$ & $1.141 \mathrm{gE}+01$ \\
\hline 36 & $6.9418 E+00$ & $1.9283 E-01$ \\
\hline 35 & $3.5130 \mathrm{E}+02$ & $1.0332 \mathrm{E}+01$ \\
\hline 32 & 4.2693E+01 & $1.3341 E+\infty 0$ \\
\hline 32 & $6.2801 E+01$ & $1.9825 E+00$ \\
\hline 30 & $0.0000 E+00$ & $0.0000 E=00$ \\
\hline 30 & 1.7412E+02 & $5.8040 E+00$ \\
\hline 30 & $1.1565 E+02$ & $3.8549 E+\infty 0$ \\
\hline 29 & 7.85COE-02 & $2.7069 \mathrm{E}-03$ \\
\hline 28 & $5.0408 E+02$ & 1.8003E+01 \\
\hline 27 & $4.2385 E+01$ & $1.5698 \mathrm{E}+\infty$ \\
\hline 27 & $1.5031 E+01$ & 5.5669E-01 \\
\hline 27 & $7.7100 E+01$ & $2.8556 \varepsilon+00$ \\
\hline 25 & $1.2879 E+00$ & S.1516E-02 \\
\hline 24 & $2.3508 E+00$ & $9.7950 E-02$ \\
\hline 24 & $3.9537 \mathrm{E}+01$ & $1.6474 \mathrm{E}+00$ \\
\hline 24 & $9.7828 \mathrm{E}+01$ & 4. $2534 \mathrm{E}+00$ \\
\hline 22 & $4.9230 \mathrm{E}+01$ & $2.2377 \mathrm{E}+\infty 0$ \\
\hline 22 & $1.3149 \mathrm{E}+02$ & $6.2612 E+00$ \\
\hline 21 & $2.8558 \mathrm{E}+01$ & $1.3599 E+00$ \\
\hline 21 & $9.8990 E \div 01$ & $4,7138 \mathrm{E}+00$ \\
\hline 20 & $63077 E+01$ & $3.1538 E+00$ \\
\hline 20 & $5.5500 E-01$ & $2.7750 E-02$ \\
\hline 20 & 1.5220E+01 & 7.6102E-01 \\
\hline
\end{tabular}
BENZENE

POTASSIUM CHROMATE

1,1-D|CHLOROETHYLENE

POTASSIUM HYDROXIDE

1,1. OICHLOROETHENE

M-XYLENE

NITRIC ACID

VWYL CHLORIDE

BASE METAL (LEAD)

2-BUTOXYETHANOL

CHLORINATED FARAFFIN

$$
\text { O-XYLENE }
$$

PHTHALIC ACID BIS(Z. ETHYYLHEXYL)ESTER

CARBON DICXIOE

NAPTHALE:NE

SODIUM CAREONATE

DIOCTYL PHTHALATE (DOP)

SODIUM SILICATE

METHYL ALCOHOL

NONYLPHENOXYPCLY ETHANOL ETHANCL

NICKEL HYDFOXIDE
STD DEV

$9.7569 E+00$

$9.0650 \mathrm{E}+01$

$1.1395 \mathrm{E}+01$

$5.534 E+\infty$

1.0011E-01

$1.7446 \mathrm{E}+01$

1.3469E+01

$2.2971 \mathrm{E}+0$

Q.6828E+00

1.1416E+00

1.8773E-01

6.8922E+00

1.5485E+01

$1.2481 E-01$

1.2481E-0

$5.6590 E-04$
$9.0760 E+00$

1.1380E+01

$5.8717 E+\infty 0$

$3.1688 E+\infty$

1.1349E+ 01

$5.6481 E-01$

$1.5786 E+\infty 0$

$1.4672 \mathrm{E}-01$

$1.5848 \mathrm{E}+00$

$1.3+56 E+01$

4.1183E-02

4.1547E-01

1.1051E-01

4. $1010 \mathrm{E}-02$

$2.0976 \mathrm{E}+01$

5.3900E-05

1.2519E+OI

1.3200 E.05

$0.0000 E+D 0$

$0,0000 E+\infty 0$

$0.0000 E+00$

$0.0000 E+00$

$0.0000 E+00$

$0.0000 E+00$

$1.6162 E+\infty 0$

$32711 E-01$

5.2079E-01

$5.5655 \mathrm{E}+\infty 0$

4.5976E.01

7.2011E+01

5.8377E-01

1.1157E-01

$5.3308 E+\infty 0$

5.7172E-01

$10495 \mathrm{E}+01$

$15422 E-01$

$1.5108 E+01$

$1.7085 \mathrm{E}+00$

2.8315E-01

$2186 \mathrm{E}+00$

$3.8266 \mathrm{E}+\infty 0$

$0.0000 \mathrm{E}+\infty 0$

$6.8007 \mathrm{E}+00$

$6.3893 E+0 D$

1.3905E-02

$3.6768 E+01$

$1.5110 \mathrm{E}+00$

7.2056E-01

$4.9325 E+00$

6.9293E-02

6.7088E-02

$1.6527 E+\infty$

3.2957E +00

1. $7554 E+00$

$1.0664 E+01$

$1.1891 E+00$

$3.4629 \mathrm{E}+00$

B. $1383 E+\infty$

9.1459E-O2

$3.3515 E+00$
MINIMUM WT, KG

$0.0000 E+00$

$0.0000 E+00$

$0.0000 E+\infty 0$

$0.0000 E+00$

$0.0000 E+00$

$0.0000 E+00$

$0.0000 E+\infty 0$

$0.0000 E+00$

$0.0000 E+\infty 0$

$2.0000 \mathrm{E}-04$

$1.0000 E-0$

8.0000 E-04

1.0000 E. 04

$0.0000 E+00$

$2.0000 \mathrm{E}-\mathrm{OA}$

3.6000E-0

9.1000E-0

2.3000E-01

1.0000E-04

6. $1000 \mathrm{E}-03$

$0.0000 E+00$

$0.0000 E+00$

1.0000 E- 04

$0,000 \mathrm{E}+\infty$

$3.3000 E+\infty 0$

$5.0000 \mathrm{E}-02$

$0.0000 E+\infty 0$

$1.0000 \mathrm{E} .04$

$0.0000 E+00$

2 . $0000 \mathrm{E}-02$

$0.0000 E+00$

B. 2000E-03

$0.0000 E+00$

$0.0000 \mathrm{E}+00$

15000 E-DO

15000 E-03

$0.0000 E+\infty 0$

$0.0000 \mathrm{E}+00$

$1.5000 \mathrm{E}-03$

$4.3000 E-03$

2.0000E-02

$1.0000 \mathrm{E}-04$

$1,1000 E-03$

$9.0000 \mathrm{E}-03$

$2.5000=03$

0 .

$1.0000 \mathrm{E}-04$

$1.0000 \mathrm{E}-03$

2. $1000 \mathrm{E}-03$

$1.0000 \mathrm{E}-04$

$0,0000 \mathrm{E}+\infty$

$4.00005-03$

$1.3200 \mathrm{E}+\infty$

$0.0000 E+\infty 0$

$8.4000 \mathrm{E}+00$

$10000 E-04$

$1,0000 E-02$

$0.0000 E+00$

$0.0000 \mathrm{E}+00$

2.1000E-02

1.0000 E.04

$3.0000 \mathrm{E}-\mathrm{OA}$

$0,0000 E+\infty 0$

$0.0000 E+00$

$0.0000 E+00$

9.0000 E- 0.4

B.0000E-04

$1.0000 \mathrm{E}-02$

8.0000 E- 04

3.0000E-02

$5.0000 E-02$

1. $4000 \mathrm{E}-02$

$4.0000 \mathrm{E}-02$

$1.5000 \mathrm{E}-02$

$0.0000 E+00$

7.3000E-03

MAXIMUM WT, KG

$1.0260 E+02$

$1376 E+03$

$7.5940 \mathrm{E}+01$

4.8280E +04

$1.2800 E+\infty$

$1.1950 E+02$

8.6130E+01

4.7000E+01

$1.1420 E+01$

1.2800E +00

$2.5000 E+01$

4.1000E+01

$3.9200 E+01$

$1.0002 E+\infty$

2.3000E-03

4.1900E +01

B.28B0E +01

$17300 \mathrm{E}+01$

3.3000E +01

$5.7046 E+01$

$5.8500 E+\infty$

$1.1810 E+01$

$1.0800 E+00$

$4.7000 E+\infty 0$

$4.0000 E+01$

4.5360E-01

$2.9800 \mathrm{E}+00$

3.9000 E- 09

$3.0000 E-01$

$7.6500 E+01$

$4.0000 \mathrm{E}-04$

$5.1392 \mathrm{E}+01$

$1.0000 \mathrm{E}-04$

$0.0000 E+\infty$

$1.5000 \mathrm{E}-03$

$1.5000 E-03$

$0.0000 E+00$

$0.0000 E+00$

1.5000E-03

$7.2983 E+00$

1. $2000 \mathrm{E}+00$

$2.1200 E+00$

$3.6800 \mathrm{E}+01$

$1.5598 \mathrm{E}+00$

$1.9000 E+02$

$1.7000 \mathrm{E}+00$

$5.0000 \mathrm{E}-01$

2.2400E+01

$3.3300 \mathrm{E}+00$

4.9086E+01

8.7000E-01

9.1949E+0 
TABLE 4

STATISTICAL ANALYSIS OF HAZARDOUS MATERIALS

IN ASCENDING ORDER BASED ON CONTAINER COUNT (* INDICATES DATA EXHIBITED NEGATIVE VARIANCE)

(10 SHEETS)

COMPONENT NAME

$$
\text { P.XYLENE }
$$

NONYPHENOXYPOLY (ETHYLENEOXY) ETHANOL THENOYLTRIFLUOROACETONE

DIOCTM PHTHALATE

\begin{tabular}{|c|c|c|}
\hline COUNT & ACCUM WT, KG & AVERAGE WT. KG \\
\hline $\begin{array}{l}20 \\
20\end{array}$ & $\begin{array}{l}2.4201 E+01 \\
4.8681 E+\infty 0\end{array}$ & $\begin{array}{l}1.2145 E+00 \\
2.4341 E-01\end{array}$ \\
\hline 10 & $3.0684 E+02$ & $1.6091 E+01$ \\
\hline 19 & $2.4001 E+02$ & $1.2632 E+01$ \\
\hline 10 & $6.9593 E+01$ & $3.6628 \varepsilon+\infty 0$ \\
\hline 18 & $1.0370 E+01$ & $5.4581 E-09$ \\
\hline 10 & $5.3720 \mathrm{E}-01$ & 2.8274E-02 \\
\hline 18 & 6.3150E-01 & $3.5003 E-02$ \\
\hline 17 & $1.0284 E+01$ & $6.0492 E-01$ \\
\hline 17 & $0.0000 E+00$ & $0.0000 \mathrm{E}+00$ \\
\hline 17 & $1.0007 E+02$ & $5.8865 E+\infty 0$ \\
\hline 16 & $7.1030 E+01$ & $4.4304 E+\infty 0$ \\
\hline 16 & $3.2072 E+02$ & $2.0045 E+01$ \\
\hline 16 & 2.1680E+01 & $1.3550 \mathrm{E}+00$ \\
\hline 16 & $8.0499 E+00$ & $5.0312 E-01$ \\
\hline 15 & 2.6539E+01 & $1.7693 E+\infty 0$ \\
\hline 15 & $3.3131 E+02$ & $2.2087 \mathrm{E}+01$ \\
\hline 15 & $7.5000 E+00$ & $5.0000 \mathrm{E}-01$ \\
\hline 15 & $6.8721 E+01$ & $4.5814 E+00$ \\
\hline 14 & $1.5530 E+01$ & 1. $1093 \mathrm{E}+00$ \\
\hline 14 & $3.6960 E+C 0$ & $2.6400 \mathrm{E}-01$ \\
\hline 14 & $4.2237 E+\infty$ & $3.0169 E-01$ \\
\hline 14 & $3.8087 E+01$ & $2.7205 E+\infty 0$ \\
\hline 14 & $1.9775 E+02$ & $1.4125 \mathrm{E}+01$ \\
\hline 13 & $9.5095 E+01$ & $7.3150 E+\infty 0$ \\
\hline 13 & $5.1658 \mathrm{E}+00$ & 3.9737E.01 \\
\hline 13 & $8.6509 E+00$ & $7.2081 E-01$ \\
\hline 13 & $9.5684 E+00$ & 7.3603E-01 \\
\hline 13 & $3.4384 E+02$ & $2.6449 \mathrm{E}+01$ \\
\hline 13 & $2.6196 \mathrm{E}+01$ & $2.0150 E+\infty$ \\
\hline 13 & $2.6105 E+02$ & $2.0081 E+01$ \\
\hline 13 & $8.0855 E+01$ & $6.2196 \mathrm{E}+00$ \\
\hline 13 & $6.3527 E+01$ & $4.8867 E+00$ \\
\hline 13 & 2.2410E-01 & $1.7238 E-02$ \\
\hline$\$ 2$ & $3.4610 \mathrm{E}+01$ & $2.8842 \mathrm{E}+00$ \\
\hline 12 & $5.2982 \mathrm{E}+00$ & $4.4152 \mathrm{E}-01$ \\
\hline 12 & $8.8080 E+01$ & $5.6717 E+\infty$ \\
\hline 12 & $7.2790 \mathrm{E}+01$ & $0.0658 \mathrm{E}+00$ \\
\hline 12 & $1.8870 \mathrm{E}+01$ & $1.5725 \mathrm{E}+\infty$ \\
\hline 12 & $1.1800 \mathrm{E}+01$ & 9.8333E-04 \\
\hline 11 & 2.4240E-01 & 2.2036E-02 \\
\hline 11 & $3.7040 E+01$ & $3.3873 E+00$ \\
\hline 11 & $6.4085 \mathrm{E}+0 \mathrm{t}$ & $5.8250 E+\infty 0$ \\
\hline 10 & $1.5288 E+01$ & $1.5288 E+00$ \\
\hline 10 & $2.3380 \mathrm{E}+01$ & $2.3380 \mathrm{E}+00$ \\
\hline 10 & $4.7007 E+00$ & 4.7007E. 01 \\
\hline 10 & $3.4090 E+01$ & $4.2613 E+00$ \\
\hline 10 & $1.9000 E+02$ & $1.9000 E+01$ \\
\hline 9 & $1.6300 \mathrm{E}+00$ & 1.8111E-01 \\
\hline 9 & $7.4609 E+00$ & $8.2899 E-01$ \\
\hline 9 & $8.0591 E+01$ & B.9545E+DO \\
\hline 9 & $1.6892 \mathrm{E}+00$ & 1.8769E-0t \\
\hline$g$ & 5.1040E+01 & $5.6711 E+\infty$ \\
\hline 9 & $6.8180 E+00$ & 9.7400E-01 \\
\hline 8 & $9.3929 E+\infty 0$ & $1.1741 E+00$ \\
\hline 8 & $3.7239 E+00$ & 4.6549E-01 \\
\hline 8 & $0.0000 E+\infty$ & $0.0000 E+00$ \\
\hline 8 & $1.0750 \mathrm{E}-0.1$ & $1.3438 E-02$ \\
\hline 8 & $7.6660 E+00$ & $1.0951 E+00$ \\
\hline 8 & $0,0000 E+\infty 0$ & $0.0000 E+00$ \\
\hline 8 & $0.0000 E+00$ & $0.0000 E+00$ \\
\hline 8 & $4.2700 E+00$ & 5.3375E-01 \\
\hline 8 & $0.0000 E+00$ & $0.0000 E+\infty 0$ \\
\hline 8 & $2.6780 E+02$ & $3.3475 E+01$ \\
\hline 8 & $2.2960 \mathrm{E}+01$ & $2.8700 E+\infty$ \\
\hline 8 & 1.3168E+02 & $1.6460 E+01$ \\
\hline 8 & $0.0000 E+\infty 0$ & $0.0000 E+00$ \\
\hline 8 & $2.7110 E+01$ & 3. $3888 E+00$ \\
\hline 8 & $89048 E+00$ & $1.1131 E+00$ \\
\hline 8 & $0.0000 E+00$ & $0.0000 E+00$ \\
\hline 8 & 4. $9378 E+00$ & $6.1723 E-01$ \\
\hline 7 & $1.7402 E+00$ & $2.4860 \mathrm{E}-01$ \\
\hline 7 & $4.8231 E+\infty 0$ & $7.0330 \mathrm{E}-01$ \\
\hline 7 & $1.0410 E+\infty$ & $1.4871 E-01$ \\
\hline 7 & $20160 E+\infty 0$ & $2.8800 \mathrm{E}-01$ \\
\hline 7 & $2.9780 E+01$ & $4.2543 E+00$ \\
\hline 7 & 1. $6830 E+01$ & $2.4043 E+00$ \\
\hline
\end{tabular}

STD DEV

2.4545E+00

1.1658E-01

$8.0851 E+00$

$1.1226 E+01$

$3.7049 E+00$

$7.3022 \mathrm{E}-01$

3.4204E-02

6.7207E-O

$0.0000 E+00$

$7.5808 \mathrm{E}+00$

$2.4277 \mathrm{E}+\infty$

1.2465E+01

$4.6200 \mathrm{E}+\infty$

$9.7250 \mathrm{E}-01$

1.1278E+00

$2.2640 \mathrm{E}+01$

$0.0000 E+00$

$8.6346 \mathrm{E}+00$

7.5543E-01

1.8088E-01

6.5941E.01

$1.3633 \mathrm{E}+00$

$1.5558 \mathrm{E}+01$

$4.0173 E+\infty$

$8.0389 E-01$

8. 4543E-01

8. 1652E-01

1. $6830 \mathrm{E}+01$

$4.4205 \mathrm{E}+00$

$25269 \mathrm{E}+01$

$1.7699 \mathrm{E}+00$

$1.5142 E+00$

$1.5729 \mathrm{E}-02$

$4.0049 \mathrm{E}+00$

$5.0188 E-01$

2.2417E +00

$1.7259 E+\infty 0$

$3.0433 E+\infty$

$4.3567 \mathrm{E}-01$

$6.5130 E-03$

$4.6356 \mathrm{E}+00$

$5.6532 E+00$

$4.0844 E+00$

$4.5812 \mathrm{E}+00$

$1.4862 E+00$

$0.0000 \mathrm{E}+00$

$1.0708 \mathrm{E}-01$

8.4502E-01

$1.8132 E+01$

2.6910E-01

2.280tE+00

$1.1684 \mathrm{E}+00$

$1.1890 \mathrm{E}+00$

$5.1464 \mathrm{E}-01$

$0.0000 \mathrm{E}+00$

$1.2521 E-02$

1. $3923 E+C O$

$0.0000 E+\infty$

$0.0000 E+\infty 0$

4.3293E-01

$0.0000 \mathrm{E}+00$

$6.3687 \mathrm{E}+\infty 0$

3.2253E+00

$5.6909 \mathrm{E}+00$

$0.0000 E+00$

$7.1573 E+00$

t. $4593 E+\infty 0$

$0.0000 E+\infty 0$

$5.6178 \mathrm{E}-01$

1.2858E-01

$8.607 E-01$

3.3616E-01

6.6740E-O

$2.3558 \mathrm{E}+\infty 0$

$2,3461 \mathrm{E}+\infty$
MINIMUM WT, KC

MAXIMUM WT, KC

$0.0000 E+00$

$1.2000 E-03$

$7,00005-02$

7.4980E-01

$3.6000 E-02$

$3.0000 E-04$

$0.0000 E+00$

$0.0000 E-04$

$3.0000 \mathrm{E}-03$

$0.0000 E+00$

7.5000E-0

4.6000E-01

$0.2000 \mathrm{E}-02$

2.0000E-01

$1.0000 E-02$

6.5000E-02

$1.0002 E+00$

5.0000E-01

1.1040E-01

2.7000E-01

1.4000E-02

3.0400E-02

$1.0002 E+00$

1.0000 E- 04

5.0000E-01

3.0000E-04

4.7000E-0.2

1.1800E-02

$3.0000 \mathrm{E}-01$

7.0000E-03

$4.0002 E+00$

$2.0384 E+\infty 0$

$2.0384 E+00$

$1.0000 \mathrm{E}-04$

1.7000E-01

1.0000 E-02

$2.8609 E+00$

$1.8000 \mathrm{E}+00$

$1.0000 \mathrm{E}-03$

4.0000E-02

2.4000E-03

7.3000E-0

1.0000 E.02

$1,3000 E-03$

$0.0000 E+\infty 0$

1. $4100 E+00$

$1.8000 \mathrm{E}+01$

3.0000E-02

$1.0000 E-04$

5.3000E-03

$0.0000 E+00$

$1.3200 E+00$

$5.0000 \mathrm{E}-02$

$1.3000 \mathrm{E}-02$

$4.4000=03$

$0.0000 E+00$

1.0000 E. 04

7.0000E-03

$0.0000 E+00$

$0.0000 \mathrm{E}+00$

$7.0000 \mathrm{E}-02$

$0.0000 \mathrm{E}+00$

1.8200E+01

2.5000E-01

$9.7800 E+00$

$0.0000 \mathrm{E}+00$

$2.3000 E-03$

4. OBOOE.02

$0.0000 \varepsilon+00$

1.0000 E-02

2.0000 E. 01

2.4000E-03

2.0000E-02

6.0000E-03

8.9900E-01

6.8000E-01

6. $5500 \mathrm{E}+\infty 0$

3.0000E-01

$3.34105+01$

4.1998E+01

$1.1000 E+01$

2.3000E $+\infty 0$

$1.05005-01$

.5454E +00

$0.0000 E+\infty 0$

$2.7760 E+01$
$7.0000 E+00$

$3.5800 \mathrm{E}+01$

1.8680E+0\%

$4.0000 E+\infty 0$

$3.8301 E+\infty 0$

8.3500E+O1

5.0000E-01

2.7000E +01

2. $1000 E+\infty 0$

$5.0000 \mathrm{E}-01$

2. $4300 E+\infty 0$

$5.9738 E+\infty 0$

3.1210E+01

1. $4601 E+01$

$3.0000 E+\infty 0$

$3.4200 E+\infty 0$

2.2627E+ +0

4. $0100 E+01$

1.6140E+01

$1.0002 E+02$

8.1283E+ 00

$7.8925 E+00$

42000 E.02

$9.6000 \mathrm{E}+00$

$1.6000 \mathrm{E}+00$

$4619 \mathrm{E}+00$

8.0300E +00

$1.1000 \mathrm{E}+01$

$1.3600 \mathrm{E}+\infty$

$24000 \mathrm{E}-02$

$1.4500 E+01$

$1.5300 E+01$

2. $4970 E+\infty$

$1.5000 \mathrm{E}+01$

$4.7000 \mathrm{E}+00$

$7.4000 \mathrm{E}+00$

$1.9000 \mathrm{E}+01$

4.0000E-01

2.1200E +00

$5.2344 E+01$

$6.4000 \mathrm{E}-01$ 
TABLE 4

STATISTICAL ANALYSIS OF HAZARDOUS MATERIALS IN ASCENDING ORDER BASED ON CONTAINER COUNT (* INDICATES DATA EXHIBITED NEGATIVE VARIANCE)

(10 SHEETS)

\author{
COMPONENT NAME \\ ECOLITE \\ HYDRAULIC OLL \\ LEAD SOLUTION \\ LECTRA CLEANER \\ LIOUID WASTE \\ MAGNESIUM CHLORIDE \\ NAPHTHALENE \\ POLYCHLORINATED BIPHENYLS (PCE) \\ POTASSIUM CHLORIDE \\ TRI BUTYL PHOSPHATE \\ TRIBUTYL PHOSPHATE \\ COAL TAR PITCH \\ DIETHYLENE GLYCOL \\ DIMETHYL SULFOXIDE \\ HEXANOL \\ KETONE \\ LEAD BRICK \\ LEAD CHROMATE MOLYBDATE \\ LIGROINE \\ METHYLTRICAPRYLYLAMMONIUM CHLORIDE \\ MIBK \\ POLYCHLORINATED BIPHENYLS \\ PROPYLENE GLYCOL \\ ZINC CHLORIDE \\ $1,1,1$-TRICHLORETHANE \\ ACETONITRILE \\ ALUMINUM NITRATE \\ AMMONIUM CITRATE \\ ASBESTOS \\ BORIC ACID \\ CARBONIEIRACHLORIDE \\ CHROMIC ACID \\ D.TAR \\ DIMETHYLSULFOXIOE \\ ETHYL ACETATE \\ FORMALIN \\ KLEEN-O-BOWL \\ LEAD CHROMATE, CHLORIN.PARAFFIN \\ METHLYISOBUTYL KETONE \\ PERCHLORIC ACIO \\ PYRIDINE \\ SODIUM METASHLICATE \\ TURCO \\ (1-BLTYLOCTYL)BENZENE \\ (1-ETHYLDECYL) BENZENE \\ (1-METHYLUNDECYL)BENZENE \\ (1-PENTYLHEPTYL)BENZENE \\ (1.PROPYLNONYL)BENZENE \\ OXYLENE \\ 1,1,1 TRICHLORETHANE \\ 1,2 BUTLYENE OXIDE \\ 1.4BIS2-5PHENYLOXAZOOLYLBENZENE \\ 2 BUTOXYETRIANOL \\ 2-METHMNAPHTHALENE \\ 2-PROPANONE \\ AMMONIUM SULFATE \\ BUTYL ALCOHOL \\ CALCIUM CHLORIDE \\ CARBON BLACK \\ CESIUM CHLORIDE \\ CHLORINATED HYDROPARAFFIN \\ COAL TAR CREOSOTE \\ CRESOLS \\ DIETHYLENETRIAMINE \\ ETHYL ETHER \\ FLUORS \\ INDENE \\ IRON \\ ISOAMY ALCOHOL \\ ISOPROPYL ACETONE \\ D-CRESOL \\ PENTAERYTHRITOL \\ POLY ARO HYDROCARBONS \\ POLYOXYALKYLENE GLYCOL \\ POTASSIUM PERMANGANATE
}

\begin{tabular}{|c|c|}
\hline DUNT & ACCUM WT, KG \\
\hline 7 & $3.8567 \mathrm{E}+02$ \\
\hline 7 & $1.0000 \mathrm{E}-01$ \\
\hline 7 & $0.0000 E+\infty 0$ \\
\hline 7 & $1.8816 E+02$ \\
\hline 7 & 3.5112E+02 \\
\hline 7 & $3.5000 E-01$ \\
\hline 7 & $7.0400 \mathrm{E}-01$ \\
\hline 7 & 4. $9580 E+00$ \\
\hline 7 & 4. $3230 \mathrm{E}+00$ \\
\hline 7 & $1.2224 E+02$ \\
\hline 7 & 2.3768E+01 \\
\hline 7 & $2.8730 E+00$ \\
\hline 6 & $1.8520 E+01$ \\
\hline 6 & $1.2874 E+01$ \\
\hline 6 & $1.1393 E+02$ \\
\hline 6 & $6.1410 \mathrm{E}+01$ \\
\hline 6 & $3.6001 E+00$ \\
\hline 6 & $3.1612 E+02$ \\
\hline 6 & 4. $0370 E+C 0$ \\
\hline 6 & 6.5000E.01 \\
\hline 6 & $7.2820 E+00$ \\
\hline 6 & $3.8740 E+00$ \\
\hline 6 & $3.2720 E+01$ \\
\hline 6 & $2.4909 E+00$ \\
\hline 6 & $1.7151 E+\infty 0$ \\
\hline 5 & $4.4100 \mathrm{E}-02$ \\
\hline 5 & $1.2540 \mathrm{E}+01$ \\
\hline 5 & $1.2855 E+01$ \\
\hline 5 & $5.2432 E+01$ \\
\hline 5 & $3.8931 E+01$ \\
\hline 5 & $1.7899 \mathrm{E}+00$ \\
\hline 5 & $4.9728 E+00$ \\
\hline 5 & 1.3658E+01 \\
\hline 5 & $1.5510 \mathrm{E}+00$ \\
\hline 5 & $2.6450 \mathrm{E}+01$ \\
\hline 5 & $1.0649 E+02$ \\
\hline 5 & $2.7769 E+00$ \\
\hline 5 & $5.0000 \mathrm{E}+00$ \\
\hline 5 & $8.6500 E+00$ \\
\hline 5 & $8.0001 E+00$ \\
\hline 5 & $1.5800 E+00$ \\
\hline 5 & $1.2326 \mathrm{E}+00$ \\
\hline 5 & $2.9740 E+\infty 0$ \\
\hline 5 & $1.1229 \mathrm{E}+01$ \\
\hline 5 & $2.6450 \mathrm{E}+01$ \\
\hline 4 & $1.8800 E+00$ \\
\hline 4 & $1.6900 E+00$ \\
\hline 4 & $1.8800 E+00$ \\
\hline 4 & $4.9700 \mathrm{E}+00$ \\
\hline 4 & $1.4500 E+00$ \\
\hline 4 & $5.4447 E+00$ \\
\hline 4 & $1.3090 \mathrm{E}-0.1$ \\
\hline 4 & $2.6559 E+00$ \\
\hline 4 & 1. $4000 E-03$ \\
\hline 4 & $3.3800 E+00$ \\
\hline 4 & 4.0000E-02 \\
\hline 4 & $4.7035 E+00$ \\
\hline 4 & $1.7670 E+01$ \\
\hline 4 & $8.0900 E-01$ \\
\hline 4 & $20949 E+01$ \\
\hline 4 & $6.6500 \mathrm{E}-01$ \\
\hline 4 & 6.3800E-02 \\
\hline 4 & 2.3405E +01 \\
\hline 4 & 1.0410E+01 \\
\hline 4 & 3.8500 E-01 \\
\hline 4 & $4.1400 E+\infty 0$ \\
\hline 4 & 1.5000E-02 \\
\hline 4 & 3.3240E+01 \\
\hline 4 & $1.0314 E+01$ \\
\hline 4 & 6. $4272 E+00$ \\
\hline 4 & 4. 4008E+01 \\
\hline 4 & $1.5330 E+01$ \\
\hline 4 & 2.3340E-01 \\
\hline 4 & $5.2000 E+01$ \\
\hline 4 & $2.2000 \mathrm{E}-02$ \\
\hline 4 & 1. $2000 E+02$ \\
\hline 4 & $2.8265 E+01$ \\
\hline
\end{tabular}

\author{
AVERAGE WT. KG
}

$5.5096 \mathrm{E}+01$

$3.3333 \mathrm{E}-02$

$0.0000 E+00$

2. $6880 \mathrm{E}+01$

5. $0160 E+01$

5.0000E-02

1.0057E-01

27971E-01

6.1757E.01

1.7482E+01

$3.8613 \mathrm{E}+00$

4.7883E-01

$3.0867 E+\infty$

$2.1456 E+\infty O$

$2.2785 E+01$

$1.0235 \mathrm{E}+01$

6.0002E-01

$5.2686 E+01$

$6.7283 E-01$

1.0833E-01

$1.2137 \mathrm{E}+00$

6.4567E-01

$5.4533 E+\infty 0$

4.1665E-01

2.8585E-01

6.2200E-03

$2.5080 \mathrm{E}+00$

$2.5711 \mathrm{E}+00$

$1.0488 \mathrm{E}+01$

$7.7862 \mathrm{E}+00$

3.5798E-01

9.9458E-01

4.5527E+00

3. 1020E-04

$5.2900 \mathrm{E}+00$

$2.1297 \mathrm{E}+01$

5.5538E-01

$1.0000 E+\infty 0$

1. $6000 \mathrm{E}+00$

3. 1600E-O1

2. 4652E-01

$5.9480 E-01$

$3.7431 E+00$

$5.2900 \mathrm{E}+00$

4.7000 E. 01

4. 2250E-01

4. $7000 \mathrm{E}-01$

4. $9250 \mathrm{E}-01$

3.6250E-01

$13812 E+00$

3.2725E-02

6.8398E-01

3.5000E-04

8.4500 E- 01

$1.0000 \mathrm{E}-02$

$1.1759 E+\infty 0$

4.4475E+00

2.2725E.01

5. $2372 E+00$

1.6625E-01

$1.5950 \mathrm{E}-02$

$5.8512 E+00$

$2.6025 E+\infty$

$8.6250 \mathrm{E}-02$

$1.0350 E+00$

3.7500E-03

8. $3100 E+00$

2.5785E + 00

1. $6068 E+\infty 0$

1.1002E+01

$3.8325 E+00$

5.8350E-02

1. $3000 \mathrm{E}+01$

5.5000 E-03

3. $0000 \mathrm{E}+01$

$6.5663 \mathrm{E}+0 \mathrm{O}$
STD DEV

2.4390E +01

$1.9245 \mathrm{E}-02$

$0.0000 \mathrm{E}+00$

$2.7776 \mathrm{E}+01$

1.2651E+01

B. 1990E-02

$28712 \mathrm{E}-01$

7.4934E-01

2.8388E+01

$1.1137 E+\infty 0$

$3.7572 \mathrm{E}-01$

$1.8562 E+00$

$2.0730 E+\infty 0$

$2.4762 \mathrm{E}+01$

2.0976E-01

1.7809E+01

7.08B1E-01

8.230BE 02

0.3983E-01

$1.5708 \mathrm{E}+00$

4.0784E-02

$3.5398 E-01$

5.7550E-03

5.4353E-01

$21305 E+01$

2.1305E+01

1.5269E-01

1.1712E+0O

$6.8127 \mathrm{E}-01$

3.8107E-01

$0,0000 E+\infty$

$6.4643 E+\infty$

4. 3090E-0 1

$0.0000 E+00$

5.475BE-01

4.2190E-02

4.2271E-01

9.7040E-01

$0.0000 E+00$

$2.0000 E-02$

$1.5000 \mathrm{E}-02$

2.0000 E-O2

5.0000E-03

2.5000E-02

$2.0543 E+00$

5.1133E-02

1. $1591 \mathrm{E}+00$

1.0000E-04

1.1267E $+\infty$

$0.0000 E+00$

$1.2878 E+\infty 0$

$7.4630 E+00$

2.2486E. 01

$5.5503 E+\infty 0$

$1.5834 E .01$

$1.8724 E-02$

$1.0981 \mathrm{E}+\infty 0$

2.8687E+00

1.7500 E- 02

5.4641E.01

5.0000E-04

7.0057E-01

$1.6300 \mathrm{E}+\infty$

$1.9861 \mathrm{E}+00$

$1.0516 \mathrm{E}+01$

$3.5660 \mathrm{E}+00$

4 5633E-02

$0.0000 \mathrm{E}+00$

1.0000E-03

$0.0000 E+00$

1.2360E+OF

MINHMUM WT, KG

MAXIMUM WT, KG

3.7040E-0

$0.0000 E+00$

$0.0000 E+00$

$1.0002 E+00$

2.2870E+01

5. OOOOE- 02

1.0000E-02

2.5000E-02

6.0000E-03

8.5500 E-02

B. $5000 E-02$

1.7000E-02

$1.3600 \mathrm{E}+\infty 0$

$1.0300 E-01$

1.6601E+01

5.0000 E-02

2.0000E-01

3. $3861 \mathrm{E}+01$

1.800OE-01

$5.8000 E-03$

$9.0000 E-02$

$0.0000 E+00$

$7.0000 \mathrm{E}-02$

4.0000E-01

7.6000E-02

1.7000 E- 03

1. $9000 \mathrm{E}+00$

$4.0000 \mathrm{E}-03$

2.0000E-01

$1.0000 E-02$

2.0000E-01

1.7090E-01

2.6000E-01

$5.0000 \mathrm{E}-02$

$5.2900 \mathrm{E}+\infty 0$

$9.7000 \mathrm{E}+\infty$

2.0000E.02

$1.0000 E+\infty$

$1.8300 \mathrm{E}+\infty 0$

$1.0002 E+00$

2.7000 E- 01

2.0000 E- 03

7.0000E-03

1.3000 E-O

5.2900E +00

4. $6000 \mathrm{E} .01$

4.0000E-01

$4.6000 E-01$

4.9000E-0

3.5000E-01

$6.0000 E .04$

$1.7000 \mathrm{E}-03$

3.0000E-02

2.0000E-04

$0.0000 E+00$

1.0000 E.02

$\triangle 0000$ E 02 
TABLE 4

STATISTICAL ANALYSIS OF HAZARDOUS MATERIALS IN ASCENDING ORDER BASED ON CONTAINER COUNT (* INDICATES DATA EXHIBITED NEGATIVE VARIANCE) (10 SHEETS)

\begin{tabular}{|c|c|c|c|c|c|c|}
\hline COMPONENT INAME & COUNT & ACCUM WT, KG & AVERAGE WT. KG & STD DEV & MINIMUM WT, KG & MAXIMUM WT, KG \\
\hline PSEUDOCUMENE-XYLENE & 4 & 4. $4540 E+01$ & $1.1135 \mathrm{E}+01$ & $5.8100 E+\infty 0$ & $8.1800 E+00$ & $2.0000 E+01$ \\
\hline SODIUM DICHRIMATE & 4 & 4.1051E+00 & $1.0263 E+\infty$ & 1. $1828 E+\infty$ & $1.0000 E-03$ & $2.1000 E+\infty 0$ \\
\hline STRONTIUM NITRATE & 4 & 1.1580E-D1 & $2.8950 \mathrm{E}-02$ & $3.2258 \mathrm{E}-02$ & 6.0000E-04 & 6. $3000 E-02$ \\
\hline TERT BUTML ALICOHOL & 4 & $1.7580 \mathrm{E}-01$ & $4.3950 \mathrm{E}-02$ & $1.1361 \mathrm{E}-02$ & $3.0000 E-02$ & $5.5800 E-02$ \\
\hline TRIMETHYLBERLZENE & 4 & $2.4342 E+00$ & $6.0855 \mathrm{E}-01$ & 7.1071E-01 & $2.0000 \mathrm{E}-03$ & $1.4300 E+00$ \\
\hline 1,1,1-DICHLOROIETHANE & 3 & $1.5000 E+\infty$ & $5.0000 E-01$ & 8.6803E.02 & $4.5000=-01$ & $6.0000 \mathrm{E}-01$ \\
\hline 1,1-DICHLOROETHANE & 3 & $1.8700 E+\infty$ & $6.2333 \mathrm{EE}-01$ & 3.8837E-01 & $1.0000 E-01$ & 8.4000E-01 \\
\hline 2-HEPTANONE & 3 & 1. $9900 \mathrm{E}+\infty 0$ & $6.6333 \mathrm{E}-01$ & 8.4678E-01 & $1.0000-02$ & $1.6200 E+00$ \\
\hline ACETIC ACID VINYL ESTER POLYMER WICHLOROETHYLENE & 3 & $2.2270 \mathrm{E}+00$ & 7.4233E-01 & $1.0156 \mathrm{E}+00$ & $1,5000 E-01$ & $1.9150 E+\infty$ \\
\hline ALIQUAT 336 & 3 & $0.0000 E+00$ & $0.0000 E+00$ & $0.0000 E+\infty$ & $0.0000 E+00$ & $0.0000 E+00$ \\
\hline ALKMLNAPHTHALENE & 3 & 4.8340E+01 & 1.61 13E+01 & $5.4610 E+00$ & $1.1230 E+01$ & $2.2010 \mathrm{E}+01$ \\
\hline ASCORBIC ACID & 3 & 9.4130E-01 & 3.1377E-01 & $2.6948 E-01$ & 6.5000E-02 & 6.0000E-01 \\
\hline BASIC ZINC CHFOMATE & 3 & $1.4184 E+00$ & 4.7280E-01 & 4.8035E-01 & $5.8400 E-02$ & $1.0000 E+\infty 0$ \\
\hline BIS-(2-ETHYLHEXY)PHTHALATE & 3 & $6.0500 \mathrm{E}+00$ & $2.0187 E+\infty 0$ & $1.0081 E+\infty$ & $1.4000 E+\infty 0$ & $3.1800 E+\infty 0$ \\
\hline EISPHENOL ANEPICHLOROHYDRIN RESIN & 3 & $2.4010 E+00$ & B.0033E-01 & $1.2413 E+00$ & $1.0000 E-02$ & $2.2310 E+00$ \\
\hline CALCIUM FLUORIDE & 3 & $2.0100 E+00$ & $6.7000 \mathrm{E}-01$ & $1.0587 E+\infty$ & $4.0000 E-02$ & $1,8900 E+\infty 0$ \\
\hline CALCIUM HYOROXIDE & 3 & $5.3000 E-01$ & $1.7687 \mathrm{E}-01$ & $2.0817 E-02$ & $1.8000 E-01$ & 2.0000E-09 \\
\hline CALCIUM NITRATE & 3 & $3.8265 E+\infty 0$ & 1.2755E+00 & 2. $2036 \mathrm{E}+00$ & $5.0000 E-04$ & $3.8200 E+00$ \\
\hline CHLORIDES & 3 & $4.9000 E-02$ & $1.6333 E-02$ & $4.7350 E-03$ & 1.1300E-02 & $2.0700 E-02$ \\
\hline DIBUTYL PHTHALATE & 3 & 9.1980E-01 & $3.0660 E-01$ & $1.9414 E-01$ & $1.4000 E-01$ & 5.1980E-01 \\
\hline ETHOXYETHANOL & 3 & A. 1608E+ +00 & $13869 \mathrm{E}+00$ & $1.8408 \mathrm{E}+00$ & $1.3100 E-01$ & $3.5000 \mathrm{E}+\infty$ \\
\hline HEXANE & 3 & 1.4645E +01 & $4.8816 E+00$ & $63732 E+\infty 0$ & $1,1100 E+00$ & 1.2240E+01 \\
\hline HEXYLENE GLYCOL & 3 & $35350 \mathrm{E}+00$ & $35350 \mathrm{E}+00$ & $\cdot$ & $3.5350 \mathrm{E}+00$ & $3.5350 \mathrm{E}+\infty 0$ \\
\hline IRON NITRATE & 3 & $2.5900 \mathrm{E}-02$ & $8.6333 \mathrm{E}-03$ & $1,4174 \mathrm{E}-02$ & $4.0000 \mathrm{E}-04$ & $2.5000 E-02$ \\
\hline LIGHT BALLASTS CONTAINING PCB & 3 & $1.4000 E-01$ & 4.6667E-02 & $4.6188 \mathrm{E}-02$ & $2.0000 E \cdot 02$ & $1.0000 E-01$ \\
\hline LIGROINE (NAFHTHA) & 3 & $6.6600 E-01$ & $2.2200 \mathrm{E}-01$ & $2.7145 E-01$ & $1.0000=-03$ & 5.2500E-01 \\
\hline MINERAL SPIRITS & 3 & $1.2343 E+01$ & $4.1143 E+\infty 0$ & $2.7814 E+\infty 0$ & $0.2000 E-01$ & $B .0000 E+00$ \\
\hline N-HEPTANE & 3 & $39820 E+00$ & $1.3273 E+\infty$ & $1.0132 E+00$ & 1.6200E-01 & $2.0000 E+00$ \\
\hline NAPTHA & 3 & 4. 4000E-01 & 1.4667E-0t & $1.4180 \mathrm{E}-01$ & $2.0000 E .02$ & $3.0000 E-01$ \\
\hline REGULATED WATER MISCIBLE LIQUID WISOUD SLUDGE RESI & 3 & $6.5030 E+01$ & 2.1677E+01 & $1.5188 \mathrm{E}+01$ & 4.1800E+00 & $3.4185 E+01$ \\
\hline PETROLEUM DISTILLATES & 3 & $1.0400 E+\infty$ & 3.4667E-01 & $5.8315 E-01$ & $5.00005-03$ & $1.0200 E+\infty$ \\
\hline PHTHALIC ACID, BIS(2-ETHYLHEXYL)ESTER (DOP) & 3 & $6.7500 \mathrm{E}-01$ & 2.2500E-01 & 2.3828E-01 & $8.0000 E-02$ & $5.0000 E-01$ \\
\hline POTASSIUM CARBONATE & 3 & $5.0759 E+00$ & 1. $6920 \mathrm{E}+00$ & $1.8607 E+\infty$ & $2.6000 \mathrm{E}-02$ & $3.6909 E+\infty$ \\
\hline PYDRAULFYRGUEL (HYDRALLIC FLUID) & 3 & 2.8880E-01 & $8.8600 \mathrm{E}-02$ & $1.3804 \mathrm{E}-01$ & $9.9000 E-03$ & 2.4900E-01 \\
\hline SILVER NITRATE & 3 & $1.2820 E-01$ & 4.2733E-02 & $5.0778 E-02$ & $32000 E-03$ & $1.0000 E-01$ \\
\hline SODIUM ACETATE & 3 & $6.0100 \mathrm{E}-01$ & 2.0033E.01 & $1.9665 \mathrm{E}-01$ & $2.0000 E-02$ & 4.1000E-01 \\
\hline SODIUM BICARESONATE & 3 & 1.2051E+01 & 4.0170E+00 & $6.3092 \mathrm{E}+00$ & 2.2000E-01 & $1.1300 \mathrm{E}+01$ \\
\hline SODIUM FLUORIDE & 3 & 4. $5400 E+\infty 0$ & $1.5133 E+00$ & $1.7620 \mathrm{E}+00$ & $1,4000 E-01$ & $3.5000 E+00$ \\
\hline SODIUM IOLHDE & 3 & $1.6304 E+00$ & 5.4347E-01 & $8.3084 E-01$ & $1.4000 E-03$ & $1.5000 E+\infty$ \\
\hline SULFATES & 3 & $2.4410 \mathrm{E}-01$ & 8.1367E-02 & 2.3561E.02 & 5. $6800 \mathrm{E}-02$ & $1.0350 E-01$ \\
\hline TERT-BUTYL METHIYL ETHER & 3 & $5.9800 E+00$ & $1.9933 E+\infty$ & $3.5798 \mathrm{E}-01$ & $1.5800 E+\infty 0$ & $2.2000 E+\infty$ \\
\hline TETRACHLOROIETHENE & 3 & $2.0000 \mathrm{E}-01$ & 6.6867E-02 & $2.3094 E-02$ & 4.0000E-02 & 8.0000E-02 \\
\hline TETRAPROPY AMMONIUM HYDROXIDE & 3 & $4.5000 \mathrm{E}-02$ & $1.5000 \mathrm{E}-02$ & $8.6600 E .03$ & $5.0000 E-03$ & $2.0000 E-02$ \\
\hline THINNEFI & 3 & $86200 E+\infty 0$ & $2.8733 E+00$ & 8.3769E-01 & $1.9098 \mathrm{E}+00$ & $3.6700 E+00$ \\
\hline TRIBUTYPHOSPHATE & 3 & $3.0040 E+\infty 0$ & $1.0013 E+\infty$ & $1.6564 \mathrm{E}+\infty 0$ & $1.3000 \mathrm{E} .02$ & $2.0160 \mathrm{E}+\infty 0$ \\
\hline TRIETHYLAMINE & 3 & $2.0310 E-01$ & $6.7700 E-02$ & $5.8032 E-02$ & 3.0000E-03 & $1.0010 E-01$ \\
\hline TRIETHYLENETETRAMINE & 3 & $2.9700 E+00$ & $8.9000 \mathrm{E}-01$ & $1.0116 E+\infty 0$ & 1.2000E-01 & $2.1000 E+00$ \\
\hline WATER & 3 & $2.0580 E+01$ & $6.8601 E+00$ & $9.0165 E+00$ & $6.3540 E-01$ & $1.72005+01$ \\
\hline 1,1,2,2-TETRABROMOETHANE & 2 & $2.2171 E+01$ & $1.1085 E+01$ & $1.3811 E+01$ & $1.3198 E+00$ & $2.0851 E+01$ \\
\hline 1,1,2-TRICHLOROETHANE & 2 & $2.5001 E+\infty 0$ & $1.2501 \mathrm{E}+00$ & $1.7677 \mathrm{E}+00$ & $1.0000 E-04$ & $2.5000 E+00$ \\
\hline 1,2-DIETHYLBENZENE & 2 & $8.0000 E-01$ & $4.0000 E-01$ & $0.0000 E+\infty 0$ & 4.0000E-01 & 4.0000E-01 \\
\hline 1.2-EPOXY BUTANE & 2 & 4.1090E-01 & 2.0545E-01 & $2.8928 E-01$ & $9.0000 E-04$ & 4. 1000E-01 \\
\hline 1,3.DIETHYLBENZENE & 2 & $4.0000 E-01$ & $2.0000 \mathrm{E}-01$ & $0.0000 E+00$ & $20000 E-01$ & $2.0000 E-01$ \\
\hline 1-METHYL-2-PYRROLIDINONE & 2 & $5.8000 E-02$ & 2.9000 E. 02 & 1.4140E-03 & $2.8000 E-02$ & $3.0000 E .02$ \\
\hline 2-(2-BUTOXYETHOXY-ETHANOL & 2 & 4.7700E+00 & $2.3850 E+00$ & $2.7224 E+00$ & 4.6000E-01 & $4.3100 E+00$ \\
\hline 2-EUTANONE OXIME & 2 & $2.0000 E .02$ & $1.0000 \mathrm{E}-02$ & $0.0000 E+00$ & $1.0000 E .02$ & $1.0000 \mathrm{E}-02$ \\
\hline 2-ETHYL-1-HEXANOL & 2 & $3.4000 E-01$ & $1.7000 E-01$ & $2.1213 E-01$ & $2.0000 E-02$ & $3.2000 \mathrm{E}-01$ \\
\hline ACETIC ACID VIN'YL ESTER & 2 & $1.1530 E+01$ & $5.7650 \mathrm{E}+00$ & $6.6863 \mathrm{E}+00$ & $1.0300 E+00$ & $1.0500 E+01$ \\
\hline ALKYL BENZENES & 2 & 3.4725E +01 & $1.7363 E+01$ & $5.3033 E-02$ & $1.7325 \mathrm{E}+01$ & $1.7400 E+01$ \\
\hline AMERCOAT 234 & 2 & $2.0004 E+00$ & $1.0002 E+00$ & $0.0000 E+\infty 0$ & $1.0002 E+00$ & $1.0002 E+00$ \\
\hline AMMONIUM MOLYYBDATE & 2 & 1. $3600 \mathrm{E}+00$ & G.8000E-01 & $8.7681 \mathrm{E}-04$ & B.0000E-02 & $1.3000 E+00$ \\
\hline ANTIMONY TRIC HLORIDE & 2 & $2.2000 \mathrm{E}-03$ & $1.1000 \mathrm{E}-03$ & $1.4100 \mathrm{E}-04$ & $1.0000 E-03$ & $1.2000 E-03$ \\
\hline ARSENIC TRIDXIDE & 2 & $3.4100 E-01$ & $1.7050 \mathrm{E}-01$ & 1.2021E-02 & 1. $6200 E-01$ & $1.7900 \mathrm{E}-01$ \\
\hline BARIUM CHLORIDE & 2 & $2.7960 E+00$ & $1.3980 E+00$ & $7.8065 E-01$ & 8. 480OE-01 & $1.0500 \mathrm{E}+00$ \\
\hline BARIUM CHROMATE & 2 & $33850 E+\infty 0$ & 1. $6925 E+00$ & $1.2268 E+\infty 0$ & $8.2500 E-01$ & $2.5800 \mathrm{E}+00$ \\
\hline BARIUM HYDFIOXIOE & 2 & $1.5000 E+00$ & $7.5000 E-01$ & 4.2426E-01 & $45000 E-01$ & $1,0500 E+\infty$ \\
\hline BIS (2-ETHYL HEXYL) HYDFIOGEN PHOSPHATE & 2 & $1.4630 E+01$ & $7.3150 E+C O$ & $1.0161 E+01$ & $1.3000 E-01$ & $1.4500 E+01$ \\
\hline BIS(2-ETHYLHEXYL) HYDROGEN PHOSPHATE & 2 & $6.0000 E+00$ & $3.0000 E+\infty 0$ & $4.0164 E+00$ & 4. $6000 \mathrm{E}-01$ & $5.8400 E+00$ \\
\hline BISPHENCL A & 2 & $1.0300 E+00$ & $5.1500 E-01$ & $6.8589 E-01$ & 3.0000E-02 & $1.0000 E+00$ \\
\hline BROMOCRESOL GREEN & 2 & $3.0000 \mathrm{E}-02$ & $1.5000 E-02$ & 1.4142E-02 & $5.0000 E-03$ & $2.5000 E-02$ \\
\hline BUTOXYETHANOL & 2 & $1.8000 E-01$ & $8,0000 E-02$ & $9.8905 E-02$ & $1,0000 E-02$ & 1.5000E-01 \\
\hline BUTYL BENZYL PHTHALATE & 2 & 1.0500E+00 & $5.2500 \mathrm{E}-01$ & $6.7175 \mathrm{E}-01$ & $5.0000 E-02$ & $1.0000 E+00$ \\
\hline BUTYL CELLOSOLVE & 2 & $9.2980 \mathrm{E}-01$ & $4.6490 E .01$ & $6.3498 E-02$ & 4.2000E-01 & 5.0980E-01 \\
\hline HLORINATED PARAFFIN WAXES AND HYDROCARBON WAXE & 2 & 1. $6000 E+\infty$ & $8.0000 E-01$ & $9.1924 \mathrm{E}-01$ & $1.5000 \mathrm{E}-01$ & $1.4500 E+00$ \\
\hline CHROMATES & 2 & $7.4000 E-01$ & $3.7000 E-01$ & $5.0912 \mathrm{E}-01$ & 1.0000E-02 & 7.3000E-01 \\
\hline CHROME & 2 & $3.8000 E+00$ & $1.8000 \mathrm{E}+\infty$ & $1.0182 E+\infty 0$ & $1.0800 E+\infty$ & $2.5200 E+\infty$ \\
\hline CHROMIUM DXIDE & 2 & $1.3100 E+00$ & 6.5500E-01 & $8.8388 \mathrm{E}-01$ & $3.0000 \mathrm{E}-02$ & $1.2800 E+\infty$ \\
\hline
\end{tabular}


TABLE 4

STATISTICAL ANALYSIS OF HAZARDOUS MATERIALS

IN ASCENDING ORDER BASED ON CONTAINER COUNT

(* INDICATES DATA EXHIBITED NEGATIVE VARIANCE)

(10 SHEETS)

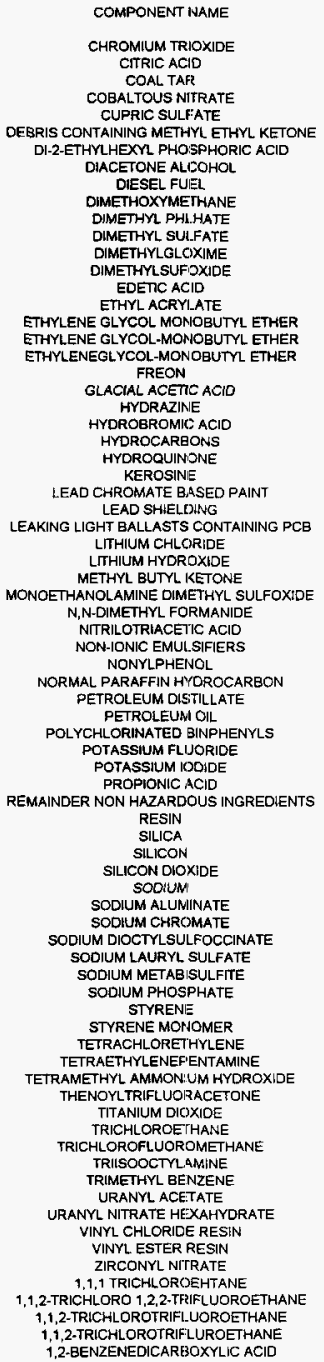

\begin{tabular}{|c|c|c|}
\hline COUNT & ACCUM WT, KG & AVERAGE WT. KG \\
\hline 2 & $4.6006 E+01$ & $2.3003 E+01$ \\
\hline 2 & $1.9900 \mathrm{E}+00$ & 9. $9500 E-01$ \\
\hline 2 & $8.7890 \mathrm{E}+00$ & $4.3995 \mathrm{E}+00$ \\
\hline 2 & $2.1000 E-03$ & $1.0500 \mathrm{E}-03$ \\
\hline 2 & $8.3950 E+00$ & 4. $1975 \mathrm{E}+\infty$ \\
\hline 2 & $2.0000 \mathrm{E}-02$ & 1.0000E-02 \\
\hline 2 & $0.0000 \mathrm{E}+00$ & $0.0000 E+00$ \\
\hline 2 & 4. $4000 \mathrm{E}-02$ & $2.2000 \mathrm{E}-02$ \\
\hline 2 & $3.8000 \mathrm{E}+\infty 0$ & $1.9000 E+00$ \\
\hline 2 & $3.0400 \mathrm{E}-02$ & $1.5200 \mathrm{E}-02$ \\
\hline 2 & 1.3500E-01 & $67500 E-02$ \\
\hline 2 & $1.8380 \mathrm{E}+01$ & 9.1800E+00 \\
\hline 2 & $1.5800 \mathrm{E}-02$ & 7.9000E.03 \\
\hline 2 & $4.5900 E+01$ & $2.2950 \mathrm{E}+01$ \\
\hline 2 & 1. $3650 E+\infty 0$ & 6.8205E-01 \\
\hline 2 & $0.0000 E+00$ & $0.0000 \mathrm{E}+00$ \\
\hline 2 & $6.0000 \mathrm{E}-02$ & $3.0000=-02$ \\
\hline 2 & $50920 \mathrm{E}-01$ & $25495 \mathrm{E}-01$ \\
\hline 2 & $2.3700 \mathrm{E}+00$ & $2.3700 \mathrm{E}+\infty$ \\
\hline 2 & $5.2000 \mathrm{E}+01$ & $2.6000 \mathrm{E}+01$ \\
\hline 2 & t.1700E-01 & 5. $3000 \mathrm{E}-02$ \\
\hline 2 & $1.6100 E+01$ & $8.0500 E+\infty 0$ \\
\hline 2 & $2.2425 \mathrm{E}+\infty$ & $1.1213 \mathrm{E}+00$ \\
\hline 2 & $2.7580 E+01$ & $1.3790 E+01$ \\
\hline 2 & $1.3910 \mathrm{E}+\infty$ & 6.9550E-01 \\
\hline 2 & $5.0000 \mathrm{E}-02$ & $5.0000 E .02$ \\
\hline 2 & 3.4500E-01 & $1.7250 \mathrm{E}-01$ \\
\hline 2 & $29540 \mathrm{E}+02$ & $14770 E+02$ \\
\hline 2 & $5.1000 E-02$ & $25500=-02$ \\
\hline 2 & 6. $9550 \mathrm{E}-01$ & $3.4775 \mathrm{E}-01$ \\
\hline 2 & 5.0010E-01 & 2.50058 .01 \\
\hline 2 & 8.0000E-03 & $4,0000 E-03$ \\
\hline 2 & 3.3498E+01 & $1.6748 E+01$ \\
\hline 2 & $1.0500 \mathrm{E}-01$ & 5.2500E-02 \\
\hline 2 & S 3300E.01 & $26650 E .01$ \\
\hline 2 & $6.5600 E+\infty 0$ & $3.2800 \mathrm{E}+\infty 0$ \\
\hline 2 & $2.3370 E+\infty 0$ & $1.1685 E+00$ \\
\hline 2 & $0.0000 \mathrm{E}+\infty$ & $0.0000 E+00$ \\
\hline$\frac{2}{2}$ & $30730 \mathrm{E}-01$ & 1.5365E-01 \\
\hline 2 & 3 O900E-01 & $1.5450 E \cdot 01$ \\
\hline 2 & $1.7000 E-01$ & 8.5000E-02 \\
\hline 2 & $1.3800 \mathrm{E}+00$ & 6. $.9000 \mathrm{E}-01$ \\
\hline 2 & $1.8000 \mathrm{E}-03$ & $9.0000 \mathrm{E}-04$ \\
\hline 2 & $2.8500 \mathrm{E}-01$ & $1.4250 \mathrm{E}-09$ \\
\hline 2 & $7.4029 E+01$ & $3.7014 \mathrm{E}+01$ \\
\hline 2 & $4.2569 E+00$ & $2.1285 \mathrm{E}+00$ \\
\hline 2 & $0.9000 E+00$ & $3.4500 \mathrm{E}+00$ \\
\hline 2 & $+.3000 E-01$ & B.5000E-02 \\
\hline 2 & $7.1767 E+00$ & $3.5884 E+00$ \\
\hline 2 & $2.9081 E+\infty$ & $1.4541 E+\infty$ \\
\hline 2 & $4.5000 E+\infty$ & $2.2500 \mathrm{E}+00$ \\
\hline 2 & $8.2500 \mathrm{E}+\infty 0$ & $3.1250 \mathrm{E}+\infty 0$ \\
\hline 2 & $8.2000 \mathrm{E}+00$ & $4.6300 \mathrm{E}+00$ \\
\hline 2 & $8.6813 \mathrm{E}+00$ & $3.3307 \mathrm{E}+\infty 0$ \\
\hline 2 & 8.7500E-01 & $48750 E .04$ \\
\hline 2 & $7.2000 \mathrm{E}+00$ & $7.2000 \mathrm{E}+\infty 0$ \\
\hline 2 & 1.7710E-01 & 8. $8550 \mathrm{E}-02$ \\
\hline 2 & $3.7503 E+00$ & $1.8752 E+\infty 0$ \\
\hline 2 & $4.0000 \mathrm{E}-04$ & 2.0000E-04 \\
\hline 2 & $1.0374 E+\infty O$ & $51870 E-01$ \\
\hline 2 & 5.2000E-01 & $2.6000 \mathrm{E}-01$ \\
\hline 2 & 3. $3500 \mathrm{E} \cdot 01$ & 1. $6750 E-01$ \\
\hline 2 & $9.4290=-01$ & 4.7145E-01 \\
\hline 2 & 2.8400E.01 & . $4200 \mathrm{E}-09$ \\
\hline 2 & $97800 E .01$ & $48900 \mathrm{E}-01$ \\
\hline 2 & 4.8600E-01 & 2. $4300 \mathrm{E}-01$ \\
\hline 2 & $6.6700 E+01$ & $3.3350 \mathrm{E}+01$ \\
\hline 2 & $1.2012 \mathrm{E}+00$ & $6.0060 \mathrm{E}-01$ \\
\hline 2 & $8.0100 E+00$ & $4.0050 \mathrm{E}+00$ \\
\hline 2 & $5.8960 \mathrm{E}+\infty 0$ & $2.9480 \mathrm{E}+00$ \\
\hline 2 & $3.7503 E+00$ & $1.8752 \mathrm{E}+\infty$ \\
\hline 2 & 1. $7080 \mathrm{E}+00$ & 8.5400E-01 \\
\hline 1 & T. $4000 \mathrm{E} .01$ & 1. $4000 \mathrm{E}-01$ \\
\hline$i$ & $1.5000 E+00$ & $1.5000 \mathrm{E}+00$ \\
\hline$i$ & $4.8200 E+\infty 0$ & $4.8200 E+00$ \\
\hline 1 & 3.6000E +00 & $3.6000 \mathrm{E}+00$ \\
\hline 1 & $2.0000 E-03$ & $20000 \mathrm{E}-03$ \\
\hline
\end{tabular}

STD DEV

3. $2523 \mathrm{E}+01$

1. $1950 E+\infty 0$

$3.0084 E+\infty$

2.1200E-04

$2.8815 E+\infty 0$

$0.0000 \mathrm{E}+00$

$0.0000 E+\infty 0$

$1.6971 \mathrm{E}-02$

8.4853E-01

2.0830E-02

$1.2170 E+01$

$1.0041 E-02$

$1.004 \mathrm{TE}-02$

9.8905E-01

$0.0000 E+00$

$0.0000 E+\infty$

3.4641E-01

$1.2728 E+01$

$1.5098 \mathrm{E}-02$

7.7782E-01

$1.3934 E+00$

1.8067E+01

2.8921E-01

\#NUMI

2.3688E-01

1. $4467 \mathrm{E}+02$

3. $4848 E-02$

4.8826E-01

3.5346E-01

$5.8570 \mathrm{E}-03$

$7.1428 \mathrm{E}+\infty 0$

3. $8891 \mathrm{E}-02$

3. $8275 E-01$

$0.0000 \mathrm{E}+\infty 0$

$2.3830 \mathrm{E}-01$

$0.0000 \mathrm{E}+00$

1.8661E-01

4. $5962 \mathrm{E}-02$

4. $9497 \mathrm{E}-02$

9 $3338 \mathrm{~B}-01$

$1.4100 \mathrm{E}-04$

2.4749E-02

2. $1020 \mathrm{E}+00$

$2.0812 E+00$

$48225 E+\infty$

$3.5355 \mathrm{E}-02$

2.3683E+OO

1. $5842 \mathrm{E}+00$

$3.5355 \mathrm{E}-01$

$3.7830 E+00$

1.4142E -02

3. $3083 E+\infty 0$

1. $7878 \mathrm{E}-02$

2509E-01

1.2374E+ +00

$1.2374 \mathrm{E}+00$

1.4100E-04

3.3041E-01

$1873 a E-01$

3. $8530 \mathrm{E}-01$

$8.2024 \mathrm{E}-02$

9.8890E-03

1. $8385 \mathrm{E}-02$

$1.3435 E+00$

5.6653E-01

$2.9788 E+00$

1 . $6235 \mathrm{E}+\infty$

$12374 E+\infty 0$

3.4790E-01

$0.0000 \mathrm{E}+00$

$0.0000 E+00$

$0.0000 \mathrm{E}+00$

$0.0000 \mathrm{E}+00$

$0.0000 E+\infty$
MINIMUMM WT, KG

MAXIMUMM WT, KG

6.0000E- 03

$2.2100 \mathrm{E}+00$

$9.0000 \mathrm{E}-04$

$2.1600 \mathrm{E}+\infty$

1.0000 E -02

$0.0000 \mathrm{E}+\infty 0$

$1.0000 \mathrm{E}-02$

1. $3000 \mathrm{E}+\infty$

$4.0000 \mathrm{E}-04$

3. 5000 E- 02

5.8000E-01

8.0000 E- 04

2.2250E+01

$1.9000 \mathrm{E}-01$

$0.0000 E+00$

3.0000E-02

$1.0000 E-02$

2.3700E $+\infty 0$

$1.7000 \mathrm{E}+01$

$4.7800 \mathrm{E}-02$

7.5000E+CO

3.7800E-01

$5.0000 \mathrm{E}-02$

5. $0000 \mathrm{E}-03$

4.5400E +01

1.0000E-03

2.5000E-03

1.0000E-0.

$0.0000 \mathrm{E}+00$

t. 1688E+01

2.5000E-02

OOOOE. 02

$3.2800 \mathrm{E}+00$

1.0000E +00

$0.0000 \mathrm{E}+00$

2.1700E-02

1. $2200 \mathrm{E} .01$

5.0000 E-02

$3.0000 E \cdot 02$

8.0000 E. 04

1.2500E-01

3.5465E+01

B.5680E-01

$4.0000 E-02$

$4.0000 E-02$

$1.8151 \mathrm{E}+00$

3.48005 .01

$2.0000 E+00$

4,5000E-01

$4.6200 E+D 0$

$0.9130 E-01$

4.7500E-01

7. $2000 \mathrm{E}+\infty 0$

$1.0000 E-04$

$1.0002 \mathrm{E}+\infty$

$1.0000=-04$

3. $7400 E-02$

2.0000E. 02

100005.01

B. $4000 \mathrm{E}-02$

8. $4000 \mathrm{E}-02$

2.3000E-01

3.2400E +01

$1.0001 \mathrm{E}+00$

$1.0002 \mathrm{E}+00$

B. $0800 E-01$

$1.4000 E-01$

$1.5000 \mathrm{E}+00$

4. $8200 \mathrm{E}+00$

$3.6000 \mathrm{E}+00$

$2.0000 \mathrm{E}-03$
4. $6000 \mathrm{E}+01$ $1.400 E+\infty 0$

$6.5890 E+\infty 0$

1. 2000E-03

$8.2350 E+\infty 0$

$1.0000 \mathrm{E} .02$

$0.0000 E+00$

3. $4000 \mathrm{E}-02$

$2.5000 E+00$

$3.0000 \mathrm{E}-02$

1.0000E-01

$1.7800 E+01$

1.5000E-02

$2.3850 \mathrm{E}+01$

$1.1750 E+00$

$0,0000 E+\infty 0$

3.0000E-02

4 9000E-01

$2.3700 E+\infty 0$

$3.5000 \mathrm{E}+01$

$7.0000 E .02$

$8.6000 E+00$

$2.1065 E+00$

$2.7202 E+01$

9.0000E-01

5.0000E-02

3.4000E-01

$2.5000 \mathrm{E}+02$

$5.0000 \mathrm{E}-02$

6.9300E.01

5.0000E-01

8.0000 E-03

2.1800E+01

8.0000E-02

$5.2300 \mathrm{E}-01$

$3.2800 E+\infty 0$

1.3370E+00

$0.0000 \mathrm{E}+00$

2.8580E-01

1.8700E-01

1.2000E-01

1. $3500 E+\infty 0$

$1.0000 \mathrm{E}-03$

$1.8000 \mathrm{E}-01$

$3.8564 \mathrm{E}+01$

$3.6001 E+00$

$6.8600 E+\infty 0$

9.0000E-02

5.281BE+ +0

$2.5601 E+00$

$2.5000 E+00$

$5.8000 \mathrm{E}+00$

$4.6400 E+\infty 0$

$5.6700 \mathrm{E}+\infty 0$

5.0000E-04

$7.2000 E+\infty 0$

1.7TOOE-01

2.7501E +00

3.0000E-04

$1.0000 \mathrm{E}+00$

5.0000 E-01

3.0000E-01

7 430OE-O

2.0000E-01

$4.9800 E-01$

2.5600E-01

3.4300E+01

$1.0012 E+00$

$6.1099 \mathrm{E}+00$

$40060 \mathrm{E}+00$

$2.7501 \mathrm{E}+\infty$

1.1000E+DO

1.4000E-01

$15000 \mathrm{E}+00$

$4.8200 E+00$

$3.6000 \mathrm{E}+00$

2.0000E-03 
TABLE 4

STATISTICAL ANALYSIS OF HAZARDOUS MATERIALS IN ASCENDING ORDER BASED ON CONTAINER COUNT (* INDICATES DATA EXHIBITED NEGATIVE VARIANCE)

(10 SHEETS)

\begin{tabular}{|c|c|c|c|c|c|c|}
\hline COMPONENT INAME & COUNT & ACCUM WT, KG & AVERAGE WT. KG & STD DEV & MINIMUMM WT, KG & MAXIMUM WT, KG \\
\hline 1,2-EENZISOTHIAZOLIN-3-ONE, 1,1-DIOXIDE, & 1 & 4.6000E-01 & 4.6000E-01 & $0.0000 E+00$ & $4.6000 E-01$ & $4.6000 E-01$ \\
\hline 2-ETHANEDIAMINE, N-(2-AMINOETH'(L)-N'-2-AMINOETHYL)AMI & $i$ & $2.8000 \mathrm{E}-01$ & $2.8000 E-01$ & $0.0000 E+00$ & $2.8000 E-01$ & $2.8000 E-01$ \\
\hline 1,3 BUTADIENE AND ACRYLONITRILE & 1 & $5.8000 E-01$ & $5.8000 E-01$ & $0.0000 E+00$ & $5.8000 E-01$ & 5.8000E-01 \\
\hline 1,3-PROPANEDIAMINE & $i$ & $1.5900 \mathrm{E}+\infty 0$ & $1.5900 E+00$ & $0.0000 \mathrm{E}+00$ & $1.5000 \mathrm{E}+00$ & $1.5900 E+00$ \\
\hline 1,4,7,10,13,18-HEXANOXACYCLOOCTADECANE & 1 & $1.0000 \mathrm{E}-01$ & $1.0000 E-01$ & $0.0000 E+00$ & $1.0000 E-01$ & $1.0000 E-01$ \\
\hline 1-CHLORO-2,3-EPOXY PROPANE & 1 & 4.8000E-01 & 4.8000E.01 & $0.0000 E+\infty 0$ & $4.8000 E-01$ & 4.8000E-01 \\
\hline 1-METHOXY 2-PROPANOL & 1 & $1.0100 \mathrm{E}+\infty 0$ & $1.0100 \mathrm{E}+00$ & $0.0000 \mathrm{E}+00$ & $1.0100 E+\infty 0$ & $1.0100 E+00$ \\
\hline 1.NITROPROFENE & 1 & $0.0000 \mathrm{E}+00$ & $0.0000 E+00$ & $0.0000 \mathrm{E}+00$ & $0.0000 E+00$ & $0.0000 E+00$ \\
\hline 2 HEPTANONE & 1 & $1.5500 E-01$ & 1.5500E-01 & $0.0000 E+00$ & 1.5500E-01 & $1.5500 \mathrm{E}-01$ \\
\hline 2,4,6-TRI(DIMETHYLAMINOMETHYL) PHENOL & $t$ & $1.5200 E+00$ & $1.5200 \mathrm{E}+00$ & $0.0000 \mathrm{E}+00$ & $1.5200 E+00$ & $1.5200 \mathrm{E}+\infty$ \\
\hline 2,4-DISOCYANATO-1-METHY BENZENE & i & $8.0000 \mathrm{E}-02$ & $8.0000 \mathrm{E}-02$ & $0.0000 E+00$ & $8.0000 \mathrm{E}-02$ & $8.0000 \mathrm{E}-02$ \\
\hline 2,5-DH-TERT-BUTYLHYMROQUINONE & 1 & $7.7800 E-01$ & $7.7800 \mathrm{E}-04$ & $0.0000 E+\infty 0$ & 7.7800E-01 & $7.7800 E-01$ \\
\hline 2-(2-BUTOXVETHOX) ETHANOL & 1 & 3.1500E-01 & 3.1500E-01 & $0.0000 E+\infty$ & 3.1500E-01 & 3.1500E-01 \\
\hline 2-(METHOXYETHOX N ETHANOL & 1 & $2.8000 \mathrm{E}-01$ & 2.8600E-01 & $0.0000 E+00$ & 2.BEDOE-01 & 2.8800E-01 \\
\hline 2-BIPHENYOL SODIUM SALT & 1 & $1.0000 \mathrm{E}-01$ & $1.0000 \mathrm{E}-01$ & $0.0000 \mathrm{E}+00$ & $1.9000 \mathrm{E}-01$ & $1.0000 E-01$ \\
\hline 2-BROMO-2-EROMOMETHYL-GLUTRARONITRILE & 1 & $1.0000 E-02$ & $1.0000 E-02$ & $0.0000 E+\infty 0$ & $1.0000 \mathrm{E}-02$ & $1.0000 E-02$ \\
\hline 2-BUTANONE & 1 & $1.0000 E-04$ & $1.0000 E-04$ & $0.0000 \mathrm{E}+\infty$ & $1.0000 \mathrm{E}-04$ & $1.0000 E-04$ \\
\hline 2-BUTOXETHANOL & 1 & 2.6000E-01 & $2.8000 \mathrm{E}-01$ & $0.0000 E+\infty 0$ & $2.0000 E-01$ & $2.6000 E-01$ \\
\hline 2-BUTOXY ETHANOL & 1 & $2.0000 \mathrm{E}+00$ & $20000 E+00$ & $0.0000 E+\infty 0$ & $2.0000 E+\infty 0$ & $2.0000 \mathrm{E}+\infty 0$ \\
\hline 2.-BUTYOXYETHANOL & $i$ & $3.4000 \mathrm{E}-02$ & $3.4000 \mathrm{E}-02$ & $0.0000 E+\infty$ & $3.4000 E-02$ & $3.4000 E-02$ \\
\hline 2-PROPOXYETHANOL & i & $7.1000 \mathrm{E}-01$ & $7.1000 \mathrm{E}-01$ & $0.0000 E+00$ & $7.1000 \mathrm{E}-01$ & $7.1000 E-01$ \\
\hline 3-10D0-2-PROPYNYL BUTYL CARBAMATE & 1 & $1.0000 E-02$ & $1.8000 \mathrm{E}-02$ & $0.0000 E+\infty$ & $1.9000 E .02$ & $1.2000 E-02$ \\
\hline 4,4-METHYLENECIIANILINE & 1 & $10000 \mathrm{E}+00$ & $1.0000 E+00$ & $0.0000 E+00$ & $1.0000 E+\infty 0$ & $1.0000 E+\infty$ \\
\hline 4-TERTBUTYLPYROCATECHOL & 1 & $0.0000 E+00$ & $0.0000 E+00$ & $0.0000 E+00$ & $0.0000 E+00$ & $0.0000 \mathrm{E}+\infty$ \\
\hline ACETAMi:ie & 9 & 1.0000E+00 & $\uparrow .0000 \mathrm{E}+00$ & $0.0000 \mathrm{E}+\infty 0$ & $1.0000 E+\infty$ & $1.0000 E+\infty$ \\
\hline ACETIC ACID ETHYL ESTER & 1 & $8.0000 E-02$ & $8.0000 E-02$ & $0.0000 E+00$ & $8.0000 \mathrm{E}-02$ & $8.0000 E-02$ \\
\hline ACEIIC ACID SODIUM SALT & 1 & $2.4000 \mathrm{E}-01$ & $2.4000 E-01$ & $0.0000 E+00$ & $2.4000 E .01$ & $2.4000=01$ \\
\hline & 1 & $37000 \mathrm{E}-01$ & $3.7000 \mathrm{E}-01$ & $0.0000 \mathrm{E}+00$ & $3.7000 E-01$ & $3.7000 \mathrm{E}-01$ \\
\hline ACETIC ACID VINYL POLYMER WITH CHLOROETHYLENE & 1 & $2.0000 E+\infty 0$ & $20000+00$ & $0.0000 E+00$ & $2.0000 E+\infty 0$ & $2.0000 \mathrm{E}+00$ \\
\hline ACETVL CHLORIDE & 1 & $6.9000 E-01$ & $6.9000 E-01$ & $0.0000 E+\infty$ & 0. $.0000 \mathrm{E}-01$ & $6.9000 E-01$ \\
\hline ACETM ENE TETRABROMIDE & 1 & $28000 \mathrm{E}+00$ & $2.8000 E+00$ & $0.0000 E+\infty 00$ & $2.8000 E+\infty 0$ & $2.8000 \mathrm{E}+\infty$ \\
\hline ACID & 1 & $2.5000 \mathrm{E} .01$ & $2.5000 \mathrm{E}-01$ & $0.0000 E+\infty 0$ & $2.5000 \mathrm{E}-01$ & 2.5000E-01 \\
\hline ACID SODIVM SALT & 1 & $2.0000 \mathrm{E}-02$ & 2.0000E-02 & $0.0000 E+\infty$ & $2.0000 \mathrm{E}-02$ & $2.0000 \mathrm{E}-02$ \\
\hline ACRYLAMIDE & 1 & $2.0000 E-02$ & $2.0000 \mathrm{E}-02$ & $0.0000 E+00$ & $2.0000 E-02$ & $2.0000 \mathrm{E}-02$ \\
\hline ACRYLIC AIID & 9 & 7. $4000 E+00$ & $7.4000 \mathrm{E}+\infty 0$ & $0.0000 E+00$ & $7.4000 E+00$ & $7.4000 E+00$ \\
\hline ALIPHATIC PETROLEUIM DISTILLATES & 1 & $1.8700 E+01$ & $1.8700 \mathrm{E}+01$ & $0.0000 E+00$ & $1.8700 E+01$ & $1.8700 \mathrm{E}+01$ \\
\hline ALKALENE CARBONATE & 1 & $0.0000 E+\infty$ & $0.0000 \mathrm{E}+00$ & $0.0000 \mathrm{E}+00$ & $0.0000 E+00$ & $0.0000 E+00$ \\
\hline ALUMINUM CHE.ORIDE & 1 & 4.0000E-0 & $4.0000 \mathrm{E}-01$ & $0.0000 \mathrm{E}+00$ & 4.0000E-01 & 4.0000E. 01 \\
\hline ALUMANUM NITRATE MONOHYDRATE & 1 & 3. $.0000 E+00$ & $3.8000 E+00$ & $0.0000 E+\infty$ & $3.9000 E+00$ & $3.9000 E+00$ \\
\hline $\begin{array}{l}\text { ALUMINUM SLLICATE } \\
\text { ALIL }\end{array}$ & 1 & $2.9850 E-01$ & $2.9850 \mathrm{E}-01$ & $0.0000 \mathrm{E}+\infty$ & $20850 E-01$ & $2.8650 E-01$ \\
\hline AMINOETHYLPIPERAZINE & 1 & $1.0000 \mathrm{E}+00$ & $1.0000 E+\infty$ & $0.0000 E+00$ & $1.0000 E+\infty 0$ & $1.0000 E+\infty 0$ \\
\hline AMINOGUANIDINE HEMISULFATE & 9 & 3.2400E-01 & $3.2400 \mathrm{E}-01$ & $0.0000 E+00$ & $3.2400 \mathrm{E}-01$ & 3.2400E.01 \\
\hline AMMONIUM BICARBONATE & 1 & $7.7400 \mathrm{E}-01$ & $7.7400 \mathrm{E}-01$ & $0.0000 E+00$ & 7.7400E-01 & $7.7400 E-01$ \\
\hline AMMONIUM CARBONATE & 1 & $3.6000 \mathrm{E}-01$ & 3.6000E.01 & $0.0000 E+00$ & 3.6000E-01 & 3.6000E-01 \\
\hline AMMONIUM CITRATE DIBASIC & 1 & $6.0000 \mathrm{E}-01$ & $6.0000 E-01$ & $0.0000 \mathrm{E}+\infty 0$ & 6.0000E-01 & $6.0000 E-01$ \\
\hline AMMONIUM NITRATE & 1 & 3.0800E-01 & $3.9600 \mathrm{E}-01$ & $0.0000 \mathrm{E}+\infty 0$ & $3.9600 \mathrm{E} .01$ & $3.0600 \mathrm{E}-01$ \\
\hline AMMONHUM PERSULFATE & 1 & $24000 E-01$ & $2.4000 \mathrm{E}-01$ & $0.0000 \mathrm{E}+00$ & $2.4000 \mathrm{E}-01$ & 2.4000E-01 \\
\hline AMONIUM CHLORIOE & 1 & $1.0000 E-02$ & $1.0000 \mathrm{E}-02$ & $0.0000 E+00$ & $1.0000 \mathrm{E}-02$ & $1.0000 E-02$ \\
\hline AMORPMOUS SILICA & 1 & $3.2500 \mathrm{E} \cdot 01$ & 3.2500E-01 & $0.0000 E+00$ & $3.2500 E-01$ & 3.2500E-01 \\
\hline ANTIMONY & 1 & $2.0000=02$ & $2.0000 E-02$ & $0.0000 E+\infty 0$ & $2.0000 \mathrm{E}-02$ & $2.0000 E-02$ \\
\hline ARSENAZO I & 1 & $3.6000 \mathrm{E} .02$ & 3. $8000 \mathrm{E}-02$ & $0.0000 E+\infty 0$ & $3.6000 \mathrm{E}-02$ & $3.6000 \mathrm{E}-02$ \\
\hline ARSENIC PENTOXIDE & 1 & $9.0000 E .01$ & $9.0000 E-01$ & $0.0000 E+00$ & $8.0000 E-01$ & $8.0000 E-01$ \\
\hline ASPHALT & $i$ & $1.5990 \mathrm{E}+01$ & $1.5800 E+01$ & $0.0000 \mathrm{E}+00$ & $1.5990 E+01$ & $1.5900 E+01$ \\
\hline AZOBENZE:NE & 1 & $2.4000 \mathrm{E}-01$ & $2.4000 E-01$ & $0.0000 E+00$ & 2.4000E-01 & 2.4000E-01 \\
\hline BAMBP & 1 & $1.9860 E+\infty 0$ & $1.8860 E+00$ & $0.0000 E+00$ & 1. $.8860 E+00$ & $1.8880 E+00$ \\
\hline BARIUM CARBONATE & 1 & $30000 E-03$ & $3.0000 \mathrm{E}-03$ & $0.0000 E+00$ & $3.0000 \mathrm{E}-03$ & $3.0000 \mathrm{E} .03$ \\
\hline BARUUM NITRATE & 1 & $4.9320 E+00$ & $4.9320 E+\infty$ & $0.0000 E+00$ & $4.8320 E+00$ & $4.8320 \mathrm{E}+00$ \\
\hline BARIUM OXIOE & 1 & $2.0000 E+00$ & $2.0000 E+00$ & $0.0000 \mathrm{E}+\infty 0$ & $2.0000 E+\infty 0$ & $2.0000 E+\infty 0$ \\
\hline BATOHOPHENATHROLINE & $i$ & $1.0000 \mathrm{E}-02$ & $1.0000 E-02$ & $0.0000 E+00$ & $1.0000 E-02$ & $1.0000 E-02$ \\
\hline TTERIES CONTAINING LEAD, LEAD OXIDES, ANO SULFURIC A & 1 & $2.0130 \mathrm{E}+01$ & $2.0130 \mathrm{E}+01$ & $0.0000 E+\infty$ & $2.0130 E+01$ & 2.0130E+01 \\
\hline BATTERIES CONTAINING POTASSIUM HYOROXIDE, NICKEL & $t$ & $1.4000 \mathrm{E}-01$ & $1.4000 E-01$ & $0.0000 E+00$ & $1.4000 \mathrm{E}-01$ & $1.4000 \mathrm{E}-01$ \\
\hline BENZENEARSENIC ACID & $i$ & $1.2600 \mathrm{E}-01$ & $1.2600 \mathrm{E}-01$ & $0.0000 E+00$ & $1.2800 \mathrm{E}-01$ & $1.2600 \mathrm{E}-01$ \\
\hline BENZENESULFONIC ACID DCIDECY SODIUM SALT & 1 & $1.8500 \mathrm{E}+\infty 0$ & $1.9500 E+00$ & $0.0000 E+00$ & $1.9500 E+00$ & $1.8500 E+00$ \\
\hline BENZIN-(NAPHTHA) & 1 & $1.8200 E-01$ & $1.6200 \mathrm{E}-01$ & $0.0000 E+\infty 0$ & $1.6200 \mathrm{E}-01$ & $1.6200 \mathrm{E}-01$ \\
\hline BENZOIC ACID & 1 & 4 5000E-02 & 4.5000E- -2 & $0.0000 E+\infty$ & $4.5000 E-02$ & 4. 5000E-02 \\
\hline BENZYL BUTYL ESTER & $i$ & $0.0000 E-02$ & $6.0000 \mathrm{E}-02$ & $0.0000 E+00$ & $6.0000 \mathrm{E}-02$ & $6.0000 \mathrm{E}-02$ \\
\hline BIS (2-ETHYLEXYL) PHTHALATE & 1 & $13100 \mathrm{E}+00$ & $1.3100 \mathrm{E}+00$ & $0.0000 E+00$ & 1.3100E+ $+\infty$ & $1.3100 \mathrm{E}+00$ \\
\hline & 1 & $1.7000 E-01$ & 1.7000E-01 & $0.0000 \mathrm{E}+00$ & $1.7000 \mathrm{E}-01$ & $1.7000 E-01$ \\
\hline BIS\{2-ETHYLHEXYL;PHTHALATE & 1 & 1.4400E+01 & $1.4400 \mathrm{E}+01$ & $0.0000 E+00$ & $1.4400 E+01$ & $1.4400 E+01$ \\
\hline BISMUTHE & 1 & Q.3600E-02 & 8.3600E-02 & $0.0000 \mathrm{E}+00$ & $8.3600 \mathrm{E}-02$ & B. $3600 E-02$ \\
\hline BISMUTH TRIOXIDE & 1 & $1.9000 \mathrm{E}-02$ & $1.9000 E-02$ & $0.0000 E+\infty$ & $1.9000 \Xi-02$ & 1.9000E-02 \\
\hline & 1 & 3.6000E-01 & $3.8000 \mathrm{E}-01$ & $0.0000 E+00$ & $3.6000 \mathrm{E}-01$ & 3.6000E-01 \\
\hline $\begin{array}{l}\text { BIX (2) (ETHYLEXY) STYR PHTHAALIC ACID (DOP) } \\
\text { BIX(2-ETHYL HEXYL) ESTER P }\end{array}$ & 1 & $2.7000 \mathrm{E}-02$ & $2.7000 \mathrm{E}-02$ & $0.0000 E+00$ & $2.7000 E-02$ & $2.7000 \mathrm{E}-02$ \\
\hline $\begin{array}{l}\text { BIX(2-ETHYL HEXY) IISE MIXTURES } \\
\text { BROMIDE }\end{array}$ & $i$ & $3.4700 E+01$ & $3.4700 E+01$ & $0.0000 E+00$ & $3.4700 E+01$ & $3.4700 \mathrm{E}+01$ \\
\hline BROMOCRESOL. PURPLE & 1 & $5.0000 E-03$ & $5.0000 E-03$ & $0.0000 E+00$ & $5.0000 E-03$ & $5.0000 \mathrm{E}-03$ \\
\hline BUTANE & 1 & $1.2000 E-03$ & $1.2000 E-03$ & $0.0000 E+\infty 0$ & 1.2000E-03 & $1.2000 E-03$ \\
\hline BUTOXYETHOXY ETHANOL & 1 & $1.5000 \mathrm{E}-01$ & $1.5000 \mathrm{E}-01$ & $0.0000 \mathrm{E}+\infty$ & 1.5000E-01 & 1.5000E-01 \\
\hline
\end{tabular}


TABLE 4

STATISTICAL ANALYSIS OF HAZARDOUS MATERIALS IN ASCENDING ORDER BASED ON CONTAINER COUNT (* INDICATES DATA EXHIBITED NEGATIVE VARIANCE) (10 SHEETS)

COMPONENT NAME

BUTYLATED HYOROXYTOLUENE BUTYLMETHYLETHER BUTYRALDEHYOE CADIUM

CADMIUM CXALIC ACID

CADMIUM HYDROXIDE CAPRIOC ACID

CARCINOGEMIC ORGANIC CHEMICAL SUBSTANCE CERIC SULFATE CERIUM NITRATE CEROUS NITRATE CESIUM IODIDE CHLORAMINE-T

CHLORINATED PARAFFIN WAX

CHLORINATED PARAFFINS

CHLOROETHYLENE

CHLOROFLUOROMETHANE

CHROMIUM (III) NITRATE NONAHYDRATE CHROMUMM SALTS COBALT HYDROXIDE COBALTOUS SULFATE COLLODION

CORROSIVE REACTOR ANALYSIS SOLUTION CRYSTALLINE.QUARTZ SILICA CUMENE HYDROXIDE CUPFERRON

CUPRIC NITRATE DEBRIS DECANE

DI(2-ETHYLHEXYL)PHTHALATE DI(2-ETHYLHEXYL)PHTHATATE DI-N-BUTYL PHTHALATE DISEC-OCTM PHTHALATE DICHILORODIFLUOROMETHANE DICHLORODIMETHYL SILANE DICHLOROOCTAMETHYLTETRA SILOXANE DICHLOROTRIFLUROETHANE DICYCLOHEXANO-1B.CROWN-6 DICYCLOPENTADIENE DIETHYL ETHER DIETHYL OXALATE

DIETHYLENE GLYCOL MONOETHYLETHER DIETHYLENE TRIAMINE DIGLYCOLIC ACID DIMETHTYL OXALATE DIMETHYL BEIVENE DIMETHYL FORIMAMIDE DIMETHYLGLYOXIME DIOXOLANE DIPHENYLAMINE

DIPROPYLENE GLYCOL MONOMETHYL ETHER DITHIOXAMIDE

DODECYLBENZENE SULFONIC ACIO DUSOCYANATE

ETHANOL (ETHYL ALCOHOL)

ETHANOL DIBENZYL MERCURY ETHANOLAMINE

ETHANOLAMINE DIMETHYLSULFOXIDE ETHIDIUM GROMIDE ETHYL CVANIDE

ETHYL MERCURIC CHLORIDE

ETHYLENEDIAMINETETRAACETIC ACID DISODIUM SALT (EDTA) ETHYLENEDIAMINETFA ACIDIC ACIO

ETHYLHEXYLPHOSPHORIC ACID FERRIC OXIDE FERROUS SULFATE FORMAMIDE FREON 113

FURFURYL-ALCOHOL

GADOLINIUM NITRATE GALLIC ACID GLYCOLS

GLYCOLS, POLYETHYLENE MONO (NONYLPHENYL) GRAPHITE

HEXACHLOROBENZNE
HEXCHLOROBENZENE

\begin{tabular}{|c|c|c|}
\hline COUNT & ACCUM WT, KG & AVERAGE WT. KG \\
\hline 1 & $1.0000 E-01$ & $1.0000 \mathrm{E}-01$ \\
\hline 1 & $1.0010 E .01$ & $1.0010 E-01$ \\
\hline 1 & $1.3000 E-02$ & $1.3000 E-02$ \\
\hline 1 & $1.0000 E-04$ & $1.0000 E-04$ \\
\hline 1 & $7.0000 E-01$ & $7.0000 E-01$ \\
\hline 1 & $2.0500 E-01$ & $2.0500 E-01$ \\
\hline 1 & $7.2000 E-01$ & $7.2000 E-01$ \\
\hline 1 & $4.5000 E+00$ & $4.5000 E+00$ \\
\hline 1 & $1.1000 E-03$ & $1.4000 E \cdot 03$ \\
\hline 1 & 1.1000E-01 & $1.1000 E-01$ \\
\hline 1 & $4.4500 E+00$ & $4.4500 E+\infty 0$ \\
\hline 1 & $2.0000 E+00$ & $2.0000 E+00$ \\
\hline 1 & $5.5000 E-01$ & $5.5000 \mathrm{E}-01$ \\
\hline 1 & $2.7000 \mathrm{E}-01$ & $2.7000 \mathrm{E}-01$ \\
\hline 1 & $2.0000 E-02$ & $2.0000 \mathrm{E}-02$ \\
\hline 1 & $3.0000 \mathrm{E}-03$ & $3.0000 \mathrm{E}-03$ \\
\hline 1 & $3.8401 E+01$ & $3.6401 E+01$ \\
\hline 1 & $2.0000 E-01$ & $2.9000 \mathrm{E}-01$ \\
\hline 1 & 1.2500E+00 & 1.2500E $+\infty$ \\
\hline 1 & $2.0000 E-02$ & $2.0000 \mathrm{E}-02$ \\
\hline 9 & $6.2280 E+00$ & $6.2280 E+\infty 0$ \\
\hline 1 & $1.1200 E+00$ & $1.1200 E+\infty 0$ \\
\hline 1 & $1.9241 E+01$ & $1.9241 E+0 \dagger$ \\
\hline 1 & $6.6700 E+C 0$ & $8.8700 E+00$ \\
\hline 1 & $2.0000 E-01$ & $2.0000 E-01$ \\
\hline 1 & $1.0000 \mathrm{E}-01$ & $1.0000 E-01$ \\
\hline 1 & $7.1010 E-01$ & $7.1010 E-01$ \\
\hline 1 & 4.0000E +01 & $4.0000 E+01$ \\
\hline 1 & $8.5000 E-02$ & 8.5000E-02 \\
\hline 1 & $2.0000 E-02$ & $2.0000 E-02$ \\
\hline 1 & $1.3500 E+\infty$ & $1.3500 E+00$ \\
\hline 1 & 1.4200E-01 & 1.4200E-01 \\
\hline 1 & $1.0000 E+00$ & $1.0000 E+00$ \\
\hline 1 & 1.1000E-01 & 1.1000E-01 \\
\hline 1 & $6.0000 E-02$ & $60000 E-02$ \\
\hline 1 & $80000 E-02$ & $8.0000 E-02$ \\
\hline 1 & $1.7998 \mathrm{E}+00$ & $1.7208 \mathrm{E}+\infty$ \\
\hline 1 & $0.0000 E+00$ & $0.0000 E+00$ \\
\hline 1 & $5.0000 E-03$ & $5.0000 E-03$ \\
\hline 1 & 3.8710E-01 & $3.9710 E-01$ \\
\hline 1 & 6. $1000 \mathrm{E}-01$ & 6. 1000E-01 \\
\hline 1 & $1.3200 E+\infty$ & $1.3200 E+00$ \\
\hline 1 & 9.8000E-02 & $9.8000 E-02$ \\
\hline 1 & $5.5000 E-02$ & $5.5000 \mathrm{E}-02$ \\
\hline$i$ & 3.2000E-01 & 3.2000E-01 \\
\hline 1 & $5.4020 E .01$ & $5.4020 E-01$ \\
\hline 4 & 1. $8000 \mathrm{E}-02$ & $1.8000 \mathrm{E}-02$ \\
\hline 1 & $90000 E-01$ & 9.0000E-01 \\
\hline 1 & 3. $3000 \mathrm{E}-03$ & 3.3000E-03 \\
\hline$i$ & $32400 E-01$ & $3.2400 \mathrm{E}-01$ \\
\hline 1 & $3.4000 \mathrm{E}-02$ & 3.4000E-02 \\
\hline 1 & 1.2200E-01 & $1.2200 \mathrm{E}-01$ \\
\hline 1 & $2.1000 E+\infty 0$ & $2.1000 E+00$ \\
\hline 1 & 3.0000E-01 & $3.0000 \mathrm{E}-01$ \\
\hline 1 & $8.0000 E-02$ & $8.0000 E-02$ \\
\hline 1 & $2.0000 \mathrm{E}-01$ & $2.0000 \mathrm{E}-01$ \\
\hline 1 & $1.3900 E+00$ & 1.39COE+00 \\
\hline 1 & $1.5799 \mathrm{E}+01$ & $1.5799 \mathrm{E}+01$ \\
\hline 1 & $3.9648 E+00$ & $3.9848 E+00$ \\
\hline 1 & $5.7000 \mathrm{E}-02$ & $5.7000 \mathrm{E}-02$ \\
\hline 1 & 2.0000E-01 & $2.0000 E-01$ \\
\hline 1 & $1.7100 \mathrm{E}-01$ & 1.7100E-01 \\
\hline 9 & $2.0100 E+00$ & $2.0100 E+00$ \\
\hline 1 & $0.0000 E+00$ & $0.0000 E+\infty 0$ \\
\hline 1 & 6.1600E-02 & $6.1800 E-02$ \\
\hline 1 & $1.2200 \mathrm{E}-01$ & $1.2200 \mathrm{E}-01$ \\
\hline 1 & $8.8170 E+00$ & 8.8170E+00 \\
\hline 1 & $1.8000 E+\infty 0$ & $1.8000 E+00$ \\
\hline 1 & $5.3600 \mathrm{E}-01$ & $5.3660 \mathrm{E}-01$ \\
\hline 1 & $2.6500 \mathrm{E}-01$ & $2.6500 E-01$ \\
\hline 1 & $8.2700 E+\infty$ & 8. $2700 E+00$ \\
\hline 1 & $2.5000 \mathrm{E}-01$ & $2.5000 \mathrm{E}-01$ \\
\hline 1 & $3.5000 \mathrm{E}-01$ & $3.5000 \mathrm{E}-01$ \\
\hline 1 & 1. $6800 \mathrm{E}+00$ & $1.8800 E+\infty 0$ \\
\hline 1 & $45000 E-01$ & 4.5000E-01 \\
\hline 1 & 2.5000E-03 & $2.5000 E-03$ \\
\hline 1 & $1.0000 \mathrm{E}-03$ & $1.0000 E-03$ \\
\hline
\end{tabular}

\begin{abstract}
STD DEV
\end{abstract}
$0.0000 \mathrm{E}+00$

$0.0000 \mathrm{E}+\infty 0$

$0.0000 \mathrm{E}+\infty 0$

$0.0000 E+00$

$0.0000 E+00$

$0.0000 E+\infty 0$

$0.0000 E+00$

$0.0000 \mathrm{E}+00$

$0.0000 E+00$

$0.0000 \mathrm{E}+00$

$0.0000 \mathrm{E}+\infty 0$

$0.0000 \mathrm{E}+\infty$

$0.0000 E+00$

$0.0000 E+00$

$0.0000 \mathrm{E}+\infty 0$

$0.0000 \mathrm{E}+\infty 0$

$0.0000 E+\infty$

$0.0000 \mathrm{E}+\infty 0$

$0.0000 \mathrm{E}+00$

$0.0000 E+00$

$0.0000 \mathrm{E}+00$

$0.0000 \mathrm{E}+00$

$0.0000 E+00$

$0.0000 E+00$

$0.0000 E+00$

$0.0000 \mathrm{E}+00$

$0.0000 \mathrm{E}+00$

$0.0000 E+\infty$

$0.0000 E+\infty$

$0.0000 E+\infty 0$

$0.0000 E+00$

$0.0000 E+\infty$

$0.0000 E+\infty$

$0,0000 E+\infty$

$0.0000 E+00$

$0.0000 E+00$

$0.0000 E+\infty 0$

$0.0000 E+\infty 0$

$0.0000 E+\infty 0$

$0.0000 E+00$

$0.0000 E+00$

0.0000 E +00

$0.0000 E+00$

$0.0000 E+\infty 0$

$0.0000 E+\infty$

$0.0000 E+\infty 0$

$0.0000 E+00$

$0.0000 E+00$

$0.0000 E+00$

$0.0000 \mathrm{E}+00$

$0.0000 E+00$

$0.0000 \mathrm{E}+00$

$0.0000 E+\infty 0$

$0.0000 E+\infty$

$0.0000 E+\infty 0$

$0.0000 E+00$

$0.0000 E+00$

$0.0000 \mathrm{E}+00$

$0.0000 \mathrm{E}+00$

$0.0000 E+00$

$0.0000 \mathrm{E}+00$

$0.0000 E+\infty 0$

$0.0000 E+00$

$0.0000 \mathrm{E}+\infty 0$

$0.0000 \mathrm{E}+00$

$0.0000 E+\infty 0$

$0.0000 \mathrm{E}+00$

$0.0000 E+\infty 0$

$0.0000 E+00$

$0.0000 E+00$

$0.0000 E+00$

$0.0000 E+00$

$0.0000 E+00$

$0.0000 E+\infty 0$

$0.0000 E+00$

$0.0000 E+\infty 0$

$0.0000 E+00$
MINIMUM WT, KG

$1.0000 \mathrm{E}-01$ 1.0010E-01

1.3000E-02

$1.0000 \mathrm{E}-04$

7.0000E-01

2.0500E-01

7. 2000E-01

4. $5000 \mathrm{E}+00$

$1.1000 \mathrm{E}-03$

1.1000 E-01

$4.4500 E+00$

$2.0000 \mathrm{E}+00$

5.5000E-01

2.7000E-01

2.0000E-02

3.0000E-03

3 . $6401 E+01$

2.0000E-01

1. $2500 \mathrm{E}+\infty \mathrm{CO}$

2.0000 E- 02

$8.2280 E+\infty 0$

$1.1200 E+\infty$

$1.9241 \mathrm{E}+01$

$6.6700 \mathrm{E}+00$

2.0000E-01

1.0000E-0

7. 1010E-OI

4.0000E+01

$8.5000 \mathrm{E}-02$

2.0000E-02

$1.3500 \mathrm{E}+\infty$

1.4200E-01

1.0000E+00

$1.1000 \mathrm{E}-\mathrm{O} 1$

$6.0000 \mathrm{E}-02$

$8.0000 E-02$

$1.7098 E+\infty 0$

$0.0000 E+\infty 0$

5 OOOOEE-CS

$3.9710 \mathrm{E}-01$

6.1000E-O

$1.3200 E+\infty$

$0.8000 \mathrm{E}-02$

$5.5000 \mathrm{E}-02$

$32000 \mathrm{E}-01$

$5.4020 E-01$

$1.6000 \mathrm{E}-02$

$0.0000 \mathrm{E}-01$

3. 3000E-03

$3.2400 E-01$

$3.4000 E-02$

1.2200E-01

2.1000E+00

3.0000E.01

8.0000E-02

$2.0000 E .01$

$1.3900 E+\infty 0$

$1.5790 \mathrm{E}+01$

$3.9648 \mathrm{E}+00$

5.700OE-02

20000 E 01

$1.7100 \mathrm{E}-01$

$2.0100 E+00$

$0.0000 \mathrm{E}+\infty$

6.1600E-02

1 . 2200E-01

$8.8170 E+00$

1. $8000 E+00$

5.3660E-01

2.6500E-01

8.2700E +00

2.5000E-01

3.5000E-01

$1.6800 \mathrm{E}+\infty$

$4.5000 \mathrm{E}-01$

2.5000E-03

1.0000 E-03

MAXIMUM WT, KG

1.0000E-01 $1.0010 \mathrm{E}-01$

1.3000E-02

$1.0000 \mathrm{E}-04$

$7.0000 E-01$

2.0500E-01

7.2000E-01

$4.5000 E+00$

$1.1000 \mathrm{E}-03$

1.1000E-01

$4.4500 E+\infty 0$

$2.0000 E+\infty 0$

5.5000E-01

2.7000E-0t

2.0000 E-02

$3.0000 \mathrm{E}-03$

$3.8401 E+01$

2. $9000 \mathrm{E}-01$

1.2500E +00

2.0000E-02

$6.2280 \mathrm{E}+00$

$1.1200 \mathrm{E}+00$

1. $241 \mathrm{E}+01$

6.6700E +00

2.0000E-01

1.0000 E- 01

$7.1010 \mathrm{E}-01$

4. $0000 \mathrm{E}+01$

B.5000E-02

2.0000E-02

$1.3500 E+00$

1. $4200 \mathrm{E}-01$

$1.0000 E+00$

1.1000 E- 01

$0.0000 \mathrm{E}-02$

8.0000E-02

$1.7908 E+\infty$

$0.0000 E+\infty 0$

5. $0000 \mathrm{E}-03$

3 9710E-01

6.1000E-01

$1.3200 E+00$

9.8000 E.02

5.5000E-02

3. 2000E-01

$5.4020 E-01$

1. $8000 E-02$

9.0000 E-O1 
TABLE 4

STATISTICAL ANALYSIS OF HAZARDOUS MATERIALS IN ASCENDING ORDER BASED ON CONTAINER COUNT (* INDICATES DATA EXHIBITED NEGATIVE VARIANCE)

(10 SHEETS)

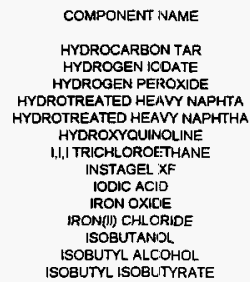

ISOPARAFFINIC PETROLEUM HYOROCARBON ISOPROPLY ALIOOHOI LEAD ACID

LEAD DIOXIDE

LEAD OXILEE

LEAD SALTS

LEAD TELLLURIOE

LEAD TETROXIDE

LEAKING BALUASTS CONTAINING $>500$ PPM PCBS

LIGHT FIXTURES CONTAMINATED WITH POLYCHLORINATED BI

LIOUIFIED PETROLEUM GAS

LITHIUM CARBONATE

LITHIUM CHROMATE

UTTHIUM MYOROSTEARATE

MAGNESIUM HYDROXIDE

MAGNESIUM METAL

MAGNESIUM PERCHLORATE MANGANESE

MANGANESE DIOXIDE

MED ALIPHATIC SOLVENT NAPHTA

MEDIUM ALIPHATIC SOLV NAPHTHA MERCURIC HYOROXIDE METAL.

METALLIC MEFCURY

METHLY ETHYL KETONE

METHYL ALCOHOL SOLUTION

METHYL ETHYL KETONE PEROXIDE

METHYL METHACRYLATE

METIYL ORANGE

METHYL PENTYL KETONE

METHYL-1-PHENYL-2.PYRAZOLIN

METHYLCYCLOHEXANONE

METHYLENE CHILORDIE

METHYLNAPHTHALENE

METHYLTRIACETOXY SILANE

METHYLTRICAPRY ALAMHONIUM CHLORIDE

METHYLTRIOCTY AMMONIUM CHLORIDE

MINERAL OIL PETROLEUM DISTILLATE HYDROTREATED

MINERAL OIL PETROLEIJM DISTILLATES

MINERAL OIL. PETROLEUNI DISTILALTE (MILO)

MINERAL OILPEIROLEIJM DISTILLLATES

MISCELLANEOUS MATERIALS MOLYBDATE ORANGE

MOLYEDIC ACID HEXAMMONIUM SALT

MONO(NONYLPHENYL) POLYETHYLENE GLYCOLS MONOEUTYL ETHER

MORPHOLINE

MULTISCIL

MURIATIC ACID

N,N DIMETHYL.FORMANIDE

N.N.N'N+TETRAMETHYL-1,3-EUTANEDEAMINE

N,N-DIMETHYL-FORMANIOE

N.N-DIMETHYLANILINE

$$
\text { N-BUTANOL }
$$

N-BUTYL ACRYYLATE

N.BUTYL ALCOOHOL

N-DODECANE METHANOL

N-METHYL-2-PYRR

NAPHTHENIC ACICI LEAD SALT

NAPHTHENIC PETROLEUM OIL NAPTHENIC OISTILLATE

\author{
COUNT ACCUM WT, KG
}

4.3500E+02

1.DOOOE-01

1.0000E-01

1.5900 E-02

1.9900E-01

$8.0000 E-02$

$3.5000 \mathrm{E} .02$

3.2998E+01

5. $3100 \mathrm{E}-01$

3. $9800 E-02$

$4.5830 \mathrm{E}+01$

$8.0000 \mathrm{E}-02$

6 4000E-02

$4.9290 \mathrm{E}-01$

3.0000E-04

2. 0000E-02

4.5000E-01

3 . O000E-02

9.5000E-02

4.0000 E -02

2. 2000E-01

$1.3000 \mathrm{E}+\infty 0$

$5.0000 \mathrm{E}+00$

8. 9990E+01

5.2000 E- 02

4 5000E-01

3. 0000 OE-02

$3.6000 E+00$

$12500 \mathrm{E}+0 \mathrm{OO}$

1 . $0000 \mathrm{E}-01$

2 OOOOE-02

95570 E.01

3.2100E-02

5.9600E-01

$2.0000 E-01$

$2.9600 \mathrm{E}+01$

$3.9000 E+00$

2.50BOE-01

$1.7509 \mathrm{E}+00$

1 0000E-03

$9.0000 E-02$

3 COOOE-02

2.3000E.02

2.4600E-01

B. OOOOE-O2

1.4000E-03

4. $4000 E-01$

$3.0000 E-03$

$2.4700 \mathrm{E}+00$

6. 6000E-01

4 AOOOE 01

9 COOOE-03

5.7000 E- 01

$1.0000 \mathrm{E}+00$

1.9000E-D2

5. 2000E-01

3. 5000E-01

3 3000E-01

2.0000E-02

1. $3499 \mathrm{E}+01$

1. $1390 \mathrm{E}+00$

2.4200E +00

1.7500E-01

4. $8000 \mathrm{E}$-0t

1.0000E-02

1.0000 E-02

$1.8410 \mathrm{E}+01$

5. DOOOE-02

$0.0000 \mathrm{E}+\infty$

3.0000E-02

4. $2000 \mathrm{E}-02$

1. 8000E-01

1.5000E-03

1.7000E-01

\author{
AVERAGE WT. KG
}

$1.0000 \mathrm{E}-01$

$1.0000 E-01$

$1.5900 \mathrm{E}-02$

1.9900E-01

8.0000E-02

$3.5000 \mathrm{E}-02$

$3.2999 \mathrm{E}+01$

5.3100E-0t

3.9800E-02

$4.5830 \mathrm{E}+01$

$4.7000 \mathrm{E}-02$

B. $0000 \mathrm{E}-02$

$6.4000 \mathrm{E}-02$

4.9290E-01

$3.0000 E-04$

2.0000E-02

4.5000E-01

$3.0000 E-02$

$9.5000 \mathrm{E}-02$

4. $0000 E-02$

2. 2000E-01

1.3000E +00

1. $1000 \mathrm{E}+00$

$5.0000 \mathrm{E}+00$

$8.9990 \mathrm{E} \cdot 01$

5.2000E-02

$4.5000 E-01$

$3.0000 E-02$

$3.6000 \mathrm{E}+00$

$1.2500 E+00$

$1.0000 \mathrm{E}-01$

2 OOOOE-O2

9.5570E-01

3.2100E-O2

5.9690E-01

2.0000E-01

$2.9000 \mathrm{E}+01$

$3.9000 \mathrm{E}+00$

2.5000 E. 01

$1.7599 \mathrm{E}+00$

1 .0000E-03

$9.0000 E-02$

3.0000E-02

2.3000E-O2

2.4800E-01

D.000E

1. $4000 \mathrm{E}-03$

4. $4000 \mathrm{E}-01$

3.0000 E- 03

$1.5900 \mathrm{E}+00$

$2.4700 E+00$

6.6000E-01

4. 4000E-01

$9.0000 E-03$

5.7000E-01

$1.0000 \mathrm{E}+00$

$1.0000 \mathrm{E}-02$

$5.2000 \mathrm{E}$.

3.5000E-Ot

3.3000 E-01

2.0000E-02

$13499 \mathrm{E}+01$

$1.1390 \mathrm{E}+\infty$

$2.4200 E+00$

1.7500E-01

4.8000E-01

$1.0000 E-02$

$1.0000 \mathrm{E}-02$

$1.8410 \mathrm{E}+01$

5.0000E-02

$0.0000 E+00$

$3.0000 E-02$

4.2000E-02

$18000 \mathrm{E}-\mathrm{O}$

$1.5000 \mathrm{E}-03$

$1.7000 \mathrm{E}$. 01

STD DEV

$0.0000 E+00$

$0.0000 E+00$

$0.0000 \mathrm{E}+00$

$0.0000 E+00$

$0.0000 E-00$

$0.0000 E+\infty 0$

$0.0000 E+\infty 0$

$0.0000 E+\infty 0$

$0.0000 \mathrm{E}+\infty 0$

$0.0000 E+00$

$0.0000 \mathrm{E}+00$

$0,0000 E+00$

$0.0000 E+00$

$0.0000 E+00$

$0.0000 E+00$

$0.0000 \mathrm{E}+00$

$0.0000 E+00$

0 OOOOE +00

$0.000 \mathrm{C}$

$0.0000 \mathrm{E}+00$

$0.0000 E+00$

$0.0000 \mathrm{E}+\infty 0$

$0.0000 E+00$

$0.0000 E+\infty 0$

$0.0000 E+\infty$

$0.0000 E+\infty 0$

$0.0000 E+\infty 0$

$0.0000 \mathrm{E}+00$

$0.0000 E+00$

$0.0000 E+00$

$0.0000 E+\infty 0$

$0.0000 \mathrm{E}+\infty 0$

$0.0000 E+\infty 0$

$0.0000 E+00$

$0.0000 \mathrm{E}+00$

$0.0000 \mathrm{E}+00$

$0.0000 E+00$

$0.0000 E+00$

$0.0000 E+\infty 0$

$0.0000 E+\infty 0$

$0.0000 E+00$

$0.0000 E+00$

$0.0000 E+\infty 0$

$0.0000 E+00$

$0.0000 \mathrm{E}+00$

$0.0000 E+00$

0,000 E +00

$0.0000 E+\infty$

$0.0000 E+\infty 0$

$0.0000 E+00$

$0.0000 E+00$

$0.0000 \mathrm{E}+00$

$0.0000 E+00$

0 O000E+00

$0.0000 E+00$

$0.0000 E+00$

$0.0000 \mathrm{E}+\infty$

$0,000 E+00$

$0,000 E+00$

$0.0000 E+00$

$0.0000 E+00$

0.0000 E +00

$0.0000 E+\infty 0$

$0.0000 E+00$

0 OOOOE+00

0.0000 E+00 
TABLE 4

STATISTICAL ANALYSIS OF HAZARDOUS MATERIALS IN ASCENDING ORDER BASED ON CONTAINER COUNT (* INDICATES DATA EXHIBITED NEGATIVE VARIANCE)

(10 SHEETS)

\begin{tabular}{|c|c|c|c|c|c|c|}
\hline COMPONENT NAME & COUNT & ACCUM WT, KG & AVERAGE WT, KG & STD DEV & MINIMUM WT, KG & MAXIMUM WT, KG \\
\hline NICKEL MONOXIDE & 1 & 6.6000E-01 & $6.6000 E-01$ & $0.0000 \mathrm{E}+00$ & $6.6000 E-01$ & $6.8000 E-01$ \\
\hline NICKEL-CADMIUM EATTERIES & 1 & $1.0000 \mathrm{E}-02$ & $1.0000 \mathrm{E}-02$ & $0.0000 E+00$ & $1.0000 \mathrm{E}-02$ & $1.0000 E-02$ \\
\hline NITROCELLULOSE & 1 & 1.6200E-01 & 1.6200E-01 & $0.0000 E+00$ & 1.6200E-01 & 1.8200E-01 \\
\hline (PHENYL)-N,N-DHSOBUTY-CAREAMOYLMETHYLPHOSPHINE & 1 & 1. $6400 \mathrm{E}-01$ & $1.0400 E-01$ & $0.0000 E+00$ & $1.6400 E-01$ & $1.8400 E-01$ \\
\hline OCTYLPHENOXYPOLYETHOXETHANOL & 1 & 8. $1000 \mathrm{E}-01$ & 8.1000E-01 & $0.0000 E+00$ & $8.1000 E-01$ & $8.1000 E-01$ \\
\hline OXINE & 1 & $2.0000 E-02$ & $2.0000 \mathrm{E}-02$ & $0.0000 \mathrm{E}+00$ & $2.0000 E-02$ & $20000 \mathrm{E}-02$ \\
\hline P-(SEC-BUTYL)-PHENOL & 1 & 4.0000E-03 & 4.0000E-03 & $0.0000 E+00$ & $4.0000 E .03$ & 4.0000E-03 \\
\hline P-DIOXANE & 1 & $4.8000 \mathrm{E}-03$ & 4.8000E.03 & $0.0000 E+00$ & $4.8000 E-03$ & 4.8000E-03 \\
\hline P.FORMALDEHYOE & 1 & $2.0000 E \cdot 02$ & $20000 E-02$ & $0.0000 E+00$ & $2,0000 E-02$ & $2.0000 \mathrm{E}-02$ \\
\hline P-NITROPHENOL & 1 & 3.24DOE-01 & 3.2400E-01 & $0.0000 E+00$ & 3.2400E-01 & 3.2400E-01 \\
\hline PAINT CONTAINING LEAD CHROMATE & 1 & $3.6482 E+01$ & $3.6482 E+01$ & $0.0000 E+00$ & $3.6482 \mathrm{E}+01$ & $3.6482 E+01$ \\
\hline PAINT CONTAININUG NONYLPHENOL & 1 & $80000 E+00$ & $8.0000 E+00$ & $0.0000 E+00$ & $8.0000 E+00$ & $8.0000 E+00$ \\
\hline PCB BALLASTS & 1 & 1.7490E+OO & $1.7490 E+00$ & $0.0000 E+\infty 0$ & $1.7490 \mathrm{E}+00$ & $1.7490 \mathrm{E}+00$ \\
\hline PENTACHLOROPHENOL & 1 & $3.0000 E-03$ & $3.0000 E-03$ & $0.0000 E+00$ & $3.0000 E .03$ & $3.0000 E-03$ \\
\hline PENTAETHYLENE-HEXAMINE & 1 & $4.0000 E-02$ & 4.0000E-02 & $0.0000 E+00$ & 4.0000E-02 & 4.0000E-02 \\
\hline PHENYL GLYCIDYL ETHER & 1 & t.0000E-02 & $1.0000 E-02$ & $0.0000 E+00$ & $1.0000 E-02$ & $1.0000 \mathrm{E}-02$ \\
\hline PIPERAZ|NE & 1 & $1.0000 \mathrm{E}+00$ & $1.0000 \mathrm{E}+00$ & $0.0000 E+\infty$ & $1.0000 E+00$ & $1.0000 \mathrm{E}+00$ \\
\hline PLASTIC & 1 & $1.0000 E+00$ & $1.0000 E+\infty$ & $0.0000 E+00$ & $1.0000 E+\infty 0$ & $1.0000 E+\infty$ \\
\hline PLASTIC ACID SUIT/GLOVESIROPEIRAGS & 1 & $9.0000 E-02$ & 9.0000E-02 & $0.0000 E+\infty$ & $9.0000 \mathrm{E}-02$ & $9.0000 E-02$ \\
\hline POLYCYCLIC AROMATIC HYDROCARBONS & 1 & $4.5000 E+00$ & $4.5000 E+00$ & $0.0000 E+\infty 0$ & $4.5000 E+00$ & $4.5000 \mathrm{E}+00$ \\
\hline POLYETHYLENE & 1 & 5.0000E-01 & $5.0000 E .01$ & $0.0000 E+\infty$ & 5.0000E-01 & $5.0000 E-01$ \\
\hline POLYETHYLENE MONO(NONYLPHENYL) GLYCOLS & 1 & $1.5500 E+00$ & $1.5500 E+\infty$ & $0.0000 E+00$ & $1.5500 E+\infty 0$ & $1.5500 E+\infty 0$ \\
\hline POLYOXPROPYLENE-POLYOXETHYLENE & 1 & $1.0000 E+00$ & $1.0000 E+\infty$ & $0.0000 E+\infty$ & $1.0000 E+\infty$ & $1.0000 E+00$ \\
\hline POLYPROPYLENE GLYCOL & 1 & 3.3000E-01 & $3.3000 E-01$ & $0.0000 E+\infty 0$ & 3.3000E-01 & $3.3000 E-01$ \\
\hline POLYPROPYLENE GLYCOLS & 1 & $2.0000 \mathrm{E}-02$ & $2.0000 E-02$ & $0.0000 E+\infty 0$ & $2.0000 E-02$ & 2.0000E-02 \\
\hline POLYVINYL ALCOHOL & 1 & $3.4000 E-01$ & 3.4000E-01 & $0.0000 E+\infty$ & $3.4000 E-01$ & $3.4000 E-01$ \\
\hline POLYVINM CHLORIDE POLYMER & 1 & $1.0000 E+\infty$ & $1,0000 E+00$ & $0.0000 E+00$ & $1.0000 E+\infty 0$ & $1.0000 E+00$ \\
\hline POTASSIUM ACETATE & 1 & 3.4000E-01 & $3.4000 E-01$ & $0.0000 E+00$ & $3.4000 E-01$ & $3.4000 E-01$ \\
\hline POTASSUUM BROMATE & 1 & 4.5000E-01 & 4.5000E-01 & $0.0000 E+00$ & 4. 5000E-01 & 4.5COOE-01 \\
\hline POTASSIUM CHLORATE & 1 & 4.5000E-01 & 4.5000E-01 & $0.0000 E+00$ & 4.5000E.01 & 4.5000E-01 \\
\hline POTASSIUM DICHROMATE & 1 & 1.2800E-01 & $1,2600 E-01$ & $0.0000 \mathrm{E}+00$ & $1.2800 E-01$ & $1.2600 \mathrm{E}-01$ \\
\hline POTASSIUM METAEISULFATE & 1 & $9.0000 E-01$ & $9.0000 E-01$ & $0.0000 E+00$ & $9.0000 E-01$ & $9.0000 \mathrm{E}-01$ \\
\hline POTASSIUM NITRATE & 1 & 2.5000E-02 & $2.5000 \mathrm{E}-02$ & $0.0000 E+\infty 0$ & $2.5000 E-02$ & $2.5000 \mathrm{E}-02$ \\
\hline POTASSILM NITRITE & 1 & 4.5000E-01 & 4.5000E-01 & $0.0000 E+00$ & 4.5000E-01 & 4.5000E-01 \\
\hline POTASSIUM PERIOOATE & 1 & $2.2000 E-04$ & $2.2000 E-01$ & $0.0000 E+00$ & 2.2000E-01 & $2.2000 \mathrm{E}-01$ \\
\hline POTASSIUM PERMANGANIE & 1 & $9.0000 E-01$ & $9.0000 \mathrm{E}-01$ & $0.0000 E+00$ & $9.0000 E-01$ & $9.0000 E-01$ \\
\hline POTASSIUM PHOSPHATE & 1 & $1.0000 E-02$ & $1.0000 E-02$ & $0.0000 E+00$ & 1.0000E-02 & $1.0000 \mathrm{E}-02$ \\
\hline POTASSIUM ZINC CHROMATE & 1 & 1.0000E-03 & $1.0000 E-03$ & $0.0000 E+00$ & 1.0000E-03 & $1.0000 E-03$ \\
\hline PROPANE 1-NITRO & 1 & $2.7500 \mathrm{E}-02$ & $2.7500 E-02$ & $0.0000 E+\infty$ & $2.7500 E-02$ & $2.7500 E-02$ \\
\hline PROPARGYL ALCOHOL & 1 & $6.9000 \mathrm{E}-01$ & $6.9000 E-01$ & $0.0000 E+\infty$ & $6.0000 \mathrm{E}-01$ & $6.0000 \mathrm{E}-01$ \\
\hline PROPYOIAMINE & 1 & 3.3000E-02 & $3.3000 \mathrm{E}-02$ & $0.0000 E+\infty 0$ & $3.3000 E-02$ & $3.3000 E-02$ \\
\hline PROPYLDIAMINE & 1 & 5.288DE-01 & $5.2880 \mathrm{E}-01$ & $0.0000 E+00$ & 5.2980E-01 & $5.2980 E-01$ \\
\hline PROPYLENE GLYCOL MONOMETHYL ETHER & 1 & $2.6500 \mathrm{E}-01$ & $2.6500 E-01$ & $0.0000 E+\infty$ & $26500 E-01$ & $2.6500 E-01$ \\
\hline PROPYLENEGLYCOL & 1 & 1. $.000 \mathrm{E}+\infty 0$ & $1.0000 E+00$ & $0.0000 E+00$ & $1.0000 \mathrm{E}+00$ & $1.0000 E+00$ \\
\hline PSUEDOCUMENE & 1 & $8.0013 E+\infty 0$ & $8.0013 E+\infty 0$ & $0.0000 E+00$ & $8.0013 E+00$ & $8.0013 E+00$ \\
\hline RUTHENIUM CHLORIOE & 1 & $2.0372 E+01$ & $2.0372 \mathrm{E}+01$ & $0.0000 \mathrm{E}+00$ & $2.0372 E+01$ & $2.0372 E+01$ \\
\hline SALLCYLIC ACID & 1 & $4.6000 E-03$ & $4.6000 E-03$ & $0.0000 \mathrm{E}+\infty 0$ & 4.6000E-03 & 4.6000E-03 \\
\hline SEC BUTYL ALCOHOL & 1 & $1.0000 E-02$ & $1.0000 \mathrm{E}-02$ & $0.0000 \mathrm{E}+\infty$ & $1.0000 E-02$ & $1.0000 \mathrm{E}-02$ \\
\hline SEC.BUTYL ALCOHOL & 1 & 3.4000 E -02 & $3.4000 \mathrm{E}-02$ & $0.0000 E+00$ & 3. $4000 \mathrm{E}-02$ & $3.4000 \mathrm{E}-02$ \\
\hline SILICA CRYSTALLINE OUARTZ & 1 & $6.7000 \mathrm{E}-02$ & $6.7000 E-02$ & $0.0000 E+00$ & 6.7000E-02 & $6.7000 \mathrm{E}-02$ \\
\hline SILVER CHLORIDE & 1 & $8.5500 \mathrm{E}-02$ & $8.5500 \mathrm{E}-02$ & $0.0000 E+00$ & $8.5500 \mathrm{E}-02$ & $8.5500 E-02$ \\
\hline SILVER ZEOLITE & 1 & $3.7300 E+\infty$ & $3.7300 E+00$ & $0.0000 \mathrm{E}+00$ & $3.7300 \mathrm{E}+\infty 0$ & $3.7300 E+00$ \\
\hline SODA ASH & 1 & $3.0000 E+00$ & $3.0000 E+00$ & $0.0000 E+00$ & $3.0000 E+00$ & $3.0000 E+00$ \\
\hline SODIUM METABORATE OCTAHYDRATE & 1 & $9.0000 E-01$ & $9.0000 \mathrm{E}-01$ & $0.0000 E+00$ & $9.0000 E-01$ & $9.0000 E-01$ \\
\hline SODIUM NITROFERRICYANIDE & 1 & $29500 \mathrm{E}-01$ & 2.8500E-01 & $0.0000 \mathrm{E}+\infty$ & 2.9500E-01 & $2.9500 E-01$ \\
\hline SOOUM PHOSPHATE TRIEASIC & 1 & $3.3570 \mathrm{E}+00$ & $3.3570 E+00$ & $0.0000 E+00$ & $3.3570 E+\infty 0$ & $3.3570 E+00$ \\
\hline SODIUM PYROPHOSPHATE & 1 & $1.3900 E+00$ & $1.3900 \mathrm{E}+00$ & $0.0000 E+\infty$ & $1.3900 E+00$ & $1,3000 \mathrm{E}+\infty$ \\
\hline SODIUM SULFIDE 9-HYDRATE & 1 & $4.9090 E-01$ & 4.9900 E-01 & $0.0000 E+00$ & 4.9800E-01 & 4.9000E-01 \\
\hline SODIUM SULFITE & 1 & 9.0000E-01 & $9.0000 E-01$ & $0.0000 E+\infty$ & $9.0000 E-01$ & $9.0000 E-01$ \\
\hline SODIUM TRIPOLY PHOSPHATE & 1 & $1.6200 E+01$ & 1.6200E+01 & $0.0000 E+00$ & $1.6200 E+01$ & $1.6200 E+01$ \\
\hline SOIL & 1 & $4.5000 E+\infty 0$ & $4.5000 E+00$ & $0.0000 E+00$ & $4.5000 E+00$ & $4.5000 E+00$ \\
\hline SOLVENT PETROLEUM DISTILLATE & 1 & $2.5000 E-01$ & 2. 5000E-01 & $0.0000 E+00$ & 2.5000E-01 & $2.5000 E-01$ \\
\hline SOLVENTS & 1 & $8.8495 E+00$ & $8.8495 \mathrm{E}+00$ & $0.0000 E+\infty$ & 8. $8495 E+00$ & $8.8495 E+\infty 0$ \\
\hline STANNOUS SULFATE & 1 & 1.7100E-01 & 1.7100E-01 & $0.0000 E+\infty 0$ & $1.7100 E-01$ & 1.7100 E-01 \\
\hline STARGLAZE & 1 & $1.0201 E+00$ & $1.0201 E+\infty$ & $0.0000 E+\infty 0$ & $1.0201 E+\infty$ & 1.0201E+O0 \\
\hline TALC & 1 & 7.3000E+00 & $7.3000 \mathrm{E}+00$ & $0.0000 E+00$ & $7.3000 E+\infty$ & $7.3000 E+00$ \\
\hline TAR & 1 & $1.0201 E+00$ & $1.0201 E+00$ & $0.0000 E+00$ & $1.0201 E+\infty 0$ & $1.0201 E+00$ \\
\hline TARTARIC ACID & 1 & 3. $7500 E-C 2$ & $3.7500 \mathrm{E}-02$ & $0.0000 E+00$ & $3.7500 \mathrm{E} .02$ & $3.7500 E-02$ \\
\hline TELLURIUM METAL & 1 & $6.0000 E \cdot 01$ & 6.CO00E.01 & $0.0000 E+00$ & $6.0000 E-01$ & $6.0000 \mathrm{E}-01$ \\
\hline TETRAACETIC ACID & 1 & 5. $0000 \mathrm{E}-01$ & $5.0000 E-01$ & $0.0000 \mathrm{E}+00$ & 5 OOOOE-01 & 5.0000E-01 \\
\hline TETRAETHYL AMMONIUM GROMIDE & 1 & $0.0000 E+00$ & $0.0000 \mathrm{E}+00$ & $0.0000 E+\infty 0$ & $0.0000 \mathrm{E}+00$ & $0.0000 E+00$ \\
\hline TETRAMETHYL 1,3-BUTANEDIAMINE & 1 & $33000 E-02$ & $3.3000 E-02$ & $0.0000 E+00$ & $3.3000 E-02$ & $3.3000 E-02$ \\
\hline TETRAMETHYLAMMONIUM EROMIDE & 1 & 8.6000E-01 & $8.6000 E-01$ & $0.0000 E+\infty$ & B.6000E-01 & B. $8000 E-01$ \\
\hline TETRAPHYENYLARSONIUM HYDROCHLORITE & 1 & 1.COOOE-04 & $1.0000 \mathrm{E}-04$ & $0.0000 E+\infty 0$ & $1.0000 \varepsilon \cdot 04$ & $1.0000 \mathrm{E} .04$ \\
\hline THENOLYTRIFLUOROACETONE & 1 & $1.1000 \mathrm{E}+00$ & $1.1000 \mathrm{E}+00$ & $0.0000 E+\infty 0$ & $1.1000 E+\infty$ & $1.1000 E+00$ \\
\hline THENOYLTRIFLUOROACETATE & 1 & $1.4000 E-03$ & $1.4000 E-03$ & $0.0000 E+00$ & $1.4000 E-03$ & $1.4000 E-03$ \\
\hline THIOACETAMIDE & 1 & $3.6000 E-03$ & 3.8000E-03 & $0.0000 E+00$ & $3.6000 E .03$ & $3.8000 \mathrm{E}-03$ \\
\hline
\end{tabular}


TABLE 4

STATISTICAL ANALYSIS OF HAZARDOUS MATERIALS

IN ASCENDING ORDER BASED ON CONTAINER COUNT

(* INDICATES DATA EXHIBITED NEGATIVE VARIANCE)

(10 SHEETS)

COMPONENT NAME
THIONYL CHLORIDE
THORIUM NITRATE TEIRAHYDRATE
TRIBUTY PHOSPHATES
TRICHLOROTRIFLUOROETHANE
TRIETHYLENERETRAMINE
TRIFLUOROACEIIC ACID
TRIOCTYLAMINE
TRIOCTYPHOSPHINE OXIDE
TRISODIUM PHOSPHATE
UNDECANE
VANDYL SULFATE
VINYL ACEIATE
VINYL ESTER
VINYLIDENE-1, 1 DIPHOSPHONIC ACIO
WHITE POWDEER CONTAINING SILOXANES
XYLENE SULFONIC ACID SOOIUM SALT
XYLENE-P
YTRIUM NITRATE
ZINC NAPHTHENATE
ZINC SALT
ZIRCONHUM SULPHATE

AVERAGE WT, KG
$1.3800 E-02$
$4.5000 E-01$
$4.2300 E+00$
$4.4000 E-01$
$2.2600 E-01$
$2.0000 E-02$
$4.8000 E-01$
$0.0000 E+00$
$3.4100 E+01$
$1.6000 E-01$
$5.4000 E-01$
$8.9090 E-01$
$3.0000 E-03$
$1.0000 E-03$
$4.2000 E+01$
$1.8400 E+00$
$1.2000 E-01$
$4.4500 E+00$
$2.5000 E-03$
$1.2200 E-01$
$2.9900 E-01$

STD DEV

$0.0000 E+\infty 0$

$0.0000 E+\infty 0$

$0.0000 \mathrm{E}+\infty 0$

$0.0000 \mathrm{E}+00$

$0.0000 E+00$

$0.0000 E+00$

$0.0000 E+00$

$0.0000 E+00$

$0.0000 \mathrm{E}+00$

$0.0000 \mathrm{E}+\infty 0$

$0.0000 E+\infty 0$

$0.0000 \mathrm{E}+00$

$0.0000 \mathrm{E}+00$

$0.0000 \mathrm{E}+\infty 0$

$0.0000 \mathrm{E}+\infty 0$

$0.0000 \mathrm{E}+\infty 0$

$0.0000 \mathrm{E}+\infty$

$0.0000 \mathrm{E}+\infty$

$0.0000 E+\infty$

$0.0000 E+\infty 0$

$0.0000 \mathrm{E}+00$
MINIMUM WT, KG

$1.3800 E-02$

$4.5000 E-01$

4. $2300 \mathrm{E}+00$

4. $4000 E-01$

2.2500E-01

2.0000E-02

4. $8000 \mathrm{E}-01$

$0.0000 \mathrm{E}+00$

3.4100E+01

$1.0000=01$

5. $4000 \mathrm{E}-01$

8.9090 E- 01

3. $0000 \mathrm{E}-03$

1. $0000 \mathrm{E}-03$

4.2000E+01

$1.8400 \mathrm{E}+00$

1.2000E-01

$4.4500 \mathrm{E}+00$

2.5000E-03

12000

2.9900E-01
MAXIMUMM WT, KO

1.3800E-02 4.5000E-01

4. $2300 \mathrm{E}+00$

4.4000E-01

2.2500E-01

2.0000E-02

4.8000E-01

O. $0000 \mathrm{E}+00$

3.4100E+01

1.6000E-01

5.4000E-01

8.9900E-01

3.0000 E-03

1.0000E-03

4. $2000 \mathrm{E}+01$

1. $2400 \mathrm{E}+\infty 0$

1.2000E-01

$4.4500 \mathrm{E}+\infty$

$2.5000 E-03$

$1.200 \mathrm{E}-01$

2.9800E-01 
TABLE 5

STATISTICAL ANALYSIS OF HAZARDOUS MATERIALS IN ASCENDING ORDER BASED ON ACCUMULATED WEIGHT (* INDICATES DATA EXHIBITED NEGATIVE VARIANCE)

(10 SHEETS)

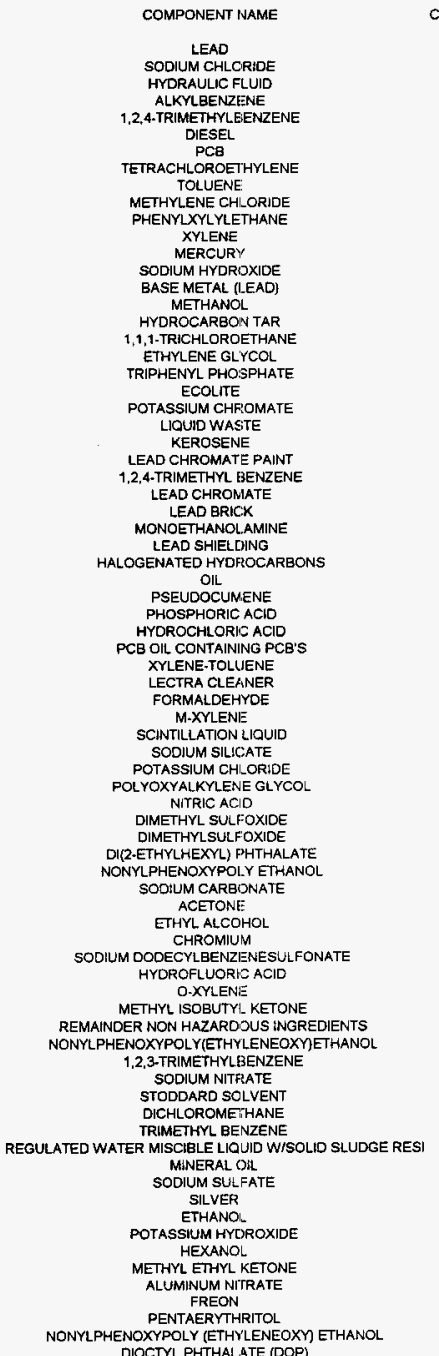

\begin{tabular}{|c|c|c|}
\hline COUNT & ACCUM WT, KG & AVERAGE WT. KG \\
\hline 423 & $9.6972 E+03$ & $2.3089 E+01$ \\
\hline 42 & $5.3830 E+03$ & $1.2817 E+02$ \\
\hline 160 & $3.7601 E+03$ & $2.3501 \mathrm{E}+01$ \\
\hline 117 & $2.9407 E+03$ & $2.5134 \mathrm{E}+01$ \\
\hline 113 & $1.7590 \mathrm{E}+03$ & $1.5705 E+01$ \\
\hline 85 & $1.3744 E+03$ & $1.6170 E+01$ \\
\hline 672 & $1.3004 E+03$ & $2.2078 E+00$ \\
\hline 252 & $1.2318 E+03$ & $5.3096 \mathrm{E}+00$ \\
\hline 205 & $1.0789 E+03$ & $5.2886 \mathrm{E}+00$ \\
\hline 346 & $9.8554 \varepsilon+02$ & $2.8484 E+00$ \\
\hline 117 & $9.4292 E+02$ & $8.0591 E+00$ \\
\hline 169 & $9.1173 E+02$ & $5.5256 E+00$ \\
\hline 262 & $7.9173 E+02$ & $3.0218 \mathrm{E}+00$ \\
\hline 61 & $7.1893 E+02$ & $1.1786 \mathrm{E}+01$ \\
\hline 28 & $5.0408 E+02$ & $1.8003 E+01$ \\
\hline 154 & 4. $4951 E+02$ & $2.9573 E+00$ \\
\hline 1 & 4.3500E+02 & $4.3500 E+02$ \\
\hline 327 & $4.3043 E+02$ & $1.3163 E+00$ \\
\hline 120 & $4.2935 E+02$ & $3.5779 \mathrm{E}+00$ \\
\hline 37 & $4.2252 \mathrm{E}+02$ & 1.1419E+01 \\
\hline 7 & $3.8567 \mathrm{E}+02$ & $5.5096 \mathrm{E}+01$ \\
\hline 35 & $3.5130 E+02$ & $1.0332 \mathrm{E}+01$ \\
\hline 7 & $3.5112 \mathrm{E}+02$ & $5.0160 E+01$ \\
\hline 13 & $3.4384 E+02$ & $2.6449 E+01$ \\
\hline 15 & $3.3131 E+02$ & $2.2087 E+01$ \\
\hline 16 & $3.2072 E+02$ & $2.0045 E+01$ \\
\hline 58 & $3.2057 \mathrm{E}+02$ & $5.5271 E+00$ \\
\hline 6 & $3.1612 E+02$ & $5.2688 E+01$ \\
\hline 19 & $3.0584 E+02$ & $1.8991 E+01$ \\
\hline 2 & $2.9540 E+02$ & $1.4770 E+02$ \\
\hline 8 & $2.6780 E+02$ & $3.3475 E+01$ \\
\hline 13 & $2.6105 \mathrm{E}+02$ & $2.0081 E+01$ \\
\hline 19 & $2.4001 \mathrm{E}+02$ & $1.2632 \mathrm{E}+01$ \\
\hline 38 & $2.3646 E+02$ & 8.3908E $+\infty$ \\
\hline 37 & $2.1603 E+02$ & $5.8386 \mathrm{E}+\infty 0$ \\
\hline 14 & $1.9775 E+02$ & $1.4125 E+01$ \\
\hline 10 & $1.0000 \mathrm{E}+02$ & $1.0000 E+01$ \\
\hline 7 & $1.8816 E+02$ & $2.6880 E+01$ \\
\hline 99 & $1.7822 \mathrm{E}+02$ & $1.8103 E+00$ \\
\hline 30 & $1.7412 \mathrm{E}+02$ & $5,8040 E+00$ \\
\hline 8 & $1.3168 E+02$ & 1. $6460 E+01$ \\
\hline 22 & $1.3149 E+02$ & $6.2612 \widetilde{E}+00$ \\
\hline 7 & $1.2224 \mathrm{E}+02$ & $1,7462 E+01$ \\
\hline 4 & $1.2000 \mathrm{E}+02$ & $3.0000 E+01$ \\
\hline 30 & $1.1565 E+02$ & $3.8549 \mathrm{E}+00$ \\
\hline 6 & 1.1393E+02 & $2.2785 E+01$ \\
\hline 5 & $1.0649 E+02$ & $2.1297 E+01$ \\
\hline 17 & 1.0007E+02 & $5.8865 \mathrm{E}+00$ \\
\hline 21 & $9.8990 E+01$ & $4.713 \mathrm{BE}+00$ \\
\hline 24 & $9.7828 E+01$ & $4.2534 E+00$ \\
\hline 232 & $9.7270 \mathrm{E}+01$ & 4. $1929 \mathrm{E}-01$ \\
\hline 13 & $9.5095 E+01$ & $7.3150 E+\infty 0$ \\
\hline 94 & $9.2783 \mathrm{E}+01$ & $1.0085 E+00$ \\
\hline 13 & $8.0855 E+01$ & $62196 \mathrm{E}+00$ \\
\hline 9 & $8.0591 E+01$ & $8.9545 \mathrm{E}+00$ \\
\hline 27 & $77100 E+01$ & $2.8556 \mathrm{E}+00$ \\
\hline 114 & $7.5529 \mathrm{E}+01$ & 6.6253E-01 \\
\hline 2 & $7.4029 E+01$ & $37014 E+01$ \\
\hline 12 & $7.2790 \mathrm{E}+01$ & $6.0658 E+00$ \\
\hline 16 & $7.1030 E+01$ & $4.4394 E+00$ \\
\hline 19 & $6.9593 E+01$ & $3.6628 E+00$ \\
\hline 15 & $6.8724 E+01$ & $45814 E+00$ \\
\hline 12 & $6.8060 E+01$ & $5.6717 E+00$ \\
\hline 2 & $6.6700 E+01$ & $3.3350 E+01$ \\
\hline 3 & $6.5030 E+01$ & 2.1677E+01 \\
\hline 11 & $6.4085 E+01$ & $5.8259 E+\infty$ \\
\hline 13 & 6. $3527 E+01$ & $48887 \mathrm{E}+\infty 0$ \\
\hline 39 & 6.3271E +01 & $1.8223 E+\infty 0$ \\
\hline 20 & $6.3077 \mathrm{E}+01$ & $3.1538 E+00$ \\
\hline 32 & $6.2801 E+01$ & $1.2625 E+00$ \\
\hline 6 & $6.1410 E+01$ & $1.0235 E+01$ \\
\hline 198 & $5.8861 E+01$ & $2.9728 \mathrm{E}-01$ \\
\hline 5 & $5.2432 E+01$ & $1.0486 E+01$ \\
\hline 2 & $5.2000 E+01$ & 2.6000E+01 \\
\hline 4 & $5.2000 E+01$ & $1.3000 E+01$ \\
\hline 9 & 5.1040E+01 & $5.6711 E+\infty 0$ \\
\hline 22 & $4.9230 E+01$ & $2.2377 \mathrm{E}+00$ \\
\hline
\end{tabular}

STD DEV

$0.0850 \mathrm{E}+0$

$7.2011 \mathrm{E}+01$

$15495 \mathrm{E}+01$

1.1380E+O1

1.1348E+01

$1.3156 \mathrm{E}+01$

$9.7569 \mathrm{E}+00$

1. $3468 \mathrm{E}+01$

$9.6828 \mathrm{E}+00$

$1.1385 \mathrm{E}+01$

$5.8717 \mathrm{E}+00$

$8.8922 \mathrm{E}+00$

1.744日E+O1

$2.0976 \mathrm{E}+0.1$

$3.6768 \mathrm{E}+01$

$5.3521 \mathrm{E}+\infty 0$

$0.0000 \mathrm{E}+00$

$5.4534 \mathrm{E}+\infty$

$9.0760 E+\infty 0$

$1.7065 \mathrm{E}+\infty 0$

$2.4390 \mathrm{E}+01$

$$
\text { . }
$$

$1.2651 \mathrm{E}+01$

$1.6830 \mathrm{E}+01$

$2.2040 \mathrm{E}+01$

$1.2465 \mathrm{E}+01$

1.2518E+01

$1.7890 E+01$

8.9851E+00

$1.4467 \mathrm{E}+02$

$6.3097 \mathrm{E}+00$

$2.5269 \mathrm{E}+01$

$1.1226 \mathrm{E} \rightarrow 01$

$1.0495 \mathrm{E}+01$

$1.5106 E+01$

$1.5559 E+01$

$0.0000 E+00$

2.7776E+01

$1.5786 E+00$

$6.8007 E+00$

$5.6909 \mathrm{E}+00$

1.0664E+01

2.8368 E +01

$0.0000 \mathrm{E}+00$

$6.3893 \mathrm{E}+\infty$

$6.4843 E+00$

7 S606E $+\infty$

$3.4629 E+\infty 0$

$3.2957 E+00$

$2.2971 E+00$

$4.0173 E+00$

$1.5848 E+00$

1. $7699 \mathrm{E}+00$

1. 8132E+01

$4.9325 E+00$

3. $1688 E+\infty$

$2.1920 E+00$

$1.7259 \mathrm{E}+\mathrm{OO}$

2.427TE+00

$3.7049 E+00$

8. $8346 \mathrm{E}+00$

2. $2417 \mathrm{E}+00$

$1.3435 E+\infty 0$

$1.5188 E+01$

$5.6532 E+\infty 0$

1.5142E+00

$5.3398 \mathrm{E}+00$

$6.1363 E+00$

$3.8268 \mathrm{E}+00$

$2.4762 \mathrm{E}+01$

$1.141 \mathrm{BE}+\infty$

$2.1305 \mathrm{E}+01$

$1.2728 \mathrm{E}+01$

0.0000 E +00

$2.2801 E+00$

1.7554E+00
MINAMUM WT, KG

$0.0000 E+\infty$

2.5000E-03

9.1000E-01

6.1000E-03

$3.3000 E+00$

$0.0000 E+00$

$0.0000 E+00$

$0.00005+00$

$0.0000 E+00$

2.3000E-01

$1.0000 E-04$

$0.0000 E+00$

2.0000E-02

3.0000E-04

$1.0000 E-04$

4.3500E+02

$0.0000 E+00$

$3.6000 E-03$

$1.3200 E+00$

$3.7040 \mathrm{E}-01$

$8.4000 \mathrm{E}+00$

2. 2670E+01

$3.0000 \mathrm{E}-01$

$10002 \mathrm{E}+00$

0.2000 E- 02

8.2000E-03

$3.3861 \mathrm{E}+01$

7.0000E-02

$1.8200 E+01$

$8200 E+01$

4.0002E +00
$7.4980 E-01$

$1.0000 \mathrm{E}-04$

4.0000E-0S

1.0000 E- 04

1.8000E +01

$1.0002 \mathrm{E}+00$

$0.0000 E+00$

$0.0000 E+00$

$9.7600 \mathrm{E}+00$

$5.0000 E-02$

$8.5500 \mathrm{E}-02$

3. $0000 \mathrm{E}+01$

2.1000E-02

1.6801E+01

$7000 E+00$

7.5000E-01

4.0000 E- 02

8.0000 .04

$0.0000 E+\infty 0$

$0.0000 E+00$

2.03B4E+00

$5.3000 \mathrm{E}-03$

$0.0000 E+00$

$1.0000 \mathrm{E}-04$

3.5465E+01

$1.9000 \mathrm{E}+\infty$

4. $6000 \mathrm{E}-01$

$3.6000 \mathrm{E}-02$

1. 1040E-01

2.B699E+00

$3.2400 \mathrm{E}+01$

4. $1600 \mathrm{E}+\infty 0$

1.0000 E-02

$2.0384 E+00$

$1.0000 \mathrm{E}-03$

1.5000 E. 02

$1.0000 \mathrm{E}-02$

5.0000E-02

$0.0000 E+00$

$2.0000 E-01$

1.7000E+01

1.3000E+01

1.3200E+00

3.000OE +02
MAXIMUM WT, KG

1.1376E+0s

$1.8990 E+02$

$6.2680 E+01$

$5.7016 \mathrm{E}+01$

4.0000E +01

$1.0260 E+02$
$8.6130 E+01$

4. $7000 \mathrm{E}+01$

7.5940E +01

$1.7390 \mathrm{E}+01$

2. $5000 E+01$

1. $1850 \mathrm{E}+02$

7. $6500 \mathrm{E}+01$

1.2400E +02

$3.9200 \mathrm{E}+01$

4.350OE +02

4.9280E +01

4. $1900 \mathrm{E}+01$

1. 1700E+01

6.5800E+01

1.0400E+01

5. 6774E+O1

4.0100E+01

$3.5800 E+01$

5. 1392E+01

B. 2508E+01

$3.3410 E+01$
$25000 E+02$

$3.6300 E+01$

$1.0002 E+02$

4.1998E+01

4.0988E+01

9.1948E+01

3.1210E+01

1.8000E+01

6.7580E+01

1.1810E+01

1.7000E+01

$2.7000 E+01$

5.3000E+01

8.5997E+01

$3.0000 E+01$

2.8080E+01

$3.3410 \mathrm{E}+01$

2.4350E+01

2.7760E+01

1.2440E+01

1.1760E+01

$1.0000 \mathrm{E}+01$

1. $4601 \mathrm{E}+01$

$4.7000 E+00$

$8.1283 E+00$

$5.2344 E+01$

1.5300E+01

$3.3000 E+01$

$3.8564 E+01$

B.0300E + 00

$7.0000 \mathrm{E}+00$

$1.1000 \mathrm{E}+01$

$2.7000 E+01$

9.4618E+DO

$3.4300 \mathrm{E}+0.1$

$3.1185 \mathrm{E}+01$

$1.5300 \mathrm{E}+01$

$7.8925 \mathrm{E}+\infty$

$2.2400 E+01$

$2.0210 E+01$

$1.8990 \mathrm{E}+01$

$6.0780 \mathrm{E}+01$

1.1420E+01

$48870 E+01$

$3.5000 E+0$

1.3000E +01

8. $2400 \mathrm{E}+00$

6. $7600 \mathrm{E}+00$ 
TABLE 5

STATISTICAL ANALYSIS OF HAZARDOUS MATERIALS IN ASCENDING ORDER BASED ON ACCUMULATED WEIGHT (* INDICATES DATA EXHIBITED NEGATIVE VARIANCE)

(10 SHEETS)

$$
\begin{aligned}
& \text { COMPONENT NAME } \\
& \text { CARBON TETRAC-HLORIDE } \\
& \text { ALKYNAPHTHALENE } \\
& \text { CHROMIUM TRIOXIDE } \\
& \text { DIMETHYLSUFOXIDE } \\
& \text { IRON(II) CHLORIOE } \\
& \text { PSEUDOCUMENE-XYLENE } \\
& \text { ISOAMYL ALCOHOL } \\
& \text { 1,1-DICHLOROETHYLENE } \\
& \text { 2-BUTOXYETHANOL. }
\end{aligned}
$$

WHITE POWDER CONTAINING SILOXANES DEBRIS

NAPTHALENE

AMMONHUM CITRATE

OCTYLPHENOXYPOLYETHOXYETHANOL DOP

PAINT CONTAINING LEAD CHROMATE

CHLOROFLUOROMETHANE

ALKYL BENZENES

BROMIDE MIXTURES

1.3.5.TRIMETHYYLENZENE

TRISODIUM PHOSPHATE STRIPCOAT

MONOETHANOLAMUNE DIMETHYL SULFOXIDE FLUORS

INSTAGEL XF

POLYCHLORINATED BIPHENYLS

HALOGENATED HYDROCARBON CREOSOTE

$$
\text { METAL }
$$

METHYL ALCOHOL

HYDROCARBONS

SODIUM NITRTE

ISOPROPANOL

D-TAR

POTASSIUM PERMANGANATE

PERCHLOROETHYLENE NAPHTHA

P.XYLENE

ETHYL BENZENE

TRI BUTYL PHOSPHATE

CHLORINATED HYOROPARAFFIN EPOXY RESIN

ISOPROPYLACETONE

1,1,2,2-TETRABROMOETHANE

2.ETHOXYETHANOL

BIS(2-ETHYLHEXYL)ESTER (DOP)

CALCIUM CHLORIDE WATER

RUTHENIUM CHLORIDE

TTERIES CONTAINING LEAD, LEAD OXIDES, AND SULFURIC A CORROSIVE REACTOR ANALYSIS SOLUTION PHENOL.

2,5-DIPHENYLOXAZOLE

ALIPHATIC PETROLEUN DISTILLATES

COAL TAR PITCH

N-BUTYL ACRYLATE

DIMETHYL SULFATE

AMMONUUM SULFATE

DIOCTYL PHTHALATE

SODIUM TRIPOLY FHOSPHATE

$$
\text { HYDRAZINE }
$$

ETHANOLAMINE OMMETHYLSULFOXIDE

1-ETHYL-2-METHYLBENZENE

SOPROPYL ACETONE

1,1, 2-TRICHLORO-1,2, 2-TRIFLUOROETHANE NITROBENZENE

CHLORINATED PARAFFIN HEXANE

BIS (2-ETHYL HEXYL) HYOROGEN PHOSPHATE

BIS(2.ETHYLHEXYL]PHTHALATE BARIUM

CARBONTETRACHLORIDE MULTISOL

DIETHYLENE GLYCOL

ACETONITFILE

\author{
COUNT ACCUM WT, KG AVERAGEWT.KG
}

$1.1379 \pm+\infty$

1. $6113 E+01$

2.3003E+01

2.2050E+01

$4.5830 \mathrm{E}+01$

1.1135E+01

1.1002E+01

$1.3341 E+\infty$

$1.5898 E+\infty 0$

4. 2000 EE+01

$4.0000 E+01$

$1.6474 E+\infty 0$

$7.7862 \mathrm{E}+00$

2.7205E $+\infty$

$3.3673 E+\infty 0$

$3.6482 E+01$

$3.6401 \mathrm{E}+04$

$1.7363 E+01$

$3.4700 \mathrm{E}+01$

$2.8842 \mathrm{E}+00$

3. $4100 E+01$

4. $2613 \mathrm{E}+00$

1. $6749 \mathrm{E}+01$

8. $3100 \mathrm{E}+00$

$32099 \mathrm{E}+01$

5.4533E+ 00

5.4582E-01

4.2543E+ 00

2.9600E+01

$1.3599 \mathrm{E}+00$

1. 3700E+Q1

3. $3888 \mathrm{E}+00$

$1.7693 \mathrm{E}+00$

$5.2900 \mathrm{E}+00$

$5.2000 E+\infty$

$6.5663 E+\infty$

$5.9554 \mathrm{E}-01$

$2.0150 \mathrm{E}+00$

1.2145E +00

5.9477E-01

$30013 E+\infty 0$

$5.8512 \mathrm{E}+00$

$2.3380 E+\infty 0$

$2.8700 E+\infty 0$

1.1085E+01

$4.9383 E .01$

1.3550E+OO

$5.2372 \mathrm{E}+00$

$6.8601 \mathrm{E}+00$

$2.0372 \mathrm{E}+01$

$2.0130 E+01$

$1.9241 E+01$

$1.5725 E+00$

4.2750E-01

$1.8700 \mathrm{E}+01$

$3.0867 \varepsilon+00$

$1.8410 E+01$

$9.1900 \mathrm{E}+00$

$4.4175 \mathrm{E}+00$

$2.4043 \mathrm{E}+00$

1.6200E+01

1. $5990 \mathrm{E}+01$

$15789 E+01$

$1.1093 E+00$

3.8325E+CO

$1.5288 E+\infty$

7.6102E-01

5.5669E-01

4. $8816 \mathrm{E}+00$

7.3150E +00

1. $4400 E+01$

7.3168E-02

4.5527 +00

1.3489E+01

$2.1456 \mathrm{E}+\infty 0$
$2.5711 \mathrm{E}+\infty 0$

STD DEV

$5.5655 E+\infty$

$5.4610 \mathrm{E}+\infty$

0.8005E-01

$0.0000 \mathrm{E}+\infty 0$

$1.0516 \mathrm{E}+01$

$3.2186 \mathrm{E}+\infty 0$

$1.5110 E+\infty 0$

$0.0000 E+00$

$0.0000 \mathrm{E}+00$

$1.8527 \mathrm{E}+00$

$7.8071 \mathrm{E}+\infty 0$

$1.3833 \mathrm{E}+00$

$4.6356 \mathrm{E}+00$

$0.0000 \mathrm{E}+00$

$0.0000 \mathrm{E}+00$

5.3033E.02

$.0000 E+00$

$.0048 \mathrm{E}+00$

$0.0000 E+00$

$7.1428 \mathrm{E}+\infty$

7.0057E- 01

$0.0000 E+00$

$1.2048 E+01$

$1.6162 \mathrm{E}+\infty 0$

$2.3559 \mathrm{E}+\infty 0$

$0.0000 E+00$

$1.1891 E+00$

$1.8967 E+0$

$7.1573 \mathrm{E}+00$

$1.1279 \mathrm{E}+\infty 0$

$0.0000 E+00$

$0.0000 E+00$

$1.2360 \mathrm{E}+01$

4. 5976E- 01

4. $4205 E+00$

$2.4545 \mathrm{E}+00$

5.8377E-01

$1.1137 \mathrm{E}+\infty$

$1.0081 \mathrm{E}+\infty$

$4.5812 E+\infty$

3. $2253 E+00$

$1.3811 E+01$

5.2078E-01

$4.6200 \mathrm{E}+00$

$55593 E+00$

$9.0165 E+00$

$0.0000 E+\infty$

$0.0000 E+\infty 0$

$0.00 C 0 E+00$

$3.0433 E+00$

$3.2711 \mathrm{E}-01$

$0.0000 E+00$

$1.8582 E+00$

$0.0000 \mathrm{E}+00$

$12176 \mathrm{E}+01$

$7.4630 E+\infty$

$23481 E+\infty 0$

$0.0000 E+00$

7.7782E-01

$0.0000 \mathrm{E}+00$

$0.0000 \mathrm{E}+00$

7.5543E-01

$3.5660 E+\infty$

1. $0844 \mathrm{E}+00$

$3.3515 E+00$

7 2056E-01

6. $3732 \mathrm{E}+\infty$

$1.0161 \mathrm{E}+01$

$0.0000 \mathrm{E}+00$

1.9773E-01

$6.8127 \mathrm{E}-0$

$0.0000 \mathrm{E}+00$

$2.0730 \mathrm{E}+00$

$3.7650 \mathrm{E}+\infty 0$

MINIMUM WT, KG

MAXIMUM WT, KG

1.1000E-

1.1230E+01

2750 .

$4.5830 E+01$

$8.1800 E+D 0$

$1.0000 \mathrm{E}-02$

t.0000E-04

$0.0000 \mathrm{E}+00$

$4.2000 \mathrm{E}+01$

$4.0000 \mathrm{E}+01$

$1.0000 \mathrm{E}-02$

$1.0000 \mathrm{E}-02$

$1.0002 E+00$

$73000 E-01$

$3.6482 E+01$

$3.6401 E+01$

$1.7325 E+01$

$3.4700 \mathrm{E}+01$

1. 7000E-01

$3.4100 E+01$

1. $4100 \mathrm{E}+00$

1.1686E+01

$7.4200 E+00$

$3.2890 \mathrm{E}+01$

$70000 E .02$

4.3000E-03

8. $9990 \mathrm{E}-04$

$2.9600 \mathrm{E}+01$

1. $4000 \mathrm{E}-02$

3.7800E-01

2 3000E-03

. $5000 \mathrm{E}-02$

$5.2000 \mathrm{E}+00$

$5.2600 \mathrm{E}+\infty$

$6.8000 \mathrm{E}-02$

9.0000E-03

$7.0000 E-03$

0.0000 E+OD

$0.0000 E+00$

8.5000E-02

$4.4402 E+00$

1.2000E-01

2.5000E-01

1.31 $120 \mathrm{E}+00$

1.0000E-04

2 OOOOE-O1

$4.5000 \mathrm{E}-01$

6.3540E-01

$2.0372 \mathrm{E}+01$

$2.0130 E+0$ 
TABLE 5

STATISTICAL ANALYSIS OF HAZARDOUS MATERIALS

IN ASCENDING ORDER BASED ON ACCUMULATED WEIGHT (* INDICATES DATA EXHIBITED NEGATIVE VARIANCE)

(10 SHEETS)

COMPONENT MIAME
1,2-DICHLORETHYLENE
MINERAL SPIFITS
SODIUM BICARSIONATE
TRICHLOROETHENE
ACETIC ACID VINYL ESTER
SODIUM METASIIICATE
TRICHLOROETHILENE
COAL TAR CREOSOTE
SULFURIC ACID
INDENE
ACETIC ACID
KLEEN-O-BOWL

ALIPHATIC PETROLEUM DISTILLATE SODIUM DIOCTYLSULFOCCINATE

SULFAMIC ACID

SOLVENTS

FERROUS SULFATE

COAL TAF

HEXONE

CUPRIC SULFATE

GADOLINIUM NITRATE CRESOL

CYCLOHEXANONE

URANYL NITRATE HECAHYDRATE PSUEDOCUMENE

LEAD CHROMATE, CHLCRIN.PARAFFIN

PAINT CONTAINING NONYLPHENOL CRESYLIC ACID

BUTYL ACETATE

AMMONIUM CHLORIDE

ACRYLIC AC: TALC

METHYLTRICAPRYLYLAMMONIUM CHLORIDE SODIUM PHOSFHATE SILICON DIOXILE HALOGENATED ORGANIIT COMPOUNOS BENZENE SILICA

11 TRICHLORO FTHANE THENOYLTRIFLUOROACETONE

CRYSTALLINE-OUARTZ SHLICA

SODIUM LAURYL SULFATE

NON-IONIC EMULSIFIERS IRON

SODIUM CHROMATE

COBALTOUS SLILFATE

TETRAHYOROFURAN

BIS-(2-ETHYLHEXYL)PHTHALATE

BIS(2-ETHYLHEXYL) HYDRCIGEN PHIOSPHATE

TERT-BUTYL METHYL ETHER

VINYL CHLORIDE RESIN O.XYLENE

CHROMC OXIDE

TASSIUM CARBONATE FORMALIIV

LIGHT FIXTURES CONTAMINATED WITH POLYCHLORINATED BI BORIC ACID

ZINC CHRONIATE

BARIUM NITFATE AMMONLM

PHTHALIC ACID

1,1,2-TRICHLOROTRIFLUOROETHANE

2-(2-BUTOXYETHOXO)-ETHANOL

2-PROPANCNE

POTASSIUM CI'ANIDE

SODIUM FLUORIDE

SODIUM ALUMINATE

CARCINOGENIC ORGANIC CHEMICAL SUBSTANCE

POLYCYCLIC AROMATIC HYDROCARBONS

$$
\text { SOIL }
$$

CEROUS NITRATE

YTTRIUM NITIRAT

4-METHYL-2-PENTANONE

\author{
COUNT ACCUMWT, KG AVERAGEWT, KG
}

$1.2343 E+01$

1.2051E+01

$1.1800 \mathrm{E}+01$

$1.1530 \mathrm{E}+01$

$1.1229 E+01$

$1.1169 \mathrm{E}+01$

$1.04 \div 0 E+01$

$1.0370 \mathrm{E}+01$

$1.0314 \mathrm{E}+01$

$1.0284 E+01$

$9.6500 \mathrm{E}+00$

$9.5684 \mathrm{E}+00$

$93929 E+00$

$9.2600 \mathrm{E}+00$

$8.9048 E+00$

$8.8495 E+00$

$8.8170 E+00$

$8.7990 \mathrm{E}+00$

$6.6500 E+\infty 0$

B. $6200 E+00$

8. 3950E $+\infty 0$

8. 2700E +00

$8.2361 \mathrm{E}+00$

$8.0489 \mathrm{E}+00$

8.0100E+00

8. $0013 \mathrm{E}+00$

B. $0001 E+00$

8. $0000 \mathrm{E}+\infty$

$7.3020 \mathrm{E}+00$

$7.666 \mathrm{OE}+\infty 0$

7. $5000 \mathrm{E}+00$

7. $4609 E+00$

7. $4000 \mathrm{E}+00$

$7.3000 \mathrm{E}+00$

$7.2820 \mathrm{E}+00$

7. 2000E+00

$7.1767 E+C O$

7.0023E+00

$6.9418 E+C 0$

$6.9000 E+C O$

$6.8513 \mathrm{E}+\mathrm{CO}$

$6.8180 E+\infty$

$88700 E+\infty 0$

$6.6613 E+00$

$6.5600 E+00$

$6.4272 \mathrm{E}+00$

$6.2500 E+00$

$6.2280 E+00$

$5.1717 \mathrm{E}+00$

$6.0500 \mathrm{E}+00$

$6.0000 \mathrm{E}+00$

$5.9800 \mathrm{E}+00$

$5.8960 \mathrm{E}+00$

5.4447E+00

$5.2982 E+\infty 0$

$5.1658 E+00$

$5.0759 \mathrm{E}+00$

$5.0000 \mathrm{E}+00$

$5.0000 \mathrm{E}+00$

$4.9729 \mathrm{E}+00$

$4.9378 E+\infty 0$

$4.9320 \mathrm{E}+00$

4.8381E+00

$48200 \mathrm{E}+00$

4.7700E +00

4.7035E+ +0

4. $7007 E+\infty$

4. $54 \mathrm{COE}+00$

$45000 E+\infty 0$

$4.5000 E+00$

$45000 E+00$

$4.5000 E+00$

4. 4500E+ 00

4. $4500 E+00$
4.4433E +00

$\begin{array}{lr}2.5080 E+00 & 5.4353 E-01 \\ 4.1143 E+00 & 2.7814 E+00 \\ 4.0170 E+00 & 6.3082 E+00 \\ 9.8333 E-01 & 4.3567 E-01 \\ 5.7650 E+\infty & 6.6963 E+00 \\ 3.7431 E+\infty & \\ 1.3789 E-01 & 5.8481 E-01 \\ 2.6025 E+\infty & 2.8687 E+00 \\ 5.4581 E-01 & 7.3022 E-01 \\ 2.5785 E+00 & 1.6300 E+00 \\ 6.0492 E-01 & 6.7207 E-01\end{array}$

6.0492E-01

7.3603E-01

1.1741E+00

4. $6300 \mathrm{E}+00$

1. $1131 E+00$

8. $6485 E+\infty 0$

$8.8170 E+\infty$

4.3895E+00

7.2091E-01

2.8733E+00

8. $2700 E+00$

2.1674E-01

$5.0312 E-01$

$4.0050 E+00$

$8.0013 E+00$

1. $6000 E+00$

$8.0000 E+\infty 0$

$8.0433 E-02$

$1.0951 \mathrm{E}+00$

5.0000E-01

6.2898E-01

$7.4000 \mathrm{E}+00$

$7.3000 E+C 0$

$1.2137 \mathrm{E}+\infty$

7.2000E+00

$3.5864 E+00$

0.3364E-02

1.9283E.01

$3.4500 E+00$

$1.1050 \mathrm{E}-01$

$9.7400 E-01$

8. $8700 \mathrm{E}+00$

$3.3307 \mathrm{E}+00$

$3.2800 \mathrm{E}+00$

$1.6068 E+\infty 0$

$3.1250 \mathrm{E}+\infty 0$

$6.2280 \mathrm{E}+00$

$9.2115 E-02$

$2.0187 \mathrm{E}+00$

$3.0000 \mathrm{E}+00$

$1.9833 \mathrm{E}+\infty 0$

$2.9480 E+00$

$1.3612 \mathrm{E}+00$

4. 4152E-01

3.9737E-01

$1.6920 \mathrm{E}+00$

$1.0000 E+\infty$

$5.0000 E+\infty 0$

9.0458E-01

6.1723E-01

$4.9320 \mathrm{E}+00$

7.0330E-01

2.4341E.01

$4.8200 E+\infty 0$

$2.3850 \mathrm{E}+00$

$1.1759 E+00$

4.7007E-01

$1.5133 E+00$

2. $2500 \mathrm{E}+00$

4.5000E +00

4. $5000 E+00$

$4.450 .0 E+00$

$4.4500 E+00$

1.2009E-01
STO DEV
$5.4353 E-0$
$2.7814 E+0$
$6.3082 E+0$
$4.3567 E-0$
$6.6963 E+0$
5
$5.6481 E-0$
$2.8687 E+0$
$7.3022 E-01$
$1.6300 E+00$
$6.7207 E-0$

8.1652E-01

1.1800E+00

1.4142E-02

1. $4593 E+00$

$0.0000 E+\infty 0$

$0.0000 E+00$

$3.0964 E+\infty 0$

8.4543E-01

8.3760E-01

2.8815E+00

$0.0000 E+00$

5.7172E-01

$0.7250 \mathrm{E}-01$

$2.8788 \mathrm{E}+00$

$0.0000 E+\infty 0$

$5.4758 \mathrm{E}-01$

$0.0000 E+00$

1.4872E-01

$1.3923 E+00$

$0.0000 E+00$

9.4502E-01

$0.0000 \mathrm{E}+\infty$

$0.0000 E+\infty$

9 3963E-01

$2.3663 E+00$

$4.1183 \mathrm{E}-02$

2.8315E-01

4.8225E+DO

1.1051E-01

$1.1684 \mathrm{E}+00$

$0.0000 E+\infty 0$

$3.3083 \mathrm{E}+\infty 0$

$0.0000 E+\infty 0$

$1.9661 \mathrm{E}+00$

$3.7830 \mathrm{E}+00$

$0.0000 \mathrm{E}+00$

4. 1547E- 01

$1.0081 E+00$

$4.0164 \mathrm{E}+\infty 0$

3.5796E-01

1. $6235 \mathrm{E}+00$

$2.0543 E+00$

5.0198E-01

8.0389E-01

$1.8807 E+00$

$0.0000 E+\infty$

$0.0000 \mathrm{E}+\infty$

$1.1712 \mathrm{E}+00$

$5.8178 E-01$

$0.0000 E+D 0$

$8.6097 \mathrm{E}-01$

$1.1658 E-01$

$0.0000 E+\infty 0$

2.7224E +00

$1.2878 \mathrm{E}+00$

$1.4882 E+00$

1.7620E +00

3.5355E-01

$0.0000 E+00$

$0.0000 E+00$

$0.0000 E+00$

$0.0000 E+00$

$0.0000 E+\infty 0$

1.5422E-01

MINIMUM WT, KG

MAXIMUM WT, KG

1. $9000 \mathrm{E}+\mathrm{CO}$

9.2000E-01

2.2000E-01

4.0000E-02

1.0300E $+\infty 0$

$0.0000 E+\infty$

$0.000 E+\infty$

3.0000E-O4

$1.0220 \mathrm{E}+00$

3.0000E-03 
TABLE 5

STATISTICAL ANALYSIS OF HAZARDOUS MATERIALS

IN ASCENDING ORDER BASED ON ACCUMULATED WEIGHT

(* INDICATES DATA EXHIBITED NEGATIVE VARIANCE)

(10 SHEETS)

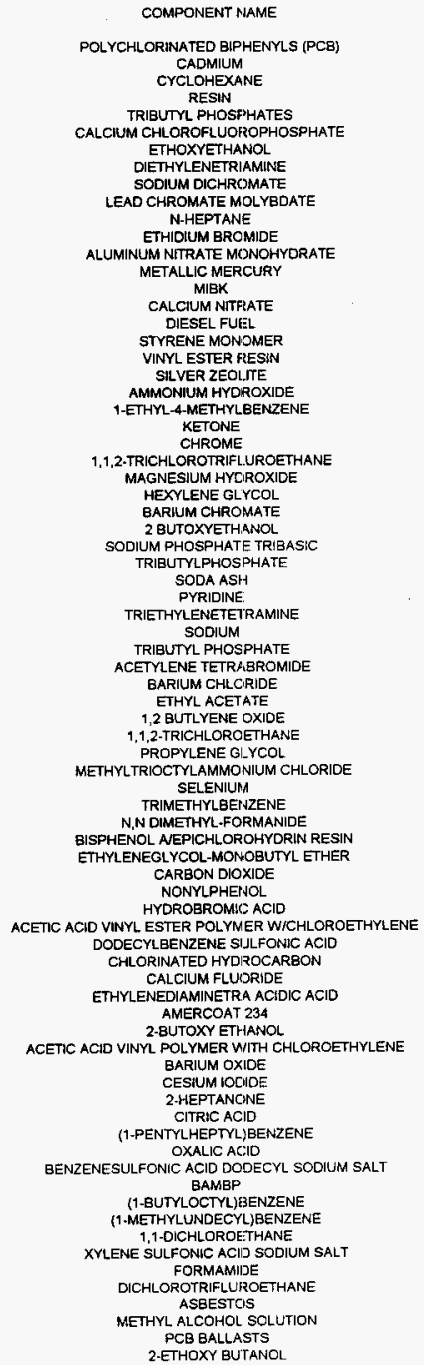

\author{
COUNT ACCUM WT, KG AVERAGEWT. KG
}

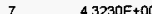

279

278
8
2

2

14

14
3
4

4

6

1

1

3

2

2

14

\begin{tabular}{|c|c|}
\hline $\begin{array}{l}4.3230 E+00 \\
4.3095 E+00\end{array}$ & $\begin{array}{l}\text { 6.1757E-01 } \\
1.5558 \mathrm{E}-02\end{array}$ \\
\hline $\begin{array}{l}4.2700 E+\infty 0 \\
4.2569 E+\infty 0\end{array}$ & $\begin{array}{l}5.3375 E-01 \\
2.1285 E+00\end{array}$ \\
\hline $4.2300 \mathrm{E}+00$ & $4.2300 \mathrm{E}+00$ \\
\hline $\begin{array}{l}4.2237 E+\infty \\
4.1608 E+\infty\end{array}$ & $\begin{array}{l}3.0169 E-01 \\
1.3869 E+00\end{array}$ \\
\hline $4.1400 E+\infty 0$ & $1.0350 E+00$ \\
\hline $4.1051 E+\infty 0$ & 1.0263E+00 \\
\hline $\begin{array}{l}4.0370 \mathrm{E}+00 \\
3.9820 \mathrm{E}+00\end{array}$ & $\begin{array}{l}6.7283 E-01 \\
1.3273 E+00\end{array}$ \\
\hline $\begin{array}{l}3.9648 E+00 \\
3.9000 E+00\end{array}$ & $\begin{array}{l}3.9648 E+\infty \\
3.9000 E+\infty 0\end{array}$ \\
\hline $3.9000 \mathrm{E}+00$ & $3.9000 \mathrm{E}+\infty$ \\
\hline $3.8740 \mathrm{E}+00$ & 6. $4587 \mathrm{E}-01$ \\
\hline $3.6265 E+\infty 0$ & $1.2755 E+\infty 0$ \\
\hline $3.8000 E+00$ & $1.9000 E+00$ \\
\hline $3.7503 E+00$ & $1.8752 E+00$ \\
\hline $\begin{array}{l}3.7503 E+00 \\
3.7300 E+00\end{array}$ & $\begin{array}{l}1.8752 E+00 \\
3.7300 E+00\end{array}$ \\
\hline $3.7239 \mathrm{E}+00$ & 4. 8549E-01 \\
\hline $3.6860 \mathrm{E}+00$ & 2.8400E-01 \\
\hline $3.6001 E+00$ & 6.0002E-01 \\
\hline $3.6000 \mathrm{E}+00$ & $1.8000 \mathrm{E}+00$ \\
\hline $3.6000 \mathrm{E}+00$ & $3.6000 \mathrm{E}+\infty$ \\
\hline $\begin{array}{l}3.6000 \mathrm{E}+00 \\
3.5350 \mathrm{E}+00\end{array}$ & $\begin{array}{l}3.6000 \mathrm{E}+\infty \\
3.5350 \mathrm{E}+\infty\end{array}$ \\
\hline $3.3850 E+\infty 0$ & $1.6925 \mathrm{E}+\infty$ \\
\hline $3.3800 E+\infty 0$ & 8.4500E-01 \\
\hline $3.3570 E+00$ & 3. $3570 E+00$ \\
\hline $30040 E+00$ & $1.0013 E+00$ \\
\hline $3.0000 E+00$ & $\begin{array}{l}3.0000 E+00 \\
5.9480 E-01\end{array}$ \\
\hline $2.9740 E+00$ & $\begin{array}{l}\text { 5. } 9480 \mathrm{E}-01 \\
9.0000 \mathrm{E}-01\end{array}$ \\
\hline $\begin{array}{l}2.9700 E+00 \\
29031 E+00\end{array}$ & \\
\hline $\begin{array}{l}2.9081 E+00 \\
2.8730 E+00\end{array}$ & $4.7883 E-01$ \\
\hline $\begin{array}{l}2.8730 E+\infty 0 \\
2.8000 E+\infty 0\end{array}$ & $28000 E+00$ \\
\hline $2.7960 E+\infty 0$ & $1.3980 E+00$ \\
\hline $2.7769 \mathrm{E}+00$ & 5.5538E-01 \\
\hline $26559 E+00$ & 6.6398E-01 \\
\hline $25001 \mathrm{E}+00$ & $1.2501 E+00$ \\
\hline $2.4999 \mathrm{E}+00$ & 4.1665E-01 \\
\hline $2.4700 E+\infty 0$ & $2.4700 E+\infty$ \\
\hline $2.4504 E+\infty 0$ & 1.9603E-02 \\
\hline $2.4342 E+\infty 0$ & $6.0855 E-01$ \\
\hline 2. $4200 \mathrm{E}+00$ & $2.4200 E+00$ \\
\hline $2.4010 \mathrm{E}+00$ & 8.0033E-01 \\
\hline $\begin{array}{l}2.3700 E+00 \\
2.3508 E+00\end{array}$ & $\begin{array}{l}2.3700 \mathrm{E}+00 \\
8.7950 \mathrm{E}-02\end{array}$ \\
\hline $23370 E+C 0$ & $1.1685 E+00$ \\
\hline $2.2425 E+00$ & $1.1213 E+00$ \\
\hline $2.2270 E+00$ & 7.4233E-01 \\
\hline $2.1000 \mathrm{E}+00$ & $2.1000 \mathrm{E}+00$ \\
\hline $2.0160 \mathrm{E}+00$ & 2.8800E.01 \\
\hline $2.0100 E+00$ & 6. $7000 \mathrm{E}-01$ \\
\hline $2.0100 E+\infty 0$ & $2.0100 \mathrm{E}+00$ \\
\hline $2.0004 E+\infty 0$ & $1.0002 E+00$ \\
\hline $2.0000 E+\infty 0$ & $2.0000 \mathrm{E}+00$ \\
\hline $2.0000 E+00$ & $2.0000 E+00$ \\
\hline $2.0000 E+00$ & $2.0000 \mathrm{E}+00$ \\
\hline $2.0000 \mathrm{E}+00$ & $2.0000 \mathrm{E}+00$ \\
\hline $1.9000 \mathrm{E}+00$ & 6. 6333E.01 \\
\hline $1.9900 E+00$ & $9.9500 E-01$ \\
\hline $1.9700 \mathrm{E}+00$ & 4.9250E-01 \\
\hline $1.9580 E+\infty 0$ & 2.7971E-01 \\
\hline $1.9500 \mathrm{E}+00$ & $1.9500 E+00$ \\
\hline 1. $8860 E+00$ & $1.8860 E+00$ \\
\hline 1.88ODE +00 & $4.7000 \mathrm{E}-01$ \\
\hline $4.8600 \mathrm{E}+00$ & 4.7000E-01 \\
\hline $1.8700 E+00$ & 6.2333E-01 \\
\hline $1.8400 \mathrm{E}+00$ & $1.8400 \leftleftarrows+00$ \\
\hline 1. $8000 E+00$ & $18000 E+00$ \\
\hline $1.7998 \varepsilon+00$ & $1.7998 E+00$ \\
\hline $1.7899 \mathrm{E}+00$ & $3.5798 \mathrm{E}-01$ \\
\hline $1.7599 \mathrm{E}+00$ & $1.7599 E+\infty$ \\
\hline $1.7490 \mathrm{E}+00$ & $1.7490 \mathrm{E}+00$ \\
\hline $17402 E+\infty$ & $2.4860 E-01$ \\
\hline
\end{tabular}

\author{
STD DEV
}

7.4934E-01 $1.0011 \mathrm{E}-01$

4.3283E-01

$2.0812 E+00$

$0.0000 E+00$

6.5041E-01

1. $8408 \mathrm{E}+00$

$5.4641 E-01$

$1.1828 E+00$

7.0661E-01

$1.0132 \mathrm{E}+\infty 0$

$0.0000 \mathrm{E}+\infty$

$0.0000 E+00$

$0.0000 \mathrm{E}+\infty$

$1.5796 \mathrm{E}+00$

$8.4853 E-01$

$4.2374 E+00$

1.2374E 00

$0.0000 E+00$

1.8083E-01

2.097eE-01

$1.0182 E+\infty$

$0.0000 E+\infty 0$

$0.0000 \mathrm{E}+00$

1. $2288 \mathrm{E}+\infty 0$

$1.1267 \mathrm{E}+00$

$0.0000 \mathrm{E}+00$

$1.6584 \mathrm{E}+00$

$0.0000 \mathrm{E}+00$

$9.7040 \mathrm{E}-01$

$1.0116 \mathrm{E}+\infty$

$1.5642 E+00$

$3.7572 \mathrm{E}-01$

$0.0000 E+00$

4. 3090E-01

1.1591E+ +00

$1.7677 \mathrm{E}+\infty$

4. $0784 \mathrm{E}-02$

12481E0

7.1071E-01

$0.0000 \mathrm{E}+00$

1.2413E $+\infty$

6. $7083 \mathrm{E}-02$

2.3830E-01

1.3834E+DO

$1.0156 E+00$

$0.0000 \mathrm{E}+00$

$6.6740 \mathrm{E}-01$

$1.0567 \mathrm{E}+00$

$0.0000 E+00$

$0.0000 E+00$

$0.0000 \mathrm{E}+00$

$0.0000 E+00$

$0.0000 E+00$

$0.0000 E+\infty$

$8.4678 \mathrm{E}-01$

1.1950E +00

5.0000E-03

2.8712E-01

$0.0000 \mathrm{E}+\infty 0$

D. $0000 \mathrm{E}+00$

2.0000E-02

2.0000E-02

3.8837E-01

$0.0000 \mathrm{E}+00$

$0.0000 E+\infty$

$0.0000 E+\infty 0$

$1.5269 \mathrm{E}-01$

$0.0000 \mathrm{E}+00$

$0.0000 \mathrm{E}+00$

1.2858E-01
MINIMUM WT. KG

MAXIMUM WT. KG

$6.0000 E-03$

$0.0000 E+00$

$7.0000 E-02$

6.5680E-01

4. $2300 \mathrm{E}+\infty 0$

$3.0400 E-02$

3.0000E-01

$1.0000 \mathrm{E}-03$

1.8000E-01

1.6200E-01

$3.9648 \mathrm{E}+00$

$3.8000 \mathrm{E}+00$

$3.0000 \mathrm{E}+\infty$

$0.0000 E+\infty 0$

$1.3000 E+\infty 0$

$1.0002 E+\infty 0$

$1.0002 E+\infty 0$

$3.7300 \mathrm{E}+00$

4. $4000 \mathrm{E}-03$

$1.4000 E-02$

2.0000E-01

$1.0000 E+00$

$3.8000 \mathrm{E}+\infty 0$

$3.8000 \mathrm{E}+\infty 0$

$3.5350 \mathrm{E}+\infty$

B. $2500 \mathrm{E}-01$

$0.0000 E+\infty 0$

$3.3570 \mathrm{E}+00$

$1.3000 E-02$

$3.0000 E+00$

7.00C0E-03

1. 2000E-01

3. $4800 \mathrm{E}-01$

1.7000E.02

$2.8000 \mathrm{E}+00$

8. $4800 \mathrm{E}-01$

2.0000E-02

3. $0000 \mathrm{E}-02$

$1.0000 \mathrm{E}-04$

4.0000E-01

$2.4700 \mathrm{E}+00$

$0.0000 \mathrm{E}+\infty$

2.0000E-03

2.4200E+00

1.0000E-02

2. $3700 \mathrm{E}+00$

8.0000E-0.4

$1.0000 E+\infty 0$

1.3600E-01

1.5000E-01

2. 1000E+ 00

B. $0000 \mathrm{E}-0 \mathrm{O}$

4.0000E-02

$2.0100 E+00$

$1.0002 \mathrm{E}+00$

$2.0000 \mathrm{E}+00$

$20000 \mathrm{E}+00$

$20000 \mathrm{E}+00$

$20000 E+00$

$1.0000 \mathrm{E}-02$

1.5000E-01

4.9000E. 01

2.5000E-02

$1.9500 \mathrm{E}+00$

$1.8860 \mathrm{E}+00$

$4.8000 E-01$

4.6000 E- 01

1.9000 E-01

$1.6400 E+00$

$1.8000 \mathrm{E}+00$

$1.7998 \mathrm{E}+\infty 0$

$2.0000 E-01$

$1.7599 \mathrm{E}+00$

$1.7490 \mathrm{E}+00$

2.0000E-01
$2.0000 E+\infty 0$

$1.2800 E+\infty 0$

$1.4500 E+\infty 0$

$3.8001 E+00$

4.2300E $+\infty 0$

$2.4309 E+\infty 0$

$3.5000 E+00$

$1.5000 E+\infty 0$

$2.1000 \mathrm{E}+\infty 0$

$2.0000 E+\infty$

$2.0000 E+\infty 0$

$3.0648 E+00$

$3.0000 E+\infty 0$

$3.9000 E+\infty$

$3.8700 E+\infty$

$3.8200 E+00$

$2.5000 E+00$

2.7501E+00

2.7501E $+\infty 0$

$3.7300 \mathrm{E}+00$

$1.4700 E+\infty 0$

$5.0000=01$

$8.0010 E-01$

2.5200E +00

$3.6000 \mathrm{E}+00$

$3.6000 \mathrm{E}+00$

$3.5350 E+\infty 0$

$2.5800 \mathrm{E}+00$

$23800 E+00$

$3.3570 \mathrm{E}+00$

$2.9160 E+00$

$3.0000 E+00$

2.3000E +00

2.1000E $+\infty 0$

$2.5801 \mathrm{E}+\infty$

$1.0000 E+00$

$2.8000 E+00$

1. $0500 \mathrm{E}+00$

1. $2000 \mathrm{E}+00$

2.3909E+00

$25000 E+\infty 0$

4.9900E-01

2. $4700 \mathrm{E}+00$

$1.0002 E+00$

$1.4300 E+00$

$2.4200 E+00$

$2.2310 E+00$

$2.3700 E+\infty 0$

2.0000E-01

1.3370E +00

2. $1065 E+\infty 0$

1. $9150 \mathrm{E}+00$

2.1000E $+\infty 0$

$8000 E+\infty 0$

$1.8900 E+\infty 0$

2.0100E $+\infty 0$

$1.0002 E+00$

$2.0000 E+\infty 0$

$2.0000 \mathrm{E}+\infty 0$

$2.0000 E+\infty 0$

$2.0000 \mathrm{E}+00$

$1.6200 \mathrm{E}+\infty 0$

1.8400E+00

5.0000E.01

B.0000E-01

$1.8500 \mathrm{E}+\infty 0$

$1.8860 \mathrm{E}+00$

5.0000E-01

5.0000E-01

9.4000E-01

$1.8400 \mathrm{E}+00$

$1.8000 \mathrm{E}+00$

$1.7098 \mathrm{E}+\mathrm{OO}$

$5.4000 \mathrm{E}-01$

$1.7599 \mathrm{E}+00$

$+.7490 \mathrm{E}+00$

5.4020E-01 
TABLE 5

STATISTICAL ANALYSIS OF HAZARDOUS MATERIALS IN ASCENDING ORDER BASED ON ACCUMULATED WEIGHT (* INDICATES DATA EXHIBITED NEGATIVE VARIANCE) (10 SHEETS)

\begin{tabular}{|c|c|c|c|c|c|c|}
\hline COMPONENT MIAME & COUNT & ACCUM WT, KG & AVERAGE WT. KG & STO DEV & MENHMUM WT, KG & MAXIMUM WT, KG \\
\hline ZINC CHLORIOE & 8 & $1.7151 E+00$ & $2.8585 \mathrm{E}-01$ & $3.5308 E-01$ & $7.6000 E-02$ & $1.0000 E+\infty 0$ \\
\hline ZIRCONYL NITRATE & 2 & 1.7080E+00 & $8.5400 \mathrm{E}-01$ & $3.4790 E-01$ & 6.0800E-04 & $1.1000 E+\infty$ \\
\hline (1.ETHYLDECYL)BENZENE & 4 & $1.6900 E+00$ & 4.2250E-01 & $1.5000 E-02$ & 4.0000E-01 & 4.3000E-01 \\
\hline MEK & 9 & $1.6892 E+00$ & 1.8769E-01 & $2.6910 \mathrm{E}-01$ & $0.0000 E+00$ & $6.4000 E-01$ \\
\hline GLYCOLS, POLYETHYLENE MCNO (NONYLPHENYL) & 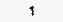 & $1.6800 \mathrm{E}+00$ & $1.6800 E+\infty$ & $0.0000 E+00$ & $1.6800 E+00$ & $1.6800 E+\infty$ \\
\hline $\begin{array}{c}\text { SODUM IODIDE } \\
\text { 2,3-DIHYDRO-1H-INDENE }\end{array}$ & $\begin{array}{l}3 \\
9\end{array}$ & $\begin{array}{l}1.6304 \mathrm{E}+00 \\
1.6300 \mathrm{E}+00\end{array}$ & $\begin{array}{l}5.4347 \mathrm{E}-01 \\
1.8111 \mathrm{E}-01\end{array}$ & $\begin{array}{l}8.3084 E-01 \\
1.0706 E-01\end{array}$ & $\begin{array}{l}\text { 1.4000E-03 } \\
3.0000 E .02\end{array}$ & $\begin{array}{l}1.5000 E+00 \\
1.0000 E-01\end{array}$ \\
\hline HLORINATED PARAFFIN WAXES AND HYDROCARGON WAXE & 2 & $1.6000 E+00$ & $8.0000 E-01$ & 0.1924E-01 & $1.5000 E-01$ & $9.4500 E+\infty$ \\
\hline 1,3-PROPANEDLAMUNE & 1 & $1.5900 E+C 0$ & $1.5900 E+00$ & $0.0000 \mathrm{E}+00$ & $1.5900 \mathrm{E}+\infty$ & $1.5000 E+00$ \\
\hline METHMTRICAPRYLALAMMONIUM CHLORIDE & 1 & $1.5900 E+\infty$ & $1.5800 E+00$ & $0.0000 E+00$ & $1.5000 E+\infty 0$ & $1.5000 E+00$ \\
\hline METHLY ISOBUTYL KETONE & 5 & $15800 E+00$ & 3.1800E-01 & $4.2190 \mathrm{E}-02$ & $2.7000 E-01$ & $3.7000 E-01$ \\
\hline CHROMIC ACIO & 5 & $1.5510 E+00$ & 3. 1020E-01 & $3.8107 E-01$ & $5.0000 E-02$ & $9.5200 E-01$ \\
\hline POLYETHYLENE MONO(NONYLPHENYL) GLYCOLS & 1 & $1.5500 E+00$ & $1.5500 \mathrm{E}+\infty$ & $0.0000 E+00$ & $1.5500 E+00$ & $1.5500 \mathrm{E}+00$ \\
\hline 2, 4,6 -TRI(DIMETHYLAMINOMETHYL) PHENOL & 1 & $1.5200 \mathrm{E}+00$ & $1.5200 E+00$ & $0.0000 E+\infty 0$ & $1.5200 E+\infty$ & $1.5200 E+00$ \\
\hline $\begin{array}{l}\text { 1,1,1-DICHLOROETHANE } \\
\text { BARIUM HYOROXIDE }\end{array}$ & $\begin{array}{l}3 \\
2\end{array}$ & $\begin{array}{l}1.5000 E+00 \\
1.5000 E+00\end{array}$ & $\begin{array}{l}5.0000 E-01 \\
7.5000 E-01\end{array}$ & $\begin{array}{l}8.6603 E-02 \\
4.2428 E-01\end{array}$ & $\begin{array}{l}\text { 4.5000E-01 } \\
\text { 4.5000E-04 }\end{array}$ & $\begin{array}{l}\text { 6.0000E-04 } \\
1.0500 E+\infty\end{array}$ \\
\hline 1,1,2-TRICHLORO 1,2,2-TRIFLUOROETHANE & 1 & $1.5000 \mathrm{E}+\infty$ & $1.5000 E+00$ & $0.0000 E+00$ & $1.50005 \div 00$ & $1.5000 E+\infty$ \\
\hline (1-PROPYLNONYL)BENZENE & 4 & $1.4500 E+00$ & $3.8250 E-01$ & $2.5000 E-02$ & 3.5000E-01 & 4.0000E-01 \\
\hline BASIC ZINC CHROMATE & 3 & $1,4184 E+00$ & 4.7280E-01 & 4.8083E-01 & $5.8400 E-02$ & $1.0000=+\infty 0$ \\
\hline HYDROQUINONE & 2 & $1.3910 E+00$ & 6.8550E-01 & 2.8921E-01 & 4.8100E-01 & $9.0000 E-01$ \\
\hline ETHANOLAMINE & 1 & $1.3000 E+00$ & $1.3000 \mathrm{E}+00$ & $0.0000 E+\infty 0$ & $1.3900 E+\infty 0$ & $1.3900 E+00$ \\
\hline SODUM PYROPHOSPHATE & $i$ & $1.3800 \mathrm{E}+00$ & $1.3900 \mathrm{E}+00$ & $0.0000 E+00$ & $1.3800 E+\infty 0$ & $1.3900 E+\infty$ \\
\hline POTASSIUM FLUORIDE & 2 & $1.3800 E+00$ & 6.8000E-01 & $9.3338 E-01$ & 3.0000E-02 & $1.3500 \mathrm{E}+00$ \\
\hline EDETIC ACID & 2 & $1.3669 E+00$ & $6.9206 \mathrm{E}-0 t$ & $6.9714 E-01$ & $1.8000 \mathrm{E}-0 \dagger$ & $1.1759 \mathrm{E}+\infty 0$ \\
\hline AMMONIUM MOLYEDATE & 2 & $1.3600 E+\infty 0$ & 6.8000E-01 & 8.7881E-01 & $0.0000 E-02$ & $1.3000 E+\infty$ \\
\hline CHLOROFORN & 39 & $1.3575 E+\infty$ & $3.4808 E-02$ & $1.1157 \mathrm{E}-01$ & $1.0000 E-04$ & $5.0000 E-01$ \\
\hline DI(2-ETHYLHEXYL)PHTHATATE & 1 & $1.3500 E+\infty$ & $1.3500 E+00$ & $0.0000 E+00$ & $1.3500 E+00$ & $1.3500 E+00$ \\
\hline DIETHYLENE GLYCOL MONOETHYLETHER & 1 & $1.3200 E+\infty$ & $1.3200 E+00$ & $0.0000 E+00$ & $1.3200 E+00$ & $\$ .3200 E+00$ \\
\hline CHROMIUM OXIDE & 2 & $1.3100 E+00$ & $6.5500 E-01$ & 8.8388E-01 & 3.0000E-02 & $1.2800 E+00$ \\
\hline BIS (2-ETHYLEXYL) PHTHALATE & 1 & $1.3100 E+00$ & $1.3100 E+\infty$ & $0.0000 E+\infty$ & $1.3100 E+00$ & $1.3100 E+\infty$ \\
\hline LEAD TETROXIDE & 1 & $1.3000 \mathrm{E}+00$ & 1.3000E+D0 & $0.0000 E+00$ & $1.3000 E+00$ & $1.3000 E+\infty$ \\
\hline PHTHALIC ACID BIS(2.ETHYLHEXYL)ESTER & 25 & 1.2879E+00 & $5.1516 \mathrm{E}-02$ & $6.9203 E-02$ & $9.0000 E-04$ & $20000 E-01$ \\
\hline CHROMIUM SALIS & 1 & $1.2500 \mathrm{E}+00$ & $1.2500 \mathrm{E}+00$ & $0.0000 E+00$ & $1.2500 E+\infty 0$ & $1.2500=+00$ \\
\hline MAGNESIUM METAL & 1 & $12500 E+00$ & $1.2500 E+00$ & $0.0000 E+00$ & $1.2500 \mathrm{E}+\infty 0$ & $1.2500 \mathrm{E}+00$ \\
\hline PERCHLORIC ACID & 5 & $1.2326 E+00$ & $2.4652 \mathrm{E}-01$ & 4.2271E-01 & $20000 E-03$ & $1.0000 E+00$ \\
\hline URANYL ACETATE & 2 & $1.2012 \mathrm{E}+\infty$ & 6.0060E-01 & $5.6653 E-01$ & $2,0000 E-01$ & $1.0012 \mathrm{~F}+00$ \\
\hline MURIATIC ACID & 1 & $1.1399 E+\infty 0$ & $11399 E+00$ & $0.0000 E+00$ & $1.1399 E+00$ & $1.1399 E+00$ \\
\hline COLLOOION & 1 & $1.1200 E+\infty$ & $1.1200 E+00$ & $0.0000 E+00$ & $1.1200 \mathrm{E}+\infty$ & $1.1200 E+00$ \\
\hline LEAKING BALLASTS CONTAINING $>500$ PPM PCBS & 1 & $1.1000 E+00$ & 1. $1000 E+00$ & $0.0000 E+00$ & $1.1000 \mathrm{E}+00$ & $1.1000 E+00$ \\
\hline THENOLYTRIFLUOROACETONE & 1 & $1.1000 E+\infty 0$ & $1.1000 \mathrm{E}+00$ & $0.0000 E+00$ & $1.1000 \mathrm{E}+00$ & $1.1000 E+00$ \\
\hline BUTYL EENZYL PHTHALATE & 2 & $1.0500 E+00$ & 5.2500E-01 & $6.7175 E-01$ & $5.0000 E-02$ & $1.0000 E+00$ \\
\hline CHLORINATEO AROMATIC HYDROCARBON & 7 & $1.0410 \mathrm{E}+00$ & $1.487+E-01$ & $3.381 \mathrm{BE}-01$ & $20000 E-02$ & $9.1100 E-01$ \\
\hline PEIROLEUM DISTLLLATES & 3 & $1.0400 E+00$ & $3.4667 E-01$ & $5.8315 E-01$ & $5.0000 \mathrm{E} .03$ & $1.0200 E+00$ \\
\hline TETRAETHYLENEPIENTAMINE & 2 & $1.0374 E+00$ & $5.1870 \mathrm{E}-\mathrm{O} 1$ & 6.8066E-01 & 3.7400E-02 & $1,0000 E+\infty$ \\
\hline BISPHENOL. A & 2 & $1,0300 E+\infty$ & 5.1500E-01 & $6.8589 E-01$ & $3.0000 \div-02$ & $1.0000 E+00$ \\
\hline STARGLAZE & 1 & $1.0201 E+00$ & $1.0201 E+00$ & $0.0000 E+00$ & $1.0201 E+00$ & $1.0201 E+00$ \\
\hline TAR & 1 & $1.0201 E+00$ & $1.0201 E+00$ & $0.0000 E+\infty 0$ & $1.0201 E+00$ & $1,0201 E+00$ \\
\hline 1-METHOXY 2.PROPANOL & 1 & $1.0100 E+00$ & $1.0100 E+\infty 0$ & $0.0000 E+\infty$ & $1.0100 E+00$ & $1.0100 E+\infty 0$ \\
\hline 4,4-METHYLENECIANILINE & 1 & $1.0000=+00$ & $1.0000 E+\infty$ & $0.0000 E+00$ & $1.0000 E+\infty$ & $1.0000 E+\infty 0$ \\
\hline ACETAMICE & 1 & $1.0000 E+\infty 0$ & $1.0000 E+00$ & $0.0000 E+00$ & $1.0000 E+\infty$ & $1.0000 E+00$ \\
\hline AMINOETHYLPIPIERAZINE & 1 & $1.0000 E+00$ & $1.0000 \mathrm{E}+00$ & $0.0000 E+00$ & $1.0000 E+00$ & $1.0000 \mathrm{E}+00$ \\
\hline DI-SEC-OCTYL PHTHALATE & 1 & $1.0000 E+00$ & $1.0000 E+00$ & $0.0000 E+00$ & $1.0000 E+00$ & $9.0000 E+00$ \\
\hline MISCELLANEOUS MATERIALS & 7 & $1.0000 \mathrm{E}+00$ & $1.0000 E+00$ & $0.0000 E+\infty$ & $1.0000 E+00$ & $1.0000 E+\infty$ \\
\hline PIPERAZINIE & 1 & $1,0000 E+00$ & $1,0000 E+\infty$ & $0.0000 E+\infty$ & $1.0000 E+\infty 0$ & $1.0000 E+\infty$ \\
\hline PLASTIC & 1 & $1.0000 E+\infty$ & $1.0000 E+\infty$ & $0.0000 E+\infty 0$ & $1.0000 E+00$ & $1.0000 E+\infty$ \\
\hline POLYOXPROPYLENE-POLYOXETHYLENE & 1 & $1.0000 E+\infty$ & $1.0000 E+00$ & $0.0000 E+00$ & $1,0000 E+\infty$ & $1.0000 E+\infty 0$ \\
\hline POLYVINYL CHLORICIE POLYMER & 1 & $1.0000 E+00$ & $1.0000 E+00$ & $0.0000 E+00$ & $1.0000 E+00$ & $1.0000 E+\infty 0$ \\
\hline PROPYLENEGI_YCOL & 1 & $1.0000 E+\infty 0$ & $1.0000 E+00$ & $0.0000 E+00$ & $1.0000 E+00$ & $1.0000 E+00$ \\
\hline TRICHLOROFLUOROMETHANE & 2 & $9.7800 E-01$ & 4.8900E-01 & 9.8990E-03 & 4.8200E-01 & $4.9600 \mathrm{E}-01$ \\
\hline SODIUM METABISULFITE & 2 & 8.7500E-01 & $4.8750 \mathrm{E}-01$ & $1.7678 \mathrm{E}-02$ & $4.7500 E-01$ & $5.0000 E-01$ \\
\hline MANGANESE DIOXIOE & 1 & $9.5570 E-01$ & 9.5570E-01 & $0.0000 E+\infty 0$ & 9.557OE-01 & 8.5570E-01 \\
\hline TITANIUM DICIXIDE & 2 & $9.4290 \mathrm{E}-01$ & $4.7145 E-01$ & $3.8530 \mathrm{E}-01$ & $1.0000 E-01$ & $7.4390 E-01$ \\
\hline ASCORBIC ACID & 3 & $8.4130 E-01$ & 3.1377E-01 & $2.6848 E-01$ & 6.5000E-02 & 6.0000E-01 \\
\hline BUTYL CELLOSOLVE & 2 & $9.2980 E-01$ & 4.6490E-01 & 6.3498E.02 & 4.2000E-01 & $5.0980 E-01$ \\
\hline DIBUTYL PHTHALATE & 3 & 9.1980E-01 & $3.0680 \mathrm{E}-01$ & $1.0414 E-01$ & $1.4000 E-01$ & $5.1880 E-0 t$ \\
\hline BUTYL ALCCIHOL & 4 & $9.0500 E-01$ & $2.2725 \mathrm{E}-01$ & 2.2486E-01 & $1.0000 \mathrm{E}-02$ & $5.0000 E-01$ \\
\hline ARSENIC PENTOXIDE & 1 & $9.0000 \mathrm{E}-01$ & $9.0000 E-01$ & $0.0000 E+00$ & $9.0000 E-01$ & $9.0000=-01$ \\
\hline DIMETHYLGE.YOXIME & 1 & $9.0000 E-01$ & $9.0000 E-01$ & $0.0000 E+00$ & $9.0000 \mathrm{E}-01$ & $9.0000 \mathrm{E}-01$ \\
\hline POTASSIUM MESABISULFATE & 1 & $9.0000 E-01$ & $0.0000 E-01$ & $0.0000 E+00$ & $9.0000 E-01$ & $9.0000 \mathrm{E}-01$ \\
\hline POTASSIUM PERNIANGANTE & 1 & 9.0000E-04 & $9.0 \mathrm{COOE}-01$ & $0.0000 E+00$ & $9.0000 E-01$ & $8.0000 \mathrm{E}-01$ \\
\hline SODIUM METABORATE OCTAHYDRATE & 1 & $9.0000 E-01$ & $9.0000 E-01$ & $0.0000 E+\infty 0$ & $9.0000 \mathrm{E}-01$ & $0.0000 E-01$ \\
\hline $\begin{array}{l}\text { SOOLUM SULFITE } \\
\text { SOL }\end{array}$ & 1 & $9.0000 \mathrm{E}-01$ & 9.0000E-01 & $0.0000 E+00$ & $9.0000 \mathrm{E}-01$ & $8.0000 E-01$ \\
\hline LIOUIFIED PETROIEUM GAS & 1 & $8.9900 \mathrm{E}-01$ & $8.9890 E-01$ & $0.0000 E+00$ & 8.8900E-01 & B.9900E-01 \\
\hline VINYL ACETATE & 1 & 8.9990 E-01 & $8.9990 \mathrm{E}-01$ & $0.0000 \mathrm{E}+00$ & $8.8990 E-01$ & 8.9800E-01 \\
\hline ARSENIC & 61 & $8.9710 \mathrm{E}-01$ & $1.4707 E-02$ & $4.1010 E-02$ & $0.0000 E+00$ & $3.0000 E-01$ \\
\hline TETRAMETHYLAMMONIUM BROMIDE & 1 & 8.6000E.01 & $8.6000 E-01$ & $0.0000 \mathrm{E}+00$ & $8.6000 E-01$ & $8.6000 \mathrm{E}-01$ \\
\hline OCTYLPHENOXYPOLYETTHOXETHANOL & 1 & $8.1000 \mathrm{E}-01$ & $8.1000 E-01$ & $0.0000 E+00$ & $8.1000 \mathrm{E}-01$ & 8. 1000E-01 \\
\hline
\end{tabular}


TABLE 5

STATISTICAL ANALYSIS OF HAZARDOUS MATERIALS
IN ASCENDING ORDER BASED ON ACCUMULATED WEIGHT
(* INDICATES DATA EXHIBITED NEGATIVE VARIANCE)

(10 SHEETS)

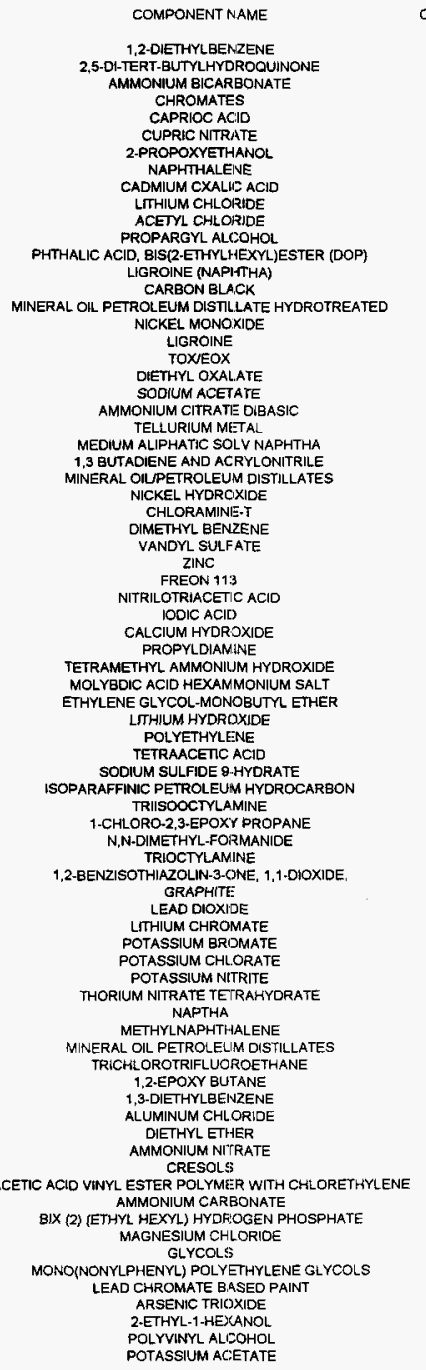

\begin{tabular}{|c|c|c|}
\hline COUNT & ACCUM WT, KG & AVERACE WT. KC \\
\hline 2 & 8.0000E-01 & $4.0000 \mathrm{E}-01$ \\
\hline 1 & $778 \mathrm{COE}-01$ & $7.7800 \mathrm{E} \cdot 01$ \\
\hline 1 & $7.7400 E-01$ & $7.7400 E-01$ \\
\hline 2 & 7.4000E-01 & 3.7000E-01 \\
\hline$t$ & $7.2000 E-01$ & $7.2000 E-01$ \\
\hline 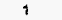 & 7. $1010 E-01$ & 7.1010E-01 \\
\hline 1 & 7.1000E-01 & 7.1000E-01 \\
\hline 7 & $7.0400 \mathrm{E}-01$ & $1.0057 E-01$ \\
\hline 1 & $7.0000 E-01$ & $7.0000 \mathrm{E}-01$ \\
\hline 2 & 6.9550E-01 & $3.4775 E-01$ \\
\hline 1 & 6.9000E-01 & 6.8000E-01 \\
\hline 1 & $6.9000 \mathrm{E}-01$ & $6.9000 E-01$ \\
\hline 3 & $6.7500=01$ & 2.2500E-01 \\
\hline 3 & $6.6600 E-01$ & 2.2200E-01 \\
\hline 4 & 6. $6500 E-01$ & $1.6025 E-01$ \\
\hline 1 & $6.6000 E-01$ & 6. $6000 E+01$ \\
\hline 1 & $66000 E-01$ & $6.6000 \mathrm{E}-01$ \\
\hline 6 & $6.5000 E-01$ & $1.0833 \mathrm{E}-01$ \\
\hline 18 & 6. $3150 E-01$ & $3,5083 E-02$ \\
\hline 1 & 6. 1000E-01 & 6. 1000E-01 \\
\hline 3 & $6.0100 E-01$ & $2.0033 E-01$ \\
\hline 1 & 6.0000E-01 & B. 0000 E. 01 \\
\hline 1 & $60000 E \cdot 01$ & $6.0000 E-01$ \\
\hline 1 & $5.9890 E-01$ & $5.9690 \mathrm{E}-01$ \\
\hline 1 & $5.8000 E-01$ & 5.8000E-01 \\
\hline 1 & $5.7000 E-01$ & 5.7000E.01 \\
\hline 20 & $5.5500 E-01$ & $2.7750 E-02$ \\
\hline 1 & $5.5000 E \cdot 01$ & 5.5000E-01 \\
\hline 1 & $5.4020 E-01$ & 5.4020E.01 \\
\hline 1 & $5.4000 E-01$ & $5.4000 E-01$ \\
\hline 19 & $5.3720 E-01$ & 2.8274E. 02 \\
\hline 1 & $5.3660 E-01$ & $5.3660 E-01$ \\
\hline 2 & 5.3300E-01 & 2.6650E-01 \\
\hline 1 & 5.3100E-01 & 5.31COE-01 \\
\hline 3 & $5.3000 E-01$ & 1.7667E-01 \\
\hline 1 & $5.2980 \mathrm{E}-01$ & 5.2980E-01 \\
\hline 2 & 5 2000E-01 & 2.6000E-01 \\
\hline 1 & 5.2000 E-01 & 5. 2000E-01 \\
\hline 2 & $5.0980 \mathrm{E}-01$ & 2.5485E-01 \\
\hline 2 & 5.0010 E -01 & $2.5005 E-01$ \\
\hline 1 & $50000 E-01$ & 5.0000E-01 \\
\hline 1 & $5.0000 E-01$ & 5.0000 E. 01 \\
\hline 1 & 4.9990E-09 & 4.9990E-01 \\
\hline 1 & $4.9290 \mathrm{E}-01$ & 4.9290E-01 \\
\hline 2 & $4.8600 \mathrm{E}-01$ & $2.4300 \mathrm{E}-01$ \\
\hline 1 & $48000 E-01$ & $4.8000 \mathrm{E}-01$ \\
\hline 1 & 4.8000E-01 & 4.8000E-01 \\
\hline 1 & 4.8000E-01 & $4.8000 E-01$ \\
\hline 1 & 4.6000E-01 & 4.8000E-01 \\
\hline 1 & $45000 E-01$ & $4.5000 E-01$ \\
\hline 1 & 4.5000E-01 & 4.5000E-01 \\
\hline 1 & 4.5000E-01 & 4.5000E-0t \\
\hline 1 & $45000 E-01$ & $4.5000 \mathrm{E}-01$ \\
\hline 1 & 4. $5000 \mathrm{E}-01$ & 4.5000E.01 \\
\hline 1 & $45000 E-01$ & 4.5000E-01 \\
\hline 1 & 4.5000E.01 & 4.5000E-01 \\
\hline 3 & 4.400OE.01 & 1.4667E-01 \\
\hline 1 & 4.4000E.01 & 4. 4000E-01 \\
\hline 1 & $4.4000 E-01$ & 4. $4000 \mathrm{E}-01$ \\
\hline 1 & 4. $4000 E-01$ & 4. $4000 \mathrm{E}-01$ \\
\hline 2 & $41090 \mathrm{E}-01$ & 2.0545E-01 \\
\hline 2 & $40000 \mathrm{E} \cdot 01$ & $2.0000 E-01$ \\
\hline 1 & 4. $0000 \mathrm{E}-01$ & 4.0000E-01 \\
\hline 1 & 3.9710E-01 & $3.8710 \mathrm{E} \cdot 01$ \\
\hline 1 & $3.9800 E-01$ & $3.9600 \mathrm{E}-01$ \\
\hline 4 & 3. $8500 \mathrm{E}-01$ & $9.6250 \mathrm{E}-02$ \\
\hline 1 & 3.7000E-01 & $3.7000 \mathrm{E}-01$ \\
\hline 1 & 3. $6000 \mathrm{E}-01$ & 3.6000E-01 \\
\hline 1 & $3.6000 E .01$ & $3.6000 E-01$ \\
\hline 7 & $3.5000 E-01$ & $5.0000 \mathrm{E} \cdot 02$ \\
\hline 1 & $3.5000 E-01$ & $3.5000 \mathrm{E}-01$ \\
\hline 1 & 3.5000E-01 & $3.50000-01$ \\
\hline 2 & $34500 \mathrm{E}-01$ & $1.7250 \mathrm{E}-01$ \\
\hline 2 & 3.4100E-01 & $1.7050 E-01$ \\
\hline 2 & 3.4000E-01 & $1.7000 E-01$ \\
\hline 1 & $34000 E-01$ & 3.4000E-01 \\
\hline 1 & $3.4000 \mathrm{E}-01$ & $3.4000 \mathrm{E}-01$ \\
\hline
\end{tabular}

STO DEV

$0.0000 E+00$

$0.0000 \mathrm{E}+00$

$0.0000 E+\infty 0$

5.0912E-01

$0.0000 E+00$

0 . DOOOE+CO

$0.0000 E+\infty 0$

8.1000E-02

$0.0000 E+\infty 0$

4.8826E-01

$0.0000 \mathrm{E}+00$

$0.0000 E+00$

2.3828E-01

$2.7145 E=01$
$1.5834 E-01$

$0.0000 \mathrm{E}+\infty 0$

$0.0000 E+\infty$

8. $2308 \mathrm{E}-\mathrm{O} 2$

3.4204E-O2

$0.0000 E+\infty 0$

1. $9065 E-01$

$0.0000 \mathrm{E}+00$

$0.0000 E+00$

$0.0000 E+00$

$0.0000 E+00$

$0.0000 E+00$

9.1459E-02

$0.0000 E+00$

$0.0000 E+00$

$0.0000 \mathrm{E}+00$

1.1260E-OT

$0.0000 E+00$

3. 6275E-01

$0.0000 \mathrm{E}+00$

2.0817E-02

$0.0000 E+00$

3.3941E-01

$0.0000 E+\infty$

3.4641E-01

$3.534 \mathrm{BE}-01$

$0.0000 E+00$

$0.0000 \bar{E}+\infty 0$

$0.0000 \mathrm{E}+00$

$0.0000 E+00$

1.8385E-O2

$0,000 \mathrm{E}+00$

$0.0000 E+00$

$0.0000 E+00$

$0.0000 E+00$

$0.0000 E+00$

$0.0000 E+\infty 0$

$0.0000 E+\infty 0$

$0.0000 E+00$

$0.0000 E+00$

$0.0000 E+00$

$0.0000 E+\infty 0$

$1,4188 E-01$

$0,0000 E+\infty$

$0.0000 E+\infty 0$

$0.0000 E+00$

2.8928E-01

0 OOOOE +00

$0.0000 E+00$

$0.0000 E+\infty 0$

$0.0000 E+\infty 0$

$1.7500 \mathrm{E}-02$

$0.0000 E+00$

$0.0000 E+00$

$0.0000 \mathrm{E}+00$

7.6000E-10

$0.0000 \mathrm{E}+00$

$0.0000 E+00$

2.3688E-01

1.2021E-02

2.1213E.01

$0.0000 E+00$

$0.0000 E+00$
MINIMUM WT, KG

MAXIMUM WT, KG

7. T.3000E-01

$7.4010 E-09 \quad 7.4010 E-01$

7.1000E-01 7.1000E-01

$1.0000 E-02 \quad 2.0000 E-01$

7.0000E.01

$2.5000 E-03$

$6.9000 E-01$

6.9000E-01

$8.0000 E-02$

$1.0000 \mathrm{E}-03$

$5.0000 E-03$

$8.0000 E-01$

$6,0000 \mathrm{E}-01$

$5.8000 E-03$

9.0000E-04

6.10C0E-01

$2.0000=-02$

6.0000E-01

6.0000 E. 01

5.0800 E-01

5.8000E-01

5.7000E-01

$0.0000 E+\infty 0$

5.5000 E-01

$5.4020 E-01$

$5.4000 \mathrm{E}-01$

$0.0000 E+\infty O$

5.3860 E-01

1.0000 E. 02

5.3100E-01

1.6000E-0

5.2980 E-01

2.0000E-02

5.2000E.01

$1.0000 \mathrm{E} \cdot 02$

$1.0000 E .04$

5.0000E:01

$5.0000 E-01$

4.0000E-01

$4.9290 \mathrm{E}-01$

2.3000E-01

4.8000E-01

4.8000E-01

4.8000 E- 01

4.6000E-01

4.5000E-0?

4.5000E-01

4.5000E-01

4.5000 E.01

4.5000E-01

4 . 5000E-01

4.5000E-01

2. $0000 \mathrm{E}-02$

4. $4000 \mathrm{E} \cdot 01$

4. $4000 \mathrm{E}-01$

4.4000 E. 01

$9.0000 \mathrm{E}-04$

2.0000E-01

4. $0000 E-01$

3.9710E-01

3.9600E-01

7. OOOOE-02

$3.7000 E .01$

3.6000E-01

3 6000E.01

$5.0000 \mathrm{E}-02$

3.5000E-01

3. 5000E-01

5. 0000E-03

$1.6200 E+01$

2.0000E-02

3.4000E-01

3. $4000 \mathrm{E}-01$ 
TABLE 5

STATISTICAL ANALYSIS OF HAZARDOUS MATERIALS

IN ASCENDING ORDER BASED ON ACCUMULATED WEIGHT

(* INDICATES DATA EXHIBITED NEGATIVE VARIANCE)

(10 SHEETS)

\begin{tabular}{|c|c|c|c|c|c|c|}
\hline COMPONENT MAME & COUNT & ACCUM WT, KG & AVERAGE WT. KG & STD DEV & MINIMUM WT, KG & MAXIMUM WT, KG \\
\hline THENOYLTRIFLUORACETONE & 2 & $3.3500 \mathrm{E}-01$ & $1.6750 \mathrm{E}-0 \mathrm{t}$ & $1.8738 E-01$ & $3.5000 \mathrm{E}-02$ & $3.0000 E-01$ \\
\hline MONOBUTYL ETHER & 1 & 3.3000E-01 & 3.3000E-01 & $0.0000 E+00$ & $3.3000 E-01$ & $3.3000 E-01$ \\
\hline POLYPROPYLENE GLYCOL & 1 & $3.3000 \mathrm{E}-01$ & $3.3000 E-01$ & $0.0000 E+00$ & $3.3000 E-01$ & $3.3000 E-01$ \\
\hline AMORPHOUS SILICA & 1 & $3.2500 E-01$ & 3.2500E-01 & $0.0000 E+00$ & 3. 2500E-01 & $3.2500 E-01$ \\
\hline AMINOGUANIDINE HEMISULFATE & 1 & $3.2400 E-01$ & $3.2400 E .01$ & $0.0000 \mathrm{E}+00$ & $3.2400 E-01$ & $3.2400 E-01$ \\
\hline DIPHENYLAMINE & 1 & $3.2400 \mathrm{E}-01$ & $3.2400 \mathrm{E}-01$ & $0.0000 E+00$ & $3.2400 E-01$ & $3.2400 E-01$ \\
\hline P.NITROPHENOL & 1 & $3.2400 \mathrm{E}-01$ & $3.2400 E-01$ & $0.0000 E+00$ & 3.2400E-01 & $3.2400 E-01$ \\
\hline DIMETTIYL OXVALATE & 1 & $3.2000 E-01$ & $3.2000 E-01$ & $0.0000 \mathrm{E}+00$ & 3.2000E-01 & $3.2000 E-01$ \\
\hline 2-(2-BUTOXYETHOXY) ETHANOL & $i$ & $3.1500 E-01$ & 3.1500E-01 & $0.0000 E+00$ & 3.1500E-01 & $3.1500 E-01$ \\
\hline PETROLEUM OIL & 2 & $3.0900 E-01$ & $1.5450 E-01$ & $4.5902 E-02$ & $1.2200 E-01$ & 1.8700E-01 \\
\hline PETROLEUM DISTILLATE & 2 & $3.0730 E-01$ & $1.5365 E-01$ & 1.8881E-01 & 2.1700E-02 & $2.6560 \mathrm{E}-01$ \\
\hline DUSOCYANATE & 1 & 3.0000E-01 & $3.0000 E-01$ & $0.0000 E+\infty 0$ & $3.0000 E-01$ & $3.0000 E-01$ \\
\hline ZIRCONIUM SULPHATE & 1 & $2.8000 \mathrm{E}-01$ & 2.8000E-01 & $0.0000 E+\infty 0$ & 2.8900E-01 & 2.8900E-01 \\
\hline ALUMINUM SHLICATE & 1 & $29850 E-01$ & $2.9850 E-01$ & $0.0000 E+\infty 0$ & 2.9850E-01 & 2.0050E-01 \\
\hline SODIUM NITROFERRICYANIDE & 1 & $2.9500 E-01$ & $2.9500 \mathrm{E}-01$ & $0.0000 E+00$ & $2.8500 E-01$ & 2.0500E-01 \\
\hline CHROMIUM (III) NITRATE NONAHYDRATE & 1 & 2.8000E-01 & $2.0000 E-01$ & $0.0000 E+00$ & 2.8000E-01 & 2.0000E-01 \\
\hline 2-(METHOXYETHOXY) ETHANOL & 1 & 2.8600E-01 & 2.8600E-01 & $0.0000 E+\infty 0$ & $28800 E-01$ & $2.8000 E-01$ \\
\hline PROPIONIC A.CID & 2 & 2.8500E-01 & $1.4250 E-01$ & 2.4749E-02 & $1.2500 E-01$ & 1.6000E-01 \\
\hline TRICHLOROETHANE & 2 & $2.8400 E-01$ & $1.4200 E-01$ & $8.2024 E-02$ & 8.4000E-02 & $2.0000 E-01$ \\
\hline $\begin{array}{c}\text { 2-ETHANEDIAMINE, N-(2-AMINOETHIL)-N*2, AMINOETHYL)AMI } \\
\text { CHLORINATED PARAFFIN WAX }\end{array}$ & 1 & $\begin{array}{l}2.8000 E-01 \\
2.7000 E-01\end{array}$ & $\begin{array}{l}2.8000 E-01 \\
2.7000 E-04\end{array}$ & $\begin{array}{l}0.0000 E+00 \\
0.0000 E+00\end{array}$ & $2.8000 E-01$ & $2.8000 E-01$ \\
\hline PYDRAULFYRGUEL (HYDRAULIC FLUID) & 3 & $2.8880 E-01$ & $8.9600 \mathrm{E}-02$ & $1.3804 E-01$ & $9.9000 E-03$ & 2.4900E-01 \\
\hline FURFURYL-ALCOHOL & 4 & $2.6500 \mathrm{E}-01$ & $2.0500 \mathrm{E}-01$ & $0.0000 E+\infty 0$ & $2.6500 \mathrm{E}-01$ & $2.6500 E-01$ \\
\hline PROPYLENE GLYCOL MONOMETHYL ETHER & 1 & $2.6500 \mathrm{E}-01$ & $2.6500 \mathrm{E}-01$ & $0.00005+00$ & $2.6500 E-01$ & $2.8500 E-01$ \\
\hline 2-BUTOXETHANOL & 1 & 2.6000E-01 & $26000 E-01$ & $0.0000 E+00$ & 2 BOOOE-01 & 2.8000E-OT \\
\hline METHLY ETHYL KETONE & 1 & $2.5080 E-01$ & $2.5080 E-01$ & $0.0000 E+00$ & $2.5080 E-01$ & $2.5060 E-01$ \\
\hline ACID & 1 & 2.5000E-01 & $2.5000 E-01$ & $0.0000 E+00$ & $2.5000 E-01$ & $2.5000 E-01$ \\
\hline GALLIC ACID & 1 & $2.5000 E-01$ & $25000 E-01$ & $0.0000 E+00$ & 2.5000E-01 & $2.5000 E-01$ \\
\hline SOLVENT PETROLEUM OISTILLATE & 1 & 2.5.000E-01 & $2.5000 E-01$ & $0.0000 E+00$ & 2.5000E-01 & 2.5000E-01 \\
\hline METHYL-1-PHENYL-2-PYRAZOLIN & 1 & 2.4600E-0\% & $2,4600 E-01$ & $0.0000 E+\infty 0$ & 2.4600E-01 & 2.4600E-01 \\
\hline SULFATES & 3 & 2.4410E-01 & 8.1367E-02 & $2.3561 E-02$ & $5.6600 E-02$ & $1.0350 E-01$ \\
\hline BIS(2-ETHYL HEXYL) ESTER PHTHALIC ACID (DOP) & 11 & $2.4240 E-01$ & 2.2036E-02 & 6.5130E-03 & $2.4000 E-03$ & $2.4000 E-02$ \\
\hline ACETIC ACID SODIUM SALT & 1 & $2.4000 E-01$ & $2.4000 E-01$ & $0.0000 E+\infty 0$ & $2,4000 \mathrm{E}-01$ & $2.4000 E-01$ \\
\hline AMMONLUM PERSULFATE & 1 & $2.4000 \mathrm{E}-01$ & $2.4000 E-01$ & $0.0000=+00$ & $2.4000 E-01$ & 2.4000E-0t \\
\hline AZOBENZENE & $i$ & $24000 \mathrm{E}-01$ & $2.4000 \mathrm{E}-0 t$ & $0.0000 E+00$ & $2.4000 \mathrm{E} \cdot 01$ & $2.4000 E-01$ \\
\hline O.CRESOL & 4 & $2.3340 \mathrm{E}-01$ & $5.8350 \mathrm{E}-02$ & $4.5633 E-02$ & $1.4000 E-03$ & $1.0000 E-01$ \\
\hline TRIETHYLENERETRAMINE & 1 & 2.2500E-01 & $2.2500 E-01$ & $0.0000 E+00$ & $2.2500 E-01$ & 2.2500E-01 \\
\hline TOTAL ORGANIC HALOGENS & 13 & $22410 E-01$ & $1.7238 \mathrm{E}-02$ & $1.5729 \mathrm{E}-02$ & $1.0000 E-04$ & 4.2000E-02 \\
\hline LEAD TELLURIDE & 1 & $2.2000 E-01$ & $2.2000 E-01$ & $0.0000 E+\infty$ & $2.2000 E-01$ & $2.2000 E-01$ \\
\hline POTASSIUM PERIODATE & 1 & 2.2000E-01 & $2.2000 E-01$ & $0.0000 E+\infty 0$ & 2.2000E-01 & $2.2000 E-01$ \\
\hline CADMIUM HYDFIOXIDE & i & 2.0500E-01 & $2.0500 \mathrm{E}-01$ & $0.0000 E+\infty 0$ & $2.0500 E-01$ & 2.0500E-01 \\
\hline TRIETHYLAMINE & 3 & 2.0310E-01 & 6.7 TOOE-02 & $5.8032 E-02$ & $3.0000 E-03$ & $1.0010 \mathrm{E}-01$ \\
\hline TETRACHLOROETHENE & 3 & $2.0000 E-01$ & $6.6667 \mathrm{E}-02$ & $2.3094 E-02$ & $4.0000 E-02$ & $8.0000 E-02$ \\
\hline CUMENE HYOROXIDE & 1 & $20000 E-01$ & 20000E-01 & $0.0000 E+00$ & 2.0000E-01 & $2.0000 E-01$ \\
\hline ETHANOL DIBENZYL MERCURY & 1 & $20000 E-01$ & 2.0000E-01 & $0.0000 E+00$ & $2.0000 E-01$ & $2.0000 E-01$ \\
\hline ETHVL MERCURIC GHLORIDE & $i$ & 2.0000E-01 & $2.0000 E-01$ & $0.0000 E+00$ & 20000E-01 & $2.0000 E-01$ \\
\hline MERCURIC HYDROXIDE & $i$ & $2.0000 E-01$ & $2.0000 \mathrm{E}-01$ & $0.0000 E+\infty 0$ & $2.0000 E-01$ & $2.0000 E-01$ \\
\hline HYOROTREATED HEAVY NAPHTHA & 1 & $1.8900 \mathrm{E}-01$ & $1.8900 \mathrm{E}-01$ & $0.0000 E+00$ & $1.8900 E-01$ & $1.0900 \mathrm{E}-01$ \\
\hline 2-BIPHENYLOL SODIUM SALT & 1 & $1.9000 \mathrm{E}-01$ & $1.0000 E-01$ & $0.0000 E+00$ & $1.8000 E-01$ & $1.9000 E-01$ \\
\hline hEXACHLOROBENZENE & 124 & $1.8170 E-01$ & 1.4853E-03 & 5.6500E-04 & $2.0000 E-04$ & $2.3000 E-03$ \\
\hline NAPHTHENIC ACID LEAD SALT & 1 & $1.8000 E-01$ & $1.8000 E-01$ & $0.0000 E+00$ & $1.8000 \mathrm{E}-01$ & $1.8000 E-01$ \\
\hline STYRENE: & 2 & 1.7710E-01 & 8.8550E-02 & $1.2500 E-01$ & $1.0000 E-04$ & $1.7700 \mathrm{E}-01$ \\
\hline TERT BUTYL ALCOHOL & 4 & $1.7500 E-01$ & $4.3950 \mathrm{E}-02$ & $1.1361 \mathrm{E}-02$ & $30000 E-02$ & $5.5800 E-02$ \\
\hline N,N,N,N'-TETRAMETHYL-1,3-BUTANEDIAMINE & 1 & $1.7500 E-01$ & $1.7500 E-01$ & $0.0000 E+00$ & $1.7500 E-01$ & $1.7500 E-01$ \\
\hline ETHYLENEDIAMINETETRAACETIC A.CID DISODIUM SALT (EDTA) & 1 & $1.7100 \mathrm{E}-01$ & $1.7100 E-01$ & $0.0000 E+00$ & $1.7100 E-01$ & $1.7100 E-01$ \\
\hline STANMOUS SULFATE & 1 & $1.7100 \mathrm{E}-01$ & $1.7100 E-01$ & $0.0000 E+00$ & $1.7100 E-01$ & $1.7100 E-01$ \\
\hline POLYCHLORINATED BINPHENYLS & 2 & 1.7000E-01 & $8.5000 E-02$ & $4.9497 \mathrm{E}-02$ & 5.0000E-02 & $1.2000 E-01$ \\
\hline BIS(2 ETHYLHEXYL) HYDROGEN PHOSPHATE & 1 & $1.7000 E-01$ & $1.7000 E-01$ & $0.0000 E+00$ & $1.7000 E-01$ & $1.7000 \mathrm{E}-01$ \\
\hline NAPTHENIC DISTILLATE & 1 & $1.7000 \mathrm{E}-01$ & $1.7000 E-01$ & $0.0000 E+\infty$ & $1,7000 E-01$ & $1.7000 \mathrm{E}-01$ \\
\hline (PHENYL)-N,N-OIISOBUTYL-CARBAMOYLMETHYLPHOSPHINE & 1 & $1.6400 E-01$ & 1.6400E-01 & $0.0000 E+\infty$ & 1. $6400 E-01$ & t. $6400 E-0 t$ \\
\hline BENZIN-(NAPHTHA) & 1 & $1.6200 \mathrm{E}-01$ & $1.6200 \mathrm{E}-01$ & $0.0000 E+00$ & 1.6200E-01 & $1.6200 \mathrm{E}-01$ \\
\hline NITROCELLUI_OSE & 1 & 1. $6200 E+01$ & 1.620CE-01 & $0.0000 E+00$ & 1. $8200 \mathrm{E}-01$ & $1.6200 \mathrm{E}-01$ \\
\hline BUTOXYETHANOL & 2 & 1.6000E-01 & $8.0000 E-02$ & $9.8995 E-02$ & $1.0000 \mathrm{E}-02$ & $1.5000 E-01$ \\
\hline UNDECANIE & $i$ & $1.6000 \mathrm{E}-01$ & $1.6000 E-01$ & $0.0000 E+00$ & $1.8000 E-01$ & $1.6000 E-01$ \\
\hline 2 HEPTANCINE & $i$ & $1.5500 \mathrm{E}-01$ & $1.5500 E-01$ & $0.0000 E+\infty 0$ & $1.5500 E-01$ & $1.5500 \mathrm{E}-01$ \\
\hline BUTOXYETHOXY ETHANOL. & 1 & $1.5000 E-01$ & $1.5000 \mathrm{E}-01$ & $0.0000 E+00$ & $1.5000 E-01$ & $1.5000 E-01$ \\
\hline DI-N-BUTYL PHTHALATE & 1 & $1.4200 E-01$ & $1.4200 E-01$ & $0.0000 E+\infty 0$ & 1.4200E-01 & $1.4200 E-01$ \\
\hline LIGHT BALLASTS CONTAINING PCB & 3 & $1.4000 \mathrm{E}-01$ & $4.6667 \mathrm{E}-02$ & 4.6188E-02 & $20000 \mathrm{E}-02$ & $1.0000 E-01$ \\
\hline $1,1,1$ TRICHLORCIEHTANE & 1 & $1.4000 \varepsilon+01$ & $1.4000 E-01$ & $0.0000 E+00$ & t. 4000E-01 & $1.4000 E-01$ \\
\hline BATIERIES CONTAINING POTASSIUM HYDROXIOE, NICKEL & 1 & $1.4000 \mathrm{E}-01$ & $1.4000 E-01$ & $0.0000 E+00$ & $1.4000 E-01$ & $1.4000 E-01$ \\
\hline $\begin{array}{l}\text { DIMETHYL PHI_HATE } \\
\text { DIMTE }\end{array}$ & 2 & $1.350 C E-01$ & $6.7500 E-02$ & $4.5962 \mathrm{E}-02$ & 3.5000E-02 & $1.0000 E-01$ \\
\hline $1,1,1$ TRICHLORETHANE & 4 & $1.3090 E-01$ & $3.2725 E-02$ & $5.1133 E-02$ & $1.7000 E-03$ & $1.0920 \mathrm{E}-01$ \\
\hline SILICON & 2 & 1.3000E-0t & $6.5000 E-02$ & $35355 \mathrm{E}-02$ & $4.0000 E-02$ & $9.0000 \mathrm{E}-02$ \\
\hline SILVER NITR:ATE & 3 & $12820 E-01$ & 4.2733E-02 & $5.0778 \mathrm{E}-02$ & $3.2000 \mathrm{E}-03$ & $1.0000 E-01$ \\
\hline BENZENEARSEINIC ACID & 1 & $1.2600 \mathrm{E}-01$ & $1.2600 \mathrm{E}-01$ & $0.0000 E+00$ & $1.2600 \mathrm{E}-01$ & $1.2600 E-01$ \\
\hline POTASSIUM DICHIROMATE & 1 & $1.2600 \mathrm{E}-01$ & $1.2600 E-01$ & $0.0000 E+00$ & $1.2600 E-01$ & $1.2600 \mathrm{E}-01$ \\
\hline DITHIOXAMIDE & 1 & $1.2200 \mathrm{E}-01$ & $1.2200 \mathrm{E}-01$ & $0.0000 \mathrm{E}+\infty$ & $1.2200 \mathrm{E}-01$ & $1.2200 \mathrm{E}-01$ \\
\hline
\end{tabular}


TABLE 5

STATISTICAL ANALYSIS OF HAZARDOUS MATERIALS IN ASCENDING ORDER BASED ON ACCUMULATED WEIGHT

(* INDICATES DATA EXHIBITED NEGATIVE VARIANCE)

(10 SHEETS)

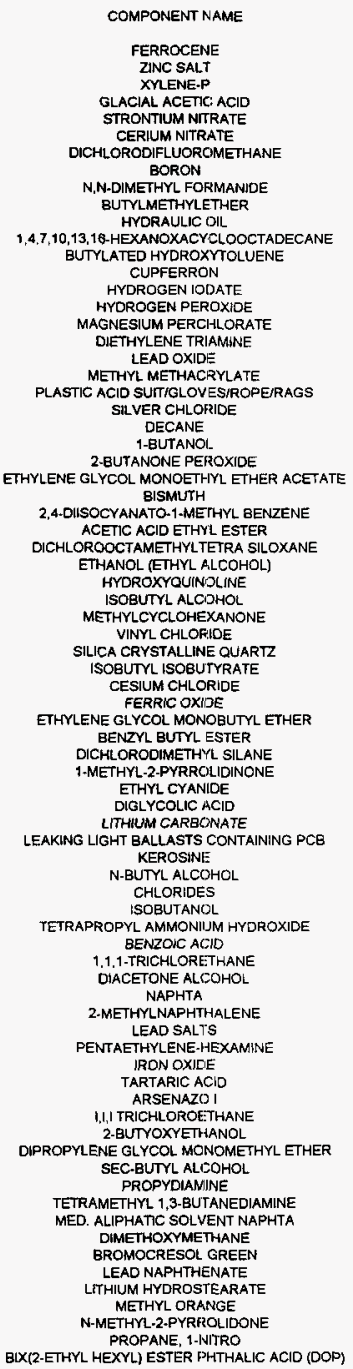
BISMUTH

2,4-OISOCYANATO-1-METHYL BENZENE ACETIC ACID ETHYL ESTER

\begin{tabular}{|c|c|c|}
\hline COUNT & ACCUM WT, KG & AVERAGE WT KG \\
\hline 1 & $1.2200 E-01$ & $1.2200 \mathrm{E}-01$ \\
\hline 1 & $1.2200 \mathrm{E}-01$ & $1.2200 E-01$ \\
\hline 1 & $1.2000 \mathrm{E}-0 \mathrm{t}$ & $1.2000 E-01$ \\
\hline 2 & 1.1780 E-01 & $5.8900 \mathrm{E}-02$ \\
\hline 4 & $1.1580 \mathrm{E}-01$ & $2.8950 \mathrm{E}-02$ \\
\hline 1 & $1.1000 E-01$ & $1,1000 E-01$ \\
\hline 1 & $1.1000 \mathrm{E}-01$ & $1.1000 E-01$ \\
\hline 8 & $1.0750 E-01$ & $1.3438 E-02$ \\
\hline 2 & $1.0500 E-01$ & $5.2500 \mathrm{E}-02$ \\
\hline 1 & $1.0010 E-01$ & $1.0010 \mathrm{E}-01$ \\
\hline 7 & $1.0000 E-01$ & 3.3333E-02 \\
\hline 1 & $1.0000 E-01$ & 1.0000 E-01 \\
\hline 1 & $1.0000 E-01$ & $1.0000 E-01$ \\
\hline 1 & I.DOOOE-01 & $1.0000 E-0 !$ \\
\hline 1 & $1.0000 E-01$ & $1.0000 \mathrm{E}-01$ \\
\hline 1 & 1.0000 E-01 & $1.0000 \mathrm{E}-01$ \\
\hline 1 & $1.0000 E-01$ & $1.0000 \mathrm{E}-01$ \\
\hline 1 & $9.8000 \mathrm{E}-02$ & $9.8000 E-02$ \\
\hline 1 & $9.5000 \mathrm{E}-02$ & 8.5000E-02 \\
\hline 1 & $9.0000 \mathrm{E}-02$ & 9.0000 E-02 \\
\hline 1 & $9.0000 \mathrm{E}-02$ & $9.0000 E-02$ \\
\hline 1 & $8.5500 E-02$ & $8.5500 E-02$ \\
\hline 1 & $8.5000 E .02$ & $8.5000 E-02$ \\
\hline 56 & $8.4000 E-02$ & $1.5000 \mathrm{E}-03$ \\
\hline 56 & $8.4000 E-02$ & t.5000E.03 \\
\hline 56 & $8.4000 E-02$ & $1.5000 \mathrm{E}-03$ \\
\hline 1 & 8.3600E-02 & $8.3600 \mathrm{E}-02$ \\
\hline$i$ & $8.0000 E-02$ & $8.0000 E-02$ \\
\hline 1 & $8.0000 \mathrm{E}-02$ & 8.0000 E-02 \\
\hline 1 & $8.0000 \mathrm{E}-02$ & $8.0000 E-02$ \\
\hline 1 & $8.0000 E-02$ & $8.0000 E-02$ \\
\hline 1 & B.0000E-02 & $8.0000 E-02$ \\
\hline 1 & 8.0000E-02 & $8.0000 E-02$ \\
\hline$i$ & $8.0000 E-02$ & $8.0000=-02$ \\
\hline 29 & 7.8500E-02 & $2.7069 \mathrm{E}-03$ \\
\hline 1 & $6.7000 E-02$ & $6.7000 \mathrm{E}-02$ \\
\hline 1 & $6.4000 \mathrm{E}-02$ & $6.4000 E-02$ \\
\hline 4 & $6.3300 \mathrm{E}-02$ & $1.5950 E-02$ \\
\hline 1 & $0.1600 E-02$ & 6. 1 BOOE-02 \\
\hline 2 & $6.0000 E-02$ & $3.0000 E-02$ \\
\hline 1 & $6.0000 \mathrm{E}-02$ & $6.0000=-02$ \\
\hline 1 & $6,0000 E-02$ & $6.0000=-02$ \\
\hline 2 & $5.8000=02$ & $2.9000=02$ \\
\hline 1 & $5.7000 \mathrm{E}-02$ & $5.7000 E-02$ \\
\hline 1 & $5.5000 \mathrm{E}-02$ & $5.5000 E-02$ \\
\hline 1 & $5.2000 \mathrm{E}-02$ & 5.2000E-02 \\
\hline 2 & 5.1000E-02 & $2.5500 E-02$ \\
\hline 2 & $5.0000 \mathrm{E}-02$ & 5. $0000 E-02$ \\
\hline 1 & $5.0000 E-02$ & $5.0000 E-02$ \\
\hline 3 & $\triangle 9000 E-02$ & $1.6333 E-02$ \\
\hline 1 & $4.7000 \mathrm{E}-02$ & $4.7000 \mathrm{E}-02$ \\
\hline 3 & $4.5000 E-02$ & $1.5000 \mathrm{E}-02$ \\
\hline 1 & $4.5000 \mathrm{E}-02$ & $4.5000 \mathrm{E}-02$ \\
\hline 5 & 4. $4100 E-02$ & $8.8200 E-03$ \\
\hline 2 & $4.4000 E-02$ & $2.2000 \mathrm{E}-02$ \\
\hline 1 & $42000 E-02$ & $4.2000 \mathrm{E}-02$ \\
\hline 4 & $4.0000 \mathrm{E}-02$ & $10000 \mathrm{E}-02$ \\
\hline$i$ & $4.0000 \mathrm{E}-02$ & $4.0000 E-02$ \\
\hline 1 & $40000 \mathrm{E}-02$ & $4.0000 E-02$ \\
\hline 1 & $3.9800 \mathrm{E}-\mathrm{O} 2$ & $3.0800 \mathrm{E}-02$ \\
\hline 1 & 3.7500E-02 & $3.7500 \mathrm{E}=02$ \\
\hline 1 & $3.6000 E-02$ & $3,6000 \mathrm{E}-02$ \\
\hline 1 & $3.5000 \mathrm{E}-02$ & $3.5000 \mathrm{E}-02$ \\
\hline 1 & 3.4000E-02 & $3.4000 \mathrm{E}-02$ \\
\hline 1 & $3.4000 \mathrm{E}-02$ & $3.4000 \mathrm{E}-02$ \\
\hline 1 & 3.4000E-02 & $3.4000 E-02$ \\
\hline 1 & $3.3000 \mathrm{E}-02$ & $3.3000 \mathrm{E}-02$ \\
\hline 1 & 3.3000E-02 & 3.3000E-02 \\
\hline 1 & $3.2100 \mathrm{E}-02$ & $3.2100 E-02$ \\
\hline 2 & $3.0400 \mathrm{E}-02$ & $1.5200 \mathrm{E}-02$ \\
\hline 2 & $3.0000 E-02$ & $1.5000 \mathrm{E}-02$ \\
\hline 1 & $3.0000 E-02$ & 3.0000 E-02 \\
\hline 1 & $3.0000 \mathrm{E}-02$ & $30000 \mathrm{E}-02$ \\
\hline 1 & $3.0000 \mathrm{E}-02$ & $3.0000 E-02$ \\
\hline 1 & $3.0000 E-02$ & $3.0000 \mathrm{E}-02$ \\
\hline 1 & $27500 E-02$ & $2.7500 \mathrm{E} .02$ \\
\hline 1 & $27000 E-02$ & $2.7000 \mathrm{E}-02$ \\
\hline
\end{tabular}

STD DEV

$0.0000 E+00$

$0.0000 E+00$

$0.0000 E+00$

1.5608E-02

3.2258E-02

$0.0000 E+\infty 0$

1.2521E-02

$3.8891 E-02$

$0.0000 \mathrm{E}+\infty 0$

$1.9245 \mathrm{E}-02$

$0.0000 \mathrm{E}+00$

$0.0000 E+00$

$0.0000 E+00$

$0.0000 E+00$

$0.0000 E+00$

$0.0000 E+00$

$0.0000 E+00$

$0.0000 E+00$

$0.0000 E+00$

$0.0000 E+00$

$0.0000 E+\infty 0$

$0.0000 E+\infty 0$

$0.0000 E+\infty 0$

$0,0000 E+00$

$0.0000 E+00$

$0.0000 E+00$

$0.0000 E+00$

$0.0000 E+00$

$0.0000 E+00$

$0.0000 E+\infty 0$

$0.0000 \mathrm{E}+00$

$0.0000 \mathrm{E}+\infty 0$

$1.3005 \mathrm{E} \cdot 02$

$0.0000 E+\infty 0$

$0.0000 E+00$

1.8724E.02

$0.0000 E+00$

$0.0000 E+00$

$0.0000 \mathrm{E}+00$

$0.0000 E+\infty 0$

1.4140E-03

$0.0000 E+00$

$0.0000 E+00$

$0,0000 \mathrm{E}+00$

3.4848E-02

WNUMI

$0.0000 \mathrm{E}+00$

4.7350E-03

$0.0000 E+\infty 0$

8. $6500 E-03$

$0.0000 E+00$

5.7550E-03

1.6974E-02

$0.0000 E+00$

$0.0000 E+00$

$0.0000 \mathrm{E}+00$

$0.0000 E+00$

$0.0000 E+00$

$0.0000 E+\infty 0$

$0.0000 E+00$

$0.0000 E+\infty 0$

$0.0000 E+00$

$0.0000 E+00$

$0.0000 \mathrm{E}+\infty$

$0.0000 E+\infty 0$

$0.0000 E+00$

$0.0000 E+00$

2.0830E.02

$1.4142 \mathrm{E}-02$

$0.0000 E+00$

$0.0000 E+00$

$0.0000 E+00$

$0.0000 E+\infty 0$

$0.0000 E+00$
MINAMUM WT, KG

MAXIMUM WT, KG

$1.2000 \mathrm{E}-01$

$1.2200 E 0$

1.2000E-01

4.780OE-02

$6.0000=04$

1.1000E-01

1.1000E-01

1.0000E-04

2.5000E-02

1.0010E-01

$0.0000 E+C 0$

$1.0000 E-01$

$1.0000 \mathrm{E}-01$

$1.0000 E-01$

1.0000E-01

1.0000E-01

1.0000 E-01

$9.8000 E-02$

9.5000E.02

$9.0000 E-02$

$9.0000 E-02$

8.5500E-02

8.5000E-02

$15000 \mathrm{E}-03$

$1.5000 E-03$

1. 5000E-03

8.3600E-02

8. $0000 E-02$

8.0000E-02

8.0000E-02

8.0000E-02

8.0000E-02

B. $0000 E-02$

1.0000E-04

6.7000E- 02

6. 4000E-02

2.0000E-04

6. 1000E-02

3. $0000 E-02$

$6.0000 \mathrm{E}-02$

6.0000E-02

2.8000E-02

5.7000 E- 02

5.5000E-02

5.2000E-02

1.0000E-03

5.0000E-02

5. $0000 \mathrm{E}-02$

1. 1300E-02

4. $7000 E-02$

5. $0000 \mathrm{E}-03$

4.5000E-02

1.7000E-03

1.0000E-02

4. $2000 \mathrm{E}-02$

4.0000E-02

4.0000E-02

4.0000E-02

3.9800E-02

3.7500E-02

3. $4000 \mathrm{E}-02$

3.400OE-02

$3.4000 E-02$

$3.3000 \mathrm{E}-02$

3.3000E-02

3.2tOOE-O2

4.0000E-04

5.0000E-03

3.0000 E- 02

3.0000E-02

3.0000E-02

3.0000E-02

$27500 E-02$

2.7000E-02

$1.2200=-01$

$1.2005-0$

1.2000E-01

7.0000f -02

0.3000E-02

$1.1000=-01$

$1.10005-01$

3.0400E-02

B.0000E-02

$1.0010 E-01$

$1.0000 E-01$

$1.0000 E-01$

1.0000E-01

$1.0000 E-01$

1.0000E-0

1.0000 E-O

$1.0000 E-01$

8.8000E-02

9.5000E-02

$9.0000 \mathrm{E}-02$

$9.0000 E-02$

$8.5500 E-02$

8.5000E-02

1.5000E-03

1.5000E-03

$1.5000 E-03$

8.3000 -02

8.0000E-02

$8.0000 E-02$

$8.0000 E-02$

$8.0000 E-02$

8.0000E-02

$8.0000 E-02$

$8.0000 E-02$

$7.5000 \mathrm{E}-02$

$6.7000 \mathrm{E}-02$

$6.4000 E-02$

$3.8000 \mathrm{E}-02$

$8.1600 E-02$

3.0000E-02

$3.0000=02$

$6.0000 \mathrm{E}-02$

3.0000E-02

$5.7000 \mathrm{E}-02$

$5.5000 E-02$

2000E-02

5.0000E-02

$5.0000 \mathrm{E}-02$

5.0000E-02

2.0700E-02

$4,7000 \mathrm{E}-02$

2.0000 E- 02

4.5000E-02

1.3000E-02 
TABLE S

STATISTICAL ANALYSIS OF HAZARDOUS MATERIALS IN ASCENDING ORDER BASED ON ACCUMULATED WEIGHT (* INDICATES DATA EXHIBITED NEGATIVE VARIANCE)

(10 SHEETS)

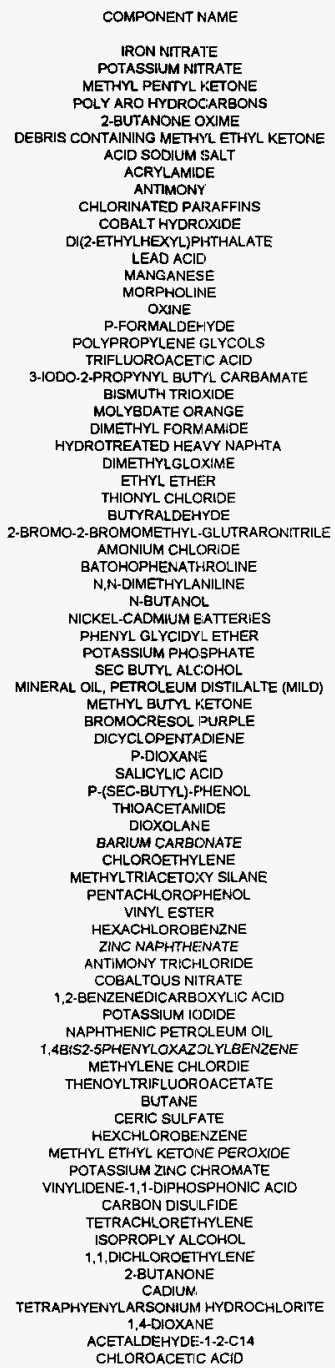

\begin{tabular}{|c|c|c|}
\hline COUNT & ACCUM WT, KG & AVERAGE $W T$ KC \\
\hline 3 & $2.5900 \mathrm{E}-02$ & 8.6333E-03 \\
\hline 1 & $2.5000 \mathrm{E}-02$ & 2.50C0E-.02 \\
\hline 1 & $2.3000 \mathrm{E}-02$ & $2.3000 E-02$ \\
\hline 4 & $2.2000 \mathrm{E}-02$ & 5.5000 E- 03 \\
\hline 2 & $2.0000 \mathrm{E}-02$ & $1.0000 \mathrm{E}-02$ \\
\hline 2 & 2.0000E-02 & 1.0000E-02 \\
\hline 1 & $2.0000 E-02$ & 2.0000 E-02 \\
\hline 1 & $2.0000 \mathrm{E}-02$ & $2.0000 E-02$ \\
\hline 1 & $2.0000 E-02$ & $2.0000 E-02$ \\
\hline 1 & $2.0000 E-02$ & $2.0000 E-02$ \\
\hline 1 & $2.0000 \mathrm{E}-02$ & $2.0000 \mathrm{E} \cdot 02$ \\
\hline 1 & $2.0000 E-02$ & $2.0000 \mathrm{E}-02$ \\
\hline 1 & $2.0000 \mathrm{E}-02$ & $2.0000 \mathrm{E}-02$ \\
\hline 1 & $2.0000 E-02$ & $2.0000 \mathrm{E}-02$ \\
\hline 9 & $2.0000 \mathrm{E}-02$ & $2.0000 \mathrm{E}-02$ \\
\hline 1 & $2.0 C 00 E-02$ & $20000 \mathrm{E}-02$ \\
\hline 1 & $2.0000 \mathrm{E}-02$ & $2.0000 \mathrm{E}-02$ \\
\hline 1 & 2.0000E-02 & $2.0000 E-02$ \\
\hline 1 & $2.0000 E-02$ & $2.0000 \mathrm{E}-02$ \\
\hline 1 & $1.9000 \mathrm{E}-02$ & $1.9000 \mathrm{E}-02$ \\
\hline 1 & $1.9000 \mathrm{E}-02$ & $1.0000 E-02$ \\
\hline 1 & $1.9000 \mathrm{E} .02$ & 1.9000 E-02 \\
\hline 1 & $1.6000 \mathrm{E}-02$ & $1.8000 \mathrm{E}-02$ \\
\hline 1 & 1. 5900E-02 & 1.5900E.02 \\
\hline 2 & $1.5800 \mathrm{E}-02$ & $7.0000 \mathrm{E}-03$ \\
\hline 4 & $1.5000 \mathrm{E} \cdot 02$ & 3.7500E-03 \\
\hline 1 & $1.3800 E-02$ & 1.3800E-02 \\
\hline 1 & 1.3000E-02 & 1.3000E-02 \\
\hline 1 & $1.0000 \mathrm{E}-02$ & $1.0000 \mathrm{E}-02$ \\
\hline 1 & $1.0000 \mathrm{E}-02$ & $1.0000 \mathrm{E}-02$ \\
\hline 1 & $1.0000 \mathrm{E}-02$ & $1.0000 \mathrm{E}-02$ \\
\hline 1 & $1.0000 \mathrm{E}-02$ & $1.0000 \mathrm{E}-02$ \\
\hline 1 & $1.0000 E-02$ & $1.0000 \mathrm{E}-02$ \\
\hline 1 & $1.0000 \mathrm{E}-02$ & $1.0000 \mathrm{E}-02$ \\
\hline 1 & $1.0000 \mathrm{E}-02$ & $1.0000 \mathrm{E}-02$ \\
\hline 1 & $1.0000 \mathrm{E}-02$ & $1.0000 E-02$ \\
\hline 1 & $1.0000 \mathrm{E}-02$ & $1.0000 \mathrm{E}-02$ \\
\hline 1 & $9.0000 E-03$ & $9.0000 \mathrm{E}-03$ \\
\hline 2 & $8.0000 \mathrm{E}-03$ & $4.0000 \mathrm{E}-03$ \\
\hline$i$ & 5.0000E-03 & $5.0000 \mathrm{E}-03$ \\
\hline 1 & $5.0000 \mathrm{E}-0.03$ & $5.0000 E-03$ \\
\hline 1 & 4.8000E-03 & $4.8000 E-03$ \\
\hline 1 & 4. $6000 \mathrm{E}-03$ & 4.6000E-03 \\
\hline 1 & $4.0000 \mathrm{E}-03$ & 4.0000E -03 \\
\hline 1 & $3.6000 \mathrm{E} \cdot 03$ & $3.6000 \mathrm{E}-03$ \\
\hline 1 & $3.3000 \mathrm{E}-03$ & $3.3000 \mathrm{E}-03$ \\
\hline 1 & 3.0000 E-03 & $3.0000 E-03$ \\
\hline 1 & $3.0000 \mathrm{E}-03$ & 3.0000 E- 03 \\
\hline 1 & $3.0000 \mathrm{E}-03$ & $3.0000 E-03$ \\
\hline 1 & $3.0000 \mathrm{E}-03$ & $3.0000 \mathrm{E} \cdot 03$ \\
\hline 1 & $3.0000 \mathrm{E}-03$ & $3.0000 \mathrm{E}-03$ \\
\hline 1 & $2.5000 \mathrm{E}-03$ & $2.5000 \mathrm{E}-03$ \\
\hline 1 & $2.5000 \mathrm{E}-03$ & $2.5000 \mathrm{E}-03$ \\
\hline 2 & $2.2000 \mathrm{E}-03$ & $1.1000 \mathrm{E}-03$ \\
\hline 2 & 2.1000E-03 & $1.0500 \mathrm{E}-03$ \\
\hline 1 & $2.0000 \mathrm{E}-03$ & $2.0000 \mathrm{E}-03$ \\
\hline 2 & 1.8000E-03 & $9.0000 E-04$ \\
\hline 1 & $1.5000 \mathrm{E}-03$ & $1.5000 \mathrm{E}-03$ \\
\hline 4 & 1.4000E-03 & 3.5000E-04 \\
\hline 1 & $1.4000 \mathrm{E}-03$ & $1,4000 E-03$ \\
\hline 1 & $1.4000 \mathrm{E}-03$ & $1.4000 \mathrm{E}-03$ \\
\hline 1 & $1.2000 \mathrm{E}=03$ & $1.2000 \mathrm{E}-03$ \\
\hline 1 & $1.1000 \mathrm{E}-03$ & 1. $1000 \mathrm{E}-03$ \\
\hline 1 & $10000 \mathrm{CE}-03$ & $1.00000-03$ \\
\hline 1 & $1.0000 E-03$ & 1.0000E-03 \\
\hline 1 & $1.0000 \mathrm{E}-03$ & $1.0000 \mathrm{E}-03$ \\
\hline 1 & $1.0000 \mathrm{E}-03$ & 1.0000 E -03 \\
\hline 58 & 5. .0000E-04 & $8.6207 \mathrm{E}-06$ \\
\hline 2 & $4.0000 E-04$ & 2.0000 E.04 \\
\hline 9 & 3.0000E-04 & $3.0000 \mathrm{E}-04$ \\
\hline 57 & 1.0000E-04 & 1.7544E-08 \\
\hline 1 & $1.0000 \mathrm{E}-04$ & $1.0000 E-04$ \\
\hline 1 & $1.0000 E-04$ & 1.0000 E-04 \\
\hline 1 & $1.0000 \mathrm{E} .04$ & $1.0000 E-04$ \\
\hline 58 & $0.0000 E+\infty$ & $0.0000 \varepsilon+\infty 0$ \\
\hline 56 & $0.0000 E+00$ & $0.0000 E+00$ \\
\hline 56 & $0.0000 E+\infty 0$ & $0.0000 \varepsilon+\infty 0$ \\
\hline
\end{tabular}

STD DEV

$1.4174 E-02$

$0.0000 E+\infty 0$

$0.0000 \mathrm{E}+00$

$1.0000 \mathrm{E}-03$

$0.0000 \mathrm{E}+00$

$0.0000 E+00$

$0.0000 E+00$

$0.0000 E+00$

$0.0000 E+\infty 0$

$0.0000 \mathrm{E}+\infty 0$

$0.0000 \mathrm{E}+\infty 0$

$0.0000 E+\infty 0$

$0.0000 E+00$

$0.0000 E+\infty 0$

$0.0000 \mathrm{E}+00$

$0.0000 E+00$
$0.0000 E+00$

$0.0000 \mathrm{E}+00$

$0.0000 \mathrm{E}+00$

$0.0000 E+00$

$0.0000 \mathrm{E}+\infty 0$

$0.0000 E+\infty 0$

$0.0000 E+\infty 0$

$0.0000 E+\infty 0$

1.0041E-02

$5.0000 E-04$

$0.0000 \mathrm{E}+00$

$0.0000 E+\infty 0$

$0.0000 E+\infty 0$

$0.0000 \mathrm{E}+00$

$0.0000 \mathrm{E}+00$

$0.0000 E+00$

$0.0000 \mathrm{E}+00$

$0.0000 E+\infty$

$0.0000 \mathrm{E}+00$

0000 .

$0.0000 E+00$

$0.0000 E+00$
$5.6570 E-03$

$0.0000 E+\infty 0$

$0.0000 E+00$

$0.0000 E+00$

$0.0000 E+00$

$0.0000 \mathrm{E}+00$

$0.0000 \mathrm{E}+00$

$0.0000 \mathrm{E}+00$

$0.0000 \mathrm{E}+00$

$0.0000 E+00$

$0.0000 \mathrm{E}+00$

$0.0000 E+\infty$

$0.0000 E+00$

$0.0000 \mathrm{E}+00$

$0.0000 \mathrm{E}+00$

$1.4100 \mathrm{E}-04$

$2.1200 \mathrm{E}-04$

$0.0000 E+00$
$14100 E-04$

$0.0000 E+00$

$1.0000 \mathrm{E}-04$

$0.0000 E+\infty$

$0.0000 \mathrm{E}+\infty 0$

$0.0000 E+00$

$0.0000 E+00$

$0.0000 E+00$

$0.0000 E+\infty 0$

$0.0000 \mathrm{E}+00$

$0.0000 \mathrm{E}+00$

$5.3900 \mathrm{E}-05$

$1.4100 \mathrm{E} .04$

$0.0000 E+00$

$13200 \mathrm{E}-\mathrm{DS}$

$0.0000 \mathrm{E}+\infty 0$

$0.0000 E+00$

$0.0000 \mathrm{E}+00$

$0.0000 E+00$

$0.0000 \mathrm{E}+00$

$0.0000 E+\infty 0$
MINIMUIM WT, KG

MAXIMUM WT, KG

(3000 EO

$4.0000 \mathrm{E}-03$

1.0000E-02

1.0000E-02

$20000 \mathrm{E}-02$

2.0500E-02

2.0000E-02

$2.0000 \mathrm{E}-02$

2.0000E-02

$20000 \mathrm{E}-02$

$20000 E-02$

$2.0000 \mathrm{E}-02$

$2.0000 \mathrm{E}-02$

2.0000 E- 02

2.0000E-02

2.0000 E. 02

20000 E.02

1.8000E-02

1.9000E-02

$1.0000 \mathrm{E}-02$

1. $.9000 \mathrm{E}-02$

1. $5900 \mathrm{E} .02$

8. $0000 \mathrm{E}-04$

$3.0000 E-03$

$1.3800 \mathrm{E}-02$

$1.3000 \mathrm{E}-02$

1.0000E-02

$1.0000 \mathrm{E} .02$

$10000 \mathrm{E}$

$1.0000 E-02$

$1.0000 \mathrm{E} .02$

1.0000E-02

$1,0000 E-02$

$1.0000 E-02$

1.0000E-02

9.0000E.03

$0.0000 E+00$

$5.0000 E-03$

5.0000 E-03

4.8000E- 03

4.8000 - 03

$4.0000 E-03$

3. $6000 \mathrm{E}-03$

3.3000E-03

3.0000E-03

3. $0000 \mathrm{E}-03$

3. $0000 \mathrm{E}-03$

$3.0000 \mathrm{E}-03$

3.0000 E- 03

2.5000E-03

2.5000E-03

1. $0000 \mathrm{E}-03$

9.0000E-04

2.0000E-03

B. O000E-OA

1. $5000 \mathrm{E}-03$

2.0000E-04

$1.4000 E-03$

1. $4000 \mathrm{E}-03$

1. $2000 \mathrm{E}-03$

1.1000E-03

$1.0000 E-03$

1.0000E-03

$1.0000 \mathrm{E}-03$

1.0000E-03

$0.0000 E+00$

1.0000E-04

3.0000 E- 04

D. $0000 \mathrm{E}+00$

1.0000E-04

1.0000 E-04

1.0000 E -04

$0.0000 E+00$

$0.0000 E+00$

O. $0000 E+C O$

2.5000E-02

2.3000E-02

B.COOOE- 03

$1.0000 \mathrm{E}-02$

$1.0000 E-02$

2.0000E-02

2.0000E-02

$2.0000 E-02$

$2.0000 E-02$

$2.0000 E-02$

$2.0000 E-02$

$2.0000 E-02$

2.0000E-02

2.0000E-02

2.0000 E- 02

2.0000 E- 02

$2.0000 E-02$

20000E-02

$1.8000 \mathrm{E}-02$

1.0000E-02

1.0000 E-02

1.6000E-02

1 5000E-02

1.5000E-02

4.0000E-03

$1.3800 \mathrm{E}-02$

1.3000E-02

$1.0000 E-02$

$1.0000 E-02$

$1.0000 \mathrm{E}-02$

1.0000E-02

$1.0000 \mathrm{E}-02$

1.0000E-02

$1.0000 E-02$

1.0000 E. 02

1.0000 E- 02

$8.0000 E-03$

$8.0000 E-03$

5.0000E-OS

5.00000 .03

4.8000 E- 03

4 6000E.03

4.0000E-03

3.6000E-03

3.3000E-03

$3.0000 E-03$

$30000-03$

$3.0000 \mathrm{E}-03$

$3.0000 E-03$

$3.0000 E-03$

$2.5000 \mathrm{E}-03$

$2.5000 \mathrm{E}-03$

$1.2000 \mathrm{E}-03$

$1.2000 \mathrm{E} .03$

$2.0000 \mathrm{E}-03$ 
WHC-SD-W-242-ES-004, Rev. 0

TABLE 5

STATISTICAL ANALYSIS OF HAZARDOUS MATERIALS

IN ASCENDING ORDER BASED ON ACCUMULATED WEIGHT

(* INDICATES DATA EXHIBITED NEGATIVE VARIANCE)

(10 SHEETS)

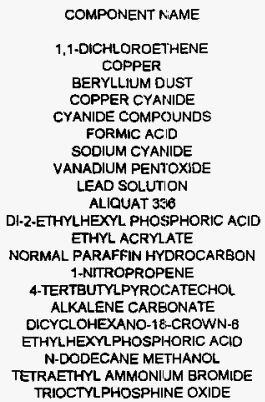

\begin{tabular}{|c|c|c|}
\hline COUNT & ACCUM WT, KG & AVERAGE WT KG \\
\hline 30 & $0.0000 E+00$ & $0.0000 E+00$ \\
\hline 17 & $0.0000 \mathrm{E}+00$ & $0.0000 E+\infty 0$ \\
\hline 8 & $0.0000 E+00$ & $0.0000 \mathrm{E}+00$ \\
\hline B & $0.0000 E+\infty 0$ & $0.0000 E+00$ \\
\hline 8 & $0.0000 E+00$ & $0.0000 \mathrm{E}+00$ \\
\hline 8 & $0.0000 E+00$ & $0.0000 E+\infty 0$ \\
\hline 8 & $0.0000 \mathrm{E}+00$ & $0.0000 \mathrm{E}+\infty$ \\
\hline 8 & $0.0000 E+\infty 0$ & $0.0000 E+00$ \\
\hline 7 & $0.0000 E+00$ & $0.0000 E+00$ \\
\hline 3 & $0.0000 E+00$ & $0.0000 E+00$ \\
\hline 2 & $0.0000 \mathrm{E}+00$ & $0.0000 E+\infty$ \\
\hline 2 & $0.0000 E+\infty 0$ & $0.0000 E+\infty 0$ \\
\hline 2 & $0.0000 E+\infty 0$ & $0.0000 E+00$ \\
\hline 1 & $0.0000 E+\infty$ & $0.0000 E+\infty$ \\
\hline 1 & $0.0000 E+00$ & $0.0000 E+00$ \\
\hline 1 & $0.0000 E+00$ & $0.0000 E+00$ \\
\hline 1 & $0.0000 E+00$ & $0.0000 \mathrm{E}+\infty 0$ \\
\hline 1 & $0.0000 E+00$ & $0.0000 E+00$ \\
\hline 1 & $0.0000 E+\infty$ & $0.0000 E+\infty 0$ \\
\hline 1 & $0.0000 E+00$ & $0.0000 E+00$ \\
\hline 1 & $0.0000 E+00$ & $0.0000 \mathrm{E}+00$ \\
\hline
\end{tabular}

STD DEV
$0.0000 E+\infty 0$
$0.0000 E+\infty 0$
$0.0000 E+00$
$0.0000 E+00$
$0.0000 E+00$
$0.0000 E+\infty$
$0.0000 E+\infty$
$0.0000 E+\infty 0$
$0.0000 E+00$
$0.0000 E+\infty 0$
$0.0000 E+\infty 0$
$0.0000 E+\infty 0$
$0.0000 E+\infty 0$
$0.0000 E+\infty 0$
$0.0000 E+\infty 0$
$0.0000 E+\infty 0$
$0.0000 E+\infty 0$
$0.0000 E+00$
$0.0000 E+\infty 0$
$0.0000 E+\infty 0$
$0.0000 E+\infty$

MINIMUM WT, KG

$0.0000 E+00$

$0.0000 E+\infty 0$

$0.0000 E+\infty 0$

$0.0000 E+00$

$0.0000 E+00$

$0.0000 E+00$

$0.0000 E+\infty 0$

$0.0000 E+00$

$0.0000 E+00$

$0.0000 E+\infty 0$

$0.0000 E+00$

$0.00005+00$

$0.0000 E+00$

$0.0000 E+\infty 0$

$0.0000 E+00$

$0.0000 E+\infty 0$

$0.0000 E+\infty 0$

$0.0000 E \div 00$

$0.0000 E+00$

$0.0000 E+00$

$0.0000 E+00$
MAXIMUM WT, KG

$0.0000 \mathrm{E}+\infty 0$

$0.0000 E+\infty$

$0.0000 E+\infty 0$

$0.0000 E+D 0$

$0.0000 \mathrm{E}+\infty 0$

$0.0000 E+\infty 0$

$0.0000 E+\infty$

$0.0000 E+\infty 0$

$0.0000 E+00$

$0.0000 \mathrm{E}+00$

$0.0000 E+\infty 0$

$0.0000 E+\infty$

$0.0000 E+\infty 0$

$0.0000 E+\infty 0$

$0.0000 E+\infty 0$

$0.0000 \mathrm{E}+\infty$

$0.0000 E+D 0$

$0.0000 E+\infty 0$

$0.0000=+\infty$

$0.0000 E+00$ 
TABLE 6

STATISTICAL ANALYSIS OF HAZARDOUS MATERLALS IN ASCENDING ORDER BASED ON MAXIMUM WEIGHT (* INDICATES DATA EXHIBITED NEGATIVE VARIANCE) (10 SHEET)

COMPONENT MAME

$$
\text { LEAD }
$$

HYOROCARBON TAR

LEAD SHIELDING

SODIUM CHLORIDE

BASE METAL (LEAD)

MERCURY

(1)

HYDROCHLORK; ACIO

TETRACHLOROETHYLENE

LEAD CHROMATE PAINT

LEAD ERICK

SODIUM HYDROXIDE

METHYLENE CHL.ORIDE

LECTRA CLEANER

POTASSIUM CHLORIDE ECOLTE

ALKYLBENZENE HEXANOL

1,2,4-TRIMETHYLBENZENE

LIQUID WASTE

SODIUM SILICATE

HYDROFLUORIC ACIO

LEAD CHROMATE

PHOSPHORIC ACID

1,1,1-TRICHLOROETHANE

ALUMINUM NITRATE TOLUENE

CHROMIUM TRIOXIDE

IRON(IB) CHLORIDE

WHITE POWOER CONTAINING SILOXANES

PSEUDOCUMENE

ETHYLENE GLYCOL

HYORAULIC FLUID

KEROSENE

DIESEL

DEBRIS

METHANOL

REMAINDER NON HAZARDOUS INGREDIENTS CARBON TETRACIHLORIDE

PAINT CONTAINING LEAD CHROMATE

CHLOROFLUOROMETHANE

HALOGENATED HYDFIOCARBONS

1,2,4-TRIMETHYL BENZENE FREON

BROMIDE MIXTURES

TRIMETHYL BEIVENE

TRISODIUM PHOISPHATE

MONOETHANOLAMINE

DIMETHYL SULFIOXIDE

METHYL ISOBUTY. KETONE INSTAGEL XF

PCB OIL CONTAINING PCE'S

REGULATED WATER NISCIBLE LIQIJID WISOLID SLUDGE RES

POLYOXYALKYLENE GLYCOL

POLYCHLORINATED BIPHENYLS

$$
\text { METAL }
$$

DI(2-ETHYLHEXYL) PHTHALATE

HYDROCARBONS

SCINTLLLATION LIQUID

STODDARD SOLYENT

POTASSIUM PERMANGANATE

$$
\text { XYLENE }
$$

DIMETHYLSULFOXIDE

DIMETHYLSUFOXIDE SiLVER

ALKYLNAPHTHALENE

MONOETHANOLAMHNE DIMETHYL SULFOXIDE

$1,1,2,2$-TETRABRON $A$ OTHANE

SODUUA NITRITE

RUTHENIUM CHLORIDE ETHANOL

TIERIES CONTAINING LEAD, LEAD OXIDES, AND SULFURIC A PSEUDOCUMENE-XYLENE ACETONE

POTASSIUM HYDROXIDE

co

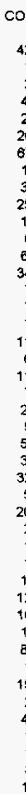

9.6972E+03

4. $3500 \mathrm{E}+02$

$2.9540 \mathrm{E}+02$

$5.3830 \mathrm{E}+03$

$5.0408 \mathrm{E}+02$

$7.0173 E+02$

$1.3004 E+03$

2.6105E+02

2.1603E +02

1.2318E+03

$3.3131 \mathrm{E}+02$

$3.1612 E+02$

7.1893E+02

$9.8554 \mathrm{E}+02$

$1.881 \mathrm{EE}+02$

1.2224E+O2

$3.8567 \mathrm{E}+02$

2.9407E+0

$+7590 \mathrm{E}+03$

$3.5112 E+02$

1. $3149 \mathrm{E}+02$

8. $0591 \mathrm{E}+01$

3. 2057E+02

$2.3646 \mathrm{E}+02$

4.3043E+02

5.2432E+01

1.0789E+03

$4.6006 E+01$

4. $5830 \mathrm{E}+0$ :

4. $2000 \mathrm{E}+01$

$2.4001 E+02$

4. $2935 \mathrm{E}+02$

$3.7601 E+03$

$3.4384 E+02$

$1.3744 E+03$

4. $0000 \mathrm{E}+01$

4. $4951 \mathrm{E}+02$

$7.4029 E+01$

$4.8930 E+01$

3. $6482 \mathrm{E}+01$

$3.6401 E+01$

$2.8780 \mathrm{E}+02$

3. $2072 \mathrm{E}+02$

$5.2000 \mathrm{E}+01$

3.4700E+01

6.6700E+01

$3.4100 E+01$

$3.0584 \mathrm{E}+02$

$1.1303 E+02$

$7.5529 \mathrm{E}+01$

$3.2909 E+01$

$1.9775 E+02$

$6.5030 \mathrm{E}+01$

$1.2000 \mathrm{E}+02$

$3.2720 \mathrm{E}+01$

$2.960 \mathrm{CE}+01$

$1.1565 \mathrm{E}+02$

$1.0007 \mathrm{E}+02$

$2.7580 \mathrm{E}+01$

$1.3168 \mathrm{E}+\mathrm{O} 2$

$8.8721 E+01$

$26265 \mathrm{E}+01$

$9.1173 \mathrm{E}+02$

1. $0649 E+02$

$4.5900 \mathrm{E}+01$

6.3271E+01

4. $8340 \mathrm{E}+01$

$3.3498 \mathrm{E}+01$

$22171 \mathrm{E}+01$

$2.7110 \mathrm{E}+01$

$2.0372 \mathrm{E}+01$

6.3077E+01

$2.0130 \mathrm{E}+01$

4.4540E+01

$9.7276 \mathrm{E}+0$

6. $2801 \mathrm{E}+01$
AVERAGE WT. KG

2.3089E+01

4.3500E +02

1. $4770 E+02$

$1.2817 \mathrm{E}+02$

1. $8003 E+01$

$3.0219 E+00$

$2.2078 \mathrm{E}+00$

$2.0081 E+01$

$5.8388 \mathrm{E}+\infty 0$

$5.3080 \mathrm{E}+00$

2.2087E+01

5.2688E+01

1.1780E+01

2.8464E +00

2.8880E+01

$1.7462 \mathrm{E}+01$

$5.5006 \mathrm{E}+01$

2.5134E+01

1. $0235 E+01$

$1.5705 \mathrm{E}+01$

$5.0160 \mathrm{E}+01$

$6.2612 \mathrm{E}+00$

8. $8545 E+00$

$5.5271 \mathrm{E}+\infty$

6. $3908 \mathrm{E}+C 0$

$1.3183 E+\infty$

$1.0486 \mathrm{E}+01$

$5.2886 E+00$

2. 3003E+01

4. $5830 \mathrm{E}+01$

4. $2000 \mathrm{E}+01$

1.2632E+01

$3.5779 E+00$

2.350tE+01

$2.6448 E+01$

1.6170E+01

4.0000E+01

$2.0573 E+\infty$

3.7014E+01

$1.1379 E+00$

$3.6462 E+01$

$3.6401 E+01$

$3.3475 E+O 1$

$2.0045 \mathrm{E}+01$

2.6000E+01

$3,4700 E+01$

$3,3350 \mathrm{E}+01$

$3.4100 E+01$

$1.6991 \mathrm{E}+01$

$2.2785 \mathrm{E}+01$

6.6253E-01

$3.2999 E+01$

$1.4125 E+01$

$2.1677 \mathrm{E}+01$

$3.0000 E+01$

$5.4533 E+\infty$

$2.0600 \mathrm{E}+01$

$38549 E+00$

$5.8865 E+00$

1.3790E+01

$1.6460 \mathrm{E}+01$

$4.5814 \mathrm{E}+00$

$6.5663 E+\infty 0$

$5.5256 E+\infty 0$

$2.1297 \mathrm{E}+01$

$2.2950 \mathrm{E}+01$

$1.6223 \mathrm{E}+00$

$1.6113 E+01$

$1.6749 E+0)$

$1.1085 \mathrm{E}+01$

$3.3888 \mathrm{E}+00$

$2.0372 \mathrm{E}+01$

$3.1538 \mathrm{E}+00$

$2.0130 \mathrm{E}+01$

$1.1135 \mathrm{E}+01$

4.1929E-01

$1.9625 \mathrm{E}+\infty$

STD DEV

9.0050E+01

$0.0000 E+\infty 0$

1.4467E+O2

7.2011E+01

$3.8768 E+01$

1.7446E+01

$9.7568 \mathrm{E}+00$

25260 + 01

1.5108E+Ot

1.3469E+01

2,2040E+01

1.7898E+01

2.097 EE+01

1.1305E+01

2.7778E+01

$2.8388 E+01$

2.4300E +01

f. 1360E +01

2.4762E+01

1.1348E+ 01

1. $2651 \mathrm{E}+01$

1. $0664 \mathrm{E}+01$

$1.8132 \mathrm{E}+01$

$1.2510 \mathrm{E}+01$

1. $0.495 E+01$

$5.4534 E+\infty 0$

2.1365E+O1

$9.6828 E+00$

3. $2523 E+01$

$0.0000 E+00$

$0.0000 \mathrm{E}+\infty 0$

$1.1226 \mathrm{E}+01$

$9.0760 E+00$

1.5495E+01

1. $6830 E+01$

1.3156E+01

$0.0000 E+\infty 0$

$5.3521 \mathrm{E}+\infty$

$2.1920 E+00$

$5.5655 E+\infty 0$

$0.0000 E+\infty$

$0.0000 E+\infty 0$

$6.3097 \mathrm{E}+00$

$1.2465 \mathrm{E}+01$

$1.2728 \mathrm{E}+\mathrm{O1}$

$0.0000 E+00$

$13435 E+\infty$

$0.0000 E+00$ 
TABLE 6

STATISTICAL ANALYSIS OF HAZARDOUS MATERLALS IN ASCENDING ORDER BASED ON MAXIMUM WEIGHT (* INDICATES DATA EXHIBITED NEGATIVE VARIANCE)

(10 SHEET)

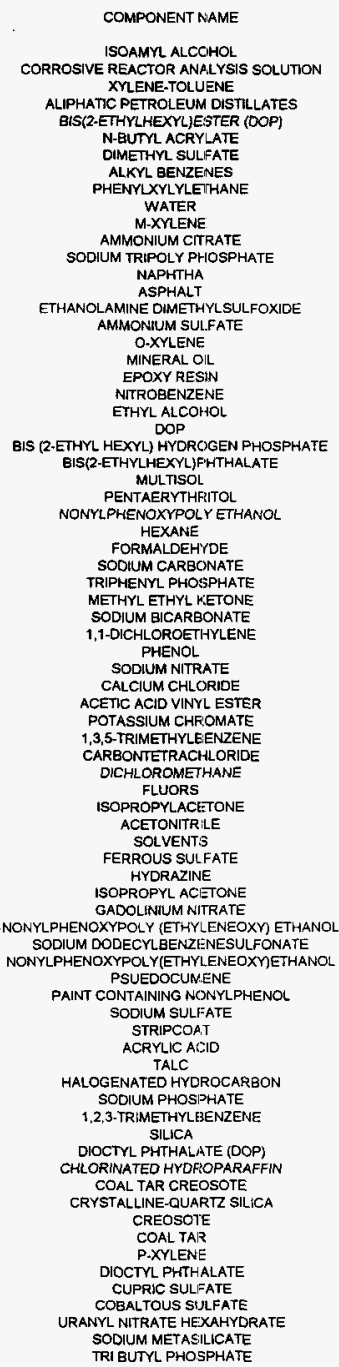

\begin{tabular}{|c|c|c|}
\hline COUNT & ACCUM WT, KG & AVERAGE WT. KG \\
\hline 4 & 4. $400 \mathrm{BE}+01$ & $1.1002 E+01$ \\
\hline 1 & $1.9241 E+01$ & $1.9241 E+01$ \\
\hline 10 & $1.9000 E+02$ & $1.9000 \mathrm{E}+01$ \\
\hline 1 & $1.8700 \mathrm{E}+01$ & $1.8700 \mathrm{E}+01$ \\
\hline 10 & $2.1890 E+01$ & $1.3550 E+\infty$ \\
\hline 1 & $1.8410 E+01$ & $1.8410 E+01$ \\
\hline 2 & $1.8380 \mathrm{E}+01$ & $9.1800 \mathrm{E}+\infty 0$ \\
\hline 2 & $3.4725 E+01$ & $1.7363 \mathrm{E}+01$ \\
\hline 117 & $9.4292 E+02$ & $8.0591 E+\infty$ \\
\hline 3 & $2.0580 E+01$ & $6.8601 E+\infty 0$ \\
\hline 30 & $1.7412 E+02$ & $5.8040 E+\infty 0$ \\
\hline 5 & $3.8831 E+01$ & $7.7862 E+00$ \\
\hline 1 & $1.6200 E+01$ & 1.6200E+01 \\
\hline 13 & $2.6106 \mathrm{E}+01$ & $2.0150 E+00$ \\
\hline 1 & $1.5990 E+01$ & $1.5990 E+01$ \\
\hline 1 & $1.5799 E+0 \dagger$ & $1.5799 \mathrm{E}+01$ \\
\hline 4 & $1.7670 E+0 \%$ & $4.4175 E+00$ \\
\hline 27 & $7.7100 E+0 ?$ & $2.8556 \mathrm{E}+00$ \\
\hline 11 & $6.4085 E+01$ & $5.8259 \mathrm{E}+00$ \\
\hline 10 & $2.3380 E+01$ & $2.3380 \mathrm{E}+00$ \\
\hline 20 & $1.5220 \mathrm{E}+01$ & $7.6102 E-01$ \\
\hline 13 & $9.5095 E+01$ & $7.3150 E+\infty 0$ \\
\hline 11 & $3.7040 E+01$ & $3.3673 E+00$ \\
\hline 2 & $1.4630 \mathrm{E}+01$ & $7.3150 E+\infty 0$ \\
\hline 1 & $1.4400 E+01$ & $1.4400 E+01$ \\
\hline 1 & 1.3499E+01 & $1.3499 \mathrm{E}+01$ \\
\hline 4 & $5.2000 \mathrm{E}+01$ & 1.3000E+01 \\
\hline 21 & S.8990E+01 & $4.7138 E+\infty 0$ \\
\hline 3 & $1.4645 \mathrm{E}+01$ & $4.8816 \mathrm{E}+00$ \\
\hline 99 & $1.7922 E+02$ & 1.8103E+OD \\
\hline 24 & $9.7828 E+0 r$ & $4.2534 E+00$ \\
\hline 37 & $4.2252 E+02$ & 1.1419E+01 \\
\hline 198 & $5.6861 E+01$ & $2.9728 \mathrm{E}-01$ \\
\hline 3 & $1.2051 E+01$ & $4.0170 E+\infty$ \\
\hline 32 & 4.2693E+01 & $1.3341 E+C 0$ \\
\hline 12 & $1.8870 E+01$ & $1.5725 E+\infty$ \\
\hline 19 & 6. $9593 E+01$ & $3.8628 E+00$ \\
\hline 4 & $2.0949 E+01$ & $5.2372 \mathrm{E}+00$ \\
\hline 2 & $1.1530 \mathrm{E}+01$ & $5.7650 \mathrm{E}+00$ \\
\hline 35 & $3.5130 E+02$ & $1.0332 E+01$ \\
\hline $\begin{array}{c}12 \\
5\end{array}$ & $\begin{array}{l}3.4610 E+01 \\
1.3858 E+01\end{array}$ & $\begin{array}{l}2.8842 E+00 \\
4.5527 E+00\end{array}$ \\
\hline 12 & $6.8060 \mathrm{E}+01$ & $5.6717 E+\infty 0$ \\
\hline 4 & $3.3240 E+01$ & $8.3100 E+00$ \\
\hline $\mathbf{8}$ & $2.2960 E+01$ & $2.8700 E+00$ \\
\hline 5 & 1.2855E+01 & $2.5711 E+\infty$ \\
\hline 1 & $8.8495 E+00$ & $8.8495 E+\infty$ \\
\hline 9 & $8.8170 E+\infty 0$ & $8.8170 \mathrm{E}+00$ \\
\hline 2 & $16100 E+01$ & $8.0500 E+00$ \\
\hline 4 & $1.5330 \mathrm{E}+01$ & $3.8325 E+00$ \\
\hline 1 & $8.2700 E+00$ & $8.2700 E+00$ \\
\hline 9 & $5.1040 E+01$ & $5.6711 \mathrm{E}+00$ \\
\hline 13 & $8.0855 E+01$ & $6.2196 \mathrm{E}+\infty$ \\
\hline 12 & $7.2790 \mathrm{E}+01$ & $6.0658 E+\infty$ \\
\hline 1 & $8.0013 E+00$ & $8.0013 E+00$ \\
\hline 1 & $8.0000 E+\infty 0$ & $8.0000 E+00$ \\
\hline 13 & $6.3527 E+01$ & $4.8887 \mathrm{E}+00$ \\
\hline 10 & $3.4090 \mathrm{E}+01$ & 4. $2613 E+\infty 0$ \\
\hline 1 & $7.4000 \mathrm{E}+00$ & $7.4000 \mathrm{E}+00$ \\
\hline 1 & $73000 E+00$ & $7.3000 E+\infty$ \\
\hline 55 & $3.0020 E+01$ & $5.4582 \mathrm{E}-01$ \\
\hline 2 & $7.2000 E+00$ & $7.2000 E+00$ \\
\hline 16 & $7.1030 E+01$ & 4.4394E +00 \\
\hline 2 & $0.9000 \mathrm{E}+00$ & $3.4500 E+\infty 0$ \\
\hline 22 & $4.9230 \mathrm{E}+01$ & $2.2377 E+00$ \\
\hline 4 & $2.3405 E+01$ & $5.8512 \mathrm{E}+00$ \\
\hline 4 & $1.0410 E+01$ & $2.6025 E+00$ \\
\hline 1 & $6.6700 \mathrm{E}+00$ & $6.6700 E+00$ \\
\hline 7 & $2.9780 E+01$ & $4.2543 E+\infty$ \\
\hline 2 & $8.7990 \mathrm{E}+00$ & 4.3995E+00 \\
\hline 20 & $2.4291 E+01$ & $1.2145 E+00$ \\
\hline 7 & $1.6830 E+01$ & $2.4043 E+00$ \\
\hline 2 & $8.3950 \mathrm{E}+00$ & $4.1975 E+\infty$ \\
\hline 1 & $8.2280 E+00$ & $6.2280 E+\infty$ \\
\hline 2 & $8.0100 E+00$ & $4.0050 \mathrm{E}+00$ \\
\hline 5 & 1.12229E+01 & $3.7431 E+00$ \\
\hline 7 & $2.3768 E+01$ & $3.9613 E+00$ \\
\hline
\end{tabular}

\begin{abstract}
STD DEV
\end{abstract}
$1.05+6 E+01$

$0.0000 E+00$

$0.0000 E+00$

$0.0000 \mathrm{E}+00$

$4.8200 E+\infty 0$

$0.0000 E+\infty 0$

1.2178E+01

5. $30335-02$

$5.8717 E+00$

$8.0165 E+00$

$6.8007 E+00$

7.8071E+00

$4.4205 E+00$

$0.0000 E+00$

$0.0000 \mathrm{E}+00$

$7.4630 \mathrm{E}+00$

$4.9325 \mathrm{E}+00$

$5.6532 \mathrm{E}+\infty$

$4.5612 E+\infty 0$

$3.3515 E+\infty 0$

$4.0173 E+00$

$4.6356 \mathrm{E}+00$

1.0161E +01

$0.0000 E+00$

$0.0000 E+00$

$0.0000 E+00$

$3.4629 E+D 0$

$6.3732 E+\infty$

$1.5786 E+00$

$3.2957 E+00$

1.7065E+00

1. $1416 \mathrm{E}+00$

B. $3092 E+\infty$

$3.2186 \mathrm{E}+00$

$3.0433 E+\infty 0$

$3.7048 E+00$

$5.5503 E+00$

$8.8983 E+\infty 0$

$4.0049 E+00$

6.8127E-01

2.2417E+00

7.0057E-01

$3.2253 E+\infty 0$

$3.7650 E+\infty 0$

$0.0000 E+00$

7.7782E-01

$3.5660 \mathrm{E}+00$

$0.0000 E+\infty 0$

$2.2801 \mathrm{E}+00$

$1.7699 \mathrm{E}+00$

$17259 \mathrm{E}+00$

$0.0000 \varepsilon+00$

$0.0000 E+00$

$1.5142 E+00$

$0000 E+00$

$0.0000 \mathrm{E}+00$

$1.6162 \mathrm{E}+00$

$2.4277 \mathrm{E}+\infty$

$4.8225 \mathrm{E}+\infty$

$1.7554 E+00$

1.0981E+00

$2.8687 \mathrm{E}+00$

$0.0000 E+00$

$2.3550 \mathrm{E}+00$

$3.0004 E+00$

2.4545E +00

$2.3461 E+\infty 0$

2.8B15E+00

$0.0000 E+\infty 0$

2.9768E+00

$1.1137 E+\infty$
MINIMUM WT, KG

1.0000E-02

$1.9241 E+01$

$1.9000 E+01$

1.8700E+01

2.0000E.01

1.8410E+01

5.8000E-01

$1.7325 E+01$

2.3000E-01

6.3540E-01

$0.0000 E+00$

1.0000E 02

$1.6200 E+0 t$

7.0000E-03

$1.5090 \mathrm{E}+01$

$1.5799 E+01$

4.4000E-01

$0.0000 E+00$

1.0000E-02

1. 2000 E- 01

7.3000E-03

$5.0000 \mathrm{E}-01$

7.3000E-01

1.3000E-01

1.4400E+01

$1.3490 \mathrm{E}+01$

$1.3000 E+01$

4.0000 E-02

$1.1100 E+\infty$

$0.0000 E+00$

8.0000E-04

1.3200E+00

0.0000 E+ 00

2.2000E-O1

$1.0000 E-04$

$1.00005-05$

3.8000E-02

4.5000E-01

$1.0300 E+00$

$8.4000 E+00$

$1.7000 E-01$

28000 E- 01

2.8090E+00

7. $4200 E+00$

2.5000E-01

4.0000E-03

$8.8170 E+00$

7. $5000 \mathrm{E}+00$

7.1000E-01

8. $2700 E+00$

1. $3200 \mathrm{E}+\infty$

$2.0384 E+C 0$

$1.8000 E+\infty 0$

$8.0013 E+00$

8.0000E +00

2.0384 E +00

$1.4100 E+00$

$14000=+00$

7. $3000 E+\infty 0$

4.3000E-03

7.2000E+00

4.8000E-01

4.0000E.02

3.0000 E -02

$4.4402 E+00$

$1.0000 \mathrm{E}-02$

6. $8700 \mathrm{E}+00$

$8.9900 \mathrm{E}-01$

2.2100E+ +0

$0.0000 \mathrm{E}+00$

B.8000E-01

2. 1600E+ +0

$6.2280 \mathrm{E}+\infty \mathrm{O}$

$1.0001 E+00$

$13000 \mathrm{E}-01$

6.5000E.02

MAXIMUM WT, KG

$1.9999 E+0$

1.9241E+0t

$1.8000 \mathrm{E}+0$

1.8700E+01

$1.2680 E+01$

1.8410E+01

$1.7800 E+01$

1.7400E+01

1.7300E+O1

1.7200E+01

$1.7000 \mathrm{E}+01$

1. $6530 \mathrm{E}+01$

$1.6200 E+01$

$1.6140 \mathrm{E}+01$

1. $5090 E+01$

$1.5790 \mathrm{E}+01$

1.5600 E+01

1.5300E+01

$1.5300 E+01$

$1.5000 \mathrm{E}+01$

1.5000E+01

$1.4601 \mathrm{E}+01$

1.4560E+01

1.4500E+01

$4400=+01$

1.3450E+01

1.3000E+01

1.2440E+01

1.2240E+01

1.1810E+01

1.1760=01

1.1700E+01

1.1420E+01

1.1300E+01

1.1190E+01

1. $1000 \mathrm{E}+01$

1.1000 E+01

1.1000E+01

$1.0500 \mathrm{E}+01$

$1.0400 \mathrm{E}+01$

$9.0000 E+00$

$9.4082 E+\infty 0$

$9.4619 E+00$

9. $1000 \mathrm{E}+00$

$9.0300 \mathrm{E}+00$

$9.0100 \mathrm{E}+00$

8. $8495 E+\infty 0$

$8.8170 E+00$

8. $6000 \mathrm{E}+00$

3. $4700 E+00$

8. $2700 \mathrm{E}+\infty$

8. $2400 \mathrm{E}+\infty 0$

$8.1283 \mathrm{E}+\infty$

$8.0300 E+00$

$8.0013 E+00$

8. $0000 \mathrm{E}+00$ 
WHC-SD-W-242-ES-004, Rev. 0

TABLE 6

STATISTICAL ANALYSIS OF HAZARDOUS MATERIALS IN ASCENDING ORDER BASED ON MAXIMUM WEIGHT (* INDICATES DATA EXHIBITED NEGATIVE VARIANCE)

(10 SHEET)

\begin{tabular}{|c|c|c|c|c|c|c|}
\hline COMPONENT NAME & COUNT & ACCUM WT, KG & AVERAGE WT. KG & STO DEV & MINIMUM WT, KG & MAXIMUM WT, KG \\
\hline MINERAL SPIFITS & 3 & $1.2343 E+01$ & $4.1143 E+00$ & $2.7814 E+00$ & $9.2000 E-01$ & $6.0000 E+00$ \\
\hline OCTYPHENOXYPOLYETHOXYETHANOL & 14 & $38087 E+01$ & $2.7205 E+00$ & $1.3633 E+00$ & $1.0002 E+00$ & $5.9738 E+00$ \\
\hline TRICHLOROETHYLENE & 111 & $1.1169 E+01$ & $1.3789 E-01$ & $5.6481 E-01$ & $0.0000 E+00$ & $5.8500 \Xi+00$ \\
\hline $\begin{array}{l}\text { EIS(2-ETHYLHEXYL) HYOROGEN PHOSPHATE } \\
\text { SODIUM CHROIMATE }\end{array}$ & $\frac{2}{2}$ & $\begin{array}{l}6.0000 E+00 \\
6.2500 E+00\end{array}$ & 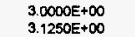 & $\begin{array}{l}4.0184 E+\infty \\
3.7890 E+\infty\end{array}$ & $\begin{array}{l}\text { 1.6000E-01 } \\
\text { 4.500OE-01 }\end{array}$ & $\begin{array}{l}5.8400 E+\infty \\
5.8000 E+00\end{array}$ \\
\hline SODIUM LAURYL SULFATE & 2 & $6.6813 E+\infty 0$ & $3.3307 E+\infty 0$ & $3,3083 E+C 0$ & $9.9130 \mathrm{E}-01$ & $5.8700 E+\infty 0$ \\
\hline COAL TAR PIJCH & 6 & $1.8520 E+01$ & $3.0867 E+00$ & $1.8582 E+\infty$ & $1.3800 E+\infty 0$ & $5.3600 E+\infty 0$ \\
\hline D-TAR & 5 & $2.6450 E+01$ & $5.2900 E+\infty 0$ & $0.0000 E+\infty 0$ & $5.2900 E+\infty$ & $5.2000 E+\infty 0$ \\
\hline TURCO & 5 & $2.6450 E+01$ & $5.2800 E+00$ & $0.0000 E+\infty$ & $5.2000 E+00$ & $5.2600 E+\infty$ \\
\hline SILICON DIOXIDE & 2 & $7.1767 E+\infty$ & $3.5884 E+00$ & $2.3663 E+00$ & $1.0151 \mathrm{E}+00$ & $5.2810 E+\infty 0$ \\
\hline & 6 & $1.2874 E+01$ & 2.1456E+00 & $2,0730 E+\infty 0$ & $1,0300 E-01$ & $5.1700 E+\infty 0$ \\
\hline $\begin{array}{l}\text { NAPTHALENE } \\
\text { LIGHT FIXTURES CONTAMINATED WITH FOLYCHLORINATEO BI }\end{array}$ & 24 & 3. $0537 E+01$ & $\begin{array}{l}\text { 1. } 6474 E+00 \\
5.0000 E+\infty\end{array}$ & $\begin{array}{l}1.8527 E+\infty 0 \\
0.0000 E+\infty 0\end{array}$ & $\begin{array}{r}1.0000 E-02 \\
5.0000 E+00\end{array}$ & $\begin{array}{l}5.0000 E+\infty \\
5.0000 E+\infty\end{array}$ \\
\hline $\begin{array}{l}\text { BARIUM NITRATE } \\
\text { BATHEOTINAIEU BI }\end{array}$ & 1 & $4.0320 E+00$ & $4.9320 \mathrm{E}+00$ & $0,0000 \mathrm{E}+\infty$ & $\begin{array}{l}5.0000 E+00 \\
4.9320 E+00\end{array}$ & $\begin{array}{l}5.0000 E+00 \\
4.9320 E+\infty\end{array}$ \\
\hline 1,1.2-TRICHLOROTRIFLUOROETHANE & 4 & $48200 E+00$ & $4.8200 E+00$ & $0.0000 E+\infty 0$ & $4.8200 E+00$ & $4.8200 E+\infty 0$ \\
\hline CHROMUUM & 94 & $9.2783 E+01$ & $1.0085 E+\infty$ & $1.5848 E+\infty$ & $0.0000=+00$ & $4.7000 E+\infty 0$ \\
\hline POTASSIUM CYANIDE & 10 & $47007 E+00$ & $4.7007 \mathrm{E}-01$ & $1.4882 E+\infty 0$ & $0.0000 E+00$ & $4.7000 E+00$ \\
\hline SOOIUM DIOCTYLSULFOCCINATE & 2 & $9.2800 E+00$ & $46300 E+00$ & $1.4142 E-02$ & $4.6200 E+00$ & $4.6400 E+\infty$ \\
\hline $\begin{array}{l}\text { CARCINOGENIC ORGANIC CHIEMICAL SUBSTANCE } \\
\text { POLYCYCEIC AROMATIC HIYDROCARBONS }\end{array}$ & $\begin{array}{l}1 \\
1\end{array}$ & $\begin{array}{l}4.5000 E+00 \\
4.5000 E+\infty 0\end{array}$ & $\begin{array}{l}4.5000 E+00 \\
4.5000 E+00\end{array}$ & $\begin{array}{l}0.0000 E+00 \\
0.0000 E+00\end{array}$ & $\begin{array}{l}4.5000 E+00 \\
4.5000 E+\infty 0\end{array}$ & $\begin{array}{l}\text { 4.5000E+00 } \\
4.5000 E+00\end{array}$ \\
\hline SOli & 1 & $45000 \mathrm{E}+00$ & $4.5000 E+00$ & $0.0000 E+00$ & $4.5000 E+00$ & $4.5000 \mathrm{E}+00$ \\
\hline CEROUS NITRATE & 1 & $44500 E+00$ & $4.4500 E+00$ & $0.0000 E+00$ & $4.4500 \mathrm{E}+00$ & $4.4500 E+00$ \\
\hline YTTRIUM NITR.ATE & 1 & $4.4500 E+\infty 0$ & $4.4500 E+00$ & $0.0000 E+00$ & $4.4500 E+\infty 0$ & $4.4500 E+00$ \\
\hline O-XYLENE & 4 & $54447 E+00$ & $1.3612 \mathrm{E}+00$ & $2,0543 E+00$ & B.0000E-04 & $4.3430 E+00$ \\
\hline 2-(2-BUTOXYETHOXY)-ETHANOL & 2 & $4.7700 E+\infty 0$ & $2.3850 \mathrm{E}+00$ & $2.7224 \mathrm{E}+00$ & $4.6000 E-01$ & $4.3100 E+00$ \\
\hline TRIBUTYL PHOSFHATES & 1 & $42300 E+00$ & $4.2300 E+00$ & $0.0000 E+00$ & $4.2300 E+00$ & $4.2300 E+00$ \\
\hline SULFAMIC ACID & 8 & $8.9048 E+00$ & $1.1131 E+00$ & $1.4593 E+\infty$ & 4.0800E-02 & $\$ 1000 E+\infty$ \\
\hline IRAN & 4 & $6,4272 \mathrm{E}+0 \mathrm{O}$ & $1.6068 \mathrm{E}+\infty$ & $1.9681 E+\infty$ & $3.5000 E .02$ & $4.1080 E+00$ \\
\hline BUTYL ACETATE & 8 & $7.6660 E+00$ & $1.0951 E+\infty 0$ & $1.3923 E+\infty 0$ & 7.0000E-03 & $4.1000 E+\infty$ \\
\hline THENOYTRIFLUOROACETONE & 9 & $6.8180 E+00$ & $9.7400 E-01$ & $1.1684 E+00$ & $5.0000 E-02$ & $4,1000 E+\infty$ \\
\hline VINYL CHLORIDE RESIN & 2 & $58960 E+00$ & $2.9480 E+00$ & $1.6235 E+00$ & $1.8000 E+\infty 0$ & $4.0000 E+00$ \\
\hline 2-BUTOXYETHANOL & 27 & $4.2385 E+01$ & $1.5698 E+D C$ & $15110 E+\infty 0$ & $0.0000 E+00$ & $4.0637 E+00$ \\
\hline INDENE & 4 & $1,0314 E+01$ & $2.5785 E+00$ & $1.6300 E+\infty$ & $1.0220 E+00$ & $4.0200 E+00$ \\
\hline CYCLOHEXANIONE & 16 & $80499 E+00$ & $5,0312 E-01$ & $9.7259 E-01$ & 1.0000E-02 & $4.0000 E+\infty$ \\
\hline ETHIOIUM BROMIDE & 1 & $3.9648 E+\infty 0$ & $3.9648 E+\infty 0$ & $0.0000 E+00$ & $3.9048 E+\infty 0$ & $3.8048 E+00$ \\
\hline ALUMINUM NITRATE MONOHYDRATE & 1 & $39000 E+\infty$ & $3.8000 E+00$ & $0,0000 E+00$ & $3.9000 E+\infty$ & $3.0000 E+\infty$ \\
\hline METALLIC MERCURY & 1 & $39000 E+00$ & $39000 E+00$ & $0.0000 E+00$ & $3.9000 \mathrm{E}+\infty 0$ & $3.0000 E+\infty 0$ \\
\hline MIEK & 6 & $3.8740 E+\infty$ & $6,4567 \mathrm{E}-01$ & $1.5796 \mathrm{E}+00$ & $0,0000 E+00$ & $3.9700 E+00$ \\
\hline CALCIUM NITRZATE & 3 & $3.8265 E+00$ & $1.2755 \mathrm{E}+00$ & $2.2036 E+00$ & $5.0000 E-04$ & $3.8200 E+00$ \\
\hline SILVER ZEOLITE & i & $3.7300=\div 00$ & $3.7300 E+\infty$ & $0.0000 E+\infty$ & $3.7300 E+00$ & $3.7300 \mathrm{E}+\infty 0$ \\
\hline POTASSIUM CARBONATE & 3 & $5.0759 E+00$ & $1.6920 E+\infty$ & $1.8607 E+\infty 0$ & 2.0000E-02 & $3.6090 E+\infty 0$ \\
\hline THINNEER & 3 & $8.6200 E+00$ & $28733 \mathrm{E}+\infty$ & B.3708E-01 & $1.0000 E+00$ & $3.6700 E+\infty 0$ \\
\hline METHYL ALCOHOL & 21 & $2.8558 E+01$ & $1.3599 E+00$ & $1,1891 E+\infty 0$ & 1.4000E-02 & $3.6301 E+\infty$ \\
\hline ISOPROPANOL & 15 & $26539 E+01$ & $1.7693 E+00$ & $1.1279 E+\infty 0$ & 6.5000E-02 & $3.6301 E+\infty 0$ \\
\hline RESIN & 2 & $4.2569 \Sigma+00$ & $2,1285 \mathrm{E}+00$ & $2.0312 E+\infty 0$ & 0.5680E-01 & $3.6001 E+00$ \\
\hline 1,1,2-TRICHLOROTRIFL.UROETHANE & 1 & $3.6000 E+00$ & $3.6000 E+\infty 0$ & $0.0000 E+\infty 0$ & $3.6000 E+\infty 0$ & $3.6000 \mathrm{E}+\infty 0$ \\
\hline MAGNESIUM HYIIROXIDE & 1 & $3.6000 E+\infty 0$ & $3.6000 E+\infty 0$ & $0.0000 E+\infty$ & $3.6000 E+00$ & $3.0000 E+\infty 0$ \\
\hline HEXYLENE GLYCOL & 3 & $35350 E+\infty$ & $3.5350 E+00$ & 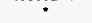 & $3.5350 E+\infty$ & $3.5350 E+00$ \\
\hline SODIUM FLUGIRIDE & 3 & $4.5400 E+\infty$ & $1.5133 E+00$ & $1.7620 E+00$ & $1.4000 E-01$ & $3.5000 E+\infty 0$ \\
\hline ETHOXYETHANOL & 3 & $4.1608 E+00$ & $1.3869 E+00$ & $1.8408 \mathrm{E}+\infty$ & $1.3100 E-01$ & $3.5000 E+\infty 0$ \\
\hline HEXONE & 13 & $8.6509 E+00$ & 7.2001E-01 & $8.4543 \mathrm{E}-01$ & $4.7000 E-02$ & $3.4200 \mathrm{E}+\infty$ \\
\hline SODIUM PHOSPHATE TRIBASIC & 1 & $3.3570 E+00$ & $3.3570 E+\infty$ & $0.0000 E+\infty$ & $3.3570 E+\infty$ & $3.3570 E+\infty$ \\
\hline $\begin{array}{l}\text { CRESOL } \\
\text { CROA }\end{array}$ & 38 & 8.2361E+00 & $2.1874 \mathrm{E}-01$ & $5.7172 E-01$ & 2.1000E-03 & $3.3300 E+\infty 0$ \\
\hline 1,2-DICHLCRETHYLENE & 5 & $1.2540 E+01$ & $25080 E+00$ & 5.4353E.01 & $1.0000 E+00$ & $32000 E+00$ \\
\hline NON-IONIC EMUISSIFIERS & 2 & $6.5600 E+\infty$ & $32800 E+00$ & $0.0000 E+00$ & $3.2800 E+00$ & 3.2800E+00 \\
\hline BIS-(2-ETHYLHEXYL)PHTHALATE & 3 & $6,0500 E+00$ & $20187 E+\infty 0$ & $1.0081 E+00$ & $1,4000 E+00$ & $3.1800 E+00$ \\
\hline ALIPHATIC PETROLEUM DISTILLATE & 8 & $939295+00$ & $1.1741 E+00$ & $1.1890 E+\infty$ & $1.3000 E-02$ & $3.0350 E+00$ \\
\hline ETHYLBENZIENE & 13 & $5.1658 E+00$ & $3.9737 E-01$ & B O389E-01 & $30000 E-04$ & $3.0000 E+\infty$ \\
\hline BORIC ACID & 5 & $49729 \mathrm{E}+00$ & $8.9458 \mathrm{E}-01$ & $1.1712 E+\infty 0$ & $1.7000 E-01$ & $3.0000 E+\infty 0$ \\
\hline SODA ASH & 1 & $30000 E+00$ & $3.0000 E+00$ & $0.0000 E+00$ & $3,0000 E+\infty$ & $3.0000 E+00$ \\
\hline TETRAHYDROFURAN & 67 & $6.1717 \mathrm{E}+00$ & $9.2115 E-02$ & 4.1547E-01 & $0.0000 E+\infty 0$ & $2.8800 E+00$ \\
\hline TRIBUTYLPHOSPHATE & 3 & $3.0040 E+\infty 0$ & $1.0013 E+00$ & $1.6584 E+00$ & 1.3000E-02 & $2,9160 E+\infty 0$ \\
\hline ACETYLENE TETRRBROMIDE & 1 & $28000 E+00$ & $2 \mathrm{BOOOE}+00$ & $0.0000 \mathrm{E}+00$ & $28000 E+00$ & $2.8000 E+00$ \\
\hline $\begin{array}{l}\text { STYRENE MOAOMER } \\
\text { STEN }\end{array}$ & 2 & $3.7503 \mathrm{E}+\infty 0$ & $1.8752 E+00$ & $1.2374 \mathrm{E}+\infty$ & $1.0002 E+00$ & $2,7501 \mathrm{E}+00$ \\
\hline VINYL ESTER IRESIN & 2 & $37503 \mathrm{E}+00$ & $1.8752 E+00$ & $1.2374 E+00$ & $1.0002 E+\infty$ & $2.7501 E+\infty$ \\
\hline CHLORINATED PARAFFIN & 27 & $1.5031 \mathrm{E}+01$ & $\$ .5669 E-01$ & 7.2056E-01 & $0.0000 E+\infty$ & $2.7300 E+00$ \\
\hline 2-PROPANONE & 4 & $4.7035 E+00$ & $1.1759 \mathrm{E}+00$ & $1.2878 E+00$ & $8.0000 E-02$ & $2.6300 E+\infty 0$ \\
\hline AMMONIA & 7 & $4.9231 E+\infty 0$ & $7.0330 \mathrm{E}-01$ & 8.6097E-01 & $2.4000 \mathrm{E}-03$ & $2.6100 E+00$ \\
\hline SODIUM & 2 & $2.9081 E+00$ & $1.4541 \mathrm{E}+00$ & $1.5642 E+00$ & $3.4800 \mathrm{E}-01$ & $2.5601 E+00$ \\
\hline EARIUM CHRCMATE & 2 & $3.3850 \mathrm{E}+00$ & $1.6925 \mathrm{E}+\infty 0$ & $1.2268 \mathrm{E}+\infty 0$ & 8.2500E-01 & $2.5600 E+00$ \\
\hline ACETIC ACID & 17 & $1.0284 E+01$ & 6.0492E-01 & $6.7207 E-01$ & $3.0000 E-03$ & $2.5451 E+\infty$ \\
\hline CHROME & 2 & $3.6000 E+00$ & $1.8000 E+00$ & $1.0182 E+00$ & $1,0800 E+\infty 0$ & $2.5200 \mathrm{E}+00$ \\
\hline SODIUM ALUNIINATE & 2 & $4.5000 E+00$ & $2.2500 E+00$ & 3.5355E-01 & $2,0000 E+00$ & $2.5000 E+\infty$ \\
\hline DIESEL FLEL & 2 & $3.8000 E+00$ & $1.9000 E+00$ & 8.4853E-01 & $1.3000=400$ & $2.5000 E+\infty 0$ \\
\hline 1,1,2-TR|CHLOROETHANE & 2 & $2.5001 \mathrm{E}+00$ & $1.2501 E+\infty$ & $1.7677 \mathrm{E}+00$ & $1.0000 E-04$ & $2.5000 E+\infty 0$ \\
\hline 1,1,2-TRICHLORO-1,2,2-TFIFLUOROETHANE & 10 & $1.5288 \mathrm{E}+01$ & $1.5268 \mathrm{E}+\infty$ & $1.0844 E+00$ & $1.3000 E-03$ & $2.4970 E+\infty$ \\
\hline METHYLTRIOCTYLAMMOINIUM CHLORIDE & 1 & $2.4700 E+\infty$ & $2.4700 E+00$ & $0.0000 E+00$ & $2.4700 E+00$ & $2.4700 E+00$ \\
\hline
\end{tabular}


TABLE 6

STATISTICAL ANALYSIS OF HAZARDOUS MATERIALS IN ASCENDING ORDER BASED ON MAXIMUM WEIGHT (* INDICATES DATA EXHIBITED NEGATIVE VARIANCE)

(10 SHEET)

\author{
COMPONENT MAME
}

CALCUM CHLOROFLUORIOPHOSPHATE

N.N DIMETHYL+FOFIMANIDE

1,2 BUTLYENE OXIDE

2 BUTOXYETHANOL

ETHYLENEGLYCOL-MONCBUTYL ETHER

METHYLIRICAPRYLYLAMMONIUM CHLORIOE SULFURIC ACID PYRIDINE

ISOPROPYL ALCOHOL

BISPHENOL AVPICHLOROHYDRIN RESIN

TERT-BUTYL METHIIL ETHER

2-ETHOXYETHANOL

AMMONEUM CHLORIOE

HYOROEROMIC ACID

1-ETHYL-2-METHYLIBENZENE

SODIUM DICHROMATE

DODECYLBENZENE SULFONIC ACID

ETHYLENEDIAMINETRA, ACIDIC ACID

POLYCHLORINATED BIPHENYLS (PCB)

LEAD CHROMATE MOLYBDATE N.HEPTANE

2.BUTOXY ETHANOL

ACETC ACIO VINYL POLYMER WITH CHLOROETHYLENE BARIUM OXIDE CESIUM IODIDE

LEAD CHROMATE, CHLORIN.PARAFFIN BARIUM CHLORIDE

BENZENESULFONIC ACID DOOECYL SODIUM SALT

KLEEN-O-BOWL
ACETIC ACID VINYL ESTER POLYMER WICHLOROETHYLENE CALCIUM FLUORIDE BAMBP
CITRIC ACID

XYLENE SULFONIC ACIO SODIUM SALT

CHLORINATED HYDROCARBON FORMAMIDE

DICHLOROTRIFLUROETHANE

METHYL ALCOHOL SOLUTION PCB BALLASTS

ETHYL BENZENE
GLYCOLS, POLYETHYLENE MONO (NONYLPHENYL) 2-HEPTANONE CHROMIC OXJDE ZINC CHROMATE DIETHYLENETRIAMINE 1,3-PROPANEDIAMINE

METHYLTRICAPRYLALAMMONIUM CHLORIDE PERCHLOROETHYLENE

POLYETHYLENE MONO(NONYLPHENYL) GLYCOLS

2,4,6-TRI(DIMETHYLAMINO|METHYL) PHENOL SODIUM HODIDE

1,1,2-TRICHLORO 1,2,2-TRIFLUORDETHANE AMMONIUM HYDROXIOE CYCLOHEXANE

HLORINATEO PARAFFIN WAXES AIND HYOROCARBON WAXE TRIMETHYLBEIJENE ETHANOLANIINE

SODIUM PYROPHIDSPHATE TRICHLOROETHENE

POTASSIUM FLUORIDE

DIQ-ETHYLHEXYLJFHTHATATE NONYLPHEINOL

DIETHYLENE GLYCOL MONOETHYLETHER

BIS (2-ETHYLEXYL) PHTHALATE AMMONIUM MOL.YBDATE

LEAD TETROXIDE$$
\text { BAFIUM }
$$

CADMHUA

CHROMIUM OXIDE

CHROMIUM SALTS

MAGNESIUM MET

$$
\text { BENZENE }
$$

2,5-DIPHENYLOXAZOLE

ETHYL ACETATE

EOETIC ACID

\begin{tabular}{|c|c|c|}
\hline COUNT & ACCUM WT, KG & AVERAGE WT. KG \\
\hline 14 & 4.2237E+00 & 3.0160E-01 \\
\hline 1 & $2.4200 E+\infty$ & $2.4200 E+00$ \\
\hline 4 & $2.6559 \mathrm{E}+\infty$ & $6.6398 E-01$ \\
\hline 4 & $3.3800 E+00$ & 8.4500E-01 \\
\hline 2 & $2.3700 \mathrm{E}+\infty$ & $2.3700 E+00$ \\
\hline 6 & $7.2820 E+\infty 0$ & $1.2137 E+00$ \\
\hline 19 & $1.0370 E+01$ & $5.4581 \mathrm{E}-01$ \\
\hline 5 & $2.9740 \mathrm{E}+00$ & $5.9480 \mathrm{E}-01$ \\
\hline 13 & $9.5684 E+\infty 0$ & 7.3603E.01 \\
\hline 3 & $2.4010 E+00$ & $20033 E-01$ \\
\hline 3 & $5.9800 E+00$ & $1.9933 \mathrm{E}+00$ \\
\hline 44 & $2.1729 E+01$ & 4. $9383 \mathrm{E}-01$ \\
\hline 9 & $7.4600 E+00$ & $8.2890 E-01$ \\
\hline 2 & $2.2425 E+D C$ & $1.1213 E+\infty 0$ \\
\hline 14 & $1.5530 \mathrm{E}+01$ & $1.1093 E+\infty$ \\
\hline 4 & 4.1051E+00 & $1.0263 E+\infty 0$ \\
\hline 3 & $2.9700 E+\infty 0$ & $9.9000 E-01$ \\
\hline 1 & $2,1000 E+\infty 0$ & $2.1000 E+00$ \\
\hline 1 & $20100 E+00$ & $2.0100 E+00$ \\
\hline 7 & $4.3230 E+00$ & 6.1757E-01 \\
\hline 6 & $4.0370 E+00$ & $6.7283 E-01$ \\
\hline 3 & $3.9820 E+00$ & $1.3273 E+\infty$ \\
\hline 1 & $2.0000 E+00$ & $2.0000 E+\infty 0$ \\
\hline 1 & $2.0000 E+\infty 0$ & $2.0000 E+\infty 0$ \\
\hline 1 & $2,0000 E+00$ & $2.0000 E+00$ \\
\hline 1 & $20000 E+00$ & $20000 E+00$ \\
\hline 5 & $8.0001 E+00$ & $1.6000 E+00$ \\
\hline 2 & $2.7980 E+00$ & $1.3980 E+\infty$ \\
\hline 1 & $1.9500 E+00$ & $1.9500 E+\infty$ \\
\hline 5 & $8.6500 E+\infty$ & $1.9300 E+\infty$ \\
\hline 3 & $22270 E+00$ & $7,4233 E-01$ \\
\hline 3 & $20100 E+00$ & $6.7000 \mathrm{E}-01$ \\
\hline 1 & $9.8860 \mathrm{E}+00$ & $1.8860 E+00$ \\
\hline 2 & $1.9900 E+00$ & $9.8500 \mathrm{E}-01$ \\
\hline 1 & 1.8400E+00 & $1.8400 E+\infty 0$ \\
\hline 7 & $2.0160 E+\infty$ & 2.8800E-01 \\
\hline 1 & $1.8000 E+\infty 0$ & $1.8000 E+00$ \\
\hline 1 & $1.7098 \mathrm{E}+\infty$ & $1.7098 E+00$ \\
\hline 1 & $1.7590 \mathrm{E}+00$ & $1.7599 E+00$ \\
\hline 1 & $17490 \mathrm{E}+00$ & $1.7480 E+00$ \\
\hline 40 & $2,3791 E+01$ & $5.0477 \mathrm{E}-01$ \\
\hline 1 & 1. $6800 E+00$ & $1.6800 E+C 0$ \\
\hline 3 & $1.9000 \mathrm{E}+00$ & 6.6333E-01 \\
\hline 12 & $5.2982 E+00$ & $4.4152 \mathrm{E}-01$ \\
\hline 8 & $4.9378 E+\infty 0$ & 6.1723E-01 \\
\hline 4 & $4.1400 E+00$ & $1,0350 E+00$ \\
\hline 1 & $15900 E+\infty 0$ & $1.5900 E+00$ \\
\hline 1 & $15900 \mathrm{E}+00$ & $1.5900 \mathrm{E}+00$ \\
\hline 44 & $26204 E+01$ & 5.955AE-01 \\
\hline 1 & $15500 \mathrm{E}+00$ & $1.5500 E+00$ \\
\hline 1 & $1.5200 E+00$ & $1.5200 E+\infty$ \\
\hline 3 & $1.6304 E+00$ & $5.4347 \mathrm{E}-09$ \\
\hline 1 & $1.5000 E+\infty$ & $1.5000 \mathrm{E}+00$ \\
\hline 8 & $3.7239 E+00$ & 4. $6549 E-01$ \\
\hline 8 & $4.2700 E+00$ & $5.3375 \mathrm{E}-01$ \\
\hline 2 & $1.6000 E+00$ & 8.000CE-01 \\
\hline 4 & $2.4342 E+00$ & 0.0855E-01 \\
\hline 1 & 1. $3900 E+00$ & 1. $3900 E+\infty 0$ \\
\hline 1 & 1. $3900 E+00$ & 1. $3900 E+00$ \\
\hline 12 & $1.1800 E+01$ & $0.8333 E-01$ \\
\hline 2 & $1,3800 E+00$ & 8.9000E-01 \\
\hline 1 & $1.3500 E+00$ & $1.3500 E+00$ \\
\hline 2 & $2.3370 E+\infty$ & 1.1685E+00 \\
\hline 1 & $1.3200 E+00$ & $1.3200 E+00$ \\
\hline 1 & $13100 E+00$ & $1.3100 E+00$ \\
\hline 2 & 1. $3600 E+00$ & $0.8000 \mathrm{E}-01$ \\
\hline 1 & $1.3000 E+\infty 0$ & $1,3000 E+00$ \\
\hline 191 & $1.3756 E+01$ & $7.3169 E-02$ \\
\hline 279 & $4.3095 E+\infty 0$ & $1.5558 \mathrm{E}-02$ \\
\hline 2 & 1. $3100 E+00$ & 8.5500E-01 \\
\hline 1 & $1.2500 E+\infty 0$ & $1.2500 E+\infty 0$ \\
\hline 1 & 1.2500E +00 & 1.2500E +00 \\
\hline 36 & $6.9418 E+\infty$ & 1.9283E-01 \\
\hline 44 & 1. $8810 E+01$ & $4.2750 \mathrm{E}-01$ \\
\hline 5 & $2.7769 E+00$ & $5.5538 \mathrm{E}-01$ \\
\hline 2 & $1.3659 \mathrm{E}+00$ & $6.8295 \mathrm{E}-01$ \\
\hline 9 & $1.1399 E+00$ & $1.1399 \epsilon+00$ \\
\hline
\end{tabular}

STD DEV

$0.0000 E+00$

$1.1591 E+00$

t. 1267E+00

9.3063E-01

$7.3020-01$

Q.7040E-01

8.1652E-OT

$1.2413 E+\infty$

3.5798E-01

5.2079E-O1

0.4502E-01

1.3934E+00

7.5543E-01

$1.1828 E+00$

$1.0116 E+00$

$0,0000 E+00$

$0.0000 E+00$

7.4934E-01

7.0661E-01

1. $0132 E+\infty 0$

$0.0000 E+00$

$0.0000 E+00$

$0.0000 E+00$

$0.0000 E+00$

5.4758E-01

7.6065E-01

$0.0000 E+\infty 0$

$1.0158 \mathrm{E}+00$

$1.0567 \mathrm{E}+00$

$0.0000 E+00$

$1.1950 E+\infty 0$

$0.0000 E+\infty 0$

Q.6740E-01

$0.0000 E+00$

$0.0000 E+\infty 0$

$0,0000 \mathrm{E}+\infty$

$0.0000 E+00$

5.8377E-01

$0.0000 E+00$

8. 4678E-01

\$.0196E-01

5.6178E-01

5. $4641 \mathrm{E}-01$

$0.0000 E+00$

D. $0000 \mathrm{E}+\mathrm{CO}$

4.5976E-01

$0.0000 E+00$

$0.0000 E+00$

$83084 E-01$

$0.0000 E+00$

5.1464E-01

4.3293E-01

Q. 1924E-01

$7.1071 E-01$

$0.0000 E+00$

$0.0000 E+D O$

$4.3567 \mathrm{E}-01$

9.3338E-01

$0.0000 E+00$

$23830 E-01$

$0.0000 \mathrm{E}+\infty 0$

$0.0000 E+\infty 0$

87681 E-01

$0.0000 \mathrm{E}+00$

1.9773E-01

$1.0011 E-01$

8. $8388 \mathrm{E}-01$

$0.0000 E+00$

$0.0000 E+00$

2.8315E-01

3 2711E-O1

4. 3090E-01

6. $9714 \mathrm{E}-01$

$0.0000 E+00$
MINIMUM WT, KG

3.0400E-02

$2.4200 \mathrm{E}+00$

3.0000E-02

$0.0000 E+00$

2.3700E+DO

9.0000E-02

$3.0000 E-04$

7.0000E-03

1. $1800 \mathrm{E}-02$

1.0000 E.02

$1.5800 \mathrm{E}+00$

1.0000E-04

1.0000E-04

1.3800 E.01

$27000 E-01$

1.8000E-03

1.2000E.01

2.1000E +00

2.0100E +00

6.0000E-03

1.8000 E-0 1

$1.6200 E-01$

$2.0000 E+00$

$2.0000 \mathrm{E}+\infty$

2.0000E +00

$2.0000 E+00$

$1.0002 E+00$

8. 4600 E-01

$1.9500 E+\infty 0$

1. $9300 E+\infty 0$

1.5000E-01

4.0000E-02

$1.8860 E+00$

1.5000E-01

$1.8400 \mathrm{E}+00$

$6.0000 E .03$

1.8000E+00

$1,7008 E+00$

$1,7509 E+00$

$1.7490 E+00$

$0.0000 \mathrm{E}+00$

$1,6800 \mathrm{E}+00$

$1.0000 \mathrm{E}-02$

$1.0000 \mathrm{E}-02$

1.0000E-02

1. OOOOE 01

$1.5000 E+00$

$1.5800 E+00$

9.0000 E-03

$1.5500 E+00$

$1.5200 E+\infty 0$

1. $4000 \mathrm{E}-03$

$1.5000 \mathrm{E}+00$

4. $4000 \mathrm{E}-03$

7.0000 E-02

1.5000E-01

20000 E.03

$1.3900 E+00$

$1.3900 \mathrm{E}+00$

4.0000E- 02

$3.0000 E-02$

$1.3500 E+C 0$

1 .0000E $+\infty$

$1.3200 E+00$

$1.3100 E+\infty 0$

6.0000E- 02

$1.3000 E+\infty 0$

2.0000E-04

$0.0000 E+00$

3.0000E-02

1. $2500 \mathrm{E}+00$

$1.2500 E * 00$

$0.0000 E+00$

$2.0000 E-02$

2.0000E-02

$1.9000 \mathrm{E}-01$

$1.1390 E+\infty 0$

MAXIMUM WT, KG

$243005+00$

2. $4200 E+00$

$2.3090 \mathrm{E}+00$

2.3600E +00

2.3700E+00

$23800 \mathrm{E}+00$

$23000=+\infty$

2.3000E+00

$2.2627 E+\infty$

$2.2310 \mathrm{E}+\infty$

2. $2000 \mathrm{E}+00$

$2.1200 E+\infty$

$2.1200 E+\infty$

2.1005E+00

2. $1000 \mathrm{E}+00$

2.1000E+00

2. $1000 E+\infty 0$

$2.1000 E+00$

$2.0100 E+00$

$2.0000 \mathrm{E} \div 00$

$2.0000 \mathrm{E}+00$

$2.0000 E+00$

$2.0000 E+\infty$

20000 +

2.0000E+00

$2.0000 \mathrm{E}+00$

$1.9990 E+00$

$1.9500 \mathrm{E}+00$

$1.0500 E+\infty$

$1.9300 E+\infty 0$

1. $9150 E+\infty 0$

$1.8000=+00$

$1.8880 \mathrm{E}+00$

1.8400E+ $+\infty$

$1.2400 E+\infty 0$

1. $8000 E+\infty 0$

$1.8000 \mathrm{E}+00$

$1.7998 \mathrm{BE}+00$

$1.7590 \mathrm{E}+00$

$1.7490 \mathrm{E}+\infty 0$

$1.7000 E+\infty 0$

1. $8800 E+\infty 0$

1. $6200 \mathrm{E}+00$

$1.6000 \mathrm{E}+00$

$1.5098 E+00$

$+5900 \mathrm{E}+00$

$1.5900 \mathrm{E}+\infty 0$

$1.5900 \mathrm{E}+00$

$1.5599 \mathrm{E}+00$

$1.5500 \mathrm{E}+\infty$ 
TABLE 6

STATISTICAL ANALYSIS OF HAZARDOUS MATERIALS IN ASCENDING ORDER BASED ON MAXIMUM WEIGHT (* INDICATES DA'TA EXHIBITED NEGATIVE VARIANCE)

(10 SHEET)

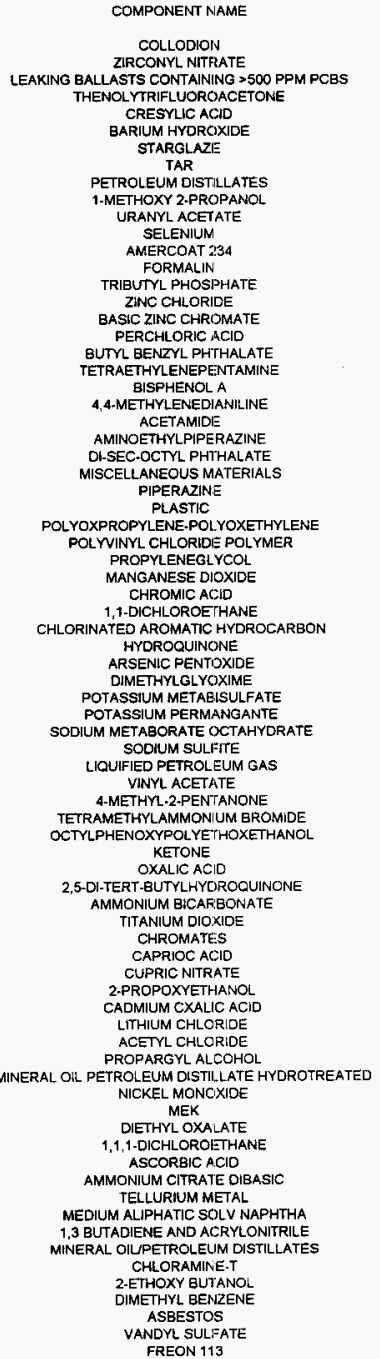

\begin{tabular}{|c|c|}
\hline COUNT & ACCUM WT, KG \\
\hline 1 & 1.1200E +00 \\
\hline 2 & $1.7080 E+\infty 0$ \\
\hline 1 & 1. $1000 \mathrm{E}+00$ \\
\hline 1 & $1.1000 \mathrm{E}+00$ \\
\hline 97 & $7.8020 E+00$ \\
\hline 2 & $1.5000 \mathrm{E}+00$ \\
\hline 1 & $1.0201 E+\infty$ \\
\hline 1 & $1.0201 E+00$ \\
\hline 3 & $1.0400 E+00$ \\
\hline 1 & $1.0100 E+00$ \\
\hline 2 & $1.2012 E+00$ \\
\hline 127 & $2.4504 E+\infty 0$ \\
\hline 2 & $20004 E+00$ \\
\hline 5 & $5.0000 E+00$ \\
\hline 7 & $2.8730 E+C 0$ \\
\hline 6 & $1.7151 E+\infty$ \\
\hline 3 & $1.4184 E+\infty 0$ \\
\hline 5 & $1.2326 \mathrm{E}+\infty 0$ \\
\hline 2 & $1.0500 E+00$ \\
\hline 2 & $1.0374 E+\infty 0$ \\
\hline 2 & $1.0300 E+\infty 0$ \\
\hline 1 & $1.0000 E+00$ \\
\hline 1 & $1.0000 E+00$ \\
\hline : & $1.0000 E+00$ \\
\hline 1 & 1.0000E+00 \\
\hline 1 & $1.0000 E+00$ \\
\hline 1 & 1.0000E +00 \\
\hline 1 & $1.0000 E+\infty$ \\
\hline 1 & 1. $0000 E+00$ \\
\hline 1 & 1.0000E +00 \\
\hline 1 & $1.0000 \mathrm{E}+00$ \\
\hline 1 & 9 5570E-01 \\
\hline 5 & $1.5510 \mathrm{E}+00$ \\
\hline 3 & $1.8700 E+00$ \\
\hline 7 & $1.0410 E+00$ \\
\hline 2 & 1.3910E+DO \\
\hline 1 & $9.0000 \mathrm{E}-01$ \\
\hline 1 & $9.0000 E-01$ \\
\hline 1 & 9.0000 E-01 \\
\hline 1 & $9.0000 \mathrm{E}-01$ \\
\hline 1 & 8.0000 E- 01 \\
\hline 1 & $9.0000 E-01$ \\
\hline 1 & 8. $9990 E-01$ \\
\hline 1 & 8 9990E-01 \\
\hline 37 & $4.4433 E+00$ \\
\hline 1 & B $6000 E-01$ \\
\hline 1 & 8. 1000E-01 \\
\hline 6 & $3.6001 E+00$ \\
\hline 7 & $1.9580 E+00$ \\
\hline 1 & 7.7800E-01 \\
\hline 1 & 7.7400E-01 \\
\hline 2 & 9.4290E 01 \\
\hline 2 & 7.4000E-01 \\
\hline 1 & 7.2000E-01 \\
\hline 1 & 7.1010E-01 \\
\hline 1 & $7.1000 \mathrm{E}-01$ \\
\hline 1 & 7.0000E-01 \\
\hline 2 & $6.9550 E-01$ \\
\hline 1 & 6.9000E-01 \\
\hline 1 & 6.9000E-01 \\
\hline 1 & 6.6000E-01 \\
\hline 9 & 6.6000E-01 \\
\hline 9 & $1.6892 E+\infty$ \\
\hline 1 & 6.1000E-01 \\
\hline 3 & $1.5000 E+\infty 0$ \\
\hline 3 & 9.4130E-01 \\
\hline 1 & 6.0000 E-01 \\
\hline 1 & 8.0000E-01 \\
\hline 1 & 5.9690E-01 \\
\hline 1 & $5.8000 \mathrm{E}-01$ \\
\hline 1 & $5.7000 \mathrm{E}-01$ \\
\hline 1 & $5.5000 \mathrm{E}-01$ \\
\hline 7 & $1.7402 E+00$ \\
\hline 1 & $5.4020 \mathrm{E}-01$ \\
\hline 5 & $1.7899 E+\infty 0$ \\
\hline 1 & $5.4000 \mathrm{E}-01$ \\
\hline & \\
\hline
\end{tabular}

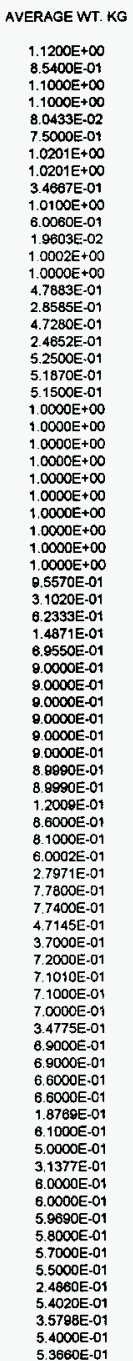

STD DEV

$0.0000 E+00$

$3.4790 \mathrm{E}-01$

$0.0000 E+00$

$0.0000 E+\infty 0$

1.4672E-Q1

4. 2426E-01

$0.0000 E+00$

$0.0000 E+00$

5.8315E-01

$0.0000 E+00$

5.6653E.01

1.2481E-01

$0.0000 E+00$

$0.0000 E+00$

3.7572E-01

3.5398E-01

4.8083E-01

4.2271E-01

6. $9066 \mathrm{E}-01$

6. $8580 E-01$
$0.0000 E+00$

$0.0000 E+00$

$0.0000 \mathrm{E}+00$

$0.0000 E+00$

$0.0000 E+\infty 0$

$0.0000 E+00$

$0.0000 E+00$

$0.0000 \mathrm{E}+00$

$0.0000 \mathrm{E}+00$

$0.0000 E+00$

$0.0000 E+00$

3.8107E-01

3.8837E-01

3.3616E-01

2.8921E-01

$0.0000 \mathrm{E}+00$

$0.0000 E+00$

$0.0000 E+\infty$

$0,0000 \mathrm{E}+00$

$0.0000 E+00$

$0.0000 E+\infty 0$

$0.0000 E+00$

$0.0000 E+00$

1.5422E-01

$0.0000 E+00$

$0.0000 E+00$

2.097 EE 01

2.8712E-01

$0.0000 E+00$

0. $0000 \mathrm{E}+00$

$3.8530 \mathrm{E}-01$

$5.0812 \mathrm{E}-01$

D. $0000 E+O 0$

$0.0000 E+00$

$0.0000 E+00$

$0.0000 E+00$

4.8826E-01

$0.0000 \mathrm{E}+00$

$0.0000 E+\infty$

$0.0000 E+\infty 0$

$0.0000 E+00$

2.6910E-01

$0.0000 \mathrm{E}+00$

8.6803E-02

$2.6946 \mathrm{E} .01$

$0.0000 \mathrm{E}+00$

$0.0000 E+00$

$0.0000 \mathrm{E}+00$

$0.0000 E+00$

$0.0000 E+00$

$0.0000 E+\infty 0$

$1.2858 \mathrm{E}-01$

$0.0000 \mathrm{E}+00$

$1.5209 E-01$

$0.0000 \mathrm{E}+00$

$0.0000 E+\infty 0$
MINIIMUM WT

$1.1200 \mathrm{E}+0$

$6.0800 E-01$

$1.1000 \mathrm{E}+\infty$

$1.1000 E+\infty 0$

$1.0000 E-04$

4.5000E-01

$1.0201 \mathrm{E}+\infty$

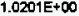

$5.0000 \mathrm{E}-03$

$1.0100 \mathrm{E}+\infty$

2.0000E-01

$0.0000 E+\infty 0$

$1.0002 E+00$

$1.0000 E+00$

1.7000E-02

$7.8000 E-02$

5.8400E-02

2.0000E-03

$5,0000-02$

3.7400E-02

3.0000E-02

$1.0000 E+00$

$1.0000 E+00$

1 OOCOE + 00

$1.0000=+00$

$1.0000 E+\infty$

$1.0000 E+00$

1.0000E+OO

$1.0000 E+\infty 0$

$1,0000 E+00$

$1.0000 E+00$

9.5570E-01

5.0000E-02

1.0000E-01

2.0000E-02

4.9100E-O1

$9.0000 \mathrm{E}-01$

9.0000 E-01

8.0000E-01

8.0000E-01

Q.0000E-01

8.0000E-01

8.0900E-01

8.9900E-01

$0.0000 E+\infty 0$

8. $\$ 000$ E 01

8.1000E-OI

2.0000E-01

2.5000E-02

7.7BOOE-01

7.7400E-01

1.9900E-01

1.0000E-02

7 2000E-01

7.1010E-01

7. 1000E-01

$7.0000 E-01$

2.5000E-03

6.0000E.01

6.8000 E. 01

$6.6000 \mathrm{E}-01$

6. 6000E-01

$0.0000 E+00$

6.1000 E. 01

4.5000E-01

6.5000E.02

6.0000E-01

6.0000E-01

5.8600E-01

5.8000E-01

5.7000E-01

5.5000E-01

2.0000E-01

5.4020E-01

2.0000E-01

5.4000E-01

5.3860E-01
MAXIMUM WT. KG

$1.1200 E+\infty 0$

1.1000E $+\infty 0$

$1.1000 E+\infty 0$

1.1000E+D

$1.0800 E+\infty 0$

$1.0500 \mathrm{E}+00$

$1.0201 E+\infty$

$1.0201 E+\infty 0$

$1.0200 E+\infty 0$

$1.0100 E+\infty$

1.C012E+ +00

$1.0002 E+00$

$1.0002 E+00$

$1.0000 E+00$

1. $0000 \mathrm{E} \cdot 00$

$1.0000 \mathrm{E}+00$

$1.0000 E+00$

$1.0000 E+00$

$1.0000 E+00$

1.0000E +00

$4.0000 E+00$

$1.0000 E+00$

$1.0000 E+00$

$1.0000 E+\infty 0$

$1.0000 \mathrm{E}+00$

$1.0000 E+00$

$1.0000 E+\infty 0$

$1.0000 E+00$

$1.0000 E+00$

$1.0000 E+\infty 0$

Q.5570E-01

9.5200E-01

9.4000E-01

0.1100E-01

$9.0000 \mathrm{E}-01$

9.0000 E-01

9.0000 E. 01

9.0000 E 01

Q.0000E-01

Q 0000E-01

9.0000 E-01

8. 9980E.01

8. 9090E-01

8. $7000 \mathrm{E}-01$

8.6000E-01

8.1000E-01

8.0010E-01

8.0000E-01

7.7800E-01

7.7400E-01

7. 4380E-0

7.3000E-01

7.2000E-01

7. 1010E-01

7.1000E-01

70000 E-01

6.8300 르-01

6.8000E-01

$6.9000 E-01$

6.6000E-01

6.6000E-01

8.4000E-01

6.1000E-01

B.0000E-01

6.0000 E-01

6.0000E-01

6.0000E-D1

5.9090E-01

5.8000E-01

$5.7000 \mathrm{E}-01$

$5.5000 \mathrm{E}-01$

.4020E-01

5.4020E-01

.4000E-01

5.4000E-01

$5.3860 \mathrm{E}-01$ 
TABLE 6

STATISTICAL ANALYSIS OF HAZARDOUS MATERIALS IN ASCENDING ORDER BASED ON MAXIMUM WEIGHT (* INDICATES DATA EXHIBITED NEGATIVE VARIANCE) (10 SHEET)

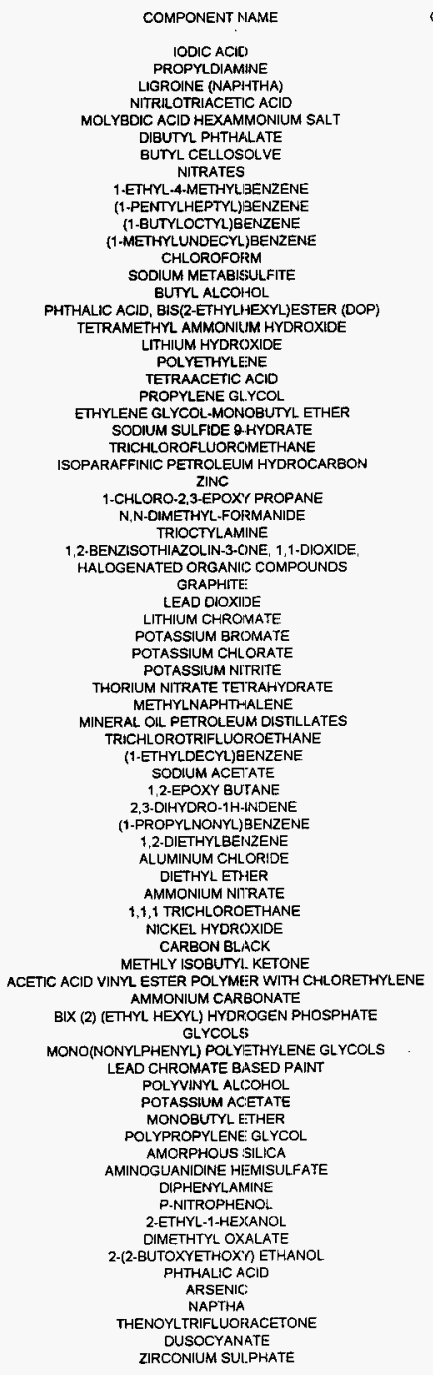

\begin{tabular}{|c|c|c|}
\hline COUNT & ACCUM WT, KG & AVERAGE $W T$ KG \\
\hline 1 & $5.3100 E-01$ & $5.3100 \mathrm{E}-0.1$ \\
\hline 1 & 5.2980E.01 & 5.2880E-01 \\
\hline 3 & 6.6800E-01 & 2.2200E-01 \\
\hline 2 & 5.3300E-01 & 2.6650E-01 \\
\hline 1 & $5.2000 E-01$ & 5.2000E-01 \\
\hline 3 & $9.1880 \mathrm{E}-01$ & $3.0860 \mathrm{E}-01$ \\
\hline 2 & $9.2880 \mathrm{E}-01$ & 4.6490E-01 \\
\hline 15 & $7.5000 \mathrm{E}+C 0$ & 5.0000 E-01 \\
\hline 14 & $3.6960 E+\infty 0$ & 2.6400E-01 \\
\hline 4 & $1.9700 \mathrm{E}+\infty$ & 4.9250E-01 \\
\hline 4 & $1.8800 \mathrm{E}+\infty 0$ & 4.7000E-01 \\
\hline 4 & $1.8800 \mathrm{E}+00$ & 4. TOCOE.01 \\
\hline 39 & $1,3575 E+00$ & 3. $4808 \mathrm{E}-02$ \\
\hline 2 & $97500 E-01$ & 4.8750E-01 \\
\hline 4 & Q . 0000E-01 & 2.2725E-01 \\
\hline 3 & 6.7500E-01 & 2.2500E-01 \\
\hline 2 & 5.2000E-01 & 2.6000E-01 \\
\hline 2 & 5.0010E-01 & $2.5005 E-01$ \\
\hline 1 & $5.0000 E-01$ & 5.0000E-01 \\
\hline 1 & $5.0000 E-01$ & $5.0000 \mathrm{E}-01$ \\
\hline 6 & $2.4999 \mathrm{E}+\infty 0$ & 4.1685E-01 \\
\hline 2 & $5.0090 E .01$ & 2.5495E.01 \\
\hline 1 & 4.9080E-01 & 4.9090E-01 \\
\hline 2 & $8.7800 E .01$ & 4. $8900 \mathrm{E} .01$ \\
\hline 1 & $4.9290 \mathrm{E}-01$ & 4.8290E-01 \\
\hline 19 & 5 3720E-04 & $2.8274 E-02$ \\
\hline 1 & 4.8000E-01 & 4.8000E-01 \\
\hline$i$ & 4. 8000E-01 & 4. $8000 \mathrm{E}-01$ \\
\hline 1 & 4.8000E-01 & $48000 E-01$ \\
\hline 1 & 4.8000E-01 & 4.6000E.01 \\
\hline 78 & $7.0023 \mathrm{E}+00$ & 9.3364E-02 \\
\hline 1 & 4. 5000E-01 & 4.5000E-01 \\
\hline 1 & $45000 E-01$ & 4. $50000-01$ \\
\hline$i$ & 4. 5000E-01 & 4.5000E-0t \\
\hline 1 & $45000 \mathrm{E}-\mathrm{C1}$ & 4.5000E-01 \\
\hline 1 & 4 50DOE-01 & 4. 5000E-01 \\
\hline 1 & 4. 5000E-01 & 4.5000E.01 \\
\hline 1 & 4. 5000E-01 & 4.5000E-01 \\
\hline 1 & 4.4000E.01 & 4.4000E-01 \\
\hline 1 & 4.4000E-01 & 4.4000E-01 \\
\hline 1 & $4.4000 E-01$ & 4. $4000 E-01$ \\
\hline 4 & $1.69000 \mathrm{E}+00$ & 4.2250E-01 \\
\hline 3 & 8.0100E-01 & $2.0033 E-01$ \\
\hline 2 & 4. $1090 \mathrm{E}-01$ & 2.0545E.01 \\
\hline$s$ & $1.6300 \mathrm{E}+\infty 0$ & $1.8 \div 11 E-01$ \\
\hline 4 & $14500 E+00$ & $3.6250 E-01$ \\
\hline 2 & 8.0000E-01 & 4.0000E-01 \\
\hline 4 & 4.0000E-01 & 4.0000E-01 \\
\hline$i$ & $3.9710 \mathrm{E}-01$ & $3.9710 \mathrm{E}-01$ \\
\hline 1 & 3.9600E-01 & $3.8600 \mathrm{E}-01$ \\
\hline 62 & $6.8513 E+\infty$ & $1.1050 \mathrm{E}-01$ \\
\hline 20 & $5.5500 \mathrm{E}-01$ & $2.7750 \mathrm{E}-02$ \\
\hline 4 & $6.6500 \mathrm{E}-01$ & $1.6625 \mathrm{E}-01$ \\
\hline 5 & $1.5800 \mathrm{E}+00$ & $3.1600 E-01$ \\
\hline 1 & $3.7000 E-01$ & $3.7000 \mathrm{E}-01$ \\
\hline 1 & 3.6000E-01 & 3.6000E-01 \\
\hline 1 & $3.6000 E-01$ & $3.6000 \mathrm{E}-01$ \\
\hline 1 & $3.5000 \mathrm{E}-01$ & $3.5000 E-01$ \\
\hline 1 & $3.5000 \mathrm{E}-01$ & 3.5000E-01 \\
\hline 2 & 3.4500E-01 & $1.7250 E-01$ \\
\hline 1 & 3.4000E-01 & $3.4000 \mathrm{E}-01$ \\
\hline 1 & 3.4000E-01 & $3.4000 \mathrm{E}-01$ \\
\hline 1 & 3. $3000 E-01$ & 3.3000E-01 \\
\hline 1 & $3.3000 \mathrm{E}-01$ & 3.3000E.01 \\
\hline 1 & 3.2500E-01 & 3.2500E-01 \\
\hline 1 & $32400 \mathrm{E}-01$ & $3.2400 \mathrm{E}-01$ \\
\hline 1 & $3.2400 E-04$ & $32400 E-01$ \\
\hline 1 & $3.2400 \mathrm{E} \cdot 01$ & $3.2400 \mathrm{E}-01$ \\
\hline 2 & 3. $4000 E-01$ & $17000 \mathrm{E}-01$ \\
\hline 1 & 3.2000E-01 & 3.2000E-01 \\
\hline 1 & 3 1500E-01 & 3.1500E-01 \\
\hline 20 & $4.8681 \mathrm{E}+00$ & 2.4341E-01 \\
\hline 61 & $8.9710 \mathrm{E}-01$ & 1.4707E-02 \\
\hline 3 & $4.4000 E \cdot 01$ & 1.4687E-01 \\
\hline 2 & 3.3500E-01 & 1. $6750 E .01$ \\
\hline 1 & $30000 \mathrm{E}-01$ & $3.0000 \mathrm{E}-01$ \\
\hline 1 & 2.9900E-01 & 2.9900E-01 \\
\hline
\end{tabular}

STDDEV

$0.0000 \mathrm{E}+\infty 0$

$0.0000 E+\infty 0$

$2.7145 E .01$

3. $6275 \mathrm{E}-01$

$0.0000 \mathrm{E}+00$

6.3488E-02

$0.0000 \mathrm{E}+00$

1.8088E-01

$5.0000 E-03$

2.0000E-02

$2.0000 \mathrm{E}-02$

1.1157E-01

2.2486E-01

2.3828E-01

$3.3941 \mathrm{E} .01$

$3.5348 E-01$

$0.0000 E+00$

$0.0000 E+\infty 0$

4.0784E-02

$0.0000 E+00$

9.8980E-03

$0.0000 E+00$

1.1280E-01

$0.0000 E+\infty 0$

$0.0000 E+00$

$0.0000 E+\infty 0$

4.1183E-02

$00000 \mathrm{E}+00$

$0.0000 \mathrm{E}+00$

$0.0000 E+00$

$0.0000 \mathrm{E}+\infty 0$

$0.0000 E+00$

$0.0000 E+00$

$0.0000 E+00$

$0.0000 \mathrm{E}+00$

$0.0000 \mathrm{E}+00$

1.5000 E. 02

1.8685E-01

2.8928E-01

1.0706E-01

2.5000E-02

$0.0000 \mathrm{E}+00$

$0.0000 \mathrm{E}+\infty$

$0.0000 E+\infty 0$

$0.0000 \mathrm{E}+00$

$1.1051 \mathrm{E}-01$

$0.1458 E-02$

1.5834E-01

4.2190E-02

$0.0000 \mathrm{E}+00$

$0.0000 E+\infty 0$

$0.0000 \mathrm{E}+00$

$0.0000 \mathrm{E}+00$

$0.0000 \mathrm{E}+00$

2.3688E-01

$0.0000 \mathrm{E}+00$

$0.0000 \mathrm{E}+00$

$0.0000 \mathrm{E}+\infty 0$

$0.0000 \mathrm{E}+00$

$0.0000 \mathrm{E}+00$

$0.0000 E+\infty 0$

$0.0000 \mathrm{E}+\infty$

2.1213E-01

$0.0000 \mathrm{E}+00$

$0.0000 \mathrm{E}+00$

1. 1658E-01

4.1019E-02

1.4189E-01

1. $8738 E-01$

$0.0000 \mathrm{E}+00$

0. $0000 \mathrm{E}+00$
MINIMUM WT, KG

5.3100E-01

5.20BOE-01

1.0000E-03

1.0000E-02

5.2000E-01

1.4000E-01

4.2000E-01

5.0000E-01

1. $4000 \mathrm{E}-02$

$4.9000 \mathrm{E}-01$

4.6000E-01

4.8000E-01

1.0000E-04

4.7500E-01

$1.0000 \mathrm{E}-02$

8.0000E-02

10000 OS

5.0000E-01

5.0000E-01

4.0000E-01

$1.0000 E-02$

4.9000E-01

4.8200E-01

$4.0200 \mathrm{E}-01$

$0.0000 E+00$

4. 80000 - 01

4.8000E-01

4.8000E-01

4.6000E-01

5.0000 E. 02

4.5000 E. 01

4.5000E-01

4.5000E-01

4.5000E-01

4.5000E-01

$4.5000 E-01$

4.5000E-01

4. 4000E-01

4.4000E. 01

4.4000E- 01

$4.0000 \mathrm{E}-01$

2.0000E-02

9.0000E.04

3.0000 E 02

3. 5000E.01

$4.0000 E-01$

4. $0000 \mathrm{E}-01$

3.8710E-01

3. 9600 E- 01

1.0000 E- 04

$0.0000 E+00$

5. $0000 \mathrm{E}-03$

2.7000E-01

3.7000E-91

$3.6000 E-01$

3. $8000 \mathrm{E}-01$

3.5000E-01

3.5000E-01

5. $0000 \mathrm{E}-03$

3. $4000 E-01$

3.4000E-01

3.3000E-01

3.3000E-01

3.2500E-01

3.2400E- 01

3.240OE 01

3.2400E-01

$2.0000 \mathrm{E}-02$

3. $2000 \mathrm{E}-01$

3.1500E-01

1.2000E-03

0 COOOE +00

$2.0000 \mathrm{E}-02$

$3.5000 \mathrm{E}-02$

3.0000E-01

2.0500E-01
MAXIMUM WT, KG

5.3100E-0t

5.2080E-01

5.2500E-01

5.2300E-01

5.2000E-01

$5.0080 E-01$

5000

5.0000E-01

5.0000E-01

5.0000E-01

5.0000E-01

5.0000E-01

5.0000E-01

5.0000E-01

5.0000E-01

5.0000E-01

$5.0000=01$

5.0000E-01

5.0000E-01

4.9090E-01

$4.8000 \mathrm{E}-01$

4.900 EE-01

4.0800E-01

4.9200 E-01

4.9120E-01

4.8000E-01

4. 8000 E-01

4.8000 E 01

4. $0000 E-01$

4.5360E-01

4. 5000E-01

4. 5000E-01

4. 5000E-01

4 5000E-01

4.5000E-01

4.5000E-01

4.5000E-01

4. $4000 E-01$

4.40005

4. 4000든 01

4.3000E-01

4. 1000 E-01

4. 1000E-01

4.0000E-01

4.0000E-01

4.0000E-01

4.0000E 01

3.9710E-01

3.9600 E- 01

3.9000E-01

3.8000E-01

3.7500E-01

3.7000E-01

$3.7000 E-01$

3.6000E-01

3.6000E-OI

3.5000E-D1

3.5000E-01

3.4000E-01

3.4000E-01

3.4000E-01

3.3000E-01

3.3000E-01

3.2500E-01

3. 2400E 01

3.2400E-01

$32400 E-01$

3. 2000 E- 01

3.2000E-01

3. 1500E-01

$3.0000 \mathrm{E}-01$

$3.0000 E-01$

3.0000E.01

3.0000E-01

30000 E-01

2.9000E.01 
TABLE 6

STATISTICAL ANALYSIS OF HAZARDOUS MATERIALS IN ASCENDING ORDER BASED ON MAXIMUM WEIGHT (* INDICATES DATA EXHIBITED NEGATIVE VARIANCE) (10 SHEET)

\begin{tabular}{|c|c|c|c|c|c|c|}
\hline COMPONENT NAME & COUNT & ACCUM WT, KG & AVERAGE WT. KG & STD OEV & MINIMUM WT, KG & MAXIMUM WT, KG \\
\hline ALUMINUM SILICATE & 1 & 2.9850E-01 & $2.8850 \mathrm{E}-01$ & $0.0000 E+00$ & 2. $2850 E-01$ & $2.0950 E-01$ \\
\hline SODIUM NITROFERRICYANIDE & 1 & $2.9500 \mathrm{E}-01$ & $2.9500 \mathrm{E}-01$ & $0.0000 E+00$ & 2.8500E-01 & 2.0500E-01 \\
\hline CHROMIUM (II) NITRATE NONAHYDRATE & 1 & $2.8000 \mathrm{E}-01$ & $29000 E-01$ & $0.0000 E+00$ & 2.0000E-01 & 2.8000E-01 \\
\hline 2-(METHOXYETHOXY) ETHANOL & 1 & $2.8600 \mathrm{E}-01$ & $2.8800 E-01$ & $0.0000 E+\infty 0$ & $2.8600 E-01$ & $2.8600 E-01$ \\
\hline $\begin{array}{l}\text { PETROLEUM DISTILLATE } \\
\text { 2-ETHANEDIAMINE N-(2-AMINOETHYL)-N'-2.AMINOETHYL)AMI }\end{array}$ & 2 & $3.0730 \mathrm{E}-01$ & $1.5365 E-01$ & $1.8661 E-01$ & 2.1700E-02 & 2.8580E-01 \\
\hline 2-ETHANEDIAMINE, N-(2-AMINOETHYL)-N'-2-AMINOETHYL)AMI & 1 & 2.8000E-01 & $2.6000 E-01$ & $0.0000 E+\infty 0$ & $2.8000 E-01$ & 2.8000E-01 \\
\hline CHLORINATED PARAFFIN WAX & 1 & 2.7000E-01 & $2.7000 E-01$ & $0.0000 \mathrm{E}+\infty 0$ & $2,7000 E-01$ & $2.7000 E-01$ \\
\hline FURFURYL-ALCOHOL & 1 & $2.6500 E-04$ & $2.6500 E-01$ & $0.0000 E+00$ & 2.6500E-01 & $2.6500 E-01$ \\
\hline PROPYLENE GLYCOL MONOMETHYL ETHER & 1 & $2.6500 E-01$ & $2.6500 \mathrm{E}-01$ & $0.0000 E+\infty 0$ & $2.6500 \mathrm{E}-01$ & $2.6500 E-01$ \\
\hline CARBON DIOXIDE & 24 & $2.3506 \mathrm{E}+00$ & 9.7950E-02 & 8.7088E.02 & $8.0000 E-04$ & 2.8000E-01 \\
\hline 2-BUTOXETHANOL & 1 & $2.6000 E-01$ & $2.6000 \mathrm{E}-01$ & $0.0000 E+00$ & $2.6000 E-01$ & 2.6000E-0t \\
\hline TRIISOOCTYLAMINE & 2 & $4.8600 \mathrm{E}-01$ & $2.4300 \mathrm{E}-01$ & $1.8395 E-02$ & 2.3000E-01 & $2.5800 E-01$ \\
\hline METHLY ETHYL KETONE & 1 & $2.5080 \mathrm{E}-01$ & $2.5080 E-01$ & $0.0000 E+00$ & 2.5080E-01 & $2.5080 E-01$ \\
\hline ACID & 1 & $2.5000 \mathrm{E}-01$ & $2.5000 \mathrm{E}-01$ & $0.0000 E+00$ & 2.5000E-01 & $2.5000 E-01$ \\
\hline GALLIC ACID & 1 & $2.5000 E-01$ & $2.5000 \mathrm{E}-01$ & $0.0000 E+00$ & 2.5000E-01 & 2.5000E-01 \\
\hline SOLVENT PETROLEUM DISTLLLATE & 1 & $2.5000 E-01$ & $2.5000 E-01$ & $0.0000 \mathrm{E}+00$ & $2.5000 E-01$ & $2.5000 E-01$ \\
\hline PYDRAUUFYRGUEL (HYORAULIC FLUID) & 3 & $2.6880 E-01$ & B.9000E-02 & 1.3804E-01 & 9.8000E-03 & $2.4900 E-01$ \\
\hline METHYL-1-PHENYL-2-PYRAZOLIN & 1 & $2.4800 E-01$ & $2.4600 E-01$ & $0.0000 E+00$ & 2.4600E-01 & 2.4000E-01 \\
\hline ACETIC ACID SODIUM SALT & 1 & $2.4000 E-01$ & 2.4000E-01 & $0.0000 E+\infty$ & $2.4000 E-01$ & $2,4000 E-01$ \\
\hline AMMONIUM PERSULFATE & 1 & $2.4000 E-01$ & $2.4000 E-01$ & $0.0000 E+\infty 0$ & 2.4000E-01 & 2.4000E-01 \\
\hline AZOBENZENE & 1 & $2.4000 E-01$ & $2.4000 \mathrm{E}-01$ & $0.00005+00$ & $2.4000 E-01$ & $2.4000 \mathrm{E}-01$ \\
\hline TRIETHYLENERETRAMINE & 1 & $2.2500 \mathrm{E} \cdot 01$ & $2.2500 \mathrm{E}-01$ & $0.0000 E+00$ & 2.2500E-01 & 2.2500E-01 \\
\hline LEAD TELLURIDE & 1 & $2.2000 \mathrm{E}-01$ & $2.2000 \mathrm{E}-01$ & $0.0000 E+00$ & 2.2000E-01 & $2.2000 E-01$ \\
\hline POTASSIUM PERIODATE & 1 & $2.2000 E-01$ & 2.2000E-01 & $0.0000 E+00$ & $2.2000 E-01$ & $2.2000 E-01$ \\
\hline LIGROINE & 6 & $6.5000 \mathrm{E}-01$ & $1.0833 E-01$ & 8.2308E-02 & $5.8000 E-03$ & 2.1000E-01 \\
\hline CADMIUM HYDROXIDE & 1 & 20500E-01 & 2.0500E-01 & $0.0000 E+\infty$ & 2.0500E-01 & $2.0500 E-01$ \\
\hline PHTHALIC ACID EIS(2-ETHYLHEXYL)ESTER & 25 & $1.2879 E+00$ & $5.1516 E-02$ & 6.9293E-02 & 8.0000E-04 & 2.0000E-01 \\
\hline NAPHTHALENE & 7 & 7.0400E-01 & $1.0057 E .01$ & $8.1090 E-02$ & $1.0000 E-02$ & 2.0000E-01 \\
\hline CALCIUM HYDROXIDE & 3 & $53000 E-01$ & 1.7667E-01 & 2.0817E-02 & $1.8000 E-01$ & $2.0000 E-01$ \\
\hline 1,3-DIETHYLBENIZENE & 2 & $4.0000 E-01$ & $2.0000 \mathrm{E}-01$ & $0.0000 E+00$ & $2.0000 E-01$ & 2.0000E-01 \\
\hline TRICHLOROETHANE & 2 & $2.8400 \mathrm{E}-01$ & $1,4200 \mathrm{E}-01$ & 8.2024E-02 & $8.4000 E-02$ & $2.0000 E .01$ \\
\hline CUMENE HYDROXIDE & 1 & $2.0000 E-01$ & $2.0000 E-01$ & $0.0000 E+00$ & $2.0000 E-01$ & $2.0000 \mathrm{E}-01$ \\
\hline ETHANOL DIBENTYL MERCURY & 1 & $2.0000 E-01$ & $2.0000 E-01$ & $0.0000 E+00$ & $2.0000 E-01$ & $2.0000 E-01$ \\
\hline ETHYL MERCURIC CHLORIDE & 1 & $20000 E-01$ & $20000 E-01$ & $0.0000 E+00$ & $2.0000 E-01$ & $2.0000 E-01$ \\
\hline MERCURIC HYDROXIOE & 1 & $2.0000 E-01$ & $2.0000 E-01$ & $0,0000 E+00$ & $2.0000 E-01$ & 2.0000E-01 \\
\hline HYOROTREATEO HEAVY NAPHTHA & 1 & 1.9900E-01 & $1.9900 \mathrm{E}-01$ & $0.0000 E+00$ & $1.8900 E-01$ & $1.8000 \mathrm{E}-01$ \\
\hline 2-BIPHENYLOL SOOIUM SALT & 1 & $1.9000 E-01$ & $1.9000 E-01$ & $0.0000 E+00$ & $1.9000 \mathrm{E}-01$ & $1.9000 E-01$ \\
\hline PETROLEUM OIL & 2 & 3.0900E-01 & $1.5450 E-01$ & 4.5962E-02 & $1.2200 E .01$ & 1.8700E-01 \\
\hline NAPHTHENIC ACID LEAD SALT & 1 & $1.8000 E-01$ & $1.8000 \mathrm{E}-01$ & $0.0000 E+\infty$ & $1.8000 \mathrm{E}-01$ & $1.8000 E-01$ \\
\hline ARSENIC JRIOXIDE & 2 & 3.4100E-01 & $1.7050 E-01$ & $1.2021 E-02$ & $1.6200 E-01$ & 1.7000E-01 \\
\hline STYRENE & 2 & 1.7710E-01 & $8.8550 E-02$ & 1. $2500 \mathrm{E}-01$ & $1.0000 E-04$ & 1.7700 E-01 \\
\hline N,N,N'N-TETRAMETHYL-1,3-BUTANEDIAMINE & $t$ & $+.7500 \mathrm{E}-01$ & $1.7500 E-01$ & $0.0000 E+00$ & $1.7500 \mathrm{E}-01$ & $1.7500 \mathrm{E}-01$ \\
\hline ETHYLENEDIAMINETETRAACETC ACID DISODIUM SALT (EDTA) & 1 & $1.7100 E-01$ & $1.7100 E-01$ & $0.0000 E+00$ & $1.7100 E-01$ & 1.7100E-01 \\
\hline STANNOUS SULFATE & 1 & $17100 E-01$ & $1.7100 E-01$ & $0.0000 E+00$ & 1.7100E-01 & $1.7100 E-01$ \\
\hline BIS(2 ETHYLHEXYL) HYDROGEN PHOSPHATE & 1 & 1.7000E-01 & $1,7000 \mathrm{E}-01$ & $0.0000 E+00$ & $1.7000 \mathrm{E}-01$ & $1.7000 E-01$ \\
\hline NAPTHENIC DISTILLATE & 1 & 1.7000E-01 & $1.7000 \mathrm{E}-01$ & $0.0000 E+\infty$ & 1.7000E-01 & 1.7000E-01 \\
\hline (PHENYL)-N,N-DHSOBUTYL-CARBAMOYLMETHYLPMOSPHINE & 1 & 1.6400E-01 & 1.6400E-01 & $0.0000 E+00$ & $1.8400 E-01$ & $1.8400 E-01$ \\
\hline BENZIN-(NAPHTHA) & $\uparrow$ & $1.6200 \mathrm{E}-01$ & 1.6200E-01 & $0.0000 E+00$ & $1.6200 E-01$ & 1.8200E-01 \\
\hline NITROCELLULLOSE & 1 & $1.6200 \mathrm{E}-01$ & 1.6200E-01 & $0.0000 E+00$ & $1.6200 \mathrm{E}-01$ & $1.8200 E-01$ \\
\hline PROPIONIC ACID & 2 & 2.8500E-01 & 1.4250 E- 01 & $2.4749 \mathrm{E}-02$ & $1.2500 E-01$ & $1.8000 E-01$ \\
\hline UNDECANE & 1 & $1.6000 E-01$ & $1.6000 \mathrm{E}-01$ & $0.0000 E+00$ & $1.6000 \mathrm{E}-01$ & $1.6000 E-01$ \\
\hline 2 HEPTANONE & 1 & $1.5500 \mathrm{E}-01$ & $1.5500 \mathrm{E}-01$ & $0.0000 E+\infty$ & $1.5500 E-01$ & $1.5500 E-01$ \\
\hline BUTOXYETHANOL & 2 & $1.6000 \mathrm{E}-01$ & $8.0000 E-02$ & $9.8095 \mathrm{E}-02$ & $1.0000 E .02$ & t.5000E-01 \\
\hline BUTOXYETHOXY ETHANOL & 1 & $1.5000 E-01$ & $1.5000 \mathrm{E}-01$ & $0.0000 E+\infty 0$ & $1.5000 \mathrm{E}-04$ & $1.5000 E-0 t$ \\
\hline DI-N-BUTVL PHTHALATE & 1 & $1.4200 E-01$ & $1.4200 E-01$ & $0.0000 E+00$ & $1.4200 E-01$ & $1.4200 E-01$ \\
\hline 1,1,1 TRICHLOROEHTANE & 1 & $1.4000 E-01$ & $1.4000 E-01$ & $0.0000 E+00$ & 1.4000E-01 & $1.4000 E-01$ \\
\hline BATTERIES CONTAINING POTASSIUM HYDROXIDE, NICKELL & 1 & $1.4000 E-01$ & $1.4000 \mathrm{E}-01$ & $0.0000 E+00$ & 1.4000E-01 & $1.4000 E-01$ \\
\hline BENZENEARSENIC ACID & 1 & $1.2600 \mathrm{E}-01$ & $1.2600 \mathrm{E}-01$ & $0.0000 E+\infty 0$ & $1.2600 \mathrm{E}-01$ & $1.2000 E-01$ \\
\hline POTASSIUM DICHROMATE & 1 & $1.2600 \mathrm{E}-01$ & $1.2000 \mathrm{E}-01$ & $0.0000 E+\infty 0$ & $1.2600 E-01$ & $1.2600 E-01$ \\
\hline DITHIOXAMIDE & 1 & $1.2200 \mathrm{E}-01$ & $1.2200 \mathrm{E}-01$ & $0.0000 E+00$ & $1.2200 E-01$ & $1.2200 E-01$ \\
\hline FERROCENE & 1 & $1.2200 \mathrm{E}-\mathrm{O} 1$ & $1.2200 \mathrm{E}-01$ & $0.0000 E+00$ & $1.2200 E-01$ & 1.2200E-01 \\
\hline ZINC SALT & 1 & $1.2200 \mathrm{E}-01$ & $1.2200 E-01$ & $0.0000 E+00$ & $1.2200 \mathrm{E}-01$ & 1.2200E-01 \\
\hline POLYCHLORINATED BINPHENYLS & 2 & $1.7000 E-01$ & $8.5000 E-02$ & $4.9487 E-02$ & 5.0000E-02 & 1.2000E-01 \\
\hline XYLENE.P & 1 & $1.2000 \mathrm{E}-01$ & $1.2000 \mathrm{E}-01$ & $0.0000 E+\infty$ & $1.2000 E-01$ & 1.2000E-01 \\
\hline CERIUM NITRATE & $i$ & 1.1000E-01 & $1.1000 E .01$ & $0.0000 E+00$ & 1.1000E-01 & $1.1000 \mathrm{E}-01$ \\
\hline DICHLORODIFLUOROMETHANE & 9 & $1.1000 \mathrm{E}-01$ & $1.1000 E-01$ & $0.0000 E+00$ & $1.1000 E-01$ & $1.1000 E-01$ \\
\hline 1.1.1 TRICHLORETHANE & 4 & $1.3090 \mathrm{E}-01$ & $32725 \mathrm{E}-02$ & 5.1133E-02 & $1.7000 E-03$ & $1.0920 E-01$ \\
\hline TOXJEOX & 18 & $6.3150 E-01$ & $3.5089 E .02$ & $34204 E-02$ & 8.0000E-04 & $1.0500 \mathrm{E}-01$ \\
\hline CRESOLS & 4 & 3.8500E-01 & $9.6250 \mathrm{E}-02$ & $1.7500 E-02$ & 7.0000E-02 & $1.0500 E-01$ \\
\hline SULFATES & 3 & $2.4410 E-01$ & $81367 E-02$ & 2.3581E-02 & $5.6800 \mathrm{E}-02$ & $1.0350 E-01$ \\
\hline TRIETHYLAMINE & 3 & $20310 E-01$ & $6.7700 E-02$ & $5.6032 \mathrm{E}-02$ & $3.0000 E-03$ & $1.0010 \mathrm{E}-01$ \\
\hline BUTYLMET\&YYLETHER & 1 & 1 OO10E.01 & $1.0010 E-01$ & $0.0000 E+00$ & $1.0010 E-01$ & $10010 \mathrm{E} .01$ \\
\hline O-CRESOL & 4 & $2.3340 E-01$ & $5.8350 \mathrm{E} .02$ & 4.5633E-02 & $1.4000 \mathrm{E}-03$ & $1.0000 E-01$ \\
\hline LIGHT EALLASTS CONTAINING PCB & 3 & $4000 E-01$ & 4.6667E-02 & 4.618aE-02 & $2.0000 E-02$ & 1.0000E-01 \\
\hline $\begin{array}{l}\text { DIMETHYL PHLLATE } \\
\text { DILTE }\end{array}$ & 2 & $1.3500 \mathrm{E}-01$ & $6.7500 E-02$ & $4.5962 E-02$ & $3.5000 E-02$ & 1.0000E-01 \\
\hline SILVER NITRATE & 3 & $1.2820 \mathrm{E}-04$ & $4.2733 E \cdot 02$ & $5.0779 E-02$ & $3.2000 \mathrm{E} \cdot 03$ & 1.0000E-01 \\
\hline HYORALLIC OIL & 7 & 1 O000E-01 & 3. 3333E-02 & $1.9245 E-02$ & $0.0000 E+\infty$ & $1.0000 E .01$ \\
\hline
\end{tabular}


TABLE 6

STATISTICAL ANALYSIS OF HAZARDOUS MATERIALS IN ASCENDING ORDER BASED ON MAXIMUM WEIGHT (* INDICATES DATA EXHIBITED NEGATIVE VARIANCE)

(10 SHEET)

COMPONENT NAME

1,4,7,10,13,16-HEXANOXACYELOOCTADECANE BUTYLATED HYDROXYTOLUENE CUPFERRON

HYDROGEN IODATE

HYOROGEN PEROXIOE

MAGNESIUM PERCHLORATE

DIETHM ENE TRIAMINE LEAD OXIDE SULICON

METHYL METMACRYLATE

PLASTIC ACID SUIT/GLOVESRROPERAGS

SILVER CHLOFIDE DECANE BISMUTH

TETRACHLOROETHENE N,N-DIMETHYL FOFMANIDE 2,4-DIISOCYANATO-1+MEI"HYL BENZENE ACETIC ACIO ETHYL ESTER

DICHLOROOCTAMETHYLTITRA SILOXANE

ETHANOL (ETHYL ALCOHOL) HYDROXYOUINIOLINE

ISOBUTYL ALCOHOL

METHYCYCLOHEXANONE VINYL CHLORIOE

GLACIAL ACETIC ACID

SILICA CRYSTALLINE QUARTZ ISOBUTYL ISOBUTYRATE

STRONTIUM NITRATE

FERRIC OXIDE

BENZYL BUTYL ESTER

DICHLORODIMETHY'L SILANE ETHYL CYANIDE

TERT BUTYL ALCOHOL

TTHUM CARBONATE

MAGNESIUM CHLORIDE

LEAKING LIGHT BALLASTS CONTAINING PCB KEROSINE

N-BUTYL ALCOHOL

ISOBUTANOL

BENZOIC ACIO

TOTAL ORGANIC HALOGENS NAPHTA

PENTAETHYLENE.HEXAMINE IRON OXIDE

CESIUM CHLORIDE TARTARIC AIIID
ARSENAZOI

I,I,I TRICHLOROETHANE

DIACETONE ALCOOHOL

2-BUTYOXYETHANOL

DIPROPYLENE GLYCOL MONOMETHYL ETHER SEC-EUTYL ALCOHOL PROPYOIAMINE

TETRAMETHYL 1,3-BUTANEDIAMINE MED ALIPHATIC SOLVENT NAPHTA BORON

ETHYLENE GLYCOL MONOBUTYL ETHER

1-METHYL-2-PYRROLIDINONE DIMETHOXYMETHANE LEAD NAPHTHENATE

LITHUM HYDROSTEARATE METHYL ORANGE

N-METHYL-2-PYREIQUIDONE PROPANE, 1-NITRO

BIX(2-ETHYL HEXYL) ESTER FHTHALIC ACIO (DOP) BROMOCRESOL GREEN IRON NITRA.TE

POTASSUMM NITRATE

BIS(2-ETHYL HEXYL) ESTER FHTHALIC ACIO (OOP) METHYL PENTYL KETONE CHLORIDES

TETRAPROPYL AMMONIUM HYOROXIDE ACID SODIUM SALT ACRYLAMIDE ANTIMONY

\begin{tabular}{|c|c|c|}
\hline COUNT & ACCUM WT, KG & AVERAGE WT. KG \\
\hline 1 & $1.0000 E \cdot 0 t$ & $1.0000 E-01$ \\
\hline 1 & 1.0000E.01 & $1.0000 \mathrm{E}-01$ \\
\hline 1 & $1.0000 E-01$ & $1.0000 E-01$ \\
\hline 1 & $1.0000 E-01$ & $1.0000 E-01$ \\
\hline$t$ & $1,0000 E-01$ & $1.0000 \mathrm{E}-01$ \\
\hline 1 & $1.0000 E-01$ & 1.0000E-0t \\
\hline 1 & $9.8000 \mathrm{E} .02$ & $9.8000 E-02$ \\
\hline 1 & $9.5000 \mathrm{E}-02$ & $9.5000 \mathrm{E}-02$ \\
\hline 2 & 1.3000E.01 & 6.5000E-02 \\
\hline 1 & $9.0000 E-02$ & $9.0000=-02$ \\
\hline 1 & $9.0000 E-02$ & $0.0000 E-02$ \\
\hline 1 & B.5500E-02 & B.5500E-02 \\
\hline 1 & $8.5000 \mathrm{E}-02$ & $8.5000 E-02$ \\
\hline 1 & 8. 3600E-02 & 8.3600E-02 \\
\hline 3 & 2.0000E-01 & 6.6687E-02 \\
\hline 2 & 1.0500E-04 & $5.2500 E-02$ \\
\hline 1 & B.0000E.02 & B. $0000 E .02$ \\
\hline 1 & $8.0000 E-02$ & $80000 E-02$ \\
\hline 1 & $8.00 C O E \cdot 02$ & $8.0000 \mathrm{E}-02$ \\
\hline 1 & $8.0000 E-02$ & 8.00005 .02 \\
\hline 1 & $8.00 \mathrm{COE}-02$ & $8.0000 \mathrm{E}-02$ \\
\hline 1 & $8.0000 E .02$ & $8.0000 \mathrm{E}-02$ \\
\hline 1 & $8,0000 E-02$ & $8.0000 \mathrm{E}-02$ \\
\hline 29 & 7. $8500 \mathrm{E}-02$ & 2.7069E-03 \\
\hline 2 & 1.1780E-01 & 5.8900 E- 02 \\
\hline 1 & $6.7000 \mathrm{E} \cdot 02$ & 6.7000E-02 \\
\hline 1 & $6.4000 \mathrm{E}-02$ & $64000 E-02$ \\
\hline 4 & $1.1580 E .01$ & $2.8950 E .02$ \\
\hline 1 & 6.1600E-02 & $6.1600 \mathrm{E}-02$ \\
\hline 1 & $60000 E \cdot 02$ & $6.0000 \mathrm{E}-02$ \\
\hline 1 & $6.0000 E-02$ & $6.0000 \mathrm{E}-02$ \\
\hline 1 & 5, 7000 E -02 & 5. $7000 E-02$ \\
\hline 4 & 1. $7580 \mathrm{E} \cdot 01$ & 4.3950E-02 \\
\hline 1 & 5.5000E-02 & $5.5000 E-02$ \\
\hline 1 & 5.2000E-02 & 5.2000E-02 \\
\hline 7 & 3,5000E-01 & $5.0000 \mathrm{E}-02$ \\
\hline 2 & \$. $1000 E-02$ & $2.5500 \mathrm{E}-02$ \\
\hline 2 & $5.0000 E-02$ & 5. $0000 \mathrm{E}-02$ \\
\hline 1 & $\$, 0000 E-02$ & $5.0000 \mathrm{E}-02$ \\
\hline 1 & 4. $7000 \mathrm{E}-02$ & 4.7000E-02 \\
\hline 1 & 4.5000 E- -02 & 4.5000E-02 \\
\hline 13 & 2.2410E-01 & 1.7238E-02 \\
\hline 1 & $4.2000 E-02$ & 4.2000E-02 \\
\hline 1 & $4.0000 E-02$ & $4,0000 \mathrm{E}-02$ \\
\hline 1 & $4.0000 E-02$ & 4.0000E-02 \\
\hline 1 & 3.9800E-02 & $3.9800 \mathrm{E}-02$ \\
\hline 4 & 8 $3800 \mathrm{E}-02$ & $1.5950 \mathrm{E}-02$ \\
\hline 1 & $3.7500 \mathrm{E}-02$ & $3.7500 E-02$ \\
\hline 1 & $3.6000 \mathrm{E}-02$ & $3.6000 E-02$ \\
\hline 1 & 35000E-02 & $3,5000 \mathrm{E}-02$ \\
\hline 2 & 4. $4000 E-02$ & 2.2000E 02 \\
\hline 1 & $3.4000 E-02$ & $3.4000 \mathrm{E}-02$ \\
\hline 1 & 3.4000E-02 & $3.4000 \mathrm{E}-02$ \\
\hline 1 & $34000 E-02$ & $34000 \mathrm{E}-02$ \\
\hline 1 & 3.3000E-02 & 3.3000E.02 \\
\hline 1 & 3. 3000E-02 & 3.3000E-02 \\
\hline$t$ & 32100E-C2 & $3.2100 E-02$ \\
\hline 8 & 1.0750E-01 & 1. $3438 \mathrm{E}-02$ \\
\hline 2 & 6.0000E-02 & $3.0000 \mathrm{E}-02$ \\
\hline 2 & 5.8000E-02 & $29000 \mathrm{E}-02$ \\
\hline 2 & 3.0400E-02 & $1.5200 \mathrm{E} \cdot 02$ \\
\hline 1 & $30000 E+02$ & 30000 E-02 \\
\hline 1 & $30000 \mathrm{E}-02$ & $3.0000 \mathrm{E}-02$ \\
\hline 1 & 3 COOOE-02 & $3.0000 \mathrm{E}-02$ \\
\hline 1 & $3.0000 E-02$ & $3.0000 E-02$ \\
\hline 1 & $27500 E-02$ & $2.7500 \mathrm{E}-02$ \\
\hline 1 & $2.7000 \mathrm{E}-02$ & $2.7000 \mathrm{E}-02$ \\
\hline 2 & $3.0000 \mathrm{E}-02$ & $1.5000 \mathrm{E}-02$ \\
\hline 3 & 2.5000E-02 & 8.6333E-03 \\
\hline 1 & 2.5000E-02 & $25000 \mathrm{E}-02$ \\
\hline 11 & 2.4240E-01 & $2.2036 \mathrm{E}-02$ \\
\hline 1 & $2.3000 \mathrm{E}-02$ & $2.3000 \mathrm{E}-02$ \\
\hline 3 & $4.9000 \mathrm{E}-02$ & 1.6333E-02 \\
\hline 3 & 4.5000E-02 & $1.5000 \mathrm{E}-02$ \\
\hline 1 & $20000 \mathrm{E}-02$ & $20000 E-02$ \\
\hline 1 & $2.0000 \mathrm{E}-02$ & $2.0000 \mathrm{E}-02$ \\
\hline 9 & $2.0000 E-02$ & $2.0000 \mathrm{E}-02$ \\
\hline
\end{tabular}

STD DEV

$0.0000 E+\infty 0$

$0.0000 E+00$

$0.0000 E+\infty$

$0.0000 E+00$

$0.0000 E+00$

$0.0000 E+C 0$

$0.0000 E+\infty 0$

$0.0000 E+00$

3.5355E-02

$0.0000 E+00$

$0.0000 E+00$

$0.0000 E+00$

$0.0000 E+00$

$0.0000 E+00$

2.3004E-02

3.8891E-02

$0.0000 E+\infty 0$

$0.0000 E+\infty 0$

$0.0000 E+D O$

$0.0000 \mathrm{E}+\infty$

$0.0000 E+\infty$

$0.0000 E+\infty$

$0,0000 \mathrm{E}+\infty$

1.3905E-02

$1.5698 \mathrm{E}-02$

$0.0000 E+00$

$0.0000 \mathrm{E}+00$

3.2258E-02

$0,0000 \mathrm{E}+00$

$0,0000 E+\infty 0$

$0.0000 E+\infty$

$0.0000 E+00$

1.1381E-02

7. $6000 \mathrm{E}-10$

3. $4648 E-02$

4NUMI

$0,0000 E+00$

$0.0000 E+00$

$0.0000 E+00$

1.5729E-02

$0.0000 E+00$

$0.0000 E+00$

$0.0000 E+\infty 0$

$0.0000 E+00$

$1.8724 E-02$

$0,0000 E+00$

$0.0000 E+00$

$0.0000 E+00$

1.6971E-02

$0,0000 E+\infty 0$

$0.0000 E+\infty 0$

$0.0000 E+00$

$0.0000 E+00$

$0.0000 \mathrm{E}+00$

$0.0000 \mathrm{E}+00$

1.2521E-02

Q $0000 \mathrm{E}+00$

$1.4140 \mathrm{E}-03$

2.0930E-02

0 OODOE +00

$0.0000 E+00$

$0.0000 E+00$

$0.0000 E+00$

$0.0000 E+\infty 0$

$0.0000 E+\infty 0$

1.4142E-O2

$1.4174 E-02$

$0.0000 E+00$

6. $5130 \mathrm{E} .03$

$0.0000 E+00$

4.7350E-03

8.6800E-03

$0.0000 E+\infty 0$

$0.0000 E+\infty 0$

$0.0000 E+O 0$
MINIMUM WT, KG

1.0000E-01

$1.0000 \mathrm{E}-01$

1.0000E-04

1.0000 E- 01

$1.0000 E-01$

1 COOOE-OS

$9.8000 E-02$

$9.5000 E-02$

$4.0000 E-02$

$0.0000 E-02$

$8.0000=02$

6.5500E- 02

8.5000E-02

8.3600 E.02

4.0000 E-02

2.5000E-02

$8.0000 E-02$

6.0000E-02

B. $0000 \mathrm{E}-02$

8.0000E-02

8.0000E-02

8.0000E.02

8.0000E-02

$1.0000=.04$

4.7800E-02

6. $7000 \mathrm{E}-02$

$6.4000 \mathrm{E}-02$

6.0000E-04

6.1800E.02

$6.0000 \mathrm{E} .02$

B.0000E-02

$5.7000 E-02$

3.0000E-02

$5.5000 \mathrm{E} .02$

5. $2000 \mathrm{E}-02$

$5.0000 \mathrm{E}-02$

$1.0000 E-03$

$5.0000 E-02$

5.0000E-02

$4.7000 E-02$

$4000=02$

1.0000E-04

4.2000E-02

4.0000E-02

$4.0000 \mathrm{E}-02$

3 . $9000-02$

2.0000E-04

$3.7500 \mathrm{E}-02$

3. $6000 \mathrm{E}-02$

3.5000E.02

1.0000E-02

3. $4000 \mathrm{E}-02$

3. 4000 E.02

$3.4000 \mathrm{E}-02$

3. 3000E-02

3. $3000=.02$

3 2100E-O2

$1.0000 \mathrm{E}-04$

3.0000E-02

2.8000E-02

4.0000E-04

3 0000E.02

3 0000E-02

3.0000E-02

$3.0000 E-02$

2.75COE-02

2.7000E.02

$5.0000 E-03$

4 OOOOE-O4

$2.5000 E-02$

2. 4000 E-03

2.3000E-02

1 1300E-OO

-

$2.0000 E-02$

$2.0000 E-02$

2.0000E-02

MAXIMUIM WT, KG

$1.0000 \mathrm{E}-01$

1.0000 E- 01

1.0000E-01

1.0000E-01

$1.0000 E-01$

$1.0000 E-01$

9.8000E-02

$9.5000 E-02$

9.0000 E-02

$9.0000 \mathrm{E}-02$

$9.0000 E-02$

8.5500E-02

8.5000E-02

8.3600E-02

8.0000E-02

8.0000E-02

8.0000 E- 02

8.0000E-02

80000 E-02

8.0000E-02

8.0000 E- 02

6.0000E-02

B. $0000 \mathrm{E}-02$

$7.5000 E-02$

7.0000E-02

6.7000E-02

6. $4000 E .02$

6.3000E- 02

6. 1000E 02

$6.0000 E-02$

$6.0000 E-02$

5.7000 E- 02

$5.5800 E-02$

$5.5000 \mathrm{E} .02$

5. $2000 \mathrm{E}-02$

$5.0000 E-02$

5.0000 E- 02

5.0000 E.02

$5.0000 E-02$

4.7000E-02

4 5000E 02

4.2000E-02

4.2000E-02

$4.0000 E-02$

4.0000E-02

$30800 E .02$

$3.8000 \mathrm{E}-02$

$3.7500 \mathrm{E}-02$

3.8000E-02

3.5000E.02

$3.4000 \mathrm{E}-02$ 
TABLE 6

STATISTICAL ANALYSIS OF HAZARDOUS MATERIALS IN ASCENDING ORDER BASED ON MAXIMUM WEIGHT (* INDICATES DATA EXHIBITED NEGATIVE VARIANCE)

(10 SHEET)

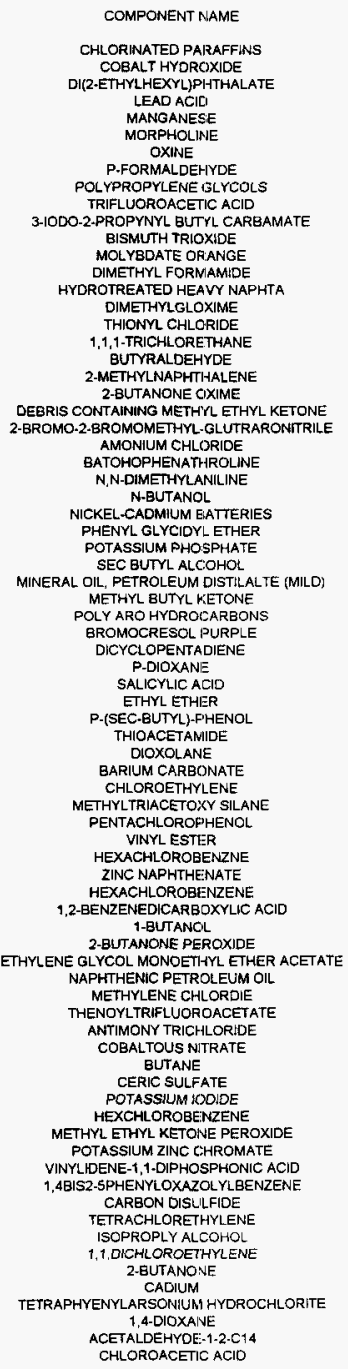

\section{STD DEV}

$0.0000 E+00$

$0.0000 \mathrm{E}+00$

$0.0000 E+\infty$

$0.0000 E+\infty 00$

$0.0000 E+\infty$

$0.0000 E+\infty 0$

$0.0000 \mathrm{E}+\infty 0$

$0.0000 E+\infty$

$0.0000 E+00$

$0.0000 E * 00$

$0.0000 E+00$

$0.0000 E+00$

$0.0000 E+00$

D. $0000 \mathrm{E}+\infty 0$

1.0041E-02

$0.0000 E+00$

$0.0000 E+00$

$0.0000 E+\infty 0$

$0.0000 E+\infty 0$

$0.0000 E+00$

$0.0000 E+00$

$0.0000 \mathrm{E}+\infty$

$0.0000 E+C 0$

$0.0000 E+D 0$

$0.0000 E+00$

$0.0000 E+00$

$0.0000 E+00$

$0.0000 E+00$

$0.0000 E+00$

$0.0000 E+00$

5.6570E-03

1.0000E-03

0.000 E

$0.0000 E+00$

5.0000E-04

$0.0000 \mathrm{E}+00$

$0.0000 E+\infty 0$

$0.0000 E+\infty$

$0.0000 E+\infty 0$

$0.0000 E+00$

$0.0000 E+00$

$0.0000 E+00$

$0.0000 \mathrm{E}+00$

$0,0000 E+\infty 0$

$0.0000 E+00$

$5.6500 E-04$

$0.0000 E+00$

$0.0000 E+\infty 0$

$0.0000 \mathrm{E}+\infty$

$0.0000 E+\infty 0$

$0.0000 E+00$

$0.0000 E+00$

$0.0000 E+00$

$1.4100 E-04$

2.1200E-04

$0.0000 E+\infty 0$

$0.0000 E+00$

$1.4100 E-04$

$0.0000 E+00$

$0.0000 E+\infty 0$

$0.0000 \mathrm{E}+\infty$

$0.0000 E+00$

1.0000 E- 04

5. $39005-05$

$1.4100 E-04$

$0.0000 E+00$

1.3200E-05

$0.0000 E+00$

$0.0000 E+00$

$0.0000 E+00$

$0.0000 \mathrm{E}+00$

$0.0000 \mathrm{E}+00$

$0.0000 E+00$
MINIMUM WT

2.0000E-02

2.0000E-02

2.0000E-02

2.0000E-02

2.0000 E.02

$2.0000 E-02$

$2.0000 \mathrm{E}-\mathrm{O} 2$

2.0000E-02

$2.0000 E-02$

2.0000E-02

$1.9000 E-02$

1.9000E-02

1.8000 E.02

1.8000E-02

1.5900E-O2

8.0000E-04

1.3800E-02

$1,7000 E-03$

1.3000E-02

1.0000 EE- 02

1.0000E-02

1.0000 E-O2

1.0000E-02

1 COOOE.02

1.0000E-02

$1.0000 E-02$

$1.0000 E .02$

t.0000E-02

1 . 0000 E.02

1 .0O0OE- 02

1.0000E-02

$9.0000 E-03$

$0.0000 E+00$

4. $0000 \mathrm{E}-03$

5.0000 E.03

5. 0000E-03

4 BOOOE-C3

4. 8000 E-03

3.0000E-03

4 . $0000 E-03$

3.8000E-03

3 3000E.03

3.0000E-03

3.0000E-03

3.0000E-03

3.0000E-03

3. 0000 E- 03

2.5000E-03

2 .5000E-03

2,0000E-04

2.0000E-03

$1.5000 \mathrm{E}-03$

1.5000E-03

1.5000E-03

1. $5000 \mathrm{E}-03$

1.4000 E -03

$1.4000 E-03$

1.0000 E- 03

Q.0000E-04

1.2000E-03

1.1000E-03

8.0000E-04

$1.0000 E-03$

1.0000 EE -03
1.0000 E -03

2.0000E-04

0 . OOCOE + 00

$1.0000 \mathrm{O}$

3.0000E-04

0 .0000E+00

1.0000E-04

1.0000E-04

$1.0000 \mathrm{E}-04$

$00000 E+00$

$0.0000 \mathrm{E}+00$

$0.0000 E+00$

MAXIMUM WT, KG

2.0000E-02

$2.0000 \mathrm{E}-02$

$2.0000 \mathrm{E}-02$

2.0000E-02

2.0000E-02

2.0000E-02

$2.0000 \mathrm{E}-02$

2.0000E-02

$2.0000 E-02$

2.0000E-02

1. $0000 \mathrm{E}-02$

1.0000E-02

$1,0000 \mathrm{E}-02$

1.6000E-02

1.5900 E-02

$1.5000 \mathrm{E}-02$

$1.3800 \mathrm{E}-02$

1. $3000 E-02$

$1.3000 E-02$

1.0000E-02

1.0000E-02

1.0000 E-02

1. .0000 E-02

$1.0000 \mathrm{E}-02$

$1.0000 \mathrm{E}-02$

$1.0000 \mathrm{E}-02$

1.0000 E. 02

$1.0000 E-02$

$1.0000 \mathrm{E}-02$

1.0000 E-02

1.0000E-02

$8.0000 E-03$

8.0000 E -03

. 0000 E-03

$5.0000 E .03$

5.0000E-03

$4.8000 E-03$

4. $8000 E-03$

4.0000E-03

4.0000E-03

$3.8000 \mathrm{E}-03$

$3.3000 E-03$

3.0000 E-03

3.0000E-05

$3.0000 \mathrm{E}-03$

$3.0000 E-03$

$3000=03$

$2.5000 \mathrm{E}-03$

$2.5000 \mathrm{E}-03$

2.3000E-03

2.0000 E -03

15000 E-03

15000 E.03

$1.5000 \mathrm{E}-03$ 
WHC-SD-W-242-ES-004, Rev. 0

TABLE 6

STATISTICAL ANALYSIS OF HAZARDOUS MATERIALS

IN ASCENDING ORDER BASED ON MAXIMUM WEIGHT

(* INDICATES DATA EXHIBITED NEGATIVE VARIANCE)

(10 SHEET)

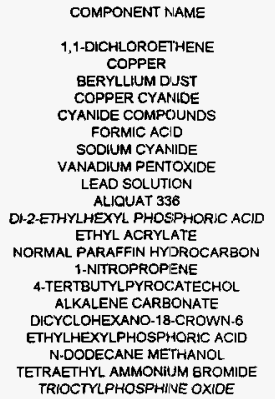

$\begin{array}{cc}\text { COUNT } & \text { ACCUM WT. KG } \\ 30 & 0.0000 E+00 \\ 17 & 0.0000 E+00 \\ 8 & 0.0000 E+00 \\ 8 & 0.0000 E+00 \\ 8 & 0.0000 E+00 \\ 8 & 0.0000 E+00 \\ 8 & 0.0000 E+00 \\ 8 & 0.0000 E+00 \\ 7 & 0.0000 E+00 \\ 3 & 0.0000 E+00 \\ 2 & 0.0000 E+00 \\ 2 & 0.0000 E+00 \\ 2 & 0.0000 E+00 \\ 1 & 0.0000 E+00 \\ 1 & 0.0000 E+\infty \\ 1 & 0.0000 E+\infty 0 \\ 1 & 0.0000 E+00 \\ 1 & 0.0000 E+00 \\ 1 & 0.0000 E+00 \\ 1 & 0.0000 E+00 \\ 1 & 0.0000 E+\infty\end{array}$

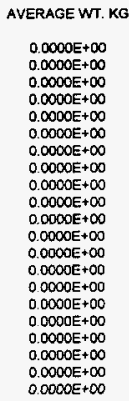

STD DEV

$0.0000 E+00$

$0.0000 E+\infty$

$0.0000 E+\infty$

$0.0000 E+\infty 0$

$0.0000 E+00$

$0.0000 E+\infty 0$

$0.0000 \mathrm{E}+\infty 0$

$0.0000 E+00$

$0.0000 \mathrm{E}+00$

$0.0000 E+00$

$0.0000 \mathrm{E}+00$

$0.0000 \mathrm{E}+00$

$0.0000 E+00$

$0.0000 E+00$

$0.0000 E+00$

$0.0000 \mathrm{E}+00$

$0.0000 \mathrm{E}+\infty$

$0.0000 E+\infty 0$

$0.0000 E+00$

$0.0000 E+00$

$0.0000 E+\infty 0$
MINIMUUM WT, KG

$0.0000 E+00$

$0.0000 \mathrm{E}+00$

$0.0000 E+00$

$0.0000 \mathrm{E}+00$

$0.0000 \mathrm{E}+\infty 0$

$0.0000 \mathrm{E}+\infty$

$0.0000 E+\infty 0$

$0.0000 E+\infty 0$

$0.0000 \mathrm{E}+00$

$0.0000 \mathrm{E}+\infty 0$

$0.0000 \mathrm{E}+00$

$0.0000 \mathrm{E}+\infty 0$

$0.0000 \mathrm{E}+00$

$0.0000 \mathrm{E}+00$

$0.0000 E+\infty$

$0.0000 \mathrm{E}+00$

$0.0000 E+00$

$0.0000 \mathrm{E}+\infty$

$0.0000 \mathrm{E}+\infty$

$0.0000 \mathrm{E}+0$

$0.0000 E+\infty$
MAXIMUM WT, KG

$0.0000 \mathrm{E}+00$ $0.0000 \mathrm{E}+\infty$

$0.0000 E+\infty 00$

$0.0000 E+00$

$0.0000 E+00$

$0.0000 E+00$

$0.0000 E+00$

$0.0000 E+00$

$0.0000 E+00$

$0.0000 \mathrm{E}+00$

$0.0000 \mathrm{E}+00$

$0.0000 E+\infty 0$

0.0000 E

$0.0000 \mathrm{E}+00$

$0.0000 \mathrm{E}+00$

$0.0000 \mathrm{E}+\infty 0$

$0.0000 \mathrm{E}+00$

$0.0000 E+\infty 0$

$0.0000 E+\infty$

$0.0000 \mathrm{E}+00$

$0.0000 \mathrm{E}+\infty 0$ 
WHC-SD-W-242-ES-004, Rev. 0

TABLE 7

\section{STATISTICAL ANALYSIS OF ALPHA-BEARING RADIONUCLIDES IN ASCENDING ORDER BASED ON CONTAINER COUNT}

(1 SHEET)

\begin{tabular}{|c|c|c|c|c|c|c|c|}
\hline ISOTOPE & UNIT & COUNT & ACCUMULATION & AVERAGE & STD DEV & MINIMUM & MAXIMUM \\
\hline AM-241 & GM & 481 & $5.1666 \mathrm{E}-01$ & 1.0741E-03 & $8.6750 \mathrm{E}-03$ & $1.2500 E-16$ & $1.1407 E-01$ \\
\hline PU-238 & GM & 449 & 1.1289E-01 & $2.5142 \mathrm{E}-04$ & $2.0360 \mathrm{E}-03$ & $3.4300 E+17$ & $2.4735 E-02$ \\
\hline PU-239 & GM & 442 & $3.6278 E+02$ & 8.2078E-01 & $6.6033 E+00$ & $1.1000 E-13$ & $7.9729 E+01$ \\
\hline PU-241 & GM & 370 & $2.2210 E+00$ & $6.0028 \mathrm{E}-03$ & $3.8457 \mathrm{E}-02$ & $6.2000 E-19$ & $3.7612 E-01$ \\
\hline TH-232 & GM & 318 & $6.4180 E+03$ & $2.0182 E+01$ & $5.8567 E+01$ & $9.8300 \mathrm{E}-14$ & $9.6010 \mathrm{E}+02$ \\
\hline PU-240 & GM & 309 & $2.2085 E+01$ & $7.1472 E-02$ & $4.7946 \mathrm{E}-01$ & $6.6900 \mathrm{E}-15$ & $4.8540 E+00$ \\
\hline $\mathrm{U}-238$ & GM & 280 & $5.5534 \mathrm{E}+03$ & $1.9834 E+01$ & $3.6758 E+01$ & $2.4000 E-16$ & $3.7497 E+02$ \\
\hline $\mathrm{PU}$ & GM & 261 & $1.0139 E+03$ & $3.8849 \mathrm{E}+00$ & $2.7503 E+01$ & 1.1980E-08 & $3.1630 \mathrm{E}+02$ \\
\hline U.235 & $\mathrm{GM}$ & 228 & $3.0260 E+01$ & $1.3272 E-01$ & 2.8471E-01 & $9.8200 \mathrm{E}-10$ & $3.4820 E+\infty 0$ \\
\hline RA-226 & $\mathrm{GM}$ & 227 & $1.1512 \mathrm{E}-02$ & 5.0713E-05 & $3.7400 E-04$ & $1.0400 \mathrm{E}-14$ & $5.6000 E-03$ \\
\hline $\mathrm{U}-234$ & $\mathrm{GM}$ & 223 & 2.7089E-01 & $1.2148 E-03$ & $2.7490 \mathrm{E}-03$ & 4.4100E-09 & $3.4500 E-02$ \\
\hline URANIUM-DEPLETED & $\mathrm{GM}$ & 222 & $1.3363 E+04$ & $6.0191 \bar{E}+01$ & $6.9547 E+02$ & $7.1000 \mathrm{E}-11$ & $1.0200 E+04$ \\
\hline $\mathrm{TH}-234$ & $G M$ & 201 & $9.0581 E-08$ & $4.5065 \mathrm{E}-10$ & $1.6400 E-09$ & $6.5200 \mathrm{E}-16$ & $2.2100 E-08$ \\
\hline TH-228 & $\mathrm{GM}$ & 190 & $1.6539 \mathrm{E}-04$ & $8.7047 E-07$ & $1.1900 E-05$ & 1.4800E-13 & $1.6400 \mathrm{E}-04$ \\
\hline PU-242 & $G M$ & 140 & $7.5813 E-02$ & 5.4152E-04 & $2.4170 E-03$ & $2.2900 \mathrm{E}-17$ & $1.6660 \mathrm{E}-02$ \\
\hline RA-224 & $\mathrm{GM}$ & 130 & $3.5140 \mathrm{E}-07$ & 2.7030E-09 & $2.3200 \mathrm{E}-08$ & $7.5000 E-13$ & 2.5000E-07 \\
\hline URANIUM-ENRICHED & $\mathrm{GM}$ & 118 & $5.2564 \mathrm{E}+03$ & $4.4546 E+01$ & 7.4065E+01 & $1.0700 E-06$ & $5.5400 \mathrm{E}+02$ \\
\hline CM-242 & GM & 85 & $2.2402 E-09$ & $2.6355 E-11$ & $1.3400 E-10$ & $2.8000 E-22$ & $9.0000 E-10$ \\
\hline U.236 & $\mathrm{GM}$ & 65 & $4.8369 \mathrm{E}-01$ & $7.4414 E-03$ & $3.3332 \mathrm{E}-02$ & $9.8200 E-09$ & $2.6900 E-01$ \\
\hline PU239 FSL GR EQUIV & GM & 61 & $0.0000 E+00$ & $0.0000 E+00$ & $0.0000 E+00$ & $0.0000 E+00$ & $0.0000 E+00$ \\
\hline NP-237 & GM & 54 & $1.7754 E+01$ & 3.2879E- 01 & $1.0332 E+00$ & $3.3100 E-09$ & $6.1400 E+00$ \\
\hline U-233 & GM & 26 & $6.9158 \mathrm{E}-03$ & $2.6599 \mathrm{E}-04$ & $2.8100 \mathrm{E}-04$ & $6.1000 E-07$ & $1.0000 E-03$ \\
\hline $\mathrm{CM}-244$ & GM & 17 & $2.0120 \mathrm{E}-05$ & $1.1835 E-06$ & 4.0000E-05 & $7.3700 \mathrm{E}-15$ & $1.6600 \varepsilon-05$ \\
\hline CM-243 & GM & $\$ 1$ & $3.1550 E-07$ & $2.8682 \mathrm{E}-08$ & 4.9700E-08 & $6.6300 \mathrm{E}-16$ & 1.3300E-07 \\
\hline U-232 & GM & 11 & $1.1870 \mathrm{E}-07$ & $1.0791 \mathrm{E}-08$ & $1.1400 E-08$ & $5.7000 E-11$ & $2.7000 E-08$ \\
\hline AM-243 & GM & 10 & $1.0740 \mathrm{E}-03$ & $1.0740 E-04$ & $3.3500 E-04$ & $3.9000 \mathrm{E}-08$ & $1.0600 \mathrm{E}-03$ \\
\hline $\mathrm{CM}-245$ & GM & 8 & $1.0579 E-05$ & 1.3224E-06 & 1.5300E-06 & $1.1000 E-08$ & $3.4000 E-06$ \\
\hline
\end{tabular}


WHC-SD-W-242-ES-004, Rev. 0

TABLE 8

STATISTICAL ANALYSIS OF ALPHA-BEARING RADIONUCLIDES IN ASCENDING ORDER BASED ON ACCUMULATED WEIGHT

(। SHEET)

\begin{tabular}{|c|c|c|c|c|c|c|c|}
\hline ISOTOPE & UNIT & COUNT & ACCUMULATION & AVERAGE & STD DEV & MINIMUM & MAXMUMM \\
\hline URANIUM-DEPLE:TED & GM & 222 & $1.3363 E+04$ & $6.0191 E+01$ & $6.9547 E+02$ & 7.1000E-11 & $1.0200 E+04$ \\
\hline $\mathrm{TH}-232$ & GM & 318 & $6.4180 E+03$ & $2.0182 E+01$ & $5.8567 E+01$ & $9.8300 E-14$ & $9.6010 E+02$ \\
\hline U-238 & GM & 280 & $5.5534 E+03$ & $1.9834 E+01$ & $3.6758 E+01$ & $2.4000 E-16$ & $3.7497 \mathrm{E}+02$ \\
\hline URANIUM-ENRICHED & $\mathrm{GM}$ & 418 & $5.2564 E+03$ & $4.4546 E+01$ & $7.4065 E+01$ & $1.0700 E-06$ & $5.5400 E+02$ \\
\hline PU & $\mathrm{GM}$ & 261 & 1.0139E +03 & $3.8849 E+\infty 0$ & $2.7503 E+01$ & 1.1980E-08 & $3.1630 E+02$ \\
\hline PU-239 & $\mathrm{GM}$ & 442 & $3.6278 E+02$ & 8.2078E-01 & $6.6033 E+00$ & 1.1000E-13 & $7.9729 E+01$ \\
\hline$U-235$ & GM & 228 & $3.0260 E+01$ & 1.3272E-01 & $2.8471 E-01$ & $9.8200 E-10$ & $3.4820 E+00$ \\
\hline PU-240 & GM & 309 & $2.2085 E+01$ & $7.1472 E-02$ & $4.7946 E-01$ & $6.6900 \mathrm{E}-15$ & $4.8540 E+00$ \\
\hline NP-237 & GM & 54 & $1.7754 E+01$ & 3.2879E-01 & $1.0332 E+00$ & 3.3100E-09 & $6.1400 E+00$ \\
\hline PU-241 & GM & 370 & $2.2210 E+\infty 0$ & $6.0028 E-03$ & $3.8457 E-02$ & 5.2000E-19 & 3.7612E-01 \\
\hline $\begin{array}{c}A M-241 \\
U-236\end{array}$ & $\begin{array}{l}\text { GM } \\
\text { GM }\end{array}$ & $\begin{array}{l}481 \\
65\end{array}$ & $\begin{array}{l}5.1666 E-01 \\
4.8369 E-01\end{array}$ & $\begin{array}{l}1.0741 \mathrm{E}-03 \\
7.4414 \mathrm{E}-03\end{array}$ & $\begin{array}{l}8.6750 E-03 \\
3.3332 E-02\end{array}$ & $\begin{array}{l}1.2500 E-16 \\
9.8200 E-09\end{array}$ & $\begin{array}{l}1.1407 E-01 \\
2.6900 E-01\end{array}$ \\
\hline U-234 & GM & 223 & $2.7089 E-01$ & $1.2148 \mathrm{E}-03$ & $2.7490 \mathrm{E}-03$ & 4.4100E-09 & 3.4500E-02 \\
\hline PU-238 & $G M$ & 449 & $1.1289 \mathrm{E}-01$ & $2.5142 \mathrm{E}-0.4$ & $2.0360 \mathrm{E}-03$ & $3.4300 \mathrm{E}-17$ & $2.4735 E-02$ \\
\hline$P U-242$ & GM & 140 & $7.5813 E-02$ & $5.4152 E-04$ & $2.4170 E-03$ & $2.2900 E-17$ & $1.6560 E-02$ \\
\hline $\mathrm{RA}-226$ & GM & 227 & 1.1512E-02 & $5.0713 E-05$ & 3.7400 E-04 & 1.0400E- 14 & 5.6000E-03 \\
\hline U-233 & $G M$ & 26 & $6.9158 \mathrm{E}-03$ & $2.6599 E-04$ & 2.8100E-04 & $6.1000 E-07$ & $1.0000 E-03$ \\
\hline AM-243 & GM & 10 & $1.0740 E-03$ & $1.0740 \mathrm{E}-04$ & 3.3500E-04 & $3.9000 E-08$ & $1.0600 E-03$ \\
\hline TH-228 & GM & 190 & $1.6539 \mathrm{E}-04$ & $8.7047 E-07$ & 1.1900E-05 & $1.4800 \mathrm{E}-13$ & 1.64OOE-04 \\
\hline $\mathrm{CM}-244$ & $G M$ & 17 & $2.0120 E-05$ & 1.1835E-06 & 4,0000E-06 & $7.3700 \mathrm{E}-15$ & $1.6600 E-05$ \\
\hline $\mathrm{CM}-245$ & GM & 8 & $1.0579 E-05$ & 1.3224E-06 & 1.5300E-06 & 1.1000E-08 & $3.4000 E-06$ \\
\hline RA-224 & $G M$ & 130 & $3.5140 E-07$ & $2.7030 \mathrm{E}=09$ & 2.3200E-08 & 7.5000 E- 13 & 2.5000E-07 \\
\hline $\mathrm{CM}-243$ & $G M$ & 11 & $3.1550 E-07$ & $2.8682 \mathrm{E}-08$ & 4.9700E-08 & $6.6300 E-16$ & 1.3300E-07 \\
\hline U-232 & $\mathrm{GM}$ & 11 & 1.1870E-07 & $1.0791 \mathrm{E}-08$ & 1.1400E-08 & $5.7000 E-11$ & 2.7000E-08 \\
\hline TH-234 & GM & 201 & $9.0581 E-08$ & $4.5065 \mathrm{E}-10$ & $1.6400 E-09$ & $6.5200 E-16$ & $2.2100 \mathrm{E}-0 \mathrm{~B}$ \\
\hline $\mathrm{CM}-242$ & GM & 85 & $2.2402 E-09$ & $2.6355 \mathrm{E}-11$ & 1.3400E-10 & 2.8000E-22 & $9.0000 E-10$ \\
\hline PU239 FSL GR EQUIV & GM & 61 & $0.0000 E+00$ & $0.0000 E+00$ & $0.0000 E+00$ & $0.0000 E+00$ & $0.0000 E+00$ \\
\hline
\end{tabular}


WHC-SD-W-242-ES-004, Rev. 0

TABLE 9

STATISTICAL ANALYSIS OF ALPHA-BEARING RADIONUCLIDES IN ASCENDING ORDER BASED ON MAXIMUM WEIGHT

(1 SHEET)

\begin{tabular}{|c|c|c|c|c|c|c|c|}
\hline ISOTOPE & UNIT & COUNT & ACCUMULATION & AVERAGE & STD DEV & MINIMUM & MAXIMUM \\
\hline URANIUM-DEPLETED & GM & 222 & $1.3363 E+04$ & $6.0191 E+01$ & $6.9547 E+02$ & $7.1000 \mathrm{E}+11$ & $1.0200 E+04$ \\
\hline TH-232 & GM & 318 & $6.4180 E+03$ & $2.0182 E+01$ & $5.8567 E+01$ & $9.8300 E-14$ & $9.6010 E+02$ \\
\hline URANIUM-ENRICHED & GM & 118 & $5.2564 E+03$ & $4.4546 \mathrm{E}+01$ & $7.4065 E+01$ & 1.0700E-06 & $5.5400 E+02$ \\
\hline U-238 & GM & 280 & $5.5534 E+03$ & $1.9834 E+01$ & $3.6758 E+01$ & $2.4000 \mathrm{E}-16$ & $3.7497 E+02$ \\
\hline PU & GM & 261 & $1.0139 E+03$ & $3.8849 E+C 0$ & $2.7503 E+01$ & $1.1980 \mathrm{E}-08$ & $3.1630 E+02$ \\
\hline PU-239 & GM & 442 & $3.6278 E+02$ & 8.2078E-01 & $6.6033 E+00$ & 1.1000E-13 & $7.9729 E+01$ \\
\hline NP-237 & $\mathrm{GM}$ & 54 & $1.7754 E+01$ & $3.2879 \mathrm{E}-01$ & $1.0332 E+00$ & 3.3100E-09 & $6.1400 E+00$ \\
\hline PU-240 & GM & 309 & $2.2085 E+01$ & 7.1472E-02 & 4.7946E-01 & $6.6900 E-15$ & $4.8540 E+00$ \\
\hline U-235 & GM & 228 & $3.0260 E+01$ & $1.3272 \mathrm{E}-01$ & $2.8471 E-01$ & $9.8200 \mathrm{E}-10$ & $3.4820 E+00$ \\
\hline$P U-241$ & GM & 370 & $2.2210 E+00$ & $6.0028 E-03$ & 3.8457E-02 & 5.2000E-19 & $3.7612 \mathrm{E}-01$ \\
\hline U-236 & GM & 65 & 4.8369E-01 & $7.4414 E-03$ & $3.3332 E-02$ & 9.8200E-09 & 2.6900E-01 \\
\hline AM-241 & GM & 481 & $5.1666 \mathrm{E}-01$ & $1.0741 E-03$ & $8.6750 E-03$ & 1.2500E-16 & $1.1407 \mathrm{E}-01$ \\
\hline U-234 & GM & 223 & $2.7089 \mathrm{E}-01$ & $1.2148 \mathrm{E}-03$ & $2.7490 \mathrm{E}-03$ & $4.4100 \mathrm{E}-09$ & $3.4500 \mathrm{E}-02$ \\
\hline PU-238 & GM & 449 & $1.1289 \mathrm{E}-01$ & $2.5142 E-04$ & $2.0360 E-03$ & $3.4300 E-17$ & $2.4735 E-02$ \\
\hline PU-242 & GM & 140 & $7.5813 \mathrm{E}-02$ & $5.4152 \mathrm{E}-04$ & 2.4170E-03 & $2.2900 \mathrm{E}-17$ & $1.6660 \mathrm{E}-02$ \\
\hline RA-226 & GM & 227 & 1.1512E-02 & $5.0713 E-05$ & $3.7400 \mathrm{E}-04$ & $1.0400 E-14$ & $5.6000 E-03$ \\
\hline AM-243 & GM & 10 & $1.0740 \mathrm{E}-03$ & $1.0740 \mathrm{E}-04$ & $3.3500 \mathrm{E}-04$ & $3.9000 E-08$ & $1.0600 E-03$ \\
\hline U-233 & $\mathrm{GM}$ & 26 & $6.9158 \mathrm{E}-03$ & 2.6599E-04 & $2.8100 \mathrm{E}-04$ & $6.1000 E-07$ & $1.0000 E-03$ \\
\hline $\mathrm{TH}-228$ & GM & 190 & 1.6539E-04 & 8.7047E-07 & 1.1900E-05 & $1.4800 E-13$ & $1.6400 \mathrm{E}-04$ \\
\hline CM-244 & GM & 17 & $2.0120 \mathrm{E}-05$ & $1.1835 \mathrm{E}-06$ & 4.0000E-06 & $7.3700 \mathrm{E}-15$ & $1.6600 E-05$ \\
\hline CM-245 & $\mathrm{GM}$ & 8 & 1.0579E-05 & 1.3224E-06 & $1.5300 \mathrm{E}-06$ & 1.1000E-08 & $3.4000 \mathrm{E}-06$ \\
\hline RA-224 & $\mathrm{GM}$ & 130 & $3.5140 E-07$ & $2.7030 \mathrm{E}-09$ & $2.3200 E-08$ & $7.5000 \mathrm{E}-13$ & $2.5000 \mathrm{E}-07$ \\
\hline CM-243 & $\mathrm{GM}$ & 11 & 3.1550E-07 & $2.8682 \mathrm{E}-08$ & $4.9700 E-08$ & $6.6300 \mathrm{E}-16$ & $1.3300 \mathrm{E}-07$ \\
\hline$U-232$ & GM & 11 & $1.1870 E-07$ & 1.0791E-08 & 1.1400E-OB & $5.7000 \mathrm{E}-11$ & $2.7000 \mathrm{E}-08$ \\
\hline TH-234 & GM & 201 & $9.0581 E-08$ & $4.5065 E-10$ & $1.6400 E-09$ & $6.5200 \mathrm{E}=16$ & $2.2100 \mathrm{E}-08$ \\
\hline $\mathrm{CM}-242$ & GM & 85 & $2.2402 \mathrm{E}-09$ & 2.6355E-11 & $1.3400 \mathrm{E}-10$ & $2.8000 \mathrm{E}-22$ & $9.0000 \mathrm{E}-10$ \\
\hline PU239 FSL GR EQUIV & GM & 61 & $0.0000 E+\infty 0$ & $0.0000 \mathrm{E}+00$ & $0.0000 \mathrm{E}+00$ & $0.0000 E+00$ & $0.0000 E+00$ \\
\hline
\end{tabular}


WHC-SD-W-242-ES-004, Rev. 0

TABLE 10

STATISTICAL ANALYSIS OF FISSION PRODUCTS IN ASCENDING ORDER BASED ON CONTAINER COUNT

(1 SHEET)

\begin{tabular}{|c|c|c|c|c|c|c|c|}
\hline ISOTOPE & UNIT & COUNT & ACCUMULATION & AVERAGE & STD DEV & MINLAMUM & MAXIMUM \\
\hline TOTAL BETAVAAMMA & $\mathrm{Cl}$ & 2764 & $7.0532 \mathrm{E}+04$ & $2.5518 \mathrm{E}+01$ & $1.3391 \mathrm{E}+03$ & $6.0000 \mathrm{E}-12$ & $7.0400 E+04$ \\
\hline$c 0-60$ & $\mathrm{Cl}$ & 1004 & 2. O084E-01 & 2.0004E-04 & $1.7200 \mathrm{E}-03$ & $4.0000 \mathrm{E}-11$ & $3.9927 \mathrm{E}-02$ \\
\hline $\operatorname{cs}-137-84-137 \mathrm{M}$ & $\mathrm{Cl}$ & 989 & $2.5059 \mathrm{E}+01$ & $2.5338 \mathrm{E}-02$ & 4.1791E-01 & $9.0000 \mathrm{E}-13$ & $9.5000 \mathrm{E}+\infty 0$ \\
\hline$S R-90-Y 90$ & $\mathrm{Cl}$ & 780 & $2.7455 E+01$ & 3.5199E-02 & $7.1501 E-01$ & 1.3600E-10 & $1.9900 E+01$ \\
\hline $\mathrm{H}-3$ & Cl & 571 & 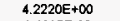 & 7.3940E-03 & 4.8866E-02 & $5.0000 E-12$ & 6.2250E-01 \\
\hline $\mathrm{C}-14$ & $\mathrm{Cl}$ & 404 & $9.6027 \mathrm{E}-02$ & $2.3769 E-04$ & $2.3410 E-03$ & $1.5000 \mathrm{E}-18$ & $3.7400 E-02$ \\
\hline $\operatorname{TC} 99$ & $\mathrm{Cl}$ & 312 & $2.3481 E-02$ & 7.5259E-05 & $6.9500 \mathrm{E}-04$ & $3.0100 \mathrm{E}-13$ & $8.8900 \mathrm{E}-03$ \\
\hline $\mathrm{PE}-212$ & cl & 216 & 1.4087E-05 & 6.5220E-08 & $3.2400 E-07$ & 9.5500E-19 & $2.4700 E-06$ \\
\hline$M N-5-4$ & $\mathrm{Cl}$ & 214 & 1.1621E-02 & $6.4303 E-05$ & $2.3300 \mathrm{E}-0.4$ & $4.2000 \mathrm{E}-11$ & $2.8900 E-03$ \\
\hline$P M-147$ & $\mathrm{Cl}$ & 210 & 4.2529E-01 & $2.0252 E-03$ & $5.3290 \mathrm{E}-03$ & $4.0600 \mathrm{E}-11$ & 3.4700E-02 \\
\hline$A C-228$ & $\mathrm{Cl}$ & 202 & 1. 1465E-07 & $5.6756 \mathrm{E}-10$ & $6.1800 \mathrm{E}-09$ & $2.9300 \mathrm{E}-15$ & 8.7000E-08 \\
\hline $1-129$ & Cl & 192 & $3.1596 E-03$ & 1.6466E-05 & 1. $1500 E-04$ & $1.7600 E-14$ & $1.2700 E-03$ \\
\hline EU-154 & $\mathrm{Cl}$ & 157 & $2.1799 E-01$ & 1.38B5E-03 & $9.6890 E-03$ & $2.3000 \mathrm{E}-14$ & $8.8400 E-02$ \\
\hline$R U-106-R H-106$ & $\mathrm{Cl}$ & 152 & 2.1779E-01 & 1.4328E-03 & $3.2640 \mathrm{E}-03$ & $1.9000 E-14$ & 2.7000E- 02 \\
\hline NI-60 & $\mathrm{Cl}$ & 151 & $1.1855 \mathrm{E}-02$ & 7.8609E-05 & $3.1800 E-04$ & $9.0000=-11$ & $2.7000 E-03$ \\
\hline NL-63 & $\mathrm{Cl}$ & 144 & $5.3706 \mathrm{E}-02$ & 3.7206E-04 & $4.2010 \mathrm{E}-03$ & $2.5300 E-12$ & $5.0421 E-02$ \\
\hline $\mathrm{BH}-212$ & $\mathrm{Cl}$ & 131 & 3.4812E-07 & 2.6574E-09 & $2.3100 E-08$ & $1.0700 \mathrm{E}-23$ & 2.5000E-07 \\
\hline$R A 228$ & $\mathrm{Cl}$ & 131 & $7.4979 \mathrm{E}-04$ & $5.7236 \mathrm{E}-06$ & $6.5400 E-05$ & $2.5300 E-10$ & $7.4800 E-04$ \\
\hline$P B-214$ & $\mathrm{Cl}$ & 130 & $2.0994 \mathrm{E}-06$ & $1.6149 E-07$ & $7.1200 E-07$ & 3. $1200 \mathrm{E}-18$ & 6.1100E- 06 \\
\hline Gl-214 & $\mathrm{Cl}$ & 129 & $1.2880 \mathrm{E}-05$ & 9.9845E-08 & $1.1400 E-07$ & $5.0700 E-18$ & $2.3000 E-07$ \\
\hline$C E-144$ - PR-144 & $\mathrm{Cl}$ & 126 & $7.5015 \mathrm{E}-01$ & 5.9536E-03 & $1.0949 E-02$ & $6.0000 \mathrm{E}-09$ & $5.0200 E-02$ \\
\hline$T L-208$ & $\mathrm{Cl}$ & 126 & 4.6483E-05 & $3.6891 \mathrm{E}-07$ & $4.1200 E-06$ & 1. $6500 \mathrm{E}-16$ & 4.6200E-0S \\
\hline FE-59 & $\mathrm{Cl}$ & 104 & 2.2767E-03 & 2. $1891 \mathrm{E}-05$ & $4.5300 E-05$ & $1.5800 E-14$ & $3.0000 \mathrm{E}-04$ \\
\hline SE-79 & $\mathrm{Cl}$ & 88 & $2.6543 \mathrm{E}-05$ & $3.0162 \mathrm{E}-07$ & $1.3200 E-06$ & t.9000E-14 & $7.9400 E-06$ \\
\hline NB-94 & $\mathrm{Cl}$ & 83 & 4.7017E- 06 & $4.9418 E-08$ & $2.5900 E=07$ & $1.2000 \mathrm{E}-17$ & $1.5900 \mathrm{E}-06$ \\
\hline$E U+152$ & Ci & 73 & 4.6666E-03 & 6. $3926 \mathrm{E}-05$ & $3.3300 \mathrm{E}-04$ & 1.5000E-12 & $2.5500 E-03$ \\
\hline $5 N-151$ & $\mathrm{Cl}$ & 73 & $8.4500 E-02$ & 1. $1575 E-03$ & $1.4840 \mathrm{E}-03$ & 4.7000E-10 & $5.7000 \mathrm{E}-0.3$ \\
\hline EU-155 & $\mathrm{Cl}$ & 64 & $2.3881 E-02$ & 3.7315E-04 & $2.1240 \mathrm{E}-03$ & $1.2000 \mathrm{E}-14$ & 1. $6500 \mathrm{E}-02$ \\
\hline PE-CI & $\mathrm{Cl}$ & 62 & $0.0000 E+00$ & $0.0000 E+\infty 0$ & $0.0000 E+00$ & $0.0000 E+\infty 0$ & $0.0000 E+00$ \\
\hline TOTAL-ALPHA & $\mathrm{Cl}$ & 60 & $0.0000 E+00$ & $0.0000 E+\infty 0$ & $0.0000 \mathrm{E}+00$ & $0.0000 E+\infty 0$ & $0.0000 E+00$ \\
\hline $\cos 58$ & $\mathrm{Cl}$ & 55 & 8.3768E-03 & $1.5231 \mathrm{E}-04$ & $4.2200 \mathrm{E} \cdot 04$ & $2.5000 \mathrm{E}-07$ & 2.8000 E- 03 \\
\hline$F E-55$ & $\mathrm{Cl}$ & 51 & 2.769бE-02 & $5.4306 E-04$ & $2.4730 \mathrm{E}-03$ & $2.8000 E \cdot 09$ & $1.7000 E-02$ \\
\hline$N A-22$ & $\mathrm{Cl}$ & 50 & $38498 E-03$ & 7.6996E-05 & $2.4600 E-04$ & $5.2000 \mathrm{E}-10$ & $1.5500 E-03$ \\
\hline NI-59 & $\mathrm{Cl}$ & 46 & 1.3595E-05 & 2.9553E-07 & 6. 1400E-07 & 1. $8300 \mathrm{E}-12$ & 3.8000E-06 \\
\hline$P+32$ & $\mathrm{Cl}$ & 43 & 5.1087E-03 & 1. $1881 E-04$ & $5.4600 E-04$ & 3. 1000E-OE & $3.6000 \mathrm{E}-03$ \\
\hline S-35 & $\mathrm{Cl}$ & 42 & 1.1986E-02 & $2.8538 E-04$ & 6.7100E-04 & $1.0200 \mathrm{E}-09$ & $3.0000 \mathrm{E}-03$ \\
\hline CS-134 & $\mathrm{Cl}$ & 41 & $48495 \mathrm{E}-03$ & 1. $1828 E-04$ & $5.7500 E-04$ & $2.2000 \mathrm{E}-12$ & $3.6200 \mathrm{E}-03$ \\
\hline SB- 126 & $\mathrm{Cl}$ & 40 & $2.3312 \mathrm{E}-03$ & 5. $8279 E-05$ & $1.4700 \mathrm{E}-04$ & $5.0000 \mathrm{E}-12$ & $9.0000 \mathrm{E}-04$ \\
\hline KR-85 & $\mathrm{Cl}$ & 39 & 1.8424E-04 & 4. $7241 \mathrm{E}-06$ & $1.2200 \mathrm{E}-05$ & $8.0000 \mathrm{E}-08$ & $7.9000 E-05$ \\
\hline$T E+125 \mathrm{M}$ & $\mathrm{Cl}$ & 35 & $13696 E-03$ & 3.9703E-05 & $5.8800 E-05$ & $2.3600 \mathrm{E}-08$ & 2. $4000 \mathrm{E}-04$ \\
\hline $\mathrm{ZN}-65$ & $\mathrm{Cl}$ & 29 & 2.8852E-04 & 9.9491E-06 & 4.2200E-05 & $1.3500 \mathrm{E}-10$ & 2.2000E-04 \\
\hline$K-40$ & $\mathrm{Gl}$ & 22 & 6. $8408 E-05$ & 3. $1095 \mathrm{E}-06$ & 5. $3500 \mathrm{E}-06$ & $5.1500 E-0 B$ & 2.0500E-05 \\
\hline$C A-45$ & $\mathrm{Cl}$ & 19 & $39458 \mathrm{E}-03$ & $2.0768 \mathrm{E}-04$ & $4.5500 \mathrm{E}-04$ & $3.9100 E-00$ & $2.0000 \mathrm{E}-03$ \\
\hline $\mathrm{BE}-7$ & $\mathrm{C}$ & 16 & $52240 \mathrm{E}-03$ & $3.2650 \mathrm{E}-04$ & $5.9300 E-04$ & 3.3000 E- 10 & $2.33806-03$ \\
\hline CR-51 & $\mathrm{Cl}$ & 16 & $3.0952 \mathrm{E}-03$ & $1.9345 E-04$ & $2.6100 \mathrm{E}-04$ & $2.8224 \mathrm{E}-10$ & $1.0000 \mathrm{E}-03$ \\
\hline TA- 182 & $\mathrm{Cl}$ & 15 & 1.1571E-01 & $7.7140 \mathrm{E}-\mathrm{DB}$ & $1.1476 \mathrm{E}-02$ & $7.6000 \mathrm{E}-0 \mathrm{~B}$ & $3.4000 E-02$ \\
\hline $\cos 57$ & $\mathrm{Cl}$ & 13 & $8.0789 \mathrm{E}-05$ & $6.2145 \mathrm{E}-06$ & $1.0800 \mathrm{E}-05$ & $2.1000 E-11$ & $3.4580 \mathrm{E}-0.5$ \\
\hline $\mathrm{PO}-210$ & $\mathrm{Cl}$ & 13 & $3.6129 E-05$ & $2.7792 \mathrm{E}-0 \mathrm{~B}$ & $9.4700 \mathrm{E}-06$ & $4.9100 \mathrm{E}-25$ & $3.4300 \mathrm{E}-05$ \\
\hline NE-93M & $\mathrm{Cl}$ & 10 & 2.2383E-04 & $2.2383 \mathrm{E}-05$ & 3.6900E-05 & $4.7300 \mathrm{E}-13$ & $9.5000 E-05$ \\
\hline$C D-109$ & $\mathrm{Cl}$ & 8 & $2.8230 \mathrm{E}-06$ & $3.5288 E-07$ & $2.3600 E-07$ & 2.0000E-08 & 6.0000E-07 \\
\hline $\cos 6 \theta$ & $\mathrm{Cl}$ & 7 & 2.6419E-06 & $3.7741 E-07$ & $2.5900 \mathrm{E}-07$ & $1.5970 E-07$ & $9.2820 E-07$ \\
\hline NB- 95 & $\mathrm{Cl}$ & 7 & $1.0543 \mathrm{E}-04$ & $1.5061 \mathrm{E}-05$ & $1.0400 \mathrm{E}-05$ & $3.20000-06$ & $2.8300 \mathrm{E}-05$ \\
\hline NI-63 METAL & $\mathrm{Cl}$ & 7 & $2.9400 E-10$ & $4.2000=-11$ & $0.0000 E+00$ & $4.2000 E-11$ & $4.2000 \mathrm{E}-11$ \\
\hline ZR-95 - NB-95M & $\mathrm{Cl}$ & 7 & $6.8821 E-04$ & $9.8316 \mathrm{E}-05$ & $1.7000 \mathrm{E}-04$ & $1.0000 E-07$ & $4.3800 E-04$ \\
\hline $1-125$ & $\mathrm{Cl}$ & 5 & $2.0500 E-05$ & 4. $1000 \mathrm{E} \cdot 06$ & $4.0700 \mathrm{E}-06$ & $1.0000 \mathrm{E}-07$ & $1.0000 \mathrm{E}-05$ \\
\hline$A G-110$ & $a$ & 4 & 4. $1353 E-06$ & $1.0338 \mathrm{E}-06$ & 6. $3200 \mathrm{E}-07$ & 6.5260E-08 & $1.3500 E-06$ \\
\hline$A G-110 M-A G=110$ & $\mathrm{cl}$ & 4 & $2.0235 E-06$ & 6.0586E-07 & $8.6300 \mathrm{E}-07$ & $7.3500 \mathrm{E}-08$ & $1.8000 E-06$ \\
\hline NB-95M & $\mathrm{Cl}$ & 3 & 1.9872E-08 & $6.6240 E-09$ & $7.8900 \mathrm{E}-09$ & 8. $1200 \mathrm{E}-10$ & $1.6600 \mathrm{E}-08$ \\
\hline RU-103 - RH-103M & $\mathrm{Cl}$ & 3 & $1.9361 E-07$ & $6.4536 \mathrm{E} .08$ & $1.0800 \mathrm{E}-07$ & 5.6800E-10 & $1.8000 \mathrm{E}-07$ \\
\hline SC- -4 & $\mathrm{Cl}$ & 3 & $4.9000 E-05$ & $1.6333 E-05$ & $1.33006-05$ & $5.0000 \mathrm{E}-06$ & 3.1000E-05 \\
\hline TA-1B3 & $\mathrm{Cl}$ & 3 & 4.3900E-04 & $1.4633 E-04$ & $5.8300 \mathrm{E}-05$ & $7.9000 E-05$ & $1.8000 E-04$ \\
\hline CS- 135 & $\mathrm{Cl}$ & 2 & 6.6200E-13 & $3.3100 \mathrm{E}-13$ & $2.0900 E-13$ & $1.8300 \mathrm{E}-13$ & $4.7900 \mathrm{E}-13$ \\
\hline $\mid-131$ & $\mathrm{Cl}$ & 2 & 3.0000E-07 & $1.5000 \mathrm{E}-07$ & $7.0700 \mathrm{E} \cdot 08$ & $1.0000 \mathrm{E}-07$ & $2.0000 \mathrm{E}-07$ \\
\hline PA-231 & $\mathrm{Cl}$ & 2 & $2.4000 \mathrm{E}-07$ & 1.2000E-07 & $1.7000 E-07$ & 4. $0100 \mathrm{E}-16$ & $2.4000 E-07$ \\
\hline PD-107 & $\mathrm{Cl}$ & 2 & 1.3360E- 13 & $66800 \mathrm{E}-14$ & 4.2100E-14 & $3.7000 \mathrm{E}-14$ & $9.6600 \mathrm{E}-14$ \\
\hline SE-75 & $\mathrm{Cl}$ & 2 & 1.4103E-03 & $7.0513 \mathrm{E}-04$ & $9.9700 E-04$ & $25400 E-07$ & $1,4100 \mathrm{E}-03$ \\
\hline$V-49$ & $\mathrm{Cl}$ & 2 & $5.4299 \mathrm{E}-08$ & $2.7150 E-08$ & $3.7300 \mathrm{E}-08$ & 8. $0640 \mathrm{E}-10$ & $5.3493 E-08$ \\
\hline ZR- 93 & $\mathrm{Cl}$ & 2 & $4.6300 \mathrm{E}-12$ & $2.3150 E-12$ & 1.4600E-12 & 1.2800E-12 & $3.3500 \mathrm{E}-12$ \\
\hline AU- 195 & $\mathrm{Cl}$ & 1 & 1. $1660 \mathrm{E}-05$ & 1.1660E-C5 & $0.0000 E+00$ & 1. $1660 \mathrm{E}-05$ & $1.1660 \mathrm{E}-05$ \\
\hline$B A-133$ & $\mathrm{Cl}$ & 1 & 3.7600E-03 & $37600 E-03$ & $0.0000 E+00$ & $3.7600 \mathrm{E}-03$ & 3.7600E-03 \\
\hline CE-141 & $\mathrm{Cl}$ & 1 & $1.4500 E-08$ & $4.4500 E-08$ & $0.0000 E+\infty$ & $1.4500 E-08$ & $1.4500 E-08$ \\
\hline $\mathrm{CL}-36$ & $\mathrm{Cl}$ & 1 & $3.5600 \mathrm{E}-05$ & $3.5600 \mathrm{E}-05$ & $0.0000 E+00$ & 3.5600E-05 & 3.5600E 05 \\
\hline GD-154 & $\mathrm{Cl}$ & 1 & 1.2500E-05 & 1. $2500 \mathrm{E}-05$ & $0.0000 \mathrm{E}+00$ & 1. $2500 \mathrm{E}-05$ & $1.2500 \mathrm{E}-05$ \\
\hline$M F-181$ & $\mathrm{Cl}$ & 1 & 2.1037E-02 & 2. $1037 \mathrm{E}-02$ & $0.0000 E+00$ & $2.1037 E-02$ & 2. $1037 \mathrm{E}-02$ \\
\hline NO-93 & $\mathrm{Cl}$ & 1 & $9.9465 \mathrm{E}-08$ & $9.9465 \mathrm{E}-08$ & $0.0000 E+00$ & $9.9465 \mathrm{E}-08$ & 9.9465E-08 \\
\hline PT-195M & $\mathrm{Cl}$ & 1 & 2.8000E-05 & $2.8000 E-05$ & $0.0000 E+00$ & $2.8000 \mathrm{E}-05$ & $2.8000 \mathrm{E}-05$ \\
\hline SB-124 & $\mathrm{Cl}$ & 1 & $2.7000 \mathrm{E}-07$ & $2.7000 E-07$ & $0.0000 \mathrm{E}+00$ & $2.7000 E-07$ & 2.7000E-07 \\
\hline$W-185$ & $\mathrm{Cl}$ & 1 & 2.0000 E- 07 & $2.0000 E-07$ & $0.0000 E+00$ & 2.0000E-07 & $2.0000 \mathrm{E}-07$ \\
\hline$X E-131 M$ & $\mathrm{Cl}$ & 1 & $2.6000 \mathrm{E}-05$ & 2. $6000 \mathrm{E}-05$ & $0.0000 E+00$ & 2. $6000 \mathrm{E}-05$ & $2.6000 \mathrm{E}-05$ \\
\hline
\end{tabular}


TABLE 11

STATISTICAL ANALYSIS OF FISSION PRODUCTS

IN ASCENDING ORDER BASED ON ACCUMULATED ACTIVITY

(1 SHEET)

\begin{tabular}{|c|c|c|c|c|c|c|c|}
\hline ISOTOPE & UNIT & COUNT & ACCUMULATION & AVERAGE & STO DEV & MININUM & MAXIMUMM \\
\hline TOTAL BETAIGAMMA & $\mathrm{Cl}$ & 2764 & $7.0532 E+04$ & $2.5518 E+01$ & $1.3391 E+03$ & $6.0000 \mathrm{E}-12$ & $7.0400 E+04$ \\
\hline SR-90 - Y90 & $\mathrm{Cl}$ & 780 & $2.7455 E+01$ & $3.5199 \mathrm{E}-02$ & $7.1501 E-01$ & $1.3600 \mathrm{E}-10$ & t.9900E+01 \\
\hline CS-137- B4 137M & $\mathrm{cl}$ & 989 & $2.5059 E+01$ & $2.5338 \mathrm{E}-02$ & 4.1791E-01 & $9.0000 \mathrm{E}-13$ & $9.5000 E+\infty$ \\
\hline $\mathrm{H}-3$ & $\mathrm{Cl}$ & 571 & $4.2220 E+00$ & $7.3940 \mathrm{E}-03$ & $4.8866 E-02$ & $6.0000 \mathrm{E}-12$ & 6.2250E-01 \\
\hline CE-144-FPR-144 & $\mathrm{Cl}$ & 126 & $7.5015 E-01$ & 5. $9536 \mathrm{E}-03$ & 1.0949E-02 & $6.0000 E-09$ & $5.0200 \mathrm{E}-02$ \\
\hline PM-14.7 & $\mathrm{Cl}$ & 210 & 4.2529E-01 & $2.0252 \mathrm{E}-03$ & $5.3290 \mathrm{E}-03$ & $4.0600 \mathrm{E}-11$ & $3.4700 E-02$ \\
\hline EU-154 & $\mathrm{Cl}$ & 157 & $2.1799 E-01$ & $1.3885 E-03$ & $9.6890 E-03$ & $2.3000 E-14$ & $8.8400 E-02$ \\
\hline$R U-106-F 2+-106$ & $\mathrm{Cl}$ & 152 & 2.1779E-01 & $1.4328 E-03$ & $3.2640 \mathrm{E}-03$ & $1,9000 \mathrm{E}-14$ & $2.7000 E-02$ \\
\hline co.65 & $\mathrm{cl}$ & 1004 & $2.0084 E-01$ & $2.0004 \mathrm{E}-04$ & $1.7200 \mathrm{E}-03$ & $40000 E-11$ & $3.9927 E-02$ \\
\hline TA-182 & $\mathrm{Cl}$ & 15 & $1.1571 \mathrm{E}-01$ & $7.7140 E-03$ & $1.1476 \mathrm{E}-02$ & 7. $6000 \mathrm{E}-08$ & $3.4000 E-02$ \\
\hline$c-14$ & $\mathrm{Cl}$ & 404 & $9.6027 \mathrm{E}-02$ & $2.3768 E-04$ & $2.3410 E-03$ & $1.5000 \mathrm{E}-18$ & $3.7400 \mathrm{E}-02$ \\
\hline SM-151 & C1 & 73 & $8.4500 \mathrm{E}-02$ & 1. $1575 E-03$ & 1. $4840 E-03$ & $47000 E-10$ & $5.7000 E-03$ \\
\hline N1-63 & $\mathrm{Cl}$ & 144 & $5.3706 E-02$ & $3.7296 E-04$ & $4.2010 \mathrm{E}-03$ & $2.5300 \mathrm{E}-12$ & $5.0421 E-02$ \\
\hline FE-5:5 & $\mathrm{Cl}$ & 51 & $2.7696 \mathrm{E}-02$ & 5. $4306 \mathrm{E}-0.4$ & 2.4730E-03 & $2.8000 E-00$ & $1.7000 E-02$ \\
\hline EU-1E5 & $\mathrm{Cl}$ & 64 & $2.3881 E-02$ & $3.7315 E-0.4$ & $2.1240 E-03$ & 1.2000E-14 & $1.6500 E-02$ \\
\hline TC-9: & $\mathrm{Cl}$ & 312 & $2.3481 E-02$ & $7.5259 E-05$ & $6.9500 \mathrm{E}-0.4$ & $3.0100 \mathrm{E}-13$ & $8.8900 E-03$ \\
\hline MF- 181 & $\mathrm{Cl}$ & 1 & $2.1037 E-02$ & $2.1037 \mathrm{E}-02$ & $0.0000 E+\infty 0$ & $2.1037 \mathrm{E}-02$ & 2. 1037E -02 \\
\hline s-35 & $\mathrm{Cl}$ & 42 & 1.1986E-02 & $2.8538 E-04$ & $5.7100 \mathrm{E}-04$ & $1.0200 E-09$ & $3.0000 E-03$ \\
\hline NL-60 & $\mathrm{Cl}$ & 151 & $1.1855 E-02$ & 7.8509E-05 & $3.1800 E-04$ & $9.0000 E-11$ & $2.7000 E-03$ \\
\hline MN-54 & $\mathrm{Cl}$ & 214 & 1.1621E-02 & 5.4303E-05 & $2.3300 E-04$ & 4.2000E-11 & $2.8900 E-03$ \\
\hline $\mathrm{CO}-5 \mathrm{~B}$ & $\mathrm{Cl}$ & 56 & 8.3768E-03 & 1.5231E-04 & $4.2200 E-04$ & 2.5000E-07 & $2.8000 \mathrm{E}-03$ \\
\hline BE-7 & $\mathrm{Cl}$ & 16 & $5.22 \mathrm{dOE}+03$ & 3.2650E-OA & $5.8300 E-04$ & $3.3000 \mathrm{E}-10$ & $2.3380 \mathrm{E}-03$ \\
\hline P-32 & $\mathrm{Cl}$ & 43 & 5. $1087 E .03$ & $1,1881 \mathrm{E}-04$ & $5.4600 E-04$ & $3.1000 E-06$ & $3.6000 E-03$ \\
\hline $\operatorname{cs}-134$ & $\mathrm{Cl}$ & 41 & $4.8495 \mathrm{E}-03$ & 1. $1828 \mathrm{E}-04$ & $5.7600 E-04$ & 2.2000E-12 & 3.6200E-03 \\
\hline$E U-152$ & $\mathrm{cl}$ & 73 & d.6656E-03 & 6. 3926E-05 & $3.3300 E-04$ & $1.5000 \mathrm{E}-12$ & $2.5500 \mathrm{E}-03$ \\
\hline CA-45 & $\mathrm{Ct}$ & 19 & $3.9458 \mathrm{E}-03$ & $2.0768 E-04$ & $4.5500 \mathrm{E}-04$ & $3.9100 \mathrm{E}-09$ & $2.0000 E-03$ \\
\hline NA-22 & $\mathrm{Cl}$ & 50 & $3.8498 E-03$ & 7.6996E-0.5 & $2.4600 E-04$ & 6. $2000 \mathrm{E}-10$ & $1.5600 \mathrm{E}-03$ \\
\hline$B 4-133$ & $\mathrm{Cl}$ & 1 & 3.7600E-03 & $37600 E-03$ & $0.0000 E+00$ & $3.7600 E-03$ & 3.7600E-03 \\
\hline $1-129$ & $\mathrm{Cl}$ & 192 & 3.1596E-03 & 1. $6456 \mathrm{E}-05$ & 1. $1500 \mathrm{E}-04$ & $1.7600 \mathrm{E}-14$ & $1.2700 E-03$ \\
\hline CR-51 & $\mathrm{Cl}$ & 16 & $3.0952 \mathrm{E}-03$ & 1.9346E-04 & $2.6100 \mathrm{E}-04$ & $2.8224 \mathrm{E}-10$ & $1.0000 \mathrm{E}-03$ \\
\hline SB-126 & $\mathrm{Cl}$ & 40 & 2.3312E-03 & 5.8279E-05 & $1.4700 E-04$ & $5.0000 \mathrm{E}-12$ & 9.0000E-04 \\
\hline$F E-5: 3$ & $\mathrm{Cr}$ & 104 & 2.2767E-03 & 2. IOSTE-O5 & 4.5300E-05 & 1.5800E-14 & $3.0000 \mathrm{E}-04$ \\
\hline SE-7:5 & $\mathrm{Cl}$ & 2 & 1.4103E-03 & $7.0513 E-04$ & $9.9700 \mathrm{E}-04$ & $25400 \mathrm{E}-07$ & $1.4100 \mathrm{E}-03$ \\
\hline TE-125:M & $\mathrm{Cl}$ & 35 & $1.3896 E-03$ & $3.9703 E-06$ & $5.8800 E-05$ & $2.3600 \mathrm{E}-08$ & $2.4000 \mathrm{E}-0.4$ \\
\hline RA2:19 & $\mathrm{Cl}$ & 131 & 7.4979E-04 & $5.7236 \mathrm{E}-06$ & $6.5400 \mathrm{E}-05$ & $2.5300 \mathrm{E}-10$ & $7.4800 \mathrm{E}-04$ \\
\hline ZR-95 - NB-95M & $\mathrm{Cl}$ & 7 & $6.8821 \mathrm{E}-04$ & $98316 \mathrm{E}-05$ & $1.7000 E-04$ & $1.0000 E-07$ & 4.3800E-04 \\
\hline TA-1E3 & $\mathrm{Cl}$ & 3 & 4.3900E-04 & 1.4633E-04 & $5.8300 \mathrm{E}-05$ & $79000 E-05$ & $1.8000 \mathrm{E}-04$ \\
\hline $2 \mathrm{~N}-65$ & $\mathrm{Cl}$ & 29 & $2.8852 \mathrm{E}-04$ & $99491 E-06$ & $4.2200 E-05$ & $1.3500 E-10$ & $2.2000 E-04$ \\
\hline NB-92M & $\mathrm{Cl}$ & 10 & 2.2383E-04 & $2.2383 E-05$ & $3.6900 E-05$ & $4.7300 \mathrm{E}-13$ & $9.5000 E-00$ \\
\hline KR-85 & $\mathrm{CI}$ & 39 & $1.8424 E-04$ & 4.7241E-06 & 1.2200E-05 & 3. $0000 E-08$ & $7.9000 E-05$ \\
\hline NB-95 & $\mathrm{Cl}$ & 7 & $1.0543 \mathrm{E}-04$ & $1.5061 \mathrm{E}-05$ & $1.0400 E-05$ & $3.2000 E-06$ & $2.8300 \mathrm{E}-05$ \\
\hline $\cos 57$ & $\mathrm{Cl}$ & 13 & 8.0789E-05 & 6. 2145E-06 & 1.0900E-05 & 2. $1000 \mathrm{E}-11$ & 3.45BOE-06 \\
\hline$k-40$ & $\mathrm{Cl}$ & 22 & $6.8408 \mathrm{E}-05$ & 3. $1095 \mathrm{E}-06$ & $3.3500 \mathrm{E}-06$ & 5. $1500 E-08$ & $2.0500 \mathrm{E} \cdot 05$ \\
\hline $5 C-47$ & $\mathrm{Cl}$ & 3 & $4.9000 \mathrm{E}-05$ & 1.6333E-05 & $1.3300 E-05$ & $5.0000 \mathrm{E}-06$ & $3.1000 \mathrm{E}-05$ \\
\hline$T L-2 C B B$ & $\mathrm{Cl}$ & 126 & 4.6483E-05 & $36891 E-07$ & 4. $1200 \mathrm{E}-06$ & 1.6500E-16 & 4.6200E-05 \\
\hline$P 0-210$ & $\mathrm{Cl}$ & 13 & $3.6129 E-05$ & 2.7792E-06 & $9.4700 \mathrm{E}-06$ & $49100 E-25$ & $3.4300 \mathrm{E}-0 \mathrm{~S}$ \\
\hline $\mathrm{CL}-36$ & $\mathrm{Cl}$ & 1 & $3.5600 \mathrm{E}-05$ & 3.5600E-05 & $0.0000 E+00$ & $3.5600 E-05$ & $3.6600 E-05$ \\
\hline PT-19.5M & $\mathrm{Cl}$ & 1 & $2.8000 \mathrm{E}-05$ & $2.8000 \mathrm{E}-05$ & $0.0000 E+00$ & $2.8000 \mathrm{E}-05$ & $2.8000 \mathrm{E}-06$ \\
\hline SE-79 & $\mathrm{Cl}$ & 88 & 2.6543E-05 & 3.0162E-07 & $1.3200 E-06$ & $1.9000 \mathrm{E}-14$ & $7.9400 \mathrm{E}-06$ \\
\hline$X E-131 M$ & CI & 1 & $2.6000 E-05$ & 2.6000E-05 & $0.0000 E+\infty 0$ & 2.6000E-05 & $2.6000 E-05$ \\
\hline PB-214 & $\mathrm{Cl}$ & 130 & $2.0994 E-05$ & 1.6149E-07 & $7.1200 E-07$ & $3.1200 E-18$ & 8.1100E-06 \\
\hline $1-1215$ & CI & 5 & $2.0500 E-08$ & 4. $1000 E-06$ & $4.0700 E-05$ & $1.0000 E-07$ & $1.0000 E-06$ \\
\hline $\mathrm{PQ}-212$ & $\mathrm{Cl}$ & 216 & $1.4087 \mathrm{E}-06$ & $6.5220 E-08$ & $3.2400 \mathrm{E}-07$ & $9.5500 \mathrm{E}-19$ & $2.4700 E-06$ \\
\hline N1-59 & $\mathrm{cl}$ & 46 & $1.3595 \mathrm{E}-05$ & $2.9553 E-07$ & $8.1400 E=07$ & $1.8300 \mathrm{E}-12$ & $3.8000 E-06$ \\
\hline $\mathrm{Bl}-2 \cdot 14$ & $\mathrm{Cr}$ & 129 & $1.2880 \mathrm{E}-0.5$ & $9.9645 E-08$ & $1.1400 E-07$ & 5.0700E-18 & $2.3000 E-07$ \\
\hline GO-154 & Cl & 1 & 1.2500E-05 & $1.2500 E-05$ & $0.0000 \mathrm{E}+\infty 0$ & 1.2500E-05 & $1.2500 E-05$ \\
\hline AU-195 & $\mathrm{Cl}$ & 1 & 1.1660E-05 & $1.1660 \mathrm{E}-05$ & $0.0000 E+00$ & 1.1680E-05 & 1. $1660 \mathrm{E}-06$ \\
\hline$A G-110$ & $\mathrm{Cl}$ & 4 & 4.1353E-D6 & $1.0338 E-06$ & 6.3200E-07 & 8. $5260 E-08$ & 1.3500E-06 \\
\hline NB-914 & $\mathrm{Cl}$ & 83 & $4.1017 \mathrm{E}-06$ & $4.9418 E-08$ & $2.5900 \mathrm{E}-07$ & $1.9000 \mathrm{E}-17$ & $1.5900 E .06$ \\
\hline CD-109 & $\mathrm{Cl}$ & 8 & 2.8230E-06 & 3.5288E-07 & $2.3500 \mathrm{E} .07$ & $2,0000 E-08$ & $6,0000 E-07$ \\
\hline $\cos \$ 6$ & $\mathrm{Cl}$ & 7 & $2.6419 E-06$ & $3.7741 E-07$ & $2.5900 \mathrm{E}=07$ & 1.5970E-07 & 9.2820E-07 \\
\hline$A G-110 M-A G-110$ & $\mathrm{Cr}$ & 4 & $2.0235 E-06$ & $5.0586 E-07$ & $8.6300 E \times 07$ & $7.3500 \mathrm{E}-0.8$ & $1.8000 E-06$ \\
\hline $\mathrm{Bl}-2 \mathrm{1} 2$ & $\mathrm{Cl}$ & 131 & $3.4812 \mathrm{E}-07$ & $2.6674 \mathrm{E}-09$ & $2.3100 E-08$ & $1.07006-23$ & $2.5000 \mathrm{E}-07$ \\
\hline $1-\$ 31$ & $\mathrm{Cl}$ & 2 & 3.0000E-07 & $1.5000 \mathrm{E}-07$ & $7.0700 E-08$ & $1.0000 E-07$ & $2.0000 E-07$ \\
\hline SB-124 & $\mathrm{Cl}$ & 1 & 2.7000E-07 & $2.7000 \mathrm{E}-07$ & $0.0000 E+00$ & 2.7000E-07 & 2.7000 E- 07 \\
\hline PA-231 & $\mathrm{Cl}$ & 2 & $2.4000 E-07$ & $\$ .2000 \mathrm{E}-07$ & $1.7000 \mathrm{E}-07$ & $4.0100 E-16$ & $2,4000 \mathrm{E}-07$ \\
\hline W-135 & $\mathrm{Cl}$ & 1 & 2.0000E-07 & $2.0000 \mathrm{E}-07$ & $0.0000 E+00$ & 2.0000E-07 & $2.0000 E-07$ \\
\hline RU- 103 - RH-103M & $\mathrm{Cl}$ & 3 & $1.9361 E-07$ & 6. $4536 E-08$ & $1.0800 E-07$ & 5. $6900 \mathrm{E}-10$ & 1.8900E-07 \\
\hline$A C-228$ & $\mathrm{Cl}$ & 202 & $1.1465 E-07$ & $5.6756 \mathrm{E}-10$ & $6.1800 E-09$ & 2. $9300 E-15$ & $8.7000 \mathrm{E}-08$ \\
\hline MO:33 & $\mathrm{Cl}$ & 1 & $99465 E-08$ & $9.9465 \mathrm{E}-08$ & $0.0000 E+00$ & $9.9465 \mathrm{E}-08$ & 9.9465E-08 \\
\hline$V-47$ & ci & 2 & $5.4299 \mathrm{E}-08$ & $2.7150 \mathrm{E} .0 \mathrm{~B}$ & $3.7300 E-08$ & $8.0640 E-10$ & 5.3493E-08 \\
\hline NB-95M & $c_{i}$ & 3 & $1.9972 E \cdot 08$ & $5.6240 E-09$ & $7.9900 E-09$ & 8. $1200 \mathrm{E}-10$ & $1.5600 \mathrm{E}-08$ \\
\hline CE-141 & $\mathrm{Cl}$ & 1 & $1.4500 E-C B$ & 1. $4500 \mathrm{E}-08$ & $0.0000 E+00$ & $1.4500 \mathrm{E}-0 \mathrm{~g}$ & $1.4500 \mathrm{E}-08$ \\
\hline NI-63 METAL & $\mathrm{Cl}$ & 7 & $2.9400 \mathrm{E}-10$ & 4. $2000 E-11$ & $0.0000 E+00$ & 4. $2000 \mathrm{E}-11$ & 4.2000E-11 \\
\hline ZR-93 & $\mathrm{Cl}$ & 2 & $4.6300 \mathrm{E}-12$ & 2. $3150 E-12$ & $1.4600 \mathrm{E}-12$ & 1. $2800 \mathrm{E}-12$ & 3.3500E-12 \\
\hline $\operatorname{cs} 135$ & $\mathrm{GI}$ & 2 & $66200 \mathrm{E}-13$ & 3. $3100 \mathrm{E}-13$ & $2.0900 E-13$ & $1.8300 \mathrm{E}-13$ & 4.79000-13 \\
\hline PD-107 & $\mathrm{C}$ & 2 & 1.3360E-13 & 6. $6800 E-14$ & 4.2100E-14 & $3.7000 \mathrm{E}-14$ & $9.6600 \mathrm{E}-14$ \\
\hline PE-Cl & $\mathrm{Cl}$ & 62 & $0.0000 E+00$ & $0.0000 E+00$ & $0.0000 E+00$ & $0.0000 \mathrm{E}+00$ & $0.0000 E+00$ \\
\hline TOTAL-ALPHA & $\mathrm{Cl}$ & 60 & $0.0000 E+00$ & $0.0000 E+00$ & $0.0000 E+00$ & $0.0000 E+00$ & $0.0000 E+00$ \\
\hline
\end{tabular}


TABLE 12

STATISTICAL ANALYSIS OF FISSION PRODUCTS

IN ASCENDING ORDER BASED ON MAXIMUM ACTIVITY

\begin{tabular}{|c|c|c|c|c|c|c|c|}
\hline ISOTOPE & UNiT & COUNT & ACCUMULATION & AVERAGE & STD DEV & MINIMUM & MAXIMUM \\
\hline TOTAL BETAIGAMMA & $\mathrm{Cl}$ & 2764 & $7.0532 E+04$ & $2.5518 \mathrm{E}+01$ & $1.3391 E+03$ & $6.0000 \mathrm{E}-12$ & $7.0400 E+04$ \\
\hline SR-90 - Y90 & $\mathrm{Ci}$ & 780 & $2.7455 E+01$ & $3.5199 \mathrm{E}-02$ & $7.1501 E-01$ & $1.3600 \mathrm{E}-10$ & $1.9900 \mathrm{E}+04$ \\
\hline $\operatorname{Cs}-137-B 4-137 M$ & $\mathrm{cl}$ & 989 & $2.6059 \mathrm{E}+01$ & $2.5338 \mathrm{E}-02$ & 4.1791E-01 & $8.0000 E-13$ & $9.5000 E+00$ \\
\hline $\mathrm{H}=3$ & $\mathrm{cl}$ & 571 & $4.2220 E+00$ & $7.3940 E-03$ & $4.88666-02$ & $5.0000 \mathrm{E}-12$ & $0.2250 E-01$ \\
\hline EU-154 & $\mathrm{Cl}$ & 157 & $2.1799 E-01$ & $1.3885 E-03$ & $9.6890 E_{-03}$ & $2.3000 E-14$ & $8.8400 E-02$ \\
\hline N1-63. & $\mathrm{cl}$ & 144 & $5.3706 E-02$ & $3.7296 \mathrm{E}-04$ & 4.2010E-03 & $2.5300 \mathrm{E}-12$ & $5.0421 \mathrm{E}-02$ \\
\hline CE-144 - F'R-144 & $\mathrm{Cl}$ & 126 & $7.50+5 E-01$ & $5.9536 \mathrm{E}-03$ & $1.0949 \mathrm{E}-02$ & $6.0000 E-00$ & $\$ .0200 \mathrm{E}-02$ \\
\hline $\cos (1)$ & $\mathrm{Cl}$ & 1004 & $2.0084 E-01$ & $2.0004 E-04$ & $1.7200 \mathrm{E}-03$ & $4.0000 E-11$ & $3.9927 \mathrm{E}-02$ \\
\hline$c-14$ & $\mathrm{cl}$ & 404 & $9.6027 \mathrm{E}-02$ & $2.3769 E-04$ & 2.3410E-03 & $1.5000 \mathrm{E}-18$ & $3.7400 E-02$ \\
\hline PH-147 & $\mathrm{Cl}$ & 210 & 4.2529E-01 & $2.0252 E-03$ & $5.3290 E_{-03}$ & A.0600E-11 & $3.4700 E-02$ \\
\hline T4-182 & $\mathrm{Cl}$ & 15 & 1. $1571 \mathrm{E}-01$ & $7.7140 \mathrm{E}-03$ & $+.1476 E-02$ & $7.6000 E-08$ & $3.4000 E-02$ \\
\hline RU-106 - FiH-106 & $\mathrm{Cl}$ & 152 & 2. 1779E-01 & $1.4328 \mathrm{E}-03$ & 3.2540E-03 & $1.9000 \mathrm{E}-14$ & 2.7000E-02 \\
\hline $\mathrm{HF}-\mathbf{4 8 1}$ & $\mathrm{Cl}$ & 1 & 2. 1037E-02 & $2.1037 E-02$ & $0.0000 E+\infty 0$ & 2.1037E-02 & 2. 1037E-02 \\
\hline$F E-5 t j$ & $\mathrm{Cl}$ & 51 & $2.7696 E-02$ & $5.4306 E-04$ & $2.4730 \mathrm{E}-03$ & $2.8000 E-09$ & $1.7000 E-02$ \\
\hline EU-155 & $\mathrm{Cl}$ & 64 & $2.3881 E-02$ & $3.7316 E-04$ & $2.1240 E-03$ & $1.2000 E-14$ & $1.6600=-02$ \\
\hline TC-9 & c। & 312 & $2.3481 E-02$ & $7.5259 E-00$ & $6.9500 \mathrm{E}-04$ & $3.0100 E-13$ & B. $8000 E-03$ \\
\hline SM-1211 & $\mathrm{Cl}$ & 73 & 8. $4500 \mathrm{E}=02$ & $1.1575 E-03$ & $1.4840 E-03$ & $4.7000 E-10$ & $5.7000 \mathrm{E}-03$ \\
\hline BA-133 & $\mathrm{Cl}$ & 1 & $3.7600 \mathrm{E}-03$ & $3.7600 \mathrm{E}-03$ & $0.0000 E+00$ & $3.7600 E-03$ & $3.7600 \mathrm{E}-03$ \\
\hline $\operatorname{cs}-134$ & $\mathrm{Cl}$ & 41 & $4.8495 \mathrm{E}-03$ & $1.1828 E-04$ & $6.7000 \mathrm{E}-04$ & $2.2000 E-12$ & 3.8200E-03 \\
\hline P-32 & $\mathrm{Cl}$ & 43 & 5. $1087 \mathrm{E}-03$ & $1.1881 E-04$ & $5.4600 \mathrm{E}-04$ & 3. $1000 \mathrm{E}-06$ & $3.6000 \mathrm{E}-03$ \\
\hline $5-35$ & $\mathrm{Cl}$ & 42 & $1.1986 \mathrm{E}-02$ & $2.8538 \mathrm{E}-04$ & $5.7100 E-04$ & $1.0200 \mathrm{E}-\infty 0$ & 3.0000 E-03 \\
\hline$M N-54$ & $\mathrm{Cl}$ & 214 & 1. $1621 \mathrm{E}-02$ & $5.4303 E-05$ & $2.3300 E-04$ & $4.2000 E-11$ & $2.8900 E-03$ \\
\hline CO-5s & $\mathrm{Cl}$ & 55 & 8. $3768 E-03$ & $1.5231 E-04$ & $42200 E-04$ & $2.5000 \mathrm{E}-07$ & $2.8000 \mathrm{E}-03$ \\
\hline NL 60 & $\mathrm{Cl}$ & 151 & 1. $1855 E-02$ & $7.8509 E-05$ & 3.1800E-04 & $9.0000 \mathrm{E} \cdot 11$ & $2.7000 \mathrm{E}-03$ \\
\hline EU-162 & $\mathrm{Cl}$ & 73 & $4.6668 \mathrm{E}-03$ & 6.3926E-05 & $3.3300 \mathrm{E}-04$ & $1.5000 E-12$ & $2.5500 E-03$ \\
\hline$B E-7$ & $\mathrm{C}$ & 16 & 5. $2240 E-03$ & $3.2650 \mathrm{E}-04$ & $5.9300 \mathrm{E}-04$ & $3.3000 E-10$ & $2.3380 \mathrm{E}-03$ \\
\hline$C A 45$ & $\mathrm{Cl}$ & 19 & $39458 \mathrm{E}-03$ & $2.0768 \mathrm{E}-04$ & $45500 E-04$ & $3.9100 \mathrm{E}-09$ & $2.0000=-03$ \\
\hline $\mathrm{NA}-2 \mathrm{Z}$ & $\mathrm{Ci}$ & 50 & $3.8498 E-03$ & $7.6996 E-05$ & $2.4600 E-04$ & $5.2000 \mathrm{E}-10$ & $1,55005-03$ \\
\hline SE-75 & $\mathrm{Cl}$ & 2 & $1.4103 \mathrm{E}-03$ & $7.0513 E-04$ & $9.9700 \mathrm{E}-04$ & $2.5400 \mathrm{E}-07$ & $1.4100 \mathrm{E}-03$ \\
\hline $1-129$ & $\mathrm{Cl}$ & 192 & $3.1596 \mathrm{E}-03$ & 1.6456E-05 & 1.150OE-04 & $1.7600 \mathrm{E}-14$ & 1.2700E-03 \\
\hline CR-51 & $\mathrm{Cl}$ & 16 & $30952 E-03$ & 1.9345E.04 & $2.6100 \mathrm{E}-04$ & $2.8224 E-10$ & t. $0000-03$ \\
\hline $58-125$ & $\mathrm{Cl}$ & 40 & 2.3312E-D3 & $5.8279 E-05$ & $1,4700 \mathrm{E}-04$ & $5.0000 E-12$ & $9.0000 E-04$ \\
\hline RA-228 & $\mathrm{Cl}$ & 131 & $7.4979 E-04$ & $5.7236 \mathrm{E}-06$ & 6.5400E-05 & $2.5300 E-10$ & $7.4800 \mathrm{E}-04$ \\
\hline ZR-95 - NE-95M & $\mathrm{Cl}$ & 7 & $68 \mathrm{~B} 21 \mathrm{E}-04$ & $9.8316 \mathrm{E}-05$ & $1.7000 E-04$ & $1.0000 \mathrm{E}-07$ & $43800 E-04$ \\
\hline FE- 59 & $\mathrm{Cl}$ & 104 & $2.2767 \mathrm{E}-03$ & $2.1891 E-05$ & 4.5300E-05 & 1.5800 E-14 & $3.0000 E-04$ \\
\hline$T E-125 \mathrm{M}$ & $\mathrm{C}$ & 35 & $1.3896 \mathrm{E}-03$ & $3.9703 \mathrm{E}-05$ & 5.8800E-05 & $2.3600 E-08$ & $2.4000 \mathrm{E}-04$ \\
\hline ZN-6.5 & $\mathrm{Cl}$ & 29 & $2.8852 E-04$ & $9.9491 E .06$ & $4.2200 \mathrm{E}-05$ & $1.3500 E-10$ & $22000 \mathrm{E}-04$ \\
\hline TA-183 & $\mathrm{Cl}$ & 3 & 4.3900E-04 & 1. $4633 \mathrm{E}-04$ & 5.8300E-05 & $7.9000 \mathrm{E}-05$ & $1,8000 \mathrm{E}-04$ \\
\hline NE-93M & $\mathrm{Cl}$ & 10 & $2.2383 E-04$ & $2.2393 \mathrm{E}-05$ & 3.6900E-05 & $4.7300 \mathrm{E}-13$ & 9.5000E-05 \\
\hline KR-85 & $\mathrm{Cl}$ & 39 & $1.8424 \mathrm{E}-04$ & $4.7241 E .06$ & $1.2200 \mathrm{E}-05$ & $8.0000 E-08$ & $7.9000 \mathrm{E}-05$ \\
\hline TL-208 & $\mathrm{Cl}$ & 128 & $4.6483 \mathrm{E}-05$ & $3.689+2-07$ & $4.1200 E-06$ & $1.6500 \mathrm{E}-16$ & $4.6200=-06$ \\
\hline $\mathrm{CL}-35$ & $\mathrm{C}$ & 1 & $3.5600 \mathrm{E}-05$ & $3.5600 \mathrm{E}-05$ & $0.0000 E+00$ & $3.5600 \mathrm{E}-06$ & $3.5600=05$ \\
\hline Co-57 & $\mathrm{Cl}$ & 13 & B. $078 \mathrm{SE}-05$ & $6.2145 E-06$ & $1.0800 E-05$ & $2.1000 \mathrm{E}-11$ & $3.4580 E-05$ \\
\hline PO-210 & $\mathrm{Cl}$ & 13 & $3.6129 E-05$ & $2.7792 E-06$ & $9.4700 \mathrm{E}-06$ & $4.9100 E-25$ & $3.4300 E-05$ \\
\hline $5 C-47$ & $\mathrm{Cl}$ & 3 & $4.9000 \mathrm{E}-05$ & $1.6333 \mathrm{E}-05$ & $1.3300 E-05$ & $5.0000 E-06$ & $3.1000 \mathrm{E}-0 \mathrm{~S}$ \\
\hline NB-95 & cl & 7 & 1. $0543 \mathrm{E}-04$ & $1.5061 \mathrm{E}-05$ & $1.0400 \mathrm{E}-05$ & $3.2000 E-06$ & $2.8300 E-06$ \\
\hline PT-19!5M & $\mathrm{Cl}$ & 1 & $2.8000 \mathrm{E}-05$ & $2.8000 \mathrm{E}-05$ & $0.0000 E+00$ & $2.8000 E-\infty$ & $2.8000 \mathrm{E}-05$ \\
\hline$X E-131 M$ & $\mathrm{Cl}$ & 1 & $2.6000 \mathrm{E}-05$ & $2.6000 \mathrm{E}-05$ & $0.0000 E+\infty$ & $2.6000 E-05$ & $2.6000 E-05$ \\
\hline$k-4 c k$ & $\mathrm{Cl}$ & 22 & $68408 E-05$ & $3.1095 \mathrm{E}-06$ & $5.3500 E-06$ & $5.1500 \mathrm{E}-08$ & $2.0500 E-05$ \\
\hline GD-154 & $\mathrm{Cl}$ & 1 & 1. $2500 \mathrm{E}-0.5$ & $1.2500=-05$ & $0.0000 E+00$ & $1.2500 \mathrm{E}-0 \delta$ & $1.2500 E-00$ \\
\hline$A \cup-196$ & $\mathrm{Cl}$ & 1 & 1. 1660 E-06 & $1.1680 \mathrm{E}-05$ & $0.0000 E+00$ & 1. $1600 E-05$ & 1. $1680 \mathrm{E}-05$ \\
\hline$-12 ! j$ & $\mathrm{Cl}$ & 5 & $2.0500 \mathrm{E}-05$ & $4.1000 \mathrm{E}-06$ & $4.0700 E-06$ & $1.0000 \mathrm{E}-07$ & $1,0000 E-00$ \\
\hline $\mathrm{PE}-214$ & $\mathrm{Cl}$ & 130 & $2.0994 \mathrm{E}-05$ & $1.6148 E-07$ & 7. $1200 E-07$ & 3.1200E-18 & 8.1100E-06 \\
\hline SE-79 & $\mathrm{cl}$ & 88 & $2.6543 \mathrm{E}-0.6$ & $3.0162 E-07$ & 1. $3200 E-06$ & 1.9000 E-14 & $7.9400 E-06$ \\
\hline N-5: & C। & 46 & $1.3596 E-05$ & $2.9553 \mathrm{E}-07$ & 8. $1400 \mathrm{E}-07$ & $1.8300 \mathrm{E}-12$ & $3.8000=-06$ \\
\hline $\mathrm{PB}-212$ & $\mathrm{Cl}$ & 210 & 1. $4087 \mathrm{E}-05$ & $6.5220 \mathrm{E}-08$ & $3.2400 \mathrm{E}-07$ & $9.5500 E-19$ & $2.4700 E-08$ \\
\hline$A G-110 M=A G-1$ to & $\mathrm{Cl}$ & 4 & $2.0235 \mathrm{E}-06$ & $5.0586 E-07$ & $8.6300 \mathrm{E}-07$ & $7.3500 E-08$ & $1.8000 \mathrm{E}-06$ \\
\hline NB-S14 & $\mathrm{Cl}$ & 83 & $+1017 E-06$ & $4.9418 E-08$ & $2.5900 \mathrm{E}-07$ & 1.9000 E-17 & $1.5900 \mathrm{E}-06$ \\
\hline$A G-110$ & cl & 4 & 4. $1353 E-06$ & $1.0338 E-06$ & $6.3200 \mathrm{E}-07$ & $8.5260 E-08$ & $1.3500 \mathrm{E}-06$ \\
\hline co- $\$ 6$ & $\mathrm{Cl}$ & 7 & $2.6419 E-06$ & $3.7741 \mathrm{E}-07$ & $2.5900 \mathrm{E}-07$ & $1.5970 \mathrm{E}-07$ & $9.2820 E-07$ \\
\hline CD-135 & $\mathrm{Cl}$ & 8 & $2.8230 E-06$ & $3.5288 \mathrm{E}-07$ & $2.3500 E-07$ & $2.0000 E-08$ & $8.0000 \mathrm{E}-07$ \\
\hline S8-1.24 & $\mathrm{Cl}$ & 1 & $2.7000 \mathrm{E}-07$ & $2.7000 \mathrm{E}-07$ & $0.0000 E+\infty$ & 2.7000 E- 07 & $2.7000 E-07$ \\
\hline $\mathrm{B} 1-212$ & $\mathrm{Cl}$ & 131 & $3.4812 \mathrm{E}-07$ & $2.6574 \mathrm{E}-09$ & $23100 E-08$ & $1.0700 E-23$ & 2.5000E-07 \\
\hline PA-2:31 & $\mathrm{Cl}$ & 2 & $2.4000 \mathrm{E}-07$ & $1,2000 \mathrm{E}-07$ & t.7000E-07 & 4.0100E-16 & $2.4000 E-07$ \\
\hline $\mathrm{BI}-214$ & $\mathrm{CI}$ & 129 & 1. $2880 \mathrm{E}-05$ & $9.9845 E-08$ & $1.1400 \mathrm{E}-07$ & $5.0700 E+18$ & $2.3000 E-07$ \\
\hline$i-131$ & $\mathrm{ci}$ & 2 & $3.0000 \mathrm{E}-07$ & $1.5000 \mathrm{E}-07$ & $7.0700 \mathrm{E}-08$ & $1.0000 E-07$ & 2.0000E-07 \\
\hline$w-145$ & $\mathrm{Cl}$ & 1 & $20000 E-07$ & $2.0000 E-07$ & $0.0000 E+00$ & $2.0000 E-07$ & $2.0000 E-07$ \\
\hline$R U-103-F ? H-103 M$ & $\mathrm{Cl}$ & 3 & $1.9361 \mathrm{E}-07$ & $6.4536 E-08$ & $1,0800 \mathrm{E}-07$ & $5.6900 E-10$ & $1.8900 E-07$ \\
\hline no- 93 & $\mathrm{Cl}$ & $i$ & $9.9465 E-08$ & $9.9465 E \cdot 00$ & $0.0000 E+\infty 0$ & $9.9465 E-08$ & $9.9465 E-08$ \\
\hline $\mathrm{AC}-228$ & cl & 202 & $1.1465 \mathrm{E}-07$ & $5.6756 \mathrm{E}-10$ & 6. $1800 \mathrm{E}-09$ & $2.9300 E-15$ & $8.7000 \mathrm{E}-08$ \\
\hline$y-49$ & $\mathrm{Cl}$ & 2 & $5.4299 \mathrm{E}-08$ & $2.7150 \mathrm{E}-08$ & $3.7300 \mathrm{E}-08$ & $8.0640 E-10$ & $5.3493 E-08$ \\
\hline NB-9.5N & $\vec{c}$ & 3 & $1.5872 E-08$ & $6.6240 E-09$ & 7. $8900 E-09$ & 8. $1200 \mathrm{E}-10$ & $1.5600 \mathrm{E}-08$ \\
\hline CE-141 & $\mathrm{Cl}$ & 1 & $1.4500 \mathrm{E}-0 \mathrm{~B}$ & $\$ .4500 \mathrm{E}-08$ & $0.0000 E+00$ & $1.4500 \mathrm{E}-08$ & $1.4500 \mathrm{E}-08$ \\
\hline NI-63 METAL & $\mathrm{Cl}$ & 7 & $2.9400 \mathrm{E}-10$ & $4,2000 \mathrm{E}-11$ & $0.0000 E+\infty 0$ & 4.2000E-11 & $4.2000 E-11$ \\
\hline$Z R-4 B$ & cl & 2 & 4. $6300 \mathrm{E}-12$ & $2.3150 \mathrm{E}-12$ & 1. 4600E-12 & $1.2800 E-12$ & $3.3600 E-12$ \\
\hline Cs-135 & $\mathrm{Cl}$ & 2 & $6.6200 \mathrm{E}-13$ & $3.3100 E-13$ & $2.0900 E-13$ & $1.8300 \mathrm{E}-13$ & $4.7900 \mathrm{E} \cdot 13$ \\
\hline PD-107 & Ci & 2 & $1.3360 \mathrm{E}=13$ & $6.6800 E-14$ & $4.2100 E-14$ & $3.7000 \mathrm{E}-14$ & $9.6600 E-14$ \\
\hline PE-IJ| & Cl & 62 & $0.0000 E+\infty 0$ & $0.0000 E+\infty$ & $0.0000 E+00$ & $0.0000 E+00$ & $0.0000 E+\infty 0$ \\
\hline TOTAL- $\mu$ FHA & $\mathrm{Cl}$ & 60 & $0.0000 E+00$ & $0.0000 E+00$ & $0.0000 E+00$ & $0.0000 \mathrm{E}+00$ & $0.0000 \varepsilon+00$ \\
\hline
\end{tabular}


WHC-SD-W-242-ES-004, Rev. 0

TABLE 13

STATISTICAL ANALYSIS OF PHYSICAL PROPERTIES

IN ASCENDING ORDER BASED ON CONTAINER COUNT

(2 SHEETS)

\begin{tabular}{|c|c|c|c|c|c|c|}
\hline COMPONENT & COUNT & ACCUM WT, KG & AVERAGE $W T, K G$ & STD DEV & MUNIMUM WT, KG & MAXIMUM WT, KG \\
\hline PLASTICPPOLYYURATHANE & 1863 & $1.9128 E+04$ & $1.1785 E+01$ & 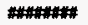 & $2.0000 E-02$ & $7.2740 E+02$ \\
\hline CONWEB PADS & 1333 & 1.0000E+04 & $7.6048 \mathrm{E}+00$ & \#\# & $1.6000 \mathrm{E}-01$ & $1.3790 E+02$ \\
\hline HAZARDOUS CONSTITUENTS & 1251 & $3.0489 E+04$ & $2.4372 E+01$ & *\#\#\# & $0.0000 E+00$ & $4.3550 E+02$ \\
\hline ABSORBENT/KITY LTRNERMICULITE & 1072 & $2.9840 E+04$ & $2.8451 E+01$ & 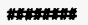 & $2.3000 E-01$ & $3.06600+02$ \\
\hline DIRT/SOILOLATOMACEOUS EARTH & 960 & $6.2731 E+04$ & $6.6664 E+01$ & \#\#\# & $3.0000 \mathrm{E}-02$ & $3.6109 E+03$ \\
\hline CLOTHURAGSNNYLON & 631 & $4.5104 E+03$ & $9.9560 E+00$ & \#\#\# & $1.0000 E-02$ & $2.7150 E+02$ \\
\hline GLASS & 606 & 9.5577E+03 & $1.6145 E+01$ & 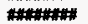 & $0.0000 E+\infty 0$ & $1.0880 E+02$ \\
\hline METAL/RON/GAL VANIZED/SHEET & 543 & $8.4591 E+04$ & $1.6685 \mathrm{E}+02$ & \#\#\# & $1.0000 \mathrm{E}-02$ & $4.9140 E+03$ \\
\hline Ons & 513 & $1.8854 E+04$ & $3.8556 \mathrm{E}+01$ & - & $0.0000 E+00$ & $1.0000 E+02$ \\
\hline LIQUID & 406 & $2.0785 E+03$ & $5.1194 E+\infty 0$ & *\#* & $0.0000 E+00$ & $7.8000 E+01$ \\
\hline RUBBER & 262 & $2.0891 \mathrm{E}+03$ & $1.1053 E+01$ & \#\#\# & $1.0000 \mathrm{E}-02$ & $2.4590 \mathrm{E}+02$ \\
\hline PAPERICARDBOARD & 260 & $3.2221 E+03$ & $1.4132 E+01$ & 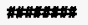 & $0.0000 E+00$ & $3.8014 E+02$ \\
\hline WOODRLUMBER/PLYWOOD & 233 & $2.2449 E+04$ & $9.7183 E+01$ & \#\#\# & $3.0000 \mathrm{E}-02$ & $1.5756 \mathrm{E}+03$ \\
\hline ANTI-CORROSIVE RADPAD & 226 & $1.0565 E+02$ & $4.6748 E-01$ & \#\# & $1.0000 E-01$ & $4.0000 E+\infty$ \\
\hline MSCELLANEOUS/UNKNOWN/OTHER & 222 & $1.7114 E+04$ & $7.8867 E+01$ & \#\#\# & $0.0000 E+00$ & $3.1900 \mathrm{E}+02$ \\
\hline $90 \mathrm{MLL}$ LINER & 207 & $1.6495 E+03$ & $7.9685 E+\infty 0$ & *\#\# & $4.4000 E+00$ & $2.6700 E+01$ \\
\hline LIQUID ORGANIC & 195 & $7.4182 \mathrm{E}+02$ & $4.1212 \mathrm{E}+01$ & $\cdot$ & $1.4000 E+01$ & $6.8040 E+01$ \\
\hline SLUDGES & 184 & $1.4536 \mathrm{E}+04$ & $7.9432 E+01$ & *\#\# & $4.4600 E+\infty 0$ & $2.3640 E+02$ \\
\hline CHEMICALS & 158 & $2.0315 E+03$ & $1.3278 E+01$ & -1* & $0.0000 E+00$ & $1.1000 E+02$ \\
\hline WATER & 137 & $1.3810 E+03$ & $1.0081 E+01$ & - & $1.6000 E+00$ & $8.2700 E+01$ \\
\hline PANTSRUCITE & 129 & $3.3198 E+03$ & $2.6558 \mathrm{E}+01$ & \#\#\#= & $1.0000 \mathrm{E}-01$ & 4.1983E+02 \\
\hline 10 ML LINER & 99 & 4.4872E+02 & $4.5325 \mathrm{E}+00$ & *:*:*:* & $2,5000 E-01$ & $2.3600 E+01$ \\
\hline STAINLESS STEEL & 95 & 1.6316E+03 & $1.8972 \mathrm{E}+01$ & **:* & $2.0000 E-02$ & $1.1487 E+03$ \\
\hline ANMALL WASTE & 87 & $1.5561 \mathrm{E}+03$ & $1.7886 \mathrm{E}+01$ & *\#\#\#\# & $3.8000 E+00$ & $3.0600 E+01$ \\
\hline FILTERS & 83 & $1.3159 E+04$ & $1.7315 E+02$ & $\div$ & 4.7000E-01 & $1.3000 E+03$ \\
\hline AIR & 56 & $9.5000 \mathrm{E}-01$ & $1.6964 E-02$ & *\# & $0.0000 E+\infty 0$ & 9.5000E-01 \\
\hline PCB & 47 & $1.8371 E+02$ & $4.0824 E+00$ & *** & $0.0000 E+00$ & $3.0000 E+01$ \\
\hline ROCKJGRAVEL & 46 & $3.7626 E+03$ & $8.1796 E+01$ & -*** & $2.6900 E+\infty 0$ & $1.0640 E+03$ \\
\hline CONCRETE & 44 & $7.1726 E+03$ & $1.7494 E+02$ & *\#\#\# & $1.7500 E+\infty 0$ & $1.3179 E+03$ \\
\hline VARIOUS SOLUTIONS SOLIDIFIED & 36 & $4.8984 E+04$ & $1.3607 \mathrm{E}+03$ & & $3.8700 E+00$ & $1.5130 E+03$ \\
\hline RESINS & 35 & $2.0670 E+02$ & $6.0794 E+00$ & **at* & $2.1000 E+00$ & $2.0000 E+01$ \\
\hline ORGANICS & 34 & $2.5275 E+02$ & $2.2977 E+01$ & & t. $3000 E+00$ & $8.0000 E+01$ \\
\hline SOLVENTS/THINNERS & 26 & $8.2150 E+01$ & $5.1344 E+\infty 0$ & *\#*\#* & $1,9000 E-01$ & $2.6000 E+0 t$ \\
\hline CLAY & 23 & $5.2000 E+01$ & $2.2609 E+\infty 0$ & 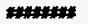 & $2.0000 E+00$ & $4.0000 E+00$ \\
\hline LEAD & 22 & $2.6071 E+03$ & $1.1850 E+02$ & 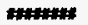 & $0.0000 E+00$ & $1.1380 E+03$ \\
\hline GREASE & 21 & $5.8023 E+02$ & $2.7630 E+01$ & ***:* & $1.0000 E+00$ & $5.5250 E+01$ \\
\hline SANO & 20 & $1.8046 E+03$ & $12890 E+02$ & & $5.4360 E+01$ & $1.9380 E+02$ \\
\hline COPPER METAL. & 16 & $1.3236 E+02$ & $8.2725 E+\infty 0$ & H\#\#\#\#: & $0.0000 E+\infty 0$ & $8.7000 E+01$ \\
\hline ALUMINUM & 13 & $2.7340 E+01$ & $2.2783 E+00$ & 1\% & $6.0000 E-02$ & $5.0000 E+\infty 0$ \\
\hline ASBESTOS & 13 & $2.5461 E+02$ & $1.9585 \mathrm{E}+01$ & 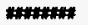 & $1.0000 E-01$ & $1.4123 \mathrm{E}+02$ \\
\hline OLL AND WATER & 13 & $4.7881 E+02$ & $3.6832 E+01$ & *ะ\#\# & $2.0000 E+00$ & $5.4820 E+01$ \\
\hline UNIVERSAL POLYPROPYLENES & 13 & $1.2986 \mathrm{E}+02$ & $9.9892 E+00$ & H:H:H* & $3.5000 E+\infty 0$ & $4.9870 E+01$ \\
\hline ANTIFREEZE & 12 & $5.9684 E+02$ & $4.9737 E+01$ & 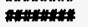 & $1.0340 E+01$ & $6.0600 E+01$ \\
\hline LIGHT BALLAST & 12 & $2.0268 \varepsilon+02$ & $1.6890 E+01$ & 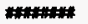 & $2.0000 E+00$ & $9.0000 E+01$ \\
\hline FOAWSTYROFOAM & 11 & $4.7881 E+02$ & $4.3528 E+01$ & 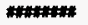 & $1.3000 E-01$ & $1.8181 E+02$ \\
\hline
\end{tabular}


WHC-SD-W-242-ES-004, Rev. 0

TABLE 13

STATISTICAL ANALYSIS OF PHYSICAL PROPERTIES

IN ASCENDING ORDER BASED ON CONTAINER COUNT

(2 SHEETS)

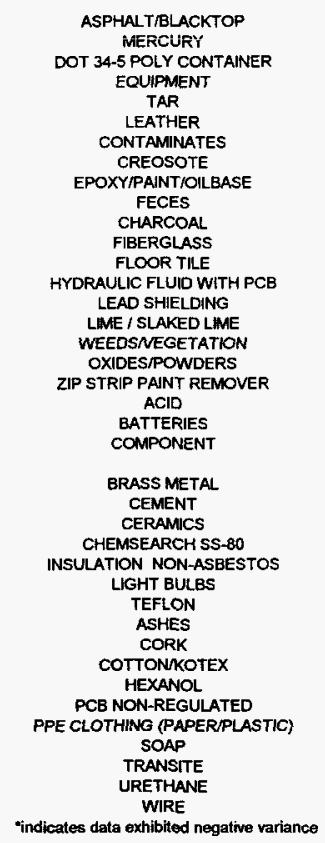

$2.0068 E+03$
$7.7000 E+02$
$1.3000 E+01$
$3.6903 E+02$
$3.6675 E+02$
$5.2600 E+00$
$4.7020 E+01$
$2.7600 E+01$
$1.2505 E+02$
$3.9760 E+01$
$3.6928 E+02$
$2.5330 E+01$
$1.1630 E+02$
$3.7500 E+00$
$7.5040 E+02$
$1.4820 E+01$
$3.5250 E+01$
$4.9200 E+01$
$1.1050 E+01$
$4.9400 E+01$
$4.4000 E+00$
$A C C M M W T, K G$
$8.3100 E+00$
$9.0600 E+01$
$3.3000 E+00$
$6.5400 E+00$
$5.9000 E+01$
$1.1600 E+00$
$7.0000 E-01$
$3.0000 E+00$
$1.0000 E-01$
$1.3700 E+00$
$0.0000 E+00$
$0.0000 E+00$
$1.2000 E+00$
$6.2700 E+\infty$
$4.3630 E+03$
$7.0800 E+00$
$1.3600 E+00$

$2.2297 E+02$
$8.5556 E+01$
$1.6250 E+00$
$5.2719 E+01$
$5.2393 E+01$
$8.7667 E-01$
$9.4040 E+00$
$5.5200 E+00$
$2.5010 E+01$
$7.9520 E+00$
$9.2320 E+01$
$6.3325 E+00$
$2.9075 E+01$
$9.3750 E-01$
$1.8760 E+02$
$3.7050 E+00$
$8.8125 E+00$
$1.6400 E+01$
$3.6833 E+00$
$2.4700 E+01$
$2.2000 E+00$
$A V R A G E$ WT.

$4.1550 E+00$
$4.5300 E+01$
$1.6500 E+00$
$3.2700 E+00$
$2.9500 E+01$
$5.8000 E-01$
$7.0000 E-01$
$3.0000 E+00$
$1.0000 E-01$
$1.3700 E+00$
$0.0000 E+00$
$0.0000 E+00$
$1.2000 E+00$
$6.2700 E+00$
$4.3630 E+03$
$7.0800 E+00$
$1.3600 E+00$

$3.0000 E+00$

10000E-

$10000+00$

2.9000E+01

2.0000E+00

2.0000E-01

O.0000E+00

3.6000E+00

$1.0000 E+01$

3.8200E+00

7.8500E+01

$3.3000 \mathrm{E}-01$
$1.3000 \mathrm{E}+01$

$1,3000 \mathrm{E}+01$
$\quad 2.500 \mathrm{E}-01$

4.5400E+01

$2.7300 \mathrm{E}+00$

$9.5000 E-01$

1.3200E+01

$\quad 5.0000 \mathrm{E}-01$

2.5000E-01

$20000 E-01$

COUNT

(1)

3.3100E+00

\#\#+

4.6000E+ +00

3.0600E-01

- $3.1500 \mathrm{E}+00$

$8.7200 E+00$

5.0000E-01

7,0000E-01

- $30000 \mathrm{E}+00$

$3.0000+00$
$\quad 10000 \mathrm{E}-01$

$1.3700 E+00$
$-\quad 0000+\infty$

- $0.0000 E+\infty 0$

$\quad 0.0000 E+\infty 0$

$12000 \mathrm{E}+\infty$

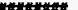

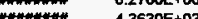

4.3630E+03

$7.0800 \mathrm{E}+\infty$
$1.3600 \mathrm{E}+\infty$

$1.0104 E+03$

$1.1950 E+02$

$2.0000 E+00$

$1.0260 E+02$

$1.0050 \mathrm{E}+02$

$2.1000 \mathrm{E}+00$

.

$6.0000 \mathrm{E}+\infty$

$46000 \mathrm{E}+01$

4.000

$1.6080 \mathrm{O}$

.TO28ET 2

2.0000E+01

5.0500E+01

$1.5000 E+\infty 0$

$4.5500 \mathrm{E}+\infty$

$2.5630 E+01$

$1.8800 E+01$

$4.9150 E+01$

4.9750 E+01

MAXIMUM WT, KG

\section{$5.0000 E+00$}

8.6000 E+01

$3.0000 E+\infty$

$3.3900 E+\infty 0$

$5.0280 E+01$

6.6000E-01

7.0000E- 01

$3.0000 E+\infty 0$

1.0000E-01

$1.3700 E+00$

$0.0000 \mathrm{E}+00$

$0.0000 \mathrm{E}+\infty 0$

$1.2000=+\infty$

$6.2700=+\infty 0$

$6.2700 \mathrm{E}+00$

$4.3630 E+03$

$7.0800 E+00$

$1.3600 E+C 0$ 
WHC-SD-W-242-ES-004 Rev. 0

TABLE 14

STATISTICAL ANALYSIS OF PHYSICAL PROPERTIES IN ASCENDING ORDER BASED ON ACCUMULATED WEIGHT

(2 SHEET)

COMPONENT

METALIRON/GAL VANIZED/SHEET DIRT/SOILDIATOMACEOUS EARTH VARIOUS SOLUTIONS SOLIDIFIED HAZARDOUS CONSTITUENTS ABSORBENT/KITY LTRRERMICULITE WOOD/LUMBERPLYWOOD PLASTICIPOLYURATHANE OILS

MISCELLANEOUS/UNKNOWN/OTHER SLUDGES FILTERS

CONWEB PADS GLASS

CONCRETE

CLOTHRAGSINLON TRANSITE

ROCKUGRAVL

PAINTSIUCITE

PAPER/CARDBOARD

LEAD

RUBBER

LIOUID

CHEMICALS

ASPHALT/BLACKTOP SAND

90 MIL LINER

STAINLESS STEEL

ANIMAL WASTE

WATER

MERCURY

LEAD SHIELDING

LIOUID ORGANIC

ANTIFREEZE

GREASE

OIL AND WATER

FOAWSTYROFOAM

10 MLL LINER

CHARCOAL

EQUIPMENT TAR

ASBESTOS

ORGANICS RESINS

LIGHT BALLAST PCB

COPPER METAL

UNIVERSAL POLYPROPYIENES

EPOXYIPAINT/OILBASE FLOOR TILE

ANTI-CORROSIVE RADPAD CEMENT

SOLVENTS/THINNEFIS

INSULATION NON-ASBESTOS CLAY ACID

OXIDES/POWDERS

CONTAMINATES FECES

WEEDSNEGETATION CREOSOTE

ALUMINUM

FIBERGLASS

LIME / SLAKED LIME:

DOT 34-5 POLY CONTAINER

ZIP STRIP PAINT REMCIVER COMPONENT
COUNT

\author{
ACCUM WT, KG
}

8.4591E+04

$6.2731 E+04$

$4.8984 E+04$

$3.0489 E+04$

$2.9840 E+04$

$2.2449 E+04$

$1.9128 \mathrm{E}+04$

$1.8854 \mathrm{E}+04$

$1.7114 E+04$

$1.4536 \mathrm{E}+04$

$1.3159 \mathrm{E}+04$

$1.0000 E+04$

$9.5577 \mathrm{E}+03$

$7.1726 \mathrm{E}+03$

$4.5101 \mathrm{E}+03$

4.3630E +03

$3.7626 \mathrm{E}+03$

$3.3198 E+03$

$3.2221 \mathrm{E}+03$

$2.6071 E+03$

$2.0891 \mathrm{E}+03$

$2.0785 \mathrm{E}+03$

$2.0315 E+03$

$2.0068 E+03$

$1.8046 E+03$

$1.6495 \mathrm{E}+03$

$1.6316 \mathrm{E}+03$

$1.5561 \mathrm{E}+03$

$1.3810 \mathrm{E}+03$

$7.7000 \mathrm{E}+02$

$7.5040 \mathrm{E}+02$

$7.4182 \mathrm{E}+02$

$5.9684 E+02$

$5.8023 E+02$

4.7881E+02

$4.7881 E+02$

$4.4872 \mathrm{E}+02$

$3.6928 \mathrm{E}+02$

$3.6903 \mathrm{E}+02$

$3.6675 \mathrm{E}+02$

$2.5461 \mathrm{E}+02$

$2.5275 \mathrm{E}+\mathrm{O} 2$

$2.0670 \mathrm{E}+02$

$2.0268 E+02$

$1.8371 E+02$

$1.3236 \mathrm{E}+02$

$1.2986 E+02$

$1.2505 E+02$

4. $1630 \mathrm{E}+02$

$1.0565 \mathrm{E}+02$

$9.0600 \mathrm{E}+01$

$8.2150 \mathrm{E}+01$

$5.9000 E+01$

5.2000E+01

$4.9400 E+01$

$4.9200 E+01$

$4.7020 \mathrm{E}+01$

$3.9760 \mathrm{E}+01$

$3.5250 \mathrm{E}+01$

$2.7600 \mathrm{E}+01$

2.7340E+D1

$2.5330 \mathrm{E}+01$

$1.4820 \mathrm{E}+01$

$1.3000 \mathrm{E}+01$

$1.1050 \mathrm{E}+01$

ACCUM WT, KG
AVERAGE WT, KG

$1.6685 \mathrm{E}+02$

$6.6664 \mathrm{E}+01$

1.3607E+03

$2.4372 E+01$

$2.8151 E+01$

$9.7183 E+01$

1.1785E+01

$3.8556 \mathrm{E}+01$

$7.8867 \mathrm{E}+01$

$7.9432 \mathrm{E}+01$

1. $7315 \mathrm{E}+02$

$7.6048 \mathrm{E}+00$

$1.6146 \mathrm{E}+01$

$1.7494 \mathrm{E}+02$

$9,9560 \mathrm{E}+00$

4.3630E +03

8.1796E +01

2.6558E+01

$1.4132 E+01$

1.1850E+02

$1.1053 E+0 t$

$5.1194 E+\infty 0$

1.3278E+01

2.2297E+02

$1.2890 \mathrm{E}+02$

$7.9685 E+00$

$1.8972 \mathrm{E}+01$

$1.7886 \mathrm{E}+01$

1.0081E+01

$8.5556 \mathrm{E}+01$

$1.8760 \mathrm{E}+02$

4.1212E+01

4.9737E+01

$2.7630 \mathrm{E}+01$

$3.6832 \mathrm{E}+01$

$4.3528 \mathrm{E}+01$

$4.5325 \mathrm{E}+00$

$9.2320 \mathrm{E}+01$

$5.2719 E+01$

5.2393E+01

$1.9585 E+01$

$2.2977 \mathrm{E}+01$

$6.0794 E+00$

$1.6890 \mathrm{E}+01$

$4.0824 E+00$

$8.2725 E+00$

$9.9892 E+00$

2.5010E+01

$2.9075 E+01$

4.6748E-01

4.5300E+01

$5.1344 \mathrm{E}+00$

$2.9500 E+01$

$2.2609 \mathrm{E}+00$

$2,4700 E+01$

$1,6400 \mathrm{E}+01$

$9.4040 E+00$

$7.9520 \mathrm{E}+00$

$8.8125 \mathrm{E}+00$

$5.5200 E+D 0$

$2.2783 \mathrm{E}+00$

$6.3325 E+00$

3. $7050 E+00$

$1.6250 \mathrm{E}+00$

$3.6833 E+00$

AVERAGE WT, KG
STD DEV

$5.1544 E+02$

$2.4270 E+02$

$3.3543 \mathrm{E}+02$

4.7914E+01

$2.7824 E+01$

$1.8417 E+02$

$3.1352 E+01$

$1.5825 E+01$

$1.1798 \mathrm{E}+02$

$5.2929 \mathrm{E}+01$

2.0070E+02

9.8737E+00

$1.4428 E+01$

$2.3686 E+02$

2.0112E+01

$0.0000 E+00$

$1.5396 E+02$

$6.4938 \mathrm{E}+01$

4.4605E+01

$2.7807 \mathrm{E}+02$

$2.5089 E+01$

$1.2906 \mathrm{E}+01$

$1.8774 E+01$

$3.3890 \mathrm{E}+02$

$3.8384 \mathrm{E}+00$

$1.1723 \mathrm{E}+02$

$5.8771 \mathrm{E}+\infty 0$

$8.3790 \mathrm{E}+00$

$4.4552 E+01$

$1.0193 \mathrm{E}+02$$$
\text { * }
$$

1.7345E+01

1.7821E+01

$1.8772 \mathrm{E}+01$

$6.3066 \mathrm{E}+01$

$4.7336 \mathrm{E}+00$

$1.5325 \mathrm{E}+01$

$3.0065 \mathrm{E}+01$

$3.9039 \mathrm{E}+01$

$3.9001 \mathrm{E}+01$

$4.4253 E+00$

2.4472E+01

$9.1634 \mathrm{E}+00$

2.2816E +01

$1.3498 \mathrm{E}+01$

1.4791E+01

1.6251E+01

4.7918E-01

$5.7558 \mathrm{E}+01$

$5.6420 \mathrm{E}+\infty 0$

$2.9387 E+01$

5.4082E-01

$3.4578 \mathrm{E}+01$

$2.8844 E+\infty 0$

$1.2873 E+01$

$5.3988 E+00$

$1.1647 \mathrm{E}+01$

$1.1394 E+00$

$1.8578 \mathrm{E}+00$

$9.1780 E+00$

$7.5430 \mathrm{E}-01$

5.1755E-01

4.6342E+00

STD DEV

MINIMUM WT, KG MAXIMUM WT, KG

1.0000E-02

$3.0000 E-02$

$3.8700 E+00$

$0.0000 E+00$

2.3000E-01

3.0000E-02

2.0000 E-02

$0.0000 E+00$

$0.0000 E+\infty 0$

4.4600E+00

4.7000E-01

1.6000E-01

$0.0000 \mathrm{E}+00$ 


\section{WHC-SD-W-242-ES-004 Rev. 0}

TABLE 14

STATISTICAL ANALYSIS OF PHYSICAL PROPERTIES

IN ASCENDING ORDER BASED ON ACCUMULATED WEIGHT

(2 SHEET)

BRASS METAL
URETHANE
CHEMSEARCH SS-80
SOAP
LEATHER
BATTERIES
HYORAULIC FLUID WITH PC8
CERAMICS
ASHES
COTTONKOTEX
WIRE
PRE CLOTHING (PAPER/PLASTIC)
LIGHT BULBS
AIR
TEFLON
CORK
HEXANOL
PCB NON-REGULATED

$8.3100 E+00$
$7.0800 E+00$
$6.5400 E+00$
$6.2700 E+00$
$5.2600 E+00$
$4.4000 E+00$
$3.7500 E+00$
$3.3000 E+00$
$3.0000 E+00$
$1.3700 E+00$
$1.3600 E+00$
$1.2000 E+00$
$1.1600 E+00$
$9.5000 E-01$
$7.0000 E-01$
$1.0000 E-01$
$0.0000 E+00$
$0.0000 E+00$

$4.1550 E+\infty 0$
$7.0800 E+\infty 0$
$3.2700 E+\infty 0$
$6.2700 E+00$
$8.7667 \mathrm{E}-01$
$2.2000 \mathrm{E}+00$
$9.3750 \mathrm{E}-01$
$1.6500 \mathrm{E}+00$
$3.0000 \mathrm{E}+00$
$1.3700 \mathrm{E}+00$
$1.3600 \mathrm{E}+00$
$1.2000 \mathrm{E}+00$
$5.8000 \mathrm{E}-01$
$1.6964 \mathrm{E}-02$
$7.0000 \mathrm{E}-01$
$1.0000 \mathrm{E}-01$
$0.0000 \mathrm{E}+00$
$0.0000 \mathrm{E}+00$

$1.1950 E+\infty 0$
$0.0000 E+\infty 0$
$1.6971 E-01$
$0.0000 E+\infty 0$
$7.5543 E-01$
$2.8284 E+00$
$5.1539 E-01$
$1.9092 E+\infty 0$
$0.0000 E+00$
$0.0000 E+00$
$0.0000 E+\infty 0$
$0.0000 E+00$
$1.1314 E-01$
$1.2695 E-01$
0.01
$0.0000 E+00$
$0.0000 E+00$
$0.0000 E+00$

$3.3100 E+00$

$7.0800 E+\infty 0$

$3.1500 \mathrm{E}+\infty 0$

$6.2700 E+\infty 0$

2.0000E-01

$2.0000 E-01$

2.5000E-01

$3.0000 E-01$

$3.0000 E+00$

$1.3700 E+00$

$1.3600 E+00$

$1.2000 E+00$

5.0000E-01

$0.0000 E+00$

7.0000 E-01

1.0000E-01

$0.0000 \mathrm{E}+00$

$0.0000 E+00$
$5.0000 E+00$
$7.0800 E+00$
$3.3900 E+00$
$6.2700 E+00$
$2.1000 E+00$
$4.2000 E+00$
$1.5000 E+00$
$3.0000 E+00$
$3.0000 E+00$
$1.3700 E+00$
$1.3600 E+00$
$1.2000 E+00$
$6.6000 E-01$
$9.5000 E-01$
$7.0000 E-01$
$1.0000 E-01$
$0.0000 E+00$
$0.0000 E+00$ 
TABLE 15

STATISTICAL ANALYSIS OF PHYSICAL PROPERTIES IN ASCENDING ORDER BASED ON MAXIMUM WEIGHT

(2 SHEETS)

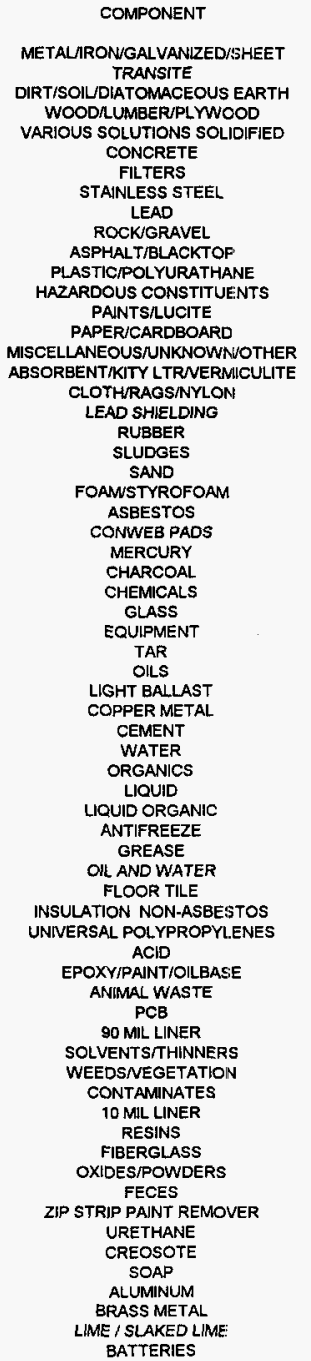

\begin{tabular}{|c|c|c|}
\hline COUNT & ACCUM WT, KG & AVERAGE WT, $K$ \\
\hline 543 & $8,4591 E+04$ & $1.6685 \mathrm{E}+02$ \\
\hline 1 & $4.3630 E+03$ & $4.3630 E+03$ \\
\hline 960 & $6.2731 \mathrm{E}+04$ & $6.6664 E+01$ \\
\hline 233 & $2.2449 E+04$ & $9.7183 E+01$ \\
\hline 36 & $4,8984 E+04$ & $1.3607 E+03$ \\
\hline 44 & $7.1726 \mathrm{E}+03$ & $1.7494 E+02$ \\
\hline 83 & $1.3159 E+04$ & $1.7315 E+02$ \\
\hline 95 & $1,6316 E+03$ & $1.8972 \mathrm{E}+01$ \\
\hline 22 & $2.6071 E+03$ & $1.1850 E+02$ \\
\hline 46 & $3.7626 E+03$ & $8.1796 \mathrm{E}+01$ \\
\hline 9 & $2.0068 \mathrm{E}+03$ & $2.2297 \mathrm{E}+02$ \\
\hline 1863 & $1.9128 E+04$ & $1.1785 \mathrm{E}+01$ \\
\hline 1251 & $3.0489 E+04$ & $2.4372 E+01$ \\
\hline 129 & $3.3198 E+03$ & $2.6558 \mathrm{E}+01$ \\
\hline 260 & $3.2221 E+03$ & $1.4132 E+01$ \\
\hline 222 & $1.7114 E+04$ & $7.8867 \mathrm{E}+01$ \\
\hline 1072 & $2.9840 E+04$ & $2.8151 E+01$ \\
\hline 631 & $4.5101 E+03$ & $8.9560 E+\infty 0$ \\
\hline 4 & $7.5040 E+02$ & $1.8760 E+02$ \\
\hline 262 & $2.0891 E+03$ & 1.1053E+01 \\
\hline 184 & $1.4536 E+04$ & $7.9432 E+01$ \\
\hline 20 & $1.8046 E+03$ & $1.2890 E+02$ \\
\hline 11 & $4.7881 E+02$ & $4.3528 E+01$ \\
\hline 13 & $2.5461 E+02$ & $1.9585 E+01$ \\
\hline 1333 & $1,0000 E+04$ & $7.6048 E+00$ \\
\hline 9 & $7.7000 E+02$ & $8.5556 E+01$ \\
\hline 4 & $3.6928 E+02$ & $9.2320 E+01$ \\
\hline 158 & $2.0315 E+03$ & $1.3278 \mathrm{E}+01$ \\
\hline 606 & $9.5577 E+03$ & $1.6145 E+01$ \\
\hline 7 & $3.6903 E+02$ & $5.2719 E+01$ \\
\hline 7 & $3.6675 E+02$ & $5.2393 E+0 r$ \\
\hline 513 & $1.8854 E+04$ & $3.8556 E+01$ \\
\hline 12 & $2.0268 E+02$ & $1.6890 \mathrm{E}+01$ \\
\hline 16 & 1.3236E +02 & $8.2725 E+00$ \\
\hline 2 & $9.0600 E+01$ & $4.5300 E+01$ \\
\hline 137 & $1.3810 E+03$ & $1.0081 E+01$ \\
\hline 34 & $2.5275 E+02$ & $2.2977 E+01$ \\
\hline 406 & $2.0785 E+03$ & $5.1194 E+00$ \\
\hline 195 & $7.4182 E+02$ & $4.1212 E+01$ \\
\hline 12 & $5.9684 E+02$ & $4.9737 \mathrm{E}+01$ \\
\hline 21 & $5.8023 E+02$ & $2.7630 E+01$ \\
\hline 13 & 4.7881E+02 & $3.6832 \mathrm{E}+01$ \\
\hline 4 & $1.1630 E+02$ & $2.9075 E+01$ \\
\hline 2 & $5.9000 E+01$ & $2.9500 E+01$ \\
\hline 13 & $1.2986 \mathrm{E}+02$ & $9.9892 E+00$ \\
\hline 2 & $4.9400 E+01$ & $2.4700 E+01$ \\
\hline 5 & $1.2505 E+02$ & $2.5010 E+01$ \\
\hline 87 & $1.5561 E+03$ & $1.7886 E+04$ \\
\hline 47 & $1.8371 E+02$ & $4.0824 E+00$ \\
\hline 207 & $1.6495 \mathrm{E}+03$ & $7.9685 E+00$ \\
\hline 26 & $8.2150 E+01$ & $5.1344 E+00$ \\
\hline 4 & $3.5250 E+01$ & $8.8125 E+00$ \\
\hline 5 & $4.7020 E+01$ & $9.4040 E+00$ \\
\hline 99 & $4.4872 E+02$ & $4.5325 E+00$ \\
\hline 35 & $2.0670 E+02$ & $6.0794 E+00$ \\
\hline 4 & $2.5330 E+01$ & $6.3325 E+00$ \\
\hline 3 & $4.9200 E+01$ & $1.6400 E+01$ \\
\hline 5 & $3.9760 E+01$ & $7.9520 E+00$ \\
\hline 3 & 1.105OE+01 & $3.6833 E+00$ \\
\hline 1 & $7.0800 E+00$ & $7.0800 E+00$ \\
\hline 5 & $2.7600 \mathrm{E}+01$ & $5.5200 E+00$ \\
\hline 1 & $6.2700 E+00$ & $6.2700 E+00$ \\
\hline 13 & $2.7340 E+01$ & $2.2783 \mathrm{E}+00$ \\
\hline 2 & $8.3100 E+00$ & $4.1550 E+00$ \\
\hline 4 & $1.4820 \mathrm{E}+01$ & $3.7050 E+00$ \\
\hline 2 & $4,4000 E+00$ & $2.2000 E+00$ \\
\hline
\end{tabular}

\begin{tabular}{l} 
STD DEV \\
\\
$5.1544 E+02$ \\
$0.0000 E+00$ \\
$2.4270 E+02$ \\
$1.8417 E+02$ \\
$3.3543 E+02$ \\
$2.3686 E+02$ \\
$2.0070 E+02$ \\
$1.1723 E+02$ \\
$2.7897 E+02$ \\
$1.5396 E+02$ \\
$3.3890 E+02$ \\
$3.1352 E+01$ \\
$4.7914 E+01$ \\
$6.4938 E+01$ \\
$4.4605 E+01$ \\
$1.1798 E+02$ \\
$2.7824 E+01$ \\
$2.0112 E+01$ \\
$1.0193 E+02$ \\
$2.5089 E+01$ \\
$5.2929 E+01$ \\
$6.3056+01$ \\
6.9061 \\
$3.9001 E+01$ \\
$9.8737 E+00$ \\
$4.4552 E+01$ \\
$1.5325 E+01$ \\
$1.9774 E+01$ \\
$1.4428 E+01$ \\
$3.0085 E+01$ \\
$3.9039 E+01$ \\
$1.5825 E+01$ \\
$2.4472 E+01$ \\
$2.2816 E+01$ \\
$5.7558 E+01$ \\
$8.3790 E+00$ \\
$1.2906 E+01$ \\
\hline \\
$1.7345 E+01$ \\
$1.7821 E+01$ \\
$1.8772 E+01$ \\
$1.6251 E+01$ \\
$2.9387 E+01$ \\
$1.3498 E+01$ \\
$3.4578 E+01$ \\
$1.4791 E+01$ \\
$5.9771 E+00$ \\
$9.1634 E+00$ \\
$3.8384 E+00$ \\
$5.6420 E+00$ \\
$1.1647 E+01$ \\
$1.2873 E+01$ \\
$4.7336 E+00$ \\
$4.4253 E+00$ \\
$9.1780 E+00$ \\
$2.8844 E+00$ \\
$5.3988 E+00$ \\
$4.6342 E+00$ \\
$0.0000 E+00$ \\
$1.1394 E+00$ \\
$0.0000 E+00$ \\
$1.8578 E+00$ \\
$1.1950 E+00$ \\
$7.5430 E-01$ \\
$2.8284 E+00$ \\
\end{tabular}

MINIMUM WT, KG

MAXIMUM WT, KG

$\begin{array}{ll}1.0000 E-02 & 4.9140 E+03 \\ 4.3630 E+03 & 4.3630 E+03 \\ 3.0000 E-02 & 3.6109 E+03 \\ 3.0000 E-02 & 1.5756 E+03 \\ 3.8700 E+00 & 1.5130 E+03 \\ 1.7500 E+00 & 1.3179 E+03 \\ 4.7000 E-01 & 1.3000 E+03 \\ 2.0000 E-02 & 1.1487 E+03 \\ 0.0000 E+00 & 1.1380 E+03 \\ 2.6900 E+00 & 1.0640 E+03 \\ 3.0000 E+00 & 1.0104 E+03 \\ 2.0000 E-02 & 7.2740 E+02 \\ 0.0000 E+00 & 4.3550 E+02 \\ 1.0000 E-01 & 4.1983 E+02 \\ 0.0000 E+00 & 3.8014 E+02 \\ 0.0000 E+00 & 3.1900 E+02 \\ 2.3000 E-01 & 3.0660 E+02 \\ 1.0000 E-02 & 2.7150 E+02 \\ 4.5400 E+01 & 2.7150 E+02 \\ 1.0000 E-02 & 2.4590 E+02\end{array}$

$2.4590 \mathrm{E}+02$

4. $4600 \mathrm{E}+00 \quad 2.3640 \mathrm{E}+02$

$5.4360 E+01 \quad 1.9380 E+02$

$1.3000 E-01 \quad 1.8181 E+02$

$1.0000 E-01 \quad 1.4123 E+02$

$1.6000 E-01 \quad 1.3790 E+02$

$1.0000 E-01 \quad 1.1950 E+02$

$7.8500 \mathrm{E}+01 \quad 1.1028 \mathrm{E}+02$

$0.0000 \mathrm{E}+00 \quad 1.1000 \mathrm{E}+02$

$0.0000 E+00 \quad 1.0880 E+02$

$2.9000 \mathrm{E}+01 \quad 1.0260 \mathrm{E}+02$

$7.0000 E+00 \quad 1.0050 E+02$

$0.0000 E+00 \quad 1.0000 E+02$

$2.0000 \mathrm{E}+00 \quad 9.0000 \mathrm{E}+01$

$0.0000 E+00 \quad 8.7000 E+01$

$4.6000 \mathrm{E}+00 \quad 8.6000 \mathrm{E}+01$

$1.6000 E+00 \quad 8.2700 E+01$

$1.3000 E+00 \quad 8.0000 E+01$

$0.0000 E+00 \quad 7.8000 E+01$

$1.4000 E+01 \quad 6.8040 E+01$

$1.0340 E+01 \quad 6.0600 E+01$

$1.0000 \mathrm{E}+00 \quad 5.5250 \mathrm{E}+01$

$2.0000 E+00 \quad 5.4820 E+01$

$1.3000 E+01 \quad 5.0500 E+01$

$8.7200 E+00 \quad 5.0280 E+01$

$3.5000 \mathrm{E}+00 \quad 4.9870 \mathrm{E}+01$

2.5000E-01 4.9150E+01

$1.0000 \mathrm{E}+01 \quad 4.6000 \mathrm{E}+01$

$3.8000 E+00 \quad 3.0600 E+01$

$0.0000 E+00 \quad 3.0000 E+01$

$4.4000 \mathrm{E}+00 \quad 2.6700 \mathrm{E}+01$

$1.9000 E-01 \quad 2.6000 E+01$

8.5000E-01 2.5630E+01

$0.0000 E+00 \quad 2.4000 E+01$

$2.5000 E-01 \quad 2.3600 E+01$

$2.1000 \mathrm{E}+00 \quad 2.0000 \mathrm{E}+01$

3.3000E-01 2.0000E+01

1.3200E+01 $1.8800 E+01$

$3.8200 E+00 \quad 1.6690 E+01$

$5.0000 \mathrm{E}-01 \quad 9.0000 \mathrm{E}+00$

$7.0800 \mathrm{E}+00 \quad 7.0800 \mathrm{E}+00$

$3.6000 E+00 \quad 6.6000 E+00$

$6.2700 \mathrm{E}+00 \quad 6.2700 \mathrm{E}+00$

$6.0000 \mathrm{E}-02 \quad 5.0000 \mathrm{E}+00$

$3.3100 \mathrm{E}+00 \quad 5.0000 \mathrm{E}+00$

$2.7300 E+00 \quad 4.5500 E+00$

$2.0000 E-01 \quad 4.2000 E+00$ 


\section{WHC-SD-W-242-ES-004, Rev. 0}

TABLE 15

STATISTICAL ANALYSIS OF PHYSICAL PROPERTIES

IN ASCENDING ORDER BASED ON MAXIMUM WEIGHT

(2 SHEETS)

COMPONENT
ANTI-CORROSIVE RADFAD
CLAY
CHEMSEARCH SS-80
CERAMICS
ASHES
LEATHER
DOT 34-5 POLY CONTAINER
HYDRAULIC FLUID WITH PCB
COTTONIKOTEX
WIRE
PPE CLOTHING (PAPER/PLASTIC)
AIR
TEFLON
LIGHT BULES
CORK
HEXANOL
PCB NON-REGULATEID

$\begin{array}{cc}\text { COUNT } & \text { ACCUM WT, KG } \\ 226 & 1.0565 E+02 \\ 23 & 5.2000 E+01 \\ 2 & 6.5400 E+00 \\ 2 & 3.3000 E+00 \\ 1 & 3.0000 E+00 \\ 6 & 5.2600 E+00 \\ 8 & 1.3000 E+01 \\ 4 & 3.7500 E+00 \\ 1 & 1.3700 E+00 \\ 1 & 1.3600 E+00 \\ 1 & 1.2000 E+00 \\ 56 & 9.5000 E-01 \\ 2 & 7.0000 E-01 \\ 2 & 1.1600 E+00 \\ 1 & 1.0000 E-01 \\ 1 & 0.0000 E+00 \\ 1 & 0.0000 E+00\end{array}$

AVERAGE WT, KG

$4.6748 E-01$
$2.2609 E+00$
$3.2700 E+00$
$1.6500 E+00$
$3.0000 E+00$
$8.7667 E-01$
$1.6250 E+00$
$9.3750 E-01$
$1.3700 E+00$
$1.3600 E+00$
$1.2000 E+00$
$1.6964 E-02$
$7.0000 E-01$
$5.8000 E-01$
$1.0000 E-01$
$0.0000 E+00$
$0.0000 E+00$

STD DEV

4.7918E-01

$5.4082 \mathrm{E}-01$

1.6971E-01

$1.9092 E+00$

$0.0000 E+00$

7.5543E -01

$5.1755 E-01$

$5.1539 E-01$

$0.0000 E+\infty 0$

$0.0000 \mathrm{E}+00$

$0.0000 \mathrm{E}+00$

1.2695E-01 ,

$1.1314 E-01$

$0.0000 \mathrm{E}+00$

$0.0000 \mathrm{E}+00$

$0.0000 E+00$
MINIMUM WT, KG

$1.0000 E-01$

$2.0000 E+\infty 0$

$3.1500 E+00$

$3.0000 E-01$

$3.0000 E+\infty 0$

$2.0000 E-01$

$1,0000 E+\infty 0$

2.5000E-01

1.3700E +00

$1.3600 \mathrm{E}+00$

$1.2000 E+00$

$0.0000 E+00$

7.0000E-01

$5.0000 \mathrm{E}-01$

1.0000 E-01

$0.0000 E+00$

$0.0000 E+00$
MAXIMUMWT, KG

$4.0000 E+00$

$4.0000 E+00$

$3.3900 E+\infty 0$

$3.0000 \mathrm{E}+00$

$3.0000 \mathrm{E}+00$

$2.1000 \mathrm{E}+00$

$2.0000 \mathrm{E}+00$

$1.5000 E+00$

$1.3700 \mathrm{E}+00$

1.3600E+00

1.2000E +00

9.5000E-01

7.0000E-01

6.6000E-01

1.0000E-01

$0.0000 E+00$

$0.0000 E+00$ 
WHC-SD-W-242-ES-004, Rev. 0

TABLE 16

STATISTICAL ANALYSIS OF DOSE-EQUIVALENT RADIONUCLIDE CONTENT

IN ASCENDING ORDER BASED ON CONTAINER COUNT

(2 SHEETS)

\begin{tabular}{|c|c|c|c|c|c|c|}
\hline ISOTOPE & COUNT & ACCUMULATION & AVERAGE & STD DEV & MINMUM & MAXIMUM \\
\hline $\begin{array}{l}\text { TOTAL BETAVGAMMA } \\
\text { CO } 60\end{array}$ & 2764 & $7.0532 E+04$ & $2.5518 E+01$ & $1.3391 \mathrm{E}+03$ & $6.0000 \mathrm{E}-12$ & $\begin{array}{l}7.0400 E+04 \\
2.5154 E-05\end{array}$ \\
\hline CS-137-BA-137M & 989 & $2.4057 E-03$ & $2.4325 E-05$ & $\begin{array}{l}1.0800 E-06 \\
4.0100 E-05\end{array}$ & $\begin{array}{l}2.5200 E-14 \\
8.6400 E-17\end{array}$ & $\begin{array}{l}2.5154 E-05 \\
0.1200 E-04\end{array}$ \\
\hline SR-90 - Y90 & 780 & $1.1806 \varepsilon-01$ & $1.5136 E-04$ & $3.0750 E-03$ & $5.8480 E-13$ & 8.5570E-02 \\
\hline $\mathrm{H}-3$ & 571 & $4.6442 \mathrm{E}-03$ & $8.1334 E-06$ & $5.3800 E-05$ & $5.5000 E-15$ & 6.8475E-0. \\
\hline AM-241 & 481 & $4.4174 E+00$ & $9.1839 E-03$ & $7.4168 \mathrm{E}-02$ & $1.0588 E-15$ & $9.7530 E-01$ \\
\hline PU-238 & 449 & $1.7567 E+\infty 0$ & 3.9124E-03 & $3.1688 E-02$ & 5.3374E-16 & $3.8490 \mathrm{E}-01$ \\
\hline PU-239 & 442 & $2.2493 E+01$ & $5.0888 \mathrm{E}-02$ & $4.0941 \mathrm{E}-01$ & $6.8200 E-15$ & $4.9432 E+00$ \\
\hline $\mathrm{C}-14$ & 404 & $6.2418 E-07$ & $1.5450 \mathrm{E}-09$ & $1.5200 E-08$ & $9.7500 \mathrm{E}-24$ & $2.4310 \varepsilon-07$ \\
\hline PU-241 & 370 & $3.6603 E+00$ & $9.8926 \mathrm{E}-03$ & $6.3376 \mathrm{E}-02$ & 8.5696E-19 & $6.1985 E-01$ \\
\hline $\mathrm{TH}-232$ & 318 & $9.1777 \mathrm{E}-03$ & $2.8861 \mathrm{E}-05$ & $8.3800 E-05$ & $1.4057 \mathrm{E}-19$ & $1.3729 E-03$ \\
\hline TC-99 & 312 & $3.0525 \mathrm{E}-06$ & $9.7836 \mathrm{E}-09$ & $9.0400 \mathrm{E}-08$ & $3.9130 E-17$ & $1.1557 E-06$ \\
\hline PU-240 & 309 & $5.0132 \mathrm{E}+00$ & $1.6224 \mathrm{E}-02$ & $1.0884 E-01$ & $1.5186 E-15$ & $1.1019 E+\infty 0$ \\
\hline $\mathrm{U}-238$ & 280 & $7.0906 \mathrm{E}-04$ & $2.5324 E-06$ & $4.6900 \mathrm{E}-06$ & $3.0643 \mathrm{E}-23$ & 4.7876E-05 \\
\hline PU & 261 & $5.3749 E+00$ & $2.0594 E-02$ & 1.4580E-01 & 6.3506E-11 & $1.6767 \mathrm{E}+00$ \\
\hline U.235 & 228 & 2.4837E-05 & $1.0893 \mathrm{E}-07$ & $2.3400 \mathrm{E}-07$ & $8.0603 E-16$ & 2.8580E-06 \\
\hline RA-226 & 227 & $2.8521 \mathrm{E}-04$ & $1.2564 E-06$ & $9.2600 \mathrm{E}-06$ & $2.5766 \mathrm{E}-16$ & $1.3874 E-04$ \\
\hline U-234 & 223 & $7.2802 E-04$ & 3.2647E-06 & $7.3900 \mathrm{E}-06$ & $1.1852 E-11$ & $9.2719 \mathrm{E}-05$ \\
\hline URANIUM-DEPLETED & 222 & $1.8487 E-03$ & $8.3275 E-06$ & $9.6200 \mathrm{E}-05$ & $9.8229 \mathrm{E}-18$ & $1.4112 E-03$ \\
\hline PB-212 & 216 & $7.8890 \mathrm{E}-09$ & $3.6523 E-11$ & $1.8100 \mathrm{E}-10$ & $5.3480 E-22$ & $1.3832 E-09$ \\
\hline MN-54. & 214 & $2.5566 \mathrm{E}-07$ & $1.1947 E-09$ & $5.1200 \mathrm{E}-09$ & $9.2400 \mathrm{E}-16$ & $6.3580 \mathrm{E}-08$ \\
\hline PM-147 & 210 & 5.1035E-05 & $2.4302 E-07$ & $6.3900 \mathrm{E}-07$ & $4.8720 \mathrm{E}-15$ & 4.1640E-06 \\
\hline$A C-2213$ & 202 & $1.9490 \mathrm{E}-10$ & $9.6486 \mathrm{E}-13$ & $1.0500 E-11$ & 4.9810E-18 & $1.4790 \mathrm{E}-10$ \\
\hline $\mathrm{TH}-234$ & 201 & 2.3116E-07 & $1.1501 E-09$ & $4.1800 \mathrm{E}-09$ & $1.6639 \mathrm{E}-15$ & $5.6399 E-08$ \\
\hline $1-129$ & 192 & $3.1596 \mathrm{E}-02$ & $1.6456 \mathrm{E}-04$ & 1.1540E-03 & $1.7600 \mathrm{E}-13$ & $1.2700 E-02$ \\
\hline $\mathrm{TH}-2213$ & 190 & $2.1725 E-01$ & $1.1434 \mathrm{E}-03$ & $1.5628 \mathrm{E}-02$ & $1.9441 E-10$ & 2.1543E-01 \\
\hline EU-154 & 157 & $1.8529 \mathrm{E}-04$ & $1.1802 E-05$ & $8.2400 E-06$ & 1.9550E-17 & $7.5140 \mathrm{E}-05$ \\
\hline RU-106 - RH-106 & 152 & $3.2669 \mathrm{E}-04$ & 2.1493E-06 & $4.9000 \mathrm{E}-06$ & $2.8500 \mathrm{E}-17$ & 4.0500E -05 \\
\hline Ni-60 & 151 & $0.0000 E+00$ & $0.0000 E+00$ & $0.0000 E+00$ & $0.0000 E+00$ & $0.0000 E+00$ \\
\hline $\mathrm{Nl}-63$ & 144 & $5.0484 E-07$ & $3.5058 \mathrm{E}-09$ & $3.9500 \mathrm{E}-08$ & 2.3782E-17 & $4.7396 \mathrm{E}-07$ \\
\hline PU-242 & 140 & $2.7113 E-04$ & $1.9366 \mathrm{E}-06$ & $8.6400 \mathrm{E}-06$ & 8.1897E-20 & $5.9581 E-05$ \\
\hline B1-212: & 131 & $2.4717 \mathrm{E}-11$ & $1.8868 E-13$ & $1.6400 \mathrm{E}-12$ & $7.5970 E-28$ & $1.7750 \mathrm{E}-11$ \\
\hline RA-22B & 131 & $2.6610 \mathrm{E}-03$ & $2.0313 E-05$ & $2.3200 E-04$ & B. $.0790 \mathrm{E}-10$ & $2.6547 E-03$ \\
\hline PB-21.4 & 130 & $5.2485 E-10$ & $4.0373 E-12$ & $1.7800 \mathrm{E}-11$ & $7.8000 E-23$ & $2.0275 E-10$ \\
\hline RA-224 & 130 & $5.2850 E-04$ & $4.0654 E-06$ & $3.4800 E-05$ & $1.1280 E-09$ & $3.7600 \mathrm{E}-0.4$ \\
\hline $81-214$ & 129 & $2.8336 \mathrm{E}-10$ & $2.1966 \mathrm{E} * 12$ & $2.5200 \mathrm{E}-12$ & 1.1154E-22 & $5.0600 \mathrm{E}-12$ \\
\hline CE-144 - PR-144 & 126 & $9.0018 E-04$ & $7.1443 \mathrm{E}-06$ & $1.3100 E-05$ & $7.2000 E-12$ & $6.0240 \mathrm{E}-05$ \\
\hline TL-208 & 126 & $4.6483 \mathrm{E}-08$ & $3.6891 \mathrm{E}-10$ & $4.1200 E-09$ & $1.6500 E-19$ & $4.6200 E-08$ \\
\hline URANIUM-ENRICHED & 118 & $7.2723 \mathrm{E}-04$ & $6.1629 \mathrm{E}-06$ & $1.0200 \mathrm{E}-05$ & $1.4804 \mathrm{E}-13$ & $7.6646 E-05$ \\
\hline FE-59 & 104 & $1.1156 \mathrm{E}-07$ & $1.0727 \mathrm{E}-09$ & $2.2200 \mathrm{E}-09$ & $7.7420 E-19$ & $1.4700 E-08$ \\
\hline SE-79 & 88 & $2.3889 \mathrm{E}-08$ & $2.7146 E-10$ & 1.1800E-09 & $1.7100 \mathrm{E}-17$ & $7.1460 \mathrm{E}-09$ \\
\hline$C M-242$ & 85 & $4.1524 E-07$ & $4.8851 E-09$ & $2.4800 \mathrm{E}-08$ & $5.1901 \mathrm{E}-20$ & $1.6682 \mathrm{E}-07$ \\
\hline NB-94 & 83 & $4.9221 E-09$ & $5.9302 \mathrm{E}-11$ & $3.1100 \mathrm{E}-10$ & $2.2800 \mathrm{E}-20$ & $1.9080 \mathrm{E}-09$ \\
\hline EU-152 & 73 & 3.0333E-06 & 4.1552E-08 & $2.1700 \mathrm{E}-07$ & $9.7500 \mathrm{E}-16$ & $1.6575 E-06$ \\
\hline $5 M-151$ & 73 & $1.3520 \mathrm{E}-05$ & 1.8521E-07 & $2.3700 \mathrm{E}-07$ & $7.5200 \mathrm{E}-14$ & $9.1200 E-07$ \\
\hline $\mathrm{U}-236$ & 65 & $1.3213 \mathrm{E}-05$ & $2.0328 \mathrm{E}-07$ & $9.1100 \mathrm{E}-07$ & $2.6825 E-13$ & $7.3483 E-06$ \\
\hline EU-155 & 64 & 4.0598E-06 & $6.3435 \mathrm{E}-08$ & $3.6100 \mathrm{E}-07$ & $2.0400 \mathrm{E}-18$ & $2.8050 E-06$ \\
\hline PE-Cl & 62 & $0.0000 E+00$ & $0.0000 E+00$ & $0.0000 E+00$ & $0.0000 E+00$ & $0.0000 \mathrm{E}+00$ \\
\hline PU239 FSL GR EQUIV & 61 & $0.0000 E+00$ & $0.0000 E+00$ & $0.0000 E+00$ & $0.0000 E+00$ & $0.0000 \mathrm{E}+00$ \\
\hline TOTAL-AL.PHA & 60 & $0.0000 \mathrm{E}+00$ & $0.0000 E+00$ & $0.0000 E+00$ & $0.0000 E+00$ & $0.0000 E+00$ \\
\hline $\mathrm{CO}-5.3$ & 55 & $3.0157 \mathrm{E}-07$ & 5.4830E-09 & $1.5200 \mathrm{E}-08$ & $9.0000 \mathrm{E}-12$ & $1.0080 \mathrm{E}-07$ \\
\hline NP-237 & 54 & $5.6326 \mathrm{E}-02$ & $1.0431 E-03$ & $3.2780 E-03$ & $1.0501 E-11$ & $1.9479 E-02$ \\
\hline FE-55 & 51 & $2.3541 \mathrm{E}-07$ & 4.6160E-09 & 2.1000E-08 & 2.3800E-14 & 1.4450E-07 \\
\hline NA. 2.2 & 50 & $2.0789 \mathrm{E}-06$ & $4.1578 E-08$ & 1.3300E-07 & $2.8080 \mathrm{E}-13$ & 8.3700E- -07 \\
\hline NI-5S & 46 & $5.4378 \mathrm{E}-11$ & 1.1821E-12 & $3.2500 \mathrm{E}-12$ & 7.3200E-18 & $1.5200 \mathrm{E}-11$ \\
\hline P.32 & 43 & $2.5033 \mathrm{E}-07$ & 5.82१6E-09 & $2.6800 E-08$ & 1.5190E-12 & $1.7640 \mathrm{E}-07$ \\
\hline s-35 & 42 & $9.3489 E-08$ & 2.2259E-09 & 4.4500E-09 & $7.9560 \mathrm{E}-15$ & $2.3400 \mathrm{E}-08$ \\
\hline CS-13:4 & 41 & 6.7893E-07 & 1.6559E-08 & $8.0500 E-08$ & $3.0800 \mathrm{E}-16$ & $5.0680 E-07$ \\
\hline SB-125 & 40 & $9.3246 \mathrm{E}-08$ & $2,3312 \mathrm{E}-09$ & $5.9000 E-09$ & $2.0000 \mathrm{E}-16$ & 3.6000E-08 \\
\hline KR-85 & 39 & $3.8690 \mathrm{E}-09$ & $9.9206 E-11$ & $2.5700 \mathrm{E}-10$ & $1.6800 \mathrm{E}-12$ & $1.6590 \mathrm{E}-09$ \\
\hline TE-125M & 35 & 3.1961E-08 & $9.1317 E-10$ & $1.3500 \mathrm{E}-09$ & $5.4280 E-13$ & 5.5200E-09 \\
\hline $\mathrm{ZN}-65$ & 29 & $1.9331 E-08$ & $6.6659 E-10$ & $2.8300 \mathrm{E}-09$ & $9.0450 \mathrm{E}-15$ & $1.4740 E-0 B$ \\
\hline $\mathrm{U}-233$ & 26 & $2.8667 \mathrm{E}-05$ & $1.1026 E-06$ & $1.1600 \mathrm{E}-06$ & 2.5286E-09 & $4.1452 E-06$ \\
\hline K- 40 & 22 & $1.2998 \mathrm{E}-08$ & $5.9080 E-10$ & $1.0200 \mathrm{E}-09$ & $9.7850 E-12$ & 3.8950E-09 \\
\hline CA-45 & 19 & $8.2863 E-08$ & 4.3612E-09 & $9.5500 \mathrm{E}-09$ & 8.2110E-14 & $4.2000 E-08$ \\
\hline CM-2.44 & 17 & 2.1187E-03 & $1.2463 \mathrm{E}-04$ & $4.2100 \mathrm{E}-04$ & $7.7606 \mathrm{E}-13$ & $1.7480 E-03$ \\
\hline
\end{tabular}




\section{WHC-SD-W-242-ES-004, Rev. 0}

TABLE 16

STATISTICAL ANALYSIS OF DOSE-EQUIVALENT RADIONUCUDE CONTENT

IN ASCENDING ORDER BASED ON CONTAINER COUNT

(2 SHEETS)

\begin{tabular}{|c|c|c|c|c|c|c|}
\hline ISOTOPE & COUNT & ACCUMULATION & AVERAGE & STD DEV & MINIMUM & MAXIMUM \\
\hline $\mathrm{BE}-7$ & 16 & $5.2241 \mathrm{E}-09$ & $3.2650 \mathrm{E}-10$ & $5.9300 \mathrm{E}-10$ & $3.3000 E-16$ & 2.3380E-D9 \\
\hline CR-51 & 16 & $3.4047 \mathrm{E}-09$ & $2.1279 \mathrm{E}-10$ & $2.8700 E-10$ & $3.1046 \mathrm{E}-16$ & 1.1000E-09 \\
\hline TA-182 & 15 & $1.6199 \mathrm{E}-05$ & $1.0800 E-06$ & 1.6100E-06 & $1.0640 E-11$ & $4.7600 \mathrm{E}-06$ \\
\hline$C O-57$ & 13 & $2.1813 \mathrm{E}-09$ & $1.6779 \mathrm{E}-10$ & $2.9200 E-10$ & $5.6700 \mathrm{E}-16$ & $9.3366 \mathrm{E}-10$ \\
\hline$P O-210$ & 13 & $1.1200 E-06$ & 8.6154E-08 & $2.9400 E-07$ & 1.5221E-26 & $1.0633 \mathrm{E}-06$ \\
\hline CM-243 & 11 & $2.7729 \mathrm{E}-05$ & $2.5208 \mathrm{E}-06$ & 4.3700E-06 & $5.8271 E-14$ & 1.1689E-05 \\
\hline $\mathrm{U}-232$ & 11 & 5.2308E-06 & $4.7552 E-07$ & $5.0300 E-07$ & 2.5119E-09 & $1.1898 E-06$ \\
\hline AM-243 & 10 & $5.3700 E-04$ & $5.3700 E-05$ & $1.6700 \mathrm{E}-04$ & $1.9500 E-08$ & $5.3000 \mathrm{E}-04$ \\
\hline NB-93M & 10 & $1.7458 \mathrm{E}-09$ & $1.7458 \mathrm{E}-10$ & $2.8800 E-10$ & $3.6894 \mathrm{E}-18$ & $7.4100 E-10$ \\
\hline CD-109 & 8 & $1.2704 \mathrm{E}-09$ & $1.5979 \mathrm{E}-10$ & $1.0500 E-10$ & $9.0000 E-12$ & $2.7000 \mathrm{E}-10$ \\
\hline$C M-245$ & 8 & $4.1592 E-06$ & 5.1990E-07 & $6.0100 E-07$ & 4.3247E-09 & $1.3367 E-06$ \\
\hline$c 0-56$ & 7 & $3.4345 \mathrm{E}-10$ & $4.9064 E-11$ & 3.3700E-11 & 2.0761E-11 & $1.2067 E-10$ \\
\hline NB-95 & 7 & $2.0032 E-09$ & $2.8617 \mathrm{E}-10$ & $1.9700 \mathrm{E}-10$ & $6.0800 E-11$ & $5.3770 \mathrm{E}-10$ \\
\hline NL-63 METAL & 7 & $2.7636 E-15$ & $3.9480 \mathrm{E}-16$ & $0.0000 \mathrm{E}+00$ & $3.9480 E-16$ & $3.9480 \mathrm{E}-16$ \\
\hline ZR-95 - NE-95M & 7 & $8.2585 E-08$ & $1.1798 E-08$ & $2.0400 \mathrm{E}-08$ & $1.2000 \mathrm{E}-11$ & $5.2560 \mathrm{E}-08$ \\
\hline I-125 & 5 & $1.7425 E-05$ & 3.4850E-06 & $3.4600 \mathrm{E}-06$ & 8.5000E-08 & $8.5000 \mathrm{E}-06$ \\
\hline$A G=110$ & 4 & $4.9623 \mathrm{E}-09$ & $1.2406=-09$ & $7.5900 \mathrm{E}-10$ & 1.0231E-10 & $1.6200 E-09$ \\
\hline$A G-110 M-A G-110$ & 4 & $2.4281 \mathrm{E}-0.9$ & $6.0704 E-10$ & $1.0400 E-09$ & 8.8200E-11 & 2.1600E-09 \\
\hline NB-95MA & 3 & $1.5500 E-13$ & $5.1667 E-14$ & $6.1500 E-14$ & $6.3336 \mathrm{E}-15$ & $1.2168 E-13$ \\
\hline RU-103 -- RH-103M & 3 & $5.6147 \mathrm{E}-12$ & $1.8716 E-12$ & $3.1300 \mathrm{E}-12$ & $1.6501 \mathrm{E}-14$ & $5.4810 E-12$ \\
\hline SC- 47 & 3 & $2.8910 \mathrm{E}-10$ & $9.6367 E-11$ & $7.8600 \mathrm{E}-11$ & 2.9500 E- 11 & $1.8290 \mathrm{E}-10$ \\
\hline TA-183 & 3 & $7.4630 E-09$ & $2.4877 E-09$ & $9.9100 \mathrm{E}-10$ & $1.3430 E-09$ & $3.0600 \mathrm{E}-09$ \\
\hline CS-135 & 2 & $9.2680 E-18$ & $4.6340 E-18$ & $2.9300 \mathrm{E}+18$ & $2.5620 \mathrm{E}-18$ & $6.7060 \mathrm{E}-18$ \\
\hline $\mid-131$ & 2 & $3.6000 \mathrm{E}-07$ & $1.8000 \mathrm{E}-07$ & $8.4900 E-08$ & $1.2000 \mathrm{E}+07$ & $2.4000 E-07$ \\
\hline PA-231 & 2 & $2.4000 E-06$ & $1.2000 \mathrm{E}-06$ & $1.7000 E-06$ & 4.0100E-15 & $2.4000 E-06$ \\
\hline PD-107 & 2 & $5.3440 E-18$ & $2.6720 \mathrm{E}-18$ & 1.6900E-18 & $1.4800 E-18$ & $3.8640 \mathrm{E}-18$ \\
\hline$S E-75$ & 2 & $3.8077 \mathrm{E}-08$ & 1.9038E-08 & $2.6900 \mathrm{E}-08$ & 6.8580E-12 & 3.8070E-08 \\
\hline$V-49$ & 2 & $5.9729 E-14$ & $2.9865 E-14$ & $4.1000 \mathrm{E}-14$ & $8.8704 \mathrm{E}-16$ & $5.8842 \mathrm{E}-14$ \\
\hline ZR-93 & 2 & $1.1575 \mathrm{E}-14$ & 5.7875E-15 & $3.6600 \mathrm{E}-15$ & $3.2000 \mathrm{E}-15$ & 8.3750E-15 \\
\hline AU-19:5 & 1 & $4.7806 \mathrm{E}-10$ & $4.7806 \mathrm{E}-10$ & $0.0000 E+00$ & $4.7806 \mathrm{E}-10$ & $4.7806 \mathrm{E}-10$ \\
\hline BA-13:3 & 1 & $9.4000 \mathrm{E}-08$ & $9.4000 \mathrm{E}-08$ & $0.0000 E+00$ & $9.4000 E-0 B$ & $9.4000 \varepsilon-08$ \\
\hline$C E-141$ & 1 & $3.9150 \mathrm{E}-13$ & $3.9150 E-13$ & $0.0000 E+00$ & $3.9150 \mathrm{E}-13$ & 3.9150E-13 \\
\hline CL-36 & 1 & 2.1360E-08 & $2.1360 E-08$ & $0.0000 E+00$ & $2.1360 E-08$ & 2.1360E-08 \\
\hline GD-154 & 1 & $0.0000 E+00$ & $0.0000 E+00$ & $0.0000 E+00$ & $0.0000 E+\infty$ & $0.0000 E+\infty 0$ \\
\hline HF-181 & 1 & 2.0196E-06 & 2.0196E-06 & $0.0000 E+00$ & $2.0196 E-06$ & $2.0196 E-06$ \\
\hline$M O-93$ & 1 & $8.9519 E-12$ & 8.9519E-12 & $0.0000 E+00$ & $8.9519 E-12$ & $8.9519 E-12$ \\
\hline PT-195M & 1 & $1.0920 E-10$ & $1.0920 \mathrm{E}-10$ & $0.0000 E+00$ & $1.0920 \mathrm{E}-10$ & $1.0920 \mathrm{E}-10$ \\
\hline SB-124 & 1 & $2.2410 \mathrm{E}-11$ & 2.2410E-11 & $0.0000 E+00$ & 2.2410E-11 & 2.2410E-11 \\
\hline$W-185$ & 1 & $5.0000 E-13$ & $5.0000 E-13$ & $0.0000 E+00$ & $5.0000 \mathrm{E}-13$ & $5.0000 \mathrm{E}-13$ \\
\hline$X E-131 M$ & 1 & $1.5860 E-09$ & $1.5860 E-09$ & $0.0000 E+\infty 0$ & 1.5860E-09 & $1.5860 \mathrm{E}-09$ \\
\hline
\end{tabular}


TABLE 17

STATISTICAL ANALYSIS OF DOSE-EQUIVALENT RADIONUCLIDE CONTENT IN ASCENDING ORDER BASED ON ACCUMULATED ACTIVITY

(2 SHEETS)

\begin{tabular}{|c|c|c|c|c|c|c|}
\hline ISOTOPE & COUNT & ACCUMULATION & AVERAGE & STD DEV & MINIMUM & MAXIMUM \\
\hline TOTAL BETA/SAMMA & 2764 & $7.0532 E+04$ & $2.5518 E+01$ & $1.3391 E+03$ & $6.0000 \mathrm{E}-12$ & $7.0400 E+04$ \\
\hline PU-239 & 442 & $2.2493 E+01$ & $5.0888 E-02$ & 4.0941E-01 & $6.8200 \mathrm{E}-15$ & $4.9432 E+00$ \\
\hline PU & 261 & $5.3749 \mathrm{E}+\infty 0$ & $2.0594 E-02$ & $1.4580 \mathrm{E}-01$ & 6.3506E-11 & $1.6767 \mathrm{E}+00$ \\
\hline PU-240 & 309 & $5.0132 E+\infty$ & $1.6224 \mathrm{E}-02$ & $1.0884 E-01$ & $1.5186 \mathrm{E}-15$ & $1.1019 E+00$ \\
\hline AM-24 1 & 481 & $4.4174 E+00$ & $9.1839 E-03$ & 7.4168E-02 & $1.0688 \mathrm{E}-15$ & $9.7530 E-01$ \\
\hline PU-24॥ & 370 & $3.6603 E+00$ & $9.8926 \mathrm{E}-03$ & $6.3376 E-02$ & $8.5696 \mathrm{E}-19$ & $6.1985 E-01$ \\
\hline PU-23\% & 449 & $1.7567 \mathrm{E}+00$ & $3.9124 E-03$ & $3.1688 E-02$ & $5.3374 E-16$ & 3.8490E-01 \\
\hline TH-22\& & 190 & $2.1725 E-01$ & 1. $1434 E-03$ & $1.5628 \mathrm{E}-02$ & $1.9441 \mathrm{E}-10$ & 2.1543E-01 \\
\hline SR-90 - Y'90 & 780 & $1.1806 E-01$ & $1.5136 E-04$ & $3.0750 E-03$ & $5.8480 E-13$ & 8.5570E-02 \\
\hline NP-237 & 54 & $5.6326 E-02$ & $1.0431 E-03$ & $3.2780 E-03$ & $1.0501 E-11$ & $1.9479 E-02$ \\
\hline$b-129$ & 192 & 3.1596E-02 & $1.6456 \mathrm{E}-04$ & $1.1540 E-03$ & $1.7600 \mathrm{E}-13$ & $1.2700 E-02$ \\
\hline TH-232 & 318 & $9.1777 \mathrm{E}-03$ & 2.8861E-05 & 8.3800E-05 & $1.4057 \mathrm{E}-19$ & $1.3729 E-03$ \\
\hline $\mathrm{H}=3$ & 571 & 4.6442E-03 & $8.1334 E-06$ & $5.3800 E-05$ & $5.5000 \mathrm{E}-15$ & 6.8475E-04 \\
\hline RA-22B & 131 & $2.6610 \mathrm{E}-03$ & 2.0313E-05 & $2.3200 E-04$ & $8.9790 E-10$ & 2.6547E-03 \\
\hline CS-137 - BA-137M & 989 & $2.4057 E-03$ & $2.4325 E-06$ & $4.0100 E-05$ & $8.6400 E-17$ & 9.12D0E-04 \\
\hline CM-244 & 17 & $2.1187 \mathrm{E}-03$ & $1.2463 E-04$ & $4.2100 \mathrm{E}-04$ & $7.7606 \mathrm{E}-13$ & $1.7480 E-03$ \\
\hline URANIIM-DEPLETED & 222 & $1.8487 E-03$ & $8.3275 E-06$ & $9.6200 \mathrm{E}-05$ & $9.8229 E-18$ & $1.4112 E-03$ \\
\hline CE-144 -- PR-144 & 126 & $9.0018 E-04$ & $7.1443 \mathrm{E}-06$ & $1.3100 \mathrm{E}-05$ & $7.2000 \mathrm{E}-12$ & $6.0240 E-05$ \\
\hline U-234 & 223 & $7.2802 E-04$ & $3.2647 \mathrm{E}-06$ & $7.3900 E-06$ & 1.1852E-11 & $9.2719 E-05$ \\
\hline URANIUM-ENRICHED & 118 & $7.2723 E-04$ & $6.1629 \mathrm{E}-06$ & $1.0200 \mathrm{E}-05$ & $1.4804 \mathrm{E}-13$ & $7.6646 E-05$ \\
\hline U-238 & 280 & $7.0906 E-04$ & $2.5324 \mathrm{E}-06$ & 4.6900E-06 & $3.0643 \mathrm{E}-23$ & $4.7876 E-05$ \\
\hline AM-243 & 10 & $5.3700 \mathrm{E}-04$ & 5.3700E-05 & $1.6700 \mathrm{E}-04$ & $1.9500 \mathrm{E}-08$ & $5.3000 E-04$ \\
\hline RA-224 & 130 & $5.2850 \mathrm{E}-04$ & $4.0654 \mathrm{E}-06$ & 3.4800E-05 & 1.1280E-09 & $3.7600 E-04$ \\
\hline RU-106 - RH-106 & 152 & $3.2669 \mathrm{E}-04$ & $2.1493 \mathrm{E}-06$ & $4.9000 \mathrm{E}-06$ & $2.8500 \mathrm{E}-17$ & $4.0500 \mathrm{E}-05$ \\
\hline RA.22(i) & 227 & $2.8521 \mathrm{E}-04$ & $1.2564 \mathrm{E}-06$ & $9.2600 E-06$ & $2.5766 E-16$ & $1.3874 E-04$ \\
\hline PU-24:2 & 140 & $2.7113 E-04$ & $1.9366 \mathrm{E}-06$ & $8.6400 E-06$ & $8.1897 E-20$ & $5.9581 \mathrm{E}-05$ \\
\hline EU-15:4 & 157 & $1.8529 \mathrm{E}-04$ & 1.1802E-06 & $8.2400 E-06$ & $1.9550 \mathrm{E}-17$ & $7.5140 E-05$ \\
\hline$c 0.60$ & 1004 & 1.2653E-04 & 1.2603E-07 & $1.0800 \mathrm{E}-06$ & $2.5200 \mathrm{E}-14$ & 2.5154E-05 \\
\hline$P M-147$ & 210 & $5.1035 E-05$ & $2.4302 E-07$ & $6.3900 \mathrm{E}-07$ & $4.8720 \mathrm{E}-15$ & $4.1640=-06$ \\
\hline U-233 & 26 & 2.8667E-0S & $1.1026 E-06$ & $1.1600 \mathrm{E}-06$ & $2.5286 E-09$ & 4.1452E-06 \\
\hline$C M-243$ & 11 & $2.7729 \mathrm{E}-05$ & 2.5208E-06 & $4.3700 \mathrm{E}-06$ & $5.8271 \mathrm{E}-14$ & 1.1689E-05 \\
\hline U-235. & 228 & $2.4837 \mathrm{E}-05$ & $1.0893 \mathrm{E}-07$ & $2.3400 E-07$ & $8.0603 E-16$ & $2.8580 E-06$ \\
\hline $\mid-125$ & 5 & $1.7425 E-05$ & 3.4850E-06 & $3.4600 \mathrm{E}-06$ & $8.5000 E-08$ & $8.5000 E-06$ \\
\hline TA-18:2 & 15 & $1.6199 \mathrm{E}-05$ & $1.0800 E-06$ & $1.6100 \mathrm{E}-06$ & $1.0640 E-11$ & $4.7600 E-06$ \\
\hline SM-151 & 73 & $1.3520 \mathrm{E}-0 \mathrm{~S}$ & $1.8521 \mathrm{E}-07$ & $2.3700 \mathrm{E}-07$ & $7.5200 E-14$ & $9.1200 E-07$ \\
\hline$U-236 i$ & 65 & 1.3213E-05 & $2.0328 \mathrm{E}-07$ & $9.1100 \mathrm{E}-07$ & $2.6825 E-13$ & $7.3483 E-06$ \\
\hline U-232: & 11 & $5.2308 \mathrm{E}-06$ & $4.7552 E-07$ & $5.0300 \mathrm{E}-07$ & 2.5119E-09 & $1.1898 E-06$ \\
\hline$C M-245$ & 8 & 4.1592E-06 & $5.4990 \mathrm{E}-07$ & $6.0100 \mathrm{E}-07$ & 4.3247E-09 & 1.3367E-06 \\
\hline EU-155 & 64 & $4.0598 \mathrm{E}-06$ & $6.3435 E-08$ & $3.6100 \mathrm{E}-07$ & $2.0400 \mathrm{E}-18$ & 2.8050E-06 \\
\hline TC-99 & 312 & $3.0525 \mathrm{E}-06$ & 9.7836E-09 & $9.0400 \mathrm{E}-08$ & $3.9130 E-17$ & $1.1557 E-06$ \\
\hline EU-152 & 73 & $3.0333 \mathrm{E}-06$ & $4.1552 E+0 B$ & 2.1700E-07 & $9.7500 E \cdot 16$ & $1.6575 E-06$ \\
\hline PA-231 & 2 & $2.4000 E-06$ & $1.2000 \mathrm{E}-06$ & $1,7000 \mathrm{E}-06$ & $4.0100 E-15$ & 2.4000E-06 \\
\hline NA-22 & 50 & $2.0789 \mathrm{E}-06$ & 4.1578E-08 & 1.3300E -07 & $2.8080 E-13$ & 8.3700E-07 \\
\hline MF-181 & 1 & $2.0196 \mathrm{E}-06$ & $2.0196 \mathrm{E}-06$ & $0.0000 E+00$ & $2.0186 E-06$ & 2.0196E-06 \\
\hline PO-210 & 13 & $1.1200 \mathrm{E}-06$ & 8.6154E-08 & $2.9400 E-07$ & $1.5221 \mathrm{E}-26$ & $1.0633 \mathrm{E}-06$ \\
\hline CS-134 & 41 & $6.7893 \mathrm{E}-07$ & 1.6559E-08 & $8.0500 E-08$ & 3.0800E- 16 & $5.0680 E-07$ \\
\hline C-14 & 404 & $6.2418 \mathrm{E}-07$ & $1.5450 \mathrm{E}-09$ & $1.5200 \mathrm{E}-08$ & $9.7500 E-24$ & $2.4310 \mathrm{E}-07$ \\
\hline $\mathrm{N} 1-63$ & 144 & $5.0484 \mathrm{E}-07$ & 3.5058E-09 & $3.9500 \mathrm{E}-08$ & $2.3782 E-17$ & 4.7396E-07 \\
\hline$C M-242$ & 85 & $4.1524 \mathrm{E}-07$ & 4.8851E-09 & $2.4800 E-08$ & $5.1901 E-20$ & $1.6682 \mathrm{E}-07$ \\
\hline $\mid-131$ & 2 & 3.6000E-07 & 1.8000E-07 & $8.4900 \mathrm{E}-08$ & $1.2000 \mathrm{E}-07$ & $2.4000 E-07$ \\
\hline$c 0.5 i 3$ & 55 & $3.0157 \mathrm{E}-07$ & $5.4830 \mathrm{E}-09$ & $1.5200 \mathrm{E}-08$ & $9.0000 E-12$ & $1.0080 E-07$ \\
\hline MN-5.4 & 214 & $2.5566 \mathrm{E}-07$ & $1.1947 \mathrm{E}-09$ & $5.1200 \mathrm{E}-09$ & $9.2400 E-16$ & $6.3580 E-08$ \\
\hline $\mathrm{P}-32$ & 43 & $2,5033 E-07$ & $5.8216 \mathrm{E}-09$ & 2.6800E-08 & $1.5190 \mathrm{E}-12$ & $1.7640 \mathrm{E}-07$ \\
\hline FE-5: & 51 & 2.3541E-07 & 4.6160E-09 & $2.1000 \mathrm{E}-08$ & $2.3800 E-14$ & $1.4450 E-07$ \\
\hline$T H-234$ & 201 & $2.3116 E-07$ & 1.1501E-09 & $4.1800 \mathrm{E}-09$ & $1.6639 \mathrm{E}-15$ & $5.6399 E-08$ \\
\hline FE-59 & 104 & $1.1156 E-07$ & $1.0727 E-09$ & $2.2200 E-09$ & $7.7420 \mathrm{E}-19$ & $1.4700 \mathrm{E}-08$ \\
\hline BA-133 & 1 & $9.4000 E-08$ & $9.4000 \mathrm{E}-08$ & $0.0000 E+00$ & $9.4000 E-08$ & 9.4COOE-OB \\
\hline S-35 & 42 & $9.3489 \mathrm{E}-08$ & $2.2259 \mathrm{E}-09$ & $4.4500 E-00$ & $7.9560 \mathrm{E}-15$ & 2.3400E-08 \\
\hline$S B-12.5$ & 40 & $9.3246 E-08$ & $2.3312 E-09$ & $5.9000=-09$ & $2.0000 \mathrm{E}-16$ & $3.6000 E-08$ \\
\hline CA-45 & 19 & 8.2863E-08 & 4.3612E-09 & $9.5500 E-09$ & $8.2110 \mathrm{E}-14$ & $4.2000 E-08$ \\
\hline ZR-95 - NB-95M & 7 & $8.2585 E-08$ & 1.1798E-08 & 2.0400E-08 & $1.2000 E-11$ & 5.2560E-08 \\
\hline TL-2C18 & 126 & 4.6483E-08 & $3.6891 E-10$ & $4.1200 \mathrm{E}-09$ & 1.6500E-19 & $4.6200 E-08$ \\
\hline SE-75 & 2 & $3.8077 \mathrm{E}-08$ & $1.9038 \mathrm{E}-08$ & 2.6900E-08 & $6.8580 E-12$ & $3.8070 E-08$ \\
\hline$T E-125 \mathrm{M}$ & 35 & $3.1961 E-08$ & $9.1317 E-10$ & 1.3500E-09 & $5.4280 \mathrm{E}-13$ & $5.5200 E-09$ \\
\hline SE-79 & 88 & $2.3889 \mathrm{E}-08$ & $2.7146 E-10$ & 1.1800E-09 & 1.7100E-17 & $7.1460 \mathrm{E}-09$ \\
\hline$C L-36$ & 1 & $2.1360 \mathrm{E}-08$ & $2.1360 \mathrm{E}-08$ & $0.0000 E+00$ & 2.1360E-08 & $2.1360 E-08$ \\
\hline
\end{tabular}


TABLE 17

STATISTICAL ANALYSIS OF DOSE-EQUIVALENT RADIONUCLIDE CONTENT IN ASCENDING ORDER BASED ON ACCUMULATED ACTIVITY

(2 SHEETS)

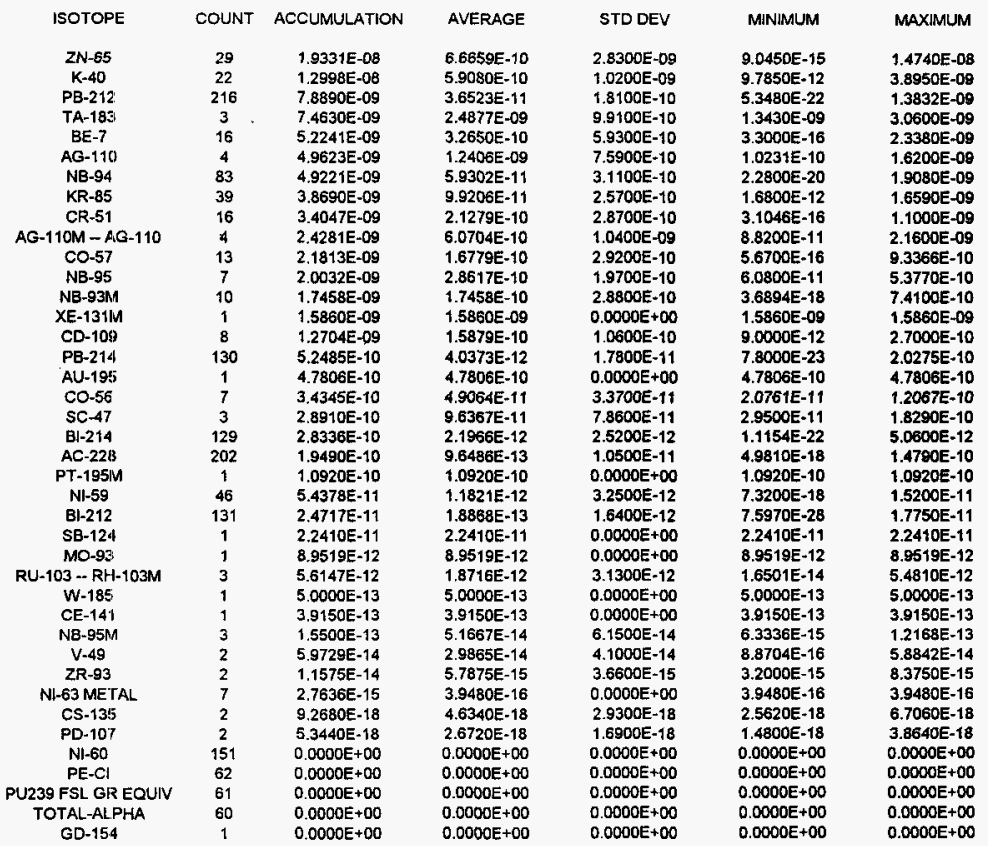


WHC-SD-W-242-ES-004, Rev. 0

TABLE 18

STATISTICAL ANALYSIS OF DOSE-EQUIVALENT RADIONUCLIDE CONTENT IN ASCENDING ORDER BASED ON MAXIMUM ACTIVITY

(2 SHEETS)

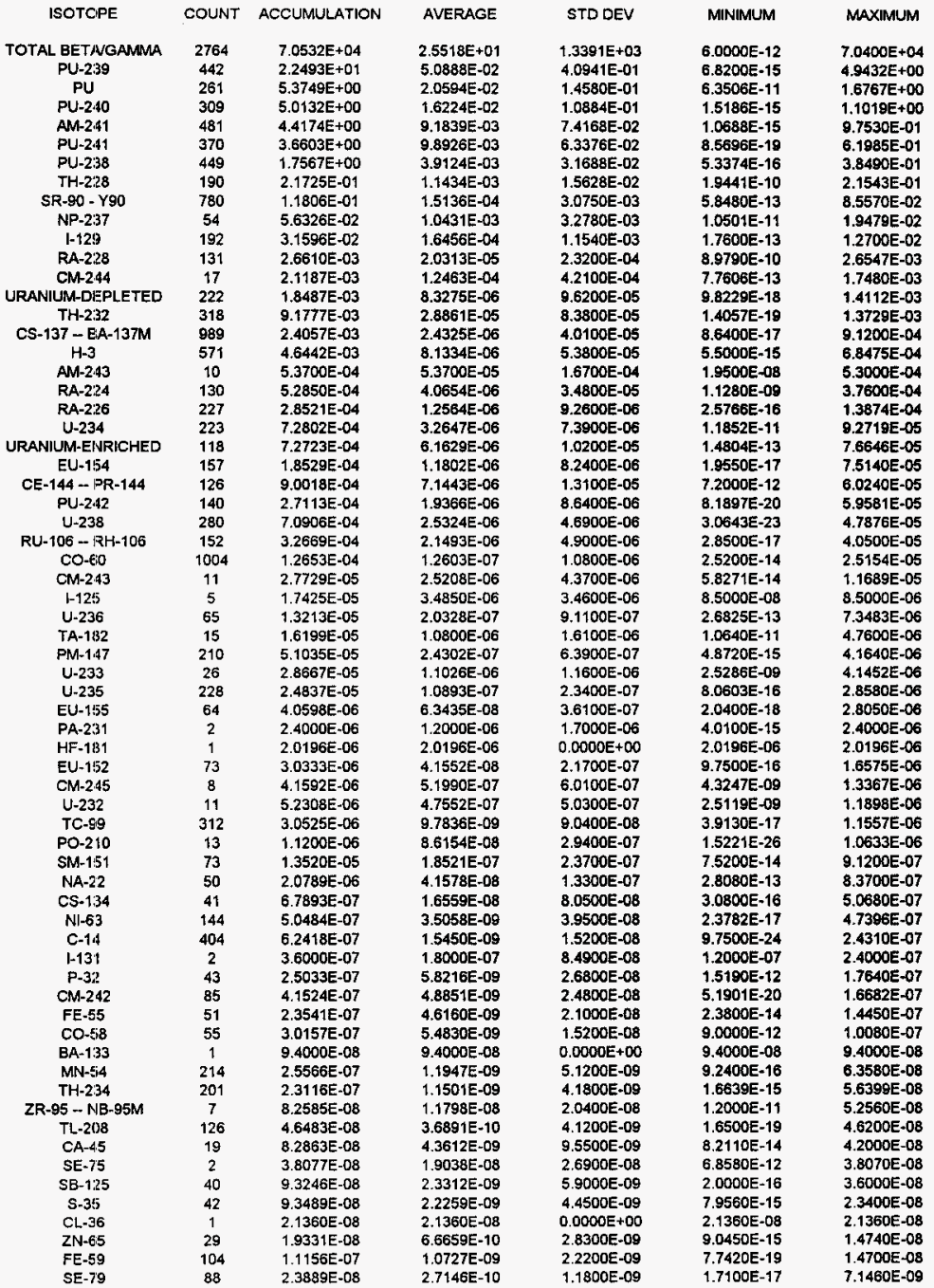


WHC-SD-W-242-ES-004, Rev. 0

TABLE 18

STATISTICAL ANALYSIS OF DOSE-EQUIVALENT RADIONUCLIDE CONTENT IN ASCENDING ORDER BASED ON MAXIMUM ACTIVITY

(2 SHEETS)

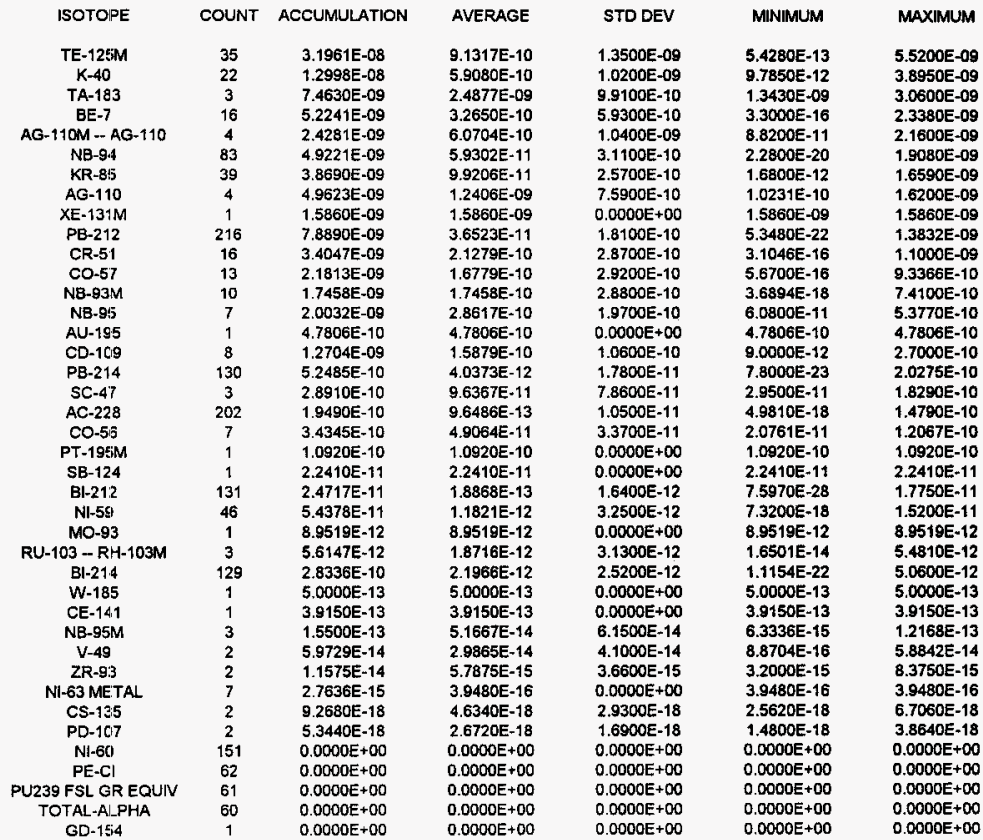


TABLE 19

ISOTOPIC DOSE-EQUIVALENT CURIE CONTENT OF CONTAINERS HAVING A DE CURIE CONTENT GREATER THAN OR EQUAL TO 1.0

(3 SHEETS)

PIN NUMBER

$\mathbf{9 4 0 0 3 2 5}$
$\mathbf{9 4 0 0 3 2 5}$
$\mathbf{9 4 0 0 3 2 5}$
$\mathbf{9 4 0 0 3 2 5}$
$\mathbf{9 4 0 0 3 2 5}$
$\mathbf{9 4 0 0 3 2 5}$
$\mathbf{9 4 0 0 3 2 5}$
$\mathbf{9 4 0 0 3 2 5}$
$\mathbf{9 4 0 0 3 2 5}$

ETF-91-310-03

ETF-91-310-03

ETF-91-310-03

ETF-91-310-03

ETF-91-310-03

ETF-91-310.03

ETF-91-310-03

ETF-91-310-03

ETF-91-310.03

ETF-91-310-03

ETF-91-310-03

ETF-91-310-03

WTFF-92-322-04

WTFF-92-322-04

WIFF-92-322-04

WTFF-92-322-04

WTFF-92-322-04

WTFF-92-322-04

WTFF-92-322-04

WTFF-92-322-04

WTFF-92-322.04

WTFF-92-322.04

WTFF-92-322-04

WTFF-92-322-04

WTFF-92-322-04

WTFF-92-322-04

WTFF-92-322-04

WTFF-92-322-04

WTFF-92-322-04

WTFF-92-322-04

WTFF-92-322-04

WTFF-92-322-04

WTFF-92-322-04

WTFF-92-322-04

WTFF-92-322-04

WTFF-92-322.04

WTFF-92-322-04

WTFF-92-322-04

WTFF-92-322-03

WTFF-92-322.03

WTFF-92-322-03

WTFF-92-322-03

WTFF-92-322-03

WTFF-92-322-03

WTFF-92-322-03

WTFF.92-322-03

WTFF-92-322-03

WTFF-92-322-03

WTFF-92-322-03

WTFF-92-322-03

WTFF.92-322-03

WTFF-92-322-03

WTFF-92-322-03

WTFF-92-322-03
ISOTOPE
TOTAL BETA/GAMMA PU. 239
CS-137 -- BA-137M
PU-240
PU-241
PU-238
SR-90 - Y9O
AM-241
PU-242

TOTAL BETA/GAMMA

SR-90 - Y9O

PU-239

AM-241

PU-240

PU. 241

PU-238

CS-137 -- BA-137M

EU-155

EU. 154

Co- 60

EU-152

TOTAL BETA/GAMMA

PU-241

AM-241

NP- 237

1-129

SR-90 - Y90

CS-137 -- BA-137M

PU. 239

CM-244

PU-240

EU. 154

PU- 238

CM- 243

Co-60

TC-99

U- 238

$\mathrm{CM}-242$

$\mathrm{U}-235$

SE-79

NI-63

PU. 242

NB-94

NB-93M

C-14

NI-59

U-236

TOTAL BETA/GAMMA

PU-241

NP. 237

I-129

SR-90 - Y9O

CS-137 -- BA-137M

PU.239

CM-244

PU-240

EU-154

PU-238

CM-243

CO-60

TC-99

U-238

CM-242
DE CURIES PEA ISOTOPE

7.9360E-08

3.4560E-OB

$1.7842 \mathrm{E}-08$

$1.1882 \mathrm{E}-\mathrm{O} 8$

5.9599E-09

3.9646E-09

1.0517E-09

9. $691 \mathrm{BE}-13$

3. $9910 E+01$

8.557OE-02

3.441 OE-O3

2.8899E-03

8.1039E-04

7. 2842E-04

$2.1319 E-04$

4.4064E-06

2. $6050 \mathrm{E}-06$

7.1145E-07

$5.2731 E-07$

6.1425E-09

$2.0840 \mathrm{E}+01$

4.5814E-01

1.5048E-02

8.9147E-03

6.9900E-03

4.7730E-03

9.1200E-O4

3.3294E-04

1.5690E-04

7.8769E-05

7.5140E-O5

1.5094E-05

1.1689E-05

7.81 20E-06

1. $1557 \mathrm{E}-06$

4.7880E-07

1.6682E-07

5.2777E-08

7.1460E-09

4. $7752 \mathrm{E} \cdot 09$

3.3581E-09

$1.3320 \mathrm{E}-09$

$7.4100 E \cdot 10$

$1.4885 \mathrm{E}-10$

1. $2680 \mathrm{E} \cdot 11$

4. 2615E-13

$1.4920 E+01$

$3.2795 \mathrm{E}-02$

6.345OE-03

$5.0000 \mathrm{E}-03$

3.4228E-03

$6.5280 \mathrm{E}-04$

2.3870E-04

$1.1162 \mathrm{E}-04$

5.6296E-05

5.3720E-05

$1.0815 \mathrm{E}-05$

8. $3583 \mathrm{E}-06$

5. 5692E-06

8. 2680E- 07

$3,4346 E-07$

1. 1993E-07
TOTAL DE CUAIES

$7.0400 \mathrm{E}+04$

$7.0400 E+04$

$7.0400 E+04$

$7.0400 E+04$

$7.0400 E+04$

$7.0400 E+04$

7.0400E + 04

$7.0400 E+04$

7. $0400 \mathrm{E}+04$

4.0004E + 01

$4.0004 E+01$

$4.0004 E+01$

4. $0004 E+01$

4.0004E + 01

$4.0004 E+01$

$4.0004 E+01$

$4.0004 \varepsilon+01$

$4.0004 E+01$

4.0004E + 01

$4,0004 E+01$

$4.0004 E+01$

$2.1335 E+01$

2.1335E + 01

2.3335E + 01

2.1335E + 01

$2.1335 E+01$

2.1335E + 01

2. $1335 \mathrm{E}+01$

2. $1335 E+01$

2. $1335 E+01$

$2.1335 E+01$

$2.1335 E+01$

$2.1335 E+01$

$2.1335 E+01$

$2.1335 E+01$

2.1335E + 11

2.1335E + 01

$2.1335 \mathrm{E}+01$

2.1335E + 01

2. $1335 E+01$

2.1335E + 01

$2.1335 E+01$

$2.1335 E+01$

$2.1335 E+01$

$2.1335 E+01$

$2.1335 E+01$

2.1335E + 01

1. $4969 E+01$

$1.4969 E+01$

1. $4969 E+01$

1. $4969 \mathrm{E}+01$

$1.4969 \mathrm{E}+01$

$1.4969 \mathrm{E}+01$

$1.4969 E+07$

$1.4969 E+01$

1. $4969 \mathrm{E}+01$

$1.4969 \mathrm{E}+01$

$1.4969 \mathrm{E}+01$

$1.4969 \mathrm{E}+01$

$1.4969 \mathrm{E}+01$

1. $4969 \mathrm{E}+01$

1. $4969 \mathrm{E}+01$

$1.4969 \mathrm{E}+01$

PERCENT DE CURIES

$1.0000 \mathrm{E}+02$

1. $1273 \mathrm{E}-10$

4.909 IE-11

2.5344E-11

1.6878E-11

8.4657E-12

5.6315E-12

1.4938E-1 2

1.3767E- 5

9.9766E + 01

$2.1391 \mathrm{E}-0$ :

8.6017E-03

7.2241E-03

2.0258E-03

1.8209E-03

5.3292E-04

1.1015E-05

7.0119E-06

1.7785E-06

1.3182E-06

1.5355E-08

$9.7678 E+01$

$2.1473 E+00$

7.0530E-02

4. $1784 \mathrm{E}-02$

3. 2762E-02

2.2371E-O2

4.2746E-03

1.5605E-03

7.3538E-04

3.6919E-04

$3.5218 \mathrm{E}-04$

7.0747E-05

5.4788E-05

3. $6615 \mathrm{E}-05$ 
TABLE 19

ISOTOPIC DOSE-EQUIVALENT CURIE CONTENT OF CONTAINERS HAVING A DE CURIE CONTENT GREATER THAN OR EQUAL TO 1.0

(3 SHEETS)

\section{PIN NUMBER}

WTFF-92-322-03

WTFF-92-322-03

WTFF-92-322-03

WTFF.92-322-03

WTFF-92-322-03

WTFF-92-322-03

WTFF-92-322-03

WTFF-92-322-03

WTFF-92-322-03

WTFF-92-322-02

WTFF-92-322-02

WTFF-92-322.02

WTFF-92-322.02

WTFF-92-322-02

WTFF-92-322-02

WTFF-92-322-02

WTFF-92-322-02

WTFF-92-322.02

WTFF-92-322.02

WTFF-92-322-02

WTFF-92-322-02

WTFF-92-322-02

WTFF-92-322-02

WTFF-92-322-02

WTFF-92-322-02

WTFF-92-322-02

WTFF-92-322-02

WTFF-92-322-02

WTFF-92-322-02

WTFF-92-322-02

WTFF.92-322-02

WTFF-92-322-02

WTFF-92-322-02

WTFF-92-322-02

WTFF-92-322-02

RHZ-87-034

RHZ-87-034

RHZ-87-034

RHZ-87-034

RHZ-87-034

RHZ-87-034

RHZ-87-034

9403711

9403711

9403711

9403711

9403711

9403711

9403711

RHZ-219-930557

RHZ-219-930557

RHZ-219-930557

RHZ-219-930557

RHZ-219-930557

RHZ-21 9-930557

RHZ-219-930557

RHZ-220-A19865

RHZ-213-A21921

RHZ-213-A21921

RHZ-213-A21921

RHZ-213-A21921

RHZ-213-A21921

RHZ-213-A21921
ISOTOPE

$$
\begin{gathered}
\text { U-235 } \\
\text { SE-79 } \\
\text { NI-63 } \\
\text { PU-242 } \\
\text { NB-94 } \\
\text { NB-93M } \\
\text { C-14 } \\
\text { NI-59 } \\
\text { U-236 }
\end{gathered}
$$

TOTAL BETA/GAMMA

PU-241

AM-241

NP-237

I-1 29

$\mathrm{SR}-90-\mathrm{Y} 90$

CS-137 - BA-137M

PU-239

CIN-244

PU-240

EU-154

PU-238

$\mathrm{CM} \cdot 243$

C0.60

TC-99

U-238

$\mathrm{CM}-242$

U-235

SE-79

$\mathrm{NI}-63$

PU-242

NB-94

NB-93M

C-14

NI-59

U-236

PU 239

PU-240

AM- 241

PU-241

PU-238

TOTAL BETA/GAMMA

PU-242

PU.239

PU-240

AM-241

PU-241

PU.238

TOTAL BETA/GAMMA PU-242

TOTAL BETA/GAMMA

PU-241

PU-238

AM-241

PU-239

PU-240

PU-242

TOTAL BETA/GAMMA

PU-239

PU-240

PU-241

AM-241

PU-238

TOTAL BETA/GAMMA
DE CURIES PER ISOTOPE

3.7757E-08

5.1120E-09

$3.4216 E-09$

2.4033E-09

1.9080 E. 09

5. 3040E-10

1. $0660 \mathrm{E}-10$

$9.0800 \mathrm{E}-12$

3. $0322 \mathrm{E}-13$

1. $3160 \mathrm{E}+01$

$2.9005 \mathrm{E} \cdot 01$

$9.4905 \mathrm{E}-03$

5.6153E-03

4. $4200 \mathrm{E}-03$

$3.0186 \mathrm{E}-03$

5.7600E-04

$2.1080 \mathrm{E}-04$

$9.8456 \mathrm{E}-05$

4. $9713 E-05$

4.7430E-05

9.5389E-06

7.3740E-06

4.9140E-O6

7.3060E-07

3.0260E-07

$1.0528 E-07$

3.3242E-08

4.5180E-09

3.0174E-09

2.1207E-09

$1.6800 \mathrm{E}-09$

4.6800E-10

9.4250E-11

8.0000E-12

$2.6825 \mathrm{E}-13$

$4.9432 \mathrm{E}+00$

1.1079E + 00

9.7530E-O1

6. $1985 \mathrm{E}-01$

3.8490E-01

5. $0000 \mathrm{E}-04$

5.9581E-O5

4. $2455 \mathrm{E}+00$

9.4634E-01

8. $7039 \mathrm{E}-01$

5. 258BE-01

3. 2989E-01

5.0000E-03

5.1141E-05

$5.0000 E+00$

1. 297OE-O4

1.2947E-05

5.7713E-06

6.0016E-07

4. 9032E-07

4.5419E-13

$5.0000 \mathrm{E}+00$

$3.0804 \mathrm{E}+00$

6.8674E-01

4.3754E-01

3.4371E-01

2.4431E-O1

5.0000E-04
TOTAL DE CURIES

1. $4969 E+01$

$1.4969 \mathrm{E}+01$

$1.4969 \mathrm{E}+01$

$1.4969 \mathrm{E}+01$

$1.4969 \mathrm{E}+01$

$1.4969 E+01$

$1.4969 \mathrm{E}+01$

$1.4969 \mathrm{E}+01$

$1.4969 \mathrm{E}+01$

1. $3474 E+01$

1. $3474 \mathrm{E}+01$

1. $3474 \mathrm{E}+01$

1. $3474 \mathrm{E}+01$

$1.3474 \mathrm{E}+01$

1. $3474 \mathrm{E}+01$

$1.3474 E+01$

1.3474E + 01

1. $3474 \mathrm{E}+01$

1. $3474 \mathrm{E}+01$

1. $3474 \mathrm{E}+01$

1. $3474 \mathrm{E}+01$

1.3474E + 01

1.3474E + 01

1. $3474 E+01$

1. $3474 \mathrm{E}+0$ ?

1. $3474 \mathrm{E}+01$

1.3474E + 01

1. $3474 \mathrm{E}+01$

$1.3474 \mathrm{E}+01$

$1.3474 \mathrm{E}+01$

$1.3474 E+01$

$1.3474 E+01$

1.3474E + 01

$1.3474 E+01$

1.3474E + 01

8.0257E + $O O$

$8.0257 E+\infty$

8.0257E + $\infty$

8.0257E + 00

$8.0257 \mathrm{E}+00$

$8.0257 \mathrm{E}+00$

$8.0257 E+00$

$6.9231 E+00$

$6.9231 E+00$

$6.9231 E+00$

$6.9231 \mathrm{E}+00$

$6.9231 E+00$

$6.9231 \mathrm{E}+00$

$6.9231 \mathrm{E}+00$

$5.0001 \mathrm{E}+00$

$5.0001 E+00$

$5.0001 E+00$

$5.0001 E+00$

$5.0001 E+00$

$5.0001 E+00$

$5.0001 \mathrm{E}+00$

$5.0000 E+00$

4.7932E + 00

$4.7932 \mathrm{E}+00$

$4.7932 E+00$

$4.7932 E+\infty 0$

$4.7932 \mathrm{E}+\infty 0$

4.7932E + 00

PERcent de curies

2.5224E-07

$3.4151 E-08$

2.2858E-08

$1.6055 \mathrm{E}-08$

$1.2747 \mathrm{E}-08$

3.6434E-09

$7.1216 \mathrm{E}-10$

6.0660E-11

2.0257E-12

9.7673E + 01

$2.1527 E+\infty$

$7.0438 \mathrm{E}-02$

4.1677E-02

3. $2805 \mathrm{E}-02$

2. 2404E-02

4. 2750E-03

$1.5645 \mathrm{E}-03$

7.3073E-04

3.6897E-04

3.5202E-04

7.0797E-05

5.4729E-05

3.6471E-05

5.4225E-06

2. 2459E-O6

$7.8141 \mathrm{E}-07$ 
TABLE 19

ISOTOPIC DOSE-EQUIVALENT CURIE CONTENT OF CONTAINERS HAVING A DE CURIE CONTENT GREATER THAN OR EQUAL TO 1.0

(3 SHEETS)

\begin{tabular}{|c|c|c|c|c|}
\hline PIN NUMBER & ISOTOPE & DE CURIES PER ISOTOPE & TOTAL DE CURIES & PERCENT DE CURIE \\
\hline RHZ-213-A21921 & PU-242 & $3.7194 E-05$ & $4.7932 \mathrm{E}+\infty$ & $7.7597 \mathrm{E}-04$ \\
\hline RHZ-87-024 & PU-239 & $2.9077 E+00$ & $4.7212 \mathrm{E}+\infty$ & $6.1589 E+01$ \\
\hline RHZ-87-024 & PU-240 & $6.4815 E-01$ & $4.7212 E+\infty 0$ & $1.3729 E+01$ \\
\hline $\mathrm{RHZ} \cdot 87-024$ & AM-241 & $5.7371 \mathrm{E} \cdot 01$ & $4.7212 \mathrm{E}+00$ & $1.2152 \mathrm{E}+01$ \\
\hline RHZ-87-024 & PU-241 & $3.6462 \mathrm{E}-01$ & $4.7212 E+\infty O$ & $7.7231 E+\infty 0$ \\
\hline RHZ-87-024 & PU. 238 & $2.2641 \mathrm{E}-01$ & $4.7212 E+\infty$ & $4.7957 E+00$ \\
\hline RHZ-87-024 & TOTAL BETA/GAMMA & $5.0000 E-04$ & $4.7212 E+00$ & $1.0591 \mathrm{E}-02$ \\
\hline RHZ-87-024 & PU- 242 & $3.5048 E-05$ & $4.7212 \mathrm{E}+00$ & $7.4235 \mathrm{E}-04$ \\
\hline RHZ-212-A22853 & PU.239 & $2.5585 \mathrm{E}+00$ & $4.1228 E+\infty 0$ & $6.2058 E+01$ \\
\hline RHZ-212-A22853 & PU-240 & $5.7034 \mathrm{E}-01$ & $4.1228 E+00$ & $1.3834 E+01$ \\
\hline RHZ-212-A22853 & AM-241 & $4.6512 \mathrm{E}-01$ & $4.1228 E+\infty 0$ & $1.1282 \mathrm{E}+01$ \\
\hline RHZ-212-A22853 & PU-241 & $3.2861 E-01$ & $4.1228 E+00$ & $7.9706 E+\infty 0$ \\
\hline RHZ-212-A22853 & PU.238 & 1.9918E-01 & $4.1228 E+00$ & 4. $6312 E+\infty$ \\
\hline RHZ-212-A22853 & TOTAL BETA/GAMMA & $1.0000 \mathrm{E}-03$ & $4.1228 E+00$ & $2.4255 \mathrm{E}-02$ \\
\hline RHZ-212-A22853 & PU-242 & $3.0756 \mathrm{E}-05$ & $4.1228 E+00$ & $7.4600 \mathrm{E}-04$ \\
\hline RHZ-86-019 & PU-239 & $2.0354 \mathrm{E}+00$ & $3.3050 E+00$ & $6.1586 E+01$ \\
\hline RHZ-86-019 & $\mathrm{PU} \cdot 240$ & $4.5370 E-01$ & $3.3050 E+00$ & $1.3728 E+01$ \\
\hline PHZ-86-019 & $A M-241$ & $4.0159 \mathrm{E}-01$ & $3.3050 E+00$ & $1.2151 E+01$ \\
\hline RHZ-86-019 & PU-241 & $2.5524 \mathrm{E}-01$ & $3.3050 E+00$ & $7.7230 E+00$ \\
\hline $\mathrm{RHZ}-86-019$ & PU-238 & $1.5849 \mathrm{E}-01$ & 3. $3050 E+00$ & $4.7955 E+00$ \\
\hline $\mathrm{RHZ}-86-019$ & TOTAL BETA/GAMMA & $5.0000 E-04$ & $3.3050 E+00$ & $1.5129 \mathrm{E}-02$ \\
\hline RHZ-86-019 & PU -242 & $2.4533 E-05$ & $3.3050 E+00$ & $7.4232 E-04$ \\
\hline AHZ-212-A22878 & PU-239 & $1.9189 E+00$ & $3.0965 E+\infty 0$ & $6.1972 E+01$ \\
\hline RHZ-212-A22878 & PU-240 & $4.2776 \mathrm{E}-01$ & $3.0965 E+\infty 0$ & $1.3814 \mathrm{E}+01$ \\
\hline RHZ-212-A22878 & $A M-241$ & $3.5397 \mathrm{E} \cdot 01$ & $3.0965 \mathrm{E}+00$ & $1.1431 E+01$ \\
\hline RHZ-212-A22878 & PU.241 & $2.4539 \mathrm{E} \cdot 01$ & $3.0965 E+00$ & $7.9248 E+00$ \\
\hline RHZZ-212-A22878 & PU. 238 & $1.4939 E-01$ & $3.0965 E+00$ & $4.8244 E+00$ \\
\hline RHZ-212-A22878 & TOTAL BETA/GAMMA & $1.0000 \mathrm{E}-03$ & $3.0965 E+00$ & $3.2295 \mathrm{E}-02$ \\
\hline RHZ-212-A22878 & PU-242 & 2.3246E-05 & $3.0965 E+00$ & $7.5073 \mathrm{E}-04$ \\
\hline ETFF-92-2B1-01 & TOTAL BETA/GAMMA & $2.0470 E+00$ & $2.0486 E+00$ & $9.9924 \mathrm{E}+01$ \\
\hline ETFF-92-281-01 & SR-90 - Y9O & $1.4577 \mathrm{E}-03$ & $2.0486 E+00$ & $7.1157 E-02$ \\
\hline ETFF-92-281-01 & CS-137 - BA-137M & $6.7488 E-05$ & $2.0486 \mathrm{E}+00$ & 3. 2944E-03 \\
\hline ETFF-92-281-01 & PU-239 & $1.5562 \mathrm{E}-05$ & $2.0486 \mathrm{E}+00$ & $7.5965 \mathrm{E} \cdot 04$ \\
\hline ETFF-92-281-01 & $A M \cdot 241$ & $1.3082 \mathrm{E}-05$ & $2.0486 \mathrm{E}+00$ & $6.3857 \mathrm{E}-04$ \\
\hline ETFF-92-281-01 & PU-240 & 3.6547E-06 & $2.0486 \mathrm{E}+00$ & $1.7840 \mathrm{E}-04$ \\
\hline ETFF-92-281-01 & PU-241 & $3.2960 \mathrm{E}-06$ & $2.0486 E+00$ & $1.6089 E-04$ \\
\hline ETFF-92-281-01 & PU-238 & $9.6478 \mathrm{E} \cdot 07$ & $2.0486 E+\infty 0$ & $4.7096 \varepsilon-05$ \\
\hline ETFF-92-281-01 & EU-154 & 8.84OOE-O8 & $2.0486 E+\infty 0$ & $4.3152 \mathrm{E}-06$ \\
\hline ETFF-92-281.01 & $\mathrm{co} \cdot 60$ & $2.1861 \mathrm{E}-08$ & $2.0486 E+00$ & $1.0671 \mathrm{E}-06$ \\
\hline ETFF-92-281-01 & $K-40$ & $3.8950 \mathrm{E}-09$ & $2.0486 \mathrm{E}+00$ & $1.9013 \mathrm{E}-07$ \\
\hline RHZ-85-002 & PU & $1.6767 \mathrm{E}+\infty$ & $1.6772 \mathrm{E}+00$ & $9.9970 E+01$ \\
\hline $\mathrm{RHZ}-85-002$ & TOTAL BETA/GAMMA & 5.0000E-04 & $1.6772 E+00$ & 2.9811E-02 \\
\hline $\mathrm{RHZ}-85-002$ & PE-CI & $0.0000 E+00$ & $1.6772 E+00$ & $0.0000 E+00$ \\
\hline $\mathrm{RHZ}-65-002$ & PU239 FSL GR EQUIV & $0.0000 E+00$ & $1.6772 \mathrm{E}+00$ & $0.0000 E+00$ \\
\hline $\mathrm{RHZ}-85-002$ & TOTAL-ALPHA & $0.0000 E+00$ & $1.6772 \mathrm{E}+\infty 0$ & $0.0000 E+00$ \\
\hline RHZ-87-044 & PU & $1.5002 E+00$ & $1.5004 \mathrm{E}+\infty 0$ & $9.9987 E+01$ \\
\hline $\mathrm{RHZ}-87 \cdot 044$ & TOTAL BETA/GAMMA & $2.0000 \mathrm{E}-04$ & $1.5004 E+\infty 0$ & $1.3330 \mathrm{E}-02$ \\
\hline RHZ-87-044 & $\mathrm{PE} \cdot \mathrm{Cl}$ & $0.0000 E+00$ & $1.5004 \mathrm{E}+\infty 0$ & $0.0000 E+\infty 0$ \\
\hline RHZ-87-044 & PU239 FSL GR EOUIV & $0.0000 E+\infty 0$ & $1.5004 E+00$ & $0.0000 E+00$ \\
\hline $\mathrm{RHZ}-87-044$ & TOTAL-ALPHA & $0.0000 E+\infty 0$ & $1.5004 \mathrm{E}+00$ & $0.0000 E+00$ \\
\hline $221 \mathrm{~T}-88-02-1$ & TOTAL BETA/GAMMA & $1.0000 E+00$ & $1.0000 E+00$ & $1.0000 E+02$ \\
\hline $221 T-88-02-2$ & TOTAL BETA/GAMMA & $1.0000 E+00$ & $1.0000 E+00$ & $1.0000 E+02$ \\
\hline $221 \mathrm{~T} \cdot 88-02-3$ & TOTAL BETA/GAMMA & $1.0000 E+00$ & $1.0000 E+00$ & $1.0000 E+02$ \\
\hline $221 \mathrm{~T}-88-02-4$ & TOTAL BETA/GAMMA & $1.0000 E+00$ & $1.0000 E+00$ & $1.0000 E+02$ \\
\hline $221 \mathrm{~T}-88-02-5$ & TOTAL BETA/GAMMA & $1.0000 E+00$ & $1.0000 E+00$ & $1.0000 E+02$ \\
\hline
\end{tabular}




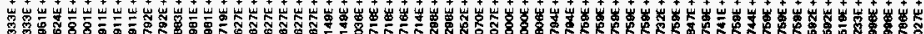

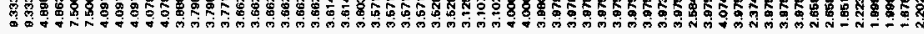

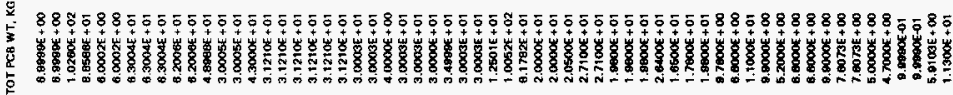

$\mathrm{g}$

$$
3
$$$$
\text { 言 }
$$$$
\vdots
$$

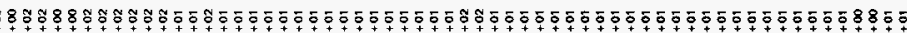

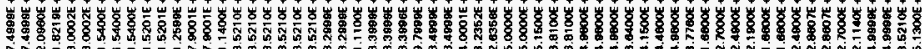

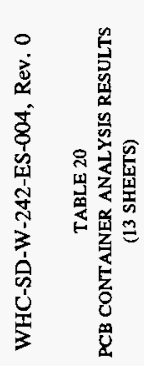

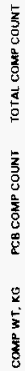

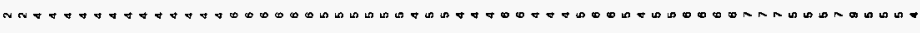

88 \%

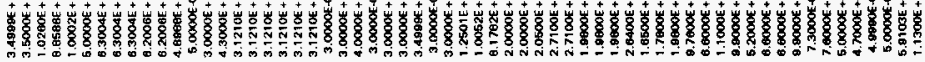

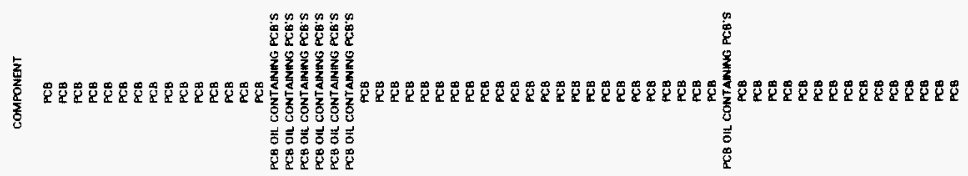




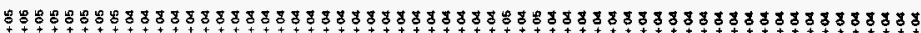

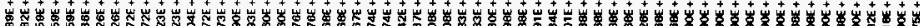

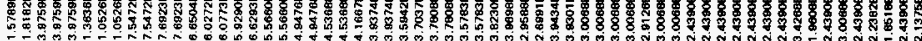

$\ddot{8}$

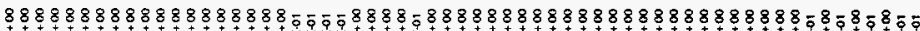

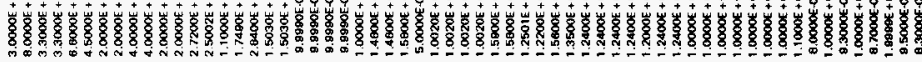

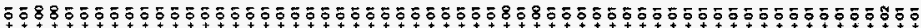

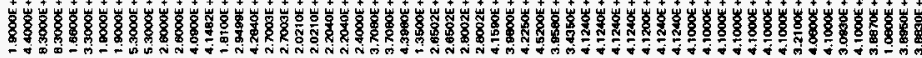

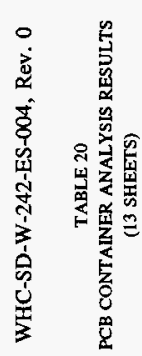

5
8
8
0
0
0
0
0
0
0

ம

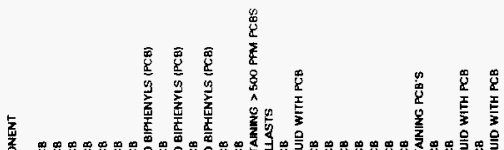

空

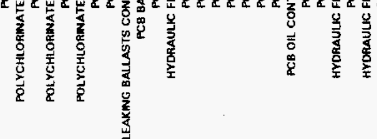

$\frac{\sqrt[5]{\frac{10}{2}}}{\frac{5}{2}}$

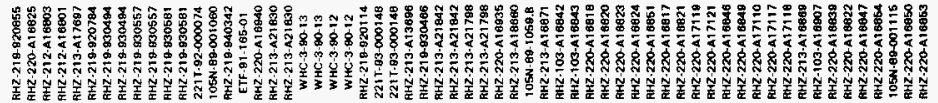



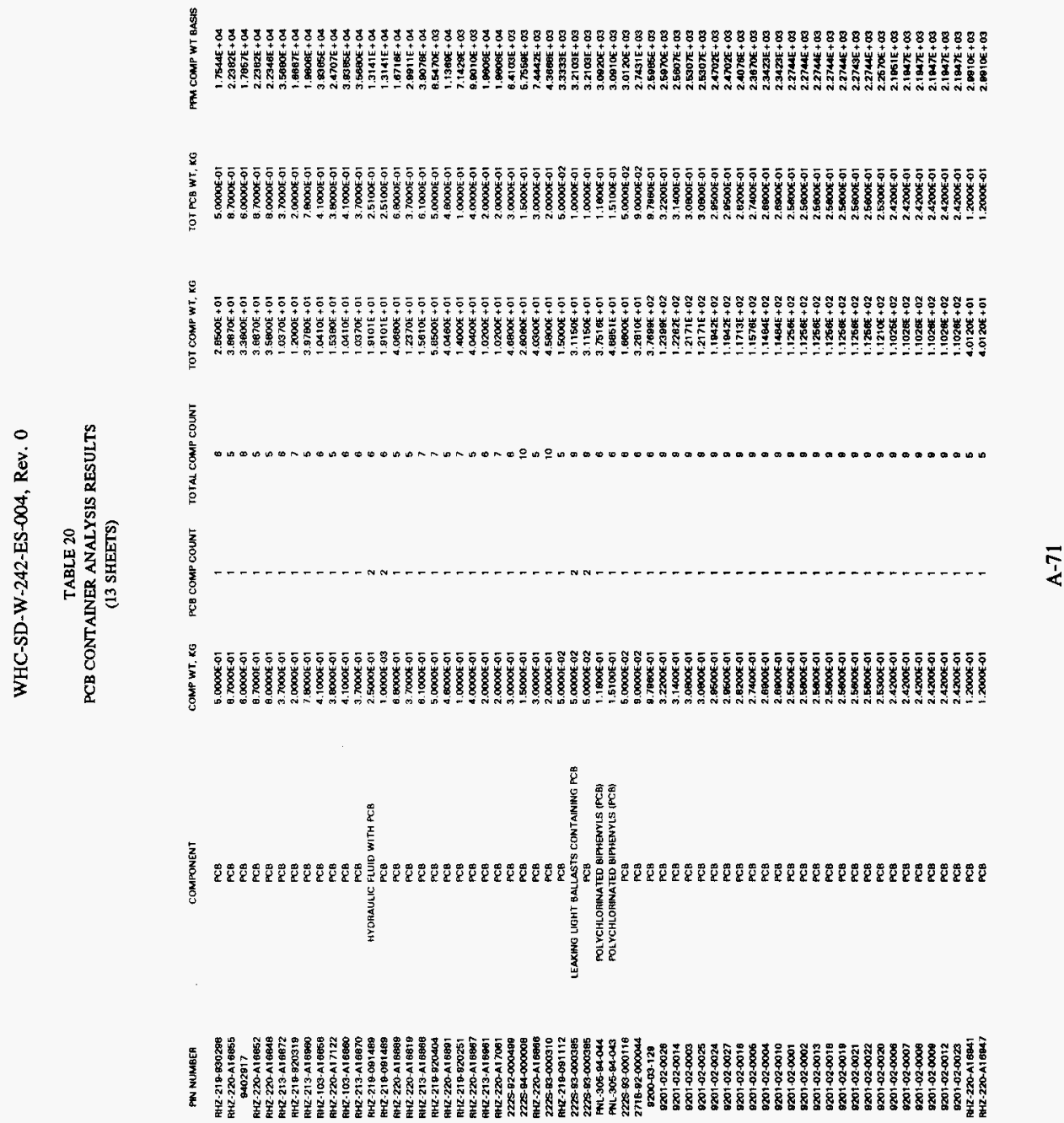

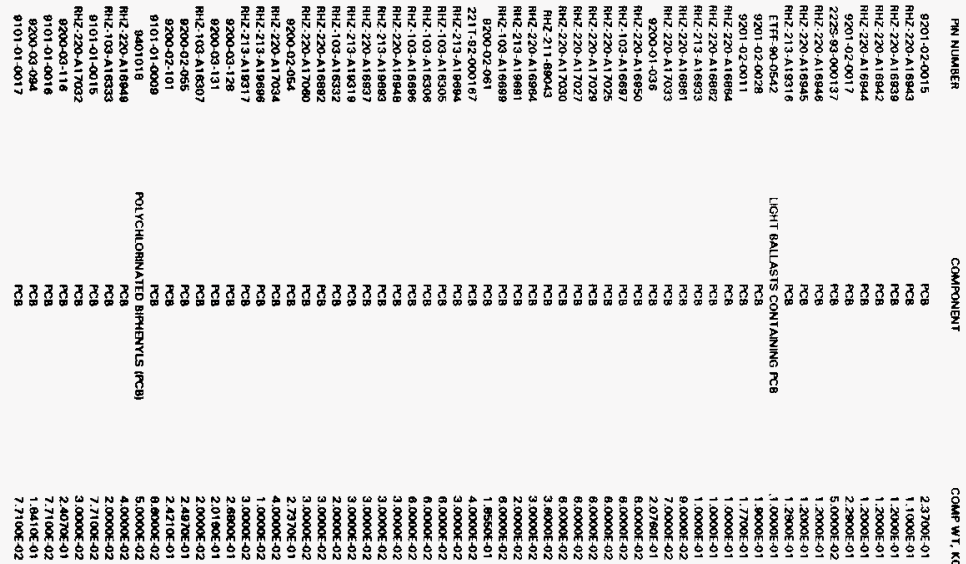

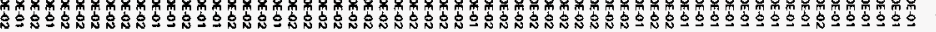

$\frac{2}{1}$
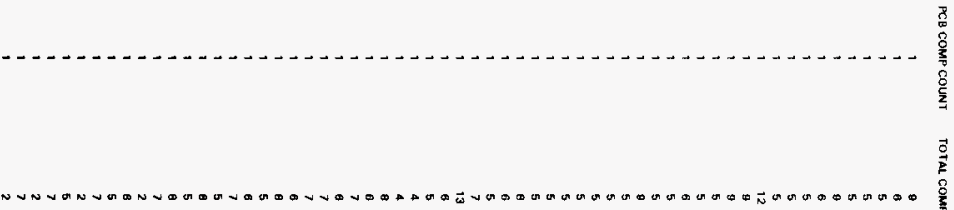

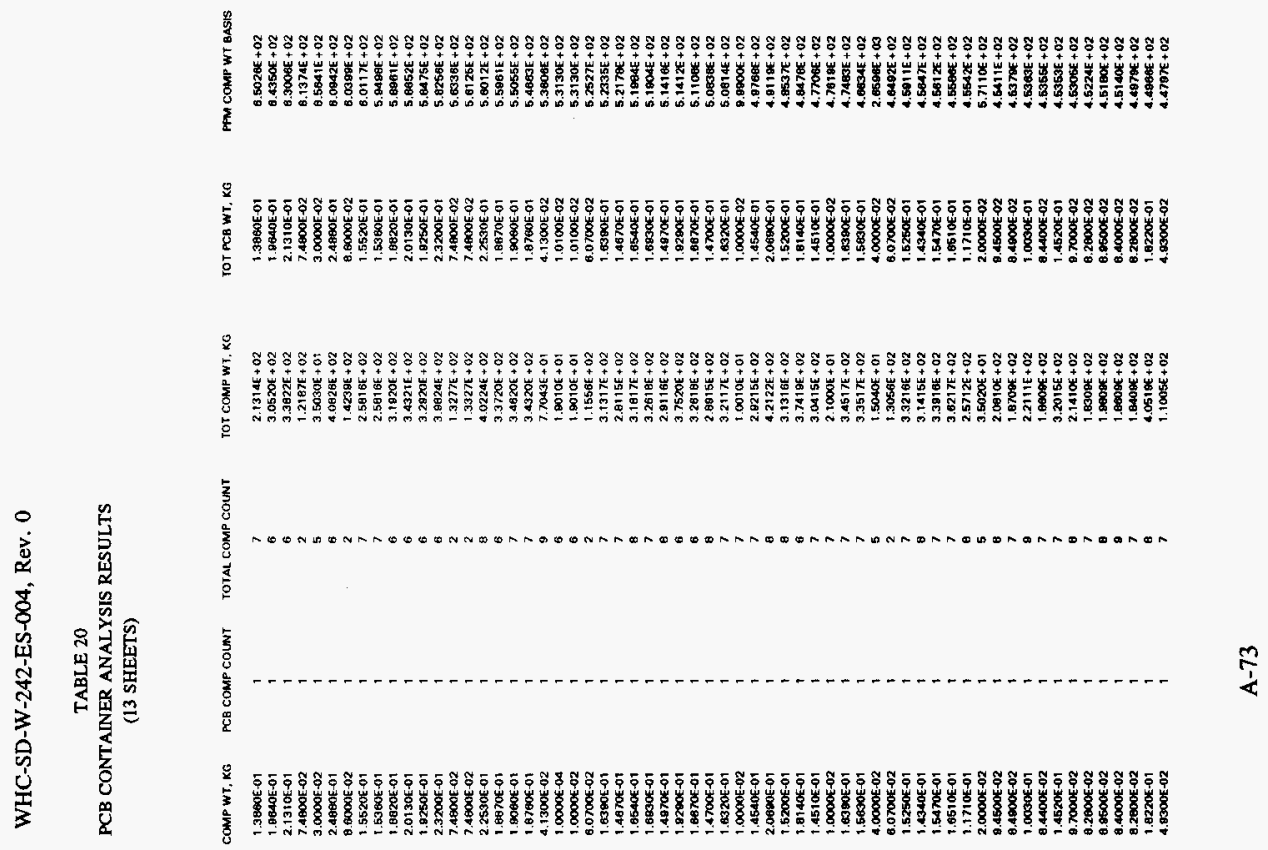

榇

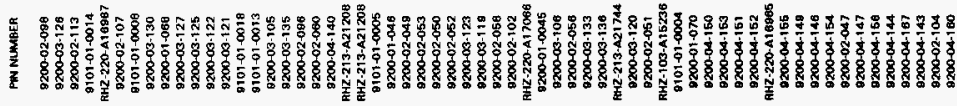




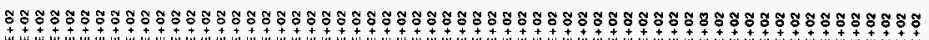

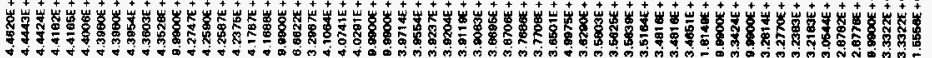

$\stackrel{8}{2}$

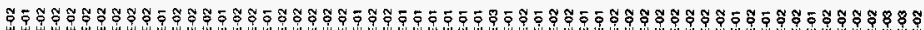

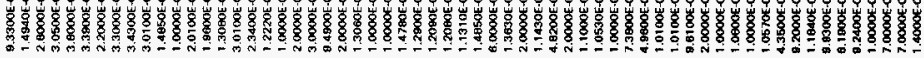

8

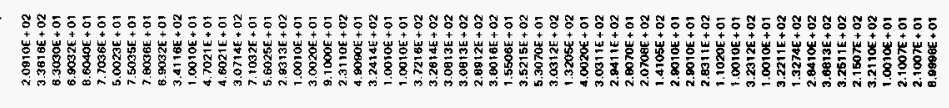

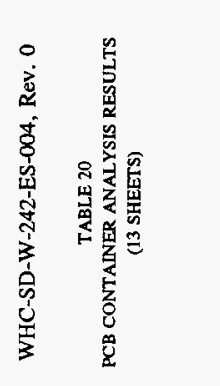

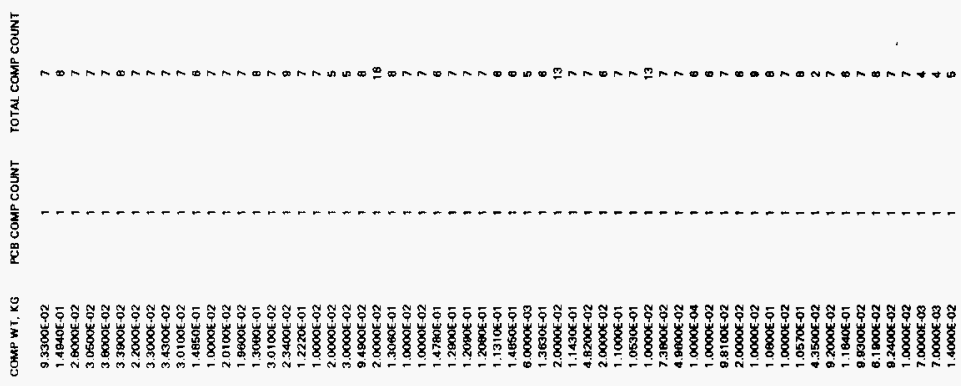

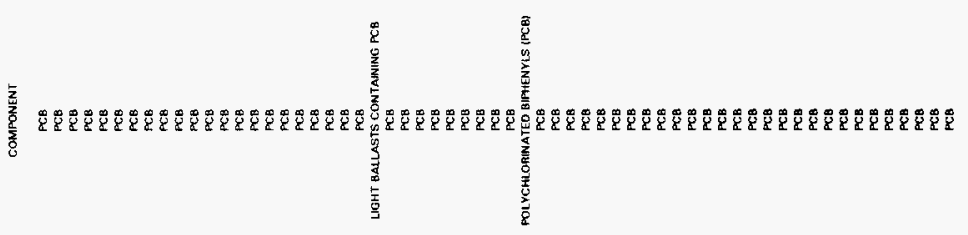

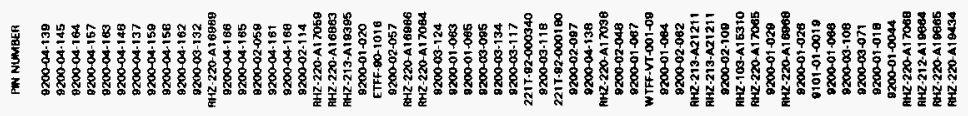


ช

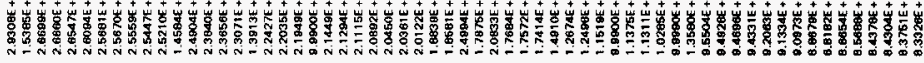

$\frac{5}{3}$

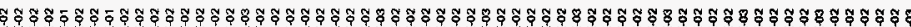

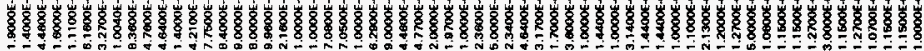

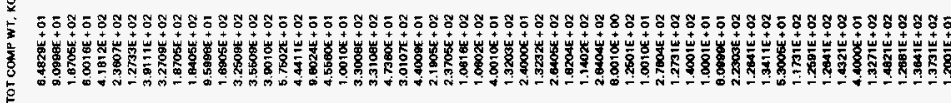

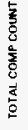

亭

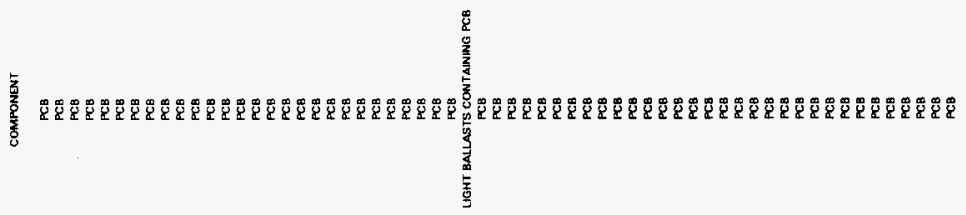



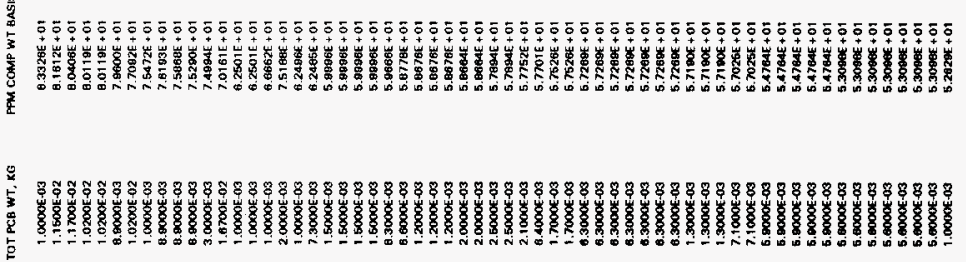

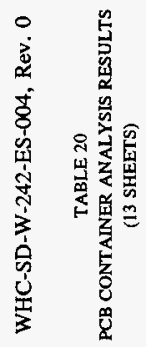

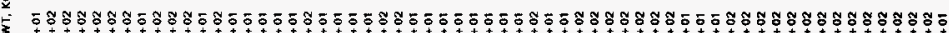

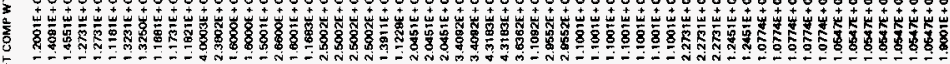
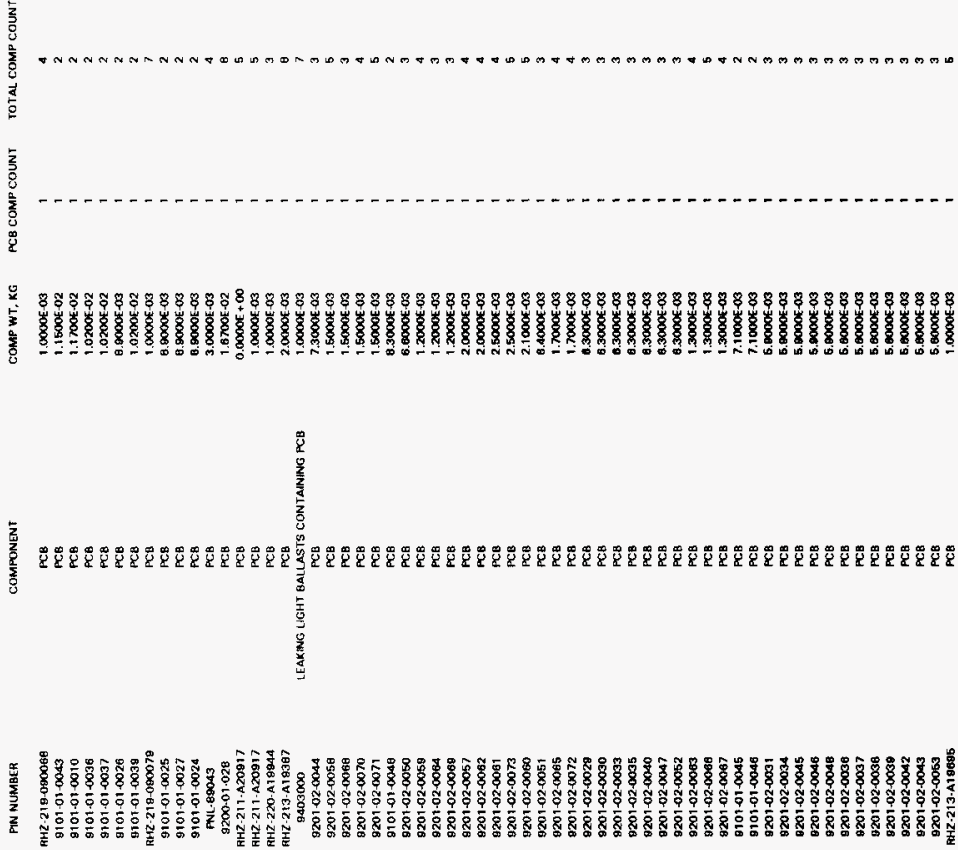

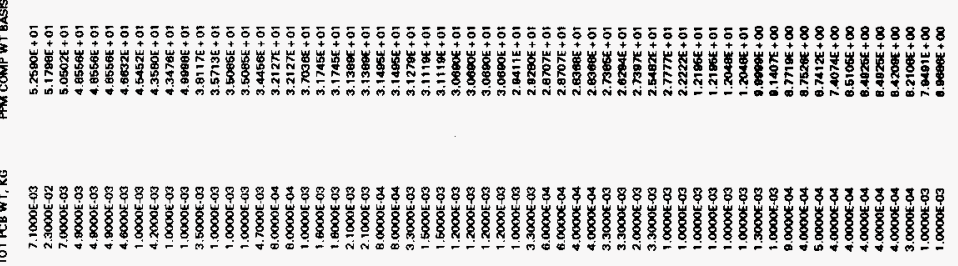

5

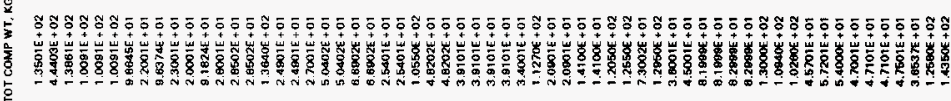

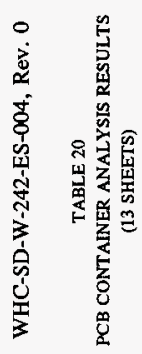
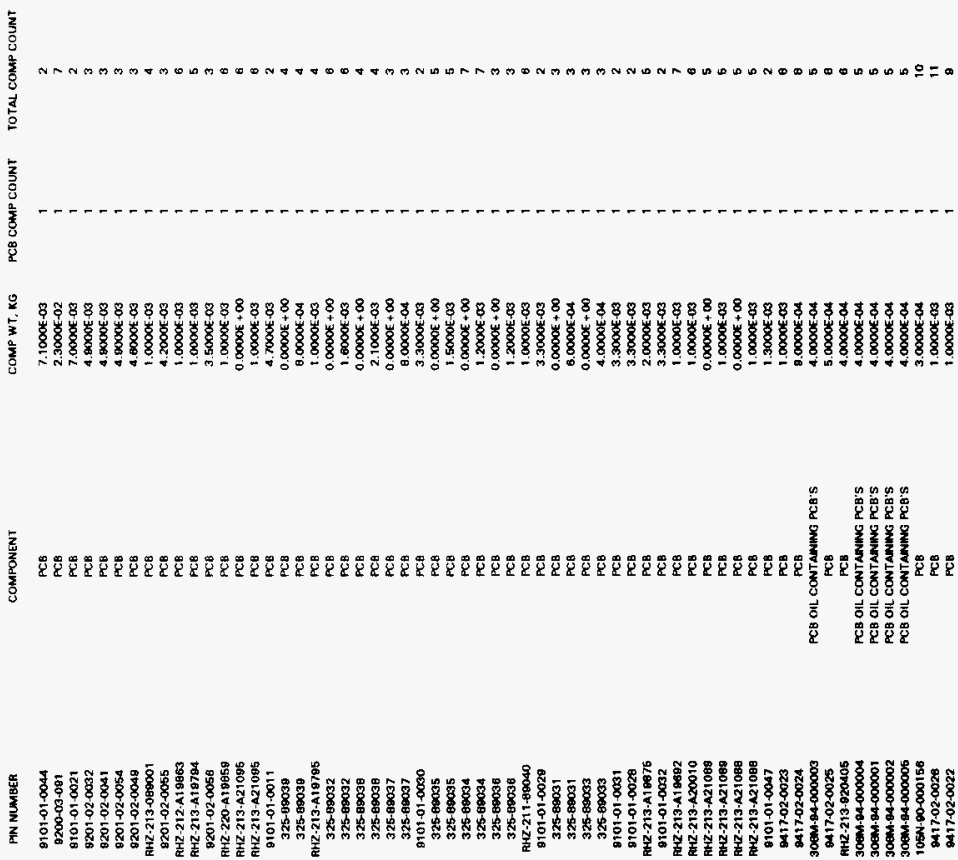

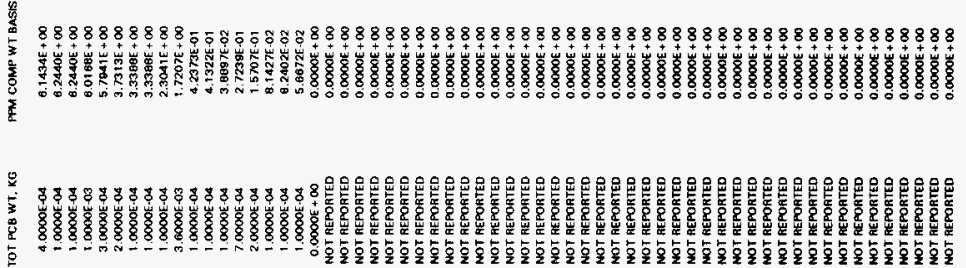

$\frac{2}{3}$

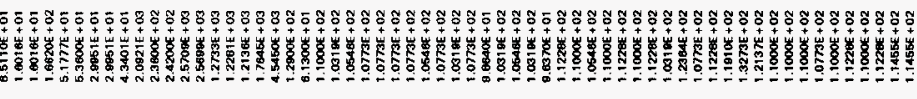

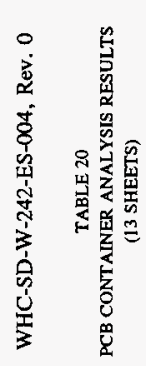

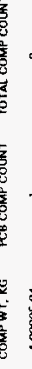

D = =

3 88 8 58 3

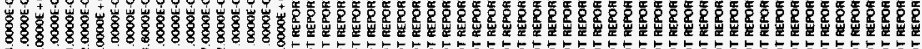

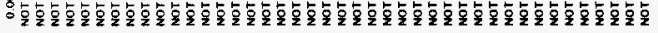

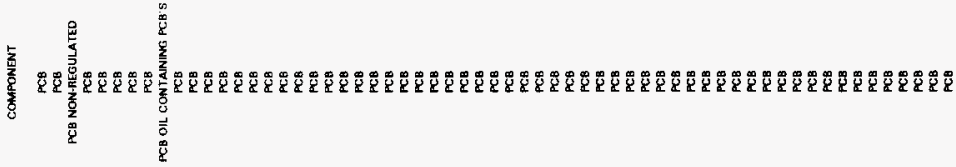

$\frac{5}{\frac{3}{2}}$

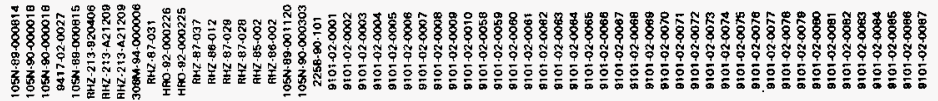


8888888888888888888888888888888888888888888888888888888888888

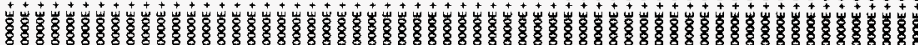
0\%

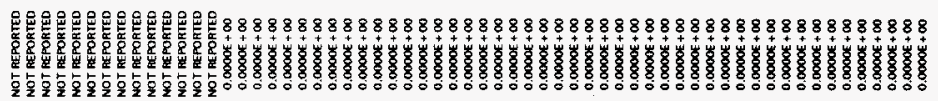

Q

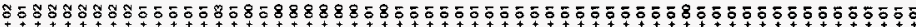

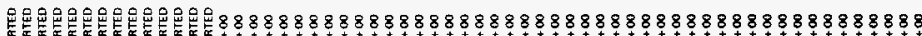

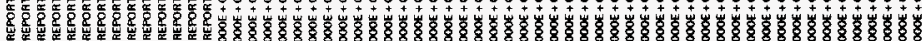

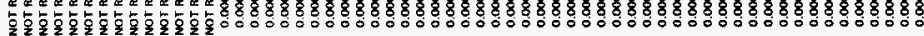

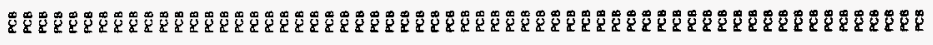

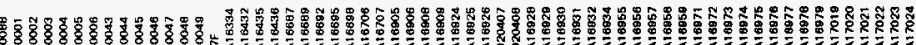

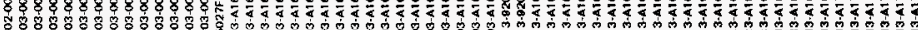

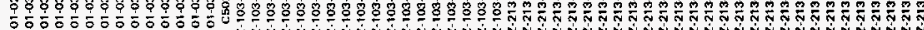

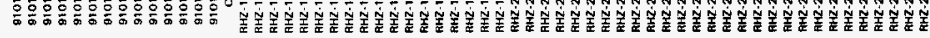




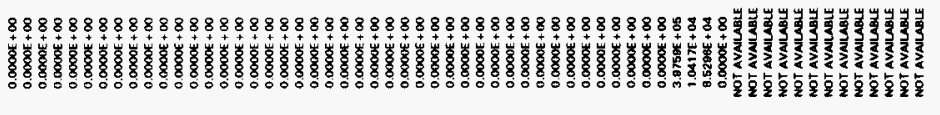
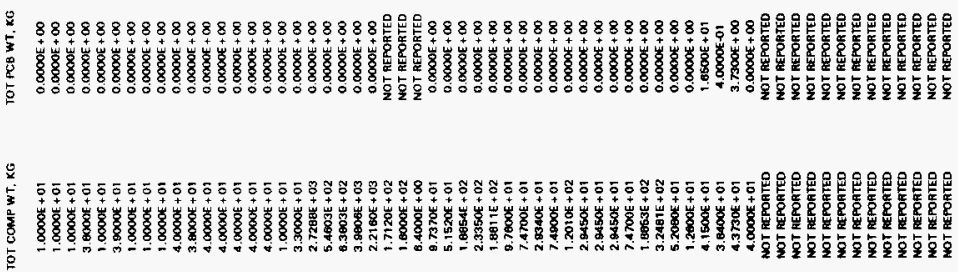

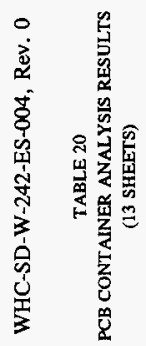

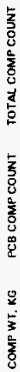

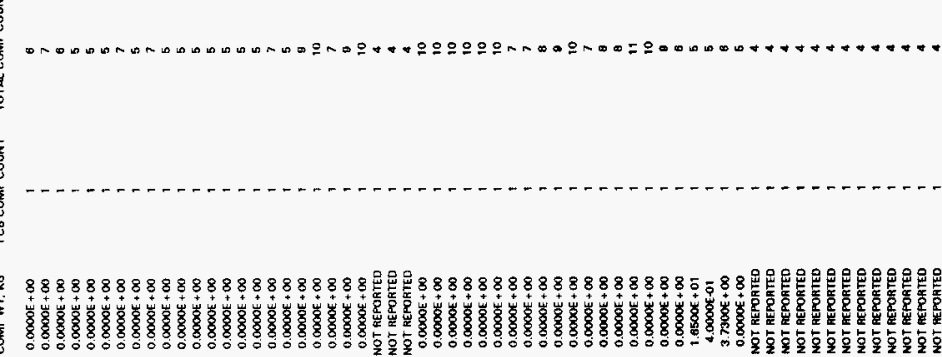



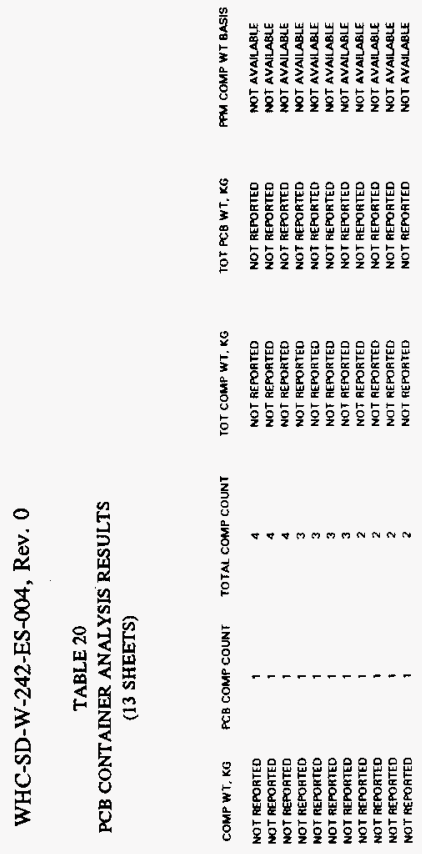

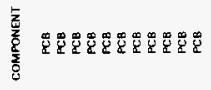

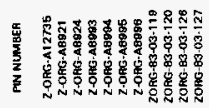


WHC-SD-W-242-ES-004, Rev. 0

TABLE 21

PIN-SPECIFIC CONCENTRATION OF PA-RELATED RADIONUCLIDES (7 SHEETS)

PIN

RSO0002493

PNL-89005

RS00000993

ASO0001693

PNL-89004

RSO0001793

RSO0000393

RS00001393

RS00011593

RS00001093

RS00000293

RS00000693

RS00002593

RSO0001493

305B-91-022

3058-91-023

305B-91-024

3058-91-026

305B-91-027

3058-91-028

RSO0001293

RS00000893

RSO0000493

3058-91-034

RSO0018894

RSO0002693

RS00012693

9403134

R500001593

RSO0012793

RS00002093

RS00000593

ASO0000193

RS00011893

RS00013293

PNL-89017

9401017

AS00002193

RS00016293

RS00012993

325-90-045

RS00001993

ASO001 2093

WTFF-92-322-04

RS00013093

RS00013693

PNL-89047

9101-01-0022

9101-01-0033

9101-01-0034

9101-01-0042

9101-01.0023

WTFF-92-322-03

9101-01-0040

9101-01-0035

PS00005693

9101-01-0041

9101.01 .0020

9101-01-0043

9101-01-0038

9101-01-0036

9t01-01-0037

9101-01-0026
TOTAL ASH VOL CU M

$2.0361 \mathrm{E}-03$

$2.8762 \mathrm{E} \cdot 03$

$2.0361 \mathrm{E}-03$

$2.0361 E-03$

2. 2519E-03

$2.0361 E-03$

2.0361E-03

$2.0361 \mathrm{E}-03$

$1.5438 \mathrm{E}-03$

2.0361E-03

2.0361E-03

$2.0361 \mathrm{E} \cdot 03$

$2.0361 \mathrm{E} .03$

$2.0361 \mathrm{E}-03$

8. 2460E-04

8.2460E-04

7.8601E-04

8. 2460E-04

8. $2460 \mathrm{E}-04$

8. 2460E-04

$2.0361 \mathrm{E}-03$

$2.0361 \mathrm{E}-03$

$2.0361 \mathrm{E}-03$

$1.4144 \mathrm{E}-03$

1.7118E-03

$2.0361 \mathrm{E}-03$

$1.5894 \mathrm{E}-03$

$6.6423 \mathrm{E}-03$

2.0361E-03

$1.7866 \mathrm{E}-03$

$2.0361 \mathrm{E}-03$

$2.0361 \mathrm{E} .03$

$2.0361 \mathrm{E}-03$

1. $6753 \mathrm{E}-03$

1. 6247E-03

$1.4024 \mathrm{E}-04$

$1.9203 \mathrm{E}-02$

$2.0361 \mathrm{E}-03$

$1.6904 \mathrm{E}-03$

$1.6753 \mathrm{E}-03$

$7.6329 \mathrm{E}-04$

$2.0361 \mathrm{E}-03$

$1.6450 \mathrm{E}-03$

3.1168E-01

1. $6803 E-03$

1. $6803 \mathrm{E}-03$

1.4176E-01

$1.3416 \mathrm{E}-03$

1.1735E-03

1. $2596 \mathrm{E}-03$

$1.2646 \mathrm{E} \cdot 03$

1.4327 E-O3

$3.0950 \mathrm{E}-01$

$1.3276 \mathrm{E}-03$

$1.4827 \mathrm{E}-03$

2.0351E-03

1. $3646 \mathrm{E}-03$

$1.3736 \mathrm{E}-03$

$1.4096 \mathrm{E}-03$

1. $2646 \mathrm{E}-03$

$1.2736 \mathrm{E}-03$

1. $2736 \mathrm{E}-03$

$1.1105 \mathrm{E}-03$
TOTAL PA CI/CU M

$1.8369 E+01$

$1.2169 \mathrm{E}+00$

1.164OE + 00

1.1566E + 00

$1.1102 E+00$

$1.0191 E+00$

B.1039E-01

8.1039E-01

7.1319E-01

$6.2081 \mathrm{E}-01$

4.3319E-01

4.1993E-01

4.1993E-01

3.9979E-01

3.8079E-01

3.8079E-01

3.4859E-01

3. 3228E-01

3.3228E-01

3.3228E-01

3.2415E-01

2.5196E-01

2.5048E-01

2.2201E-01

2.0446E-01

$1.9597 \mathrm{E}-01$

$1.5792 \mathrm{E}-01$

$1.5028 \mathrm{E}-01$

$1.2524 \mathrm{E}-01$

1.2197E-01

1.1345E-01

$1.1051 \mathrm{E}-01$

$1.0314 \mathrm{E}-01$

7.5210E-02

7.5091E-O2

7.1306E-02

6.6136E-02

6.0509E-02

6.0151E-02

$5.9690 \mathrm{E}-02$

4.5592E-02

3.1626E-02

3. $1611 \mathrm{E}-02$

3.0869E-02

2.9756E-02

2.9756E-02

2.6196E-02

$2.4336 \mathrm{E}-02$

2.3646E-02

2.3329E-02

2.3236E-02

2.2790E-02

2. 2240E-02

2. $2133 \mathrm{E}-02$

2.2021E-02

2.1805E-02

2.1533E-02

2.1392E-O2

2.0845E-02

2.0654E-02

2.0508E-02

2.0508E-02

2.0431E-02 
WHC-SD-W-242-ES-004, Rev. 0

TABLE 21

PIN-SPECIFIC CONCENTRATION OF PA-RELATED RADIONUCLIDES (7 SHEETS)

PIN

9101-01-0039

9101-01-0025

WTFF-92-322-02

9101-01-0027

9101-01-0024

331-90-072

RS00013893

9101-01-0048

9101-01-0045

9101-01-0046

RS00005593

9101-01-0044

2225-92-000507

9101-01-0021

PNL-88052

RS00005193

PNL-LSL-93-042

PNL-LSL-93-043

224U-89-0093

224U-89-0094

224U-89-0095

224U-89-0096

PNL-88051

222S-92-000064

RALOOOO194

o\$9401-18-0001

9101-01-0030

RS00009593

RS00015793

RS00005393

9101-01-0029

RS00009793

RS00013393

9200-04-166

9101-01-0031

9302-06-0001

9200-04-165

9101-01-0028

940200025

$\mathbf{9 4 0 2 0 0 0 2 6}$

Os9401-18-0005

9101.01-0032

os9401-18-0004

RS00007693

RS00015193

RS00001893

9101.01.0009

RS00014093

059401-18-0007

RS21794

9101.01.0015

RS00009093

9101.01.0016

RS00009293

RS00008793

9101.01.0017

RS00008293

3720-91.040

9101-01-0014

PNL-89048

9101-01-0008

9101.01-0018

9101-01-0013
TOTAL ASH VOL CU M

$1.3236 \mathrm{E}-03$

$1.1685 \mathrm{E}-03$

3.1153E-01

1.1735E-03

$1.1825 E-03$

$2.7276 E-02$

$1.6147 E-03$

$1.3916 \mathrm{E}-03$

1.2456E-03

1. $2456 \mathrm{E}-03$

2.0502E-03

$1.3506 \mathrm{E}-03$

$1.7169 E-02$

$1.3866 \mathrm{E}-03$

2.2631E-03

2.0300E-03

7.6989E-04

1.7807E-02

3.5824E-02

3. 5824E-02

3.5824E-02

3.5824E-02

2. $3081 \mathrm{E}-03$

2.0594E-02

$1.0996 \mathrm{E} \cdot 03$

$2.1178 \mathrm{E}-03$

$1.0555 \mathrm{E}-03$

$1.8797 \mathrm{E}-03$

$1.4051 \mathrm{E} .03$

$1.9489 \mathrm{E}-03$

$1.1275 \mathrm{E}-03$

$1.8949 \mathrm{E}-03$

$1.6298 E-03$

$9.0029 \mathrm{E}-04$

$1.2056 E-03$

4. $9094 \mathrm{E}-02$

8. $9372 \mathrm{E} \cdot 04$

1. $2556 \mathrm{E}-03$

$6.1546 \mathrm{E}-04$

$6.0131 \mathrm{E}-04$

$2.6797 \mathrm{E} \cdot 03$

1. $2956 \mathrm{E}-03$

$2.4904 \mathrm{E}-03$

$1.8746 \mathrm{E}-03$

$1.5539 \mathrm{E}-03$

$2.0361 \mathrm{E}-03$

$1.1 B 25 E-03$

$1.5186 \mathrm{E}-03$

$1.6931 \mathrm{E}-03$

$1.6721 \mathrm{E}-03$

$1.1455 \mathrm{E}-03$

$1.9051 \mathrm{E}-03$

$1.1685 \mathrm{E}-03$

$1.8848 \mathrm{E}-03$

$1.9304 \mathrm{E}-03$

$1.1825 \mathrm{E} .03$

$1.8594 \mathrm{E}-03$

1.3278E-02

1. $2186 \mathrm{E}-03$

$1.4176 \mathrm{E}-01$

$1.4237 \mathrm{E}-03$

$1.3276 \mathrm{E}-03$

$1.3326 \mathrm{E}-03$
TOTAL PA CI/CU M

1.9733E-02

1.9557E-02

1.9524E-02

1.9473E-02

$1.9325 \mathrm{E}-02$

$1.8500 E-02$

$1.5483 \mathrm{E}-02$

$1.5249 \mathrm{E}-02$

$7.4547 \mathrm{E}-02$

1. $4547 \mathrm{E}-02$

1.3754E-02

1.3416E-02

1. 2996E-02

1. 2949E-O2

1.2373E-02

1.2316E-02

$1.2246 \mathrm{E}-02$

1.1388E-02

1.0077E-02

$1.0077 \mathrm{E}-02$

1.0077E-02

1.0077E-02

9.5317E-03

9.4203E-03

B.3892E-03

8. 3088E-03

8.0438E-03

7.9802E-03

7.8287E-03

7.7207E-03

7.5299E-03

7.3881E-03

7.3627E-03

$7.0746 \mathrm{E}-03$

7.0425E-03

6.9256E-03

6.8095E-03

$6.7619 \mathrm{E}-\mathrm{O} 3$

6.661 6E-O3

6. $6522 \mathrm{E}-03$

6.5667E-03

$6.5531 \mathrm{E}-03$

6.5368E-03

$6.4706 \mathrm{E}-03$

6.4354E-O3

5.8937E-03

5.8465E-O3

5.8100E-03

5.7842E-O3

5.6814E-03

5.41 25E-03

5.3226E-O3

5.3059E-O3

5.3056E-03

5. 2528E-03

5. 243OE-O3

$5.0824 E-03$

4.9705E-03

4.9357E-03

4.8797E-03

4.8563E-03

4.5303E-03

4.51 $33 \mathrm{E}-03$ 
WHC-SD-W-242-ES-004, Rev. 0

TABLE 21

PIN-SPECIFIC CONCENTRATION OF PA-RELATED RADIONUCLIDES (7 SHEETS)

PIN

9101-01-0005

$9417.02-0008$

9417-02-0016

9101.01.0004

RS 20994

RS00003693

OS9401-18-0025

RSO0014193

RS00013993

224U-94.000070

$331-90-013$

059401-18-0026

$9200-04-160$

059401-18-0008

$059401 \cdot 18.0021$

$9302 \cdot 11.0003$

9101.01 .0019

RS00004493

9200-04-157

AS00005093

RS00007793

9101-01-0047

RS00008893

0\$9401-18.0009

9200-04-183

RSO0017194

9200-04-15B

9200-04-159

9101-01-0006

RS00017094

9200-04-161

9200-04-162

9200-04-164

059401-18-0003 16-069

WH-333-94-0O22

RS00018294

16-056

9101-01-0012

WHC-3-90-0026

9417.02-0009

9417.02.0005

RS00016993

16-070

16-071

ASO0014993

9302-08-0005

RS00017394

RS000020394

9408381

RS 00004793

RS00012493

9317-03-0008

RALOOO0294

9408753

9417.02 .0001

9101-01-0003

303K-93-000050

9101.01 .0001

9417-02-0011

9101-01-0002

RS00006793

329-90-34
TOTAL ASH VOL CU M

1.1555E-03

4.1984E-03

4.0133E-03

$1.3056 \mathrm{E} \cdot 03$

1.2084E-03

$2.0705 \mathrm{E}-03$

$2.7392 \mathrm{E}-03$

$1.5641 \mathrm{E}-03$

$1.5641 \mathrm{E}-03$

9.7343E-04

$2.9612 \mathrm{E}-02$

2.3807E-03

$1.7283 E-03$

2. $7064 \mathrm{E}-03$

2. $7064 \mathrm{E}-03$

3. $7990 \mathrm{E}-02$

$1.3276 \mathrm{E}-03$

$2.0047 E-03$

9.2657E-04

$1.9995 \mathrm{E}-03$

$1.8544 \mathrm{E}-03$

1. 3006E-03

$1.9202 \mathrm{E}-03$

$3.1684 \mathrm{E}-03$

1.4641E-03

$1.1220 \mathrm{E}-03$

$1.3287 \mathrm{E}-03$

$1.2913 \mathrm{E}-03$

$1.2736 \mathrm{E}-\mathrm{O3}$

$1.1356 \mathrm{E} \cdot 03$

$1.2473 \mathrm{E} \cdot 03$

$1.2223 E-03$

$1.1237 \mathrm{E}-03$

$2.6112 \mathrm{E}-03$

$1.4688 \mathrm{E}-02$

$3.3455 \mathrm{E}-03$

$1.0362 \mathrm{E}-03$

$1.4748 \mathrm{E}-02$

$1.3236 \mathrm{E}-03$

$1.4224 \mathrm{E}-01$

5.6536E-03

$1.3676 \mathrm{E}-02$

$1.4942 \mathrm{E}-03$

$1.4758 \mathrm{E}-02$

$1.4748 \mathrm{E}-02$

1.5742E-03

3.7145E-02

$1.1116 \mathrm{E}-03$

2.4659E-03

9.0170E-03

2.0604E-03

$1.7056 \mathrm{E}-03$

$1.0266 \mathrm{E}-01$

1. 2508E-03

1. 11 OGE-02

7.534OE-03

1. 2506E-O3

3.8649E-02

$1.2736 \mathrm{E}-03$

6.9232E-03

$1.4006 \mathrm{E}-03$

$1.8746 \mathrm{E}-\mathrm{O} 3$

$1.0667 \mathrm{E}-03$
TOTAL PA CI/CU M

4.2282E-O3

4.1456E-03

3.8345E-03

3.7422E-03

$3.7240 \mathrm{E}-\mathrm{O} 3$

$3.3228 \mathrm{E}-03$

$3.2126 \mathrm{E}-\mathrm{O} 3$

3.1968E-03

3.1968E-03

3.047OE-03

$3.0393 \mathrm{E}-03$

2.7723E-03

2.71 10E-03

2.6890E-03

2.6890E-03

2.6850E-03

$2.6329 \mathrm{E}-03$

$2.6129 \mathrm{E}-03$

2.5933E-03

2.5406E-03

2.5184E-03

2.5104E-03

2.4632E-03

2. 2969E-03

2. $2653 \mathrm{E}-03$

2.1853E-03

2.1486E-03

2.0950E-03

2.0669E-03

2.0609E-03

2.0048E-03

$1.9260 \mathrm{E}-\mathrm{O} 3$

$1.6663 \mathrm{E}-03$

$1.5536 \mathrm{E}-03$

$1.5414 \mathrm{E}-03$

$15061 \mathrm{E}-03$

$1.4978 \mathrm{E} \cdot 03$

$1.4409 \mathrm{E}-03$

$1.4199 \mathrm{E}-03$

$1.4180 \mathrm{E}-03$

$1.4026 \mathrm{E}-03$

$1.3439 \mathrm{E}-03$

$1.3385 \mathrm{E}-03$

$1.2935 \mathrm{E}-03$

$1.2903 \mathrm{E}-03$

$1.2705 \mathrm{E}-03$

$1.2384 \mathrm{E}-03$

$1.2235 \mathrm{E}-03$

$1.2166 \mathrm{E}-03$

$1.2147 \mathrm{E}-03$

$1.1085 \mathrm{E}-03$

$1.0612 \mathrm{E} \cdot 03$

$1.0536 \mathrm{E}-03$

1.0291E-03

9. $8626 \mathrm{E}-04$

$9.8561 \mathrm{E}-04$

9. $8050 E-04$

$9.6915 \mathrm{E}-04$

$9.6278 \mathrm{BE}-04$

9.5267E-04

8.7545E-04

8. 5883E-04

8.4373E. 04 
WHC-SD-W-242-ES-004, Rev. 0

TABLE 21

PIN-SPECIFIC CONCENTRATION OF PA-RELATED RADIONUCLIDES (7 SHEETS)

PIN

RS0001729

303K-93-000047

9417-02-0007

9417-02-0019

$303 \mathrm{~K}-93-000037$

331-90-071

RS00016393

9417-02-0014

303K-93-000035

221T-93-000112

RS00014393

9317-03-0007

RSO0018994

303K-93-000039

9101-01-0007

9417.02 .0013

OS9401-18.0012

9417-02.0018

RS00015293

9101-01-0010

AS00015593

9317-03.0006

RS00011193

R\$00016893

RS00015393

RS00005893

950800225

950800226

OS9401-18-0010

RFLO000294

9417-02-0002

303K-93-000036

OS9401-18-0011

RSO0020794

9417-02-0017

059401-18-0030

303K-93-000034

PNL-305-94-041

R\$00008193

9417-02-0010

R500019394

9417-02-0003

9417.02.0012

9201-02-0063

R521194

9201-02-0070

9201-02-0066

R500005493

950800228

RS00020594

303K-93-000046

RSO0005793

9417-02-0004

950800229

950800230

9417-02-0021

950800231

303K-93-000042

$303 \mathrm{~K} \cdot 93.000043$

303K-93-000040

9417-02-0025

9200-04-139
TOTAL ASH VOL CU M

1.1168E-03

1.3187E-02

1.7021E-02

1.1916E-02

1.0755E-02

2.8836E-02

$2.7166 \mathrm{E}-02$

$1.6399 \mathrm{E}-03$

1. $1693 \mathrm{E}-02$

3. $8220 \mathrm{E}-02$

1.8929E-03

2.1102E-03

1.5642E-01

2.4387E-03

3. $6660 \mathrm{E}-02$

1. $2686 \mathrm{E}-03$

1.4143E-02

$2.5473 \mathrm{E}-03$

1. $4019 \mathrm{E}-02$

1.5489E-03

1.4557E-03

$1.5641 \mathrm{E}-03$

1.7279E-01

$1.5742 \mathrm{E}-03$

$1.5893 \mathrm{E}-03$

1.6147E-03

1. 8544E-03

2. 1449E-02

2. 1449E-02

2.8844E-03

$1.0139 \mathrm{E}-03$

1.7433E-02

4. 2382E-02

3.0092E-03

1.7504E-O3

$1.7151 \mathrm{E}-02$

2.2757E-O3

3.8334E-O2

2.1737E-O2

1.8949E-03

$1.9188 \mathrm{E}-02$

1.9239E-03

2. $1350 \mathrm{E}-02$

2.2996E.02

1.5504E-04

2.5838E-03

1.6995E-04

1.5906E-04

2.0908E-03

2.4608E-02

$3.4172 \mathrm{E}-\mathrm{O} 3$

4.5884E-O2

1.9793E-03

2.5205E-02

2.7521E-02

2.7521E-02

2.0137E-02

2. $1254 \mathrm{E}-02$

2.9043E-02

2.8420E-02

2.7905E-02

3.7598E-02

7.6807E-02
TOTAL PA CI/CU M

8.3954E-04

8.3062E-04

8. 2494E-04

8.0645E-04

7.9976E-04

7.7782E-04

7.7671E-04

$7.6226 \mathrm{E}-04$

7.6147E-04

7.5307E-04

7.1183E-04

7. $1083 \mathrm{E}-04$

$7.0618 \mathrm{E}-04$

7.0120E-04

6.9241E-04

6.8131E-04

6.7946E-04

6.7524E-04

6.5672E-O4

6.4562E-O4

$6.4552 \mathrm{E}-04$

6.3936E-04

6. $3754 \mathrm{E}-04$

6.3524E-04

6. 2921E-04

6.1932E-O4

6.0938E-04

5.9707E-04

5.9707E-O4

5.9632E-O4

5.9049E-O4

5.8206E-O4

5.7799F-04

5.7158E-04

$5.7130 \mathrm{E}-04$

5.6813E-04

5. $6686 \mathrm{E}-04$

5.6483E-04

5.5206E-04

5.5200E-04

5.3058E-04

5.1979E-O4

5.0675E-04

6.0116E-04

4.9314E-04

4.9307E-04

4.927OE-04

4. 8067E-04

4.7828E- 04

4.7578E-04

4.6938E-04

4. $6442 \mathrm{E}-04$

4.5470E-O4

4.545BE-04

4. $2542 \mathrm{E}-04$

4. 2542E-0.4

4.1881E-04

4. $1643 \mathrm{E}-04$

4. $1556 \mathrm{E}-04$

4.0979E-04

3. $9611 E-04$

3.8785E-04

3.8749E-04 
WHC-SD-W-242-ES-004, Rev. 0

TABLE 21

PIN-SPECIFIC CONCENTRATION OF PA-RELATED RADIONUCLIDES (7 SHEETS)

PIN

\begin{tabular}{|c|}
\hline $303 K-93-000041$ \\
\hline RAL1194 \\
\hline $222 S 2 W-91-0498$ \\
\hline $2225-94-000008$ \\
\hline RS00009993 \\
\hline $9417-02-0020$ \\
\hline $9200-04-138$ \\
\hline RSO00007593 \\
\hline $303 K-93-000038$ \\
\hline 9417.02 .0027 \\
\hline 222S-93-000131 \\
\hline 222S-93-000134 \\
\hline Rs00006993 \\
\hline RS00014493 \\
\hline $16-055$ \\
\hline $9201 \cdot 02-0071$ \\
\hline RS00017994 \\
\hline $9101-01-0011$ \\
\hline Rs00010093 \\
\hline $303 K-93-000044$ \\
\hline $303 K \cdot 93-000048$ \\
\hline $303 \mathrm{~K}-93-000049$ \\
\hline $303 K-93-000051$ \\
\hline 9302.06 .0002 \\
\hline $221 \mathrm{~T}-94-000048$ \\
\hline $9302-06-0003$ \\
\hline $9201-02-0073$ \\
\hline $224 U-92 \cdot 000007$ \\
\hline $303 K-93-000025$ \\
\hline 9101.02 .0076 \\
\hline $9201-02-0062$ \\
\hline $303 K-93-000045$ \\
\hline $9417-02-0024$ \\
\hline 950800234 \\
\hline 9406767 \\
\hline $224 U-91-004$ \\
\hline $9101-02-0072$ \\
\hline $16-057$ \\
\hline $9101+02-0077$ \\
\hline RF93400493 \\
\hline RAL0494 \\
\hline $9101-02-0075$ \\
\hline $9417-02-0015$ \\
\hline $224 \cup-92-000041$ \\
\hline $9101-02-0064$ \\
\hline $9101-02-0068$ \\
\hline $9107-02-0070$ \\
\hline $9101-02-0074$ \\
\hline GEL-94-183-037 \\
\hline $224 U-92.000020$ \\
\hline 9200-04-153 \\
\hline 9417-02-0006 \\
\hline $9101-02-0065$ \\
\hline $9101-02-0067$ \\
\hline $9101-02-0069$ \\
\hline RS00011993 \\
\hline $224 U-92-000013$ \\
\hline PF93400193 \\
\hline $224 U-93-000114$ \\
\hline $9101-02-0058$ \\
\hline $9101-02-0073$ \\
\hline 2225-93-000135 \\
\hline
\end{tabular}

TOTAL ASH VOL CU M

4. 2153E-02

$5.6704 E-03$

2.3733E-02

4.5108E-04

1.6399E-03

2.3591E-02

4. $2712 \mathrm{E}-02$

1.8594E-03

3.7973E-02

3. 2025E-01

7. $6850 \mathrm{E}-03$

1. 2319E.02

$1.9253 \mathrm{E}-03$

$1.6905 \mathrm{E}-03$

$1.4768 \mathrm{E}-02$

2.9188E-04

$1.0980 \mathrm{E}-03$

1.3646E-03

1.7006E-O3

5. $1300 \mathrm{E}-\mathrm{O} 2$

4.864OE-O2

4. 3804E-02

4.7698E-02

3.0798E-02

5. $7073 \mathrm{E}-02$

3.5318E-02

6. $1045 E-04$

2. 2556E-03

9.8755E-O3

1.4257E-01

5.6015E-04

5. $1882 \mathrm{E}-02$

8.3391E-02

2. $6668 \mathrm{E}-02$

1. $2442 \mathrm{E}-02$

9. 2528E-O4

$1.4248 \mathrm{E}-\mathrm{O}$

$1.4748 \mathrm{E}-\mathrm{O} 2$

1.4245E-01

5.4129E-03

7. 250OE-O3

1.4243E-01

$5.7981 \mathrm{E}-\mathrm{O} 2$

3.9609E-03

1. $4236 \mathrm{E}-01$

$1.4236 \mathrm{E}-01$

1.4236E-01

1.4236E-O1

2. $3508 E-04$

3.4156E-03

1. 1679E-OI

6.4828E-02

$1.4234 \mathrm{E}-01$

$1.4234 \mathrm{E}-01$

1. $4234 \mathrm{E}-01$

1. $6855 \mathrm{E}-03$

3.5787E-03

1. $4028 \mathrm{E}-02$

1. $1053 \mathrm{E}-03$

1. $4232 \mathrm{E}-\mathrm{O}$ ]

1.4232E-O

1. $5945 \mathrm{E}-\mathrm{OS}$

1. 7682E-03
TOTAL PA Cl/CU M

3. $7864 \mathrm{E} \cdot 04$

3. $6566 \mathrm{E} .04$

3.6386E-04

3.4515E-04

3.4467E-04

3. $2756 \mathrm{E}-04$

3. $2269 \mathrm{E}-04$

3. $1338 E-04$

3.1224E-04

2.9928E-04

2.9672E-04

$2.9555 \mathrm{E} .04$

2.8924E-04

2.8778E-04

2.8688E-04

2.8233E-04

2.756OE-04

2.7343E-04

2.661OE-04

2.6339E-04

2.5376E-04

2.4976E-04

2. $4353 E+04$

2.3976E-04

2.3331E-04

2. $3259 \mathrm{E}-04$

2. 2895E-04

2. $2753 E-04$

2. $2549 \mathrm{E}+04$

2.0149E-04

2.0114E-04

2.0025E-04

$1.9798 \mathrm{E}-04$

1. $9691 \mathrm{E}-04$

1. $9673 \mathrm{E}-04$

1. $9385 \mathrm{E}-04$

$1.9257 \mathrm{E}-04$

1.8585E-04

1. 8474E-04

1. $7906 \mathrm{E}-04$

$1.7786 \mathrm{E}-04$

1.6806E-04

1.6370E-04

1.5416E-04

1.5416E-04

1.5416E-04

1.5416E-O4

1.5193E-O4

1.51 19E-04

1.4979E-04

1.4719E-04

1.4615E-04

1.4615E-O4

$1.4615 \mathrm{E}-04$

1.4567E-O4

1.443 OE-O 4

$1.4267 \mathrm{E}-04$

1.4222E-O4

1.3847E-04

1.3847E-04

1. 3734E-04

1.3460E-04 
WHC-SD-W-242-ES-004, Rev. 0

TABLE 21

PIN-SPECIFIC CONCENTRATION OF PA-RELATED RADIONUCLIDES (7 SHEETS)

PIN

9302-07.0005

PNL-RTL-92-018

9302-06-0005

9101-02-0061

9101-02-0066

9201-02-0065

RS00004593

9101.02.0086

9101-02-0087

PNL-RTL-92-017

9201-02-0026

AS00010493

9201-02-0072

RS00010693

9201-02.0014

RS00010793

9101-02-0083

$9101-02-0085$

9101-03-0005

9101-02-0060

9101-02-0062

9101-02-0071

9201-02-0003

9201-02-0025

9201-02-0067

9302-07-0001

2225-93-000025

9201.02 .0060

9417.02-0026

RS00010593

RS00007893

9101-03-0003

9101-03-0006

9101-02-0078

9101-02-0079

9101-02-0080

9101-02-0082

9101.02 .0084

9101-02-0088

224U-92-000014

9201-02-0024

9201-02-0027

9201-02-0061

GEL-94-183-053

9200-03-092

303K-93-0000 11

9201-02-0016

9101-02-0081

9101-03-0004

9200-03-093

9201-02-0005

9200-03-1 34

9201-02-0064

9201-02-0069

9200-03-1 22

9201-02-0068

9101-03-0002

9101-02-0059

9201-02-0004

9201-02-0010

9200-03-094

9200-01-028

222S-92.000489
TOTAL ASH VOL CU M

2. 2940E-O2

4. $3944 \mathrm{E}-02$

3. $2729 E-02$

1.4229E-01

$1.4229 \mathrm{E}-01$

7.5427E-04

2.014BE-03

$1.4238 \mathrm{E}-01$

$1.4238 \mathrm{E}-01$

4. 3905E-02

$1.4246 \mathrm{E}-01$

$1.8119 \mathrm{E}-03$

7.8710E-04

1.8574E-03

1.4244E-01

$1.8624 \mathrm{E}-03$

$1.4236 \mathrm{E}-01$

1.4236E-01

$1.4234 \mathrm{E}-01$

1.4227E-01

1.4227E-01

1.4227E-O1

$1.4243 \mathrm{E}-01$

$1.4243 \mathrm{E}-01$

6. $2648 E-04$

2.4963E-02

1. 7087E-02

1. $0029 E-03$

1.4882E-01

$1.9382 \mathrm{E}-03$

$1.9304 \mathrm{E}-03$

$1.4230 \mathrm{E}-01$

$1.4230 \mathrm{E}-01$

1.4234E.01

$1.4234 \mathrm{E}-01$

$1.4234 \mathrm{E}-01$

1. 4234E-01

$1.4234 \mathrm{E}-01$

$1.4234 \mathrm{E} \cdot 01$

4. $4094 \mathrm{E}-03$

1.4241E-01

$1.4241 \mathrm{E}-01$

1.2172E-03

3. $0965 \mathrm{E}-04$

7.2051E-01

$2.9210 \mathrm{E}-02$

$1.4239 \mathrm{E}-01$

$1.4232 \mathrm{E}-\mathrm{O} 1$

$1.4228 \mathrm{E}-01$

5.9565E.01

1.4237E-01

5.9357E-01

6.3833E-04

6.3833E-04

6.7681E-01

7. $8036 \mathrm{E}-04$

1.4228E-01

$1.4223 \mathrm{E}-01$

$1.4236 \mathrm{E}-01$

$1.4236 \mathrm{E}-01$

4.9369E-01

3.5005E-01

7.4958E-04
TOTAL PA CI/CU M

1.3426E-04

$1.3426 E-04$

1.3261E-04

1.3046E-04

1.3046E-04

1. $3032 \mathrm{E}-04$

1. $3004 \mathrm{E}+04$

1.3002E-04

$1.3002 \mathrm{E}-04$

1.2755E-04

$1.2743 \mathrm{E}-04$

1.2694E-04

1.2489E-04

$1.2437 \mathrm{E}-04$

1.2432E-04

1. $2403 \mathrm{E}-04$

1. $2360 \mathrm{E}-04$

1. $2360 \mathrm{E} \cdot 04$

1.2359E-04

$1.2242 \mathrm{E}-04$

1.2242E-04

1.2242E-04

$1.2226 E-04$

$1.2226 \mathrm{E}-04$

$1.2204 \mathrm{E}-04$

$1.2178 \mathrm{E}-04$

1.2056E-04

1.1979E-04

1.1966E-04

$1.1918 E-04$

1.1915E-O4

$1.1726 \mathrm{E}-04$

1.1726E-O4

$1.1717 E-04$

$1.1717 \mathrm{E}-04$

$1.1717 \mathrm{E}-04$

1.1717E-O4

$1.1717 \mathrm{E}-04$

$1.1717 \mathrm{E}-04$

$\uparrow .1711 \mathrm{E}-04$

$1.1706 \mathrm{E}-04$

$1.1706 \mathrm{E}-04$

$1.1665 \mathrm{E}-\mathrm{O} 4$

$1.1534 \mathrm{E}-04$

1,1351 E-04

$1.1343 \mathrm{E}-04$

$1.1184 \mathrm{E}-04$

$1.1102 \mathrm{E}-04$

1.1090E-04

$1.1054 \mathrm{E}-04$

1.087OE-04

$1.0864 \mathrm{E}-04$

$1.0837 \mathrm{E}-04$

$1.0837 \mathrm{E}-04$

$1.0759 \mathrm{E}-04$

1.0730 E-04

$1.0706 \mathrm{E}-04$

$1.0672 \mathrm{E}-04$

$1.0664 \mathrm{E}-04$

1.0664E-04

$1.0503 E-04$

$1.0406 \mathrm{E}-04$

$1.0170 \mathrm{E}-04$ 
WHC-SD-W-242-ES-004, Rev. 0

TABLE 21

PIN-SPECIFIC CONCENTRATION OF PA-RELATED RADIONUCLIDES (7 SHEETS)

PIN

$9201 \cdot 02 \cdot 0001$

$9201-02-0002$

9201-02-0013

9201-02-0018

9201-02-0019

9201-02+0021

9201-02-0022

RS00018394

9201-02-0020
TOTAL ASH VOL CU M

1.4234E-01

$1.4234 \mathrm{E}-01$

$1.4234 \mathrm{E}-01$

$1.4234 \mathrm{E} \cdot 01$

$1.4234 \mathrm{E}-01$

$1.4234 \mathrm{E}-01$

$1.4234 \mathrm{E}-01$

9.9337E-04

$1.4234 \mathrm{E} \cdot 01$
TOTAL PA CI/CU M
$1.0141 \mathrm{E}-04$
$1.0141 \mathrm{E}-04$
$1.0141 \mathrm{E}-04$
$1.0141 \mathrm{E}-04$
$1.0141 E-04$
$1.0141 \mathrm{E}-04$
$1.0141 \mathrm{E}-04$
$1.0067 E-04$
$1.0034 \mathrm{E}-04$ 
WHC-SD-W-242-ES-004, Rev. 0

TABLE 22

STATISTICAL ANALYSIS OF PA-RELATED RADIONUCLIDES

IN ASCENDING ORDER BASED ON CONTAINER COUNT

CONCENTRATIONS IN CURIES PER CU M

(1 SHEET)

$\begin{array}{cccc}\text { ISOTOPE } & \text { COUNT } & \text { AVG PACIPER CUM } & \text { STD DEV } \\ & & & \\ \text { C-14 } & 404 & 8.4169 E-02 & 9.2543 E-01 \\ \text { TC-99 } & 312 & 1.3299 E-03 & 9.3190 E-03 \\ \text { U-238 } & 280 & 1.1214 E-03 & 9.3390 E-03 \\ \text { U-235 } & 228 & 1.2371 E-03 & 1.0330 E-02 \\ \text { U-234 } & 223 & 9.4600 E-04 & 1.0077 E-02 \\ \text { URANIUM-DEPLETED } & 222 & 1.7794 E-04 & 1.7860 E-03 \\ \text { I-129 } & 192 & 9.3707 E-04 & 5.7660 E-03 \\ \text { URANIUM-ENRICHED } & 118 & 4.8330 E-03 & 7.7720 E-03 \\ \text { SE-79 } & 88 & 1.3541 E-03 & 6.5620 E-03 \\ \text { U-236 } & 65 & 3.7201 E-03 & 1.9176 E-02 \\ \text { U-233 } & 26 & 1.6258 E-03 & 2.4310 E-03 \\ \text { U-232 } & 11 & 2.1034 E-04 & 2.3400 E-04\end{array}$

MIN PA CI PER CUM MAXPA CI PER CUM

4.4088E-17

$1.8369 E+01$

3.2265E-10 $1.5028 \mathrm{E}-01$

8.8483E-21 $1.5028 \mathrm{E}-01$

$5.8311 \mathrm{E}-13 \quad 1.5028 \mathrm{E}-01$

$6.8051 \mathrm{E}-10 \quad 1.5028 \mathrm{E}-01$

5.6213E-15 2.6196E-02

3.2265E-10 6.6136E-02

7.4464E-12 2.4336E-02

8.8918E-10 4.5592E-02

3.2457E-06 $\quad 1.5028 E-01$

2.2357E-07 - 8.3088E-03

$1.1539 E-08 \quad 5.9707 E-04$ 
WHC-SD-W-242-ES-004, Rev. 0

TABLE 23

TRITIUM WASTE CONTAINERS CONTAINING GREATER THAN OR CLOSE TO

0.02 CURIES PER CU M OF TRITIUM

(1 SHEET)

\begin{tabular}{|c|c|c|c|c|c|}
\hline PIN & ISOTOPE & UNIT & QUANTITY & CONTAINER VOLUME, CU M & TRITIUM. CURIES/CU N \\
\hline RS00000393 & $\mathrm{H}-3$ & $\mathrm{Cl}$ & $6.2250 \mathrm{E}-01$ & 0.2080 & $2.9928 E+\infty$ \\
\hline RSO00001393 & $\mathrm{H}-3$ & $\mathrm{Cl}$ & $6.2235 \mathrm{E}-01$ & 0.2080 & $2.9921 E+\infty$ \\
\hline RS00000793 & $\mathrm{H}-3$ & $\mathrm{Cl}$ & $3.9150 E-01$ & 0.2080 & $1.8822 \mathrm{E}+00$ \\
\hline RSO0000293 & $\mathrm{H}-3$ & $\mathrm{Cl}$ & $2.9562 \mathrm{E} \cdot 01$ & 0.2080 & $1.4213 E+\infty 0$ \\
\hline RS000001493 & $\mathrm{H}-3$ & $\mathrm{Cl}$ & $2.5614 \mathrm{E}-01$ & 0.2080 & $9.2314 \mathrm{E}+0 \mathrm{O}$ \\
\hline RSO00001293 & $\mathrm{H}-3$ & $\mathrm{Cl}$ & $2.5080 E-01$ & 0.2080 & $1.2058 \mathrm{E}+00$ \\
\hline RS00000893 & $\mathrm{H}-3$ & $\mathrm{Cl}$ & $2.3310 E-01$ & 0.2080 & $1.1207 E+00$ \\
\hline RSO0000993 & $\mathrm{H}-3$ & $\mathrm{Cl}$ & $1.9350 E-01$ & 0.2080 & $9.3029 \mathrm{E}-01$ \\
\hline RSO00001693 & $\mathrm{H}-3$ & $\mathrm{Cl}$ & $1.935 O E-01$ & 0.2080 & $9.3029 \mathrm{E}-01$ \\
\hline RSO00001093 & $H-3$ & $\mathrm{Cl}$ & $1.8230 \mathrm{E}-01$ & 0.2080 & $8.7644 \mathrm{E}-01$ \\
\hline RSO00001793 & $\mathrm{H}-3$ & $\mathrm{Cl}$ & $1.6930 E-01$ & 0.2080 & $8.1394 E-01$ \\
\hline RSO0000493 & $\mathrm{H}-3$ & $\mathrm{Cl}$ & $1.3158 \mathrm{E}-01$ & 0.2080 & $6.3260 \mathrm{E}-01$ \\
\hline RSO00002593 & $H-3$ & $\mathrm{Cl}$ & $1.2760 E-01$ & 0.2080 & $6.1346 \mathrm{E}-01$ \\
\hline RS00000693 & $\mathrm{H}-3$ & $\mathrm{Cl}$ & $1.2750 E-01$ & 0.2080 & $6.1298 \mathrm{E}-01$ \\
\hline RSO00002693 & $\mathrm{H}-3$ & $\mathrm{Cl}$ & $6.9500 E-02$ & 0.2080 & 3.3413E-01 \\
\hline 950800221 & $\mathrm{H}-3$ & $\mathrm{Cl}$ & $1.9000 E-02$ & 0.2082 & $9.1258 \mathrm{E}-02$ \\
\hline 950800223 & $\mathrm{H}-3$ & $\mathrm{Cl}$ & $1.9000 E-02$ & 0.2082 & $9.1258 \mathrm{E}-02$ \\
\hline 950800222 & $\mathrm{H}-3$ & $\mathrm{Cl}$ & $1.8000 E-02$ & 0.2082 & $8.6455 \mathrm{E}-02$ \\
\hline RS00001593 & $\mathrm{H}-3$ & $\mathrm{cl}$ & $1.2000 E .02$ & 0.2080 & $5.7692 \mathrm{E}-02$ \\
\hline RSO0000193 & $\mathrm{H}-3$ & $\mathrm{cl}$ & $9.6000 E-03$ & 0.2080 & $4.6154 \mathrm{E}-02$ \\
\hline RS00000593 & $\mathrm{H}-3$ & $\mathrm{Cl}$ & $8.8500 \mathrm{E}-03$ & 0.2080 & $4.2548 \mathrm{E}-02$ \\
\hline RS00013793 & $\mathrm{H}-3$ & $\mathrm{Cl}$ & $8.8333 \mathrm{E}-03$ & 0.2082 & $4.2427 \mathrm{E}-02$ \\
\hline RS00002093 & $\mathrm{H}-3$ & $\mathrm{Cl}$ & 8.7900E-03 & 0.2080 & $4.2260 E-02$ \\
\hline RSO0002893 & $\mathrm{H}-3$ & $\mathrm{Cl}$ & $8.5500 \mathrm{E}-03$ & 0.2080 & $4.1106 \mathrm{E}-02$ \\
\hline RS00003193 & $\mathrm{H}-3$ & $\mathrm{Cl}$ & $8.5500 E-03$ & 0.2080 & $4.1106 \mathrm{E}-02$ \\
\hline RS00002993 & $\mathrm{H}-3$ & $\mathrm{Cl}$ & $8.5100 E-03$ & 0.2080 & $4.0913 E-02$ \\
\hline RS00003093 & $\mathrm{H}-3$ & $\mathrm{Cl}$ & $8.5100 E-03$ & 0.2080 & $4.0913 E-02$ \\
\hline RSOO001993 & $\mathrm{H}-3$ & $\mathrm{Cl}$ & $8.3170 E-03$ & 0.2080 & $3.9986 \mathrm{E}-02$ \\
\hline RS00001893 & $\mathrm{H}-3$ & $\mathrm{Cl}$ & $8.1450 E-03$ & 0.2080 & $3.9159 \mathrm{E}-02$ \\
\hline 950800231 & $\mathrm{H}-3$ & $\mathrm{Cl}$ & $7.9000 E-03$ & 0.2082 & $3.7944 \mathrm{E}-02$ \\
\hline RSO0002193 & $\mathrm{H}-3$ & $\mathrm{Cl}$ & $6.8370 E-03$ & 0.2080 & $3.2870 \mathrm{E}-02$ \\
\hline RS00002393 & $\mathrm{H}-3$ & $\mathrm{Cl}$ & $4.6100 E-03$ & 0.2080 & $2.2163 E-02$ \\
\hline RSO0002293 & $\mathrm{H}-3$ & $\mathrm{Cl}$ & $4.6050 E-03$ & 0.2080 & $2.2139 \mathrm{E}-02$ \\
\hline $16-034$ & $\mathrm{H}-3$ & $\mathrm{Cl}$ & $4.5768 \mathrm{E}-03$ & 0.2082 & $2.1983 \mathrm{E}-02$ \\
\hline HEXO-92-000003 & $\mathrm{H}-3$ & $\mathrm{Cl}$ & 4.2000E-03 & 0.2000 & $2.1000 \mathrm{E}-02$ \\
\hline HEXO-92-0000O4 & $\mathrm{H}-3$ & $\mathrm{Cl}$ & $4.2000 \mathrm{E}-03$ & 0.2000 & $2.1000 E-02$ \\
\hline HEXO-92-000005 & $\mathrm{H}-3$ & $\mathrm{Cl}$ & $4.2000 \mathrm{E}-03$ & 0.2000 & $2.1000 E-02$ \\
\hline HEXO-92-000006 & $\mathrm{H}-3$ & $\mathrm{Cl}$ & $4.2000 \mathrm{E}-03$ & 0.2000 & 2.1000E-02 \\
\hline HEXO-92-000007 & $\mathrm{H}-3$ & $\mathrm{Cl}$ & $4.2000 \mathrm{E}-03$ & 0.2000 & $2.1000 \mathrm{E}-02$ \\
\hline HEXO-92-000011 & $\mathrm{H}-3$ & $\mathrm{Cl}$ & $4.2000 \mathrm{E}-03$ & 0.2000 & $2.1000 \mathrm{E}-02$ \\
\hline HEXO-92-000012 & $\mathrm{H}-3$ & $\mathrm{Cl}$ & $4.2000 \mathrm{E}-03$ & 0.2000 & $2.1000 \mathrm{E}-02$ \\
\hline $16-030$ & $\mathrm{H} \cdot 3$ & $\mathrm{Cl}$ & $4.1006 \mathrm{E}-03$ & 0.2082 & $1.9695 \mathrm{E}-02$ \\
\hline $16-033$ & $\mathrm{H}-3$ & $\mathrm{Cl}$ & $4.1006 \mathrm{E}-03$ & 0.2082 & $1.9695 \mathrm{E}-02$ \\
\hline RS00005293 & $\mathrm{H} \cdot 3$ & $\mathrm{Cl}$ & $3.8833 \mathrm{E}-03$ & 0.2082 & $1.8652 E-02$ \\
\hline 16.035 & $\mathrm{H}-3$ & $\mathrm{Cl}$ & $3.5440 \mathrm{E}-03$ & 0.2082 & $1.7022 \mathrm{E}-02$ \\
\hline $16-036$ & $\mathrm{H}-3$ & $\mathrm{cl}$ & $3.5440 E-03$ & 0.2082 & $1.7022 \mathrm{E}-02$ \\
\hline $16-037$ & $\mathrm{H}-3$ & Cl & $3.5440 \mathrm{E}-03$ & 0.2082 & $1.7022 \mathrm{E}-02$ \\
\hline RSO0001193 & $H \cdot 3$ & $\mathrm{Cl}$ & $3.1500 \mathrm{E}-03$ & 0.2080 & $1.5144 \mathrm{E}-02$ \\
\hline
\end{tabular}


WHC-SD-W-242-ES-004, Rev. 0

TABLE 24

DEBRIS CONTAINERS FOR WHICH THE VOLUMETRIC PERCENTAGE DISCREPANCY (DESIGNATED "COLB-COLC") BETWEEN REPORTED AND CALCULATED VOLUMETRIC PERCENTAGES EXCEEDS 25 VOLUME PERCENT

(5 SHEETS)

PIN

\begin{tabular}{|c|c|}
\hline 202A-94.000303 & 95.0000 \\
\hline WHC-RR-90-16 & 84.0000 \\
\hline ETFF-93-196-02 & 86.0000 \\
\hline $9200-04-137$ & 89.0000 \\
\hline $105 \mathrm{~N}-89-000796$ & 95.0000 \\
\hline RAZZ-213.A19875 & 76.0000 \\
\hline $105 \mathrm{~N}-89.000797$ & 96.0000 \\
\hline $2225-93.001020$ & 85.0000 \\
\hline RHZ-213-A21211 & 69.0000 \\
\hline ETFF.94-117.06 & 96.0400 \\
\hline WHC-RR-90.03 & 94.0000 \\
\hline FHZ-219-920855 & 69.0000 \\
\hline $9200-04-148$ & 79.0000 \\
\hline WHC-RA-90-05 & 64.0000 \\
\hline WHC.RP-90-04 & 75.0000 \\
\hline WHC-A-89-817 & 85.0000 \\
\hline RHZ-213-A21 209 & 64.0000 \\
\hline $2718-90-0646$ & 74.0000 \\
\hline 271B-90-0641 & 94.0000 \\
\hline WTF-92-003.01 & 89.0000 \\
\hline WHC-RR-90-01 & 66.0000 \\
\hline 9417.02 .0006 & 76.9000 \\
\hline 340-91-00024 & 80.0000 \\
\hline WHC-RR-90-15 & 79.0000 \\
\hline $2718-90-0626$ & 94.0000 \\
\hline WTFF-93-140-03 & 81.7400 \\
\hline WHC-A-89-816 & 85.0000 \\
\hline $9417-02-0015$ & 72.0000 \\
\hline RHZ-211-89039 & 54.0000 \\
\hline RHZZ.211-A19954 & 55.0000 \\
\hline 2225-92-000040 & 62.0000 \\
\hline FHZ:219-920404 & 54.0000 \\
\hline 2211 -92-000088 & 69.0000 \\
\hline $105 \mathrm{~N}-90-000009$ & 78.0000 \\
\hline 2225-93-001021 & 84.0000 \\
\hline $202 A-92-000328$ & 86.0000 \\
\hline WHC-A.90-844 & 70.0000 \\
\hline $105 \mathrm{~N}-89-001102$ & 59.0000 \\
\hline 9403037 & 60.0000 \\
\hline 2312.92 .000018 & 90.0000 \\
\hline $100 \mathrm{~N}-94-018600$ & $\mathbf{5 7 . 4 0 0 0}$ \\
\hline $\mathrm{RHZ} \cdot 213-\mathrm{A} 21808$ & 61.0000 \\
\hline $105 \mathrm{~N}-89-001101$ & 58.0000 \\
\hline FHZ-219-920029 & 49.0000 \\
\hline HEXO-93-000300 & 9.0000 \\
\hline ETFF-94-180-02 & 94.2400 \\
\hline RHZZ-219-920251 & 50.0000 \\
\hline
\end{tabular}

COLB-COLC REP TOT VOL, M3

TOTAL CALC COMP VOL, M3

\begin{tabular}{|c|c|}
\hline 73.7543 & 2.7200 \\
\hline 70.7693 & 0.2100 \\
\hline 70.6989 & 1.1200 \\
\hline 69.8511 & 0.3220 \\
\hline 69.8122 & 0.2100 \\
\hline 67.4891 & 0.2100 \\
\hline 65.2979 & 0.2100 \\
\hline 64.3353 & 6.3710 \\
\hline 63.8571 & 0.2100 \\
\hline 63.2273 & 1.1200 \\
\hline 63.2093 & 0.2100 \\
\hline 62.2317 & 0.2080 \\
\hline 61.0701 & 0.3220 \\
\hline 59.9129 & 0.2100 \\
\hline 59.0566 & 0.2100 \\
\hline 58.9346 & 0.2100 \\
\hline 58.7834 & 0.2100 \\
\hline 58.5698 & 0.2100 \\
\hline 58.1456 & 0.2100 \\
\hline 57.7209 & 7.0000 \\
\hline 57.4924 & 0.2100 \\
\hline 76.9000 & 0.2082 \\
\hline 55.7539 & 0.2100 \\
\hline 55.5996 & 0.2100 \\
\hline 55.4394 & 0.2100 \\
\hline 55.3355 & 0.2082 \\
\hline 54.0587 & 0.2100 \\
\hline 53.4117 & 0.2082 \\
\hline 52.7967 & 0.1520 \\
\hline 52.7135 & 0.2100 \\
\hline 50.7075 & 0.2000 \\
\hline 50.4666 & 0.2080 \\
\hline 50.4614 & 0.2100 \\
\hline 48.3361 & 0.2100 \\
\hline 48.2989 & 6,3710 \\
\hline 48.2801 & 6.3700 \\
\hline 48.1405 & 0.1500 \\
\hline 48.0815 & 0.2100 \\
\hline 47.7295 & 0.2082 \\
\hline 47.5619 & 3.1400 \\
\hline 47.4853 & 0.2082 \\
\hline 47.1774 & 0.2100 \\
\hline 47.0383 & 0.2100 \\
\hline 46.8642 & 0.2000 \\
\hline 46.8144 & 15.0000 \\
\hline 46.6958 & 2.2100 \\
\hline 46.3132 & 0.2000 \\
\hline
\end{tabular}

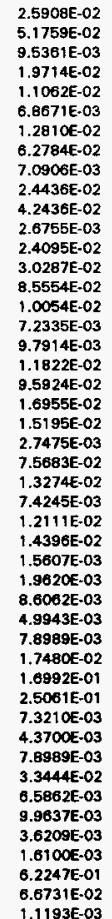

TOTAL REP COMP VOL CU M

CALC TOTAL VOL, M3

$1.2194 E-01$
$3.9121 E-01$
$6.2323 E-02$
$1.0295 E-01$
$4.3920 E-02$
$8.0686 E-02$
$4.1725 E-02$
$3.0382 E-01$
$1.3787 E-01$
$7.4472 E-02$
$1.3782 E-01$
$3.9530 E-02$
$1.3439 E-01$
$7.4104 E-01$
$5.3661 E-01$
$3.8673 E-02$
$1.3866 E-01$
$6.3456 E-02$
$3.2972 E-02$
$3.0667 E-01$
$1.9929 E-01$
$7.5379 E-02$
$1.1332 E-02$
$3.2343 E-01$
$3.4425 E-02$
$2.8119 E-02$
$3.9142 E-02$
$7.7447 E-02$
$1.2970 E-01$
$8.5811 E-02$
$7.6212 E-02$
$1.4134 E-01$
$4.2608 E-02$
$5.8927 E-02$
$4.7596 E-01$
$6.6439 E-01$
$3.3491 E-02$
$4.0024 E-02$
$6.4373 E-02$
$7.8806 E-02$
$6.6429 E-02$
$7.2082 E-02$
$3.3032 E-02$
$7.5382 E-02$
$1.1152 E+00$
$1.4036 E-01$
$3.0361 E-02$


WHC-SD-W-242-ES-004, Rev. 0

TABLE 24

DEBRIS CONTAINERS FOR WHICH THE VOLUMETRIC PERCENTAGE DISCREPANCY (DESIGNATED "COLB-COLC") BETWEEN REPORTED AND CALCULATED VOLUMETRIC PERCENTAGES EXCEEDS 25 VOLUME PERCENT

(5 SHEETS)

\begin{tabular}{|c|c|}
\hline $221 T \cdot 92-000054$ & 53.0000 \\
\hline RHZ-212-A21603 & 71.0000 \\
\hline RHZ-220-A19865 & 58.0000 \\
\hline RHZZ-219-091497 & 57.0000 \\
\hline $221 \mathrm{~T}-92-000150$ & 51.0000 \\
\hline $105 \mathrm{~N}-89-001100$ & 54.0000 \\
\hline PNL-89046 & 90.0000 \\
\hline RHZ-219-091495 & 50.0000 \\
\hline $271 B-90-0017$ & 72.0000 \\
\hline RHZ-219-920114 & 50.0000 \\
\hline $105 \mathrm{~N}-90-000137$ & 59.0000 \\
\hline $105 N-90.000138$ & 59.0000 \\
\hline ETFF-93-105-04 & 85.0000 \\
\hline FHZ-220-A19859 & 76.0000 \\
\hline ETF-91-036-13 & 65.0000 \\
\hline 9404422 & 51.8200 \\
\hline WHC-A-91-820 & 70.0000 \\
\hline $221 T-92-000181$ & 46.0000 \\
\hline 9417.02 .0005 & 80.7000 \\
\hline RHZ-219-930531 & 87.0000 \\
\hline 202A-92-000313 & 77.0000 \\
\hline 221 T-92-000151 & 47.0000 \\
\hline $325-90-045$ & 47.0000 \\
\hline RHZ-211-090038 & 80.0000 \\
\hline RHZ-219-930526 & 87.0000 \\
\hline $\mathrm{RHZ} \cdot 219-091640$ & 52.0000 \\
\hline ETFF-94-084-01 & 98.8900 \\
\hline 2225-94-000008 & 57.0000 \\
\hline $2714-91-90601$ & 57.0000 \\
\hline RHZZ-211.090057 & 49.0000 \\
\hline $\mathrm{RHZ}-219-930298$ & 40.0000 \\
\hline WHC-AB9-518 & 40.0000 \\
\hline WHC-PR-90-02 & 75.0000 \\
\hline $9417-02-0007$ & 59.7000 \\
\hline 202L-94-000701 & 10.0000 \\
\hline $9200-02-110$ & 40.0000 \\
\hline $9200-04-149$ & 99.0000 \\
\hline $9200-04155$ & 40.0000 \\
\hline $221 T-92-000100$ & 52.0000 \\
\hline RHZZ-219.091112 & 40.0000 \\
\hline RHZ-219.091490 & 50.0000 \\
\hline PHZZ-219.930468 & 73.0000 \\
\hline $9417-02-0012$ & 82.5000 \\
\hline 9417.02 .0001 & 64.3000 \\
\hline RAHZ-213-A20010 & 46.0000 \\
\hline KEH-92-079-05 & 53.0000 \\
\hline $9200-04-143$ & 99,0000 \\
\hline
\end{tabular}

TOTAL CALC COMP VOL \%

\begin{tabular}{|c|c|}
6.7671 \\
24.8876 \\
12.0116 \\
11.0178 \\
5.0780 \\
8.7061 \\
44.9036 \\
5.1300 \\
27.4665 \\
6.1151 \\
15.8189 \\
15.8423 \\
42.0269 \\
33.5325 \\
22.8717 \\
9.7215 \\
27.9147 \\
4.0251 \\
38.7400 \\
45.3846 \\
35.6396 \\
5.7595 \\
6.2510 \\
39.2570 \\
46.5789 \\
11.7535 \\
58.8312 \\
17.0401 \\
17.8232 \\
10.1662 \\
1.2162 \\
1.2854 \\
36.3316 \\
21.1901 \\
48.3831 \\
1.8940 \\
60.9923 \\
2.1892 \\
14.3057 \\
2.3401 \\
12.4126 \\
35.4210 \\
44.9816 \\
27.0258 \\
8.7758 \\
15.7922 \\
61.9810 \\
\\
\hline
\end{tabular}

COLB-COLC

46.2329

46.1124

45.9884

45.9822

45.2939

45.0964

44.8700

44.5335

43.8849

43.1811
43.1577

42.9731

42.4675

42.1283

42.0985

42.0853

41.9749

41.9600

41.3604

41.3604
41.2405

40.7490

40.7430

40.4211

40.2465

40.0588

39.9599

39.1768

38.8338

38.7838

38.7146

38.6684

38.5099

38.3831

38.1060

38.0077

37.8108

37.8943

37.6599

37.5790

37.5184

37.2742
37.2242

37.2078

37.0190
REP TOT VOL, M3 TOTAL CALC COMP VOL, M3

TOTAL REP COMP VOL CU M

\begin{tabular}{l}
0.2100 \\
0.2100 \\
0.2100 \\
0.2080 \\
0.2100 \\
0.2100 \\
0.2100 \\
0.2080 \\
0.2100 \\
0.2080 \\
0.2100 \\
0.2100 \\
0.2082 \\
0.2100 \\
0.1500 \\
0.3218 \\
0.2100 \\
0.2100 \\
0.2082 \\
0.2082 \\
0.2000 \\
0.2100 \\
0.1400 \\
0.2100 \\
0.2082 \\
0.2080 \\
2.2100 \\
0.2082 \\
0.2000 \\
0.2100 \\
0.2082 \\
0.1520 \\
0.2100 \\
0.2082 \\
0.2082 \\
0.3220 \\
0.3220 \\
0.3220 \\
0.2100 \\
0.2100 \\
0.2080 \\
0.2082 \\
0.2082 \\
0.2082 \\
0.2100 \\
0.2080 \\
0.3220 \\
\hline
\end{tabular}

$1.1130 E-01$
$1.4910 E-01$
$1.2180 E-01$
$1.1856 E-01$
$1.0710 E-01$
$1.1340 E-01$
$1.8900 E-01$
$1.0400 E-01$
$1.5120 E-01$
$1.0400 E-01$
$1.2390 E-01$
$1.2390 E-01$
$1.7697 E-01$
$1.5960 E-01$
$9.7500 E-02$
$1.6676 E-01$
$1.4700 E-01$
$9.6600 E-02$
$1.6802 E-01$
$1.8113 E-01$
$1.5400 E-01$
$9.8700 E-02$
$6.5800 E-02$
$1.6800 E-01$
$1.8113 E-01$
$1.0816 E-01$
$2.1865 E+00$
$1.1867 E-01$
$1.1400 E-01$
$1.0290 E-01$
$8.3280 E-02$
$6.0800 E-02$
$1.5750 E-01$
$1.2430 E-01$
$2.0820 E-02$
$1.2880 E-01$
$3.1878 E-01$
$1.2880 E-01$
$1.0920 E-01$
$8.4000 E-02$
$1.0400 E-01$
$1.5199 E-01$
$1.7177 E-01$
$1.3387 E-01$
$9.6600 E-02$
$1.1024 E-01$
$3.1878 E-01$

CALC TOTAL VOL, M3

$$
\begin{aligned}
& 1.4178 \mathrm{E}-01 \\
& 7.8836 \mathrm{E}-02 \\
& 8.4544 \mathrm{E}-02 \\
& 6.2626 \mathrm{E}-02 \\
& 1.2294 \mathrm{E}-01 \\
& 4.5892 \mathrm{E}-02 \\
& 1.8129 \mathrm{E}-01 \\
& 8.2752 \mathrm{E}-02 \\
& 2.2302 \mathrm{E}-02 \\
& 5.0221 \mathrm{E}-02 \\
& 6.6176 \mathrm{E}-02 \\
& 5.3841 \mathrm{E}-02 \\
& 2.2844 \mathrm{E}-02 \\
& 4.7654 \mathrm{E}-02 \\
& 5.2967 \mathrm{E}-02 \\
& 5.5875 \mathrm{E}-02 \\
& 6.3561 \mathrm{E}-02 \\
& 1.6732 \mathrm{E}-01 \\
& 3.6378 \mathrm{E}-02 \\
& 8.1432 \mathrm{E}-03 \\
& 3.4035 \mathrm{E}-02 \\
& 1.0383 \mathrm{E}-01 \\
& 7.6329 \mathrm{E}-02 \\
& 2.8597 \mathrm{E}-02 \\
& 1.0829 \mathrm{E}-02 \\
& 7.1985 \mathrm{E}-02 \\
& 1.3483 \mathrm{E}-01 \\
& 4.4984 \mathrm{E}-02 \\
& 2.5590 \mathrm{E}-01 \\
& 7.4078 \mathrm{E}-02 \\
& 1.6132 \mathrm{E}-01 \\
& 1.1462 \mathrm{E}-01 \\
& 5.1534 \mathrm{E}-02 \\
& 5.5823 \mathrm{E}-02 \\
& 2.1564 \mathrm{E}-02 \\
& 4.8574 \mathrm{E}-01 \\
& 1.2803 \mathrm{E}-01 \\
& 4.6025 \mathrm{E}-01 \\
& 1.8434 \mathrm{E}-01 \\
& 6.9361 \mathrm{E}-02 \\
& 8.6506 \mathrm{E}-02 \\
& 1.7977 \mathrm{E}-02 \\
& 5.1185 \mathrm{E}-02 \\
& 2.2222 \mathrm{E}-02 \\
& 1.7854 \mathrm{E}-01 \\
& 3.5003 \mathrm{E}-02 \\
& 1.3136 \mathrm{E}-01
\end{aligned}
$$


WHC-SD-W-242-ES-004, Rev. 0

TABLE 24

DEBRIS CONTAINERS FOR WHICH THE VOLUMETRIC PERCENTAGE DISCREPANCY (DESIGNATED "COLB-COLC") BETWEEN REPORTED AND CALCULATED VOLUMETRIC PERCENTAGES EXCEEDS 25 VOLUME PERCENT

(5 SHEETS)

PIN

\begin{tabular}{|c|c|}
\hline $9417-02-0003$ & 87.5000 \\
\hline $\mathrm{AHZ}-21$ 2-A21790 & 59.0000 \\
\hline RHZ-219-090079 & 50.0000 \\
\hline FHZ-213-920408 & 44,0000 \\
\hline RHZ-219-920784 & 42.0000 \\
\hline $9417-02-0009$ & 52.5000 \\
\hline ETFF-92-356-02 & 94.0000 \\
\hline $\mathrm{RHZ} \cdot 213-\mathrm{A} 21798$ & 49.0000 \\
\hline $9200-04.168$ & 49.0000 \\
\hline $9201-02-0062$ & 40.0000 \\
\hline $305 \mathrm{~B}-91-022$ & 60.0000 \\
\hline $305 \mathrm{~B}-91-023$ & 60.0000 \\
\hline $3058-91-026$ & 60.0000 \\
\hline $3058-91-027$ & 60.0000 \\
\hline $3058-91-028$ & 60.0000 \\
\hline ETF-91.051.01 & 40.0000 \\
\hline ETFF-94-195-03 & 96.1400 \\
\hline WTFF-93.110-18 & 81.0000 \\
\hline PHZ-213-A21842 & 49.0000 \\
\hline $105 \mathrm{~N}-90-000135$ & 40.0000 \\
\hline RHZZ-219.090068 & 79.0000 \\
\hline 9403711 & 50.0000 \\
\hline 305B-91.024 & 60.0000 \\
\hline $224 U-94-170002$ & 46.0000 \\
\hline 16.069 & 80.0000 \\
\hline $221 T-92.000180$ & 52.0000 \\
\hline RHZZ-219-920472 & 35.0000 \\
\hline 9406824 & 53.7200 \\
\hline $224 U-94-170003$ & 46.0000 \\
\hline FHZ-219-920319 & 42.0000 \\
\hline $303 K-93-000047$ & 41.0000 \\
\hline $329-90-34$ & 53.0000 \\
\hline $221 T-92-000083$ & 56.0000 \\
\hline ETFF-93-090-02 & 46.0000 \\
\hline WTFF-93.147-03 & 65.0000 \\
\hline PAHZ-213-A21830 & 38.0000 \\
\hline WTFF-93-098-05 & 85.0000 \\
\hline 9203.01 .0002 & 46.0000 \\
\hline FHZ-211-A21529 & 45.0000 \\
\hline $221 T-93-000112$ & 20.0000 \\
\hline WTFF-93-145-01 & 56.0000 \\
\hline ETFF-94-153-01 & 98,9000 \\
\hline RHZ-219-091491 & 35.0000 \\
\hline WTFF-93-147-02 & 56.0000 \\
\hline WTFF-93-263-01 & 93.9900 \\
\hline ETF-241.900204 & 50.0000 \\
\hline WTFF-93-232-03 & 37.0000 \\
\hline
\end{tabular}

TOTAL CALC COMP VOL \%

\begin{tabular}{|l|}
\hline 50.6701 \\
22.2805 \\
13.2840 \\
7.3487 \\
5.4347 \\
16.1603 \\
57.6892 \\
12.9216 \\
12.9866 \\
76.0089 \\
24.0101 \\
24.0101 \\
24.0101 \\
24.0101 \\
24.0101 \\
4.3243 \\
60.5672 \\
45.4350 \\
13.7238 \\
4.8655 \\
43.9776 \\
84.9556 \\
25.1888 \\
11.1897 \\
45.3430 \\
17.4834 \\
0.9807 \\
19.7523 \\
12.1813 \\
8.2081 \\
7.2205 \\
19.2298 \\
22.3019 \\
12.3225 \\
31.5456 \\
4.6223 \\
51.7968 \\
12.8150 \\
12.1526 \\
52.6811 \\
23.3588 \\
66.2879 \\
2.4297 \\
23.4425 \\
61.5338 \\
17.6307 \\
4.8617 \\
\hline
\end{tabular}

3.2840

5.4347

57.6892

12.9216

76.0089

24.0101

24.0101

24.0101

4.3243

60.5672

13.7238

4.8655

84.9555

25.1888

45.3430

17.4834

0.9807
19.7523

12.1813

7.2205

19.2298

12.3225

31.5458

12.8150
12.1526

12.1526

52.6811

66.2879

2.4297

61.5338

17.6307
4.6617
COLB-COLC REP TOT VOL, M3

$\begin{array}{lc}36.8299 & 0.2082 \\ 36.7195 & 0.2100 \\ 36.7160 & 0.2100 \\ 36.6513 & 0.2080 \\ 36.5653 & 0.2080 \\ 36.3397 & 0.2082 \\ 36.3108 & 10.0300 \\ 36.0784 & 0.2100 \\ 36.0134 & 0.3220 \\ 36.0089 & 0.2000 \\ 35.9899 & 0.2100 \\ 35.9899 & 0.2100 \\ 35.9899 & 0.2100 \\ 35.9899 & 0.2100 \\ 35.9899 & 0.2100 \\ 35.6757 & 0.1500 \\ 35.5728 & 0.2082 \\ 35.5650 & 0.2082 \\ 35.2762 & 0.2100 \\ 35.1345 & 0.2100 \\ 35.0224 & 0.1500 \\ 34.9555 & 0.2082 \\ 34.8112 & 0.2100 \\ 34.8103 & 0.2082 \\ 34.6570 & 0.2082 \\ 34.5166 & 0.2100 \\ 34.0193 & 0.2080 \\ 33.9677 & 0.2082 \\ 33.8187 & 0.2082 \\ 33.7919 & 0.2000 \\ 33.7795 & 0.2082 \\ 33.7702 & 0.2100 \\ 33.6981 & 0.2100 \\ 33.6775 & 8.3000 \\ 33.4544 & 1.1200 \\ 33.3777 & 0.2100 \\ 33.2032 & 0.2082 \\ 33.1850 & 0.2100 \\ 32.8474 & 0.2100 \\ 32.6811 & 0.2082 \\ 32.6412 & 1.1200 \\ 32.6121 & 8.3000 \\ 32.5703 & 0.2000 \\ 32.5575 & 1.1200 \\ 32.4562 & 8.3000 \\ 32.3693 & 0.2100 \\ 32.3383 & 0.2082\end{array}$

TOTAL CAIC COMP VOL, M3

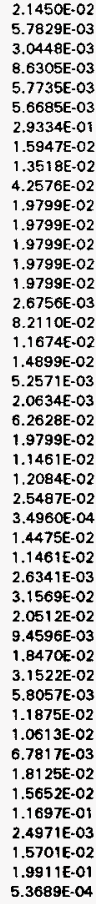

TOTAL REP COMP VOL CU M

$1.8218 E-01$
$1.2390 E-01$
$1.0500 E-01$
$9.1520 E-02$
$8.7360 E-02$
$1.0931 E-01$
$9.4282 E+00$
$1.0290 E-01$
$1.5778 E-01$
$8.0000 E-02$
$1.2600 E-01$
$1.2600 E-01$
$1.2600 E-01$
$1.2600 E-01$
$1.2600 E-01$
$6.0000 E-02$
$2.0016 E-01$
$1.6864 E-01$
$1.0290 E-01$
$8.4000 E-02$
$1.1850 E-01$
$1.0410 E-01$
$1.2600 E-01$
$9.5772 E-02$
$1.6656 E-01$
$1.0920 E-01$
$7.2800 E-02$
$1.1185 E-01$
$9.5772 E-02$
$8.4000 E-02$
$8.5362 E-02$
$1.1130 E-01$
$1.1760 E-01$
$3.8180 E+00$
$7.2800 E-01$
$7.9800 E-02$
$1.7697 E-01$
$9.6600 E-02$
$9.4500 E-02$
$4.1640 E-02$
$6.2720 E-01$
$8.2087 E+00$
$7.0000 E-02$
$6.2720 E-01$
$7.8012 E+\infty 0$
$1.0500 E-01$
$7.7034 E-02$

CALC TOTAL VOL, MB

$$
\begin{aligned}
& \text { 2.5955E-02 } \\
& \text { 2.2921E-02 } \\
& 1.1744 \mathrm{E}-01 \\
& 1.0623 \mathrm{E}-01 \\
& \text { 3.5077E-02 } \\
& \text { 5.0848E-01 } \\
& 1.2341 \mathrm{E}-01 \\
& 1.0409 \mathrm{E}-01 \\
& \text { 5.6015E-02 } \\
& \text { 8. 246CE-02 } \\
& \text { 8. } 2460 \mathrm{E}-02 \\
& \text { 8. 2460E-02 } \\
& \text { 8.2460E-02 } \\
& \text { 6.1872E-02 } \\
& \text { 1.3557E-01 } \\
& \text { 2.5694E-02 } \\
& \text { 2.5694E-02 } \\
& 1.0805 \mathrm{E}-01 \\
& 4.6920 \mathrm{E}-03 \\
& \text { 4.6920E-03 } \\
& \text { 7.3719E-02 } \\
& 1.0243 \mathrm{E}-01 \\
& 1.0243 E-01 \\
& \text { 2.6651E-02 } \\
& \begin{array}{l}
1.4578 \mathrm{E}-01 \\
3.5648 \mathrm{E}-02
\end{array} \\
& \text { 7. } 3284 \mathrm{E}-02 \\
& \begin{array}{l}
7.3284 \mathrm{E}-02 \\
9.4090 \mathrm{E}-02
\end{array} \\
& \text { 3. } 2091 \mathrm{E}-02 \\
& \text { 4.3722E-01 } \\
& \text { 1.0667E-01 } \\
& \text { 4. } 2416 \mathrm{E}-02 \\
& \text { 1.4989E-01 } \\
& 9.9925 \mathrm{E}-02 \\
& \text { 1.2560E-O1 } \\
& \text { 2.2926E-02 } \\
& 5.5805 \mathrm{E}-02 \\
& \text { 3.4405E-02 } \\
& 6.7006 \mathrm{E}-02 \\
& \begin{array}{l}
1.7646 \mathrm{E}-01 \\
1.0277 \mathrm{E}-01
\end{array} \\
& \begin{array}{l}
1.7646 \mathrm{E}-01 \\
1.0277 \mathrm{E}-01
\end{array} \\
& \begin{array}{r}
1.0277 \mathrm{E}-01 \\
6.6976 \mathrm{E}-02
\end{array} \\
& \text { 6.6976E-02 } \\
& \text { 3.2358E-01 } \\
& \text { 3.0452E-03 }
\end{aligned}
$$$$
\text { 4. 2334E-02 }
$$ 
WHC-SD-W-242-ES-004, Rev. 0

TABLE 24

DEBRIS CONTAINERS FOR WHICH THE VOLUMETRIC PERCENTAGE DISCREPANCY (DESIGNATED "COLB-COLC") BETWEEN REPORTED AND CALCULATED VOLUMETRIC PERCENTAGES EXCEEDS 25 VOLUME PERCENT

(5 SHEETS)

\begin{tabular}{|c|c|}
\hline $9201-02-0073$ & 40.0000 \\
\hline RHZ-211-A21187 & 60.0000 \\
\hline RHZ-219-A21682 & 39.0000 \\
\hline 9417.02 .0014 & 80.5000 \\
\hline RHZ-219-930584 & 37.0000 \\
\hline RHZZ-213-A21744 & 34.0000 \\
\hline $16-049$ & 80.0000 \\
\hline 16-062 & 80.0000 \\
\hline $18-063$ & 80.0000 \\
\hline $16-064$ & 80.0000 \\
\hline $105 \mathrm{~N}-90-000136$ & 37.0000 \\
\hline $9201-02-0071$ & 30.0000 \\
\hline $\mathrm{RHZ} \cdot 219 \cdot 930461$ & 50.0000 \\
\hline $9305-03-0032$ & 73.0000 \\
\hline $9200-04-144$ & 99.0000 \\
\hline PNL-RTL-92-019 & 35.0000 \\
\hline PNL-RTL-92-016 & 35.0000 \\
\hline PNL-RTL-92-018 & 35.0000 \\
\hline PNL-RTL-92-017 & 35.0000 \\
\hline WESF-89-209 & 50.0000 \\
\hline WESF-89-303 & 50.0000 \\
\hline WESF-89-210 & 50.0000 \\
\hline WESF-89-283 & 50.0000 \\
\hline $9417-02-0011$ & 75.8000 \\
\hline FHZ-219.090061 & 40.0000 \\
\hline FHZ-211-A21824 & 35.0000 \\
\hline ETFF. 93.082 .03 & 50.0000 \\
\hline $16-030$ & 80.0000 \\
\hline 16.036 & 80.0000 \\
\hline $16-041$ & 80.0000 \\
\hline 9417.02 .0010 & 91.8000 \\
\hline WTFF-93-116-02 & 85.0000 \\
\hline 221 T.92.000122 & 39.0000 \\
\hline 940200012 & 87.0000 \\
\hline FHZ-212.A21959 & 35.0000 \\
\hline $9200-04-167$ & 49.0000 \\
\hline $9417-02-0002$ & 82.9000 \\
\hline WTFF-VT-001-01 & 58.0000 \\
\hline EXEH-92-135-01 & 14.3500 \\
\hline KEH-92-134.01 & 37.0000 \\
\hline FEZZ-212-A21228 & 69.0000 \\
\hline $22252 W-90-230$ & 30.0000 \\
\hline ETFF-92-356-03 & 96.0000 \\
\hline ETF-91-190-02 & 97.0000 \\
\hline 9417.02 .0004 & 91.7000 \\
\hline RHZ-219-091496 & 32.0000 \\
\hline $16-031$ & 80.0000 \\
\hline
\end{tabular}

\begin{tabular}{|c|}
\hline 72.1081 \\
27.9594 \\
7.1072 \\
48.7197 \\
5.3684 \\
2.4612 \\
49.1701 \\
49.1701 \\
49.1701 \\
49.1701 \\
6.1927 \\
60.6004 \\
19.4063 \\
42.4533 \\
68.7586 \\
4.8093 \\
4.8521 \\
4.8802 \\
4.9513 \\
20.0460 \\
20.0481 \\
20.0487 \\
20.0564 \\
46.3060 \\
10.5079 \\
5.6075 \\
20.7019 \\
50.9264 \\
50.9264 \\
50.9264 \\
62.7553 \\
58.0329 \\
10.1091 \\
58.1873 \\
6.3127 \\
20.5354 \\
54.4512 \\
29.5832 \\
42.6864 \\
8.7789 \\
40.8058 \\
1.8160 \\
68.0266 \\
69.1398 \\
64.0029 \\
4.3758 \\
52.4984 \\
\\
\hline
\end{tabular}

$\begin{array}{ll}32.1081 & 0.2000 \\ 32.0406 & 0.2100 \\ 31.8928 & 0.2100 \\ 31.7803 & 0.2082 \\ 31.6316 & 0.2082 \\ 31.5388 & 0.2100 \\ 30.8299 & 0.2082 \\ 30.8299 & 0.2082 \\ 30.8299 & 0.2082 \\ 30.8299 & 0.2082 \\ 30.8073 & 0.2100 \\ 30.6004 & 0.2000 \\ 30.5937 & 0.2082 \\ 30.5467 & 0.2082 \\ 30.2414 & 0.3220 \\ 30.1907 & 0.2100 \\ 30.1479 & 0.2100 \\ 30.1198 & 0.2100 \\ 30.0487 & 0.2100 \\ 29.9540 & 0.2100 \\ 29.9519 & 0.2100 \\ 29.9513 & 0.2100 \\ 29.9436 & 0.2100 \\ 29.4940 & 0.2082 \\ 29.4921 & 0.2100 \\ 29.3925 & 0.2100 \\ 29.2981 & 8.3000 \\ 29.0736 & 0.2082 \\ 29.0736 & 0.2082 \\ 29.0736 & 0.2082 \\ 29.0447 & 0.2082 \\ 28.9671 & 0.2082 \\ 28.8909 & 0.2100 \\ 28.8127 & 0.2082 \\ 28.6873 & 0.2080 \\ 28.4646 & 0.3220 \\ 28.4488 & 0.2082 \\ 28.4168 & 0.2082 \\ 28.3364 & 8.7000 \\ 28.2211 & 0.2080 \\ 28.1942 & 0.2100 \\ 28.1840 & 0.2100 \\ 27.9734 & 9.6000 \\ 27.8602 & 0.0300 \\ 27.6971 & 0.2082 \\ 27.6242 & 0.2080 \\ 27.5036 & 0.2082 \\ & \\ & \end{array}$

$4.4018 E-02$
$8.9340 E-03$
$8.5724 E-03$
$1.8058 E-02$
$2.4884 E-03$
$2.9668 E-03$
$1.4091 E-02$
$1.4091 E-02$
$1.4091 E-02$
$1.4091 E-02$
$5.9061 E-03$
$1.7688 E-02$
$1.8827 E-02$
$5.5011 E-02$
$1.0992 E-01$
$1.3378 E-02$
$1.3378 E-02$
$1.3378 E-02$
$1.3378 E-02$
$1.0688 E-02$
$1.1350 E-02$
$9.9866 E-03$
$1.3922 E-02$
$1.7680 E-02$
$6.5714 E-03$
$5.2571 E-03$
$1.4769 E-01$
$1.5117 E-02$
$1.5117 E-02$
$1.5117 E-02$
$2.3521 E-02$
$1.4439 E-02$
$1.7118 E-02$
$1.3072 E-02$
$5.4444 E-03$
$4.9333 E-02$
$2.3796 E-02$
$2.3698 E-02$
$4.4286 E-01$
$3.6388 E-03$
$1.4008 E-02$
$5.3510 E-03$
$5.0332 E-01$
$5.9110 E-03$
$2.5371 E-02$
$1.9977 E-03$
$1.6098 E-02$

\begin{tabular}{|l} 
8.OOOOE-O2 \\
$1.2600 E-01$ \\
$8.1900 E-02$ \\
$1.6760 E-01$ \\
$7.7034 E-02$ \\
$7.1400 E-02$ \\
$1.6656 E-01$ \\
$1.6656 E-01$ \\
$1.6656 E-01$ \\
$1.6656 E-01$ \\
$7.7700 E-02$ \\
$6.0000 E-02$ \\
$1.0410 E-01$ \\
$1.5199 E-01$ \\
$3.1878 E-01$ \\
$7.3500 E-02$ \\
$7.3500 E-02$ \\
$7.3500 E-02$ \\
$7.3500 E-02$ \\
$1.0500 E-01$ \\
$1.0500 E-01$ \\
$1.0500 E-01$ \\
$1.0500 E-01$ \\
$1.5782 E-01$ \\
$8.4000 E-02$ \\
$7.3500 E-02$ \\
$4.1500 E+00$ \\
$1.6656 E-01$ \\
$1.6656 E-01$ \\
$1.6656 E-01$ \\
$1.9113 E-01$ \\
$1.7697 E-01$ \\
$8.1900 E-02$ \\
$1.8113 E-01$ \\
$7.2800 E-02$ \\
$1.5778 E-01$ \\
$1.7260 E-01$ \\
$1.2076 E-01$ \\
$1.2485 E+00$ \\
$7.6960 E-02$ \\
$1.4490 E-01$ \\
$6.3000 E-02$ \\
$9.2160 E+00$ \\
$2.9100 E-02$ \\
$1.9092 E-01$ \\
$6.6560 E-02$ \\
$1.6656 E-01$ \\
\end{tabular}

$6.1045 E-02$
$3.1954 E-02$
$1.2062 E-01$
$3.7064 E-02$
$4.6353 E-02$
$1.2054 E-01$
$2.8658 E-02$
$2.8658 E-02$
$2.8658 E-02$
$2.8658 E-02$
$9.5372 E-02$
$2.9188 E-02$
$9.7016 E-02$
$1.2958 E-01$
$1.5986 E-01$
$2.7816 E-01$
$2.7571 E-01$
$2.7412 E-01$
$2.7018 E-01$
$5.3316 E-02$
$5.6612 E-02$
$4.9822 E-02$
$6.9412 E-02$
$3.8180 E-02$
$6.2538 E-02$
$9.3752 E-02$
$7.1340 E-01$
$2.9683 E-02$
$2.9683 E-02$
$2.9683 E-02$
$3.7481 E-02$
$2.5769 E-02$
$1.6933 E-01$
$2.2465 E-02$
$8.6246 E-02$
$2.4023 E-01$
$4.3702 E-02$
$8.0107 E-02$
$1.0375 E+000$
$4.1449 E-02$
$3.4329 E-02$
$2.9465 E-01$
$7.3989 E-01$
$8.5494 E-03$
$3.9640 E-02$
$4.5654 E-02$
$3.0664 E-02$


WHC-SD-W-242-ES-004, Rev. 0

TABLE 24

DEBRIS CONTAINERS FOR WHICH THE VOLUMETRIC PERCENTAGE DISCREPANCY (DESIGNATED "COLB-COLC") BETWEEN REPORTED AND CALCULATED VOLUMETRIC PERCENTAGES EXCEEDS 25 VOLUME PERCENT

(5 SHEETS)

PIN

16-061

WTF-92-083-02

WTFF.VT.01-131

$271 \mathrm{~B}-90-064$

9402859

RZ-219.091026

RHZ-211-090037

RHZZ-219.090067

RHZ-213-A21306

WTFF-93-116-0

2225-91-0328

16-073

RHZ-213-A21954

RHZ-211-A20983

221T-93-000148

16.048

16.048
$16-051$

$16-051$
16.065

HEX0-92-00002

RAHZ-213-A19795

RHZ-21 2-A2146

RHZ-219-920111

ETFF-92-337-02

ETFF-92-343-0

$9417.02-0017$
$2225-93-000385$
REP COMP VOL $\%$

43.0000

66.0000

75.0000

90.0000

59.0000

41.0000

52.0000

35.0000

82.0000

68.0000

80.0000

30.0000

45.0000

65.0000

80.0000

80.0000

80.0000

80.0000

27.0000

50.0000

5.0000

7.0000

11.0000

70.0000

95.5000
44.6000
TOTAL CALC COMP VOL \%

COLB-COLC

REP TOT VOL, M3

TOTAL CALC COMP VOL, M3

$1.6098 \mathrm{E}-02$
$4.5639 \mathrm{E}-03$
$1.4715 \mathrm{E}-02$
$9.8572 \mathrm{E}-03$
$6.8538 \mathrm{E}-03$
$3.0700 \mathrm{E}-01$
$9.8597 \mathrm{E}-03$
$1.2339 \mathrm{E}-02$
$7.8551 \mathrm{E}-03$
$2.1613 \mathrm{E}-02$
$1.6429 \mathrm{E}-03$
$1.4720 \mathrm{E}-02$
$1.6298 \mathrm{E}-02$
$1.6945 \mathrm{E}-02$
$3.9429 \mathrm{E}-03$
$9.5286 \mathrm{E}-03$
$3.5397 \mathrm{E}-03$
$6.7160 \mathrm{E}-03$
$1.7123 \mathrm{E}-02$
$1.7123 \mathrm{E}-02$
$1.7123 \mathrm{E}-02$
$1.1237 \mathrm{E}-03$
$7.4914 \mathrm{E}-03$
$1.2752 \mathrm{E}-02$
$5.3956 \mathrm{E}-02$
$3.5616 \mathrm{E}-01$
$1.2571 \mathrm{E}-02$
$2.4772 \mathrm{E}-02$
$7.5933 \mathrm{E}-03$

$\begin{array}{ll}27.5036 & 0.2082 \\ 27.1386 & 0.2080 \\ 27.0502 & 0.2082 \\ 26.9011 & 0.2100 \\ 26.7330 & 0.2082 \\ 26.7122 & 9.6110 \\ 26.6687 & 0.2100 \\ 26.6134 & 0.2100 \\ 26.5671 & 0.2100 \\ 26.4396 & 0.2100 \\ 26.3934 & 0.2080 \\ 26.3737 & 0.2082 \\ 26.2318 & 0.2100 \\ 26.2264 & 0.2082 \\ 26.0461 & 0.2080 \\ 26.0209 & 0.2100 \\ 26.0022 & 0.2082 \\ 25.9864 & 0.2100 \\ 25.9662 & 0.2082 \\ 25.9662 & 0.2082 \\ 25.9662 & 0.2082 \\ 25.8910 & 0.2000 \\ 25.6641 & 0.2100 \\ 25.5666 & 0.2100 \\ 25.4793 & 2.7180 \\ 25.4654 & 8.7000 \\ 25.4404 & 0.2082 \\ 25.2273 & 0.2082 \\ 25.0183 & 0.2082 \\ & \end{array}$

52.4964
15.8614
68.6898
39.0989
48.2670
63.2878
9.3313
32.3866
14.4329
25.5604
8.6066
55.6263
41.7682
53.7736
3.9539
18.9791
38.9978
31.0136
54.0338
54.0338
54.0338
1.1090
7.3359
24.4334
49.5207
36.4654
44.5596
70.2727
19.5817

0.208
TOTAL REP COMP VOL CU M

$1.6656 E-01$
$8.9440 E-02$
$1.9933 E-01$
$7.3860 E-01$
$1.5615 E-01$
$8.6499 E+00$
$7.5600 E-02$
$1.2390 E-01$
$8.6100 E-02$
$1.0920 E-01$
$7.2800 E-02$
$1.7072 E-01$
$1.4280 E-01$
$1.6656 E-01$
$6.2400 E-02$
$9.4500 E-02$
$1.3533 E-01$
$1.1970 E-01$
$1.6656 E-01$
$1.6656 E-01$
$1.6656 E-01$
$5.4000 E-02$
$6.9300 E-02$
$1.0500 E-01$
$2.0385 E+00$
$9.5700 E-01$
$1.4574 E-01$
$1.9883 E-01$
$9.2857 E-02$

CALC TOTAL VOL, M3

3.0664 E-02
$2.8773 E-02$
$2.1422 E-02$
2.5211 E-02
$1.4200 E-02$
$4.8509 E-01$
$1.0566 E-01$
$3.8099 E-02$
$5.4425 E-02$
$8.4558 E-02$
$1.9088 E-02$
$2.6462 E-02$
$3.9020 E-02$
$3.1512 E-02$
$9.9722 E-02$
$5.0206 E-02$
$9.0767 E-03$
$2.1655 E-02$
$3.1690 E-02$
$3.1690 E-02$
$3.1690 E-02$
$1.0133 E-01$
$1.0212 E-01$
$5.2190 E-02$
$1.0896 E-01$
$9.7669 E-01$
$2.8211 E-02$
$3.5251 E-02$
$3.8777 E-02$


WHC-SD-W-242-ES-004, Rev. 0

TABLE 25

SUMMARY ANALYSIS OF SUSPECT "FREE LIQUID" VOLUMETRIC PERCENTAGES IN ASCENDING ORDER BASED ON CONTAINER COUNT

COMPONENT

HAZARDOUS CONSTITUENTS OILS

LIQUID

SLUDGES

WATER

PAINTSRUCITE

PCB

GREASE

LIQUID ORGANIC

SOLVENTSTTHINNERS

OIL AND WATER

ANTIFREEZE

LIGHT BALLAST

ORGANICS

MERCURY

CONTAMINATES

CREOSOTE

EPOXY/PAINT/OILBASE

HYDRAULIC FLUID WITH PCB

ZIP STRIP PAINT REMOVER ACID

CHEMSEARCH SS-80

PCB NON-REGULATED

TRANSITE

HEXANOL
(1 SHEET)

\begin{tabular}{|c|c|c|c|c|}
\hline COUNT & AVERAGE VOL PERCENT & VOL PERCENT STD DEV & MIN VOL PERCENT & MAX VOL PERCENT \\
\hline 1251 & $1.6587 E+01$ & $2.3017 \mathrm{E}+01$ & $1.0000 E-02$ & $1.0000 E+02$ \\
\hline 489 & $4.9590 E+01$ & $2.3602 E+01$ & $1.0000 E+\infty 0$ & $9.8000 E+01$ \\
\hline 406 & $5.9568 \mathrm{E}+00$ & $1.3503 E+01$ & $1.0000 E+\infty$ & $8.2000 E+01$ \\
\hline 183 & $4.9217 \mathrm{E}+01$ & $3.7776 \mathrm{E}+01$ & $1.0000 E+\infty$ & $1.0000 E+02$ \\
\hline 137 & $1.3076 E+01$ & $1.1403 E+01$ & $1.0000 E+\infty 0$ & $8.4000 E+01$ \\
\hline 125 & $2.3172 E+01$ & $2.6090 \mathrm{E}+01$ & $1.0000 E+00$ & $1.0000 E+02$ \\
\hline 45 & $9.6756 E+00$ & $2.0386 E+01$ & $0.0000 E+00$ & $9.5000 \mathrm{E}+01$ \\
\hline 21 & $1.7719 \mathrm{E}+01$ & $1.8971 E+01$ & $1.0000 E+00$ & $7.5000 E+01$ \\
\hline 18 & $5.2374 E+01$ & $1.9048 E+02$ & $1.0000 E+00$ & $8.5000 E+01$ \\
\hline 16 & $1.0077 \mathrm{E}+01$ & $2.3380 E+01$ & $1.0000 E+00$ & $6.5000 E+01$ \\
\hline 13 & $4.7385 E+01$ & $2.0966 E+01$ & $5.0000 \mathrm{E}+00$ & $6.5000 \mathrm{E}+01$ \\
\hline 12 & $2.5250 E+01$ & $3.8168 E+00$ & $1.5000 \mathrm{E}+01$ & $2.7000 \mathrm{E}+01$ \\
\hline 12 & $2.4300 E+01$ & $1.6740 E+01$ & $4.0000 E+00$ & $6.9000 E+01$ \\
\hline 11 & $3.4206 E+01$ & $6.5865 E+01$ & $1.0000 \mathrm{E}-02$ & $7.7000 E+01$ \\
\hline 9 & $3.4889 E+00$ & $1.1591 E+00$ & $1.0000 E+00$ & $4.3000 E+00$ \\
\hline 5 & $4.6000 E+00$ & $4.9295 E+00$ & $1.0000 E+00$ & $1,0000 E+01$ \\
\hline 5 & 1.1000E+01 & $7.0711 E-01$ & $1.0000 \mathrm{E}+01$ & $1.2000 \mathrm{E}+01$ \\
\hline 5 & $2.8800 E+01$ & $1.4464 E+01$ & $1.2000 \mathrm{E}+01$ & $5.0000 E+01$ \\
\hline 4 & $1.2500 E+00$ & 5.0000 E-01 & $1.0000 E+00$ & $2.0000 E+C 0$ \\
\hline 3 & $6.0000 E+00$ & $7.8102 \mathrm{E}+00$ & $1.0000 E+00$ & $1.5000 E+01$ \\
\hline 2 & $1.3000 E+01$ & $1.5556 \mathrm{E}+01$ & $2.0000 E+00$ & $2.4000 E+01$ \\
\hline 2 & $4.0000 E+00$ & $1.4142 E+00$ & $3.0000 E+00$ & $5.0000 E+\infty 0$ \\
\hline 1 & $0.0000 E+00$ & $0.0000 E+00$ & $0.0000 E+\infty$ & $0.0000 E+00$ \\
\hline 1 & $9.7000 \mathrm{E}+01$ & $0.0000 E+00$ & $9.7000 E+01$ & $9.7000 E+01$ \\
\hline 1 & $1.0000 E+01$ & $0.0000 E+00$ & $1.0000 \mathrm{E}+01$ & $1.0000 E+01$ \\
\hline
\end{tabular}


WHC-SD-W-242-ES-004, Rev. 0

TABLE 26

U-235 CONTAINERS IN ASCENDING ORDER BASED ON THE WEIGHT OF U-235 (4 SHEETS)

\begin{tabular}{|c|c|c|c|c|c|c|c|}
\hline PIN NUMBEER & U-235, GMS & PIN NUMBER & U-235, GMS & PIN NUMBER & U-235, GMS & PIN NUMGER & U.235, GMS \\
\hline PNL-89047 & $1.0200 E+02$ & $9200-03-120$ & $7.5400 \mathrm{E}-01$ & $9101-02.0061$ & 5.0990E-01 & $9101-03.0002$ & 4.1840E-01 \\
\hline PNL-89048 & $1.9000 E+01$ & $9200-03-125$ & $7.4200 \mathrm{E}-01$ & $8101-02-0066$ & $5.0990 \mathrm{E}-01$ & $9201-02-0004$ & $4.1700 \mathrm{E}-01$ \\
\hline 9403134 & $7.2317 E+\infty 0$ & $9200-03-135$ & $7.3700 \mathrm{E}-01$ & $9200-01-069$ & $5.0900 E-01$ & $9201-02-0010$ & 4.1700E-01 \\
\hline WHC-3-90-0026 & $5.5400 E+\infty 0$ & $9200-03-091$ & $7.3100 E-01$ & $9101-02-0086$ & $5.0850 E-01$ & $9101-02-0059$ & 4.1690E-01 \\
\hline $224 U \cdot 88001$ & $5.0000 E+\infty$ & $9101-02-0077$ & $7.2720 \mathrm{E}-01$ & $9101-02-0087$ & $5.0850 E-01$ & $9200-03-130$ & 4.1400E-O1 \\
\hline URMW2-1 & $3.0000 E+\infty$ & $9200-04-139$ & $7.2100 \mathrm{E}-01$ & $9201-02-0026$ & $4.9860 E-01$ & $9200-03-127$ & $4.0700 E-01$ \\
\hline $9417-02-0027$ & $2.9760 E+\infty 0$ & $9101-01-0036$ & $7.1740 \mathrm{E}-01$ & $9101-01-0044$ & 4.977OE-O1 & 9201-02-0001 & 3.9650E-01 \\
\hline $9317-03-0007$ & $2.3631 E+\infty$ & $9101-01-0037$ & $7.1740 \mathrm{E}-01$ & $9101.01-0045$ & 4.9770E.01 & $9201-02-0002$ & 3.9650E-01 \\
\hline $9317-03-0006$ & $2.3542 E+\infty$ & $9101-01-0038$ & $7.1740 \mathrm{E}-01$ & $9101-01-0046$ & 4.977OE-O1 & $9201-02-0013$ & $3.9650 \mathrm{E}-01$ \\
\hline $9317-03-0008$ & $2.2950 E+\infty$ & $9101.01-0039$ & $7.1740 E-01$ & $9200-03-105$ & $4.9720 \mathrm{E}-01$ & $9201-02-0018$ & $3.9650 E-01$ \\
\hline $9200-03-090$ & $2.0560 E+00$ & $9101-02-0075$ & $6.9580 \mathrm{E}-01$ & $9417-02-0024$ & 4.9700E-01 & $9201-02-0019$ & 3.9650E-01 \\
\hline $9200-03-122$ & $1.7640 E+\infty 0$ & $303 K-93-000050$ & $6.5350 \mathrm{E}-01$ & $9101-01-0021$ & $4.9320 \mathrm{E}-01$ & $9201-02-0021$ & $3.9650 \mathrm{E}-01$ \\
\hline $9200-03-093$ & $1.5960 E+\infty$ & $9200-02-113$ & 6.4900E-01 & $9200.02 \cdot 103$ & $4.8900 \mathrm{E}-01$ & 9201.02 .0022 & $3.9650 E-01$ \\
\hline $9200-03-092$ & $1,5640 E+\infty$ & $9200-01-063$ & $6.4600 \mathrm{E}-01$ & $9201-02-0014$ & 4.8640E-01 & 9200-01-070 & 3.9300E-01 \\
\hline $9200-03-134$ & $1.5630 E+\infty$ & $9101-01-0024$ & $6.2770 \mathrm{E}-01$ & $9101-02-0083$ & $4.8330 \mathrm{E}-01$ & $9201-02-0020$ & $3.9230 E-01$ \\
\hline $9200-03-118$ & $1.5110 E+\infty$ & $9101-01.0025$ & $6.2770 \mathrm{E}-01$ & $9101-02-0085$ & $4.8330 \mathrm{E}-01$ & $9200-02-101$ & 3.9200E-O1 \\
\hline $9200-02-107$ & 1. $2650 E+\infty 0$ & $9101-01-0026$ & $6.2770 \mathrm{O}-01$ & $9101-03-0005$ & $4.8320 \mathrm{E}-01$ & $9101-02-0063$ & 3.8550E-01 \\
\hline $9200-03-094$ & $1.2560 E+\infty$ & $9101-01-0027$ & $6.2770 \mathrm{E}-01$ & 9101.02 .0060 & $4.7840 \mathrm{E}-01$ & 9200.02 .049 & $3.8100 \mathrm{E}-01$ \\
\hline $9200-03-124$ & $1.1430 E+\infty$ & $9200-02-057$ & $6.1800 E-01$ & $9101-02-0062$ & 4.7840E-01 & $9201-02-0006$ & $3.7600 \mathrm{E}-01$ \\
\hline $9200-02-104$ & $1.1110 E+\infty$ & $9101-02-0064$ & $6.0280 E-01$ & $9101-02-0071$ & 4.7840E-O1 & $9201-02-0007$ & 3.7600E-01 \\
\hline $9200-03-106$ & $1,0520 E+\infty$ & $9\{01-02-0068$ & $6.0280 \mathrm{E}-01$ & $9201-02-0003$ & $4.7830 E-01$ & $9201-02-0008$ & $3.7600 \mathrm{E}-01$ \\
\hline $9200-01-028$ & $1.0458 E+\infty$ & $9101-02-0070$ & $6.0280 \mathrm{E}-01$ & $9201-02-0025$ & $4.7830 \mathrm{E}-01$ & $9201-02-0012$ & $3.7600 \mathrm{E}-01$ \\
\hline $9200-02-054$ & $9.9900 \mathrm{E}-01$ & $9101-02.0074$ & $6.0280 E-01$ & $9200-03.119$ & 4.7100E-01 & $9201-02-0023$ & 3.7600E-01 \\
\hline $9200-02-055$ & $9.8200 E-01$ & $9200-03-089$ & $6.0200 \mathrm{E}-01$ & $9200-01-068$ & 4.5900E-01 & 9200-03-095 & $3.6800 E-01$ \\
\hline $9200-03-108$ & $9.7000 \mathrm{E}-01$ & $9200-03-071$ & $5.8700 \mathrm{E}-01$ & $9 t 01-03-0003$ & 4.5B30E-01 & $9201-02-0015$ & $3.6790 E-01$ \\
\hline $9200-02-051$ & $9.6300 E-01$ & $9101-01-0048$ & $5.8290 \mathrm{E}-01$ & $9101-03-0006$ & 4.5830E-01 & $9201-02-0017$ & 3.5570E-01 \\
\hline $9200-03-136$ & $9.1100 E-01$ & $9200-02-097$ & $5.8100 \mathrm{E}-01$ & $9101-02-0078$ & $4.5810 \mathrm{E}-01$ & $303 K-93-000037$ & 3.4709E-01 \\
\hline $9101-01-0022$ & 8.9680E-01 & $9200-02-052$ & $5.7800 E-01$ & $9101-02-0079$ & $4.5810 E-01$ & $9201-02-0009$ & $3,4600 E-01$ \\
\hline $9101-01-0023$ & 8.968CE-01 & $9200-03-123$ & 5.7600E-01 & $9101-02-0080$ & $4.5810 \mathrm{E}-\mathrm{O} 1$ & $9417-02-0012$ & $3.4300 E-01$ \\
\hline $9101-01-0035$ & 8.9680E-01 & 9101.02 .0065 & $5.7140 \mathrm{E}-01$ & 9101.02 .0082 & 4.5810E-01 & $9417-02-0004$ & $3.4100 E-01$ \\
\hline $9101-02-0076$ & 8.8300E-01 & $9101-02-0067$ & $5.7140 E-01$ & $9101-02-0084$ & 4.5810E-O1 & $9200-04-138$ & 3.4000E-01 \\
\hline $9200-03-126$ & $8.3000 E-01$ & $9101-02-0069$ & $5.7140 \mathrm{E} \cdot 01$ & $9101-02-0088$ & 4.5810E-01 & $9200-02-114$ & 3.3700E-01 \\
\hline $9200-02-050$ & 8. $2500 E-01$ & $9200-02-096$ & 5.6800E-01 & $9417-02-0016$ & 4.5800E-01 & $9200-01-065$ & $3.3300 E-01$ \\
\hline $9200-02-053$ & $8.2500 E-01$ & $9417-02-0022$ & $5.5600 E-01$ & $9201-02-0024$ & 4.5790E-01 & $9200-02-109$ & $3.3300 E-01$ \\
\hline $9200-03-121$ & $8.1800 E-01$ & $9417-02-0005$ & $5.4700 E-01$ & $9201-02-0027$ & 4.5790E-O1 & $9200-01-064$ & 3. 2500E-01 \\
\hline $9101-01-0020$ & $8.0710 E-01$ & $9101-02-0058$ & 5.4130 E-01 & $303 K-93-000039$ & 4.4936E-01 & $9417-02-0003$ & 3.2200E-01 \\
\hline $9101-01-\infty 034$ & $8.0710 E-01$ & $9101-02-0073$ & $5.4130 \mathrm{E}-01$ & $9200-02-059$ & 4.4000E-01 & $303 k-93-000034$ & 3.2053E-01 \\
\hline $9101-01-0040$ & $8.0710 E-01$ & 9200.02 .060 & $5.4000 \mathrm{E}-01$ & $9201-02-0016$ & 4.3740E-01 & $9200-04-151$ & 3.1460E-O1 \\
\hline $9101-01-0041$ & $8.0710 E-01$ & $9417-02-0026$ & $5.3000 E-01$ & $9101-02-0081$ & 4.3400E-O1 & $9200-02-102$ & 3.1100E-01 \\
\hline $9101-01-0042$ & $8.0710 E-01$ & $9200-02-061$ & $5.2700 \mathrm{E}-01$ & $9417-02-0025$ & 4.3400E-01 & 9408381 & 3.0960E-01 \\
\hline $9101-01-0043$ & 8.0710E-01 & $9200-03-128$ & 5. $2500 \mathrm{E}-01$ & $9101-03-0004$ & 4.3340E-01 & 9408382 & $3.0960 E-01$ \\
\hline $9101-01-0033$ & $7.6220 E-01$ & $9200-02.058$ & $5.1900 \mathrm{E}-01$ & $9201-02-0005$ & $4.2510 E-01$ & $940 \mathrm{~B} 753$ & 3.0960E-01 \\
\hline $9101-02-\infty 072$ & $7.5860 E-01$ & $9417-02-0008$ & $5.1800 \varepsilon-01$ & $303 K-93-000036$ & $4.1882 E-01$ & $9101-03-000 t$ & 3.0880E-01 \\
\hline
\end{tabular}


WHC-SD-W-242-ES-004, Rev. 0

TABLE 26

U-235 CONTAINERS IN ASCENDING ORDER BASED ON THE WEIGHT OF U-235 (4 SHEETS)

\begin{tabular}{|c|c|c|c|c|c|c|c|}
\hline PIN NUMBER & U-235, GMS & PIN NUMBER & U-235, GMS & PIN NUMBER & U-235, GMS & PIN NUMBER & U-235, GMS \\
\hline $303 K-93-000046$ & $3.0800 \mathrm{E}-01$ & $303 K-93-000042$ & $2.0404 \varepsilon-01$ & $9200-01-040$ & 9.4100 E-02 & $9200-01-041$ & 4.4300E-02 \\
\hline $9417-02-0010$ & $3.0300 \mathrm{E}-01$ & $9200-01.066$ & $2.0370 E-01$ & $9101.01-0047$ & $8.9680 E-02$ & $9200-04-161$ & 4.1920E-02 \\
\hline $9417-02-0002$ & 3.0200E-01 & $303 K-93-000051$ & $1.9807 \mathrm{E}-01$ & $9200-01-037$ & $8.6800 \mathrm{E}-02$ & $9201-02-0044$ & 4.1300E-O2 \\
\hline $9200-01-067$ & 2.9900E-01 & $303 k-93-000038$ & $1.9797 \mathrm{E}-01$ & $9200-01-025$ & 7.9000E-02 & $303 K-93-000024$ & 4.072OE-O2 \\
\hline $9201.02-0028$ & $2.9430 E-01$ & $9200-01-029$ & $1.9700 \mathrm{E}-01$ & $9200-04-160$ & 7.8500E.02 & $9200-04-157$ & 4.027OE-02 \\
\hline $9200-04-153$ & $2.9350 E-01$ & $9101-01-0008$ & 1.8990E-01 & $9200.01-0045$ & $7.6200 \mathrm{E}-02$ & $9200-04-162$ & 3.944OE-02 \\
\hline $9200 \cdot 03-133$ & $2.9300 \mathrm{E}-01$ & $9101-01-0009$ & $1.8990 E-01$ & $9200-04-137$ & $7.5700 E-02$ & RALO0000294 & $3.8310 E-02$ \\
\hline $9200-02-100$ & $2.9200 E-01$ & 303K-93-000043 & $1.8812 E-01$ & $9200-01-036$ & $7.5000 E-02$ & $9201-02-0050$ & 3.7400E-02 \\
\hline $9417-02-0015$ & 2.9000E-01 & $9200-03-132$ & $1.8330 E-01$ & WTFF-92-322-03 & 7.2900E-02 & $9200-04-144$ & 3.6800E-O2 \\
\hline $9417-02-0017$ & $2.9000 E-01$ & $9200-02.099$ & $1.7620 \mathrm{E}-01$ & $9101.01-0006$ & 7.227 OE-O2 & $9201-02-005$ & 3.6200E-02 \\
\hline $9200-04-167$ & $2.8600 \mathrm{E}-01$ & $303 K-93-000049$ & $1.7121 \mathrm{E}-01$ & $9200-01-046$ & $7.0400 E-02$ & PNLD-93-000426 & 3.594OE-O2 \\
\hline $9417-02-0007$ & $2.8600 E-01$ & $9101-01-0015$ & $1.7030 E-01$ & $9200-01-032$ & 7.0300E-02 & PNLD-93-000427 & 3.5940E-O2 \\
\hline $9417-02-0013$ & $2.8600 E-01$ & $9101-01-0016$ & $1.7030 E-01$ & $303 k-93-000014$ & 6.5340E-02 & PNLD-93-000428 & 3.5940E-02 \\
\hline $9417-02-0006$ & 2.8400E-01 & $9101-01-0017$ & $1.7030 \mathrm{E}-01$ & $9200-04-145$ & 6.4900E-02 & PNLD-93-000429 & 3.594OE-O2 \\
\hline $9200-02-098$ & $2.7600 E-01$ & $9200-04 \cdot 155$ & $1.7030 E-01$ & WTFF-92-322-02 & $6.4200 E-02$ & PNLD-93.000430 & 3.594OE-02 \\
\hline $9417-02-0018$ & $2.7400 \mathrm{E}-01$ & $303 K-93-000040$ & $1.6902 E-01$ & $303 K-93-000027$ & $5.9580 \mathrm{E}-02$ & PNLD-93-000443 & 3.594OE-02 \\
\hline $9201-02-0011$ & $2.7380 E-01$ & $9200-02-115$ & $1.6670 \mathrm{E}-01$ & $9200-01-035$ & $5.8300 E-02$ & PNLD-93-000444 & $3.5940 E-02$ \\
\hline $303 K-93-000047$ & $2.7198 E-01$ & 9101-01-0013 & $1.6520 E-01$ & $9200-04-147$ & $5.7600 E-02$ & PNLD-93-000445 & 3.5940E-02 \\
\hline $9200-01-027$ & 2.7100E-01 & $9101-01-0014$ & $1.6520 \mathrm{E}-01$ & $9200-04-140$ & $5.6800 E-02$ & PNLD-93-000446 & 3.594OE-02 \\
\hline $9200-03-129$ & $2.7100 E-01$ & $9101-01-0018$ & $1.6520 E-01$ & $9200-04-163$ & 5.564OE-O2 & PNLD-93-000447 & 3.594OE-02 \\
\hline $9200-04-150$ & $2.7010 \mathrm{E}-01$ & $9200-03-116$ & $1.5640 E-01$ & $303 k-93-000026$ & $5.5170 E-02$ & PNLD-93-000448 & 3.594OE-02 \\
\hline 9200-03-131 & $2.6700 \mathrm{E}-01$ & $9200-04-154$ & $1.5030 \mathrm{E}-01$ & $9200-01-034$ & 5.4500E-02 & 9201-02-0029 & 3.5400E-02 \\
\hline $9417-02-0014$ & $2.6500 \mathrm{E}-01$ & 9200-01-038 & $1.5010 \mathrm{E}-01$ & $9200-01-033$ & 5.4400E-02 & 9201-02-0030 & 3.540OE-O2 \\
\hline $303 K-93-000044$ & $2.5766 \mathrm{E}-01$ & $9200-01.039$ & $1.4820 \mathrm{E}-01$ & $303 k-93-000033$ & $5.3580 E-02$ & $9201-02-0033$ & $3.5400 E-02$ \\
\hline $9417-02-0019$ & 2.5600E-O1 & $9200-04-156$ & $1.4670 E-01$ & $303 K-93-000020$ & 5.3260E- 02 & 9201-02-0035 & 3.5400E-02 \\
\hline $9200-02-048$ & $2.5400 E-01$ & $303 K \cdot 93-000045$ & $1.4534 \mathrm{E}-01$ & $303 K-93-000023$ & 5.2640E-02 & 9201-02-0040 & $3.5400 E-02$ \\
\hline $9417-02-0011$ & $2.5300 E-01$ & $9200-01-026$ & $1.3630 E-01$ & $9101-01-\infty 012$ & $5.1620 E-02$ & 9201-02-0047 & 3.5400E-02 \\
\hline $9417-02-0023$ & $2.5300 E-01$ & 9101-01-0004 & $1.3420 E-01$ & $9200-02-056$ & 5.1400E-02 & $9201-02-0052$ & 3.5400E-02 \\
\hline $9417-02-0021$ & $2.5100 E-01$ & 9101-01-0005 & $1.3420 E-01$ & $303 k-93-000032$ & $5.1300 E-02$ & $9200-04-164$ & $3.5110 E-02$ \\
\hline $9200-01-030$ & 2.4760E-01 & $9200-02-110$ & $1.3390 E-01$ & $303 K-93-000011$ & 5.1000E-02 & 303K-93-000025 & 3.4770E-02 \\
\hline $9417-02-0020$ & 2.4200E-01 & $9200-01-014$ & $1.2570 E-01$ & $9200-04-146$ & 5.0900E-02 & $9101-01-0001$ & 3.3680E-02 \\
\hline $9417-02-0009$ & 2.3600E-01 & $9200-02-047$ & 1.1440 E-01 & $303 K-93-000019$ & $5.0660 E-02$ & $9101-01-0002$ & 3.3680E-02 \\
\hline $9101-01-0028$ & $2.3320 E-01$ & $9200-02.062$ & $1.1400 E-01$ & $9200-04-149$ & $5.0400 E-02$ & $9101-01-0003$ & 3.3680E-02 \\
\hline $9101-01-0029$ & $2.3320 E-01$ & $9200-01-002$ & $1.1240 E-01$ & $9200-01-0044$ & 4.980OE-02 & $9201-02-0031$ & 3. 3500E-02 \\
\hline $9101-01-0030$ & 2.3320E-01 & $9200-02-111$ & 1.1110 E-01 & $303 K-93-000015$ & 4.954OE-02 & $9201-02-0034$ & 3. 3500E-02 \\
\hline $9101-01-0031$ & $2.3320 E-01$ & WTFF-92-322-04 & $1.0180 E-01$ & $9200-01-018$ & 4.9500E-02 & $9201-02-0045$ & 3. $3500 E-02$ \\
\hline $9101-01-0032$ & $2.3320 E-01$ & $9200-04-143$ & $1.0 t$ tOE-OT & $9200-04-168$ & $4.9500 E-02$ & $9201-02-0046$ & $3.3500 E-02$ \\
\hline $303 K-93-000048$ & $2.2891 E-01$ & WH-333-94-0022 & $9.9310 E-02$ & $303 k-93-000017$ & $4.9110 \mathrm{E}-02$ & $9201-02-0048$ & 3. 350OE-02 \\
\hline $9200-03-117$ & $2.2620 \mathrm{E}-01$ & $9200-01-031$ & $9.7600 E-02$ & $303 K-93-000013$ & 4.8800E-02 & $9101-03-0043$ & 3.3400E-02 \\
\hline $9417-02-0001$ & 2.2100E-01 & 9101-01-0019 & $9.6010 E-02$ & $9200-04-158$ & 4.7900E-02 & 9101-03-0044 & 3. $3400 E-02$ \\
\hline $9200-04-152$ & $2.1710 E-01$ & $202 A \cdot 92-002370$ & $9.5800 E-02$ & $303 k-93-000018$ & $4.7200 E-02$ & $9201-02-0036$ & 3.1600E-02 \\
\hline $303 K-93-000041$ & $2.1667 \mathrm{E}-01$ & $202 A-92-002371$ & $9.58 C O E-02$ & 9200-04-159 & $4.5320 E-02$ & $9201-02-0037$ & $3.1600 E-02$ \\
\hline $303 K-93-000035$ & $2.0991 \mathrm{E}-01$ & $9200-01-020$ & 9.440OE-02 & $303 K-93-000016$ & 4.4950E-02 & $9201-02-0038$ & $3.1600 E-02$ \\
\hline
\end{tabular}


WHC-SD-W-242-ES-004, Rev. 0

TABLE 26

U-235 CONTAINERS IN ASCENDING ORDER BASED ON THE WEIGHT OF U-235

(4 SHEETS)

\begin{tabular}{|c|c|c|c|c|c|c|c|}
\hline PIN NUMBER & U-235, GMS & PIN NUMBER & U-235, GMS & PIN NUMBER & U-235, GMS & PIN NUMBER & U-235, GMS \\
\hline $9201-02-0039$ & $3.1600 \mathrm{E} \cdot 02$ & GEL-94-183-037 & 4. 200OE-03 & $224 U-90-00132$ & $1.0000 \mathrm{E} \cdot 03$ & 222S-93-000106 & $1.2354 \mathrm{E}-04$ \\
\hline $9201-02-0042$ & $3.1600 \mathrm{E}-02$ & GEL-94-183-052 & $4.2000 \mathrm{E} \cdot 03$ & $224 U-91-00012$ & $1.0000 \mathrm{E}-03$ & 222S-93-000137 & $1.1000 \mathrm{E}-04$ \\
\hline $9201-02-0043$ & 3. $1600 E-02$ & GEL-94-183-053 & $4.2000 E-03$ & $224 U-92-000007$ & 1.0000 E.03 & $2225 \cdot 93-000107$ & 8.9289E-05 \\
\hline $9201 \cdot 02-0053$ & $3.1600 \mathrm{E}-02$ & $202 A-92-000328$ & $4.107 \mathrm{OE}-03$ & $224 U-92 \cdot 000013$ & $1.0000 \mathrm{E}-03$ & PNL-PSL-93-003 & 8.1000E-05 \\
\hline $303 K-93-000012$ & $3.0740 \mathrm{E} \cdot 02$ & $9201-02-0061$ & $3.9000 E-03$ & $224 U-92-000014$ & $1.0000 E .03$ & $2225-93-000303$ & $5.9359 \mathrm{E} \cdot 05$ \\
\hline 9408386 & $3.0650 \mathrm{E}-02$ & $9201-02-0073$ & 3.900OE-03 & $224 U-92-000020$ & $1.0000 E-03$ & $2225-92-000499$ & 5.0350E-05 \\
\hline $9201-02-0032$ & $2.7800 \mathrm{E}-02$ & $9201-02-0060$ & $3.3000 E-03$ & $224 U-92-000041$ & $1.0000 \mathrm{E}-03$ & GEL-94-183-031 & 4.6000E-05 \\
\hline $9201.02-0041$ & $2.7800 \mathrm{E}-02$ & $9201-02-0057$ & 3.1000E-03 & $224 \mathrm{U}-92-000067$ & $1.0000 \mathrm{E}-03$ & $2225-92-000038$ & 4.5266E-05 \\
\hline $9201-02-0054$ & $2.7800 E-02$ & $9201-02-0062$ & 3.1000E-03 & 224 U-92-000068 & 1.0000E-03 & 222S-92-000194 & 4.2007E-O5 \\
\hline $9101-01-0010$ & $2.5810 E-02$ & 9408743 & $2.8700 E-03$ & $224 U-92-000116$ & $1.0000 \mathrm{E}-03$ & 222S-93-000385 & $4.021 \mathrm{OE}-\mathrm{O5}$ \\
\hline $9201-02-0049$ & $2.5800 \mathrm{E}-02$ & 9408744 & 2.8700 - -03 & $224 U-92-000127$ & $1.0000 E-03$ & PNL-325-92-048 & $3.494 \mathrm{OE} \cdot \mathrm{O} 5$ \\
\hline $9201-02-0055$ & $2.3900 \mathrm{E}-\mathrm{O} 2$ & 9408745 & $2.8700 \mathrm{E}-03$ & 224U-93.000001 & $1.0000 E-03$ & $222 S-92-000500$ & 3.0953E-05 \\
\hline $9101-01-0007$ & $2.3740 \mathrm{E}-02$ & 9408746 & $2.8700 \mathrm{E}-03$ & $224 \mathrm{U}-93-000030$ & 1.0000 E-03 & RBLO000294 & $2.9697 \mathrm{E}-05$ \\
\hline $9101-03-0047$ & $2.1900 \mathrm{E}-02$ & 9408747 & $2.8700 E-03$ & WHC-3-89-0064 & $1.0000 E-03$ & WTFF-93-084-02 & $2.5437 \mathrm{E}-05$ \\
\hline $9101.03-0048$ & $2.1900 \mathrm{E}-02$ & 9408748 & 2.8700E-03 & WHC-3-90-12 & 1.0000E-03 & $2225-92-000321$ & $2.5269 \mathrm{E}-\mathrm{O} 5$ \\
\hline $9201-02-0056$ & $2.0100 \mathrm{E}-02$ & 9408749 & $2.8700 E-03$ & WHC-3-90-13 & 1.0000E-03 & 202A-93-00032B & 2.5000E-05 \\
\hline $9101-03-0045$ & $1.9600 E-02$ & 9408750 & $2.8700 E-03$ & WHC.3.90-14 & $1.0000 E-03$ & 202A-93-321001 & $2.2000 \mathrm{E}-05$ \\
\hline $9200-04 \cdot 166$ & $1.9010 \mathrm{E}-02$ & 9408751 & $2.8700 \mathrm{E}-\mathrm{O} 3$ & WHC-3-90-15 & $1.0000 \mathrm{E}-03$ & 202A-93-321002 & $2.2000 \mathrm{E}-05$ \\
\hline $9200-04-165$ & $1.8080 \mathrm{E}-02$ & $9201-02-0065$ & $2.7000 \mathrm{E}-03$ & WHC-3-90-16 & $1.0000 E-03$ & $202 A-93-321003$ & $2.2000 E-05$ \\
\hline $9101-03-0046$ & $1.8000 \mathrm{E}-02$ & $9201-02-0072$ & $2.7000 E-03$ & WHC-3-90-63 & 1.0000E-03 & 202A-93-321004 & $2.2000 \mathrm{E}-05$ \\
\hline 9101-03-0049 & $1.8000 \mathrm{E}-02$ & $2225-93-001021$ & $2.6697 \mathrm{E}-03$ & 222S-93-000382 & 9.3200E-04 & 202A-93-322001 & 2.2000E-05 \\
\hline RFLO000294 & $1.7818 E-02$ & $9201-02-0058$ & $2.3000 \mathrm{E}-03$ & $222 S-93-000310$ & $6.6000 \mathrm{E}-04$ & 202A-93-322002 & 2.2000E-05 \\
\hline $9200-04-148$ & $1.441 \mathrm{OE}-02$ & $9201-02-0068$ & $2.3000 E-03$ & $9305-03-0032$ & $6.0000 E-04$ & 202A-93-322003 & $2.2000 \mathrm{E}-05$ \\
\hline $321-91-0001$ & $1.2630 \mathrm{E}-02$ & $9201-02-0070$ & $2.3000 E-03$ & $2240 \cdot 90-111$ & 5.5000E-04 & 202A-93-322004 & $2.2000 \mathrm{E}-05$ \\
\hline 9101-01-0011 & $1.0330 \mathrm{E}-02$ & $9201-02-0071$ & $2.3000 E-03$ & 222S-93-000135 & 5.4470E-O4 & 202A-93-323001 & $2.2000 \mathrm{E}-05$ \\
\hline EFSG-92-000819 & $6.7900 \mathrm{E}-03$ & $9305-03-0027$ & $2.2000 \mathrm{E}-\mathrm{O} 3$ & 222S-93-000302 & $5.3736 E-04$ & 202A-93-323002 & $2.2000 \mathrm{E}-05$ \\
\hline RALOO00194 & $6.6819 \mathrm{E}-03$ & $9201-02-0063$ & $2.1000 \mathrm{E}-03$ & 224U-90-114 & $5.1000 E-04$ & 9402892 & 2.1000E-05 \\
\hline $9101-02-0001$ & 5.2000E-03 & $9201-02-0066$ & $2.1000 \mathrm{E}-03$ & $22252 W-91-0498$ & $5.0319 E-04$ & $2225-93-000136$ & 2.0900E-05 \\
\hline $224 U-91-004$ & $5.0000 E-03$ & $9201-02-0067$ & $2.1000 \mathrm{E}-\mathrm{O} 3$ & 224U-94-000010 & 4.2200E-04 & 2225-93-000304 & $1.7070 E-05$ \\
\hline 9101-02-0004 & 4.9000E-03 & $224 U-94-000070$ & $1.9100 E \cdot 03$ & $2225-94-000008$ & 4.0930E-04 & 202A-93-C00330 & 1.7000E-O5 \\
\hline $9101-02-0005$ & $4.9000 \mathrm{E}-03$ & $224 U-94-170002$ & $1.9100 \mathrm{E}-03$ & ANL93-MW-00086 & 3.7000E-04 & 202A-93-317003 & $1.7000 \mathrm{E}-05$ \\
\hline $9101-02-0006$ & $4,9000 E-03$ & 224U-94-170003 & $1.9100 E-03$ & ANL93-MW-00102 & 3.7000E-04 & 202A-93-317004 & $1.7000 E-05$ \\
\hline $9101-02-0007$ & $4.9000 \mathrm{E}-03$ & $9201-02-0059$ & $1.9000 E-03$ & ANL93-MW-00137 & 3.7000E-04 & 202A-93-317005 & $1.7000 \mathrm{E}-05$ \\
\hline $9101-02-0009$ & 4.9000E-03 & $9201-02-0064$ & $1.9000 E-03$ & 222S-93-000132 & 3.5090E-04 & 202A-93-317006 & $1.7000 \mathrm{E}-05$ \\
\hline $9101-02-0003$ & $4.6000 \mathrm{E}-03$ & $9201-02-0069$ & $1.9000 E-03$ & 222S-93-000384 & $3.3150 E-04$ & 202A-93-31700B & $1.7000 \mathrm{E}-05$ \\
\hline $9101-02-0008$ & 4.6000E-03 & $224 U-93-000114$ & $1.8910 E-03$ & WTFF-93-090-04 & 2.9700E-04 & 202A-93-317010 & $1.7000 \mathrm{E}-05$ \\
\hline $9305-03-0028$ & 4.4000E- 03 & 222S-93-001020 & $1.6121 \mathrm{E}-03$ & 222S-93-000116 & $1.8112 \mathrm{E}-04$ & $2225-94-000016$ & $1.6290 E-05$ \\
\hline 9101-02-0002 & 4.3000E-03 & 222S-92-000202 & $1.0946 E-03$ & 222S-93-000319 & $1.8040 \mathrm{E}-04$ & 202L-92-000710 & $1.5200 \mathrm{E}-05$ \\
\hline $9101-02-0010$ & 4.3000E-03 & 2225-94-000010 & $1.0300 \mathrm{E}-03$ & 222S-93-000386 & $1.6870 E-04$ & 202A-92-000313 & 1.5000 E-05 \\
\hline GEL-94-183-026 & 4. $2000 E-03$ & 222S-94-00001 2 & $1.0240 E-03$ & $2225-93-000113$ & $1.6017 \mathrm{E}-04$ & $2225-92-000375$ & $1.4109 \mathrm{E}-05$ \\
\hline GEL-94-183-027 & 4.2000E-03 & $224 U-90-00128$ & 1.0000E-03 & $222 S-93-000153$ & $1.5020 E-04$ & 2225-92-000501 & 1.3687E-O5 \\
\hline GEL-94-183-030 & 4. $2000 \mathrm{E}-03$ & $224 U-90-00129$ & $1.0000 E-03$ & 222S-93-000318 & 1.3520E-04 & $202 L-92-000702$ & 1.3100E-OS \\
\hline GEL-94-1 83-036 & 4.2000E-03 & $224 U-90-00131$ & $1.0000 E-03$ & $222 S-93-000134$ & $1.2480 E-04$ & 202L-92-000709 & 1.3100E-05 \\
\hline
\end{tabular}


WHC-SD-W-242-ES-004, Rev. 0

TABLE 26

U-235 CONTAINERS IN ASCENDING ORDER BASED ON THE WEIGHT OF U-235 (4 SHEETS)

\begin{tabular}{|c|c|c|c|c|c|}
\hline PIN NUMBER & U-235, GMS & PIN NUMBER & U-235, GMS & PIN NUMBER & U.235, GMS \\
\hline $222 S-92-000286$ & $1.1897 E-05$ & RELOOOOO194 & $2.9697 \mathrm{E}-07$ & 2225-92-000325 & $4.0638 E-09$ \\
\hline 2225-93-000052 & $1.1254 \mathrm{E}-05$ & $2225-93-000037$ & $2.3042 \mathrm{E}-07$ & $2225-92-000290$ & 3.284OE-O9 \\
\hline $222 S-92-000376$ & $1.0948 E-05$ & HEXO.92.000023 & $1.6200 \mathrm{E}-07$ & 313-93-00009 & $2.7000 \mathrm{E}-09$ \\
\hline $2225-92-000320$ & $1.0947 E-05$ & 2225-92-000037 & $1.5160 \mathrm{E}-07$ & 222S-92-000609 & $1.7268 \mathrm{E}-09$ \\
\hline $202 \mathrm{~L}-92-000704$ & $1.0900 E-05$ & $2225-92-000610$ & $1.2210 \mathrm{E}-07$ & 9402923 & $1.1450 \mathrm{E}-09$ \\
\hline 2225-93-000039 & 9.5810E-06 & 2225-92-000292 & $1.1687 E-07$ & WTFF-93-007-03 & $1.9019 E-10$ \\
\hline 222S-93-000030 & 9.359OE-06 & $2225-92-000004$ & $1.0456 \mathrm{E}-07$ & REO0001090 & $5.0000 \mathrm{E}-11$ \\
\hline $202 k-93-000705$ & B.9300E-06 & 2225-92-000324 & $9.7810 \mathrm{E}-08$ & HEXO-92-000003 & $2.1000 \mathrm{E}-12$ \\
\hline $202 L-92.000701$ & 8.9200E-06 & $2225-93-000031$ & $9.1590 E-08$ & HEXO-92-000004 & $2.1000 \mathrm{E}-12$ \\
\hline $2225-92-000608$ & 8.6440E-06 & 222S-92-000045 & $8.7720 E-08$ & HEXO-92-000005 & $2.1000 \mathrm{E}-12$ \\
\hline $202 A-92-000308$ & 8.4200E-06 & 222S-92-000296 & $8.0430 \mathrm{E}-08$ & HEXO-92-000006 & $2.1000 \mathrm{E}-12$ \\
\hline 202A-93-000309 & 8.0000E-06 & 222S-92-000189 & $7.7500 \mathrm{E}-08$ & HEXO-92-000007 & $2.1000 \mathrm{E}-12$ \\
\hline 202A-93-000316 & $8.0000 E-06$ & 2225-92-000040 & 7.7080E-08 & HEXO-92-000011 & $2.1000 \mathrm{E}-12$ \\
\hline $202 \mathrm{~L}-92-000706$ & $7.9500 \mathrm{E}-06$ & $2225-92-000046$ & 7. 7080E-08 & HEXO-92-000012 & $2.1000 \mathrm{E}-12$ \\
\hline $2225-92-000190$ & 7.6330E-06 & $222 S-92-\infty 00163$ & $7.7070 \mathrm{E}-08$ & HEXO-92-000013 & 1.300OE- 12 \\
\hline $202 A-93-000331$ & 7.0000E-06 & 222S-92-000005 & $5.7810 ̣ \mathrm{E}-08$ & HEXO-92-000008 & 7.1000 E- 13 \\
\hline ETFF-94-084-01 & 6.9600E-06 & 222S-92-000616 & $5.7803 \mathrm{E}-08$ & PNL-325-93-054 & $2.4000 E-18$ \\
\hline 222S-93-000395 & $6.7300 \mathrm{E}-06$ & WTFF-93-007-01 & $4.9938 E \cdot 08$ & & \\
\hline 222S-92-000305 & $6.5702 E-06$ & $2225-92-000032$ & 4.6220E-08 & & \\
\hline 222S-93-000305 & $6.4174 E-06$ & 222S-92-000319 & $4.5367 \mathrm{E}-08$ & & \\
\hline 222S-92-000330 & 6.2533E-06 & 2225-92-000348 & $4.0749 E-08$ & & \\
\hline 2225-93-000044 & 5.5804E-06. & 222S-92-000289 & $3.3908 \mathrm{E}-08$ & & \\
\hline $222 S-92-000205$ & 4.9479E-06 & 9402927 & 3. $2835 \mathrm{E}-08$ & & \\
\hline 222S-92-000612 & 4.3905E-06 & 2225-92-000192 & $3.0850 \mathrm{E}-08$ & & \\
\hline PNL-325-93-002 & 3.5200E-06 & 2225-92-000204 & $2.2951 E-08$ & & \\
\hline $2225-92-000033$ & $3.0739 \mathrm{E}-06$ & WTFF-93-145-03 & $2.2079 \mathrm{E}-08$ & & \\
\hline 222S-93-000011 & $2.8638 E-06$ & WTFF-93-054-01 & $1.9201 E-08$ & & \\
\hline 2225-92-000191 & 2.6527E-06 & 2225-92-000279 & $1.5374 \mathrm{E}-08$ & & \\
\hline 202L-93-000704 & $2.1600 E-06$ & $2225-92-000283$ & $1.3475 E-08$ & & \\
\hline 202L-94-000701 & $2.1600 \mathrm{E}-06$ & 2225-92-000293 & $1.3200 \mathrm{E}-08$ & & \\
\hline 2225-92-000301 & $2.1475 E-06$ & $2225-92-000284$ & $1.1791 \mathrm{E}-08$ & & \\
\hline 222S-92-000498 & $2.1380 \mathrm{E}-06$ & 222S-92-000352 & $1.1581 E-08$ & & \\
\hline 2225-92-000336 & $1.5582 \mathrm{E}-06$ & $2225-92-000138$ & $1.1190 \mathrm{E}-08$ & & \\
\hline $2225-92-000155$ & 1.3687E-06 & 2225-92-000294 & $1.0951 \mathrm{E}-08$ & & \\
\hline WHC-A-91-822 & $1.1917 \mathrm{E}-06$ & WHC-A-90-850 & $1.0700 \mathrm{E}-08$ & & \\
\hline 202A-93-000310 & $1.0000 E-06$ & $2225-92-000322$ & $9.7390 E-09$ & & \\
\hline $2225-92-000358$ & $9.9270 E-07$ & $2225-92-000298$ & $9.1600 E-09$ & & \\
\hline $2225-92-000493$ & $9.7910 \mathrm{E}-07$ & 222S-92-000195 & 8. 8340E-09 & & \\
\hline $2225-92-\infty 00166$ & $7.3590 E-07$ & $2225-92-000323$ & $7.7070 \mathrm{E}-09$ & & \\
\hline 2225-93-000130 & $6.9930 \mathrm{E}-07$ & 2225-92-000360 & $6.70706-09$ & & \\
\hline $2225-92-000076$ & 6.6750E-07 & $2225-92-000611$ & $6.0962 \mathrm{E}-09$ & & \\
\hline $2225-92-000077$ & $6.3487 E-07$ & 222S-92-000297 & $5.1150 E-09$ & & \\
\hline WHC-A-91-825 & $5.0066 \mathrm{E}-07$ & $222 S-92-000282$ & $4.0638 \mathrm{E}-09$ & & \\
\hline
\end{tabular}




\section{WHC-SD-W-242-ES-004, Rev. 0}

TABLE 27 DOT-REGULATED CONTAINERS IN EXCESS OF 15 GM OF PU-238, PU-239, PU-241, U-233, AND U235 DATA SORTED IN ASCENDING ORDER BASED ON FISSILE QUANTITY (1 SHEET)

PIN NUMBER

RHZ-85-002

RHZ-87-044

PNL-89047

RHZ-87-034

RHZ-86-002

9403711

RHZ-87-028

RHZ-87-041

$\mathrm{RHZ}-86-010$

$\mathrm{RHZ}-213-\mathrm{A} 21921$

$\mathrm{RHZ}-87-039$

$\mathrm{RHZ}-87-024$

RHZ-212-A22853

$\mathrm{RHZ}-86-019$

RHZ-212-A22878

$\mathrm{RHZ}-87-031$

RHZ-87-025

PNL-89048
TOTAL FISSILE QUANTITY, GM

$3.1630 \mathrm{E}+02$

$2.8300 E+02$

$1.0200 E+02$

$7.9729 E+01$

$7.2000 E+01$

$6.8477 \mathrm{E}+01$

$6.8000 \mathrm{E}+01$

$6.1000 E+01$

$5.6000 \mathrm{E}+01$

4.9683E + 01

$4.8000 E+01$

$4.6899 \mathrm{E}+01$

4.1267E + 01

$3.2829 \mathrm{E}+01$

$3.0951 \mathrm{E}+01$

$2.6000 \mathrm{E}+01$

$2.5000 E+01$

$1.9000 \mathrm{E}+01$ 
WHC-SD-W-242-ES-004, Rev. 0

TABLE 28

HYDROGEN/FISSILE ATOMIC RATIO FOR PINS CONTAINING WATER AND DOT FISSILE ISOTOPES (1 SHEET)

PIN NUMBER

9406778
ETFF-93-133-03
WTFF-93-155-05
ANL93-MW-00131
WTFF-93-018-02
WTFF-93-015-08
WTFF-93-018-01
ANL93-MW-00128
WHC-3-89-0064
ANL93-MW-00105
ANL93-MW-00102
ANL93-MW-00086
ANL93-MW-00137
9317-03-0007
9317-03-0008
9317-03-0006

\author{
FISSILE QUANTITY, GM \\ LIQUID MEDIUM LIQUID WEIGHT, KG
}
4.3500E-08
5.57DOE-08
$1.57 D O E-06$
3.1000E-06
$1.1800 \mathrm{E}-05$
$1.1 \mathrm{BOOE}-05$
$1.1800 E-05$
$1.8000 \mathrm{E}-05$
$1.000 \mathrm{OE}-03$
$1.5000 \mathrm{E}-04$
3.7000E-04
4. $2600 \mathrm{E}-04$
3. $7000 \mathrm{E}-04$
2.3631E + 00
2. $2950 E+00$
2. $3542 \mathrm{E}+00$

WATER
WATER
WATER
WATER
WATER
WATER
WATER
WATER
ACID
WATER
WATER
WATER
WATER
WATER
WATER
WATER

$5.5000 E+00$
$2.0000 E+00$
$6.1900 E+00$
$6.6000 E+00$
$1.5000 E+01$
$5.0000 E+00$
$5.0000 E+00$
$4.8000 E+00$
$4.9150 E+01$
$6.0000 E+00$
$6.8000 E+00$
$5.4000 E+00$
$3.5000 E+00$
$8.2700 E+01$
$3.8000 E+01$
$1.6100 E+01$

FISSILE CONC, GM/LITER

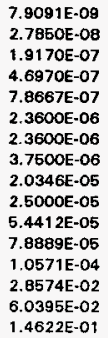

WATER/FISSILE ATOM RATIO

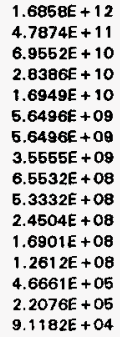


WHC-SD-W-242-ES-004, Rev. 0

TABLE 29

SUMMARY OF ORGANIC SOLVENT INVENTORY SORTED IN ASCENDING ORDER BASED ON CONTAINER COUNT

(1 SHEET)

COMPONENT
PLASTIC/POLYIJRATHANE
PCB
HAZARDOUS CONSTITUENTS
WOOD/LUMBER/PLYWOOD
GLASS
PAPER/CARDBOARD
CONWEB PADS
RUBBER
HEXACHLOROBENZENE
CLOTH/RAGS/NYLON
OILS
DIOCTYL PHTHA,LATE IDOPI
CRESOL
O-CRESOL
CARBON TETRACHLORIDE
CHEMICALS
METHYLENE CHLORIDE
STRIPCOAT
9O MIL LINER
CARBONTETRA.CHLORIDE
DII2-ETHYLHEXYLIPHTHALATE
HALOGENATED HYDROCARBON
HEXACHLOROBENZNE
LEATHER
OIL. AND WATER
TRI BUTYL PHOSPHATE
METHANOL
PAINTS/LUCITE
FESINS
SOLVENTS/THINNERS

$\begin{array}{cc}\text { COUNT } & \text { ACCUM, KG } \\ 32 & 1.9494 E+03 \\ 21 & 1.2882 E+00 \\ 20 & 2.4312 E+02 \\ 17 & 1.1692 E+03 \\ 13 & 1.2891 E+02 \\ 13 & 3.8980 E+02 \\ 11 & 6.1600 E+01 \\ 9 & 1.5233 E+02 \\ 8 & 1.5400 E-02 \\ 7 & 2.0980 E+01 \\ 6 & 3.1110 E+01 \\ 4 & 3.5300 E+00 \\ 3 & 2.1610 E-01 \\ 3 & 1.4340 E-01 \\ 2 & 1.5000 E+00 \\ 2 & 4.4000 E+01 \\ 2 & 5.8000 E+00 \\ 2 & 8.1700 E+00 \\ 1 & 6.9000 E+00 \\ 1 & 9.4982 E+00 \\ 1 & 2.0000 E-02 \\ 1 & 1.6000 E-02 \\ 1 & 2.5000 E-03 \\ 1 & 2.1000 E+00 \\ 1 & 2.3940 E+01 \\ 1 & 5.9012 E+00 \\ 0 & 0.0000 E+00 \\ 0 & 0.0000 E+00 \\ 0 & 0.0000 E+00 \\ 0 & 0.0000 E+00\end{array}$

AVERAGE, KG
$6.0918 E+01$
$6.1343 E-02$
$1.2156 E+01$
$6.8774 E+01$
$9.9162 E+00$
$2.9985 E+01$
$5.6000 E+00$
$1.6926 E+01$
$1.9250 E-03$
$2.9971 E+00$
$5.1850 E+00$
$8.8250 E-01$
$7.2033 E-02$
$4.7800 E-02$
$7.5000 E-01$
$2.2000 E+01$
$2.9000 E+00$
$4.0850 E+00$
$6.9000 E+00$
$9.4982 E+00$
$2.0000 E-02$
$1.6000 E-02$
$2.5000 E-03$
$2.1000 E+00$
$2.3940 E+01$
$5.9012 E+00$
$0.0000 E+00$
$0.0000 E+00$
$0.0000 E+00$
$0.0000 E+00$

MIN. KG

MAX, KG

$1.0000 E+00$
$0.0000 E+00$
$0.0000 E+00$
$3.0000 E-02$
$0.0000 E+00$
$1.0000 E-02$
$4.0000 E+00$
$5.0000 E-01$
$1.6000 E-03$
$1.2000 E-01$
$1.0000 E-02$
$3.0000 E-02$
$2.1000 E-03$
$1.4000 E-03$
$5.0000 E-01$
$2.2000 E+01$
$2.3000 E+00$
$1.4100 E+00$
$6.9000 E+00$
$9.4982 E+00$
$2.0000 E-02$
$1.6000 E-02$
$2.5000 E-03$
$2.1000 E+00$
$2.3940 E+01$
$5.9012 E+00$
$0.0000 E+00$
$0.0000 E+00$
$0.0000 E+00$
$0.0000 E+00$

$2.7100 E+02$ 2.4880E-01 $3.4340 E+01$ 3.500OE + 02 8.1000E + 01 1. $1700 E+02$ $2.0000 E+01$ $3.0000 E+01$ 2.3000E-03 $1.5000 E+01$

1.3400E + 01

$1.5000 E+\infty$ 1.5000E-01 $1.0000 E-01$ $1.0000 E+00$ 2. $2000 E+01$ $3.5000 E+\infty 0$ 6. $7600 E+\infty 0$ $6.9000 E+00$ 9.4982E + 00 2.0000E-02 $1.6000 \mathrm{E}-02$ 2.5000E-03 2. $1000 E+\infty 0$ 2.3940E + 01 $5.9012 E+00$ $0.0000 E+\infty 0$ $0.0000 E+00$ $0.0000 E+\infty 0$ $0.0000 E+00$ 
WHC-SD-W-242-ES-004, Rev. 0

TABLE 30

LOW ACTIVITY CONTAINERS

(1 SHEET)

PIN

\begin{tabular}{|c|c|}
\hline ETF-91.190-02 & $9.0000 E \cdot 12$ \\
\hline ETF-91-227-03 & $1.3552 \mathrm{E}-11$ \\
\hline PNL. 88044 & 2.0000E-11 \\
\hline ETF-91-224-01 & $9.7760 \mathrm{E}-10$ \\
\hline $100 \mathrm{~N}-91-0104$ & $2.2400 \mathrm{E}-09$ \\
\hline $221 \mathrm{~T}-92-000324$ & 2.4520E-09 \\
\hline $100 \mathrm{~N}-91-0371$ & $2.7710 E-09$ \\
\hline $100 \mathrm{~N}-91-0372$ & $2.7710 \mathrm{E}-09$ \\
\hline $100 \mathrm{~N}-91-0373$ & $2.7710 E-09$ \\
\hline $100 \mathrm{~N}-91-0374$ & $2.7710 E-09$ \\
\hline 100 N-91.0375 & 2.7710 -09 \\
\hline $100 \mathrm{~N}-91-0380$ & $2.7710 \mathrm{E}-09$ \\
\hline $100 \mathrm{~N}-91-0370$ & 2.7710E-O9 \\
\hline RS0001 2593 & $4.0000 E-09$ \\
\hline 9402899 & 4.0001E-09 \\
\hline $2211.92-000187$ & 5.8020E-09 \\
\hline $100 K-92-004000$ & $6.7680 \mathrm{E}-09$ \\
\hline RS000009693 & $1.0000 \mathrm{E}-08$ \\
\hline $100 N-90-003800$ & $1.3106 \mathrm{E}-0 \mathrm{~B}$ \\
\hline $100 \mathrm{~N} \cdot 99.032600$ & $1.3400 \mathrm{E}-08$ \\
\hline $100 \mathrm{~N}-92-014000$ & $1.3584 \mathrm{E}-\mathrm{OO}$ \\
\hline $100 \mathrm{~N}-93.032400$ & $1.4744 \mathrm{E}-08$ \\
\hline GEL-94-183-031 & $1.5822 \mathrm{E}-08$ \\
\hline $100 K-91-021700$ & $1.7402 E-08$ \\
\hline $2211 \cdot 92-000357$ & $1.7760 \mathrm{E}-08$ \\
\hline 940200012 & $1.8791 \mathrm{E}-08$ \\
\hline $100 \times-92.017500$ & $2.0128 \mathrm{E}-08$ \\
\hline $100 K-92-017400$ & $2.0400 E-08$ \\
\hline 9402853 & $2.4000 E-08$ \\
\hline $221-T-91-00066$ & $2.5950 E-08$ \\
\hline 221-T-91-00065 & $2.5950 E-0 B$ \\
\hline 221.T.91.00071 & $2.5950 E-06$ \\
\hline 221 T-91-00067 & $2.5950 E-08$ \\
\hline $2217 \cdot 91.00068$ & 2.595OE-OB \\
\hline $2211.92-000090$ & $2.6130 E-08$ \\
\hline 221 T-92-000149 & $2.6130 E-08$ \\
\hline 9402852 & $2.63800-08$ \\
\hline $100 N-93-032600$ & $2.7192 E-08$ \\
\hline 221 T.92.000034 & $2.77506-08$ \\
\hline $221 \mathrm{~T} \cdot 92-000055$ & $2.7750 E-08$ \\
\hline $221 T-92-000056$ & $2.7750 E-06$ \\
\hline $221 \mathrm{~T} \cdot 92.000073$ & $2.7750 E-06$ \\
\hline 221 T-92-000074 & $2.7750 \mathrm{OE}-08$ \\
\hline $221 \mathrm{~T} \cdot 92.000083$ & $2.7750 E-0 B$ \\
\hline $221 \mathrm{~T}-92-000088$ & $2.7750 E-08$ \\
\hline $221 \mathrm{~T} \cdot 92.000089$ & $2.7750 E-08$ \\
\hline $221 T-92-000139$ & $2.7750 \mathrm{E}-08$ \\
\hline
\end{tabular}

PIN 221T-92-000145 117-92-000146 $211-02-000147$ 121 92000148 (217.920014 $2217-92-000180$ $2217-92.00045$ $221 T-92.000036$ $221 T \cdot 92.000054$ $221 T-92-000122$ $221 \mathrm{~T}-92 \cdot 000140$ $221 T-92-000142$ 221T-92-000150 221T-92.000161 $1706-94-000600$ $1706-94-000800$

$1706-94-000900$

$1706-94-001400$

$1706-94-002200$

$1706-94-00$

$1706-94000300$

$1706-94000400$

$$
9402833
$$

$100 \mathrm{~N}-93.032500$

WHC-RR-90-01

WHC-PR-90-03

WHC-RA-90.05

WHC-RR-90-07

WHC-RR-90-09

WHC-RP-90-10

WHC-Rr-90-10

WHC-PR-90-11

WHC-RR-90-13

WHC-RR-9O-17

WHC-RR-90-02

WHC.PR-900

WHC-RR-90-O

WHC-RR-90-OB

WHC-RA-9O-12

WhC

100N-94-004300
TOTAL CURIES

2.7750E-08 $2.7750 \mathrm{E}-08$ 2.7750E-08 2.7750 -08 $2.7750 E-08$ $2.7750 \mathrm{E}-08$ $2.7750 E-08$ $2.7750 \mathrm{E}-08$ $2.7750 \mathrm{E}-\mathrm{O} 8$ $2.7750 \mathrm{E}-08$ $2.7750 \mathrm{E}-08$ $2.7750 \mathrm{E}-08$ $2.7750 \mathrm{E}-08$ 2.7750E-08 2.7750E-08 $2.7750 E-08$ $2.7750 \mathrm{E}-08$ $2.7750 \mathrm{E}-\mathrm{OB}$ 2.8950E-08 2.8950E-08 2.8950E-OB .8950E-OB $2.8950 \mathrm{E}-08$ 2.8950E-08 $2.9040 E-O B$ 2.9100E-0B 2.916OE-OO $2.9100 E-08$ $2.9160 E-08$ $2.9270 E-0 B$ $3.9006 \mathrm{E}-08$ $3.9006 E-08$ 3.9006E-08

$3.9006 \mathrm{E}-08$

3.9006E-08

$3.9006 \mathrm{E}-08$

$3.9006 \mathrm{E}-08$

$3.9006 \mathrm{E}-08$

$3.9006 \mathrm{E}-08$

$3.9006 \mathrm{E}-08$

3.9006E-08

3.9006E-08

$3.9006 \mathrm{E}-08$

.

.

3.9006E-OB

3.9006E-08
PIN

TOTAL CUFAES GEL-94-1 183-030 GEL-94-183-036 GEL-94-183-037 GEL-94-183-052 GEL-94-183.053 GEL-94-183-026 .94-183.027 9402892

2225-92.000609 221 T-92-000303 $100 \mathrm{~N}-90.038200$ $221 \mathrm{~T}-92-000304$ 221 $-92-000302$ OS9401-18-0013 059401-18-0022 PNL-331-93-038 PNL-331.93-039

PNL-331-93-040 9408743

9408744

9408745

9408746

940874

(9) 940879 9408750

9408751

PNL.325-92.057

PNL-329-94-030

$228-92-000290$

2225-92-000293

WTF-92-083-02

KEH-92-079-05

WTFF- $93-110-18$

RS00016693

ETF-91.051-01

9201-02.0059

9201-02-0069

9201.02 .0064

WTFF-93.007.03

ETF-241.900474

ETF-24 1-900732

2225-92-000282
$4.1570 \mathrm{E}$ 4.4716E-08 4.4716E-08 4.4716E-08 $4.4716 \mathrm{E}-08$ $4.4716 \mathrm{E}-08$

$4.4716 \mathrm{E}-08$

$4.4716 \mathrm{E}-08$

$5.4551 \mathrm{E}-08$

$6.4077 \mathrm{E}-08$

6.4510E-08

.8878E-08

$7.0420 \mathrm{E}-08$

$7.5760 \mathrm{E}-08$

8.0000E-08

8.0000 - 08

$8.6950 \mathrm{OE}-08$

8.6950E-08

8.6950E.08

$9.0241 E-0 B$

.0241E-08

$.0241 E-08$

$9.0241 E-08$

$.0241 E-08$

$9.0241 E-08$

$9.0241 \mathrm{E}-08$

$9.0241 E-08$

$9.0241 \mathrm{E}-08$

$9.0241 \mathrm{E}-06$

$9.2600 E-08$

$.7254 E-08$

$1.0199 \mathrm{E}-07$

.0694E-07

$1.0919 E-07$

$1.1005 E-07$

$1.1273 \mathrm{E}-07$

$1.1434 E-07$

$1.2929 E-07$

$1.4134 E-07$

$1.4134 E-07$

$1.4134 \mathrm{E}-07$

$1.4134 E-07$

$1.4744 E .07$

$1.5000 \mathrm{E}-07$

1.5000E-07

$1.5025 \mathrm{E}-07$

$1.5200 E-07$
$1.5874 E-07$
PIN

9201-02-0066 9201.02 .0067 224U-90-00128 $2240-90-00129$ 24U-90-0013 $224 U-90-00132$ 224U-91.00012 224U-92.000068 224U-92-000116 224U-92.00012 224U-93-00000 $224 \mathrm{U}+93.00003$ $100 \mathrm{~N}-93-020000$ 9201.02-007i 9201-02-0058 9201-02-0068 9201.02-0070 $1706-94-001800$ 2225-92-000297 WTFF.93-132-01 RHZ-213-A19696

9201-02-0065

9201-02-0072

9402810

224U-93-000114 224U-94-170002 24U. 04170003 ETFF-93-1 27.05 $225-92.000365$ 2225-92-000611 9402851 9402858

100 N-93-031600 9201-02-0057

9201-02-0062

16-014

308M-94-000006

9201-02-0060

WTFF-93-116.0 9402901

TFF-94.082.05

$2225-92.000323$

2225-93-000304

KEK-92.134-01

9201-02-0061

TOTAL CUFIES

$1.5674 E .07$

$1.5674 \mathrm{E}-07$

$1.5881 \mathrm{E}-07$

$1.5881 \mathrm{E}-07$

$1.5881 E-07$

$1.5881 \mathrm{E}-07$

$1.5881 E-07$

$1.5881 \mathrm{E}-07$

$1.5881 E-07$

1.5881E-07

$1.5881 \mathrm{E}-07$

$1.5881 \mathrm{E}-07$

$1.6000 \mathrm{E}-07$

$1.721 \mathrm{SE}-07$

$1.7216 \mathrm{E}-07$

$1.7216 E-07$

$1.7367 E-07$

$1.8788 \mathrm{E}-07$

$20178 E-07$

2.0272E-07

$2.0272 \mathrm{E}-07$

2.0400E-07

2.1840 E-07

$2.1841 E-07$

$2.1841 \mathrm{E}-07$

2.2157E-O

(24)

.25

2.2800 E- 07

2.3013E-07

2.3329E-07

2.3600E-07

2.3610E-07

$2.4780 E-07$

2.4844E-07

2.5708E-0?

2.6880E-O

$2.8390 \mathrm{E}-07$

2.8536E-07 
WHC-SD-W-242-ES-004, Rev. 0

TABLE 31

UNIQUE DANGEROUS WASTE CODES IN THERMALLY TREATABLE INVENTORY (1 SHEET)

\begin{tabular}{|c|c|}
\hline DW CODES & DW CODES \\
\hline D001 & P106 \\
\hline $\mathrm{DOO} 2$ & P120 \\
\hline D003 & РСB2 \\
\hline D004 & U001 \\
\hline D005 & U002 \\
\hline D006 & U006 \\
\hline D007 & U019 \\
\hline D008 & บ025 \\
\hline Doog & U031 \\
\hline D010 & U080 \\
\hline D011 & U103 \\
\hline D012 & U108 \\
\hline D018 & U117 \\
\hline D019 & U121 \\
\hline D022 & U123 \\
\hline D023 & U144 \\
\hline D026 & U159 \\
\hline 0028 & U160 \\
\hline D029 & U161 \\
\hline D031 & U165 \\
\hline D035 & U169 \\
\hline D036 & U170 \\
\hline 0037 & U188 \\
\hline D038 & U211 \\
\hline D039 & U213 \\
\hline Do40 & U218 \\
\hline D043 & U220 \\
\hline F001 & U226 \\
\hline $\mathrm{FOO} 2$ & U239 \\
\hline F003 & W001 \\
\hline F004 & WC01 \\
\hline F005 & WCO2 \\
\hline P012 & WL01 \\
\hline P022 & WLO2 \\
\hline $\mathrm{PO} 23$ & WP01 \\
\hline $\mathrm{PO} 29$ & WPO2 \\
\hline P030 & WP03 \\
\hline P098 & WT01 \\
\hline P102 & WT02 \\
\hline
\end{tabular}


WHC-SD-W-242-ES-004, Rev. 0

TABLE 32

CONTAINERS OF ASBESTOS WASTE

(1 SHEET)

PIN

COMPONENT

COMPONENT WEIGHT,KG

202L-92-000702

ASBESTOS

5.4000E-01

271B-91-0026

ASBESTOS

2.5000E-01

ASBESTOS

3.0000E-01

2.0000E-01

325-89034

$325-89038$

9406778

EKEH-92-135-01

ASBESTOS

5.0000E-0?

ASBESTOS

$1.0000 \mathrm{E}-01$

ASBESTOS

$5.3100 E+00$

EKEH-92-337-01

ASBESTOS

$4.0330 \mathrm{E}+01$

ETFF-91-315-06

ETFF-92-337-02

ETFF-93-070-01

RHZ-213-A22872

WTFF-93-147-03

ASBESTOS

$1.6700 \mathrm{E}+00$

$1.4123 E+02$

ASBESTOS

$2.0000 E+01$

ASBESTOS

$3.4000 E+0 t$

ASBESTOS

8.7200E + OO

WTFF-94-005-01

ASBESTOS

$2.0000 E+00$ 
TABLE 33

HIGH MERCURY PINS - CONTAINERS HAVING GREATER THAN 260 PPM MERCURY (1 SHEET)

\section{PIN NUMBER}

PNL-325-93-054 PNL-325-93-053 PNL-325-92-081 PNL-325-92-070 PNL-325-92-071 PNL-325-92-072 PNL-325-92-080 2225-92-000205 271U-91-90601 ETF-241-901020 202L-94-000701 940200012

221-T-91.00065 WTF-BW-94-0001 RHZ-220-A19400 RHZ-212-A 18996 105N-90-000012

PNL-89017

PNL-89017

WHC-A-90-804

222S-93-000387

ANL93-MW-00048

ANL93-MW-00047

271U-91-90602 16-014

WHC-A-90-804 ANL93-MW-00016 ANL93-MW-00010 ANL93-NW.00013 ANL93-MW-00006 ANL93-MW-00014 ANL93-MW-00012 ANL93-MW-00015 ANL93-MW-00005 ANL93-MW-00007 ANL 93-MW-00009 ANL93-MW-00002 ANL93-MW-00003 ANL93-MW-00008 ANL93-MW-00004 ANL93-MW-00017 ANL93-MW-00001 ANL93-MW-00011 ANL93-MW-00018 ANL93-MW-00019 ANL93-MW-00020 RHZ-213-A22805 221T-92-000100
COMPONENT NAME

MERCURY
MERCURY
MERCURY
MERCURY
MERCURY
MERCURY
MERCURY
MERCURY
MERCURY
MERCURY
MERCURY
MERCURY
ALLIC MERCURY
MERCURY
MERCOAT 234
MERCOAT 234
MERCURY

ETHANOL DIBENZYL MERCURY

ETHYL MERCURIC CHLORIDE

MERCURIC HYDROXIDE

MERCURY

MERCURY

MERCURY

MERCURY

MERCURY

MERCURY

MERCURY

MERCURY

MERCUAY

MERCURY

MERCURY

MERCURY

MERCURY

MERCURY

MERCURY

MERCURY

MERCURY

MERCURY

MERCURY

MERCURY

MERCURY

MERCURY

MERCURY

MERCURY

MERCUAY

MERCURY

MERCURY MERCURY
COMPONENT WEIGHT, KG

1. $1950 \mathrm{E}+02$

$1.0960 E+02$

$1.0510 E+02$

$1.0510 E+02$

$1.0510 \mathrm{E}+02$

1. $0510 E+02$

1. 051 OE + 02

7. $1000 \mathrm{E}+00$

1. $5300 \mathrm{E}+01$

$1.0002 E+00$

1. $9500 \mathrm{E}+00$

3. $3400 \mathrm{E}+00$

$3.9000 E+00$

2.3100E + 00

$1.0002 \mathrm{E}+00$

$1.0002 \mathrm{E}+00$

$1.8600 \mathrm{E}-01$

2.0000E-01

2.0000E-01

2.0000E-01

2.2500E-01

5.3000E-01

4. O000E-01

$1.0000 \mathrm{E}-01$

$1.0000 E-01$

3.0000E-02

2.1000E-01

2.1000E-01

2. 2000E-01

2.0000E-01

2.0000E-01

$1.9000 \mathrm{E}-01$

2.0000E-01

2.0000E-01

$1.8000 \mathrm{E}-01$

1. 8000E-01

1. $7000 \mathrm{E}-01$

$1.7000 \mathrm{E}-01$

$1.8000 \mathrm{E}-01$

1. 7000E-01

1. 5000E-01

$1.6000 \mathrm{E}-01$

2.0000E-01

$1.8000 \mathrm{E}-01$

2.0000E-01

1. $6000 \mathrm{E}-01$

$5.0000 \mathrm{E}-02$

4.0000E-02
TOTAL WEIGHT, KG

PPM MERCUAY

1.955OE + 02

$1.7950 E+02$

$2.0270 E+02$

$2.0450 E+02$

$2.0450 E+02$

$2.0450 E+02$

2. $0450 E+02$

$6.5600 E+01$

$1.6800 E+02$

1.1400E + O1

4. 727OE + 01

9.6100E + 01

$1.651 \mathrm{OE}+\mathrm{O} 2$

1.3600E + 02

$5.6970 E+01$

$6.4000 E+O$

1.4000E + 01

2.9500E + 01

$2.9500 E+01$

$3.2720 \mathrm{E}+01$

5.8160E + 01

$2.5270 E+O 2$

$2.0000 E+02$

8. 1700 E + 01

9.5340E + 01

3. $2720 E+01$

2.3360E + 02

$2.3550 E+02$

$2.4770 E+02$

2. $2640 \mathrm{E}+\mathrm{O} 2$

2.264OE + O2

$2.1550 E+02$

2. $2730 E+02$

2. $2950 E+02$

$2.0680 E+02$

$2.0680 E+02$

$1.9640 \mathrm{E}+02$

$2.0000 E+02$

$2.1360 E+02$

$2.0270 E+02$

$1.8000 E+02$

$1.9550 E+02$

2.555OE + 02

$2.5000 E+02$

2. $8090 \mathrm{E}+02$

$2.2550 E+02$

1. 11 OOE + 02

1.273OE + O2
$6.1125 E+05$

6.1058E + 05

5.1850E + 05

$5.1394 E+O 5$

$5.1394 \mathrm{E}+$ OD

$5.1394 \mathrm{E}+05$

$5.1394 \mathrm{E}+05$

1.0823E + O5

$9.1071 \mathrm{E}+04$

8. $7737 \mathrm{E}+04$

4.1252E + 04

$3.4755 E+04$

2. $3622 E+04$

1. $6985 E+04$

1. $6961 E+04$

t. $562 \mathrm{BE}+04$

1. $3286 E+04$

$6.7797 \mathrm{E}+03$

$6.7797 E+03$

$6.1125 E+03$

3. $8673 E+03$

$2.0973 E+03$

2. $0000 E+03$

1. $2240 E+03$

1. $04 \mathrm{BgE}+03$

$9.1687 \varepsilon+02$

B. $9897 E+02$

8. $9172 E+02$

$8.6817 E+02$

$8.8339 E+02$

$6.8339 E+02$

8.8167E + 02

8. $7989 E+02$

8. $7146 E+02$

B. $7041 E+02$

$8.7041 E+02$

$8.655 \mathrm{BE}+02$

$8.5000 E+02$

$8.4270 E+02$

8. $3868 E+02$

8. $3333 E+02$

$8.1841 E+02$

$7.8278 E+02$

7. $2000 E+02$

$7.1200 \mathrm{E}+02$

$7.0953 \mathrm{E}+02$

$4.5045 \mathrm{E}+02$

3.1422E + 02 
WHC-SD-W-242-ES-004, Rev. 0

TABLE 34

PINS REPORTED TO CONTAIN BERYLLIUM DUST

(1 SHEET)

PIN NUMBER

GEL-94-183-026

GEL-94-183-027

GEL-94-183-030

GEL-94-183-031

GEL-94-183-036

GEL-94-183-037

GEL-94-183-052

GEL-94-183-053
COMPONENT NAME

BERYLLIUM DUST BERYLLIUM DUST BERYLLIUM DUST BERYLLIUM DUST BERYLLIUM DUST BERYLLIUM DUST BERYLLIUM DUST BERYLLIUM DUST
COMPONENT WEIGHT, KG

$0.0000 E+00$

$0.0000 E+00$

$0.0000 E+00$

$0.0000 E+00$

$0.0000 E+00$

$0.0000 E+00$

$0.0000 E+00$

$0.0000 E+00$ 
WHC-SD-W-242-ES-004, Rev. 0

TABLE 35

PINS CONTAINING LEAD

SORTED IN ASCENDING ORDER BASED ON PPM LEAD

PIN

WHC-A-90-828 WHC-A-90-826 2240-91.004 BPLANT-89-487

WHC-A-90-827

WHC-A-90-825

WHC-A-90-811

WHC.A.90-813

WHC-A-90-818

WHC-A-90-817

WHC-A-90-815

WHC-A-90-819

WHC-A-90-814

WHC-A.90-820

WHC-A-90-855

WHC-A.90-812

WHC-A-90-808

9417.02.0016

WHC-A-90-82

ETFF.93-315.02

ETFF-93-315-04

(2305-03-0030

9305-03.0030

WTFF-93-135-01

2718-89-0734

2716-89-0734

RHIZ-219-930462

WTFF-93-152-01

$\mathrm{RHZ}-87.037$

305-03-0029

$\mathrm{RHZ}-87-037$

ETFF.94-082.06

WTFF-VT-001-07

WHC-A-90-853

HC-A.89-84

9406824

WH-333-94-0022

$\mathrm{RHZ}-213-\mathrm{A} 21803$

RHZ-213-A21306

WHC-A-90-837
RHZ-212-A21603

(13 SHEET)

COMPONENT NAME

\section{LEAD BRICK}

$$
\text { LEAD }
$$

LEAD CHROMATE PAINT

LEAD BRICK

LEAD BRICK

LEAD

LEAD

EAD

LEAD

LEAD

EAD

LEAD

EAD

IEAD

IEAD

EAD

IEAD

LEAD

LEAD

EAD

LEAD

EAD

EAD

LEAD

EAD

LEAD

PAINT CONTAINING LEAD CHROMATE

LEAD

EAD

EAD

EAD

LEAD

EAD

LEAD

LEAD

LEAD

EAD

LEAD

LEAD

LEAD SHIEDING

LEAD

EAD

EAD

LEAD

\section{COMPO}

$6.0690 E+01$
$8.2508 E+01$
$8.3007 E+01$
$8.3506 E+01$
$3.3861 E+01$
$3.8859 E+01$
$4.3409 E+01$
$3.4477 E+02$
$3.3157 E+02$
$3.1797 E+02$
$3.2432 E+02$
$3.1751 E+02$
$3.1751 E+02$
$3.1751 E+02$
$3.1887 E+02$
$3.1207 E+02$
$6.0191 E+01$
$2.5387 E+02$
$3.5460 E+02$
$1.6842 E+02$
$3.1380 E+02$
$1.5023 E+02$
$2.5385 E+02$
$2.0603 E+02$
$2.0003 E+02$
$3.3160 E+02$
$1.7503 E+02$
$2.4312 E+02$
$3.6482 E+01$
$3.6480 E+01$
$1.0400 E+02$
$1.6080 E+02$
$9.0000 E+01$
$2.6984 E+02$
$2.0702 E+02$
$1.2840 E+02$
$2.0700 E+02$
$1.0500 E+02$
$3.2880 E+01$
$1.1290 E+02$
$1.0718 E+02$
$4.5400 E+01$
$6.0000 E+01$
$3.2309 E+01$
$3.4301 E+01$
$5.6699 E+01$
$2.5501 E+01$

WASTE WEIGHT, KG

PPM UEAD

$1.0002 E+06$ $1.0001 E+06$

$1.0001 E+\infty$

$1.0001 E+06$

$1.000 E+\infty 0$

$9.9998 E+05$

$9.9997+05$

$9.9338 E+05$

$9.9312 E+05$

$9.9312 E+05$

$9.9294 E+05$

$9.9294 E+05$

$9.9294 E+05$

$9.9285 E+05$

$9.9268 \mathrm{E}+05$

$9.9162 E+05$

$9.9104 \mathrm{~F}+05$

$9.8747 E+05$

$9.8747+05$

$9.868 E+05$

$.7365+05$

9.7368 + 05

$9.6521 E+05$

$9.5828 E+05$

$.5715+05$

$9.5315 E+05$

$9.5125 E+0.5$

$9.4970 E+05$

$9.4416 \mathrm{E}+05$

9.4410E+05

$9.1228 \mathrm{E}+05$

$8.9933 \mathrm{E}+05$

B.8496E + 05

$8.7896 E+05$

$8.5036 E+05$

$8.5033 E+0.5$

$8.5031 \mathrm{E}+05$

8. $1395 \mathrm{E}+05$

$7.4727 \mathrm{E}+05$

$6.6139 \mathrm{E}+05$

$5.9993 E+05$

$5.8205 E+05$

$5.8205 \mathrm{E}+05$

$5.7692 E+05$

$6.3849 \varepsilon+05$

$4.6981 E+05$

$4.0557 E+05$ 
WHC-SD-W-242-ES-004, Rev. 0

TABLE 35

PINS CONTAINING LEAD

SORTED IN ASCENDING ORDER BASED ON PPM LEAD

(13 SHEET)

PIN

BPLANT-89-486

BPLANT-89-490

BPLANT-89-489

AHZ-213-A22805

9417-02-0003

ETFF-94-082-05

RHZ-86-002

RHZ-86-002

WTFF-93-063-01

WHC-A-90-853

RHZ-21 2-A21763

PHZ-212-A22878

RHZZ-213-A23126

A

RH:Z-87.04

WHC.A.90-839

PNIL-329-92-007

BPLANT-89-61

2A-92-00237

313-93-00009

BPLANT-89.610

2716-90-0430

BPLANT-89-612

$2718-90-0105$

2718-90-0106

BPLANT-89.652

271 18-90.0432

BPLANT-89-65

2718-90-0098

BPLANT-89-653

2718-90-0099

BPLANT-89-614

2718-90-0019

RHZ-212-A21462

WTF-BW-94-0001

HRO-92.000521

HC.A-90.83

RHZ-220-A20194

AT2-211-0a9045

TTFF-93-090-4

PHZ-219-930376

WTFF-VT-001.0

221 T-92-00045
COMPONENT NAME

IEAD CHROMATE PAINT EAD CHROMATE PAINT EAD CHROMATE PAINT

LEAD

EAD

EAD

EAD

EAD

LEAD

LEAD

LEAD BRICK

LEAD

LEAD

EAD

LAD

LEAD

IEAD

LEAD SHIELDING

LEAD CHROMATE

LEAD

LEAO CHROMATE

LEAD CHROMATE PAINT

LEAO CHROMATE

LEAD CHROMATE PAINT

IEAD CHADMATE

LEAD CHAOMATE

EAD CHFIOMATE PAINT

LEAD CHROMATE

EAD CHROMATE PAINT

EAD CHFOMATE PAINT

LEAD CHROMATE

LEAD

BATTERIES CONTAINING LEAD, LFAD OXIDES, AND SULFURIC ACID

IEAD

IEAD

LEAD CMROMATE

LEAD CHROMATE

LEAD

EAD SHIELDING

LEAD

BASE METAL (LEAD)

LAD
COMPONENT WEIGHT, KG

$1.7100 E+01$

1.2301E+01

$8.6999 E+\infty 0$

$1.1600 E+01$

$1.0000 E+0$

$3.2000 E+01$

$7.2400 E+02$

$7.2393 E+02$

$5.4000 E+00$

$5.6790 E+01$

$1.4701 E+01$

$2.9000 E+01$

2.2000E + 01

$1.5681 E+01$

$1.1380 E+03$

$1.1378 E+03$

$3.6700 E+01$

4.5400E + 01

3.4700E + 01

$3.5800 E+01$

$5.9000 E+\infty 0$

$8.5003 E+00$

2.8595E+O

4.8716E + O1

$4.4602 E+01$

$2.9302 \mathrm{E}+01$

$2.8200 E+01$

$3.8201 E+0$

$3.3198 E+0$

$3.0100 E+01$

$4.7718 E+0$

$3.0899 E+01$

$5.1392 E+01$

$1.0201 E+01$

9.5481E +00

$2.0130 E+01$

$1.0000 E+01$

$1.6098 E+01$

$9.0000 E+00$

$3.0000 E+\infty 0$

$1.9999 E+\infty$

$2.3455 \mathrm{E}+02$

$2.5000 E+02$

$3.0000 E+\infty 0$

$1.0000 E+01$

B. $6400 E+\infty O$
WASTE WEIGHT, KG

PPM LEAD

$3.9954 E+05$

$3.9940 E+05$

$3.9908 \mathrm{E}+05$

$3.7438 E+05$

3.7061E + 05

3.5714E+05

3.5165E + 05

$3.4553 \mathrm{E}+05$

$3.4550 E+05$

$3.3748 \mathrm{E}+$ O5

$3.3269 \mathrm{E}+05$

$3.1279 E+05$

$3.1183 E+05$

$3.0986 \mathrm{E}+0.5$

$2.9033 E+05$

$2.8425 E+05$

2.8415E+05

$2.7866 E+05$

$2.4488 E+05$

$2.4215 E+05$

$2.3599 E+05$

$2.3599 E+\infty 5$

$2.3137 \mathrm{E}+05$

$2.0095 \mathrm{E}+05$

$2.0027 \mathrm{E}+05$

$2.0023 E+05$

$2.0019 \mathrm{E}+\mathrm{CS}$

$2.0015 E+05$

$2.0014 E+05$

$2.0011 E+05$

$2.0011 E+05$

$2.0000 E+05$

$1.9999 E+05$

$1.9986 \mathrm{E}+05$

$1.9981 E+05$

$1.9732 E+05$

$1.9486 E+05$

$1.9171 E+05$

$1.8519 \mathrm{~F}+05$

$1.4661 E+\infty 5$

$1.4286 E+C 5$

$1.4286 E+05$

$1.4285 E+05$

$1.4285 E+05$

$1.3177 E+05$

$1.2639 E+05$

$1.2500 E+05$

$1.0309 E+05$
$1.0026 E+05$ 
WHC-SD-W-242-ES-004, Rev. 0

TABLE 35

PINS CONTAINING LEAD

SORTED IN ASCENDING ORDER BASED ON PPM LEAD

(13 SHEET)

COMPONENT NAME

$$
\begin{aligned}
& \text { LEAD } \\
& \text { LEAD } \\
& \text { LEAD SHIELDING } \\
& \text { LEAD SHIELDING } \\
& \text { IEAD } \\
& \text { LEAD CHROMATE } \\
& \text { LFAD CHROMATE } \\
& \text { LEAD } \\
& \text { LFAD CHROMATE } \\
& \text { LEAD }
\end{aligned}
$$

LEAD CHROMATE

LEAD

LEAD

LEAD

LEAD

LEAO

LEAO

IEAD

LEAD

LEAD

LEAD

EAD

EAD

EAD

EAD

EAD

LEAD

LEAD CHROMATE PAINT

LEAD CHAOMAT

9417.02 .0019

FHZ-220-090004

FHZZ-220-090024

PHZ-211-09003?

ZZ-219-A2158

PATZ-86-010

2225-92-000155

2225-92.000155

WhZ-211-A20985

PHZ-219-920779

DTFF $180-02$

ETF-94-180-02

(12.212.420239

ETFF-92-356-02

ETFF-93-084-06

WTFF-93-356-01

PHLZ-219-090067

105N-90-00031

EKEH-92-147-01

\section{COMPON}

WASTE WEIGHT, KG

$4.6000 E+01$

$3.4000 E+0$

$2.8580 E+03$

$1.9510 \mathrm{E}+03$

$1.2250 \mathrm{CE}+03$

$1.2000 E+01$

$9.0000 E+00$

$1.0271 \mathrm{E}+02$

$5.5100 E+01$

$2.7330 E+01$

$1.2000 E+01$

8.4500E + 01

$1.0940 E+02$

$1.5260 E+02$

$1.5260 \mathrm{E}+02$

$1.5260 \mathrm{E}+02$
$2.1610 \mathrm{OE}+02$

2.1610E+02

$1.7300 E+02$

$1.3670 E+02$

$1.3670 E+02$

$1.5030 \mathrm{E}+02$

..5030e+02

. 1800 E + 1

1.2530E +02

$2.1390 E+02$

$1.5710 E+02$
$1.5710 E+02$

1.5710E +02

$1.7070 E+02$
$1.0720 E+02$

$1.0720 E+02$

1.1400E +02

$2.6000 E+O$

$3.5000 E+01$

$3.8000 E+01$

$2.0603 E+03$

2.0603E +03

$7,5900 E+01$

$3.2000 E+01$

$6.0000 E+01$

$2.9000 E+01$

$4.9600 E+02$

$1.7000 E+01$

$2.4500 E+03$

$2.9000 E+01$

$2.5000 E+01$
$5.3000 E+01$

$1.1300 E+O$

$5.5100 E+O$
PPM LEAD

$1.0000 E+05$ $1.0000 E+05$ $9.4997 \mathrm{E}+04$ $9.4054 \mathrm{E}+04$ $9.2424 E+04$ $8.3350 E+04$ $8.3311 \mathrm{E}+04$

8. $1784 \mathrm{E}+04$

$7.7314 E+O 4$

$7.7205 E+04$

$6.2483 E+04$

$6.0355 \mathrm{E}+04$

$6.0329 E+04$

$6.0288 \mathrm{E}+04$

$6.0288 \mathrm{E}+04$

$6.0157 \mathrm{E}+04$

$6.0116 \mathrm{E}+04$

$5.985+04$

$5.9985+04$

$5.9893 E+04$

$5.9805+0$

$5.971 E+04$

$5.085+04$

.

$5.9835 E+04$

$5.9835 E+04$

$5.9754 E+04$

$5.9701 \mathrm{E}+04$

$5.9649 E+04$

$5.8824 \mathrm{E}+04$

$5.7692 \mathrm{E}+04$

$5.7140 E+04$

$5.2629 \mathrm{E}+04$

$5.0197 E+04$

$5.0188 E+04$

$5.0066 E+04$

$4.6875 E+04$

$4.5170 E+04$

$4.4828 \mathrm{E}+04$

$4.4828 E+04$

4.4194E+ 04

$4,4106 E+04$

$4.3136 E+04$

$4.2414 E+04$

$4.0000 E+04$

$3.7734 E+04$

$3.6504 E+04$

$3.6298 \mathrm{E}+04$ 
WHC-SD-W-242-ES-004, Rev. 0

TABLE 35

PINS CONTAINING LEAD

SORTED IN ASCENDING ORDER BASED ON PPM LEAD

(13 SHEET)

PIN

271B-92-000381 RHZZ-211-A20983 $105 \mathrm{~N}-90-000314$ $105 \mathrm{~N}-90-000310$ 9305-03-0028 AHZ-211-090038 $105 N-90-000313$ 222S-92-000358 ETFF-93-055-01 ETFF-93-055-02 ETFF-93-055-03 ETFF-92-356-03 ETFF-93.055-04 PHZ-220-A20195 RHZ-219-091384 $105 \mathrm{~N}-90-000312$ ETFF-93-133-03 RHZ-211.090039 $105 \mathrm{~N}-90-000308$ RHZ-211.090056 RHZ-213-A22872 PtiZ-219-091250 AH2-211.09005 RHZ-219-091319 $105 \mathrm{~N}-90.000304$ RHZ-219-091385 $100 \mathrm{~N}-94.018600$ RHZ-220-A20120 $105 N-90-000306$ 202A-94-000324 105N-90-000309 RHZ-219-09138 202L-92-000704 ANL93-MW-00018 105N-90-000307 WTFF-93.089-02 WTFF-93-089-02 WTF-93-089-02 (21T) 2217.92.00015 221T-92-000054 2217.92-000150 224U-94-000010 $224 \mathrm{U}-94-000010$
$271 \mathrm{~B}-90-0017$ 100 N-94-004300 271B-93-000128
WTFF-93-109-10
COMPONENT NAME

LEAD TETROXIDE

LEAD CHROMATE PAINT

LEAD CHROMATE

LEAD

LEAD CHROMATE,CHLOFIN.PARAFFIN

LEAD CHROMATE

LEAD

BASE METAL (LEAD)

BASE METAL (LEAD)

BASE METAL (LEAD)

BASE METAL (LEAD)

BASE METAL (LEAD)

IEAD CHROMATE

LEAD CHROMATE

EAD CHROMATE

$$
\text { LEAD }
$$

LEAD CHROMATE, CHLOFIN.PARAFFIN

IFAD CHROMATE

IEAD CHROMATE PAINT

IEAD CHROMATE

LEAD CHROMATE

LEAD CHROMATE PAINT

LAD OXIDE

LEAD CHFOMATE

LEAD CHROMATE

LEAD CHROMATE

LEAD CHROMAIE

LEAD CHROMATE

LEAD CMROMATE

LEAD CHROMATE

LEAD CHROMATE

LEAD DIOXIDE

LEAD

IEAD CHROMATE

EAD CHFOMATE MOLYBDATE

LEAD CHROMATE

IFAD

LEAD

LEAD CHROMATE MOLYBDATE

NAPFIHENIC ACID LAD SALT

AINT

LEAD CHROMATE

LEAD CHROMATE

LEAD CHROMATE
COMPONENT WEIGHT, KG

WASTE WEIGHT, KG

PPM LEAD

4.2000E+01

$4.5000 E+01$

$9.1000 \mathrm{E}+01$

3.0800E+01

$3.2000 E+01$
$1.1300 E+02$

4.9700E +01

$1.9510 E+03$

2.767OE + 03

$2.8580 E+03$

4.1510E +03

$1.2250 E+03$

$2.6000 E+01$

$5.4000 E+01$

$1.3600 E+02$

$1.8000 E+01$

$1.8000 E+01$
$4.0000 E+01$

4.000OE +01

1.1500E + O2

4.3000E+01

$5.0000 E+01$

$4.6000 E+01$

$4.8000 E+01$

4.9962E + 00

$1.1300 E+02$

$4.9000 E+01$

2.990OE +01

4.400OE + O1

$1.0400 E+02$

3.0300E + 01

$1.2000 E+02$

$3.5000 E+01$

$2.9910 E+01$

2. $2230 E+02$

$1.2900 E+02$

7.0000E + 01

$7.0000 \mathrm{E}+01$

7.0000E +01

$1.1100 E+02$
$3.9100 E+01$

3.54OOE +

$4.5400 E+01$

$4.5400 E+01$

$2.9000 E+01$

$1.7600 E+0$

$3.4100 E+O$

$5.2100 E+01$

$3.4500 \mathrm{E}+\mathrm{O}$

$3.8000 \mathrm{E}+\mathrm{O}$
$3.5813 E+04$

$3.5714 E+04$
$3.4998 E+04$

$3.2967 \mathrm{E}+04$

$3.2967 \mathrm{E}+04$

$3.2468 E+04$

$3.1256 E+04$

$3.0531 E+04$

$3.0181 E+04$

$3.0138 E+04$

$2.9996 \mathrm{E}+04$

$2.9874 E+04$

$2.9872 \mathrm{E}+04$

$2.9388 E+04$

$2.8838 E+04$

$2.7778 E+04$

$2.7572 E+04$

$2.5333 E+04$

$2.5005 E+04$

$2.3914 \mathrm{E}+04$

$2.3260 E+04$

2.3200 + 04

$2.1743 E+04$

$2.1743 E+04$

$2.0838 E+04$

$1.9014 E+04$

$2.4337 E+04$

$1.8365 \mathrm{E}+04$

1. $7057 \mathrm{E}+04$

$1.7041 \mathrm{E}+04$

$1.6587 \mathrm{E}+04$

$1.6502 \mathrm{E}+04$

$1.6250 E+04$

$1.5429 \mathrm{E}+04$

$1.5045 \mathrm{E}+04$

$1.4845 E+04$

1. $3952 \mathrm{E}+04$

1. $3600 E+04$

$1.3600 \mathrm{~F}+04$

$1.2840 E+04$

$1.2020 E+04$

$1.03525+04$

$1.0362 \mathrm{E}+04$

$1.0352 \mathrm{E}+04$

$1.0345 E+04$

$1.0227 E+04$

$9.9707 E+03$

8.9251E+03

$8.6957 \mathrm{E}+03$

$8.5526 \mathrm{E}+03$ 
WHC-SD-W-242-ES-004, Rev. 0

TABLE 35

PINS CONTAINING LEAD

SORTED IN ASCENDING ORDER BASED ON PPM LEAD

PIN

COMPONENT NAME

(13 SHEET)

\author{
COMPONENT WEIGHT, KG
}

WASTE WEIGHT, KG

PPM LEAD

LEAD CHROMATE MOLYBDATE

$$
\text { LEAD }
$$

WTFF-93-109-10
ETFF-93-067-01

ETFF-93-067-01
ETFF-93-067-01

$105 \mathrm{~N}-89-000523$

$221 \mathrm{~T}-92.000064$

RHZ-213-A21544

2225-93.000011

$2225-93.000011$
$221 T \cdot 92.000340$

EKEH-92-176-02

940200012

RHZ-213-A21546

EKEH-92-161-02

WKEH-92-198-03

$100 \mathrm{~N}-94-004300$

$9402000+2$

2718-93-000128

2225-93-001021

$2024-93-000328$

$221 \mathrm{~T}-92.000074$

RHZ-219-A21573

RTZ-219-A21573

WTF- $3-098-0$

WN193-00-017

ANL93-NW-0004

WTFF-VT-001-98

ANL93-MW-00015

ANL93-MW.00010

ANL93-MW-00013

ANL93.MW.00005

ANE93-MW-00011

ANL 93-MN-00048

FHZ-212-A21763

ANL93-MW-00016

ANL93-MW-00008

ANL93-MW-00006

ANE93-MW-00014

ANL93-MW-00004

ANL93-MW-00007

ANL93-MW.00009

ANL93-MW.00012

ANL93-MW.00001

ANL93-MW-00003

ANL93-MW-00002

ANL93-MW-00017

PHZ-21 2-A1995
LEAD CHROMATE

LEAD

LEAD CHROMATE

EAD

LEAD TELLURIDE

LEAD

LEAD CHROMATE

LEAD

LEAD CHROMATE
LEAD CHROMATE

LEAD CHROMATE

LEAD CHROMATE MOLYBDATE

EAD

LEAD CHROMATE MOLYBDATE

LEAD

LEAD

LEAD

LEAD CHROMATE

LEAD CHROMATE

LEAO

LEAD

LEAO

LEAD

LEAD

LEAD
LFAD

LEAD

LEAD

LEAD
LEAD

LEAD

LEAD

UEAD

LEAD

LEAD

LEAD

LFAD

LEAD

LEAD

LEAD CHROMATE
LEAD CHROMATE
$3.2500 E-01$
$3.6000 E-01$
$3.6000 E-01$
$4.5360 E-01$
$6.0000 E-01$
$1.8000 E-01$
$6.8100 E-01$
$2.2000 E-01$
$1.0000 E-01$
$3.1000 E-01$
$4.0000 E-01$
$1.8000 E-01$
$1.7000 E-01$
$9.9300 E+00$
$2.8000 E-01$
$3.5000 E-01$
$1.8000 E-01$
$7.7800 E+00$
$3.3400 E+00$
$2.9910 E-01$
$1.2000 E-01$
$1.4000 E-01$
$1.4000 E-01$
$1.2200 E-01$
$4.7000 E-01$
$2.0400 E+00$
$8.7000 E-02$
$5.0000 E-01$
$5.2000 E-01$
$5.5000 E-01$
$5.0000 E-01$
$4.9000 E-01$
$6.3000 E-01$
$1.3000 E-01$
$5.1000 E-01$
$4.6000 E-01$
$4.9000 E-01$
$4.9000 E-11$
$4.3000 E-01$
$4.4000 E-01$
$4.4000 E-01$
$4.6000 E-01$
$4.1000 E-01$
$4.2000 E-01$
$4.1000 E-01$
$3.7000 E-01$
$7.0000 E-02$
$3.8000 E+01$

$4.6000 \mathrm{E}+01$

4.6000E + 01

$6.5000 E+O$

8.6200E +01

$2.7000 E+01$
$1.0340 E+02$

$3.3630 E+01$

$1.5400 \mathrm{E}+01$

$4.8000 E+01$

$6.5700 E+01$

$3.0000 E+01$

$3.0000 E+O 1$

$1.7600 E+03$

$5.2100 E+01$

$6.5700 E+01$

$3.4500 E+01$

$1.5582 \mathrm{E}+03$

$6.6960 \mathrm{E}+02$

$7.2000 \mathrm{E}+01$

$7.2000 E+O 1$

$3.6900 \mathrm{E}+01$
$4.4000 \mathrm{E}+01$

$4.6000 \mathrm{E}+01$

$4.6000 \mathrm{E}+01$

1.2000E + 01

$1.7230 E+02$

$8.0100 E+02$

$3.4500 E+01$

$1.9960 E+02$

$2.0780 E+02$

$2.2000 E+02$

$2.0180 E+02$

$1.9780 E+0$

2.250OE + 02

4. $7000 E+01$

$2.0590 \mathrm{E}+02$

$1.8590 \mathrm{~F}+02$

$1.9870 E+02$

$1.9670 E+02$

$1.7500 E+02$

$1.7910 E+02$

$1.7910 E+02$

$1.8780 \mathrm{E}+02$

$1.6780 E+O 2$

$1.7230 \mathrm{E}+02$

$1.6370 \mathrm{C}+02$

$1.687 \mathrm{OE}+02$

$1.5230 E+02$
$3.1800 E+01$
$6.5526 E+03$

$7.8261 E+03$

$7.8261 E+03$

$6.9785 \mathrm{E}+03$

$6.9606 \mathrm{E}+03$

$6.6667 \mathrm{E}+03$

$6.5861 \mathrm{E}+03$

$6.5418 E+03$

$6.4936 E+03$

$6.4583 E+03$

$6.0883 E+03$

$6.0000 E+03$

$5.6667 \mathrm{E}+03$

$5.6420 E+03$

$5.3743 \mathrm{E}+03$

$5.3272 E+03$

$5.2174 \mathrm{E}+03$

$4.9929 E+03$

$4.9881 E+03$

$4.1542 E+03$

$3.2520 E+03$

$3.1818 E+03$

$3.0435 E+03$

$29048 \mathrm{E}+03$

$2.7278 \mathrm{E}+03$

$2.5468 \mathrm{E}+03$

$2.5217 \mathrm{E}+03$

$2.5050 E+03$

$2.5024 \mathrm{E}+03$

$2.5000 E+03$

$2.4777 \mathrm{E}+03$

$2.4772 E+03$

$2.8000 E+03$

$2.7660 E+03$

$2.4769 \mathrm{E}+03$

$2.4744 E+03$

$2.4660 E+03$

$2.4660 E+03$

$2.4571 E+03$

$2.4567 \mathrm{E}+03$

$2.4567 E+03$

$2.4494 E+03$

$2.4494 E+03$

$2.4434 E+03$

$2.4376 E+03$

$2.4303 E+03$

$2.4294 E+03$
$2.2013 E+03$ 
WHC-SD-W-242-ES-004, Rev. 0

TABLE 35

PINS CONTAINING LEAD

SORTED IN ASCENDING ORDER BASED ON PPM LEAD

(13 SHEET)

COMPONENT NAME

LEAD
LEAD
LEAD CHROMATE
LEAD
LFAD
LEAD
LEAD
LEAD CHROMATE
IEAD SALTS
LEAD CHROMATE
LEAD
LEAD
LEAD
LEAD
LEAD NAPHTHENATE
LEAD
LEAD
LEAD
LEAD CHROMATE
LEAD
LEAD CHROMATE
LEAD
LEAD
LEAD
LEAD
LEAD
LEAD
LEAD
LEAD
LEAD
LEAD
LEAD
LEAD
LEAD
LEAD
LEAD
LEAD
LEAD
LEAD
LEAD
LEAD
LEAD
LEAD
LEAD
LEAD
LEAD ACIO
LEAD

COMPONENT WEIGHT, KG

\begin{tabular}{|c|}
\hline \\
\hline $\begin{array}{l}3.3000 \mathrm{E}-02 \\
6.400 \mathrm{E}-02\end{array}$ \\
\hline $2.5000 \mathrm{~F}+00$ \\
\hline 4.5600E-02 \\
\hline $3.8000 \mathrm{E}-01$ \\
\hline $2.9000 E-01$ \\
\hline $6.4000 \mathrm{E}-02$ \\
\hline 4.0000E-02 \\
\hline $7.0000 \mathrm{E}-02$ \\
\hline $1.3000 \mathrm{E}-01$ \\
\hline $1.2740 \mathrm{E}-01$ \\
\hline $2.8000 E+00$ \\
\hline $3.5000 \mathrm{E}-02$ \\
\hline 3.0000E-02 \\
\hline $6.0000 \mathrm{E}-02$ \\
\hline $6.0000 \mathrm{E}-01$ \\
\hline $5.0000 E-02$ \\
\hline $4.0000 E-02$ \\
\hline $1.0000 E+00$ \\
\hline 5.0000 E. 02 \\
\hline $7.2100 \mathrm{E}-02$ \\
\hline $7.2100 \mathrm{E}-02$ \\
\hline $7.2100 \mathrm{E}-02$ \\
\hline $7.2100 \mathrm{E}-02$ \\
\hline $7.2100 \mathrm{E}-02$ \\
\hline $7.2100 E-02$ \\
\hline $7.2100 \mathrm{E}-02$ \\
\hline $7.2100 E-02$ \\
\hline $7.2100 E-02$ \\
\hline 3.0000 E- 02 \\
\hline $2.0000 \mathrm{E}-02$ \\
\hline $3.0000 E-02$ \\
\hline $2.0000 \mathrm{E}-02$ \\
\hline $1.7300 \mathrm{E}-01$ \\
\hline $1.2000 \mathrm{E}-01$ \\
\hline $1.7000 E-01$ \\
\hline 1.1840E-01 \\
\hline $2.1000 \mathrm{E}-02$ \\
\hline 4.0000 E-02 \\
\hline $7.2100 E-02$ \\
\hline $7.2100 E-02$ \\
\hline 3.0000E-02 \\
\hline $1.7000 E-01$ \\
\hline 1.6630 E-01 \\
\hline $2.0000 \mathrm{E}-02$ \\
\hline $1.0000 \mathrm{E}-\mathrm{D}$ \\
\hline
\end{tabular}

WASTE WEIGHT, KG

PPM LEAD

EKEH-92-161-02 224U-94-000010 FiHZ-219-091443 $221 T-94-000048$ 2225-93.000387 ANL93-MW.00019 ANL93-MW-CO020 RHAZ-219.091442 RHZ-213-A21808 224U-91-00012 $221 \mathrm{~T}-94-000114$ 9403711

202A-92.000308 221T-92-000182 ETFF-93.179.05 WTFF VT 01-153 PHZ 212 A21603 NLD-93-000426 PNL-93-000427

PNLD-93-000428

PNLD-93-000429

PNLD-93-000430

PLL-93-000443

PNLD-33-000444

PNLD-93-000445

ETFF-93-305-06

221T-92-000167

05N-89-0008

2240-90-00132

224U-90-00131

224U-90-00132

2244-90-00131

(2)

PNLD-93-000447

NLD-93-00044

EKEH-92-176-02

2240-90-00128

RHZ-213-A21808

2718-91-0168
$3.0000 E+01$

$1.7600 E+01$

3. $5000 E+01$

$3.5010 E+03$
$2.8180 E+01$

$2.5320 E+02$

$1.9780 \mathrm{E}+02$

$4.6000 E+O$

$3.0000 E+01$

$5.4010 E+O$

$1.0400 E+02$

$1.0400 E+02$

$2.3623 E+03$

$3.3000 E+01$

$3.0000 E+01$

$6.3140 \mathrm{E}+01$

$6.3140 E+01$

$6.3700 \mathrm{E}+02$

$5.4000 E+01$

$4.9000 E+O$

SOEE

$6,3000 E+01$

$9.1000 E+01$

9.1000E + 01

9. $1000 E+01$

$9.1000 E+01$

$9.1000 \mathrm{E}+01$

$9.1000 E+01$

9. $1000 \mathrm{E}+01$

$9.1000 E+O$

$9.1000 E+01$

$5.3000 E+01$

$3.6000 E+01$

$6.4000 E+01$

4.3000E + 01

$3.9000 E+02$

$2.7400 \mathrm{E}+02$

3.900OE + 0

$3.7400 E+02$

$2.7400 E+02$

.

9.9100e + 01

$9.1000 E+01$

$9.1000 E+01$

$4.8000 E+01$

$4.2300 E+02$

$4.2300 E+02$

$5.4010 E+01$

$2.9560 E+01$
$2.0000 E+03$

$1.8750 E+03$

$1.8286 E+03$

$1.6656 \mathrm{E}+03$

1. $6182 \mathrm{E}+03$

$1.5008 E+03$

$1.4661 E+03$

$1.3913 E+03$

$1.3333 E+03$

$1.2961 E+03$

$1.2500 E+03$

$1.2250 E+03$

$1.1853 E+03$

$1.0606 E+03$

$1.0000 E+03$

$9.5027 \mathrm{E}+02$

$9.4192 \mathrm{E}+02$

$9.2593 E+02$

$.1633 E+02$

$7.9618 E+02$

$7.901 \mathrm{E}+02$

$7.9231 \mathrm{E}+02$

$7.9231 E+02$

.

$7.9231 E+02$

$7.9231 \mathrm{E}+02$

$7.9231 E+02$

$7.9231 \mathrm{E}+02$

$7.9231 \mathrm{E}+02$

$7.9231 \mathrm{E}+02$

$7.9231 E+02$

5. $6604 \mathrm{E}+02$

$5.5556 \mathrm{E}+02$

$4.6875 E+02$

$4.6512 \mathrm{E}+02$

4.4359E + 02

4.3796E + 02

4. $3590 E+02$

$4.3512 E+02$

$4.1176 E+02$

$4.176 E+02$

4.0363E+02

$7.9231 \mathrm{E}+02$

$7.9231 E+02$

$6.2500 E+02$

$4.0189 \mathrm{E}+02$

$3.9314 E+02$

$3.7030 E+02$

$3.3829 E+02$ 
WHC-SD-W-242-ES-004, Rev. 0

TABLE 35

PINS CONTAINING LEAD SORTED IN ASCENDING ORDER BASED ON PPM LEAD

(13 SHEET)

COMPONENT NAME

$221 \mathrm{~T}-92 \cdot 000200$ 221T-92-000202 $221 \mathrm{~T}-92.000203$ $221 \mathrm{~T}-92-000204$ $221 \mathrm{~T} \cdot 92.00020$ $221 \mathrm{~T}-92-00019$ $2240-90-00129$ 224U-90-00129 2718.91.0168 9408381 9408382 221T-92-000122 $105 \mathrm{~N}-89-00112$ 9408386

$21 T-92-00018$ ETFF-90-0542 221T.92.000i80 221T.92.000180 BHZ-219-09144 RHL-219.09144 RHZ-219-091494 321.91 .0001

$105 \mathrm{~N}-69.00059$

$105 \mathrm{~N}-89-000561$

303K-93-000039

$105 \mathrm{~N}-89-000560$

$105 \mathrm{~N}-89.000562$

RHZ-213-A21306

ETFF-91.192.02

$2211 \cdot 92-000269$

ETFF-92-337-02

KEH-91-108-112

303K-93-000041

221T-92-000048

WTFF-91-259-08

WTFF-VT-001.73

202A-94-000310

(1)

(1)

$105 N-89-00111$

105N

303K-93-000037

05N-89-001114

303K-93-000038
WASTE WEGHT, KG

PPM LEAD

$3.1786 E+02$

$3.0562 E+02$

$3.0198 E+02$

$2.0340 \mathrm{C}+02$

$2.8031 \mathrm{E}+02$

$2.6042 \mathrm{E}+02$

$2.5714 \mathrm{E}+02$

$2.5063 E+02$

$2.3681 \mathrm{E}+02$

$2.3288 E+02$

$2.2667 E+02$

$2.2368 \mathrm{E}+02$

$2.1978 E+02$

$2.1429 E+02$

$2.1429 E+02$

$2.1142 E+02$

$2.0408 E+02$

$1.9608 \mathrm{E}+02$

$1.8868 \mathrm{E}+02$

$1.8868 \mathrm{E}+02$

$1.8788 E+02$

$1.8788 E+02$

$16949 E+02$

$1.0949 E+02$

$1.5802 E+02$

$1.5610 E+02$

$1.5504 E+02$

$1.5059 \mathrm{E}+02$

$1.4167 \mathrm{E}+02$

$1.3697 \mathrm{E}+02$

$1.0870 E+02$

$1.0808 \mathrm{E}+02$

$1.0526 \mathrm{E}+02$

$1.0526 \mathrm{E}+02$

$1.0417 E+02$

$1.0394 E+02$

$1.0279 E+02$

9. $2593 \mathrm{E}+01$

$9.0090 \mathrm{~F}+01$

$8.7404 \mathrm{E}+01$

$8.4112 \mathrm{E}+01$

$8.4112 E+01$

$7.6923 \mathrm{E}+01$

$7.4074 E+01$

$7.4074 E+0$

$7.2581 E+0$

$6.9767 E+01$

$5.7143 E+01$
$5.2032 E+01$ 
WHC-SD-W-242-ES-004, Rev. 0

TABLE 35

PINS CONTAINING LEAD

SORTED IN ASCENDING ORDER BASED ON PPM LEAD

(13 SHEET)

COMPONENT NAME

EAD

BASE METAL (LEAD)

BASE METAL. (LEAD)

BASE METAL (LEAD)

LEAD

EAD

LEAD

LEAD

LEAD

LEAD

LEAD

LEAD

LEAD

LEAD

LEAD

IEAD

IEAD

LEAD

LEAD

LEAD

LEAD

LEAD

LEAD

LEAD

LAD

BASE METAL (LEAD)

IFAD

9408744

$105 \mathrm{~N}-89-000512$

9408743

$\$ 408746$

9408747

$105 \mathrm{~N}-89-000612$

$05 \mathrm{~N}-89-00038$

$105 \mathrm{~N}-89-00038$

$105 \mathrm{~N}-69-000387$

$105 \mathrm{~N}-89-000844$

$105 \mathrm{~N}-89-000384$

(05N

$105 \mathrm{~N}-89-000532$

$105 \mathrm{~N}-89-000514$

$105 \mathrm{~N}-89-000514$
COMP

\begin{tabular}{ll}
$4.9000 E-03$ & $9.6200 E+01$ \\
$2.0000 E-01$ & $1.9530 E+03$ \\
$1.8000 E-01$ & $1.7600 E+03$ \\
$1.5000 E-01$ & $1.4670 E+03$ \\
$1.6000 E-01$ & $1.5650 E+03$ \\
$3.0000 E-03$ & $5.9000 E+01$ \\
$3.0000 E-03$ & $6.8000 E+01$ \\
$3.6000 E-03$ & $8.2000 E+01$ \\
$3.6000 E-03$ & $8.3000 E+01$ \\
$3.0000 E-03$ & $7.4000 E+01$ \\
$2.4000 E-03$ & $6.4000 E+01$ \\
$4.0000 E-03$ & $1.1100 E+02$ \\
$1.4000 E-03$ & $3.9000 E+01$ \\
$4.0000 E-03$ & $1.1300 E+02$ \\
$1.2000 E-03$ & $3.4000 E+01$ \\
$4.0000 E-03$ & $1.1359 E+02$ \\
$1.6000 E-03$ & $4.6260 E+01$ \\
$7.0000 E-04$ & $2.2000 E+01$ \\
$3.0000 E-03$ & $9.4600 E+01$ \\
$4.0000 E-04$ & $1.6000 E+01$ \\
$1.0000 E-03$ & $4.5460 E+01$ \\
$8.0000 E-04$ & $3.7000 E+01$ \\
$4.0000 E-04$ & $1.8800 E+01$ \\
$1.5000 E-03$ & $8.2000 E+01$ \\
$1.5000 E-03$ & $8.2000 E+01$ \\
$1.5000 E-03$ & $8.2000 E+01$ \\
$1.5000 E-03$ & $8.3000 E+01$ \\
$1.5000 E-03$ & $8.4000 E+01$ \\
$2.0000 E-03$ & $1.1600 E+02$ \\
$1.2000 E-03$ & $7.5000 E+01$ \\
$1.0000 E-03$ & $6.4000 E+01$ \\
$1.0000 E-03$ & $6.4000 E+01$ \\
$1.2000 E-03$ & $7.7000 E+01$ \\
$1.2000 E-03$ & $7.7000 E+01$ \\
$1.2000 E-03$ & $7.7000 E+01$ \\
$1.2000 E-03$ & $7.7000 E+01$ \\
$1.1000 E-03$ & $7.1000 E+01$ \\
$1.0000 E-03$ & $6.6000 E+01$ \\
$1.0000 E-03$ & $6.9000 E+01$ \\
$1.0000 E-03$ & $7.0000 E+01$ \\
$8.0000 E-04$ & $5.6000 E+01$ \\
$1.0000 E-03$ & $7.1000 E+01$ \\
$1.1000 E-03$ & $8.2000 E+01$ \\
$1.1000 E-03$ & $8.3000 E+01$ \\
$1.1000 E-03$ & $8.4000 E+01$ \\
$1.1000 E-03$ & $8.6000 E+01$ \\
$9.0000 E-04$ & $7.1000 E+01$ \\
& \\
& \\
\hline &
\end{tabular}

PPM LEAD

$5.0936 E+01$ $1.0241 E+02$ $1.0227 \mathrm{E}+02$ $1.0225 \mathrm{E}+02$ $1.0224 E+02$ $5.0847 \mathrm{E}+01$ $4.4118 \mathrm{E}+01$ 4.3902E + 01 $4.3373 \mathrm{E}+01$ $4.0541 \mathrm{E}+01$ $3.7500 E+01$ $3.6036 \mathrm{E}+01$ $3.5897 \mathrm{E}+$ or $3.5398 \mathrm{E}+01$ $3.5294 \mathrm{E}+01$ $3.5214 E+01$ $3.4587 \mathrm{E}+01$ $3.1818 E+0$ $3.1712 E+O$ $2.5000 E+01$ $2.1997 E+O$ $2.1622 \mathrm{E}+0$ $2.1277 \mathrm{E}+01$ 2.1277E+0 $1.8293 \mathrm{E}+0$ $1.8293 \mathrm{E}+0$ ? $1.8293 \mathrm{E}+01$ $1.8072 \mathrm{E}+01$ $1.7857 \mathrm{E}+01$ $1.7241 E+01$

$1.6000 \mathrm{E}+01$

$1.5625 E+0$

$1.5625 \mathrm{E}+0$

$1.5584 E+01$

$1.5584 E+01$

$1.5584 E+01$

$1.5584 E+01$

$1.5493 \mathrm{E}+01$

$1.5152 \mathrm{E}+01$

$1.4493 E+01$

$1.4286 E+01$

$1.4286 E+0$

$1.4085 \mathrm{E}+0$

$1.4085 E+01$

$1.3415 E+01$

$1.3253 E+01$

$1.3095 E+01$ $1.2791 E+01$
$1.2676 E+01$ 
WHC-SD-W-242-ES-004, Rev. 0

TABLE 35

PINS CONTAINING LEAD

SORTED IN ASCENDING ORDER BASED ON PPM LEAD

(13 SHEET)

COMPONENT NAME

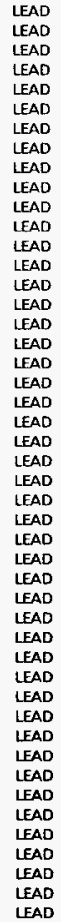

COMPONENT WEIGHT, KG

\begin{tabular}{|c|}
\hline $\begin{array}{l}1.0000 \mathrm{E}-03 \\
1.1000 \mathrm{E}-03\end{array}$ \\
\hline $\begin{array}{l}1.1000 E-03 \\
7.0000 E-04\end{array}$ \\
\hline $1.0000 \mathrm{E}-03$ \\
\hline $1.0000 E-03$ \\
\hline $1.0000 \mathrm{E}-03$ \\
\hline $1.0000 \mathrm{E}-03$ \\
\hline $9.0000 E-04$ \\
\hline $9.0000 E-04$ \\
\hline $1.1000 E-03$ \\
\hline $1.1000 \mathrm{E} .03$ \\
\hline 1.0000 E- 03 \\
\hline $6.0000 E-04$ \\
\hline 1. 1000 E -03 \\
\hline $9.0000 \mathrm{E}-04$ \\
\hline 9.0000 E -04 \\
\hline $1.0000 E-03$ \\
\hline 8.0000 E.04 \\
\hline $8.0000 \mathrm{E}-04$ \\
\hline $9.0000 \mathrm{E}-04$ \\
\hline $8.0000 E-04$ \\
\hline $8.0000 \mathrm{E}-04$ \\
\hline $9.0000 \mathrm{E}-04$ \\
\hline 9.0000E-04 \\
\hline $9.0000 E-04$ \\
\hline $9.0000 \mathrm{E}-04$ \\
\hline $8.0000 E-04$ \\
\hline 9.0000 - 04 \\
\hline $9.0000 E-04$ \\
\hline $9.0000 E-04$ \\
\hline $9.00006-04$ \\
\hline $9.00006-04$ \\
\hline $9.0000 \mathrm{E}-04$ \\
\hline $9.0000 E-04$ \\
\hline $9.0000 \mathrm{E}-04$ \\
\hline $7.0000 \mathrm{E}-04$ \\
\hline 9.0000 E- 04 \\
\hline $9.0000 E-04$ \\
\hline $9.0000 E-04$ \\
\hline $9.0000 E-04$ \\
\hline 1.20006 .03 \\
\hline $9.0000 \mathrm{E}-04$ \\
\hline $9.0000 \mathrm{E}-04$ \\
\hline $9.0000 \mathrm{E}-04$ \\
\hline 8.0000E-04 \\
\hline $\begin{array}{l}9.0000 \text { E-04 } \\
9.0000 \text {-04 }\end{array}$ \\
\hline \\
\hline
\end{tabular}

PPM LEAD

$1.2658 E+01$ $1.2644 E+01$ $1.2500 E+01$ $1.2195 E+01$ $1.2048 \mathrm{E}+01$ $1.2048 \mathrm{E}+01$ $1.1905 E+01$ $1.1842 E+01$ $1.1688 E+01$ $1.3924 E+01$ $1,3924 E+01$ $1.3889 E+01$ $1.3780 E+01$ $1.3580 \mathrm{E}+01$ $1.1538 E+01$ $1.1538 \mathrm{E}+01$ $1.1494 E+01$ $1.1429 \mathrm{E}+01$ $1.1429 E+01$ $1.1392 \mathrm{E}+01$ $1.1288 \mathrm{E}+01$ $1.1268 \mathrm{E}+01$ $1.1268 E+01$ $1.0976 E+01$ $1.0976 E+01$ $1.0976 E+01$ $1.0976 E+01$ $1.0843 E+01$ $1.0843 E+01$ $1.0843 E+01$ $1.0843 E+01$ $1.0843 E+01$ $1.0843 \mathrm{E}+01$ $1.0843 E+01$ $1.0843 E+01$ $1.0843 E+01$ $1.0769 E+01$ $1.0714 E+01$ $1.0714 E+01$ $1.0714 E+01$ $1.0714 E+01$ $1.0310 \mathrm{E}+01$ $1.0588 \mathrm{E}+01$ $1.0588 \mathrm{E}+01$ $1.0465 E+01$ $1.0465 E+01$ $1.0390 E+01$ $1.0345 E+01$ 
WHC-SD-W-242-ES-004, Rev. 0

TABLE 35

PINS CONTAINING LEAD

SORTED IN ASCENDING ORDER BASED ON PPM LEAD

(13 SHEET)

PIN

$105 \mathrm{~N}-89-000493$

$05 \mathrm{~N}-89-000490$

$105 \mathrm{~N}-89-000489$

WHC-A-91-811

$105 \mathrm{~N}-89-000566$

$105 \mathrm{~N}-89-00049$

RBLO000194

$105 \mathrm{~N} \cdot 89-00037$

$105 \mathrm{~N}-89-000483$

$105 \mathrm{~N}-89 \cdot 000504$

$105 \mathrm{~N}-89-000835$

$105 \mathrm{~N}-89-000582$

$105 \mathrm{~N}-89.000507$

$105 \mathrm{~N}-80.000488$

$105 \mathrm{~N}-89-000503$

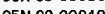

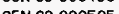

105N-89-000506

$105 \mathrm{~N}-89-000508$

105N-89-000495

$105 \mathrm{~N}-89-000574$

05N-89-000586

$105 \mathrm{~N}-89.000496$

105N-89-000584

$105 \mathrm{~N}-89.000585$

$105 \mathrm{~N}-89-000521$

$105 \mathrm{~N}-89-000630$

$105 \mathrm{~N}-89-000492$

$105 \mathrm{~N}-89-000629$

$105 \mathrm{~N}-89-000540$

$105 \mathrm{~N}-89-000583$

$105 \mathrm{~N}-89.000388$

$105 \mathrm{~N}-89-000632$

ERO-93-000725

$05 N-89.00063$

9408751

PNL-372-92.046

$105 N-89-000383$

$105 \mathrm{~N}-89-000779$

$105 \mathrm{~N}-89 \cdot 000414$

$105 \mathrm{~N}-\mathrm{B9}-000778$

$105 \mathrm{~N}-89-000780$

$105 \mathrm{~N}-89-000415$

$105 \mathrm{~N}-89-000416$
COMPONENT NAME

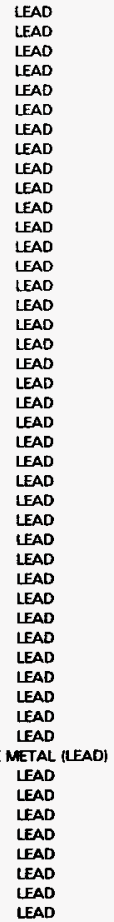

COMPONENT WEIGHT, KG

9.0000E-04

$9.0000 E-04$

$9.0000 E-04$

3.0000E-04

$6.0000 \mathrm{E}-04$

$9.0000 E-04$

$6.0000 E-04$

$6.0000 E-04$

$5.0000 E-04$

$9.0000 \mathrm{E}-04$

$6.0000 \mathrm{E}-\mathrm{O}$

6.0000 - 04

$6.0000 \mathrm{E}-04$

$6.0000 \mathrm{E}-04$

$7.0000 \mathrm{E}-04$

$6.0000 \mathrm{E}-04$

$6.0000 \mathrm{E}-04$

$6.0000 \mathrm{E}-04$

.

.

$9.0000 \mathrm{E}-04$

.0OOE-O4

$9.0000 \mathrm{E} \cdot 04$

$9.0000 E-04$

$8.0000 \mathrm{E}-04$

$6.0000 \mathrm{E}-04$

$6.0000 \mathrm{E}-04$

$7.0000 \mathrm{E}-04$

$7.0000 \mathrm{E}-04$

7.0000 E- 04

$7.0000 \mathrm{E}-04$

$6.0000 \mathrm{E}-04$

7.0000 E. 04

$6.0000 E-04$

7.0000E-04

$5.0000 E .04$

$5.0000 \mathrm{E}-04$

$1.0000 E-03$

$5.0000 E-04$

.

.

5.0000 E 04

4.0000E-O4

5.0000E-04

4.0000E- 04

$5.0000 E-04$

5.0000E-04

4.0000E. 04

4.0000E-04
WASTE WEIGHT, KG

PPM LEAO

8. $7000 E+01$

$9.0000 E+01$

9.1000E+01

$3.0800 \mathrm{E}+0$

$6.2010 \mathrm{E}+01$

$9.4000 \mathrm{E}+01$

$6.3000 \mathrm{E}+\mathrm{O}$

$6.5000 E+0$

$5.5000 E+O$

$9.0000 \mathrm{E}+01$

$6.6000 \mathrm{E}+0$

$6.6000 \mathrm{E}+01$

$6.6070 \mathrm{E}+01$

$6.7000 E+01$

$7.9000 E+01$

$6.8000 \mathrm{E}+01$

$6.8000 E+01$

$6.8000 E+01$

$6.6000 E+01$

$8.7000 E+O$

$8.7000 E+O$

8.

$8.8000 E+01$

$8.9000 E+01$

$6.8000 E+01$

6.9000E + 01

$7.0000 E+01$

$8.2000 E+01$

8.2000E + 01

8.300OE + O

8.3000E + 01

$7.2000 \mathrm{E}+01$

8.5000E + 01

$7.3000 E+01$

$8.8000 E+01$

$6.3000 E+01$

$6.3000 \mathrm{E}+01$

$1.3720 E+02$

1.3720E

.

4.2000E+O

$7.8301 E+01$

$6.5000 E+01$

$8.2000 E+O$

$0.0000 E+01$

$8.3000 E+01$

$8.3000 E+O$

6.8000E+O1
$1.0345 E+01$

$1.0000 E+0$

$9.8901 E+00$

$9.7403 E+00$

$9.5745 E+00$

$9.5238 \mathrm{E}+00$

$9.2308 E+00$

$9.0909 \mathrm{E}+00$

$1.0000 \mathrm{E}+0$

$9.0909 E+\infty 0$

$9.0909 E+00$

$9.0813 \mathrm{E}+00$

$8.9552 E+00$

$8.8608 \mathrm{E}+00$

8.8235E +00

$8.8235 \mathrm{E}+00$

$9.0909 E+00$

$1.0345 \mathrm{E}+01$

$1.0345 E+01$

$1.0345 \mathrm{E}+\mathrm{O}$

$1.0227 \mathrm{E}+0$

$1.0112 \mathrm{E}+0$

8.8235E + 00

$6.6957 \mathrm{E}+00$

$8.5714 \mathrm{E}+\infty$

$8.5366 \mathrm{E}+\infty 0$

8.5366E $+\infty$

$8.4337 E+\infty$

$8.4337 \mathrm{E}+\infty 0$

$8.3333 E+\infty 0$

$8.2353 E+\infty$

$8.2192 E+\infty$

$7.9545 E+\infty$

$7.9365 \mathrm{E}+\infty 0$

$7.9365 \mathrm{E}+\infty$

$7.2886 E+\infty$

$7.2464 E+\infty$

$7.1420 \mathrm{E}+\infty$

$7.1429 E+\infty$

$0.335 E+\infty$

$0.1530 \mathrm{C}+\infty$

$6.0976 \mathrm{E}+\infty$

$6.0606 \mathrm{E}+\infty$

$6.0241 E+\infty$

$8.0241 E+\infty$

$5.8824 E+\infty$

$5.8824 E+00$ 
WHC-SD-W-242-ES-004, Rev. 0

TABLE 35

PINS CONTAINING LEAD

SORTED IN ASCENDING ORDER BASED ON PPM LEAD

(13 SHEET)

PIN

$105 \mathrm{~N}-89-000417$

$105 \mathrm{~N}-89-000382$

$105 \mathrm{~N}-89-000380$

$105 \mathrm{~N}-90-000156$
$105 \mathrm{~N}-89-000379$

$105 \mathrm{~N}-89-000539$

$105 \mathrm{~N} \cdot 89-00038$

$100 \mathrm{~N}-93-032400$

$105 \mathrm{~N}-89-000458$

$105 \mathrm{~N}-89-00078$

$271 \mathrm{~B}-93-000176$

$100 \mathrm{~N} \cdot 93.032500$

$105 \mathrm{~N}-89-000830$

$303 \mathrm{~K} \cdot 93-000025$

$303 \mathrm{~K}-93.000026$

$105 \mathrm{~N} \cdot 89-000457$

$105 \mathrm{~N}-89-000517$

$105 \mathrm{~N}-89.000820$

$105 \mathrm{~N}-89.00$

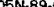

$105 \mathrm{~N}-89-000854$

$55 \mathrm{~N}-89.000855$

OON-93-032600

05N-89-000458

$105 \mathrm{~N}-89-000821$

105N-89-000923

$105 N \cdot 89-000924$

05N-69-000459

$163 \mathrm{~N}-91-0002$

$63 \mathrm{~N}-91.0003$

$105 \mathrm{~N}-89-000418$

$105 \mathrm{~N}-89-000822$

$105 \mathrm{~N}-89.000828$

$105 \mathrm{~N}-89-000829$

105 N-89-000831

$303 \mathrm{~K} .93 .000027$

$105 \mathrm{~N}-89-000645$

$05 \mathrm{PO}-000455$

105N-89-000455

$105 N-89-00046$

$105 \mathrm{~N}-89-000824$

$105 \mathrm{~N}-89.000825$

$105 \mathrm{~N}-89-000826$

$105 N-89.000846$

I05N-89-000849

$105 \mathrm{~N} \cdot 89.000850$

105N-89-000851
COMPONENT NAME

LEAD

LEAD

LEAD

LEAD

LEAD

LEAD

LEAD

LEAD

LEAD

LEAD

LEAD

IEAD

LEAD

IEAD

LEAD

LEAD

LEAD

LEAD

UEAD

LEAD

LEAD

LEAD

LEAD

LEAD

LEAD

IEAD

LEAD

LEAD

LEAD

IEAD

IEAD

IEAD

LEAD

EAD

LEAD

LEAD

LEAD

LEAD

LEAD
COMPONENT WEIGHT, KG

$4.0000 E \cdot 04$

4.0000E-04

4.0000E-04

$2.0000 \mathrm{E}-04$

$4.0000 E-04$

3.0000E-04

4.0000E-04

2.0000 E- 04

3.0000E-04

3.0000E-04

$2.0000 \mathrm{E} .04$

$2.0000 \mathrm{E}-\mathrm{OA}$

$3.0000 \mathrm{E}+04$

4.0000E-04

5.0000E-04

$3.0000 \mathrm{E}-04$

$3.0000 \mathrm{E}-04$

3.0000 - 04

2.0000 E. 04

2.0000E-04

.0000-04

.000

$3.0000 E-04$

1.0000E-04

$3.0000 \mathrm{E}-04$

3.0000E-C4

$5.0000 E-04$

$5.0000 \mathrm{E}-04$

$3.0000 \mathrm{E}-04$

4.0000E- 0

4.0000E-O4

4.0000E. 04

3.0000E-O4

3.0000E.04

3.0000E-04

3.0000E-04

3.0000E-04

4.0000 - -2

3.0000 EO

3.00005

3.0000504

$3.00005-04$

3.0000E-04

3.0000E-04

3.0000E-04
WASTE WEIGHT, KG

PPM LEAD

$6.8000 \mathrm{E}+01$

$7.0000 E+0$

$7.1000 E+01$

$3.6000 E+0$

7.3000E + O

$5.5000 \mathrm{E}+0$

$7.4000 E+0$

$4.2000 E+0$

$6.3000 E+0$

$6.4000 E+O$

4.3990E + 01

4. $6000 \mathrm{E}+\mathrm{O}$

$7.1000 E+01$

$9.9000 E+01$

1. $2900 E+02$

$7.8000 E+0$

$7.8000 E+O$

$7.8000 E+01$

$5.3000 E+01$

5.300E +0

$8.0000 E+O$

$8.0000 E+01$

$8.0000 E+01$

$2.7000 E+01$

$8.2000 E+01$

$8.2000 E+01$

$9.3000 E+01$

$9.3000 E+01$

$5.6000 E+01$

$7.5000 E+0$

$8.1000 E+01$

$8.1000 E+0$

$6.1000 E+0$

8.2000E + 01

8.2000E + 01

8. $2000 E+O 1$

8. $2000 E+01$

$1.1000 E+02$

1.

$8.4000 E+01$

$8.4000 E+O$

$8.4000 E+01$

0.4000 + 01

$8.4000 E+01$

$8.4000 E+01$

8. $4000 E+01$

$8.4000 E+0$

$8.4000 E+01$
$8.4000 E+01$
$5.8824 \mathrm{E}+00$

$5.7143 \mathrm{E}+00$

$5.5556 \mathrm{E}+00$

$5.5556 E+00$

$5.4795 E+00$

$5.4545 \mathrm{E}+00$

$5.4054 \mathrm{E}+00$

$4.7619 \mathrm{E}+\infty$

$4.7619 \mathrm{E}+\infty$

$4.6875 E+\infty$

$4.5465 E+\infty$

$4.3478 E+\infty 0$

4. $2254 E+00$

$4.0404 E+\infty 0$

$3.8760 E+00$

$3.8462 E+00$

$3.8462 \mathrm{E}+00$

$3.8462 E+O 0$

$3.7736 \mathrm{E}+\infty 0$

$3.7500 \mathrm{E}+00$

$3.7500 \mathrm{E}+00$

$3.7500 \mathrm{E}+0$

$3.7037 \mathrm{E}+00$

3.7037E +

$3.6505 \mathrm{E}+00$

$5.3763 \mathrm{E}+00$

$5.3763 \mathrm{E}+00$

$5.3571 \mathrm{E}+\infty$

$5.3333 E+\infty$

$4.9383 E+\infty$

$4.9383 E+\infty$

$4.9180 E+\infty$

$3.6585 E+\infty 0$

$3.6585 E+\infty$

$3.6585 E+\infty 0$

$3.6585 E+\infty 0$

$3.6364 \mathrm{E}+00$

$3.6145 \mathrm{E}+\infty$

$3.5714 E+\infty$

$3.5714 E+\infty$

$3.5714 E+\infty$

$3.5714 E+\infty$

$3.5714 E+\infty$

$35714 E+\infty$

$35714 E+\infty 0$

$3.5714 E+00$

$3.5714 E+\infty 0$ 
WHC-SD-W-242-ES-004, Rev. 0

TABLE 35

PINS CONTAINING LEAD

SORTED IN ASCENDING ORDER BASED ON PPM LEAD

(13 SHEET)

PIN

$105 \mathrm{~N}-89-000647$ $105 \mathrm{~N}-89-00087$

$105 \mathrm{~N}-89-000679$

$105 \mathrm{~N}-89-000845$

$100 \mathrm{~N}-90-003800$

$105 \mathrm{~N}-89-000852$

$105 \mathrm{~N}-89-000827$

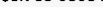

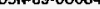

$05 N-89-000856$

$308 \mathrm{M}-94-000006$

$308 \mathrm{M}-94-000003$

$303 K-93.000023$

308 M. 94.000004

(208M-94-00000

308M-94-00000

(05N-89-000464

$03 \mathrm{~K} 00002$

(0)

$100 \mathrm{k} \cdot 91.021700$

$100 \% .91 .021700$

100n-90-003800

$100 \mathrm{~N}-90-038200$

$100 \mathrm{~N}-91.032600$

$105 \mathrm{~N}-89-000403$

$105 \mathrm{~N}-89-000404$

$105 \mathrm{~N}-89.000405$

$105 \mathrm{~N}-89-000406$

$105 \mathrm{~N}-89-000407$

$105 \mathrm{~N}-89-000554$

$105 \mathrm{~N}-89-000867$

$105 \mathrm{~N}-89-000519$

$105 \mathrm{~N}-89.000462$

$105 \mathrm{~N}-89-000463$

$105 \mathrm{~N}-89-000460$

$105 \mathrm{~N}-89-000461$

$105 N-89-000878$

RALO000294

OOK-91-021700

$221 T-92-000275$

$21 \mathrm{~T}-92-00030$

$221 \mathrm{~T}-92-000303$

ETF-91. 184-01
COMPONENT WE!GHT, KG

$3.0000 E-04$
$3.0000 E-04$
$3.000 E-04$
$3.0000 E-04$
$3.000 E-04$
$1.0000 E-04$
$2.000 E-04$
$2.000 E-04$
$2.000 E-04$
$2.0000 E-04$
$2.000 E-04$
$2.000 E-04$
$2.0000 E-04$
$1.0000 E-04$
$1.0000 E-04$
$3.0000 E-04$
$1.0000 E-04$
$1.0000 E-04$
$1.0000 E-04$
$1.0000-04$
$1.0000 E-04$
$2.0000 E-04$
$1.0000 E-04$
$0.0000 E+00$
$0.0000 E+00$
$0.0000 E+00$
$0.0000 E+00$
$0.0000 E+00$
$0.0000 E+00$
$0.0000 E+00$
$0.0000 E+00$
$0.0000 E+00$
$0.0000 E+00$
$0.0000 E+00$
$2.0000 E-04$
$2.0000 E-04$
$2.0000 E-04$
$2.0000 E-04$
$2.0000 E-04$
$2.0000 E-04$
$2.0000 E-04$
$1.0000 E-04$
$0.0000 E+00$
$0.0000 E+00$
$0.0000 E+00$
$0.0000 E+00$
$0.0000 E+00$

WASTE WEIGHT, KG

PPM LEAD

$3.4884 E+\infty$

$3.4884 E+\infty$

$3.4884 E+\infty 0$

$3.4884 E+\infty$

$3.4483 \mathrm{E}+\infty$

$3.2258 \mathrm{E}+00$

$3.2258 \mathrm{E}+\infty$

$3.1746 \mathrm{E}+\infty$

$3.1250 E+\infty 0$

$3.0303 E+\infty$

$3.0303 E+\infty 0$

$3.0303 E+\infty$

$2.9851 E+\infty 0$

$2.3041 E+\infty 0$

2. $1882 \mathrm{E}+\infty$

$2.1739 E+00$

$2.1277 \mathrm{E}+00$

$2.1231 \mathrm{E}+\infty 0$

$2.1231 E+\infty$

$2.1231 E+\infty$
$2.1053 E+\infty$

$1.9608 \mathrm{E}+\infty$

$1.9608 \mathrm{E}+\infty$

1. $2500 E+\infty$

.

0.0000 + 00

$0.0000 E+\infty$

$0.0000 E+\infty 0$

$0.0000 E+\infty 0$

$0.0000 E+00$

$0.0000 E+\infty$

$0.0000 E+\infty 0$

$0.0000 E+\infty$

$0.0000 E+\infty$

$0.0000 E+\infty$

$0.0000 E+\infty 0$

$2.9412 E+\infty$

$2.8169 E+\infty 0$

$2.7778 E+\infty 0$

$2.7397 \mathrm{E}+\infty$

$2.7397 E+\infty$

$2.7397 \mathrm{E}+\infty$

$2.7397 E+\infty$

$2.7397 E+\infty$

$0.0000 E+\infty$

0.0000 +

$0.0000+\infty$

$0.000 \mathrm{C}+\infty$

$0.0000+\infty$ 
WHC-SD-W-242-ES-004, Rev. 0

TABLE 35

PINS CONTAINING LEAD

SORTED IN ASCENDING ORDER BASED ON PPM LEAD

(13 SHEET)

PIN

\section{ETFF-92-343-01}

WHC-RR-90-01

WhC-RR-90-02

WHC-RR-90-03

WHC-RR-90-04

WHC-RR-90-05

WHC-RR-90-06

WHC-RR-90-07

WHC-RR-90.08

WHC-RR-90-09

WHC-RR-90-10

WHC-RR-90-11

WHC.RR-90.12

WHC-RF-90-13

WHC.RR-90-14

WHC.RP-90-15

WHC-RR-90-16

WHC.RPr90-17

224U-8800

URMW2-1
COMPONENT NAME

LEAD
LEAD
LEAD
LEAD
LEAD
LEAD
LEAD
LEAD
LEAD
LEAD
LEAD
LEAD
LEAD
UEAD
IEAD
IEAD
IEAD
LEAD
LEAD
LEAD

LEAO

EAD

EAD

EAD

AD

EAD

EAD

EAD

IEAD

LEAD

LEAD
COMPONENT WEIGHT, KG

$0.0000 E+00$

$0.0000 E+00$

$0.0000 \mathrm{E}+00$

$0.0000 \mathrm{E}+00$

$0.0000 E+00$

$0.0000 \mathrm{E}+00$

$0.0000 E+00$

$0.0000 E+00$

$0.0000 E+00$

$0.0000 E+00$

$0.0000 E+00$

$0.0000 E+00$

$0.0000 \mathrm{E}+00$

$0.0000 E+00$

$0.0000 \mathrm{E}+00$

$0.0000 \mathrm{E}+00$

$0.0000 E+00$

$0.0000 E+00$

$0.0000 E+00$

$0.0000 E+00$
$0.0000 E+00$
WASTE WEIGHT, KG

$3.5000 E+01$

$9.7370 E+01$

$5.1520 E+01$

$1.8854 E+02$

$2.3350 E+02$

$1.8811 E+02$

$9.7600 E+01$

$7.4700 E+01$

$2.9340 E+0$

$7.4900 E+0$

$1.2010 E+O 2$

$2.9450 E+01$

$2.9450 E+01$

$2.9450 E+01$

$7.4700 E+01$

$1.8853 E+02$

$3.2481 \mathrm{E}+02$

$5.2080 \mathrm{E}+01$

0.000 + +00

0000
PPM LEAD

$0.0000 \mathrm{E}+00$

$0.0000 \mathrm{E}+00$

$0.0000 E+00$

$0.0000 E+00$

$0.0000 E+00$

$0.0000 \mathrm{E}+00$

$0.0000 \mathrm{E}+00$

$0.0000 E+00$

$0.0000 E+00$

$0.0000 E+00$

$0.0000 E+00$

$0.0000 E+00$

$0.0000 E+00$

$0.0000 E+00$

$0.0000 E+00$

$0.0000 \mathrm{E}+00$

$0.0000 \mathrm{E}+00$

$0.0000 E+00$
$0.0000 E+00$

0.000 T

$0.000 \mathrm{E}+00$ 
WHC-SD-W-242-ES-004, Rev. 0

TABLE 36

SUMMARY OF WASTE VOLUMES BY GENERATOR

SORTED IN ASCENDING ORDER BASED ON CONTAINER COUNT (* INDICATES DATA EXHIBITED NEGATIVE VARIANCE)

(2 SHEETS)

\begin{tabular}{|c|c|c|c|c|c|c|c|}
\hline COMPANY & GENERATOR & COUNT & ACCUM VOLUME, CUM & AVG VOLUME, CUM & STDDEV & MIN VOLUME, CU M & MAX VOLUME, CUM \\
\hline WHC & $2345 z$ & 455 & $3.5040 E+02$ & $7.7010 E-01$ & $2.1335 E+\infty 0$ & $3.0000 \mathrm{E}-02$ & $1.2921 E+01$ \\
\hline WHC & $100 \mathrm{~N}$ & 408 & $8.5622 \mathrm{E}+01$ & $2.0986 \mathrm{E}-01$ & $2.8714 \mathrm{E}-03$ & $1.5200 \mathrm{E}-01$ & $2.1000 E-01$ \\
\hline LBL & LBLAB & 215 & $4.4870 E+01$ & $2.0870 \mathrm{E}-01$ & $7.7499 \mathrm{E}-03$ & $2.0800 \mathrm{E}-01$ & $3.2180 \mathrm{E}-01$ \\
\hline ANL & ARGON & 210 & $4.3722 E+01$ & $2.0820 \mathrm{E}-01$ & $0.0000 E+00$ & $2.0820 E-01$ & 2.0820E-01 \\
\hline BATC & BATCO & 205 & 4.2274E +01 & $2.0622 \mathrm{E}-01$ & $4.6254 E-03$ & $2.0000 E-01$ & $2.1000 E-01$ \\
\hline WHC & $222 \mathrm{~s}$ & 144 & $4.1773 E+01$ & $2.9009 \mathrm{E}-01$ & $7.2420 \mathrm{E}-01$ & $2.0000 \mathrm{E}-01$ & $6.3710 E+00$ \\
\hline ALB & ALBNY & 126 & $4.0572 \mathrm{E}+01$ & $3.2200 \mathrm{E}-01$ & 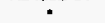 & $3.2200 E-01$ & $3.2200 E-01$ \\
\hline WHC & $202 A$ & 95 & $2.7978 E+01$ & $2.9450 \mathrm{E}-01$ & $6.8202 \mathrm{E}-01$ & $1.1000 \mathrm{E}-01$ & $6.3700 E+00$ \\
\hline WHC & $221 \mathrm{~T}$ & 69 & $2.0638 E+01$ & $2.9911 \mathrm{E}-01$ & $7.4172 \mathrm{E}-01$ & $2.0820 \mathrm{E}-01$ & $6.3710 E+00$ \\
\hline BAPL & BAPL & 49 & $1.0202 \mathrm{E}+01$ & $2.0820 \mathrm{E}-01$ & $4.3016 \mathrm{E}-09$ & $2.0820 \mathrm{E}-01$ & $2.0820 \mathrm{E}-01$ \\
\hline BNL & BNLAB & 49 & $1.0202 \mathrm{E}+01$ & $2.0820 \mathrm{E}-01$ & $4.3016 \mathrm{E}-09$ & $2.0820 \mathrm{E}+01$ & $2.0820 \mathrm{E}-01$ \\
\hline WHC & $224 U$ & 44 & $8.8842 E+00$ & $2.0191 E-01$ & $2.6848 \mathrm{E}-02$ & $3.0000 \mathrm{E}-02$ & 2.1200E-01 \\
\hline FERM & NALAB & 37 & $7.7484 E+\infty 0$ & $2.0942 \mathrm{E}-01$ & 8.5424E-04 & $2.0820 \mathrm{E}-01$ & $2.1000 E-01$ \\
\hline WHC & $303 K$ & 35 & $7.1924 E+00$ & $2.0550 \mathrm{E}-01$ & $1.5990 \mathrm{E}-02$ & $1.1360 \mathrm{E}-01$ & $2.0820 E-01$ \\
\hline PSN & PSNS & 34 & $9.9348 E+01$ & $2.9220 \mathrm{E}+00$ & $1.0981 E-07$ & $2.9220 E+\infty 0$ & $2.9220 E+00$ \\
\hline PNL & 331 & 30 & $6.2802 E+00$ & $2.0934 \mathrm{E}-01$ & 8.8224E-04 & $2.0820 \mathrm{E}-01$ & $2.1000 E-01$ \\
\hline WHC & $271 B$ & 28 & $1.2281 E+01$ & 4.3861E-01 & $1.2868 E+\infty 0$ & $3.0000 \mathrm{E}-02$ & $7.0000 E+00$ \\
\hline WHC & 163PA & 26 & $4.9050 E+00$ & $1.8865 \mathrm{E}-01$ & $6.0301 \mathrm{E}-02$ & $1.8000 E-02$ & 2.1000E-01 \\
\hline WHC & 2415 & 26 & $7.7438 \mathrm{E}+01$ & $2.9784 E+00$ & $3.8972 E+\infty 0$ & $2.0800 E-01$ & $8.7000 \mathrm{E}+00$ \\
\hline PNL & $305 \mathrm{~B}$ & 23 & $5.5710 E+00$ & $2.4222 \mathrm{E}-01$ & $1.5647 \mathrm{E}-01$ & 2.0820E-01 & $9.6000 E-01$ \\
\hline WHC & $221 B$ & 22 & $4.2104 \mathrm{E}+00$ & $1.9138 E-01$ & $2.7544 \mathrm{E}-02$ & $1.5200 \mathrm{E}-01$ & $2.1000 E-01$ \\
\hline WHC & 221TS & 18 & $3.7800 E+00$ & $2.1000 E-01$ & $0.0000 E+00$ & $2.1000 \mathrm{E}-01$ & $2.1000 E-01$ \\
\hline WHC & $241 \mathrm{~A}$ & 18 & $7.1051 E+01$ & $3.9473 E+00$ & $4.2840 E+00$ & $1.5000 E-01$ & $8.7000 E+\infty 0$ \\
\hline PNL & 325 & 17 & $3.3128 E+00$ & $1.9487 \mathrm{E}-01$ & 3.3932E-02 & $8.0000 \mathrm{E}-02$ & $2.1000 E-01$ \\
\hline WHC & $242 A$ & 17 & $8.1663 E+01$ & $4.8037 \mathrm{E}+00$ & $5.1361 E+00$ & $3.0000 E-02$ & $1.3300 E+01$ \\
\hline WHC & BG3AE & 17 & $3.5700 E+00$ & $2.1000 \mathrm{E}-01$ & $0.0000 E+00$ & $2.1000 \mathrm{E}-01$ & 2.1060E-01 \\
\hline WHC & $1310 \mathrm{P}$ & 15 & $2.8470 E+\infty O$ & $1.8980 \mathrm{E}-01$ & $4.9691 \mathrm{E}-02$ & $2.7000 \mathrm{E}-02$ & $2.1000 \mathrm{E}-01$ \\
\hline WHC & $105 \mathrm{~N}$ & 14 & $2.7510 E+\infty 0$ & $1.9650 E-01$ & $2.5214 E-02$ & $1.1000 E-01$ & 2.0820E-01 \\
\hline WHC & 241AY & 14 & $2.8196 E+00$ & $2.0140 \mathrm{E}-01$ & 2.6315E-02 & $1.1000 E-01$ & $2.1000 \mathrm{E}-0\}$ \\
\hline WHC & $241 T X$ & 12 & $1.4423 \mathrm{E}+01$ & $1.2019 \mathrm{E}+00$ & $2.3931 E+00$ & $2.0820 E-01$ & $8.7000 E+00$ \\
\hline WHC & 324 & 12 & $2.5200 E+00$ & $2.1000 E-01$ & . & $2.1000 \mathrm{E}-01$ & $2.1000 \mathrm{E}-01$ \\
\hline PNL & 324 & 11 & $1.0560 E+01$ & $9.6000 E-01$ & • & $9.6000 E-01$ & $9.6000 \mathrm{E}-01$ \\
\hline PNL & LSLII & 11 & $2.2956 \mathrm{E}+\infty 0$ & $2.0869 E-01$ & $8.4078 \mathrm{E}-04$ & $2.0820 \mathrm{E}-01$ & $2.1000 \mathrm{E}-01$ \\
\hline WHC & $163 \mathrm{~N}$ & 11 & $2.2974 E+00$ & $2.0885 \mathrm{E}-01$ & $9.0814 E-04$ & 2.0820E-01 & 2.1000E-01 \\
\hline WHC & 2025 & 11 & $2.2902 \mathrm{E}+00$ & $2.0820 \mathrm{E}-01$ & 2.3561E-09 & $2.0820 \mathrm{E}-01$ & $2.0820 E-01$ \\
\hline WHC & $241 C$ & 11 & $5.1809 E+01$ & $4.7099 \mathrm{E}+00$ & $4.3029 E+00$ & $1.5000 E-01$ & $8.7000 \varepsilon+00$ \\
\hline WHC & $241 \mathrm{~T}$ & 11 & $2.7765 E+01$ & $2.5241 \mathrm{E}+00$ & $3.9665 E+00$ & $2.0800 \mathrm{E}-01$ & $8.7000 E+00$ \\
\hline WHC & 2765 & 11 & $1.7000 E+01$ & $1.5455 E+00$ & $4.4624 E+00$ & $2.0000 \mathrm{E}-01$ & $1.5000 E+01$ \\
\hline KER & MCGEE & 10 & $2.1000 E+00$ & $2.1000 \mathrm{E}-01$ & , & $2.1000 \mathrm{E}-01$ & $2.1000 E-01$ \\
\hline WHC & 202AL & 9 & $1.8846 E+00$ & $2.0940 \mathrm{E}-01$ & $9.0000 E-04$ & 2.0820E-01 & $2.1000 E-01$ \\
\hline WHC & $2415 Y$ & 9 & $4.4161 \mathrm{E}+01$ & $4.9068 E+00$ & $6.8263 E+00$ & 2.0820E-01 & $1.4000 E+01$ \\
\hline WHC & 333 & 9 & $1.8882 \mathrm{E}+00$ & $2.0980 E-01$ & 6.0000E-04 & $2.0820 \mathrm{E}-01$ & 2.1000E-01 \\
\hline WHC & $105 \mathrm{KW}$ & 8 & $1.6656 E+00$ & $2.0820 E-01$ & $3.9825 E-09$ & $2.0820 E-01$ & $2.0820 E-01$ \\
\hline WHC & 377 & 8 & $1.6656 E+00$ & $2.0820 E-01$ & $3.9825 E-09$ & $2.0820 E-01$ & $2.0820 E-01$ \\
\hline WHC & 308 & 7 & $1.4574 E+00$ & $2.0820 E-01$ & $4.3016 E-09$ & $2.0820 E-01$ & $2.0820 E-01$ \\
\hline WHC & MIXTF & 7 & $4.9255 \mathrm{E}+01$ & $7.0364 E+00$ & $1.1364 E+00$ & $6.3710 E+00$ & $8.7000 E+00$ \\
\hline PNL & RTL & 6 & $1.2600 E+00$ & $2.1000 E-01$ & . & 2.1000E-01 & 2.1000E-01 \\
\hline WHC & $1706 \mathrm{~K}$ & 6 & $1.2492 E+00$ & $2.0820 \mathrm{E}-01$ & 3.3320E-09 & 2.0820E-01 & 2.0820E-01 \\
\hline WHC & 2258 & 6 & $1.2600 E+00$ & $2.1000 \mathrm{E}-01$ & 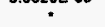 & 2.1000E-01 & 2.1000E-01 \\
\hline WHC & $241 A P$ & 6 & $4.1628 \mathrm{E}+00$ & $6.9380 \mathrm{E}-01$ & $8.2749 \mathrm{E}-01$ & 2.0820E-01 & $2.2100 E+00$ \\
\hline WHC & $241 B X$ & 6 & $1.1864 E+00$ & $1.9773 E-01$ & 9.3815E-02 & $3.0000 \mathrm{E}-02$ & $3.2180 E-01$ \\
\hline PNL & 329 & 5 & $1.0428 \mathrm{E}+00$ & $2.0856 E-01$ & $8.0498 E-04$ & 2.0820E-01 & $2.1000 \mathrm{E}-01$ \\
\hline vou & UURD & 5 & $1.0400 \mathrm{E}+00$ & $2.0800 E-01$ & . & 2.0BOOE-01 & $2.0800 \mathrm{E}-01$ \\
\hline WHC & $109 \mathrm{~N}$ & 5 & $1.0410 \mathrm{E}+00$ & $2.0820 \mathrm{E}-01$ & $0.0000 E+00$ & 2.0820E-01 & $2.0820 E-01$ \\
\hline WHC & 241AN & 5 & $1.1542 \mathrm{E}+00$ & $2.3084 E-01$ & $5.0848 \mathrm{E}-02$ & 2.0800E-01 & $3.2180 E-01$ \\
\hline WHC & $241 U$ & 5 & $1.0408 \mathrm{E}+00$ & $2.0816 E-01$ & $8.9443 \mathrm{E}-05$ & $2.0800 \mathrm{E}-01$ & $2.0820 \mathrm{E}-01$ \\
\hline KEH & 2312 & 4 & $1.1760 E+01$ & $2.9400 E+00$ & $0.0000 E+00$ & $2.9400 \mathrm{E}+\infty 0$ & $2.9400 E+00$ \\
\hline PNL & ISV & 4 & $1.1790 \mathrm{E}+00$ & $2.9475 \mathrm{E}-01$ & $5.6500 \mathrm{E}-02$ & $2.1000 \mathrm{E}-01$ & $3.2300 E-01$ \\
\hline WHC & $241 B$ & 4 & $9.3162 E+00$ & $2.3291 \mathrm{E}+00$ & $4.2473 E+00$ & $2.0000 \mathrm{E}-01$ & $8.7000 \mathrm{E}+\infty$ \\
\hline WHC & $2415 x$ & 4 & $8.9246 E+00$ & $2.2312 E+00$ & $4.0459 \mathrm{E}+00$ & 2.0820E-01 & $8.3000 E+00$ \\
\hline WHC & $241 T Y$ & 4 & 8.3280E-01 & $2.0820 \mathrm{E}-01$ & $0.0000 E+00$ & $2.0820 E-01$ & $2.0820 \mathrm{E}-01$ \\
\hline WHC & 244AR & 4 & $8.3280 \mathrm{E}-01$ & $2.0820 E-01$ & $0.0000 E+00$ & $2.0820 \mathrm{E}-01$ & $2.0820 \mathrm{E}-01$ \\
\hline WHC & 100KE & 3 & $1.1382 E+00$ & 3.7940E-01 & $3.0366 \mathrm{E}-01$ & $2.0000 E-01$ & $7.3000 \mathrm{E}-01$ \\
\hline
\end{tabular}


WHC-SD-W-242-ES-004, Rev. 0

TABLE 36

SUMMARY OF WASTE VOLUMES BY GENERATOR

SORTED IN ASCENDING ORDER BASED ON CONTAINER COUNT

(* INDICATES DATA EXHIBITED NEGATIVE VARIANCE)

(2 SHEETS)

\begin{tabular}{|c|c|c|c|c|c|c|c|}
\hline COMPANY & GENERATOR & COUNT & ACCUM VOLUME, CUM & AVG VOLUME, CUM & STO DEV & MIN VOLUME, CUM & MAX VOLUME, CUM \\
\hline WHC & 1706KE & 3 & $6.2460 \mathrm{E}-01$ & $2.0820 \mathrm{E}-01$ & * & $2.0820 E-01$ & $2.0820 E-01$ \\
\hline WHC & $2706 \mathrm{~T}$ & 3 & $6.2460 \mathrm{E}-01$ & $2.0820 E-01$ & - & $2.0820 E-01$ & $2.0820 E-01$ \\
\hline WHC & 271CR & 3 & $9.8482 E+00$ & $3.2827 \mathrm{E}+00$ & $5.4803 E+00$ & $3.0000 \mathrm{E}-02$ & $9.6+00 E+D 0$ \\
\hline PNL & 326 & 2 & 4.1640E-01 & $2.0820 \mathrm{E}-01$ & $0.0000 E+00$ & $2.0820 E-01$ & $2.0820 E-01$ \\
\hline PNL & 3720 & 2 & 4.1720E-01 & $2.0860 E-01$ & $5.6569 \mathrm{E}-04$ & $2.0820 E-01$ & $2.0900 \mathrm{E}-01$ \\
\hline PNL. & 3745 & 2 & $4.2000 E-01$ & 2.1000E-01 & $0.0000 \mathrm{E}+00$ & $2.1000 \mathrm{E}-01$ & $2.1000 E-01$ \\
\hline WHC & $1015 Y$ & 2 & $1,7000 E+01$ & $8.5000 E+00$ & $2.8284 E-01$ & $8.3000 E+00$ & $8.7000 E+00$ \\
\hline WHE & 105KE & 2 & $1.1109 \mathrm{E}+01$ & $5.5545 E+00$ & $7.5583 E+00$ & $2.1000 \mathrm{E}-01$ & $1.0899 \mathrm{EE}+01$ \\
\hline WHC & 163PAD & 2 & 4.1640E-01 & $2.0820 \mathrm{E}-01$ & $0.0000 E+00$ & $2.0820 E-01$ & $2.0820 E-01$ \\
\hline WHC & $200 \mathrm{~W}$ & 2 & 6.4360E-01 & $3.2180 E-01$ & $0.0000 E+00$ & 3.2180E-01 & $3.2180 \mathrm{E}-01$ \\
\hline WHC & $241 \mathrm{AW}$ & 2 & $8.5080 E+00$ & $4.2540 \mathrm{E}+00$ & $5.7219 \mathrm{E}+00$ & $2.0800 E-01$ & 8. $3000 \mathrm{E}+00$ \\
\hline WHE & $241 A X$ & 2 & $4.2000 \mathrm{E}-01$ & $2.1000 E-01$ & $0.0000 E+00$ & $2.1000 E-01$ & $2.1000 \mathrm{E}-01$ \\
\hline WHC & 241BY & 2 & $4.1000 \mathrm{E}-01$ & $2.0500 \mathrm{E}-01$ & $7.0711 E-03$ & $2.0000 \mathrm{E}-01$ & $2.1000 E-01$ \\
\hline WHC & $242 \mathrm{~S}$ & 2 & $1.5300 \mathrm{E}+01$ & $7.6500 E+00$ & $9.1924 E-01$ & $7.0000 \mathrm{E}+00$ & 8. $3000 E+00$ \\
\hline WHC & 2710 & 2 & $4.0000 E-01$ & $2.0000 \mathrm{E}-01$ & $0.0000 E+00$ & $2.0000 E-01$ & $2.0000 E-01$ \\
\hline WHC & 340 & 2 & 4.2000E-01 & $2.1000 \mathrm{E}-01$ & $0.0000 E+00$ & 2.1000E-01 & $2.1000 \mathrm{E}-01$ \\
\hline PNL & 1234 & 1 & $3.2800 E-01$ & 3.2800E-01 & $0.0000 E+00$ & $3.2800 E-01$ & $3.2800 \mathrm{E}-01$ \\
\hline SLA & SLACU & 1 & $2.0820 \mathrm{E}-01$ & $2.0820 \mathrm{E}-01$ & $0.0000 \mathrm{E}+00$ & 2.0820E-01 & $2.0820 \mathrm{E}-01$ \\
\hline WHC & $105 \mathrm{H}$ & 1 & $3.2180 \mathrm{E}-01$ & $3.2180 E-01$ & $0.0000 E+\infty$ & $3.2180 E-01$ & $3.2180 \mathrm{E}-01$ \\
\hline WHC & 151ER & 1 & $2.0820 E-01$ & $2.0820 \mathrm{E}-01$ & $0.0000 \mathrm{E}+00$ & 2.0820E-01 & $2.0820 E-01$ \\
\hline WHC & 204AR & 1 & $1.1200 E+00$ & $1.1200 E+00$ & $0.0000 E+00$ & $1.1200 E+00$ & $1.1200 E+00$ \\
\hline WHC & $231 Z$ & $i$ & $3.1400 E+00$ & $3.1400 \mathrm{E}+00$ & $0.0000 E+00$ & $3.1400 E+00$ & $3.1400 \mathrm{E}+00$ \\
\hline WHC & 2400 & 1 & $2.0820 \mathrm{E}-01$ & $2.0820 \mathrm{E}-01$ & $0.0000 E+00$ & $2.0820 \mathrm{E}-01$ & $2.0820 E-01$ \\
\hline WHC & $241 A Z$ & 1 & $8.3000 E+00$ & $8.3000 E+\infty 0$ & $0.0000 E+00$ & $8.3000 \mathrm{E}+00$ & $8.3000 E+00$ \\
\hline WHC & $241 \mathrm{EW}$ & 1 & $2.0820 E-01$ & $2.0820 \mathrm{E}-01$ & $0.0000 E+00$ & $2.0820 E-0 t$ & $2.0820 E-01$ \\
\hline WHC & $243 G$ & 1 & $2.2100 \mathrm{E}+00$ & $2.2100 E+00$ & $0.0000 E+00$ & $2.2100 E+00$ & $2.2100 E+\infty 0$ \\
\hline WHC & $2713 \mathrm{~W}$ & 1 & $2.0820 \mathrm{E}-01$ & $2.0820 E-01$ & $0.0000 E+00$ & $2.0820 E-01$ & $2.0820 E-01$ \\
\hline WHC & $271 T$ & 1 & $1.7925 E+01$ & $1.7925 E+01$ & $0.0000 E+00$ & $1.7925 E+01$ & $1.7925 E+0 t$ \\
\hline WHC & $276 \mathrm{U}$ & 1 & $2.0000 E-01$ & $2.0000 \mathrm{E}-01$ & $0.0000 E+00$ & $2.0000 E-01$ & $2.0000 E-01$ \\
\hline WHC & 2ETF & 1 & $2.1000 E-01$ & $2.1000 E-01$ & $0.0000 E+00$ & $2.1000 E-01$ & $2.1000=-01$ \\
\hline WHC & 309 & 1 & $2.0820 E-01$ & $2.0820 E-01$ & $0.0000 E+00$ & $2.0820 E-01$ & $2.0820 E-01$ \\
\hline WHC & 313 & 1 & $2.0000 E-01$ & $2.0000 E-01$ & $0.0000 E+00$ & $2.0000 E-01$ & $2.0000 \mathrm{E}-01$ \\
\hline WHC & 321 & 1 & $1.1000 E-01$ & $1.1000 \mathrm{E}-01$ & $0.0000 E+00$ & $1.1000 \mathrm{E}-01$ & $1.1000 E-01$ \\
\hline WHC & $399-3-1$ & 1 & $2.0000 E-01$ & $2.0000 \mathrm{E}-0.1$ & $0.0000 E+\infty$ & $2.0000 E-01$ & 2.0000 E-01 \\
\hline WHC & T PLANT & $i$ & $6.3700 E+00$ & $6.3700 E+00$ & $0.0000 E+00$ & $6.3700 E+00$ & $6.3700 E+00$ \\
\hline
\end{tabular}


WHC-SD-W-242-ES-004, Rev. 0

TABLE 37

SUMMARY OF GROSS CONTAINER WEIGHTS BY GENERATOR

SORTED IN ASCENDING ORDER BASED ON CONTAINER COUNT

(2 SHEETS)

\begin{tabular}{|c|c|c|c|c|c|}
\hline COMPANY & GENERATOR & COUNT & ACCUM GROSS WT, KG & AVG GROSS WT, KG & STD DEV \\
\hline WHC & $2345 Z$ & 455 & $9.5372 \mathrm{E}+04$ & $2.0961 E+02$ & $5.7906 \mathrm{E}+02$ \\
\hline WHC & $100 N$ & 408 & $3.9626 \mathrm{E}+04$ & $9.7122 \mathrm{E}+01$ & $1.8506 E+01$ \\
\hline LBt & LBLAB & 215 & $2.1975 E+04$ & $1.0221 E+02$ & 8.0835E+01 \\
\hline ANL & ARGON & 210 & $3.6609 \mathrm{E}+04$ & $1.7433 E+02$ & $9.4417 \mathrm{E}+01$ \\
\hline BATC & BATCO & 205 & $2.9859 E+04$ & $1.4565 \mathrm{E}+02$ & $3.7277 \mathrm{E}+01$ \\
\hline WHC & 2225 & 144 & $1.5469 \mathrm{E}+04$ & $1.0742 E+02$ & $2.1566 \mathrm{E}+02$ \\
\hline ALB & ALBNY & 126 & $3.8596 E+04$ & $3.0632 E+02$ & $1.0113 E+02$ \\
\hline WHC & $202 A$ & 95 & $1.3077 \mathrm{E}+04$ & $1.3765 \mathrm{E}+02$ & $1.6751 \mathrm{E}+02$ \\
\hline WHC & $221 \mathrm{~T}$ & 69 & $1.0924 \mathrm{E}+04$ & $1.5832 E+02$ & $3.6980 E+02$ \\
\hline BAPL & BAPL & 49 & $5.3681 \mathrm{E}+03$ & $1.0955 E+02$ & $4.6204 E+01$ \\
\hline BNL & BNLAB & 49 & $3.8716 \mathrm{E}+03$ & $7.9013 E+01$ & $7.0808 \mathrm{E}+\infty 0$ \\
\hline WHC & $224 \mathrm{U}$ & 44 & $5.2904 \mathrm{E}+03$ & $1.2024 E+02$ & $9.7316 \mathrm{E}+01$ \\
\hline FERM & NALAB & 37 & $6.7408 \mathrm{E}+03$ & $1.8218 E+02$ & $4.3869 E+01$ \\
\hline WHC & $303 K$ & 35 & $5.2570 E+03$ & $1.5020 E+02$ & 1.3295E+01 \\
\hline PSN & PSNS & 34 & $7.0369 E+04$ & $2.0697 E+03$ & $7.0047 E+01$ \\
\hline PNL & 331 & 30 & $3.7377 \mathrm{E}+03$ & $1.2459 E+02$ & $2.3826 \mathrm{E}+01$ \\
\hline WHC & $271 B$ & 28 & $4.2506 \mathrm{E}+03$ & $1.5181 E+02$ & $3.3388 \mathrm{E}+02$ \\
\hline WHC & 163PA & 26 & $1.9930 E+03$ & $7.6655 E+01$ & $3.5903 \mathrm{E}+01$ \\
\hline WHC & 2415 & 26 & $1.8882 \mathrm{E}+04$ & $7.2624 \mathrm{E}+02$ & $9.5800 E+02$ \\
\hline PNL & $305 B$ & 23 & $2.1294 E+03$ & $9.2581 E+01$ & $4,1294 E+01$ \\
\hline WHC & $221 \mathrm{~B}$ & 22 & $2.4610 E+03$ & $1.1187 \mathrm{E}+02$ & $7.9070 \mathrm{E}+01$ \\
\hline WHC & 221TS & 18 & $5.8605 E+03$ & $3.2558 \mathrm{E}+02$ & $9.5032 E+01$ \\
\hline WHC & $241 A$ & 18 & $1.6604 \mathrm{E}+04$ & $9.2244 E+02$ & $9.7057 E+02$ \\
\hline PNL & 325 & 17 & $2.1889 E+03$ & $1.2876 \mathrm{E}+02$ & $6.6234 \mathrm{E}+01$ \\
\hline WHC & $242 \mathrm{~A}$ & 17 & $2.9092 \mathrm{E}+04$ & $1.7113 E+03$ & $1.9033 E+03$ \\
\hline WHC & BGJAE & 17 & $2.1568 \mathrm{E}+03$ & $1.2687 E+02$ & $8.5395 E+01$ \\
\hline WHC & $1310 \mathrm{P}$ & 15 & $1.6919 E+03$ & $1.1280 E+02$ & $4.8708 E+01$ \\
\hline WHC & $105 \mathrm{~N}$ & 14 & $9.6935 \mathrm{E}+02$ & $6.9239 \mathrm{E}+01$ & $1.4585 E+01$ \\
\hline WHC & 241AY & 14 & $1.3920 E+03$ & $9.9430 E+01$ & 6.6877E+01 \\
\hline WHC & 241TX & 12 & $5.0996 \mathrm{E}+03$ & $4.2497 E+02$ & $6.9126 E+02$ \\
\hline WHC & 324 & 12 & $1.0580 E+03$ & $8.8170 E+01$ & $4.2874 \mathrm{E}+01$ \\
\hline PNL & 324 & 11 & $3.0030 E+03$ & $2.7300 \mathrm{E}+02$ & $0.0000 E+00$ \\
\hline PNL & LSLII & 11 & $1.3791 \mathrm{E}+03$ & $1.2537 \mathrm{E}+02$ & $1.9194 E+01$ \\
\hline WHC & $163 \mathrm{~N}$ & 11 & $8.4406 E+02$ & $7.6733 E+01$ & $2.3662 E+01$ \\
\hline WHC & 2025 & 11 & $1.6015 \mathrm{E}+03$ & $1.4559 \mathrm{E}+02$ & $1.3946 E+01$ \\
\hline WHC & $241 C$ & 11 & $1.3427 \mathrm{E}+04$ & $1.2206 \mathrm{E}+03$ & $1.1278 E+03$ \\
\hline WHC & $241 \mathrm{~T}$ & 11 & $8.5640 E+03$ & $7.7855 \mathrm{E}+02$ & $1.2004 E+03$ \\
\hline WHC & 2765 & 11 & $9.0600 E+03$ & $8.2364 E+02$ & $2.3630 \mathrm{E}+03$ \\
\hline KER & MCGEE & 10 & $1.4909 E+03$ & $1.4909 E+02$ & $2.7041 E+01$ \\
\hline WHC & $202 \mathrm{AL}$ & 9 & $5.1520 \mathrm{E}+02$ & $5.7244 E+01$ & $1.2618 E+01$ \\
\hline WHC & $2415 Y$ & 9 & $2.3594 E+04$ & $2.6216 E+03$ & $3.7069 E+03$ \\
\hline WHC & 333 & 9 & $1.1761 E+03$ & $1.3067 E+02$ & 1.3897E+02 \\
\hline WHC & $105 \mathrm{KW}$ & 8 & $6.0389 E+02$ & $7.5486 E+01$ & $2.9114 E+01$ \\
\hline WHC & 377 & 8 & 4.7990E+02 & $5.9988 \mathrm{E}+01$ & $6.7226 \mathrm{E}+00$ \\
\hline WHC & 308 & 7 & $5.1690 E+02$ & $7.3843 E+01$ & $9.2552 \mathrm{E}+00$ \\
\hline WHC & MIXTF & 7 & $2.6488 E+04$ & $3.7840 E+03$ & $9.3305 E+02$ \\
\hline PNL & RTL & 6 & $7.4004 E+02$ & $1.2334 \mathrm{E}+02$ & $3.7188 E+00$ \\
\hline WHC & $1706 \mathrm{~K}$ & 6 & $4.0518 E+02$ & $6.7530 E+01$ & $1.8299 \mathrm{E}+01$ \\
\hline WHC & 2258 & 6 & $9.6342 \mathrm{E}+02$ & $1.6057 \mathrm{E}+02$ & $8.2809 E+01$ \\
\hline WHC & 241AP & 6 & $1.3920 E+03$ & $2.3200 \mathrm{E}+02$ & $2.8879 \mathrm{E}+02$ \\
\hline WHC & $241 B X$ & 6 & $7.5340 E+02$ & $1.2557 \mathrm{E}+02$ & $1.3256 \mathrm{E}+02$ \\
\hline PNL & 329 & 5 & $7.5568 \mathrm{E}+02$ & $1.5114 \mathrm{E}+02$ & $4.0353 E+01$ \\
\hline UOU & UURD & 5 & $4.7764 E+02$ & $9.5528 \mathrm{E}+01$ & $8.8966 E+00$ \\
\hline WHC & $109 \mathrm{~N}$ & 5 & $3.4400 \mathrm{E}+02$ & $6.8800 \mathrm{E}+01$ & $7.1903 E+00$ \\
\hline WHC & $241 \mathrm{AN}$ & 5 & $6.7300 E+02$ & $1.3460 E+02$ & $1.3644 E+02$ \\
\hline WHC & $241 \mathrm{U}$ & 5 & $3.4000 \mathrm{E}+02$ & $6.8000 E+01$ & $4.4721 E+\infty 0$ \\
\hline KEH & $231 Z$ & 4 & $1.5363 \mathrm{E}+03$ & $3.8408 E+02$ & $1.9744 E+01$ \\
\hline PNL & ISV & 4 & $3.5698 \mathrm{E}+02$ & $8.9245 E+01$ & $2.1267 E+01$ \\
\hline WHC & $241 B$ & 4 & $1.4120 \mathrm{E}+03$ & $3.5300 \mathrm{E}+02$ & $5.7339 \mathrm{E}+02$ \\
\hline WHC & $241 s x$ & 4 & $3.2820 \mathrm{E}+03$ & $8.2050 \mathrm{E}+02$ & $1.4686 E+03$ \\
\hline WHC & $241 T Y$ & 4 & $1.5200 E+02$ & $3.8000 E+01$ & $8.8694 E+00$ \\
\hline WHC & 244AR & 4 & $9.9500 E+02$ & $2.4875 E+02$ & $3.3019 E+01$ \\
\hline WHC & 100KE & 3 & $2.0699 \mathrm{E}+02$ & $6.8997 E+01$ & $1.0000 E+00$ \\
\hline
\end{tabular}

MIN GROSS WT, KG

$9.5000 E+00$

2.9940E+01

$4.9000 E+01$

$5.1800 E+01$

$5.6820 \mathrm{E}+01$

$3.5000 E+01$

$8.5000 E+01$

$2.1400 E+01$

4.3600E+01

$6.1200 E+01$

$6.3600 E+01$

$3.6290 E+01$

$7.0800 E+01$

$1.0900 \mathrm{E}+02$

$1.7710 \mathrm{E}+03$

$2.9500 E+01$

$1.2300 \mathrm{E}+01$

$1.1000 \mathrm{E}+01$

$5.6000 \mathrm{E}+01$

4.3200E +01

$3.1980 \mathrm{E}+01$

$1.4742 E+02$

$6.0000 E+01$

$2.3850 E+01$

$1.3000 \mathrm{E}+01$

4.5350E+01

$9.0000 E+00$

$4.5360 \mathrm{E}+01$

2.3000E +01

4.5600E+01

6.1190E+01

$2.7300 E+02$

$1.0270 E+02$

$4.3000 E+01$

$1.1070 E+02$

4.8990E +01

$4.7000 E+01$

6.3000E+01

$1.0201 \mathrm{E}+02$

$3.4500 E+01$

5.2010E +01

$3.3640 E+01$

$5.0000 E+01$

4.7700E +01

$5.3100 E+01$

2.2500E+03

$1.1900 E+02$

$4.8000 E+01$

$5.6060 E+01$

$5.8000 E+01$

$1.1400 E+0 t$

1.0780E+02

8.1600E+01

$5.7000 E+01$

6.1000E+01

$6.3000 E+01$

$3.6683 E+02$

$6.0330 E+01$

$5.9000 E+01$

$5.9000 \mathrm{E}+01$

$2.9000 E+01$

2.1500E +02

$6.8000 \mathrm{E}+01$
MAX GROSS WT, KG

$5.3600 E+03$

1.8416E+02

$1.1998 E+03$

$3.5450 \mathrm{E}+02$

$3.9040 E+02$

$2.2968 E+03$

$4.8300=+02$

$1.4550 \mathrm{C}+03$

$3.1561 E+03$

$3.7830 \mathrm{E}+02$

$9.5340 E+01$

4.5300E+02

2.2702E+02

1.7400E +02

2.1790E+03

1.7010E+02

1.8297E+03

1.4501E+02

2.9690E+03

2.3766E+02

$2.8000 E+02$

4.4057E+02

$2.8830 E+03$

2.0450E+02

$5.3760 E+03$

3.4092E+02

1.5898E+02

9.2000E+01

2.1200E+02

$2.5540 E+03$

1.7241E+02

2.7300E+02

1.4523E+02

$1.0401 \mathrm{E}+02$

$1.6720 \mathrm{E}+02$

2.6620 $E+0$

2.7920E+0

$7.9480 \mathrm{E}+03$

1.9899E+02

$7.7700 E+01$

$7.5700 E+03$

4.4089E+02

t.3600E+02

$7.2700 E+01$

$7.85005+01$

$4.8150 \mathrm{E}+03$

$12900 E+02$

$9.1000 E+0$

$2.4548 \mathrm{E}+02$

$7.9400 E+02$

$3.4100 E+02$

2.0640E+02

1.0340E+02

7. $6000 E+01$

$3.7700 \mathrm{E}+02$

$7.4000 E+01$

4.1223E+02

$1.1068 \mathrm{E}+02$

1.2130E+03

$3.0230 E+03$

$4.9000 E+01$

$2.9400 E+02$

$7.0000 E+01$ 
WHC-SD-W-242-ES-004, Rev. 0

TABLE 37

SUMMARY OF GROSS CONTAINER WEIGHTS BY GENERATOR SORTED IN ASCENDING ORDER BASED ON CONTAINER COUNT (2 SHEETS)

\begin{tabular}{|c|c|c|c|c|c|c|c|}
\hline COMPANY & GENERATOR & COUNT & ACCUM GROSS WT, KG & AVG GROSS WT, KG & STD DEV & MIN GROSS WT, KG & MAX GROSS WT, KG \\
\hline WHC & 1706KE & 3 & $2.6200 \mathrm{E}+\mathrm{O} 2$ & $8.7333 E+01$ & $1.7786 E+01$ & $6.7000 E+01$ & $1.0000 E+02$ \\
\hline WHC & $2706 \mathrm{~T}$ & 3 & $2.6251 E+02$ & $8.7503 E+01$ & $4.4353 \mathrm{E}+01$ & $4.9000 E+01$ & $1.3600 E+02$ \\
\hline WHC & $271 \mathrm{CR}$ & 3 & $2.5160 E+03$ & $8.3867 E+02$ & $1.3611 E+03$ & $2.5000 E+01$ & $2.4100 E+03$ \\
\hline PNL & 326 & 2 & $1.0980 E+02$ & $5.4900 \mathrm{E}+01$ & $1.3435 E+01$ & $4.5400 E+01$ & $6.4400 E+01$ \\
\hline PNL & 3720 & 2 & $2.0871 \mathrm{E}+02$ & $1.0436 E+02$ & $7.1488 E+\infty 0$ & $9.9300 \mathrm{E}+01$ & $1.0941 E+02$ \\
\hline PNL & 3745 & 2 & $2.0749 E+02$ & $1.0375 E+02$ & $2.0160 E+01$ & $8.9490 E+01$ & $1.1800 E+02$ \\
\hline WHC & $1015 Y$ & 2 & $5.7740 E+03$ & $2.8870 E+03$ & $9.6167 E+01$ & $2.8190 E+03$ & $2.9550 E+03$ \\
\hline WHC & 105KE & 2 & $5.6402 E+03$ & $2.8201 E+03$ & $3.8185 E+03$ & $1.2002 E+02$ & $5.5202 E+03$ \\
\hline WhC & 163PAD & 2 & $1.4800 E+02$ & $7.4000 E+01$ & $1.4142 E+01$ & $6.4000 E+01$ & $8.4000 E+01$ \\
\hline WHC & $200 \mathrm{~W}$ & 2 & $5.4800 \mathrm{E}+02$ & $2.7400 \mathrm{E}+02$ & $4.2426 \mathrm{E}+\infty 0$ & $2.7100 E+02$ & $2.7700 E+02$ \\
\hline Whic & 241AW & 2 & $1.0320 \mathrm{E}+03$ & $5.1600 E+02$ & $6.2367 E+02$ & $7.5000 E+01$ & $9.5700 E+02$ \\
\hline WHC & $241 A X$ & 2 & 3.1080E+02 & $1.5540 E+02$ & $3.7335 E+01$ & $1.2900 E+02$ & $1.8180 E+02$ \\
\hline WhC & 241BY & 2 & $2.8501 \mathrm{E}+02$ & $1.4251 \mathrm{E}+02$ & $1.2799 E+02$ & $5.2000 E+01$ & $2.3301 E+02$ \\
\hline WHC & $242 S$ & 2 & $2.2300 \mathrm{E}+03$ & $1.1150 E+03$ & $2.8426 E+02$ & $9.1400 E+02$ & $1.3160 E+03$ \\
\hline WHC & 2710 & 2 & $2.4970 E+02$ & $1.2485 E+02$ & $6.1023 \mathrm{E}+01$ & $8.1700 E+01$ & $1.6800 E+02$ \\
\hline WHC & 340 & 2 & $1.2303 \mathrm{E}+02$ & $6.1515 E+01$ & $2.2790 E+01$ & 4.54COE+01 & $7.7630 E+01$ \\
\hline PNL & 1234 & 1 & $3.1706 \mathrm{E}+02$ & $3.1706 E+02$ & $0.0000 E+00$ & $3.1706 \mathrm{E}+02$ & $3.1706 \mathrm{E}+02$ \\
\hline SLA & SLACU & $t$ & $1.7000 \mathrm{E}+02$ & 1.7000E +02 & $0.0000 E+00$ & $1.7000 E+02$ & 1.7000E +02 \\
\hline WhC & $105 \mathrm{H}$ & 1 & $9.0000 E+01$ & $9.0000 E+01$ & $0.0000 E+00$ & $8.0000 E+01$ & $9.0000 E+01$ \\
\hline WHC & 151ER & 1 & $1.0900 \mathrm{E}+02$ & $1.0900 E+02$ & $0.0000 E+\infty 0$ & $1.0900 E+02$ & $1.0900 E+02$ \\
\hline WHC & 204AR & 1 & $2.6000 \mathrm{E}+02$ & $2.6000 E+02$ & $0.0000 E+00$ & $2.6000 E+02$ & $2.6000 E+02$ \\
\hline WhC & 2312 & 1 & $3.8800 E+02$ & $3.8800 E+02$ & $0.0000 E+00$ & $3.8800 \mathrm{E}+02$ & $3.8800 E+02$ \\
\hline WHC & 2400 & 1 & $5.4000 E+01$ & $5.4000 E+01$ & $0.0000 E+00$ & 5.4000E+01 & $5.4000 E+01$ \\
\hline WHC & $241 A Z$ & 1 & $1.4302 E+03$ & $1.4302 E+03$ & $0.0000 E+00$ & $1.4302 E+03$ & $1.4302 E+03$ \\
\hline WHC & $241 \mathrm{EW}$ & 1 & $5.6000 E+01$ & $5.6000 E+01$ & $0.0000 E+00$ & $5.6000 E+01$ & $5.6000 E+01$ \\
\hline WHC & 2436 & 1 & $4.9900 \mathrm{E}+02$ & $4.9900 E+02$ & $0.0000 E+00$ & $4.9900 \mathrm{E}+02$ & $4.9900 E+02$ \\
\hline WHC & $2713 W$ & 1 & $5.1500 E+01$ & $5.1500 E+01$ & $0.0000 \mathrm{E}+00$ & 5.1500E+01 & $5.1500 E+01$ \\
\hline WHC & $271 \mathrm{~T}$ & 1 & $6.2728 E+03$ & $6.2728 E+03$ & $0.0000 E+00$ & $6.2728 \mathrm{E}+03$ & $6.2728 \mathrm{E}+03$ \\
\hline WHC & $276 \mathrm{U}$ & 1 & $5.6700 E+01$ & $5.6700 E+01$ & $0.0000 E+00$ & $5.6700 E+01$ & $5.6700 E+01$ \\
\hline WHC & $2 E T F$ & 1 & $1.8144 E+02$ & $4.8144 E+02$ & $0.0000 E+00$ & $1.8144 E+02$ & $1.8144 E+02$ \\
\hline WHC & 309 & 1 & 1.1800E+02 & $1.1800 E+02$ & $0.0000 E+00$ & $1.1800 E+02$ & $1.1800 E+02$ \\
\hline WHC & 313 & 1 & $5.5500 \mathrm{E}+01$ & $5.5500 E+01$ & $0.0000 E+00$ & $5.5500 \mathrm{E}+01$ & $5.5500 E+01$ \\
\hline WhC & 321 & 1 & $3.2700 E+01$ & $3.2700 E+01$ & $0.0000 E+00$ & $3.2700 E+01$ & $3.2700 E+01$ \\
\hline WHC & $399-3-1$ & 1 & $2.5000 \mathrm{E}+02$ & $2.5000 \mathrm{E}+02$ & $0.0000 \mathrm{E}+00$ & $2.5000 \mathrm{E}+02$ & $2.5000 \mathrm{E}+02$ \\
\hline WHC & T PLANT & 1 & $2.2950 E+03$ & $2.2950 \mathrm{E}+03$ & $0.0000 E+00$ & $2.2950 E+03$ & $2.2950 \mathrm{E}+03$ \\
\hline
\end{tabular}


TABLE 38

SUMMARY OF CONTAINER TARE WEIGHTS BY GENERATOR SORTED IN ASCENDING ORDER BASED ON CONTAINER COUNT

(2 SHEETS)

\begin{tabular}{|c|c|c|c|c|c|c|c|}
\hline COMPANY & GENERATOR & COUNT & ACCUM TARE WT, KG & AVG TARE WT, KC & STD DEV & MIN TARE WT, KG & MAX TARE WT, KG \\
\hline WHC & 23452 & 449 & $3.6486 \mathrm{E}+04$ & 8.1261E+01 & $2.2660 \mathrm{E}+02$ & $5.0000 E+00$ & $1.2250 E+03$ \\
\hline WHC & $100 \mathrm{~N}$ & 408 & $1.1005 E+04$ & $2.6973 E+01$ & 5.4458E-01 & $1.6000 E+01$ & $2.7000 E+01$ \\
\hline LBL & LBLAB & 215 & $5.5124 E+03$ & $2.5639 E+01$ & $1.2975 E+\infty 0$ & $2.0000 E+01$ & $3.7000 E+01$ \\
\hline ANL & ARGON & 210 & $5.8170 E+03$ & $2.7700 E+01$ & $2.3003 E_{-06}$ & $2.7700 E+01$ & $2.7700 E+01$ \\
\hline BATC & BATCO & 205 & $6.2918 E+03$ & $3.0692 \mathrm{E}+01$ & $3.9420 \mathrm{E}+\infty 0$ & $2.7000 E+01$ & $3.6360 E+01$ \\
\hline WHC & 2225 & 144 & $5.6664 E+03$ & $3.9350 E+01$ & $8.3285 E+01$ & $2.2700 E+01$ & $7.3860 E+02$ \\
\hline ALB & ALBNY & 126 & $4.9140 E+03$ & $3.9000 \mathrm{E}+01$ & $0.0000 E+\infty 0$ & $3.9000 E+01$ & $3.9000 E+01$ \\
\hline WHC & $202 \mathrm{~A}$ & 95 & $3.5617 E+03$ & $3.7492 \mathrm{E}+01$ & $7.7202 E+01$ & $1.6000 E+01$ & $7.3800 E+02$ \\
\hline WHC & $221 \mathrm{~T}$ & 69 & $2.4821 E+03$ & $3.5972 \mathrm{E}+01$ & $9.2621 E+01$ & $2.2700 E+01$ & $7.9380 E+02$ \\
\hline BAPL & BAPL & 49 & $1.4982 E+03$ & $3.0576 E+01$ & $1.2286 E+00$ & $3.0400 E+01$ & $3,9000 E+01$ \\
\hline BNL & BNLAB & 49 & $1.4455 E+03$ & $2.9500 E+01$ & $0.0000 E+00$ & $2.9500 E+01$ & $2.9500 E+01$ \\
\hline WHC & $224 U$ & 44 & $1.2710 E+03$ & $2.8886 E+01$ & $3.8653 E+00$ & $5.0000 E+00$ & $3.0000 E+01$ \\
\hline FERM & NALAB & 37 & $1.0380 E+03$ & $2.8054 E+01$ & $1.4519 E+00$ & $2.7000 E+01$ & $3.0000 E+01$ \\
\hline WHC & $303 K$ & 35 & $1.0850 \mathrm{E}+03$ & $3.1000 E+01$ & $0.0000 \mathrm{E}+00$ & $3.1000 E+01$ & $3.1000 E+01$ \\
\hline PSN & PSNS & 34 & $1.2342 E+04$ & $3.6300 E+02$ & $0.0000 E+00$ & $3.6300 \mathrm{E}+02$ & $3.6300 E+02$ \\
\hline PNL & 331 & 30 & $7.4400 \mathrm{E}+02$ & $2.4800 E+01$ & $2.9408 E+00$ & $2.1000 E+01$ & $2.7000 E+01$ \\
\hline WHC & $271 B$ & 28 & $1.9149 E+03$ & $6.8388 E+01$ & $2.2129 E+02$ & $5.0000 E+00$ & $1.1970 E+03$ \\
\hline WHC & $163 P A$ & 26 & $6.3000 \mathrm{E}+02$ & $2.4231 E+01$ & $7.8348 \mathrm{E}+00$ & $2.0000 E+00$ & $2.7000 E+01$ \\
\hline WHC & $241 S$ & 26 & $7.6490 \mathrm{E}+03$ & $2.9419 \mathrm{E}+02$ & $3.6891 E+02$ & $3.1000 E+01$ & $7.9400 E+02$ \\
\hline PNL & $305 \mathrm{~B}$ & 23 & $7.4986 E+02$ & $3.2603 E+01$ & $2.8944 E+01$ & $2.1000 E+01$ & $1.6466 \mathrm{E}+02$ \\
\hline WHC & $221 B$ & 22 & $5.2200 E+02$ & $2.3727 E+01$ & $5.4505 E+00$ & $1.6000 E+01$ & $2.9500 E+01$ \\
\hline WHC & $221 \mathrm{TS}$ & 18 & $4.8600 \mathrm{E}+02$ & $2.7000 E+01$ & $0.0000 E+00$ & $2.7000 \mathrm{E}+01$ & $2.7000 E+01$ \\
\hline WHC & $241 \mathrm{~A}$ & 18 & $6.6250 \mathrm{E}+03$ & $3.6806 E ̈+02$ & $3.9206 E+02$ & $1.6000 E+01$ & $7.9400 E+02$ \\
\hline WHC & $242 \mathrm{~A}$ & 17 & $9.5919 E+03$ & $5.6423 E+02$ & $5.9866 E+02$ & $5.0000 E+\infty 0$ & $1.2270 E+03$ \\
\hline WHC & BG3AE & 17 & $4.5900 E+02$ & $2.7000 E+01$ & $0.0000 E+00$ & $2.7000 E+01$ & $2.7000 E+01$ \\
\hline PNL & 325 & 16 & $5.3250 \mathrm{E}+02$ & 3.3281E+01 & $7.3920 \mathrm{E}+00$ & $1.6000 E+01$ & $4.1400 E+01$ \\
\hline WHC & $1310 \mathrm{P}$ & 15 & $3.5800 E+02$ & $2.3867 E+01$ & $7.1700 E+\infty 0$ & $2.0000 E+00$ & $2.7000 E+01$ \\
\hline WHC & $105 N$ & 14 & $4.0600 E+02$ & $2.9000 E+01$ & $3.7417 E+\infty 0$ & $1.6000 E+01$ & $3.0000 E+01$ \\
\hline WHC & 241AY & 14 & $4.1100 \mathrm{E}+02$ & $2.9357 E+01$ & $4.1064 E+\infty 0$ & $1.6000 E+01$ & $3.1000 E+01$ \\
\hline WHC & $241 T X$ & 12 & $2.0210 E+03$ & $1.6842 E+02$ & $2.1655 E+02$ & $2.2000 E+01$ & $7.9400 \mathrm{E}+02$ \\
\hline WHC & 324 & 12 & $3.2400 E+02$ & $2.7000 E+01$ & $0.0000 E+\infty 0$ & $2.7000 E+01$ & $2.7000 E+01$ \\
\hline PNL & 324 & 11 & $2.0020 \mathrm{E}+03$ & $1.8200 E+02$ & $0.0000 E+\infty 0$ & $1.8200 E+02$ & $1.8200 E+02$ \\
\hline PNL & LSLII & 11 & $2.8465 E+02$ & $2.5877 E+01$ & $4.4838 E+\infty 0$ & $2.1000 E+01$ & $3.0230 E+01$ \\
\hline WHC & $163 N$ & 11 & $3.1800 E+02$ & $2.8909 E+01$ & $1.5136 E+\infty 0$ & $2.7000 E+01$ & $3.0000 E+01$ \\
\hline WHC & $202 S$ & 11 & $3.3040 E+02$ & $3.0036 E+01$ & 1.2060E-01 & 3.0000E+01 & $3.0400 E+01$ \\
\hline WHC & $241 C$ & 11 & $5.3400 E+03$ & $4.8545 E+02$ & $4.5443 E+02$ & 1.6O0OE+04 & $1.2250 E+03$ \\
\hline WHC & $241 T$ & 11 & $2.6300 \mathrm{E}+03$ & $2.3909 E+02$ & $3.5640 E+02$ & $3.1000 E+01$ & $7.9400 \mathrm{E}+02$ \\
\hline WHC & 2765 & 11 & $2.8850 E+03$ & $2.6227 E+02$ & $7.6704 \mathrm{E}+02$ & $3.1000 \varepsilon+01$ & $2.5750 E+03$ \\
\hline KER & MCGEE & 10 & $2.7000 \mathrm{E}+02$ & $2.7000 E+01$ & $0.0000 E+00$ & $2.7000 E+01$ & $2.7000 \mathrm{E}+01$ \\
\hline WHC & $202 \mathrm{AL}$ & 9 & $2.7710 E+02$ & $3.0789 E+01$ & $2.3709 E+00$ & $2.8500 \mathrm{E}+01$ & $3.6300 E+01$ \\
\hline WHC & $241 S Y$ & 9 & $1.5869 E+04$ & $1.7632 E+03$ & $2.5542 E+03$ & $3.1000 E+01$ & $5.1680 E+03$ \\
\hline WHC & 333 & 9 & $2.3900 E+02$ & $2.6556 E+01$ & $1.3333 E+00$ & $2.3000 E+01$ & $2.7000 E+0 t$ \\
\hline WHC & $105 \mathrm{KW}$ & 8 & $2.4000 E+02$ & $3.0000 E+01$ & $0.0000 E+00$ & $3.0000 E+01$ & $3.0000 E+01$ \\
\hline WHC & 377 & 8 & $2.4000 \mathrm{E}+02$ & $3.0000 E+01$ & $0.0000 E+00$ & $3.0000 E+01$ & $3.0000 E+01$ \\
\hline WHC & 308 & 7 & $2.1700 \mathrm{E}+02$ & $3.1000 E+01$ & $0.0000 E+00$ & $3.1000 E+01$ & $3.1000 E+01$ \\
\hline WHC & MIXTF & 7 & $5.5580 \mathrm{E}+03$ & $7.9400 E+02$ & $0.0000 E+00$ & $7.9400 E+02$ & $7.9400 \mathrm{E}+02$ \\
\hline PNL & RTL & 6 & $1.3800 E+02$ & $2.3000 E+01$ & $3.0984 E+00$ & $2.1000 E+01$ & $2.7000 E+01$ \\
\hline WHC & $1706 \mathrm{~K}$ & 6 & $1.7300 E+02$ & $2.8833 E+01$ & $2.8577 E+00$ & $2.3000 E+01$ & $3.0000 E+01$ \\
\hline WHC & $225 B$ & 6 & $1.6200 E+02$ & $2.7000 \mathrm{E}+01$ & $0.0000 E+00$ & $2.7000 E+01$ & $2.7000 E+01$ \\
\hline WHC & 241AP & 6 & $6.2300 E+02$ & $1.0383 E+02$ & $1.1965 E+02$ & $2.2000 E+01$ & $2.9800 \mathrm{E}+02$ \\
\hline WHC & $241 B X$ & 6 & $1.5400 \mathrm{E}+02$ & $2.5667 E+01$ & $1.1413 E+01$ & $5.0000 E+00$ & $3.8000 E+01$ \\
\hline PNL & 329 & 5 & $1.1440 E+02$ & $2.2880 E+01$ & $2.4550 E+00$ & $2.1000 E+01$ & $2,7000 E+01$ \\
\hline UOU & UURD & 5 & $1.5000 E+02$ & $3.0000 E+01$ & $0.0000 E+00$ & $3.0000 E+01$ & $3.0000 \mathrm{E}+01$ \\
\hline WHC & $109 N$ & 5 & $1.5000 E+02$ & $3.0000 E+01$ & $0.0000 E+00$ & $3.0000 E+01$ & $3.0000 E+01$ \\
\hline WHC & 241AN & 5 & $3.4100 E+02$ & $6.8200 E+01$ & $7.9327 E+01$ & $3.1000 E+01$ & $2.1000 E+02$ \\
\hline WHC & $241 U$ & 5 & $1.5500 E+02$ & $3.1000 E+01$ & $0.0000 E+00$ & $3.1000 E+01$ & $3.1000 E+01$ \\
\hline $\mathrm{KEH}$ & 2312 & 4 & $8.1720 E+02$ & $2.0430 E+02$ & $0.0000 E+00$ & $2.0430 E+02$ & $2.0430 E+02$ \\
\hline PNL. & ISV & 4 & $1.3200 E+02$ & $3.3000 E+01$ & $4.0000 E+00$ & $2.7000 E+0 t$ & $3.5000 E+01$ \\
\hline WHC & $241 B$ & 4 & $8.7800 E+02$ & $2.1950 E+02$ & $3.8302 E+02$ & $2.2000 E+01$ & $7.9400 E+02$ \\
\hline WHC & $2415 x$ & 4 & $8.8700 E+02$ & $2.2175 E+02$ & $3.8150 E+02$ & $3.1000 E+01$ & $7.9400 E+02$ \\
\hline WHC & $241 \mathrm{TY}$ & 4 & $8.8000 E+01$ & $2.2000 E+01$ & $0.0000 E+00$ & $2.2000 E+01$ & $2.2000 E+01$ \\
\hline WHC & 244AR & 4 & $1.2400 E+02$ & $3.1000 E+01$ & $0.0000 E+00$ & $3.1000 E+01$ & $3.1000 E+01$ \\
\hline WHC & 100KE & 3 & $7.6000 E+01$ & $2.5333 E+01$ & $8.0829 E+00$ & $1.6000 \mathrm{E}+01$ & $3.0000 E+01$ \\
\hline
\end{tabular}


WHC-SD-W-242-ES-004, Rev. 0

TABLE 38

SUMMARY OF CONTAINER TARE WEIGHTS BY GENERATOR SORTED IN ASCENDING ORDER BASED ON CONTAINER COUNT

(2 SHEETS)

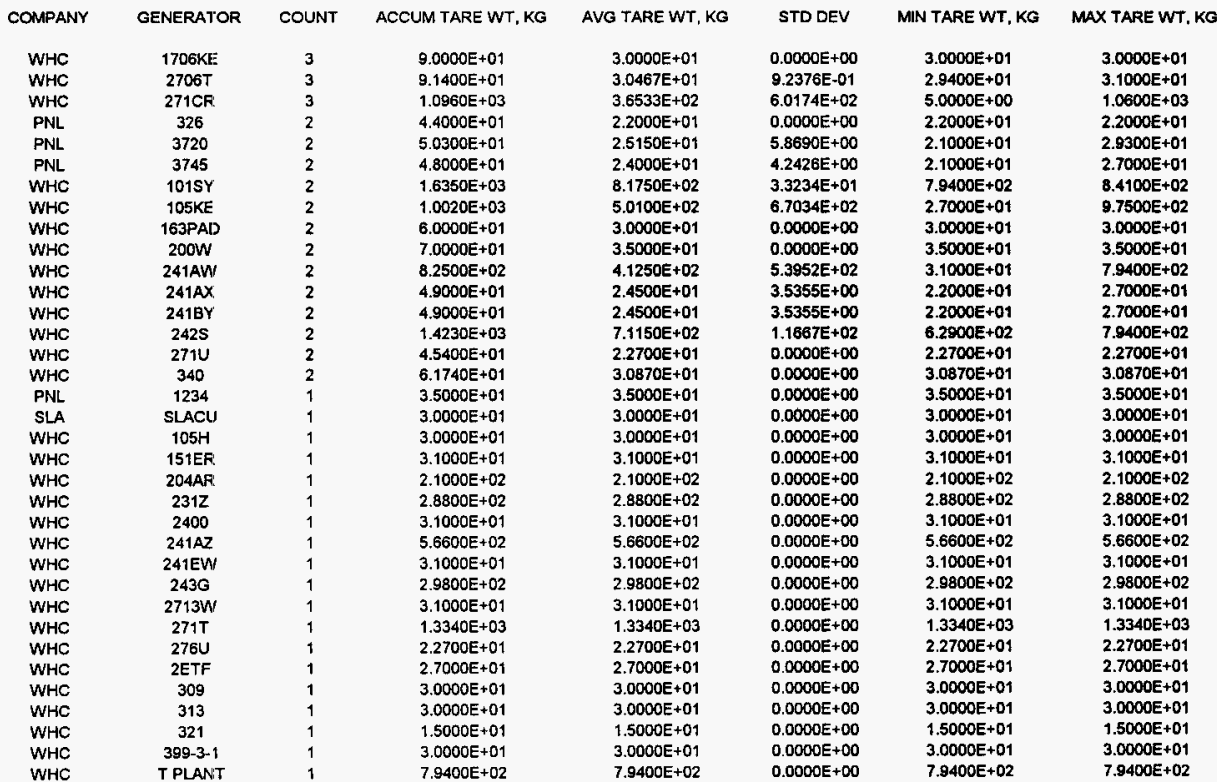


TABLE 39

SUMMARY OF WASTE WEIGHTS BY GENERATOR SORTED IN ASCENDING ORDER BASED ON CONTAINER COUNT

(2 SHEETS)

\begin{tabular}{|c|c|c|c|c|c|c|c|}
\hline COMPANY & GENERATOR & COUNT & ACCUM WASTE WT, KG & AVG WASTE WT, KG & STD DEV & MIN WASTE WT, KG & MAX WASTE WT, KG \\
\hline WHC & $2345 Z$ & 426 & $5.3039 E+04$ & $1.2450 E+02$ & $3.8860 \mathrm{E}+02$ & $4.0000 E+00$ & $4.1350 E+03$ \\
\hline WHC & $100 \mathrm{~N}$ & 403 & $2.9970 E+04$ & $7.4367 \mathrm{E}+01$ & $1.8387 E+01$ & $9.3000 E+00$ & $1.6100 E+02$ \\
\hline LBL & LBLAB & 215 & $1.5381 E+04$ & $7.1540 E+01$ & $2.8837 E+01$ & $2.5000 E+01$ & $3.9000 E+02$ \\
\hline ANL & ARGON & 210 & $3.0753 E+04$ & $1.4644 E+02$ & $9.4317 E+01$ & $2.4100 E+01$ & $3.2680 E+02$ \\
\hline BATC & BATCO & 205 & $2.2930 E+04$ & $1.1185 E+02$ & $3.8655 E+01$ & $2.0450 E+01$ & $3.5910 E+02$ \\
\hline WHC & $222 S$ & 144 & $9.7920 \mathrm{E}+03$ & $6.8000 E+01$ & $1.3717 E+02$ & $5.0000 E+\infty 0$ & $1.5582 E+03$ \\
\hline ALB & ALBNY & 126 & $3.3308 E+04$ & $2.6435 \mathrm{E}+02$ & $1.0123 E+02$ & $4.6000 E+01$ & $4.4400 E+02$ \\
\hline WHC & $202 A$ & 88 & $8.1968 E+03$ & $9.3146 E+01$ & $1.0538 E+02$ & $6.4000 E+00$ & $5.6200 E+02$ \\
\hline WHC & $221 \mathrm{~T}$ & 69 & $8.3136 E+03$ & $1.2049 \mathrm{E}+02$ & $2.7844 E+02$ & $1.5400 E+01$ & $2.3623 E+03$ \\
\hline BAPL & BAPL & 49 & $3.8699 \mathrm{E}+03$ & $7.8978 \mathrm{E}+01$ & $4.6001 E+01$ & $3.0800 \mathrm{E}+01$ & $3,4790 E+02$ \\
\hline BNL & BNLAB & 49 & $2.4215 E+03$ & $4.9419 E+01$ & $7.0437 E+00$ & $3.4100 E+01$ & $6.5840 \mathrm{E}+01$ \\
\hline WHC & $224 \mathrm{U}$ & 42 & $3.9395 \mathrm{E}+03$ & $9.3797 E+01$ & $9.9107 E+01$ & $1.5100 E+01$ & $4.2300 E+02$ \\
\hline FERM & NALAB & 37 & $5.7988 E+03$ & $1.5672 \mathrm{E}+02$ & 4.6414E+01 & $4.0800 E+01$ & $2.0400 \mathrm{E}+02$ \\
\hline WHC & $303 \mathrm{~K}$ & 35 & $4.1710 E+03$ & $1.1917 \mathrm{E}+02$ & $1.3252 E+01$ & $7.8000 E+01$ & $1.4300 E+02$ \\
\hline PSN & PSNS & 34 & $5.8017 E+04$ & $1.7064 E+03$ & $7.0084 E+01$ & $1.4080 E+03$ & $1.8160 E+03$ \\
\hline WHC & $271 B$ & 28 & $2.3632 E+03$ & $8.4400 E+01$ & $1.2238 \mathrm{E}+02$ & $1.5900 E+00$ & $6.3270 E+02$ \\
\hline WHC & $163 P A$ & 26 & $1.4429 \mathrm{E}+03$ & $5.5496 E+01$ & $3.2525 \mathrm{E}+01$ & $8.0000 E+00$ & $1.2200 E+02$ \\
\hline WHC & $241 S$ & 26 & $1.1221 E+04$ & $4.3158 \mathrm{E}+02$ & $6.3612 E+02$ & $2.5000 E+01$ & $2.1750 E+03$ \\
\hline PNL & 3058 & 23 & $1.3906 \mathrm{E}+03$ & $6.0461 E+01$ & $2.9374 E+01$ & $1.4100 E+01$ & $1.2640 E+02$ \\
\hline WHC & $221 B$ & 22 & $2.0336 \mathrm{E}+03$ & $9.2436 E+01$ & $7.3313 E+01$ & $1,3600 \mathrm{E}+01$ & $2.5720 E+02$ \\
\hline PNL & 331 & 20 & $1.9342 E+03$ & $9.6708 E+01$ & $3.0673 E+01$ & $2.5000 \mathrm{E}+\infty 0$ & $1.4910 E+02$ \\
\hline WHC & $241 \mathrm{~A}$ & 18 & $9.9800 E+03$ & $5.5444 E+02$ & $6.2872 E+02$ & $3.3000 E+01$ & $2.0890 E+03$ \\
\hline WHC & $242 A$ & 17 & $1.9504 E+04$ & $1.1473 E+03$ & $1.3463 E+03$ & $8.0000 E+00$ & 4.1510E+03 \\
\hline WHC & BG3AE & 17 & $1.8842 E+03$ & $1.1083 E+02$ & $8.5405 \mathrm{E}+01$ & $2.9340 E+01$ & $3.2481 E+02$ \\
\hline WHC & $1310 \mathrm{P}$ & 15 & $1.3770 \mathrm{E}+03$ & $9.1800 E+01$ & $4.3473 E+01$ & $7.0000 E+00$ & $1.3600 E+02$ \\
\hline PNL & 325 & 14 & $1.5190 E+03$ & $1.0850 E+02$ & $5.7976 E+01$ & $1.9090 \mathrm{E}+01$ & $1.6630 E+02$ \\
\hline WHC & $105 N$ & 14 & $5.5300 \mathrm{E}+02$ & $3.9500 E+01$ & $1.6696 \mathrm{E}+01$ & $4.0000 E+00$ & $6.2000 \mathrm{E}+01$ \\
\hline WHC & $241 \mathrm{AY}$ & 14 & $9.9000 E+02$ & $7.0714 E+01$ & $6.4601 E+01$ & $8.0000 E+00$ & $1.8100 \mathrm{E}+02$ \\
\hline WHC & 221TS & 13 & $4.3252 E+03$ & $3.3271 E+02$ & $7.4102 E+01$ & $1.3590 E+02$ & $4.0640 E+02$ \\
\hline WHC & $241 T X$ & 12 & $3.0786 E+03$ & $2.5655 \mathrm{E}+02$ & $4.8761 E+02$ & $2.3604 E+01$ & $1.7600 E+03$ \\
\hline WHC & 324 & 12 & $8.1236 E+02$ & $6.7697 E+01$ & $4.5030 E+01$ & $2.8060 E+01$ & $1.4974 E+02$ \\
\hline PNL & 324 & 11 & $1.0010 E+03$ & $9.1000 \mathrm{E}+01$ & $0.0000 E+00$ & $9.1000 E+01$ & $9.1000 E+01$ \\
\hline WHC & $163 \mathrm{~N}$ & 11 & $5.1100 E+02$ & $4.6455 \mathrm{E}+01$ & $2.8264 E+01$ & $1.0000 E+01$ & $8.1000 E+01$ \\
\hline WHC & 2025 & 11 & $1.2711 E+03$ & $1.1555 \mathrm{E}+02$ & $1.3966 E+01$ & $8.0700 E+01$ & 1.3720E +02 \\
\hline WHC & $241 C$ & 11 & $8.8594 \mathrm{E}+03$ & $8.0540 \mathrm{E}+02$ & $7.9456 E+02$ & $2.2000 E+01$ & t. $.8680 E+03$ \\
\hline WHC & $241 T$ & 11 & $5.9340 E+03$ & $5.3945 E+02$ & $8.4528 E+02$ & $1.6001 E+01$ & $1.9980 E+03$ \\
\hline WHC & 2765 & 11 & $6.1660 E+03$ & $5.6055 E+02$ & $1.5962 E+03$ & $3.2000 \varepsilon+01$ & $5.3730 E+03$ \\
\hline PNL & LSLII & 10 & $9.6430 \mathrm{E}+02$ & $9.6430 E+01$ & $2.0278 \mathrm{E}+01$ & $5.5600 E+01$ & $1.1500 \mathrm{E}+02$ \\
\hline WHC & $202 \mathrm{AL}$ & 9 & $2.3760 E+02$ & $2.6400 \mathrm{E}+01$ & $1.0869 E+01$ & $5.4000 E+00$ & $4.1400 E+01$ \\
\hline WHC & $241 S Y$ & 9 & $7.7242 E+03$ & $8.5824 E+02$ & $1.1533 E+03$ & $2.1005 E+01$ & $2.4012 E+03$ \\
\hline WHC & 333 & 9 & $9.6504 \mathrm{E}+02$ & $1.0723 E+02$ & $1.3896 \varepsilon+02$ & $1.0140 E+01$ & $4.1736 E+02$ \\
\hline WHC & $105 \mathrm{~kW}$ & 8 & $3.6389 \mathrm{E}+02$ & $4.5486 \mathrm{E}+01$ & $2.9114 E+01$ & $2.0000 E+01$ & $1.0600 E+02$ \\
\hline WHC & 377 & 8 & $2.3990 E+02$ & $2.9988 E+01$ & $6.7226 \varepsilon+00$ & $1.7700 \mathrm{E}+01$ & $4.2700 E+01$ \\
\hline WHC & 308 & 7 & $2.9990 \mathrm{E}+02$ & $4.2843 E+01$ & $9.2552 \mathrm{E}+00$ & $2.2100 E+01$ & $4.7500 E+01$ \\
\hline WHC & MIXTF & 7 & $2.0930 E+04$ & $2.9900 \mathrm{E}+03$ & $9.3305 \mathrm{E}+02$ & $1.4560 E+03$ & $4.0210 E+03$ \\
\hline PNL & RTL & 6 & $5.9600 \mathrm{E}+02$ & $9.9333 E+01$ & $7.2296 E+\infty 0$ & $9.1000 E+01$ & $1.0800 E+02$ \\
\hline WHC & $1706 \mathrm{~K}$ & 6 & $2.2500 E+02$ & $3.7500 E+01$ & $1.8262 E+01$ & $1.8000 \mathrm{E}+01$ & $6.1000 E+01$ \\
\hline WHC & $225 B$ & 6 & $8.7056 \mathrm{E}+02$ & $1.4509 E+02$ & $6.7366 E+01$ & $3.3660 E+01$ & $2.2300 \mathrm{E}+02$ \\
\hline WHC & $241 A P$ & 6 & $7.6900 E+02$ & $1.2817 E+02$ & $1.8290 \mathrm{E}+02$ & $2.7000 E+01$ & $4.9600 E+02$ \\
\hline$W H C$ & $241 B X$ & 6 & $6.0340 E+02$ & $1.0057 E+02$ & $1.2925 E+02$ & $6.4000 E+00$ & $3.1900 E+02$ \\
\hline PNL & 329 & 5 & $6.3930 E+02$ & $1.2786 E+02$ & $4.1950 E+01$ & $8.6800 E+01$ & $1.8540 E+02$ \\
\hline UOU & UURD & 5 & $3.2757 E+02$ & $6.5514 E+01$ & $8.9240 E+00$ & $5.1530 E+01$ & $7.3400 \mathrm{E}+01$ \\
\hline WHC & $109 \mathrm{~N}$ & 5 & $1.9400 E+02$ & $3.8800 E+01$ & $7.1903 \mathrm{E}+00$ & $2.7000 E+01$ & $4.6000 E+01$ \\
\hline WHC & $241 \mathrm{AN}$ & 5 & $3.3200 \mathrm{E}+02$ & $6.6400 E+01$ & $5.7713 E+01$ & $3.0000 E+01$ & $1.6700 \mathrm{E}+02$ \\
\hline WHC & $241 U$ & 5 & $1.8500 E+02$ & $3.7000 E+01$ & $4.4721 E+\infty 0$ & $3.2000 E+01$ & $4.3000 E+01$ \\
\hline $\mathrm{KEH}$ & $231 Z$ & 4 & $7.1913 E+02$ & $1.7978 E+02$ & $1.9744 E+01$ & $1.6253 E+02$ & $2.0793 E+02$ \\
\hline PNL & ISV & 4 & $1.9700 E+02$ & $4.9250 E+01$ & $8.9954 E+00$ & $4.0000 E+01$ & $6.0000 E+01$ \\
\hline WHC & $241 B$ & 4 & $5.3375 E+02$ & $1.3344 E+02$ & $1.9054 E+02$ & $2.7750 \mathrm{E}+01$ & $4.1900 E+02$ \\
\hline WHC & $2415 x$ & 4 & $2.3950 E+03$ & $5.9875 E+02$ & $1.0872 E+03$ & $2.8000 E+01$ & $2.2290 \mathrm{E}+03$ \\
\hline WHC & $241 T Y$ & 4 & $6.4000 E+01$ & $1.6000 \mathrm{E}+01$ & $8.8694 E+\infty 0$ & $7.0000 E+00$ & $2.7000 E+01$ \\
\hline WHC & 244AR & 4 & B.7100E +02 & $2.1775 E+02$ & $3.3019 E+01$ & $1.8400 E+02$ & $2.6300 E+02$ \\
\hline WHC & $100 \mathrm{KE}$ & 3 & $1.3200 E+02$ & $4.4000 E+01$ & $8.7178 E+00$ & $3.8000 E+01$ & $5.4000 E+01$ \\
\hline WHC & $1706 \mathrm{KE}$ & 3 & $1.7200 E+02$ & $5.7333 E+01$ & $1.7786 E+01$ & $3.7000 E+01$ & $7.0000 E+01$ \\
\hline
\end{tabular}


WHC-SD-W-242-ES-004, Rev. 0

TABLE 39

SUMMARY OF WASTE WEIGHTS BY GENERATOR SORTED IN ASCENDING ORDER BASED ON CONTAINER COUNT

(2 SHEETS)

\begin{tabular}{|c|c|c|c|c|c|c|c|}
\hline COMPANY & GENERATOR & COUNT & ACCUM WASTE WT, KG & AVG WASTE WT, KG & STD DEV & MIN WASTE WT. KG & MAX WASTE WT, KG \\
\hline WHC & $2706 \mathrm{~T}$ & 3 & $1.7111 \mathrm{E}+02$ & $5.7035 E+01$ & $4.4182 E+01$ & $1.8000 \mathrm{E}+01$ & $1.0500 \mathrm{E}+02$ \\
\hline WHC & $271 C R$ & 3 & $1.4200 \mathrm{E}+03$ & $4.7333 E+02$ & $7.5936 \mathrm{E}+02$ & $2.0000 E+01$ & $1.3500 \mathrm{E}+03$ \\
\hline PNL & 326 & 2 & $6.5800 E+01$ & $3.2900 E+01$ & $1.3435 E+01$ & $2.3400 E+01$ & $4.2400 E+01$ \\
\hline PNL & 3720 & 2 & $1.5841 \mathrm{E}+02$ & $7.9206 \mathrm{E}+01$ & $1.2792 \mathrm{E}+00$ & $7.8301 E+01$ & $8.0110 E+01$ \\
\hline PNL & 3745 & 2 & $1.5750 \mathrm{E}+02$ & $7.8750 E+01$ & $2.5809 E+01$ & $6.0500 E+01$ & $9.7000 E+01$ \\
\hline WHC & $101 S Y$ & 2 & $4.1390 E+03$ & $2.0695 E+03$ & 1.2940E+02 & $1.9780 E+03$ & $2.1610 E+03$ \\
\hline WHC & $105 \mathrm{KE}$ & 2 & $4.6420 \mathrm{E}+03$ & $2.3210 \mathrm{E}+03$ & $3.1452 E+03$ & $9.7000 \mathrm{E}+01$ & $4.5450 \mathrm{E}+03$ \\
\hline WHC & 163PAD & 2 & $8.8000 E+01$ & $4.4000 E+01$ & $1.4142 \mathrm{E}+01$ & $3.4000 E+01$ & $5.4000 E+01$ \\
\hline WHC & $200 w$ & 2 & $4.7800 E+02$ & $2.3900 E+02$ & $4.2426 E+\infty$ & $2.3600 E+02$ & $2.4200 E+02$ \\
\hline WHC & 241AW & 2 & $2.0700 E+02$ & $1.0350 \mathrm{E}+02$ & $8.4146 E+01$ & $4.4000 E+01$ & $1.6300 \mathrm{E}+02$ \\
\hline WHC & $241 A X$ & 2 & $2.0982 E+02$ & $1.0491 E+02$ & 7. $6240 E+01$ & 5.1000E+01 & $1.5882 E+02$ \\
\hline WHC & 241BY & 2 & $2.4100 E+02$ & $1.2050 \mathrm{E}+02$ & $1.2799 \mathrm{E}+02$ & $3.0000 E+01$ & $2.1100 E+02$ \\
\hline WHC & $242 \mathrm{~S}$ & 2 & $8.0700 E+02$ & $4.0350 E+02$ & $1.6758 E+02$ & $2.8500 E+02$ & $5.2200 E+02$ \\
\hline WHC & 2710 & 2 & $2.0430 \mathrm{E}+02$ & $1.0215 E+02$ & $6.1023 E+01$ & $5.9000 \mathrm{E}+01$ & $1.4530 E+02$ \\
\hline WHC & 340 & 2 & $6.1290 \mathrm{E}+01$ & $3.0645 E+01$ & $2.2790 \mathrm{E}+01$ & $1.4530 \mathrm{E}+01$ & $4.6760 E+01$ \\
\hline SLA & SLACU & 1 & $1.4000 E+02$ & $1.4000 E+02$ & $0.0000 E+00$ & $1.4000 E+02$ & $1.4000 E+02$ \\
\hline WHC & $105 \mathrm{H}$ & 1 & $6.0000 E+01$ & $6.0000 E+01$ & $0.0000 E+00$ & $6.0000 E+01$ & $6.0000 E+01$ \\
\hline WHC & 151ER & 1 & $7.8000 E+01$ & $7.8000 E+01$ & $0.0000 E+00$ & $7.8000 \mathrm{E}+01$ & $7.8000 E+01$ \\
\hline WHC & 204AR & 1 & $5.0000 E+01$ & $5.0000 E+01$ & $0.0000 E+00$ & $5.0000 \mathrm{E}+01$ & $5.0000 E+01$ \\
\hline WHC & 2312 & 1 & $1.0000 E+02$ & $1.0000 \mathrm{E}+02$ & $0.0000 E+00$ & $1.0000 \mathrm{E}+02$ & $1.0000 E+02$ \\
\hline WHC & 2400 & 1 & $2.3000 E+01$ & $2.3000 E+01$ & $0.0000 E+00$ & $2.3000 E+01$ & $2.3000 E+01$ \\
\hline WHC & $241 A Z$ & 1 & $7.4020 \mathrm{E}+02$ & $7.4020 \mathrm{E}+02$ & $0.0000 E+00$ & $7.4020 \mathrm{E}+02$ & $7.4020 E+02$ \\
\hline WHC & 241EW & 1 & $2.5000 E+01$ & $2.5000 E+01$ & $0.0000 E+00$ & $2.5000 E+01$ & $2.5000 E+01$ \\
\hline WHC & 2436 & 1 & $2.0100 E+02$ & $2.0100 E+02$ & $0.0000 E+00$ & $2.0100 E+02$ & $2.0100 E+02$ \\
\hline WHC & $2713 \mathrm{~W}$ & 1 & $2.0500 E+01$ & $2.0500 E+01$ & $0.0000 E+00$ & $2.0500 E+01$ & $2.0500 E+01$ \\
\hline WHC & $271 \mathrm{~T}$ & 1 & $4.9388 E+03$ & $4.9388 \mathrm{E}+03$ & $0.0000 E+00$ & $4.9388 E+03$ & $4.9388 E+03$ \\
\hline WHC & 2760 & 1 & $3.4000 \mathrm{E}+01$ & $3.4000 E+01$ & $0.0000 E+00$ & $3.4000 E+01$ & $3.4000 E+01$ \\
\hline WHC & 309 & $i$ & $8.8000 E+01$ & $8.8000 E+01$ & $0.0000 E+00$ & $8.8000 E+01$ & $8.8000 E+01$ \\
\hline WHC & 313 & 1 & $2.5500 E+01$ & $2.5500 \mathrm{E}+01$ & $0.0000 \mathrm{E}+00$ & $2.5500 E+01$ & $2.5500 E+01$ \\
\hline WHC & 321 & 1 & $1.7700 E+01$ & $1.7700 E+01$ & $0.0000 E+00$ & $1.7700 E+01$ & $1.7700 \mathrm{E}+01$ \\
\hline WHC & $399-3-1$ & 1 & $2.2000 E+02$ & $2.2000 E+02$ & $0.0000 E+00$ & $2.2000 E+02$ & $2.2000 E+02$ \\
\hline WHC & T PLANT & 1 & $1.5010 \mathrm{E}+03$ & $1.5010 E+03$ & $0.0000 E+\infty 0$ & $1.5010 E+03$ & $1.5010 E+03$ \\
\hline KER & MCGEE & 0 & $0.0000 E+00$ & $0.0000 E+00$ & $0.0000 E+00$ & $0.0000 E+00$ & $0.0000 E+\infty 0$ \\
\hline PNL & 1234 & 0 & $0.0000 E+00$ & $0.0000 E+00$ & $0.0000 E+00$ & $0.0000 E+\infty 0$ & $0.0000 E+00$ \\
\hline WHC & 2ETF & 0 & $0.0000 E+00$ & $0.0000 E+00$ & $0.0000 E+00$ & $0.0000 E+00$ & $0.0000 E+00$ \\
\hline
\end{tabular}


TABLE 40

SUMMARY OF CALCULATED WASTE WEIGHTS BY GENERATOR SORTED IN ASCENDING ORDER BASED ON CONTAINER COUNT

(2 SHEETS)

\begin{tabular}{|c|c|c|}
\hline COMPANY & GENERATOR & COUN \\
\hline WHC & $2345 Z$ & 455 \\
\hline WHC & $100 \mathrm{~N}$ & 408 \\
\hline LBL & LBLAB & 215 \\
\hline ANL & ARGON & 210 \\
\hline BATC & BATCO & 205 \\
\hline WHC & $222 S$ & 144 \\
\hline ALB & ALBNY & 126 \\
\hline WHC & $202 A$ & 95 \\
\hline WHC & $221 \mathrm{~T}$ & 69 \\
\hline BAPL & BAPL & 49 \\
\hline BNL & BNLAB & 49 \\
\hline WHC & $224 U$ & 44 \\
\hline FERM & NALAB & 37 \\
\hline WHC & $303 K$ & 35 \\
\hline PSN & PSNS & 34 \\
\hline PNL & 331 & 30 \\
\hline WHC & $271 B$ & 28 \\
\hline WHC & 163PA & 26 \\
\hline WHC & $241 S$ & 26 \\
\hline PNL. & 3058 & 23 \\
\hline WHC & $221 \mathrm{~B}$ & 22 \\
\hline WHC & 221TS & 18 \\
\hline WHC & $241 \mathrm{~A}$ & 18 \\
\hline PNL & 325 & 17 \\
\hline WHC & $242 \mathrm{~A}$ & 17 \\
\hline WHC & BG3AE & 17 \\
\hline WHC & $1310 P$ & 15 \\
\hline WHC & $105 \mathrm{~N}$ & 14 \\
\hline WHC & $241 \mathrm{AY}$ & 14 \\
\hline WHC & 241TX & 12 \\
\hline WHC & 324 & 12 \\
\hline PNL & 324 & 11 \\
\hline PNL & LSLII & 11 \\
\hline WHC & $163 \mathrm{~N}$ & 11 \\
\hline WHC & 2025 & 11 \\
\hline WHC & $241 C$ & 11 \\
\hline WHC & $241 T$ & 11 \\
\hline WHC & $276 S$ & 11 \\
\hline KER & MCGEE & 10 \\
\hline WHC & $202 \mathrm{AL}$ & 9 \\
\hline WHC & 241SY & 9 \\
\hline WHC & 333 & 9 \\
\hline WHC & $105 \mathrm{KW}$ & 8 \\
\hline WHC & 377 & 8 \\
\hline WHC & 308 & 7 \\
\hline WHC & MIXTF & 7 \\
\hline PNL & RTL & 6 \\
\hline WHC & $1706 \mathrm{~K}$ & 6 \\
\hline WHC & $225 B$ & 6 \\
\hline WHC & 241AP & 6 \\
\hline WHC & 241BX & 6 \\
\hline PNL & 329 & 5 \\
\hline Uou & UURD & 5 \\
\hline WHC & $109 \mathrm{~N}$ & 5 \\
\hline WHC & 241AN & 5 \\
\hline WHC & $241 U$ & 5 \\
\hline KEH & 2312 & 4 \\
\hline PNL & ISV & 4 \\
\hline WHC & $241 B$ & 4 \\
\hline WHC & 2415X & 4 \\
\hline WHC & $241 T Y$ & 4 \\
\hline WHC & 244AR & 4 \\
\hline WHC & 10OKE & 3 \\
\hline
\end{tabular}

\section{ACCUM WASTE WT, KG \\ $5.8886 \mathrm{E}+04$}

$2.8621 E+04$

$1.6463 \mathrm{E}+04$

$3.0792 \mathrm{E}+04$

$2.3567 \mathrm{E}+04$

$9.8025 \mathrm{E}+03$

$3.3682 \mathrm{E}+04$

$9.5151 \mathrm{E}+03$

8.4423E+03

$3.8699 \mathrm{E}+03$

$2.4261 E+03$

$4.0194 E+03$

$5.7028 E+03$

4.1720E+03

$5.8027 E+04$

$2.9937 E+03$

$2.3357 \mathrm{E}+03$

$1.3630 E+03$

$1,1233 E+04$

$1.3795 E+03$

$1.9390 \mathrm{E}+03$

$5.3745 \mathrm{E}+03$

$9.9790 E+03$

$1.6564 \varepsilon+03$

$1.9500 \mathrm{E}+04$

$1.6978 \mathrm{E}+03$

$1.3339 \mathrm{E}+03$

$5.6335 E+02$

$9.8102 \mathrm{E}+02$

$3.0786 \mathrm{E}+03$

$7.3404 \mathrm{E}+02$

$1.0010 \mathrm{E}+03$

$1.0944 E+03$

$5.2606 \mathrm{E}+02$

$1.2711 \mathrm{E}+03$

$8.0870 \mathrm{E}+03$

$5.9340 E+03$

$6.1750 E+03$

$1.2209 E+03$

$2.3810 E+02$

$7.7251 E+03$

9.3707E+02

$3.6389 \mathrm{E}+02$

$2.3990 \mathrm{E}+02$

$2.9990 \mathrm{E}+02$

$2.0930 E+04$

6.0204E +02

$2.3218 E+02$

8.0142E+02

$7.6900 E+02$

$5.9940 E+02$

$6.4128 E+02$

$3.2764 E+02$

$1.9400 \mathrm{E}+02$

$3.3200 \mathrm{E}+02$

1.8500E+02

$7.1913 \mathrm{E}+02$

2.2498E+02

$5.3400 \mathrm{E}+02$

$2.3950 E+03$

$6.4000 \mathrm{E}+01$

$8.7100 E+02$

$1.3099 E+02$
AVG WASTE WT, KG

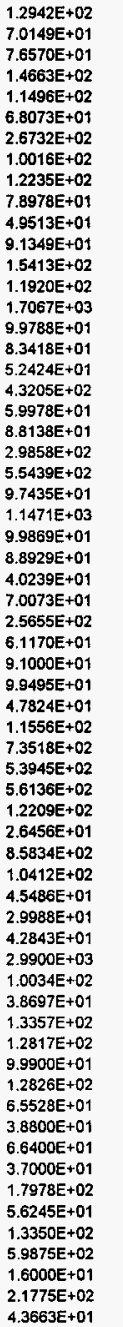

STD DEV

1.8416E+01

B. $0527 E+01$

9.4417E+01

$3.8589 \mathrm{E}+01$

$1.3718 E+02$

$1.0113 E+02$

$1.1258 E+02$

$2.7863 E+02$

$4.6001 E+01$

$7.0808 E+\infty 0$

$9.7089 \mathrm{E}+01$

4.4947E+D1

$1.3295 \mathrm{E}+01$

$7.0047 \mathrm{E}+01$

$2.4409 \mathrm{E}+0.1$

$1.2162 \mathrm{E}+02$

3. 1417E+01

6. $3582 \mathrm{E}+02$

$2.7878 E+01$

$7.7452 E+01$

$9.5032 \mathrm{E}+01$

6.2877 E+02

$5.7797 E+01$

$1.3465 E+03$

$8.5395 E+01$

4.2731E+01

$1.4968 \mathrm{E}+01$

6.5157E+01

$4.8761 \mathrm{E}+02$

$4.2874 E+01$

$0.0000 E+00$

$1.4926 \mathrm{E}+01$

$2.4933 E+01$

$1.3967 \mathrm{E}+01$

$7.2752 \mathrm{E}+02$

8.4528E+02

$1.5959 E+03$

$2.7041 E+01$

$1.0749 E+01$

$1.1534 \mathrm{E}+03$

$4.3896 \mathrm{E}+02$

$2.9114 \mathrm{E}+01$

$6.7226 E+00$

$9.2552 \mathrm{E}+00$

$9.3305 \mathrm{E}+02$

$5.8792 \mathrm{E}+00$

$1.6980 \mathrm{E}+01$

8.2809E +01

$+.8290 \mathrm{E}+02$

$1.2977 \mathrm{E}+02$

4.1474E +01

$8.8966 \mathrm{E}+00$

7.1903E+00

5.7713E+01

4.4721E+00

1.9744E+01

$1.7722 \mathrm{E}+01$

$1.9050 \mathrm{E}+02$

$1.0872 E+03$

$8.8694 E+00$

$3.3019 E+01$

$8.1388 \mathrm{E}+00$
MIN WASTE WT, KG

$9.2900 \mathrm{E}+\infty 0$

$2.5000 \mathrm{E}+01$

$2.4100 E+01$

$2.0460 E+01$

$5.0000 E+00$

$4.6000 E+01$

$3.9000 E+00$

1.5400E+01

$3.0800 \mathrm{E}+01$

3.4100E+01

$9.2900 E+00$

$4.0800 E+01$

$7.8000 E+01$

$1.4080 E+03$

$2.5000 \mathrm{E}+00$

$7.3000 E+00$

$9.0000 E+00$

$2.5000 \mathrm{E}+01$

$1.6200 \mathrm{E}+01$

$4.9800 \mathrm{E}+00$

1. $2042 \mathrm{E}+02$

$3.3000 \mathrm{E}+01$

2.2600E +01

$4.7000 E+00$

$1.8350 E+01$

$7.0000 E+00$

$1.5360 \mathrm{E}+01$

$7.0000 E+00$

2.3600E+01

$3.4190 E+01$

S.1000E+01

8.1200E+01

1.3000E+0t

$8.0700 E+01$

$2.2000 E+01$

$1.6000 \mathrm{E}+01$

$3.2000 E+01$

$7.5010 \mathrm{E}+01$

$5.9000 E+\infty 0$

2.1010E+01

$6.6400 E+00$

$2.0000 E+01$

$1.7700 E+01$

$2.2100 E+01$

$1.4560 E+03$

$9.4020 E+01$

1.8000E+01

$2.9060 E+01$

$2.7000 E+01$

$6.4000 E+00$

$8.6800 E+01$

$5.1600 \mathrm{E}+\mathrm{O} 1$

$2.7000 E+01$

$3.0000 E+01$

$3.2000 \mathrm{E}+01$

$1.6253 \mathrm{E}+02$

3.3330E+01

$2.8000 E+01$

$2.8000 \mathrm{E}+01$

$7.0000 \mathrm{E}+00$

$1.8400 \mathrm{E}+02$

$3.8000 \mathrm{E}+01$
MAX WASTE WT, KG

1.5716E +02

1.1744E+03

$3.2680 E+02$

$3.5010 E+02$

$1.5582 E+03$

4.4400E+02

7.1700E+02

2.3623E+03

$3.4790 \mathrm{E}+02$

$6.5840 E+01$

4.2300E+02

$2.0002 E+02$

$1.4300 \mathrm{E}+02$

$1.8160 E+03$

$1.4910 E+02$

$6.3270 \mathrm{E}+02$

$1.1801 E+02$

2.1750E+03

1.2640E +02

$2.5300 E+02$

4.1357E+02

$2.0890 E+03$

1.6630E+02

$4.1510 E+03$

3.1392E+02

1.3198E+02

$6.2000 \mathrm{E}+01$

$1.8100 \mathrm{E}+02$

1.7600E+03

1.4541E+02

9.1000E+01

$1.1500 \mathrm{E}+02$

$7.7010 E+01$

$1.3720 \mathrm{E}+02$

$1.86805+03$

$1.9980 E+03$

$5.3730 E+03$

1.7199E+02

$4.1400 E+01$

$2.4020 E+03$

4.1389E+02

$1.0600 E+02$

$4.2700 E+01$

$4.7500 E+01$

$4.0210 E+03$

$1.0800 \mathrm{E}+02$

$6.1000 E+01$

$2.1848 E+02$

$4.9600 E+02$

$3.1900 E+02$

1.8540E+ 02

$7.3400 E+01$

4. $6000 \mathrm{E}+01$

1.6700E +02

4.3000E+01

2.0793E+02

$7.5680 \mathrm{E}+01$

$4.1900 E+02$

$2.2290 \mathrm{E}+03$

2.7000E+01

2.6300E+02

$5.2990 E+01$ 
WHC-SD-W-242-ES-004, Rev. 0

TABLE 40

SUMMARY OF CALCULATED WASTE WEIGHTS BY GENERATOR SORTED IN ASCENDING ORDER BASED ON CONTAINER COUNT

(2 SHEETS)

\begin{tabular}{|c|c|c|}
\hline COMPANY & GENERATOR & COUNT \\
\hline WHC & $1706 \mathrm{KE}$ & 3 \\
\hline WHC & $2706 \mathrm{~T}$ & 3 \\
\hline WHC & 271CR & 3 \\
\hline PNL & 326 & 2 \\
\hline PNL & 3720 & 2 \\
\hline PNL & 3745 & 2 \\
\hline WHC & $1015 Y$ & 2 \\
\hline WHC & $105 \mathrm{KE}$ & 2 \\
\hline WHC & 163PAD & 2 \\
\hline WHE & $200 \mathrm{~W}$ & 2 \\
\hline WHC & 241AW & 2 \\
\hline WHC & $241 A X$ & 2 \\
\hline WHC & 241BY & 2 \\
\hline WHC & $242 S$ & 2 \\
\hline WHC & $271 U$ & 2 \\
\hline WHC & 340 & 2 \\
\hline PNL & 1234 & 1 \\
\hline SLA & SLACU & 1 \\
\hline WHC & $105 \mathrm{H}$ & 1 \\
\hline WHC & 151ER & 1 \\
\hline WHC & 204AR & 1 \\
\hline WHC & $231 Z$ & 1 \\
\hline WHC & 2400 & 1 \\
\hline WHC & 241AZ & 1 \\
\hline WHC & 241EW & 1 \\
\hline WHC & $243 G$ & 1 \\
\hline WHC & $2713 W$ & 1 \\
\hline WHC & $271 T$ & 1 \\
\hline WHC & $276 \mathrm{U}$ & 1 \\
\hline WHC & 2ETF & 1 \\
\hline WHe & 309 & 1 \\
\hline WHC & 313 & 1 \\
\hline WHC & 321 & 1 \\
\hline WHC & $399-3-1$ & 1 \\
\hline WHC & T PLANT & 1 \\
\hline
\end{tabular}

ACCUM WASTE WT, KG

$$
\begin{aligned}
& 1.7200 E+02 \\
& 1.7111 E+02 \\
& 1.4200 E+03 \\
& 6.5800 E+01 \\
& 1.5841 E+02 \\
& 1.5949 E+02 \\
& 4.1390 E+03 \\
& 4.6382 E+03 \\
& 8.8000 E+01 \\
& 4.7800 E+02 \\
& 2.0700 E+02 \\
& 2.6180 E+02 \\
& 2.3601 E+02 \\
& 8.0700 E+02 \\
& 2.0430 E+02 \\
& 6.1290 E+01 \\
& 2.8206 E+02 \\
& 1.4000 E+02 \\
& 6.0000 E+01 \\
& 7.8000 E+01 \\
& 5.0000 E+01 \\
& 1.0000 E+02 \\
& 2.3000 E+01 \\
& 8.6420 E+02 \\
& 2.5000 E+01 \\
& 2.0100 E+02 \\
& 2.0500 E+01 \\
& 4.9388 E+03 \\
& 3.4000 E+01 \\
& 1.5444 E+02 \\
& 8.8000 E+01 \\
& 2.5500 E+01 \\
& 1.7700 E+01 \\
& 2.2000 E+02 \\
& 1.5010 E+03
\end{aligned}
$$

STD DEV

$4.4182 \mathrm{E}+01$

$7.5936 \mathrm{E}+02$

1.3435E+01

$1.2799 \mathrm{E}+00$

$2.4402 E+01$

$1.2940 \mathrm{E}+02$

$3.1482 E+03$

$1.4142 E+01$

4.2426E+00

$8.4146 E+01$

$3.3800 \mathrm{E}+01$

$1.2446 \mathrm{E}+02$

1.6758E+02

6.1023E+01

2.279OE+01

$0.0000 \mathrm{E}+\infty$

$0.0000 E+\infty 0$

$0.0000 E+00$

$0.0000 E+00$

$0.0000 E+00$

$0.0000 E+00$

$0.0000 E+00$

$0.0000 E+00$

$0.0000 E+00$

$0.0000 E+00$

$0.0000 E+00$

$0.0000 E+00$

$0.0000 E+00$

$0.0000 E+00$

$0.0000 E+00$

$0.0000 E+00$

$0.0000 E+00$

$0.0000 E+00$

$0.0000 \mathrm{E}+00$
MIN WASTE WT, KG

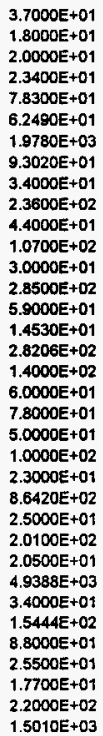

MAX WASTE WT, KG

$7.0000 E+01$
$1.0500 E+02$
$1.3500 E+03$
$4.2400 E+01$
$8.0110 E+01$
$9.7000 E+01$
$2.1610 E+03$
$4.5452 E+03$
$5.4000 E+01$
$2.4200 E+02$
$1.6300 E+02$
$1.5480 E+02$
$2.0601 E+02$
$5.2200 E+02$
$1.4530 E+02$
$4.6760 E+01$
$2.8206 E+02$
$1.4000 E+02$
$6.0000 E+01$
$7.8000 E+01$
$5.0000 E+01$
$1.0000 E+02$
$2.3000 E+01$
$8.6420 E+02$
$2.5000 E+01$
$2.0100 E+02$
$2.0500 E+01$
$4.9388 E+03$
$3.4000 E+01$
$1.5444 E+02$
$8.8000 E+01$
$2.5500 E+01$
$1.7700 E+01$
$2.2000 E+02$
$1.5010 E+03$


WHC-SD-W-242-ES-004, Rev, 0

TABLE 41

STATISTICAL ANALYSIS OF CONTAINER TYPES

IN ASCENDING ORDER BASED ON CONTAINER COUNT

( 1 SHEET)

CONTANER TYPE COLINT TOTAL VOLUME, CUM AVERAGE VOLUME, CUM

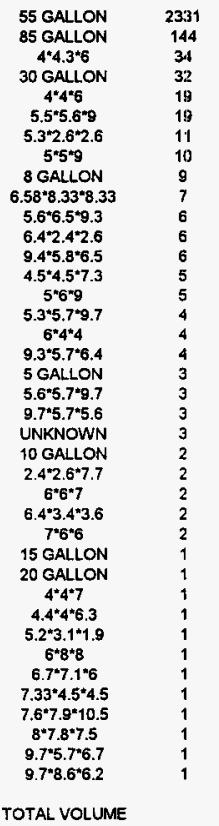

2.0836E-01

3.2230E-01

$2.9220 \mathrm{E}+00$

1.5277E-01

2.8934E+00

$8.7000 E+00$

9.6000E-01

$6.3708 E+00$

3.0000E-02

$9.6108 \mathrm{E}+00$

9.3200E-01

9.6717E +0O

4.2050E+00

8.3000E +00

8.3000E+OO

$2.9400 E+00$

$9.6070 E+00$

2.4000E-02

$8.4333 E+00$

8.5667E+00

1.4000E+01

4.0000E-02

1. $1200 E+00$

$7.1175 \mathrm{E}+00$

$2.2100 E+00$

$7.0000 \mathrm{E}+00$

4.0000E-02

8.0000E-02

$3.1720 \mathrm{E}+00$

$3.1400 E+00$

9.6000E-01

$1.0899 E+01$

6.7000E+00

4.2000E +00

1.7925E+01

1.3300E+O

$8.3000 \mathrm{E}+00$

1.5000E+ 01
1.2921E +01
STD DEV

2. 7053E- 03

7.5888E-03

1.0981E-07

1.0711E-01

9.2777E-02

1.1239E-07$$
\text { * }
$$

4.2164E-04

3.3717E-07

4.0825E-04

2.9125E-01

1.7555E-01

$0.0000 E+00$

$0.0000 E+00$

$0.0000 E+\infty 0$

$0.0000 E+00$

$0.0000 E+00$

5.1962E-03

2.3094E $=01$

2.3094E-01

$0.0000 E+00$

$1.4142 \mathrm{E}-02$

$0.0000 E+00$

2.6163E-02

$0.0000 E+00$

$0.0000 E+00$

$0.0000 E+00$

$0.0000 E+00$

$0.0000 E+00$

$0.0000 E+00$

$0.0000 E+00$

$0.0000 E+00$

$0.0000 E+00$

$0.0000 E+00$

$0.0000 E+00$

$0.0000 E+\infty 0$

$0.0000 E+00$

$0.0000 E+00$
MINIMUM VOLUME, CUM MAXMUM VOLUME, CUM

$$
\begin{aligned}
& 2.0000 E-01 \\
& 2.8300 E-01 \\
& 2.9220 E+00 \\
& 1.1000 E-01 \\
& 2.7180 E+00 \\
& 8.7000 E+00 \\
& 9.6000 E-01 \\
& 6.3700 E+00 \\
& 3.0000 E-02 \\
& 1.2921 E+01 \\
& 9.6100 E+00 \\
& 5.5600 E-01 \\
& 9.6000 E+00 \\
& 4.2050 E+00 \\
& 8.3000 E+00 \\
& 8.3000 E+00 \\
& 2.9400 E+00 \\
& 9.6070 E+00 \\
& 1.8000 E-02 \\
& 8.3000 E+00 \\
& 8.3000 E+00 \\
& 1.4000 E+01 \\
& 3.0000 E-02 \\
& 1.1200 E+00 \\
& 7.0990 E+00 \\
& 2.2100 E+00 \\
& 7.0000 E+00 \\
& 4.0000 E-02 \\
& 8.0000 E-02 \\
& 3.1720 E+00 \\
& 3.1400 E+00 \\
& 9.6000 E-01 \\
& 1.0899 E+01 \\
& 6.7000 E+00 \\
& 4.2000 E+00 \\
& 1.7925 E+01 \\
& 1.3300 E+01 \\
& 8.3000 E+00 \\
& 1.5000 E+01
\end{aligned}
$$

2.1000E-01

4,0400E-01

$2.9220 E+00$

7.3000E-01

$2.9400 E+00$

8.7000E+00

9.6000E-01

$6.3710 E+00$

3.0000E-02

$1.2921 E+01$

9.6110E+00

1.7200E+OD

$1.0030 E+01$

4.2050E+00

$8.3000 E+00$

$8.3000 E+00$

$2.9400 E+00$

$9.6070 E+00$

2.7000E- 02

8.7000E+00

$8.7000 E+00$

1. $4000 E+01$

5.0000E-02

1. $1200 \mathrm{E}+00$

$7.1360 E+00$

$2.2100 E+00$

$7.0000 E+00$

4.0000E-02

8.0000E-02

$3.1720 E+00$

$3.1400 E+00$

9.6000E-01

1.0899E+01

$6.7000 \mathrm{E}+00$

$4.2000 E+00$

$1.7925 E+01$

1.3300E+01

$8.3000 E+00$

$1.5000 E+01$ 
WHC-SD-W-242-ES-004, Rev. 0

TABLE 42

STATISTICAL ANALYSIS OF CONTAINER TYPES

IN ASCENDING ORDER BASED ON TOTAL GROSS WEIGHT

(1 SHEET)

CONTAINER TYPE COUNT TOTAL GROSS WT, KG AVERAGE GROSS WT, KG

\begin{tabular}{|c|c|}
\hline 55 GALLON & 2331 \\
\hline $4^{* 4} 43^{* 6}$ & 34 \\
\hline $5.5^{* 5} 5.6^{* \mathrm{~g}}$ & 19 \\
\hline 85 GALLON & 144 \\
\hline $5^{*} 5^{*} 9$ & 10 \\
\hline $9.4^{*} 5.8 * 6.5$ & 6 \\
\hline UNKNOWN & 3 \\
\hline $5.6^{*} 6.5^{*} 9.3$ & 6 \\
\hline $6.58 * 8.33 * 8.33$ & 7 \\
\hline $9.3^{*} 5.7 * 6.4$ & 4 \\
\hline $4.5^{*} 4.5^{*} 7.3$ & 5 \\
\hline $4^{*} 4^{*} 6$ & 19 \\
\hline $9.7^{*} 8.6^{*} 6.2$ & 1 \\
\hline $5+6 * 9$ & 5 \\
\hline $5.3 * 5.7 * 9.7$ & 4 \\
\hline $5.6^{*} 5.7^{\star} 9.7$ & 3 \\
\hline $7.6^{*} 7.9^{*} 10.5$ & 1 \\
\hline $9.7 \star^{5} .7 \star 5.6$ & 3 \\
\hline $6^{*} 8 * 8$ & 1 \\
\hline $6 * 6 * 7$ & 2 \\
\hline $8 * 7.8 * 7.5$ & 1 \\
\hline $5.3^{*} 2.6 * 2.6$ & 11 \\
\hline $6.7^{*} 7.1^{\prime \prime} 6$ & 1 \\
\hline $7^{*} 6 * 6$ & 2 \\
\hline $4^{*} 4^{* 7}$ & 1 \\
\hline $6.4 * 2.4 * 2.6$ & 6 \\
\hline 30 GALLON & 32 \\
\hline $9.7^{*} 5.7^{*} 6.7$ & 1 \\
\hline $6^{*} 4^{*} 4$ & 4 \\
\hline $7.33^{*} 4.5^{\star} 4.5$ & 1 \\
\hline $6.4^{*} 3.4^{*} 3.6$ & 2 \\
\hline $2.4 * 2.6 * 7.7$ & 2 \\
\hline $4.4^{*} 4^{*} 6.3$ & 1 \\
\hline $5.2 * 3.1 * 1.9$ & 1 \\
\hline 8 GALLON & 9 \\
\hline 5 GALLON & 3 \\
\hline 10 GALLON & 2 \\
\hline 20 GALLON & 1 \\
\hline 15 GALLON & 1 \\
\hline
\end{tabular}

TOTAL WEIGHT

\begin{tabular}{|c|c|}
\hline $2.5686 \mathrm{E}+05$ & $1.1020 E+02$ \\
\hline $7.0369 \mathrm{E}+04$ & $2.0697 E+03$ \\
\hline $4.3791 E+04$ & $2.3048 E+03$ \\
\hline $4.1963 E+04$ & $2.9141 E+02$ \\
\hline $\begin{array}{l}3.2012 E+04 \\
2.2752 E+04\end{array}$ & $\begin{array}{l}3.2012 E+03 \\
3.7920 E+03\end{array}$ \\
\hline $2.2685 E+04$ & $7.5617 \mathrm{E}+03$ \\
\hline $1.9490 E+04$ & $3.2484 E+03$ \\
\hline $1.2726 E+04$ & $1.8180 E+03$ \\
\hline $1.2296 \mathrm{E}+04$ & $3.0740 \mathrm{E}+03$ \\
\hline $9.3440 E+03$ & $1.8688 E+03$ \\
\hline $8.8720 \mathrm{E}+03$ & $4.6695 \mathrm{E}+02$ \\
\hline $7.9480 E+03$ & $7.9480 \mathrm{E}+03$ \\
\hline $7.9350 E+03$ & $1.5870 E+03$ \\
\hline $6.4522 E+03$ & $1.6131 E+03$ \\
\hline $6.3530 \mathrm{E}+03$ & 2.1177E +03 \\
\hline $6.2728 \mathrm{E}+03$ & $6.2728 E+03$ \\
\hline $6.0980 E+03$ & $2.0327 E+03$ \\
\hline $5.5202 E+03$ & $5.5202 E+03$ \\
\hline $4.7266 \mathrm{E}+03$ & $2.3633 E+03$ \\
\hline $3.0070 E+03$ & $3.0070 E+03$ \\
\hline $3.0030 E+03$ & $2.7300 E+02$ \\
\hline $2.8480 E+03$ & $2.8480 E+03$ \\
\hline $2.7437 E+03$ & $1.37+9 E+03$ \\
\hline $2.4513 E+03$ & $2.4513 E+03$ \\
\hline $2.2830 E+03$ & $3.8050 E+02$ \\
\hline $1.6334 E+03$ & $5.1044 E+01$ \\
\hline $1.5970 E+03$ & $1.5970 \mathrm{E}+03$ \\
\hline $1.5363 E+03$ & $3.8408 E+02$ \\
\hline $1.5310 E+03$ & $1.5310 \mathrm{E}+03$ \\
\hline $1.2930 \mathrm{E}+03$ & $6.4650 E+02$ \\
\hline $5.4600 \mathrm{E}+02$ & $2.7300 \mathrm{E}+02$ \\
\hline $3.8800 \mathrm{E}+02$ & $3.8800 \mathrm{E}+02$ \\
\hline $2.3766 \mathrm{E}+02$ & $2.3766 E+02$ \\
\hline $2.0287 E+02$ & $2.2541 E+01$ \\
\hline $3.4000 E+01$ & $1.1333 E+01$ \\
\hline $3.2870 E+01$ & $1.6435 E+01$ \\
\hline $2.3850 E+01$ & $2.3850 E+01$ \\
\hline $9.5000 \mathrm{E}+00$ & $9.5000 \mathrm{E}+00$ \\
\hline
\end{tabular}

$6.2987 E+05$
STD DEV

MINIMUM GROSS WT, KG MAXMUM GROSS WT, KG

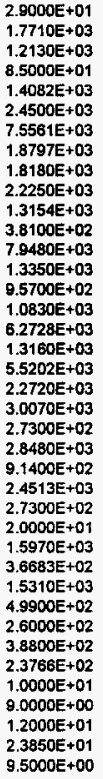

$1.1998 \mathrm{E}+03$

2.1790E+03

$2.9690 \mathrm{E}+03$

$4.8300 \mathrm{E}+02$

$4.8150 \mathrm{E}+03$

$5.3760 \mathrm{E}+03$

$7.5700 \mathrm{E}+03$

$5.2286 \mathrm{E}+03$

$1.8180 \mathrm{E}+03$

$5.3600 \mathrm{E}+03$

$2.6308 E+03$

$6.4900 E+02$

$7.9490 \mathrm{E}+03$

$2.0500 E+03$

$2.8190 E+03$

$3.0230 \mathrm{E}+03$

$6.2728 \mathrm{E}+03$

$2.9550 \mathrm{E}+03$

$5.5202 \mathrm{E}+03$

$2.4546 \mathrm{E}+03$

$3.0070 E+03$

$2.7300 E+02$

$2.8480 E+03$

$1.8297 \mathrm{E}+03$

$2.4513 E+03$

$5.1700 \mathrm{E}+02$

$1.3800 \mathrm{E}+02$

$1.5970 E+03$

4.1223E +02

$1.5310 E+03$

$7.9400 \mathrm{E}+02$

2.8600E +02

$3.8800 \mathrm{E}+02$

2.3766E +02

$8.8990 \mathrm{E}+01$

$1.4000 \mathrm{E}+01$

$2.0870 \mathrm{E}+01$

$2.3850 E+01$

$9.5000 E+00$ 
WHC-SD-W-242-ES-004, Rev. 0

TABLE 43

STATISTICAL ANALYSIS OF CONTAINER TYPES

IN ASCENDING ORDER BASED ON TOTAL TARE WEIGHT

(1 SHEET)

CONTAINER TYPE COUNT TOTAL TARE WT, KG AVERAGE TARE WT, KG

STD DEV MINIMUM TARE WT, KG MAXIMUM TARE WT, KG

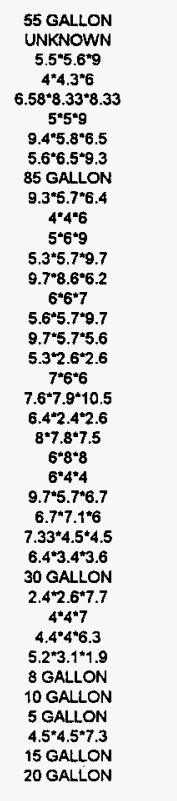

$\begin{array}{lll}6.5462 E+04 & 2.8083 E+01 & 4.6230 E+00 \\ 1.5504 E+04 & 5.1680 E+03 & 0.0000 E+00 \\ 1.5086 E+04 & 7.9400 E+02 & 0.0000 E+00 \\ 1.2342 E+04 & 3.6300 E+02 & 0.0000 E+00 \\ 8.0640 E+03 & 1.1520 E+03 & 0.0000 E+00 \\ 7.7730 E+03 & 7.7730 E+02 & 2.6844 E+01 \\ 7.3500 E+03 & 1.2250 E+03 & 0.0000 E+00 \\ 7.1850 E+03 & 1.1975 E+03 & 6.7361 E+01 \\ 5.5627 E+03 & 3.8630 E+01 & 1.2151 E+00 \\ 4.9000 E+03 & 1.2250 E+03 & 0.0000 E+00 \\ 4.2016 E+03 & 2.2114 E+02 & 3.4161 E+01 \\ 3.9700 E+03 & 7.9400 E+02 & 0.0000 E+00 \\ 2.9950 E+03 & 7.4875 E+02 & 1.2383 E+02 \\ 2.5750 E+03 & 2.5750 E+03 & 0.0000 E+00 \\ 2.4500 E+03 & 1.2250 E+03 & 0.0000 E+00 \\ 2.3820 E+03 & 7.9400 E+02 & 0.0000 E+00 \\ 2.3820 E+03 & 7.9400 E+02 & 0.0000 E+00 \\ 2.0020 E+03 & 1.8200 E+02 & 0.0000 E+00 \\ 1.8260 E+03 & 9.1300 E+02 & 4.0164 E+02 \\ 1.3340 E+03 & 1.3340 E+03 & 0.0000 E+00 \\ 1.2600 E+03 & 2.1000 E+02 & 0.0000 E+\infty 0 \\ 1.2270 E+03 & 1.2270 E+03 & 0.0000 E+00 \\ 9.7500 E+02 & 9.7500 E+02 & 0.0000 E+00 \\ 8.4720 E+02 & 2.0430 E+02 & 0.0000 E+00 \\ 7.9400 E+02 & 7.9400 E+02 & 0.0000 E+00 \\ 7.7000 E+02 & 7.7000 E+02 & 0.0000 E+00 \\ 5.9900 E+02 & 5.9900 E+02 & 0.0000 E+00 \\ 5.9600 E+02 & 2.9800 E+02 & 0.0000 E+00 \\ 5.2600 E+02 & 1.6438 E+01 & 2.6632 E+00 \\ 4.2000 E+02 & 2.1000 E+02 & 0.0000 E+00 \\ 3.5600 E+02 & 3.5600 E+02 & 0.0000 E+00 \\ 2.8800 E+02 & 2.8800 E+02 & 0.0000 E+00 \\ 1.6466 E+02 & 1.6466 E+02 & 0.0000 E+00 \\ 4.5000 E+01 & 5.0000 E+00 & 0.0000 E+00 \\ 1.0000 E+01 & 5.0000 E+00 & 0.0000 E+00 \\ 6.0000 E+00 & 2.0000 E+00 & 0.0000 E+00 \\ 0.0000 E+00 & 0.0000 E+00 & 0.0000 E+\infty 0 \\ 0.0000 E+00 & 0.0000 E+00 & 0.0000 E+00 \\ 0.0000 E+00 & 0.0000 E+00 & 0.0000 E+00\end{array}$

2.0000E +01

2.1000E+02

$5.1680 \mathrm{E}+03 \quad 5.1680 \mathrm{E}+03$

$7.9400 \mathrm{E}+02$

$3.6300 E+02 \quad 3.6300 E+02$

$1.1520 E+03 \quad 1.1520 E+03$

$7.3800 \mathrm{E}+02 \quad 7.9400 \mathrm{E}+02$

$1.2250 E+03$

$1.0600 E+03$

$3.0000 \mathrm{E}+01$

$1.2250 E+03$

$2.0400 \mathrm{E}+02$

$7.9400 E+02$

$5.6600 E+02$

$2.5750 E+03$

$1.2250 E+03$

$7.9400 E+02$

$7.9400 \mathrm{E}+02$

$1.8200 E+02$

$6.2900 \mathrm{E}+02$

1.334OE+03

2.1000E+02

1.2270E+03

9.7500E+02

$2.0430 E+02$

7.9400E+02

7.7000E+02

$5.9900 \mathrm{E}+02$

$2.9800 \mathrm{E}+02$

1.5000E+01

2.1000E+02

$3.5600 \mathrm{E}+02$

$2.8800 \mathrm{E}+02$

$1.6466 \mathrm{E}+02$

$5.0000 E+00$

$5.0000 E+00$

$2.0000 E+00$

$0.0000 E+00$

$0.0000 \mathrm{E}+00$

1.2250E+03

1.2250E+03

3.9000E+01

1.2250E+03

$2.8800 \mathrm{E}+02$

$7.9400 E \div 02$

8.4100E+02

2.5750E+03

1.2250E+03

$7.9400 \mathrm{E}+02$

$7.9400 E+02$

$1.8200 E+02$

$1.1070 E+03$

$1.3340 E+03$

2.1000E+02

$1.2270 E+03$

$9.7500 E+02$

$2.0430 \mathrm{E}+02$

$7.9400 E+02$

7.7000E+02

$5.9900 E+02$

2.9800E +02

3.1000E+01

2.1000E +02

$3.5800 E+02$

$2.8800 \mathrm{E}+02$

1.6466E+02

$5.0000 E+00$

$5.0000 E+00$

2.0000E+00

$0.0000 E+00$

$0.0000 E+00$

$0.0000 E+00$

TOTAL WEIGHT

$1.8420 E+05$ 
WHC-SD-W-242-ES-004, Rev. 0

TABLE 44

STATISTICAL ANALYSIS OF CONTAINER TYPES

IN ASCENDING ORDER BASED ON TOTAL WASTE WEIGHT

(1 SHEET)

\begin{tabular}{|c|c|c|}
\hline CONTANER TYPE & COUNT & TOTAL WASTE W \\
\hline 55 GALLON & $\begin{array}{c}2331 \\
34\end{array}$ & $\begin{array}{l}1.9047 E+05 \\
5.8017 E+04\end{array}$ \\
\hline 85 GALLON & 144 & $3.5944 E+04$ \\
\hline $5.5 * 5.6 * 9$ & 19 & $2.8705 E+04$ \\
\hline 5*5*9 & 10 & $2.4084 \mathrm{E}+04$ \\
\hline $9.4^{*} 5.8 * 6.5$ & 6 & $1.5402 E+04$ \\
\hline $5.6^{*} 6.5^{*} 9.3$ & 6 & $1.2838 E+04$ \\
\hline $9.3^{* 5.7 * 6.4}$ & 4 & $7.3960 E+03$ \\
\hline UNKNOWN & 3 & $7.4802 E+03$ \\
\hline $9.7^{*} 8.6^{*} 6.2$ & 1 & $5.3730 E+03$ \\
\hline $7.6^{* 7.9 * 10.5}$ & 1 & $4.9388 E+03$ \\
\hline $4.5^{*} 4.5^{*} 7.3$ & 5 & $4.8991 E+03$ \\
\hline $4^{*} 4^{* 6}$ & 19 & $4.6704 E+03$ \\
\hline $6.58 * 8.33 * 8.33$ & 7 & $4.6620 E+03$ \\
\hline $6 * 8 * 8$ & 1 & $4.5450 E+03$ \\
\hline $5.6 * 5.7 \mathrm{mg} .7$ & 3 & $3.9710 E+03$ \\
\hline $5^{*} 6^{* 9}$ & $\mathbf{5}$ & $3.9650 E+03$ \\
\hline $9.7 * 5.7 * 5.6$ & 3 & $3.7160 \mathrm{E}+03$ \\
\hline $5.3 * 5.7 * 9.7$ & 4 & $3.3332 E+03$ \\
\hline $6^{*} 6 * 7$ & 2 & $3.0480 E+03$ \\
\hline $4^{n} 4^{n 7}$ & 1 & $2.0953 E+03$ \\
\hline $6.7^{\star} 7.1^{\star} 6$ & 1 & $2.0780 E+03$ \\
\hline $8 * 7.8^{*} 7.5$ & 1 & $1.7800 E+03$ \\
\hline 30 GALLON & 32 & $1.1837 \mathrm{E}+03$ \\
\hline $6.4^{*} 2.4^{*} 2.6$ & 6 & $1.0230 \mathrm{E}+03$ \\
\hline $5.3^{*} 2.6 * 2.6$ & 11 & $1.0010 E+03$ \\
\hline $7.33^{*} 4.5^{*} 4.5$ & 1 & $9.3200 E+02$ \\
\hline $7^{*} 6 * 6$ & 2 & $9.1770 E+02$ \\
\hline $9.7 * 5.7 * 6.7$ & 1 & $8.0300 E+02$ \\
\hline $6^{*} 4^{*} 4$ & 4 & $7.1913 E+02$ \\
\hline $6.4^{* 3.4 * 3.6}$ & 2 & $6.9700 \mathrm{E}+02$ \\
\hline 8 GALLON & 9 & $1.5643 E+02$ \\
\hline $2.4^{*} 2.6^{*} 7.7$ & 2 & $1.2600 \mathrm{E}+02$ \\
\hline $4.4^{*} 4^{*} 6.3$ & 1 & $1.0000 \mathrm{E}+02$ \\
\hline $5.2 * 3.1 * 1.9$ & 1 & $7.3000 E+01$ \\
\hline 5 GALLON & 3 & $2.6010 E+01$ \\
\hline 10 GALLON & 2 & $2.2000 E+01$ \\
\hline 20 GALLON & 1 & $1.9090 \mathrm{E}+01$ \\
\hline 15 GALLON & 1 & $4.5000 E+00$ \\
\hline TOTAL WEIGHT & & $4.4091 E+05$ \\
\hline
\end{tabular}

STD DEV

6.0756E+01 $7.0084 \mathrm{E}+01$

$1.0872 \mathrm{E}+02$

$5.4660 E+02$

$1.2707 E+03$

9.8263E +02

1.1445E+03

$1.5255 E+03$

$6.9363 E+00$

$0.0000 E+00$

$0.0000 E+00$

5.6745E+02

$5.0386 E+01$

$0.0000 E+00$

$0.0000 E+00$

9.7645E+02

2.7670E+02

8.3863E+02

$7.9869 E+02$

4.1634E+02

$0.0000 E+00$

$0.0000 E+00$

$0.0000 E+00$

2.5876E+01

$1.0565 E+02$

$0.0000 E+00$

$0.0000 E+00$

2.4586E +02

$0.0000 E+00$

$1.9744 E+01$

2.0860E+02

$2.4995 E+01$

$1.8385 E+01$

$0.0000 E+00$

$0.0000 E+00$

$2.0873 E+00$

$5.6569 E+00$

$0.0000 E+00$

$0.0000 E+00$
MINIMUM WASTE WT, KG MAXIMUM WASTE WT, KG

$$
\begin{aligned}
& 1.5900 E+00 \\
& 1.4080 E+03 \\
& 4.0000 E+01 \\
& 4.1900 E+02 \\
& 5.6200 E+02 \\
& 1.2250 E+03 \\
& 6.3800 E+02 \\
& 1.0000 E+03 \\
& 2.3881 E+03 \\
& 5.3730 E+03 \\
& 4.9388 E+03 \\
& 2.4344 E+02 \\
& 1.0340 E+02 \\
& 6.6600 E+02 \\
& 4.5450 E+03 \\
& 2.8900 E+02 \\
& 5.4100 E+02 \\
& 5.2200 E+02 \\
& 1.6300 E+02 \\
& 1.2296 E+03 \\
& 2.0063 E+03 \\
& 2.0780 E+03 \\
& 1.7800 E+03 \\
& 4.0000 E+00 \\
& 6.3000 E+01 \\
& 9.1000 E+01 \\
& 9.3200 E+02 \\
& 2.8500 E+02 \\
& 8.0300 E+02 \\
& 1.6253 E+02 \\
& 2.0100 E+02 \\
& 4.9962 E+00 \\
& 5.0000 E+01 \\
& 1.0000 E+02 \\
& 7.3000 E+01 \\
& 7.0000 E+00 \\
& 7.0000 E+00 \\
& 1.9090 E+01 \\
& 4.5000 E+00
\end{aligned}
$$

4.2300E+02

$1.8160 E+03$

4.4400E+02

$2.17505+03$

$4.0210 E+03$

$4.1510 E+03$

4.0035E+03

$4.1350 E+03$

2. $4012 \mathrm{E}+03$

$5.3730 \mathrm{E}+03$

$4.9388 E+03$

1.7637E+03

3.6100E+02

6.6600E+02

4.5450E+03

2.2290E+03

$1.2560 \mathrm{E}+03$

2.1610E +03

$1.9780 E+03$

1.8184E+03

2.0953E+03

2.0780E+03

$1.7800 E+03$

$1.0700 E+02$

$3.0700 E+02$

9.1000E+01

9.3200E+02

$6.3270 E+02$

8.0300E+02

$2.0793 \mathrm{E}+02$

4.9600E+02

8.3000E+01

$7.6000 E+01$

$1.0000 E+02$

$7.3000 E+01$

1.1010E+01

1.5000E+01

$1.9090 E+01$

4.5000E+00 
WHC-SD-W-242-ES-004, Rev. 0

TABLE 45

GENERATOR-SPECIFIC WASTE VOLUMES

SORTED IN ASCENDING ORDER BASED ON ACCUMULATED VOLUME

(2 SHEETS)

\begin{tabular}{|c|c|c|c|c|c|}
\hline COMPANY & GENERATOR & CONTAINER COUNT & ACCUM VOLUME. CU M & PERCENT TOTAL VOLUME & ACCUMULATED PERCENT \\
\hline WHC & 23452 & 454 & $3.5019 E+02$ & 23.0963 & 23.0963 \\
\hline PSN & PSNS & 34 & $9.9348 E+01$ & 6.5524 & 29.6487 \\
\hline WHC & $100 \mathrm{~N}$ & 408 & $8.5622 E+01$ & 5.6471 & 35.2958 \\
\hline WHC & $242 A$ & 17 & $8.1663 E+01$ & 5.3860 & 40.6818 \\
\hline WHC & $241 \mathrm{~s}$ & 26 & $7.7438 E+01$ & 5.1074 & 45.7892 \\
\hline WHC & $241 \mathrm{~A}$ & 18 & $7.1051 E+01$ & 4.6861 & 50.4753 \\
\hline WHC & $241 \mathrm{C}$ & 11 & 5.1809E +01 & 3.4170 & 53.8924 \\
\hline WHC & MIXTF & 7 & $4.9255 E+01$ & 3.2486 & 57.1409 \\
\hline LBL & LBLAB & 215 & $4.4870 E+01$ & 2.9594 & 60.1003 \\
\hline WHC & $2415 Y$ & 9 & $4.4161 E+01$ & 2.9126 & 63.0129 \\
\hline ANL & ARGON & 210 & $4.3722 E+01$ & 2.8837 & 65.8966 \\
\hline BATC & BATCO & 205 & 4. $2274 E+01$ & 2.7882 & 68.6848 \\
\hline WHC & $222 \mathrm{~S}$ & 145 & $4.1982 E+01$ & 2.7669 & 71.4536 \\
\hline ALB & ALBNY & 126 & $4.0572 E+01$ & 2.6759 & 74.1295 \\
\hline WHC & $202 A$ & 95 & $2.7978 E+01$ & 1.8453 & 75.9748 \\
\hline WHC & $241 \mathrm{~T}$ & 11 & $2.7765 E+01$ & 1.8312 & 77.8060 \\
\hline WHC & $221 \mathrm{~T}$ & 69 & $2.0638 \mathrm{E}+01$ & 1.3612 & 79.1672 \\
\hline WHC & $271 T$ & 1 & $1.7925 E+01$ & 1.1822 & 80.3495 \\
\hline WHC & P01SY & 2 & $1.7000 E+01$ & 1.1212 & 81.4707 \\
\hline WHC & $276 s$ & 11 & $1.7000 E+01$ & 1.1212 & 82.5919 \\
\hline WHC & $242 S$ & 2 & $1.5300 E+01$ & 1.0091 & 83.6010 \\
\hline WHC & $241 T X$ & 12 & $1.4423 E+01$ & 0.9513 & 84.5523 \\
\hline WHC & 2718 & 28 & $1.2281 E+01$ & 0.8100 & 85.3622 \\
\hline $\mathrm{KEH}$ & 2312 & 4 & $1.1760 E+01$ & 0.7756 & 86.1379 \\
\hline WHC & $105 \mathrm{KE}$ & 2 & $1.1109 E+01$ & 0.7327 & 86.8706 \\
\hline PNL & 324 & 11 & $1.0560 E+01$ & 0.6965 & 87.5670 \\
\hline BAPL & BAPL & 49 & $1.0202 E+01$ & 0.6729 & 88.2399 \\
\hline BNL & BNLAB & 49 & $1.0202 E+01$ & 0.6729 & 88.9127 \\
\hline WHC & $271 \mathrm{CR}$ & 3 & $9.8482 E+00$ & 0.6495 & 89.5623 \\
\hline WHC & $241 B$ & 4 & 9. $3162 E+00$ & 0.6144 & 90.1767 \\
\hline WHC & $2415 x$ & 4 & $8.9246 \mathrm{E}+00$ & 0.5886 & 90.7653 \\
\hline $\mathrm{WHC}$ & $224 U$ & 44 & $8.8842 E+00$ & 0.5860 & 91.3513 \\
\hline WHC & $241 \mathrm{AW}$ & 2 & $8.5080 E+00$ & 0.5611 & 91.9124 \\
\hline WHC & $241 A Z$ & 1 & $8.3000 E+00$ & 0.5474 & 92.4598 \\
\hline FERM & NALAB & 37 & $7.7484 E+00$ & 0.5110 & 92.9709 \\
\hline WHC & 303к & 35 & $7.1924 E+00$ & 0.4744 & 93.4453 \\
\hline WHC & T PLANT & 1 & $6.3700 E+00$ & 0.4201 & 93.8654 \\
\hline PNL & 331 & 30 & $6.2802 E+00$ & 0.4142 & 94.2796 \\
\hline PNL & 3058 & 23 & $5.5710 E+00$ & 0.3674 & 94.6470 \\
\hline WHC & $163 \mathrm{PA}$ & 26 & $4.9050 E+00$ & 0.3235 & 94.9705 \\
\hline WHC & $221 \mathrm{~B}$ & 22 & $4.2104 \mathrm{E}+00$ & 0.2777 & 95.2482 \\
\hline WHC & $241 \mathrm{AP}$ & 6 & $4,1628 E+00$ & 0.2746 & 95.5228 \\
\hline WHC & $221 \mathrm{TS}$ & 18 & $3.7800 E+00$ & 0.2493 & 95.7721 \\
\hline WHC & BG3AE & 17 & $3.5700 \mathrm{E}+00$ & 0.2355 & 96.0075 \\
\hline PNL & 325 & 17 & $3.3128 E+00$ & 0.2185 & 96.2260 \\
\hline WHC & 2312 & 1 & $3.1400 E+\infty 0$ & 0.2071 & 96.4331 \\
\hline WHC & $1310 P$ & 15 & $2.8470 E+00$ & 0.1678 & 96.6209 \\
\hline WHC & $241 \mathrm{AY}$ & 14 & $2.8196 E+\infty$ & 0.1860 & 96.8069 \\
\hline WHC & $105 \mathrm{~N}$ & 14 & $2.7510 E+00$ & 0.1814 & 96.9893 \\
\hline WHC & 324 & 12 & $2.5200 E+00$ & 0.1662 & 97.1545 \\
\hline WHC & $163 N$ & 11 & $2.2974 \mathrm{E}+00$ & 0.1515 & 97.3060 \\
\hline PNL & LSLII & 11 & $2.2956 \mathrm{E}+00$ & 0.1514 & 97.4574 \\
\hline WHC & 2025 & 11 & $2.2902 \mathrm{E}+00$ & 0.1510 & 97.6085 \\
\hline WHC & 2436 & 1 & 2. $2100 E+00$ & 0.1458 & 97.7543 \\
\hline KER & MCGEE & 10 & 2. $1000 E+00$ & 0.1385 & 97.8928 \\
\hline WHC & 333 & 9 & 1. $8882 E+00$ & 0.1245 & 98.0173 \\
\hline WHC & $202 \mathrm{AL}$ & 9 & $1.8846 E+00$ & 0.1243 & 98.1416 \\
\hline WHC & $105 \mathrm{KW}$ & 8 & $1.6656 E+00$ & 0.1099 & 98.2514 \\
\hline WHC & 377 & 8 & $1.6656 \mathrm{E}+\infty 0$ & 0.1099 & 98.3613 \\
\hline WHC & 308 & 7 & $1.4574 \mathrm{E}+00$ & 0.0961 & 98.4574 \\
\hline PNL & RTL & 6 & 1.2600E + OO & 0.0831 & 98.5405 \\
\hline WHC & $225 \mathrm{~B}$ & 6 & $1.2600 E+00$ & 0.0831 & 98.6236 \\
\hline Whe & $1706 \mathrm{~K}$ & 6 & $1.2492 \mathrm{E}+00$ & 0.0824 & 98.7060 \\
\hline
\end{tabular}


WHC-SD-W-242-ES-004, Rev. 0

TABLE 45

GENERATOR-SPECIFIC WASTE VOLUMES

SORTED IN ASCENDING ORDER BASED ON ACCUMULATED VOLUME

(2 SHEETS)

\begin{tabular}{|c|c|c|c|c|}
\hline COMPANY & GENERATOR & CONTAINER COUNT & ACCUM VOLUME, CU M & PERCENT TOTAL VOLUME \\
\hline WHC & $241 B X$ & 6 & $1.1864 E+\infty 0$ & 0.0782 \\
\hline PNL & ISV & 4 & $1.1790 \mathrm{E}+\infty 0$ & 0.0778 \\
\hline WHC & 241 AN & 5 & $1.1542 E+00$ & 0.0761 \\
\hline WHC & $100 \mathrm{KI}$ & 3 & $1.1382 \mathrm{E}+00$ & 0.0761 \\
\hline WHC & 204AR & 1 & $1.1200 E+00$ & 0.0739 \\
\hline PNL & 329 & 5 & $1.0428 \mathrm{E}+00$ & 0.0688 \\
\hline WHC & $109 \mathrm{~N}$ & 5 & $1.0410 E+\infty 0$ & 0.0687 \\
\hline WHC & $241 U$ & 5 & $1.0408 E+00$ & 0.0686 \\
\hline vou & UURE & 5 & $1.0400 E+00$ & 0.0686 \\
\hline WHC & $241 \mathrm{TY}$ & 4 & 8.3280E-01 & 0.0549 \\
\hline WHC & 244AI7 & 4 & 8.3280E-01 & 0.0549 \\
\hline WHC & $200 \mathrm{~W}$ & 2 & $6.4360 \mathrm{E}-01$ & 0.0424 \\
\hline WHC & 1706KE & 3 & 6. 2460E-O 1 & 0.0412 \\
\hline WHC & 2706\lceil & 3 & $6.2460 \mathrm{E}-01$ & 0.0412 \\
\hline PNL & 3745 & 2 & 4.2000E-01 & 0.0277 \\
\hline WHC & $241 A X$ & 2 & 4. 200OE-01 & 0.0277 \\
\hline WHC & 340 & 2 & 4.2000E-01 & 0.0277 \\
\hline PNL & 3720 & 2 & 4.1720E-01 & 0.0275 \\
\hline PNL & 326 & 2 & 4.164OE 01 & 0.0275 \\
\hline WHC & 163PAD & 2 & 4. $1640 \mathrm{E}-01$ & 0.0275 \\
\hline WHC & 241By & 2 & 4. $1000 E-01$ & 0.0270 \\
\hline WHC & 2710 & 2 & 4.0000E-01 & 0.0264 \\
\hline PNL & 1234 & 1 & $3.2800 E-01$ & 0.0216 \\
\hline WHC & $105 \mathrm{H}$ & 1 & 3.21BOE-01 & 0.0212 \\
\hline WHC & 2ETF & 1 & $2.1000 E-01$ & 0.0139 \\
\hline SLA & SLACIJ & 1 & $2.0820 \mathrm{E}-01$ & 0.0137 \\
\hline WHC & 151EA & 1 & 2.0820E-01 & 0.0137 \\
\hline WHC & 2400 & 1 & $2.0820 \mathrm{E}-\mathrm{O} 1$ & 0.0137 \\
\hline WHC & 241EW & 1 & $2.0820 E-01$ & 0.0137 \\
\hline WHC & $2713 \mathrm{~W}$ & 1 & $2.0820 \mathrm{E}-01$ & 0.0137 \\
\hline WHC & 309 & 1 & 2.0820E-01 & 0.0137 \\
\hline WHC & $276 U$ & 1 & $2.0000 E-01$ & 0.0132 \\
\hline WHC & 313 & 1 & 2.0000E-01 & 0.0132 \\
\hline WHC & $399-31$ & 1 & 2.0000E-01 & 0.0132 \\
\hline WHC & 321 & 1 & 1.1000E-01 & 0.0073 \\
\hline TOTAL & & & $1.5162 E+03$ & \\
\hline
\end{tabular}

ACCUMULATED PERCENT

98.7843

98.8620

98.9381

99.0132

99.0871

99.1559

99.2245

99.2932

99.3618

99.4167

99.4716

99.5141

99.5553

99.5965

99.6242

99.6519

99.6796

99.7071

99.7345

99.7620

99.7890

99.8154

99.8371

99.8563

99.8721

99.8859

99.8996

99.9133

99.9271

99.9408

99.9646

99.9677

99.9808

99.9941

100.0013 
WHC-SD-W-242-ES-004, Rev. 0

TABLE 46

WASTE VOLUMES BY GROUP AND BY GENERATOR (1 SHEET)

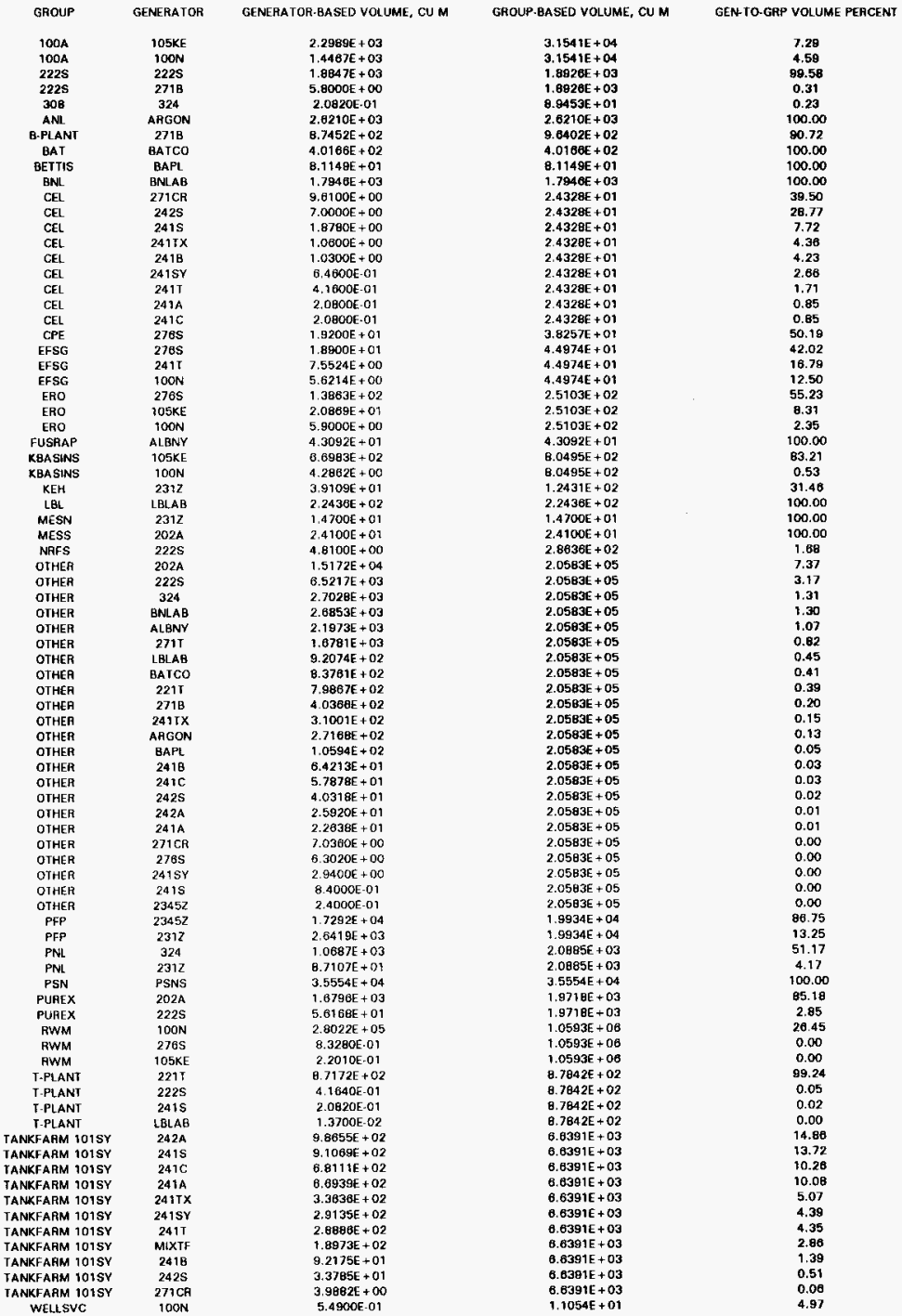


WHC-SD-W-242-ES-004, Rev. 0

TABLE 47

WASTE WEIGHTS BY GROUP AND BY GENERATOR

(1 SHEET)

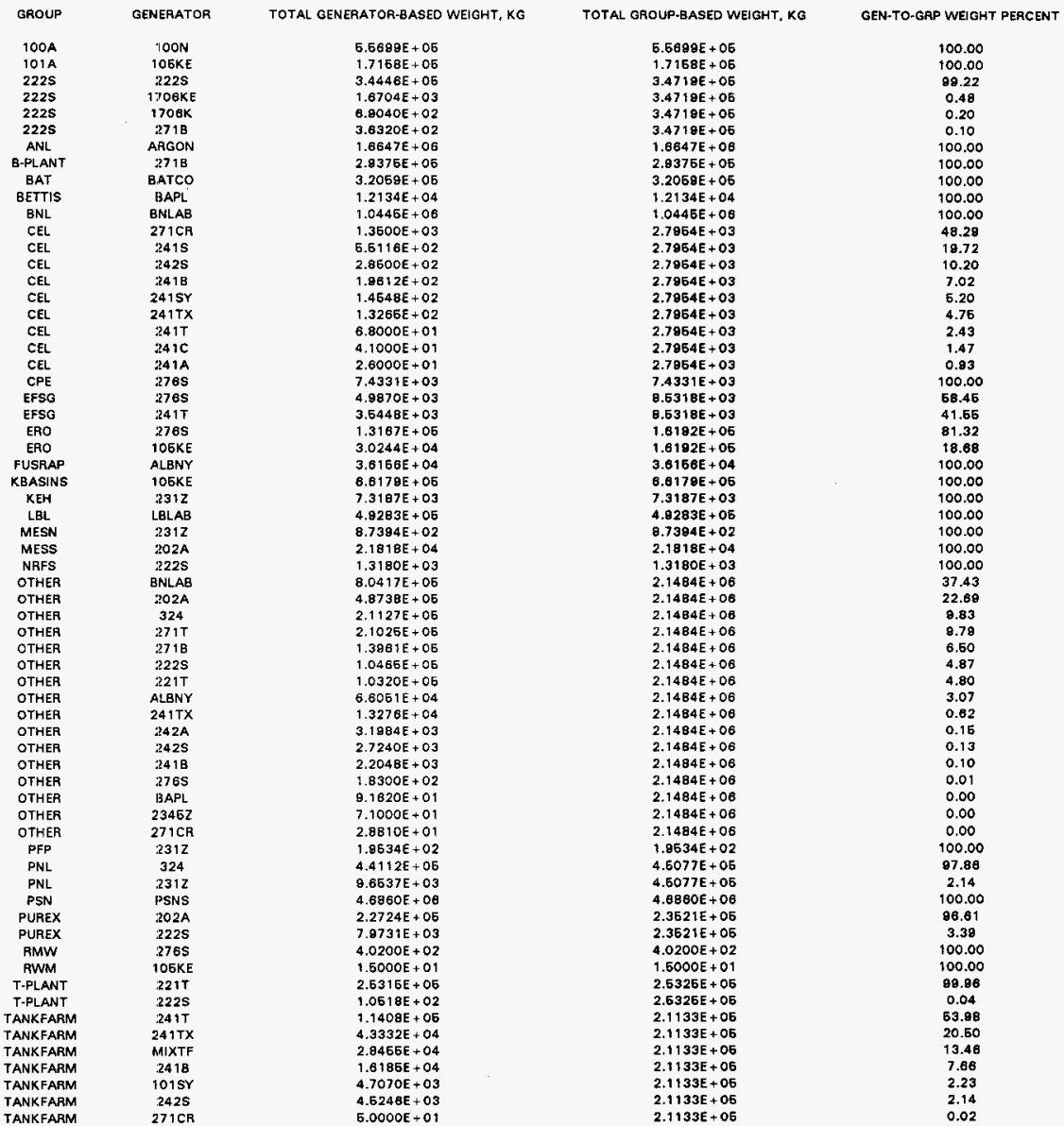


WHC-SD-W-242-ES-004, Rev. 0

TABLE 48

GROUP FACILITY DESCRIPTIONS

(1 SHEET)

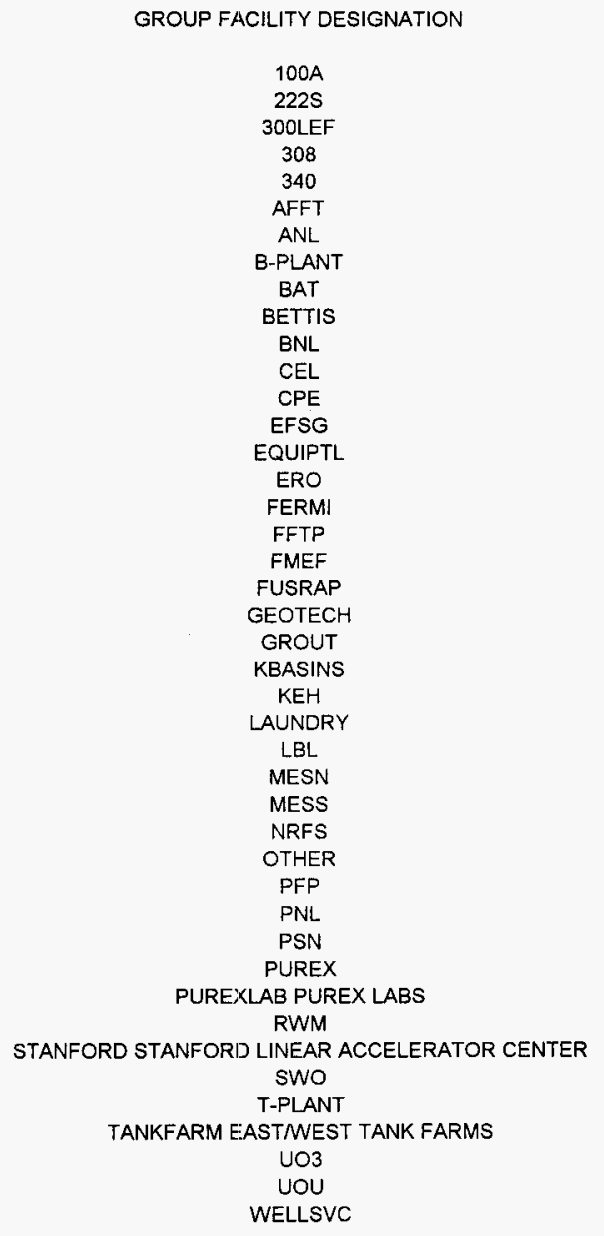


WHC-SD-W-242-ES-004, Rev. 0

TABLE 49

LIST OF QUANTITATIVELY SIGNIFICANT GROUPS AND GENERATORS

(1 SHEET)

$\begin{array}{cc}\text { GROUP } & \text { GENERATOR } \\ 100 A & 100 \mathrm{~N} \\ 101 \mathrm{~A} & 101 \mathrm{SY} \\ 222 \mathrm{~S} & 105 \mathrm{KE} \\ \text { ANL } & 202 \mathrm{~A} \\ \text { B-PLANT } & 221 \mathrm{~T} \\ \text { BAT } & 222 \mathrm{~S} \\ \text { BNL } & 231 \mathrm{Z} \\ \text { ERO } & 241 \mathrm{~B} \\ \text { KBASINS } & 241 \mathrm{~T} \\ \text { LBL } & 241 \mathrm{TX} \\ \text { OTHER } & 242 \mathrm{~S} \\ \text { PNL } & 271 \mathrm{~B} \\ \text { PSN } & 271 \mathrm{~T} \\ \text { PUREX } & 276 \mathrm{~S} \\ \text { T-PLANT } & 324 \\ \text { TANKFARM } & \text { ALBNY } \\ & \text { ARGON } \\ & \text { BATCO } \\ & \text { BNLAB } \\ & \text { LBLAB } \\ & \text { MIXTF } \\ & \text { PSNS }\end{array}$


WHC-SD-W-242-ES-004, Rev. 0

TABLE 50

SIGNIFICANT PHYSICAL PROPERTIES CORRELATED BY GROUP AND BY GENERATOR

(4 SHEETS)

\begin{tabular}{|c|c|}
\hline GROUP & GENERATOR \\
\hline $100 \mathrm{~A}$ & $100 \mathrm{~N}$ \\
\hline $100 \mathrm{~A}$ & $100 \mathrm{~N}$ \\
\hline $100 \mathrm{~A}$ & $100 \mathrm{~N}$ \\
\hline $100 \mathrm{~A}$ & $100 \mathrm{~N}$ \\
\hline $100 \mathrm{~A}$ & $100 \mathrm{~N}$ \\
\hline $100 \mathrm{~A}$ & $100 \mathrm{~N}$ \\
\hline $100 \mathrm{~A}$ & $100 \mathrm{~N}$ \\
\hline $100 \mathrm{~A}$ & $100 \mathrm{~N}$ \\
\hline $101 \mathrm{~A}$ & 105KE \\
\hline $101 \mathrm{~A}$ & 10EKE \\
\hline $101 \mathrm{~A}$ & 106KE \\
\hline $101 \mathrm{~A}$ & 106KE \\
\hline $101 \mathrm{~A}$ & 105KE \\
\hline $222 S$ & 2225 \\
\hline 2225 & $222 \mathrm{~S}$ \\
\hline $222 \mathrm{~S}$ & 2225 \\
\hline 2225 & $222 \mathrm{~S}$ \\
\hline 2225 & 2225 \\
\hline $222 \mathrm{~S}$ & 2225 \\
\hline 2225 & $222 \mathrm{~S}$ \\
\hline $222 \mathrm{~S}$ & $222 \mathrm{~S}$ \\
\hline 2225 & 2225 \\
\hline 2225 & $222 \mathrm{~S}$ \\
\hline $222 \mathrm{~S}$ & 2225 \\
\hline 2225 & $222 S$ \\
\hline ANL & ARGON \\
\hline ANL & ARGON \\
\hline ANL & ARGON \\
\hline ANL & ARGON \\
\hline ANL & ARGON \\
\hline ANL & ARGON \\
\hline ANL & ARGON \\
\hline ANL & ARGON \\
\hline ANL & ARGON \\
\hline ANL & ARGON \\
\hline ANL & ARGON \\
\hline ANL & ARGON \\
\hline BPLANT & 2718 \\
\hline B.PLANT & $271 \mathrm{~B}$ \\
\hline BPLANT & 2718 \\
\hline B-PLANT & $271 B$ \\
\hline B-PLANT & 2718 \\
\hline B-PLANT & 2718 \\
\hline B.PLANT & 2718 \\
\hline BPLANT & 2718 \\
\hline B-PLANT & 2718 \\
\hline BPLANT & $271 B$ \\
\hline BPLANT & $271 \mathrm{~B}$ \\
\hline BPLIANT & 2718 \\
\hline B-PLANT & $271 B$ \\
\hline BAT & BATCO \\
\hline
\end{tabular}

PHYSICAL PROPERTY

DIRT/SOILOIATOMACEOUS EARTH METALARON/GALVANIZED/SHE

$$
\text { OILS }
$$

PAPERUCARDBOARD

WOODRUMBER/PLYWOOD

ABSORBENT/KITY LTRNERMICULITE

WEEDSNEGETATION

METALIRON/GALVANIZED/SHEET RESINS

DIRT/SOLLDIATOMACEOUS EARTH METALIRON/GALVANIZED/SHE PLASTIC,POLYURATHANE

$$
\text { CONCRETE }
$$

DIRTISOIUDIATOMACEOUS EARTH

$$
\begin{aligned}
& \text { GLASS } \\
& \text { CLOTHRAGSNYLN }
\end{aligned}
$$

$$
\begin{aligned}
& \text { RUBBER } \\
& \text { LEAD }
\end{aligned}
$$

ASPHALT/BLACKTOP

WOODRUMBERYFI WWOOD

ABSORBENTKITY LTR/VERMICUUTT CONCRETE

DIRT/SOILDIATOMACEOUS EARTH

FOAM/STYROFOAM/PYROFOAM

WOODRUMBER/PTYWOOD

NON-HAZARDOUS METALS

ASTICFOLYURA
SLUDGES

PAPER/CARDBOARO

DIRT/SOILOIATOMACEOUS EARTH

$$
\begin{aligned}
& \text { CONCRETE } \\
& \text { ROCK/GRAVEL }
\end{aligned}
$$

PLASTICPOOLYURATHANE

CLOTHARAGSNYLON

WOODNUMBER/PLYWOOD

ABSORBENTRITY LTRNERMICULITE

FERROUS METAL

$$
\begin{aligned}
& \text { RUBBER } \\
& \text { FLTERS }
\end{aligned}
$$

FOAM/STYROFOAM/PYROFOAM

MET AL AFON/GALVANIZEDISHEET
WEIGHT, KG

$3.7434 E+05$
$6.8228 E+04$ $2.8161 E+04$ $2.3039 E+04$ $1.7211 E+04$ $1.0567 \mathrm{E}+04$

8.6348E+03

$9.4632 E+03$

$8.0296 E+04$

$6.4782 \mathrm{E}+04$

$1.7420 E+04$

$4.3630 E+03$

$9.2527 E+04$

$5.0403 E+04$

$3.8733 E+04$

$2.4441 \mathrm{E}+04$

$1.9129 E+04$

$1.3894 \mathrm{E}+04$

$1.3693 E+04$

$7.7022 \mathrm{E}+03$

$7.4400 \mathrm{E}+03$

$5.7613 E+03$

$3.9823 E+03$

$3.9820 E+05$

$3.2366 \mathrm{E}+05$

$2.4460 \mathrm{E}+0 \mathrm{OB}$

$1.6394 E+05$

$8.0557 \mathrm{E}+04$

$7.9739 E+04$

T.6598E + 04

$7.5852 E+04$

$6.4882 E+04$

$3.0930 \mathrm{E}+04$

$2.9807 E+04$

$1.9669 \mathrm{E}+04$

8.7949E+ +04

$4.8614 E+04$

$4.0024 E+04$

..3865e + 04

$1.6493 \mathrm{E}+04$

$1.1341 \mathrm{E}+04$

$9.2196 E+03$

$9.2196 E+03$
$8.2651 E+03$

$7.4342 \mathrm{E}+03$

$6.8014 E+03$

$4.8963 E+03$

4.3824E+ +03

$1.2633 E+05$
TOTAL GENERATOR-BASED $W T$, KG

6.6699E +06

$5.6699 E+05$
$5.5699 E+05$

$6.5699 \mathrm{E}+0$

6. $5699 \mathrm{E}+\mathrm{O}$

$6.6699 \mathrm{E}+06$

$5.5699 E+O 5$

6. $6699 \mathrm{E}+05$

$1.7168 E+06$

$1.7168 E+05$

$1.715 B E+O 5$

$1.7168 \mathrm{E}+05$

$4.768 \mathrm{C}$

$3.4719 E+06$

$3.4798+06$

3.477OE +OE

$3.4719 E+05$

$3.47195+05$

$3.4718 E+05$

$3.4719 E+05$

$3.4719 E+05$

$3.4719 E+05$

$3.4719 E+05$

$3.4718 E+05$

$1,6647 \mathrm{E}+06$

$1.6647 \mathrm{E}+08$

$1.6647 \mathrm{E}+\mathrm{O}$

$1.6647 \mathrm{E}+06$

$1.6847 \mathrm{E}+0 \mathrm{O}$

$1.6647 \mathrm{E}+06$

$1.8647 \mathrm{E}+06$

$1.6647 \mathrm{E}+06$

1. $6647 \mathrm{E}+\mathrm{OB}$

$1.6647 \mathrm{E}+06$

$1.6647 \mathrm{E}+06$

$1.6847 \mathrm{E}+06$

$2.9376 \mathrm{E}+06$

2.0376E+

2.0376E +

$2.9376 \mathrm{E}+\mathrm{O}$

$2.9375 E+05$

$2.9375 \mathrm{E}+\mathrm{O}$

$2.9376 E+05$

$2.9376 E+05$

$2.9376 E+05$

$2.9376 \mathrm{E}+06$

$2.9376 \mathrm{E}+\mathrm{OS}$

$2.8376 \mathrm{E}+05$

$3.2059 \mathrm{E}+05$
GROUP-BASED WT PERCENT

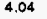

4.04

4.04
4.04

4.04

4.04

1.24
1.24

1.24

1.24
1.24

2.62

.62

2.62

2.62

.62

2.62

2.62

2.06

12.06

12.06

12.06

12.06

12.06

12.06

12.06

2.13

2.13

2.13

2.13

2.13

2.13

2.13

2.13
2.13

2.32
PHYS PROP - BASED WT PERCENT

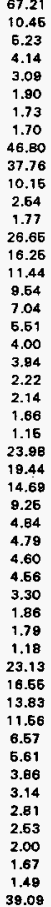


WHC-SD-W-242-ES-004, Rev. 0

TABLE 50

SIGNIFICANT PHYSICAL PROPERTIES CORRELATED BY GROUP AND BY GENERATOR

(4 SHEETS)

\begin{tabular}{|c|c|c|c|c|c|c|}
\hline GROUP & GENERATOR & PHYSICAL PROPEATY & WEIGHT, KG & TOTAL GENERATOR-BASED WT, KG & GROUP-BASED WT PERCENT & PHYS PROP - BASED WT PERCENT \\
\hline BAT & BATCO & CONCRETE & $4.6340 E+04$ & $3.2059 \mathrm{E}+06$ & 2.37 & 14.45 \\
\hline BAT & BATCO & DIRT/SOILOIATOMACEOUS EARTH & $4.0763 E+04$ & $3.2059 E+05$ & 2.32 & 12.71 \\
\hline BAT & BATCO & PLASTIC/POLYURATHANE & $1.3216 E+04$ & $3.2059 E+05$ & 2.32 & 4.12 \\
\hline BAT. & BATCO & ASBESTOS & $1,1365 E+04$ & $3.2069 E+06$ & 2.32 & 3.65 \\
\hline BAT & BATCO & BRICK/FIREBRICK & $1.0003 E+04$ & $3.2059 E+06$ & 2.32 & 3.12 \\
\hline BAT & BATCOO & NON-HAZARDOUS METALS & $9.4616 E+03$ & $3.2069 E+05$ & 2.32 & 2.95 \\
\hline BAT & BATCO & soll & $8.9473 E+03$ & $3.2059 E+05$ & 2.32 & 2.78 \\
\hline BAT & BATCO & SAND & $6.4266 E+03$ & $3.2069 \mathrm{E}+06$ & 2.32 & 1.69 \\
\hline BAT & BATCO & WOOONUMBERPLYWOOD & $6.1148 E+03$ & $3.2059 \mathrm{E}+06$ & 2.32 & 1.60 \\
\hline BAT & BATCO & SLAG & 4.8547E+O3 & $3.2069 E+06$ & 2.32 & 1.61 \\
\hline BAT & BATCO & PAPER/CARDBOARO & $4.6032 E+03$ & $3.2059 E+05$ & 2.32 & 1.44 \\
\hline BAT & BATCO & SLUDGES & $4.4864 E+03$ & $3.2068 E+06$ & 2.32 & 1.40 \\
\hline BAT & BATCO & INSULATION NON-ASBESTOS & $4.2018 E+03$ & $3.2059 E+05$ & 2.32 & 1.31 \\
\hline BAT & BATCO & ROCK/GRAVEL & $3.4483 E+03$ & $3.2068 E+05$ & 2,32 & 1.08 \\
\hline BAT & BATCO & ABSORBENT/KITY LTRMERMICULITE & $3.2662 \mathrm{E}+03$ & $3.2059 E+06$ & 2.32 & 1.02 \\
\hline BNL & BNLAB & METAL/RON/GALVANIZED/SHEET & $4.8603 E+05$ & $1.0446 E+06$ & 3.57 & 46.63 \\
\hline 8NL & BNLAB & CONCRETE & $8.8063 E+04$ & $1.0446 E+06$ & 7.67 & 8.43 \\
\hline BNL & BNLAB & STAINLESS STEEL & $8.4612 E+04$ & $1.0446 E+06$ & 7.67 & 8.10 \\
\hline 8NL & BNLAB & FOAM/STYROFOAM/PYROFOAM & 6.7866E +04 & $1.0446 E+06$ & 7.67 & 5.56 \\
\hline BNL & BNLAB & MISCELLLANEOUS/UNKNOWN/OTHER & $3.8611 E+04$ & $1.0446 E+06$ & 7.67 & 3.78 \\
\hline BNA. & BNLAB & DIRT/SOILOIATOMACEOUS EARTH & $3.3811 E+04$ & $1.0446 E+06$ & 7.67 & 3.26 \\
\hline BNL & BNLAB & COPPER METAL & $3.0791 E+04$ & $1.0446 E+06$ & 7.67 & 2.96 \\
\hline BNIL. & BNLAB & WRE & $2.6646 E+04$ & $1.0446 E+06$ & 7,67 & 2.54 \\
\hline BNL & BNLAB & PLASTICRPOLYURATHANE & $2.6831 E+04$ & $1.0446 E+06$ & 7.67 & 2.48 \\
\hline BNL & BNLAB & SLUDGES & $2.1812 E+04$ & $1.0445 E+06$ & 7.67 & 2.10 \\
\hline BNL & BNLAB & ALUMINUM & $1.6816 E+04$ & $1.0446 E+06$ & 7.67 & 1.61 \\
\hline BNL & BNLAB & CARBON STEEL & $1.6016 E+04$ & $1.0446 E+06$ & 7.67 & 1.63 \\
\hline BNL & BNLAB & PAPER/CARDBOARD & $1.6283 E+04$ & $1.0446 E+06$ & 7.67 & 1.46 \\
\hline BNL & BNLAB & RUBBER & $1.1801 E+04$ & $1.0446 E+06$ & 7.67 & 1.14 \\
\hline BNL & BNLAB & GLASS & $1.164 \mathrm{IE}+04$ & $1.0446 E+06$ & 7,67 & 1.11 \\
\hline BNL & BNLAB & ROCK/GRAVEL & $1.0614 E+04$ & $1.0446 E+06$ & 7.67 & 1.02 \\
\hline ERO & 10EKE & CHEMICALS & $2.6006 E+04$ & $1.6182 \mathrm{E}+05$ & 1.17 & 15.44 \\
\hline ERO & 10EKE & ABSORBENTKITYY LTRVVERMICULITE & $1.7640 E+03$ & $1.6192 E+06$ & 1.17 & 1.09 \\
\hline ERO & 106KE & WOODLUMBERAPLYWOOD & $1.7610 E+03$ & $1.6192 E+05$ & 1.17 & 1.08 \\
\hline ERO & 27 es & ORGANICS & $1.2848 E+05$ & $1.6182 \mathrm{E}+06$ & 1.17 & 79.35 \\
\hline ERO & 2785 & METALARON/GALVANIZED/SHEET & $1.8665 E+03$ & $1.6192 \mathrm{E}+06$ & 1.17 & 1.15 \\
\hline KBASINS & 105KE & CONCRETE & $3.1825 E+05$ & $6.6179 E+06$ & 4.80 & 48.09 \\
\hline KBASINS & 106KE & METAL/RRON/GALVANIZED/SHEET & $2.4086 E+06$ & $6.6179 E+06$ & 4.80 & 36.40 \\
\hline KBASINS & 106KE & $\begin{array}{l}\text { AESINS } \\
\text { A }\end{array}$ & $5.6907 E+04$ & $6.6179 E+06$ & 4.80 & 8.46 \\
\hline KBASINS & 105KE & PLASTIC/POLYURATHANE & $7.74696+03$ & $6.6179 E+06$ & 4.80 & 1.17 \\
\hline KBASINS & 106KE & STAINLESS STEEL & $6.7821 E+03$ & $6.6179 E+06$ & 4.80 & 1.02 \\
\hline LBL & LBLAB & METALARON/GAL VANIZED/SHEET & $4.2616 E+05$ & $4.9283 E+06$ & 3.67 & 80.47 \\
\hline LBL & LELAB & COPPER METAL & $1.4273 E+04$ & $4.9283 E+06$ & 3.67 & 2.90 \\
\hline LBL & LBLAB & HAZARDOUS CONSTITUENTS & $7.0854 E+03$ & $4.9283 \mathrm{E}+06$ & 3.67 & 1.44 \\
\hline LBL & LBLAB & ABSOREENT/KITY LTRVERMICULITE & $6.9781 E+03$ & $4.9283 E+06$ & 3.67 & 1.42 \\
\hline LBL & LELAB & MISCELLANEOUS/UNKNNOWN/OTHER & $6.6271 \mathrm{E}+03$ & $4.9283 E+06$ & 3.67 & 1.32 \\
\hline LBL & LELAB & GLASS & $6.3747 \mathrm{E}+03$ & $4.9283 E+06$ & 3.67 & 1.20 \\
\hline LBL & LBLAB & PLASTICIPOLYURATHANE & $5.6039 E+03$ & $4.9283 E+06$ & 3.67 & 1.12 \\
\hline OTHER & $202 \mathrm{~A}$ & METALARON/GAL VANIZED/SHEET & $3.1092 E+05$ & $2.1494 E+06$ & 16.67 & 14.47 \\
\hline OTHER & $202 A$ & WOODRUMBERPLYYWOOD & $7.7826 E+04$ & $2.1484 E+06$ & 15.57 & 3.62 \\
\hline OTHER & $202 \mathrm{~A}$ & PLASTICIPOLYURATHANE & $2.3898 E+04$ & $2.1484 E+06$ & 15.67 & 1.11 \\
\hline
\end{tabular}


WHC-SD-W-242-ES-004, Rev. 0

TABLE 50

SIGNIFICANT PHYSICAL PROPERTIES CORRELATED BY GROUP AND BY GENERATOR

(4 SHEETS)

\begin{tabular}{lc} 
GROUP & GENERATOR \\
OTHER & $221 T$ \\
OTHER & 2718 \\
OTHER & $271 T$ \\
OTHER & 324 \\
OTHER & 324 \\
OTHER & 324 \\
OTHER & ALBNY \\
OTHER & BNLAB \\
OTHER & BNLAB \\
OTHER & BNAAB \\
OTHER & BNLAB \\
PNL & 2312 \\
PNL & 324 \\
PNL & 324 \\
PNL & 324 \\
PNL & 324 \\
PNL & 324 \\
PNL & 324 \\
PNL & 324 \\
PNL & 324 \\
PNL & 324 \\
PNL & 324 \\
PNL & 324 \\
PNL & 324 \\
PNL & 324 \\
PNL & 324 \\
PSN & PSNS \\
PSN & PSNS \\
PSN & PSNS \\
PSN & PSNS \\
PSN & PSNS \\
PSN & PSNS \\
PUREX & $202 A$ \\
PUREX & $202 A$ \\
PUREX & $202 A$ \\
PUREX & $202 A$ \\
PUREX & $202 A$ \\
PUREX & $202 A$ \\
PUREX & $202 A$ \\
PUREX & $202 A$ \\
PUREX & $202 A$ \\
PUREX & $202 A$ \\
PUREX & $202 A$ \\
PUAEX & $202 A$ \\
TPLANT & $221 T$ \\
TPLANT & $221 T$ \\
TPLANT & $221 T$ \\
TPLANT & $221 T$ \\
TPLANT & $221 T$ \\
TPLANT & $221 T$ \\
TPLANT & $221 T$ \\
& \\
\hline
\end{tabular}

PHYSICAL PAOPERTY

METALIRON/GALVANIZEO/SHEET DIRT/SOILDIATOMACEOUS EARTH METALARONTOMACEOUS EARTH CONCRETE

HANE OART/SOIL/DIATOMACEOUS EARTH METALIRON/GALVANIZED/SHEET CEMENT
CONCRETE

DIRT/SOILOIATOMACEOUS EARTH

METAIMRONIGALACEOUS EARTH

$$
\text { GROUT }
$$

METALARON/GALVANIZED/SHEET

PLASTICIPOLYURATHANE

PAPER/CARDBOARD

STAINLESS STEEL

FERROUS METAL

LEAD

RUBBER
CONCRETE

CLOTHARAGSNYLION

10 MIL LINER

ABSORBENTKITY LTRNERMICULTTE WIRE

IEAD SHIELDING

NON-HAZARDOUS METALS ASBESTOS

$$
\text { LEAD }
$$$$
\text { CONCRET }
$$

VARIOUS SOLUTIONS SOLIOIFIED METALARON/GALVANIZED/SHEET

PLASTICAPOLYURATHAN

$$
\begin{aligned}
& \text { RUBBER } \\
& \text { OTHRAGSNYION }
\end{aligned}
$$

WOODAUMBERAPLYWOOD

PAPER/CARDBOARD

$$
\text { CONCRETE }
$$

DIRT/SOLIOIATOMACEOUS EARTH ASEESTOS$$
\text { WEEDSNEGETATION }
$$

ABSORBENTKITY LTRNERMICULIT METALARON/GALVANIZED/SHEET

WOOD TOMACEOUS EARTH$$
\begin{aligned}
& \text { CONCRETE } \\
& \text { UMBLEWEEDS }
\end{aligned}
$$

LASTICPOLIYURATHANE

PAPER/CARDBOARD

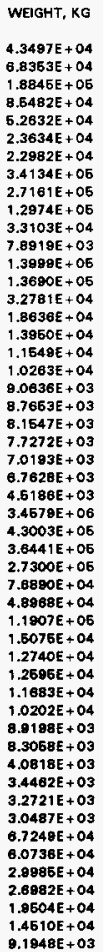

\begin{tabular}{|c|}
\hline \multirow{2}{*}{$\begin{array}{l}2.1484 E+06 \\
2.1484 E+06\end{array}$} \\
\hline \\
\hline $2.1484 E+06$ \\
\hline $2.1484 E+06$ \\
\hline $2.1484 E+06$ \\
\hline $2.1484 E+06$ \\
\hline $2.1484 E+06$ \\
\hline $2.1484 E+06$ \\
\hline $2.1484 E+06$ \\
\hline $2.1484 \mathrm{E}+06$ \\
\hline $4.6077 \mathrm{E}+06$ \\
\hline $4.6077 E+06$ \\
\hline $4.5077 E+05$ \\
\hline $4.5077 E+06$ \\
\hline $4.6077 \mathrm{E}+06$ \\
\hline $4.6077 E+05$ \\
\hline $4.5077 E+06$ \\
\hline $4.6077 E+05$ \\
\hline $4.5077 E+O 6$ \\
\hline $4.6077 \mathrm{E}+05$ \\
\hline $4.5077 E+06$ \\
\hline $4.5077 E+06$ \\
\hline $4.5077 E+06$ \\
\hline $4.6077 E+06$ \\
\hline $4.6077 E+05$ \\
\hline $4.6860 E \div 06$ \\
\hline $4.6860 E+06$ \\
\hline $4.6860 E+06$ \\
\hline $4.6860 E+06$ \\
\hline $4.6860 E+06$ \\
\hline 4.68BOE +06 \\
\hline $2.3621 E+06$ \\
\hline $2.3621 E+06$ \\
\hline $2.3621 E+06$ \\
\hline $2.3521 E+05$ \\
\hline $2.3621 E+06$ \\
\hline $2.3521 E+05$ \\
\hline $2.3621 E+06$ \\
\hline $2.3621 E+05$ \\
\hline $2.3621 E+05$ \\
\hline $2.3521 E+06$ \\
\hline $2.3521 E+06$ \\
\hline $2.3621 E+06$ \\
\hline $2.5326 E+06$ \\
\hline $2.6325 E+05$ \\
\hline $2.6325 E+06$ \\
\hline $2.6325 E+05$ \\
\hline $2.6325 E+06$ \\
\hline $2.6326 E+06$ \\
\hline $2.6326 E+06$ \\
\hline
\end{tabular}

TOTAL GENERATOR-BASEO WT, Ko

GROUP-BASED WT PERCENT

PHYS PROP - BASED WT PERCENT
2.02

3.18
6.77

3.98

1.10

1.07
15.88

12.64

6.04

1.76

31.06

7.27

4.13

2.66

2.28

1.94

1.81

1.68

1.00

73.79

7.78

5.83
1.64

1.04

60.62

5.42

6.36
4.97

4.34

3.79
3.63

1.39

1.30
26.55

23.88

11.84
10.65

7.70

6.73
3.63 
WHC-SD-W-242-ES-004, Rev. 0

TABLE 50

SIGNIFICANT PHYSICAL PROPERTIES CORRELATED BY GROUP AND BY GENERATOR

(4 SHEETS)

\begin{tabular}{|c|c|}
\hline GROUP & GENERATOA \\
\hline T-PLANT & $221 T$ \\
\hline T-PLANT & $221 \mathrm{~T}$ \\
\hline T-PLANT & $221 T$ \\
\hline T.PLANT & $221 \mathrm{~T}$ \\
\hline TANKFARM & rotsY \\
\hline TANKFARM & $241 \mathrm{~B}$ \\
\hline TANKFARM & $24 \mathrm{iB}$ \\
\hline TANKFARM & 249 \\
\hline TANKFARM & $241 T$ \\
\hline TANKFARM & $241 T$ \\
\hline TANKFARM & $241 T$ \\
\hline TANKFARM & $241 T$ \\
\hline TANKFARM & $241 T X$ \\
\hline TANKFARM & $241 T x$ \\
\hline TANKFARM & $241 T x$ \\
\hline TANKFARM & $2411 X$ \\
\hline TANKFARM & $241 T X$ \\
\hline TANKFARM & $241 T X$ \\
\hline TANKFARM & $242 \mathrm{~S}$ \\
\hline TANKFARM & MIXTF \\
\hline TANKFARM & MIXTF \\
\hline TANKFARM & MIXTF \\
\hline
\end{tabular}

PHYSICAL PROPERTY

ROCK/GRAVEL

RUBBER
ABSORBENT/KITYLTRNERMICULITE
CLOTHRAGSNYLON THARAGSNYLON

DIRT/SOLLDIATOMACEOUS EARTH WOODIATOMACEOUS EARTH

DIRT/SOLLDIATOMACEOUS EARTH WOODRUMBERPLYWOOD

METALARON/GALVANIZED/SHEET
ABSORBENTKITY LTRNERMICULITE

MBSORBENTKITY LTR/NERMICULIT
PLASTIC.POLYURATHANE PLASTICPPOLYURATHANE
WOODAUMBERPLYYOOD

DIRT/SOLLDIATOMACEOUS EARTH

METALARON/GALVANIZED/SHEET

STAIPOLYURATHANE

CONCFETE
CONLSS STE

METALIRON/GALVANIZED/SHEET

DIRTISOILDIATOMACEOUS EARTH

WOOD UUMBERUPLYWOOD

METALARON/GALVANIZED/SHEET
WEIGHT, KG

$8.0646 \mathrm{E}+03$

6.9624E+03

$3.2484 E+03$

$2.9376 E+03$

$5.9960 E+03$

$2.6322 E+03$

B. $6176 E+04$

$1.0678 E+04$

$5.5061 E+03$

$2.9890 E+03$

$2.5446 E+03$

$1.4336 \mathrm{E}+04$

9.4670E+03

$6.9428 E+03$

$2.4197 E+03$

$2.270 E+03$

$2.2110 E+03$

.

3.6249E+04

$2.8931 \mathrm{E}+03$
TOTAL GENERATOR-BASED WT, KG

$2.5325 \mathrm{E}+0 \mathrm{06}$

$2.5325 \mathrm{E}+0$

$2.5326 \mathrm{E}+\mathrm{O}$

$2.1133 E+05$

$2.1133 \mathrm{E}+$ O5

$2.1133 E+O 5$

$2.1133 E+O F$

$2.1133 E+05$

$2.1133 E+05$

$2.1133 E+06$

$2.1133 E+05$

$2.1133 E+05$

$2.1133 E+O B$

$2.1133 E+06$

$2.1133 E+05$

$2.1133 E+06$

$2.1133 E+06$

$2.1133 \mathrm{E}+\mathrm{OF}$

$2.1133 \mathrm{E}+0$

$2.1133 E+05$
GROUP-BASED WT PEACENT

1.84
1.84
1.84
1.84
1.53
1.53
1.53
1.63
1.63
1.63
1.63
1.63
1.63
1.63
1.63
1.63
1.63
1.63
1.63
1.53
1.53
1.53

PHYS PROP - BASED WT PERCENT

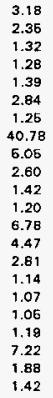


WHC-SD-W-242-ES-004, Rev. 0

TABLE 51

COUNT OF WASTE DESCRIPTIONS BY GROUP AND BY GENERATOR

(1 SHEET)

\begin{tabular}{|c|c|c|c|c|}
\hline GROUP & GENERATOR & GROUP BASED COUNT & GENERATOR BASED COUNT & PERCENT OF COUNT (GEN/GRP) \\
\hline TANKFARM & $242 A$ & 1736 & 643 & 37.04 \\
\hline TANKFARM & $241 \mathrm{~S}$ & 1736 & 319 & 18.38 \\
\hline TANKFARM & 241A & 1736 & 230 & 13.25 \\
\hline TANKFARM & $241 \mathrm{C}$ & 1736 & 201 & 11.58 \\
\hline TANKFARM & $241 \mathrm{~T}$ & 1736 & 198 & 11.41 \\
\hline TANKFARM & $241 \mathrm{~B}$ & 1736 & 47 & 2.71 \\
\hline TANKFARM & 241SY & 1736 & 43 & 2.48 \\
\hline TANKFARM & $241 \mathrm{TX}$ & 1736 & 39 & 2.25 \\
\hline TANKFARM & MIXTF & 1736 & 15 & 0.86 \\
\hline TANKFARM & $242 S$ & 1736 & 1 & 0.06 \\
\hline PFP & $2345 Z$ & 373 & 373 & 100.00 \\
\hline $222 S$ & 2225 & 103 & 94 & 91.26 \\
\hline 2225 & $1706 \mathrm{KE}$ & 103 & 8 & 7.77 \\
\hline $222 \mathrm{~S}$ & 2718 & 103 & 1 & 0.97 \\
\hline T-PLANT & $221 \mathrm{~T}$ & 74 & 74 & 100.00 \\
\hline PUREX & $202 A$ & 57 & 57 & 100.00 \\
\hline KBASINS & $105 \mathrm{KE}$ & 28 & 28 & 100.00 \\
\hline B-PLANT & $271 \mathrm{~B}$ & 23 & 23 & 100.00 \\
\hline PNL & 324 & 15 & 15 & 100.00 \\
\hline PSN & PSNS & 14 & 14 & 100.00 \\
\hline $\mathrm{CEL}$ & $241 B$ & 10 & 3 & 30.00 \\
\hline $\mathrm{CEL}$ & $241 T X$ & 10 & 3 & 30.00 \\
\hline CEL & $241 \mathrm{~S}$ & 10 & 2 & 20.00 \\
\hline $\mathrm{CEL}$ & $2415 Y$ & 10 & 1 & 10.00 \\
\hline CEL & $271 \mathrm{CR}$ & 10 & 1 & 10.00 \\
\hline RWM & $100 \mathrm{~N}$ & 9 & 9 & 100.00 \\
\hline $100 \mathrm{~A}$ & $105 \mathrm{KE}$ & 8 & 5 & 62.50 \\
\hline $100 \mathrm{~A}$ & $100 \mathrm{~N}$ & 8 & 3 & 37.50 \\
\hline ERO & 2765 & 7 & 7 & 100.00 \\
\hline OTHER & 324 & 7 & 4 & 57.14 \\
\hline OTHER & $221 \mathrm{~T}$ & 7 & 1 & 14.29 \\
\hline OTHER & $2345 Z$ & 7 & 1 & 14.29 \\
\hline OTHER & $2.71 \mathrm{~T}$ & 7 & 1 & 14.29 \\
\hline BETTIS & BAPL & 6 & 6 & 100.00 \\
\hline LBL & LBLAB & 6 & 6 & 100.00 \\
\hline BNL & BNLAB & 5 & 5 & 100.00 \\
\hline ANL & ARGON & 3 & 3 & 100.00 \\
\hline BAT & BATCO & 3 & 3 & 100.00 \\
\hline RMW & $105 \mathrm{KE}$ & 3 & 3 & 100.00 \\
\hline FUSRAP & ALBNY & 2 & 2 & 100.00 \\
\hline KEH & $231 z$ & 2 & 2 & 100.00 \\
\hline EFSG & $276 \mathrm{~S}$ & $\overline{1}$ & 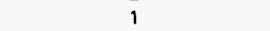 & 100.00 \\
\hline PUREXLAB & $202 \mathrm{~A}$ & 1 & $i$ & 100.00 \\
\hline
\end{tabular}


WHC-SD-W-242-ES-004, Rev. 0

TABLE 52

PRINCIPAL WORDS USED FOR WASTE DESCRIPTIONS

(1 SHEET)

\begin{tabular}{|c|c|c|c|}
\hline WASTE WORDS & COUNT & WASTE WORDS & COUNT \\
\hline OVERPACK & 338 & WCO2 & 7 \\
\hline WASTE & 106 & CAT & 6 \\
\hline BOX & 50 & CENTRAL & 6 \\
\hline SDAR & 50 & D001 & 6 \\
\hline ROOM & 44 & D008 & 6 \\
\hline TRU & 27 & KAISER & 6 \\
\hline GENERATED & 26 & LEAD & 6 \\
\hline DRUM & 24 & LIOUID & 6 \\
\hline ASSAYED & 23 & MISC & 6 \\
\hline SUSPECT & 23 & R-3 & 6 \\
\hline LEVEL & 22 & WTO1 & 6 \\
\hline LOW & 21 & ACTIVITIES & 5 \\
\hline CONTAMINATED & 20 & ATG & 5 \\
\hline DETERMINED & 20 & BONEYARD & 5 \\
\hline ROUTINE & 18 & CHEMICALS & 5 \\
\hline LLW & 15 & CLOTH & 5 \\
\hline HEPA & 14 & CORRECT & 5 \\
\hline FILTERS & 13 & DESCRIPTION: & 5 \\
\hline $2736 \mathrm{ZB}$ & 12 & F001-F005 & 5 \\
\hline OLD & 12 & LAB & 5 \\
\hline SHIPPED & 12 & MATRIX & 5 \\
\hline NEW & 11 & METAL & 5 \\
\hline CANYON & 10 & MISC. & 5 \\
\hline CHANGED & 10 & OIL & 5 \\
\hline MAINTENANCE & 10 & OPERATIONS & 5 \\
\hline CONSTRUCTION & 9 & PAINT & 5 \\
\hline MULTIPLE & 9 & RELATED & 5 \\
\hline SHIP & 9 & REMOVAL & 5 \\
\hline SIZE & 9 & REPACKAGED & 5 \\
\hline SOLID & 9 & SDAR\# & 5 \\
\hline$T$ & 9 & STEEL & 5 \\
\hline USE & 9 & SWT & 5 \\
\hline WEIGHT & 9 & WP01 & 5 \\
\hline COMPACTED & 8 & & \\
\hline CONTAINER & 8 & & \\
\hline F-LISTED & 8 & & \\
\hline NAREAWASTE & 8 & & \\
\hline OVERPACKED & 8 & & \\
\hline PLASTIC & 8 & & \\
\hline WTO2 & 8 & & \\
\hline BURIAL & 7 & & \\
\hline CONCRETE. & 7 & & \\
\hline MBA & 7 & & \\
\hline PLANT & 7 & & \\
\hline RSR & 7 & & \\
\hline SOIL & 7 & & \\
\hline
\end{tabular}




\section{DISTRIBUTION SHEET}

\begin{tabular}{|c|c|c|c|c|c|}
\hline \multirow{2}{*}{$\begin{array}{l}\text { To } \\
\text { Distribution }\end{array}$} & \multirow{2}{*}{\multicolumn{3}{|c|}{$\begin{array}{l}\text { From } \\
\text { BG Place }\end{array}$}} & \multicolumn{2}{|l|}{ Page 1 of 1} \\
\hline & & & & \multicolumn{2}{|c|}{ Date $9-30-96$} \\
\hline \multicolumn{4}{|l|}{ Project Title/Work Order } & \multicolumn{2}{|c|}{ EDT No. $618488<19341$} \\
\hline \multicolumn{4}{|c|}{$\begin{array}{l}\text { Characterization of the Solid Low Level Mixed Waste Inventory } \\
\text { for the Solid Waste Thermal Treatment Activity - III }\end{array}$} & \multicolumn{2}{|c|}{ 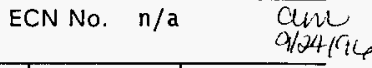 } \\
\hline Name & MSIN & $\begin{array}{c}\text { Text } \\
\text { With All } \\
\text { Attach. }\end{array}$ & Text Only & $\begin{array}{l}\text { Attach./ } \\
\text { Appendix } \\
\text { Only }\end{array}$ & $\begin{array}{l}\text { EDT/ECN } \\
\text { Only }\end{array}$ \\
\hline TL Baker & $T 3-01$ & $x$ & & & \\
\hline RJ Bottenus & $T 4-52$ & & $x$ & & \\
\hline BJ Broomfield & T3-04 & $\mathrm{x}$ & & & \\
\hline FM Coony & T3-01 & $x$ & & & \\
\hline RF Guercia & $S 7-55$ & & $x$ & & \\
\hline PL Hapke & $T 4-05$ & & $x$ & & \\
\hline KL Hladek & T3-01 & $x$ & & & \\
\hline RM Irwin & T4-03 & $x$ & & & \\
\hline WS Josephson & T3-04 & $x$ & & & \\
\hline MR Kerns & T3-0I & $x$ & & & \\
\hline DE McKenney & T3-01 & $x$ & & & \\
\hline DE Nester & T3-01 & $x$ & & & \\
\hline RD Pierce & $T 4-03$ & & $x$ & & \\
\hline BG Place & $\mathrm{T} 3-01$ & $x$ & & & \\
\hline DB Powe11 & T4-03 & & $x$ & & \\
\hline JG Riddelle & T3-01 & & $x$ & & \\
\hline JJ Valero & T3-01 & $x$ & & & \\
\hline JJ Waring & $\$ 7-55$ & $x$ & & & \\
\hline MI Wood & T3-01 & $x$ & & & \\
\hline
\end{tabular}

